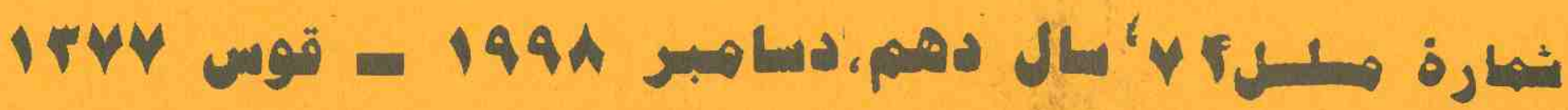
SERIAL NO.74, VOLUME 10, DECEMBER 1998 - QAUS 1377

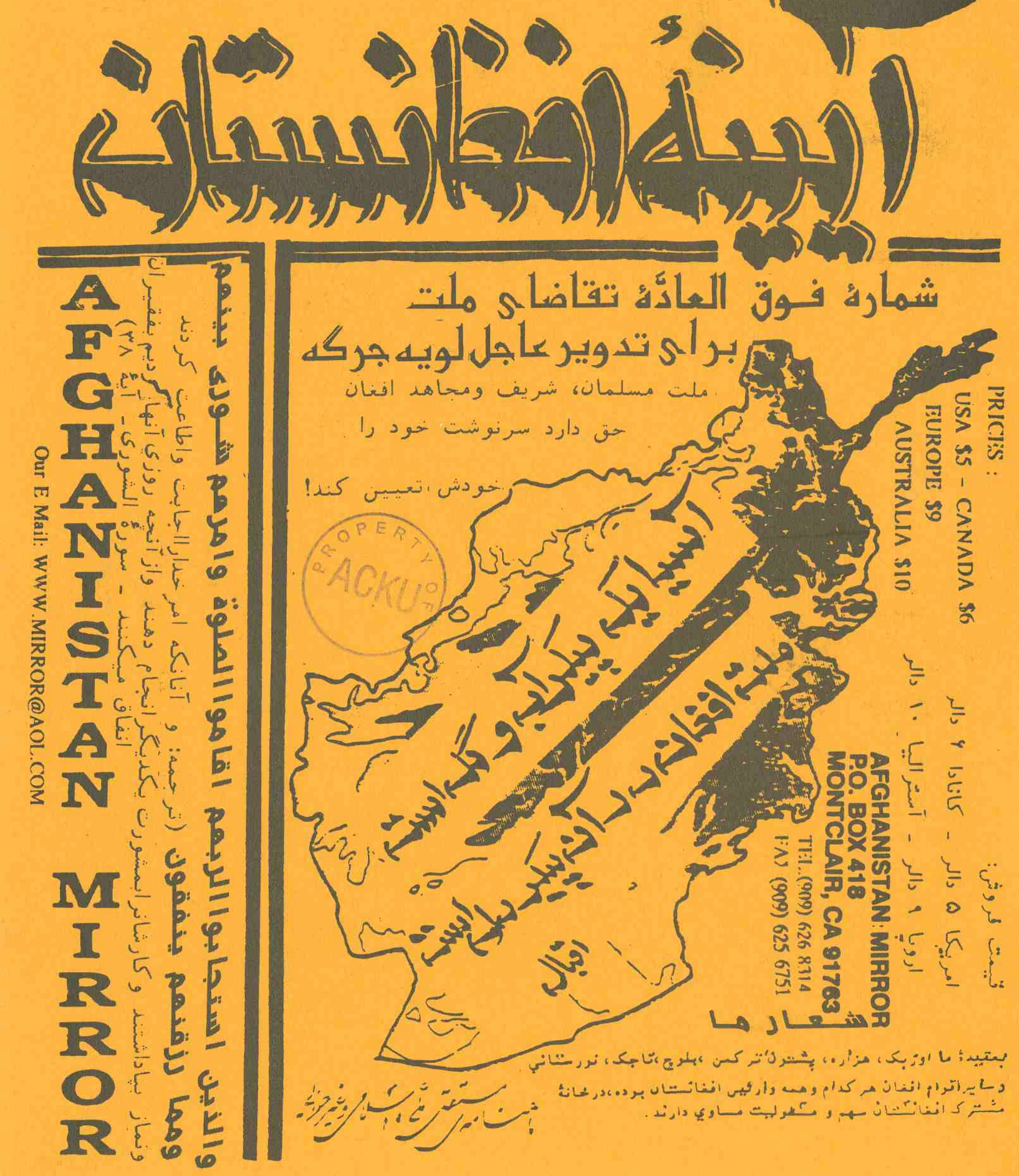




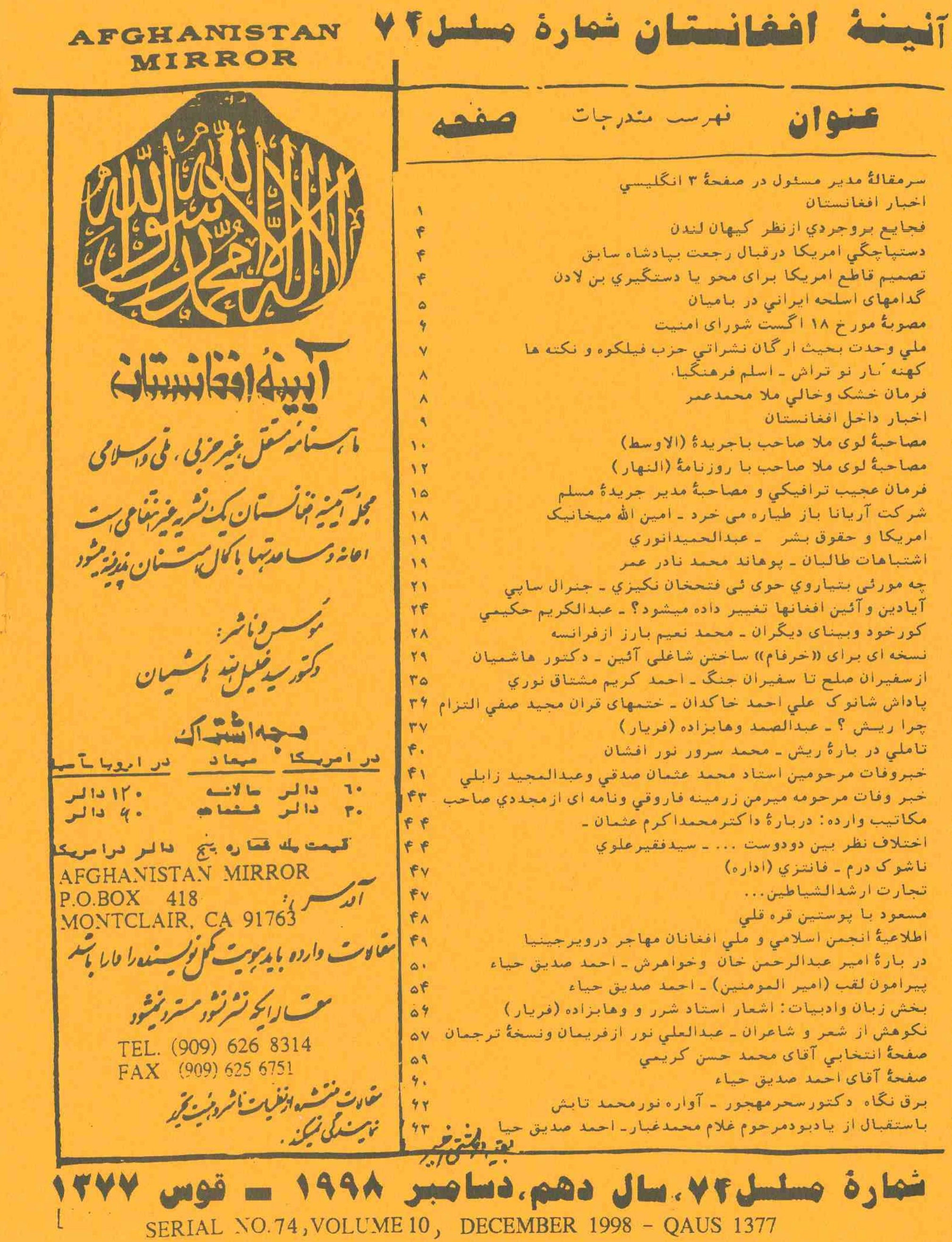




\section{اخبار هربوط برويدادها درافيانستان}

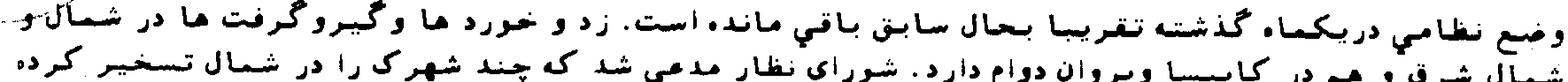

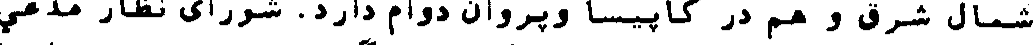

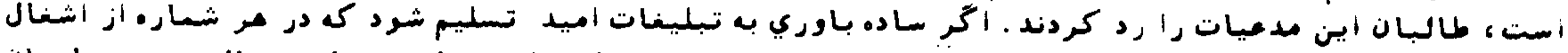

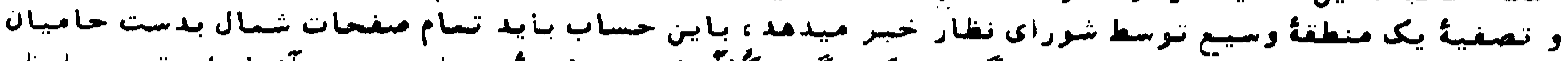

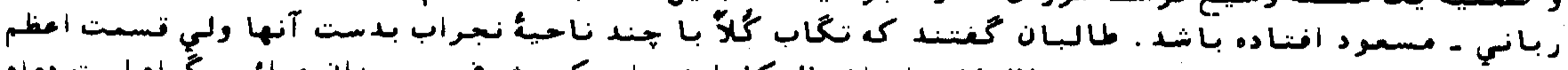

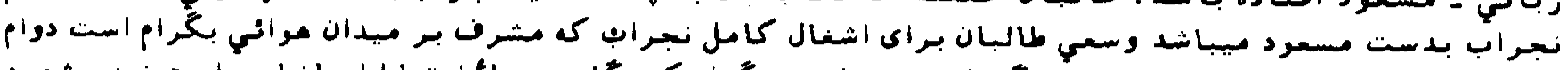

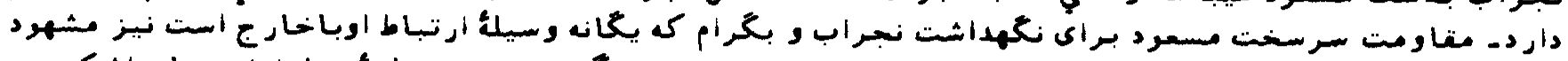

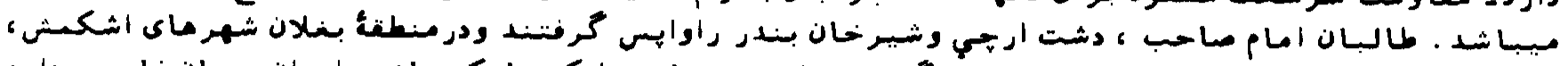

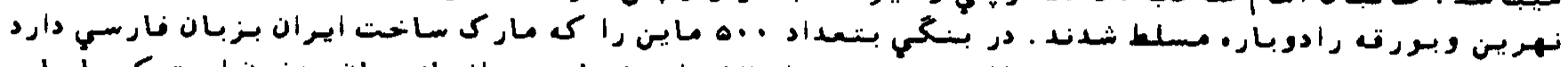

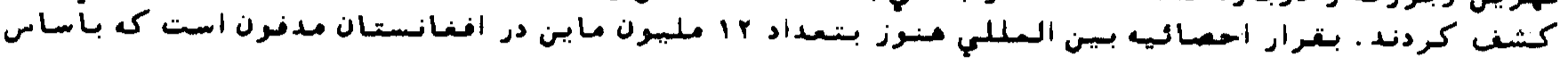

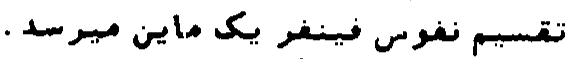

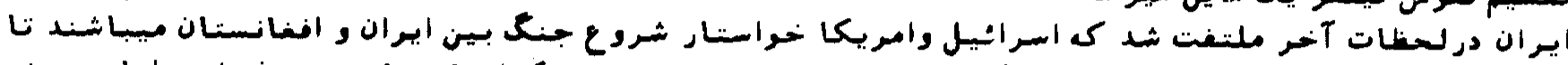

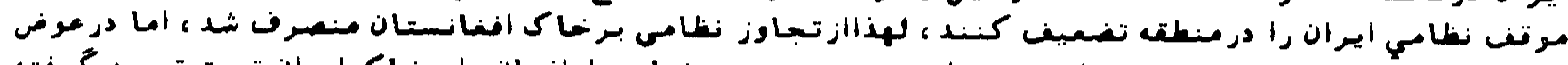

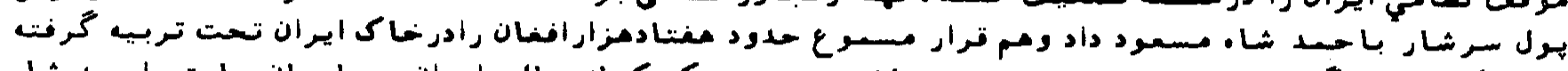

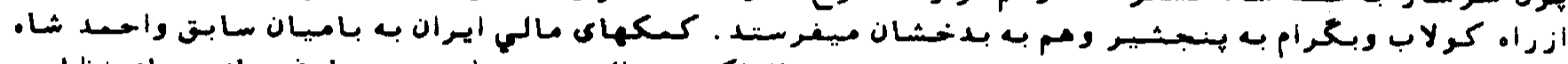

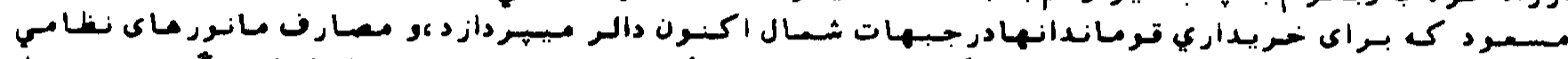

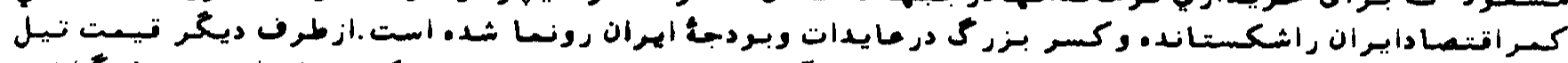

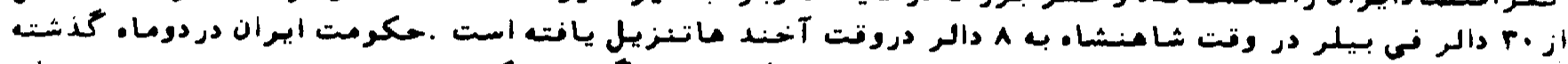

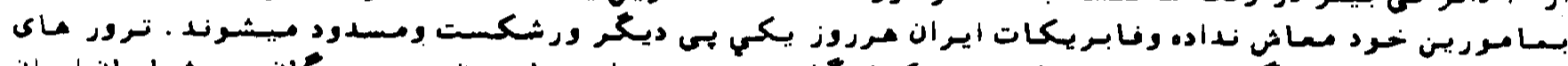

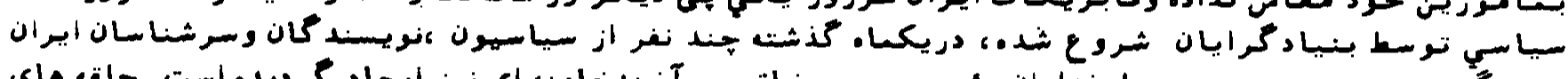

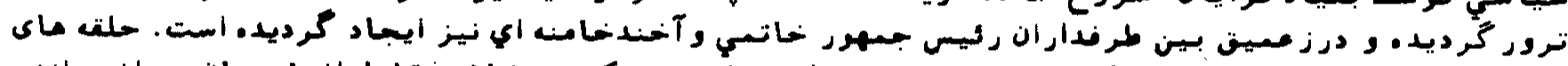

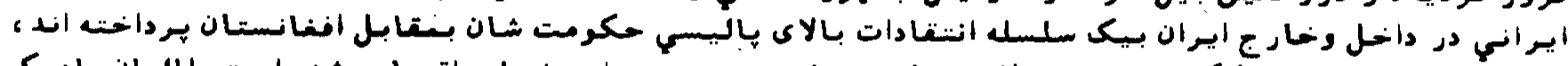

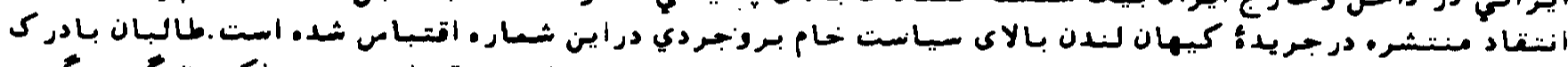

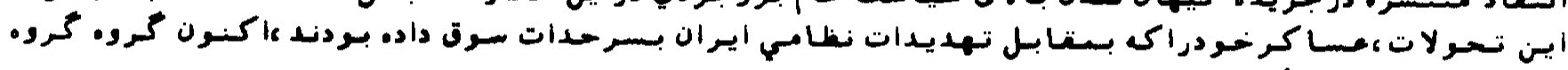

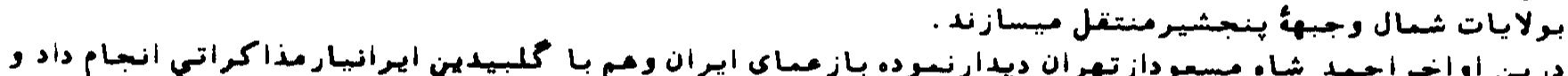

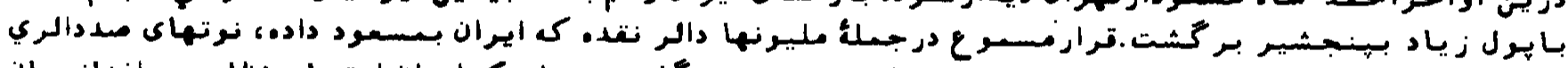

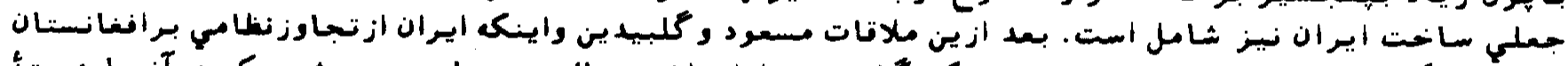

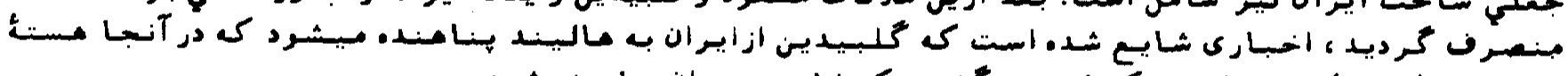

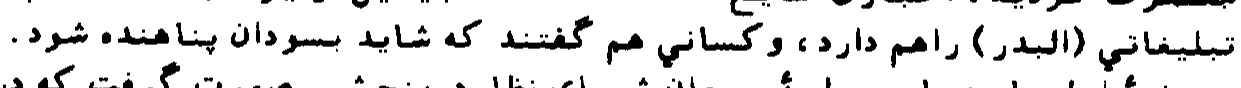

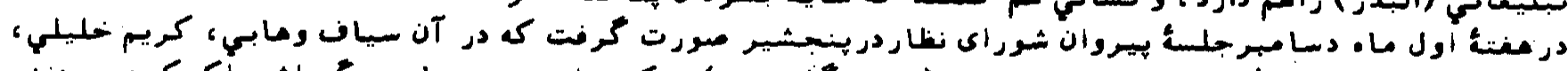

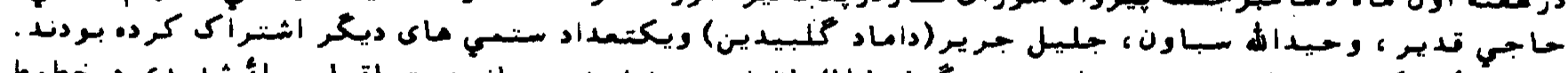

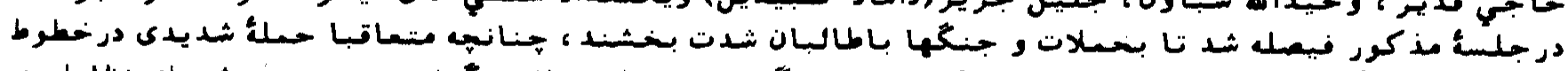

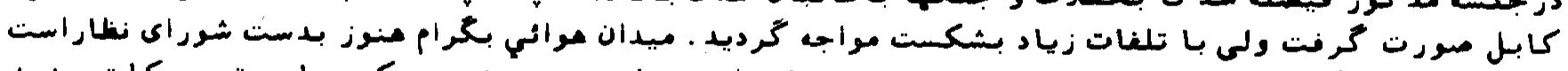

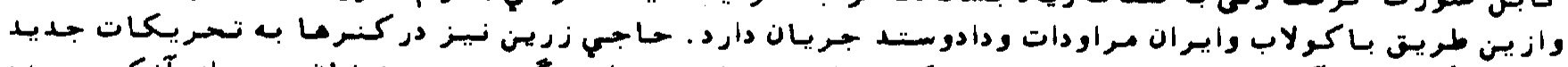

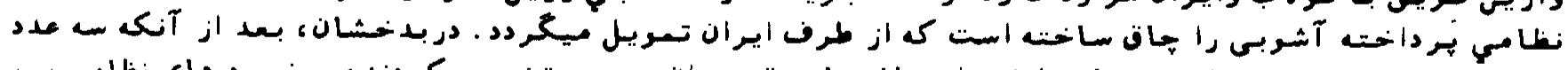

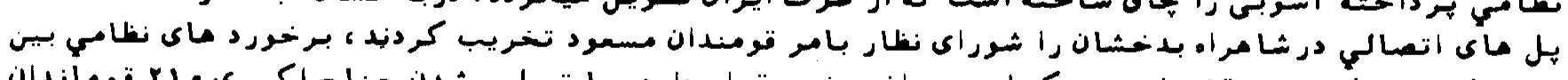

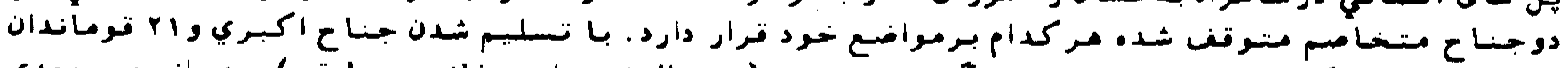

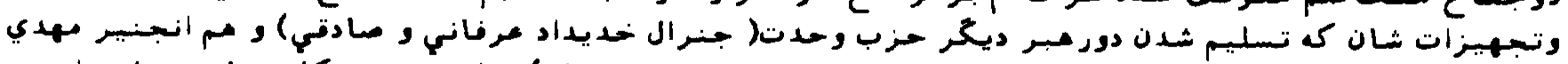

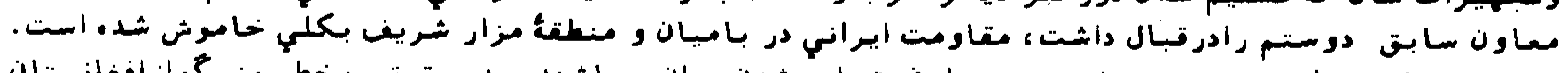

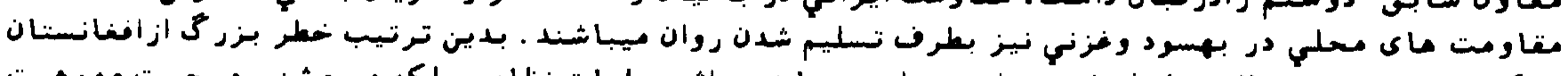

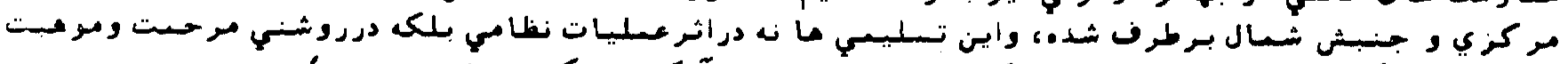

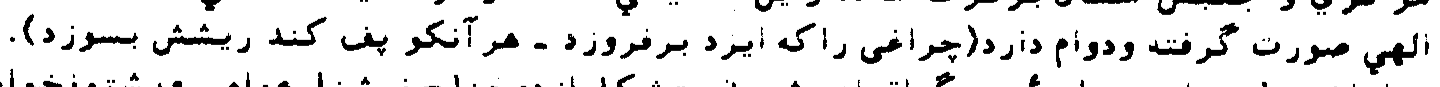

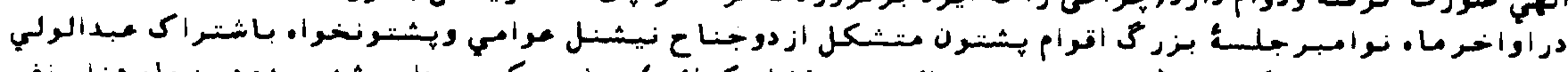

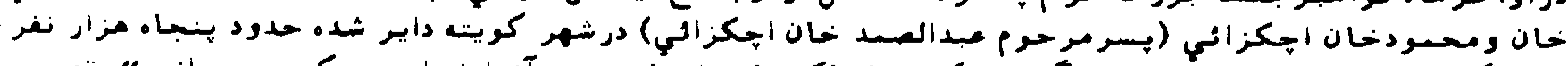

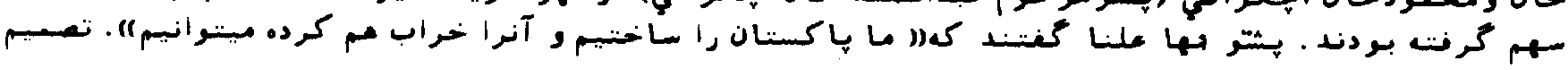

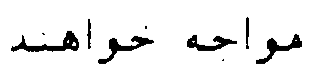

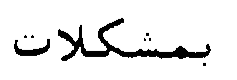
$6 ـ ـ$ انتتقـال را بمر دم قدرت سـيـاسي اكر طالبان 


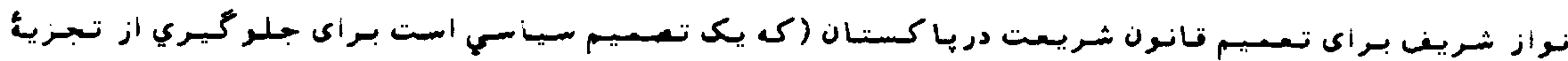

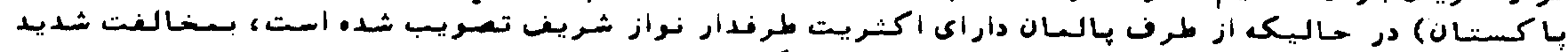

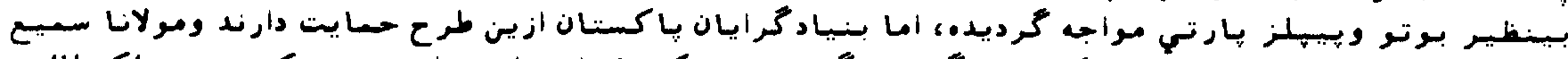

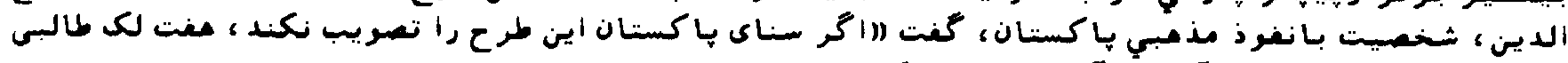

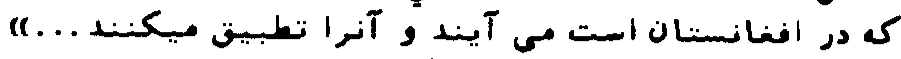

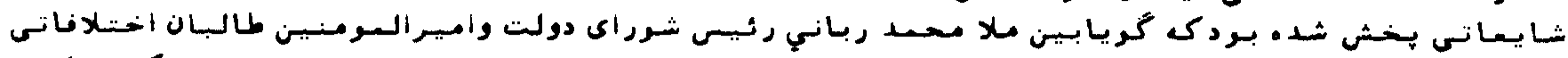

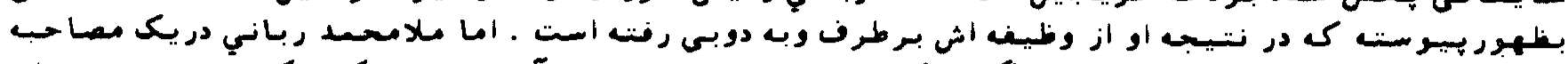

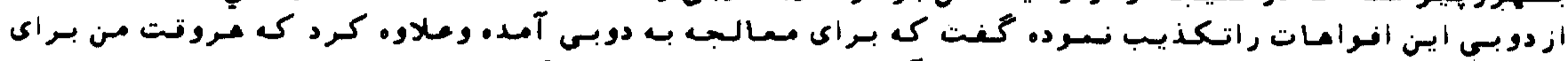

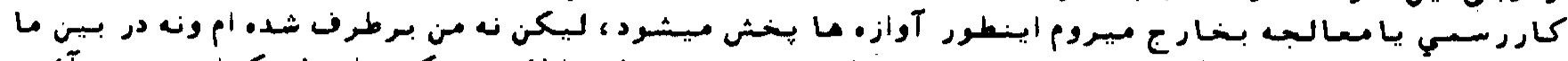

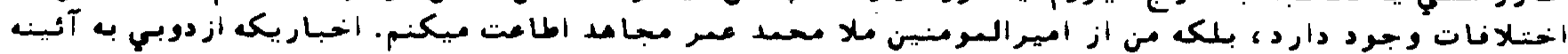

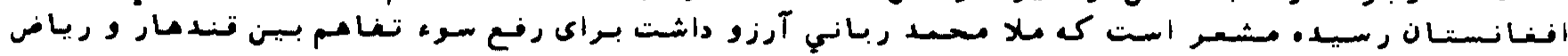

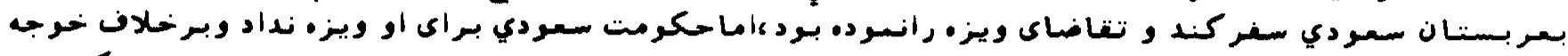

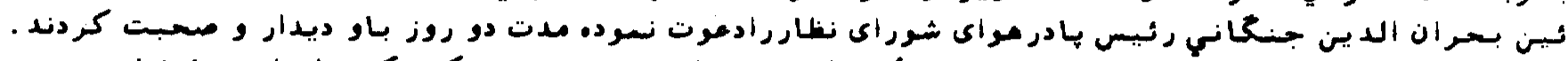

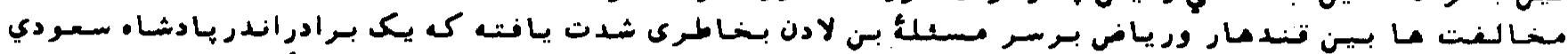

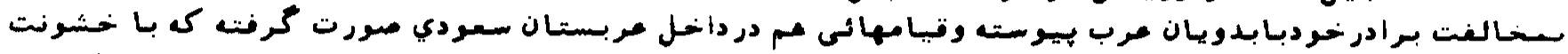

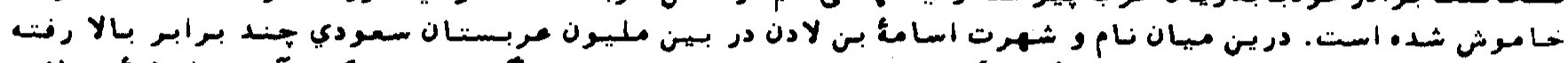

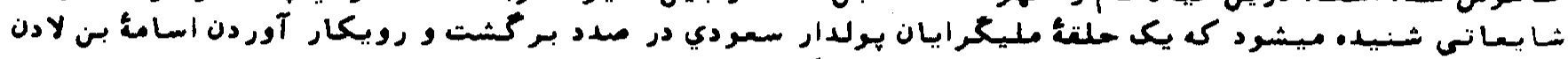

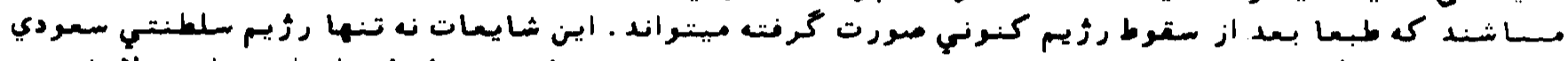

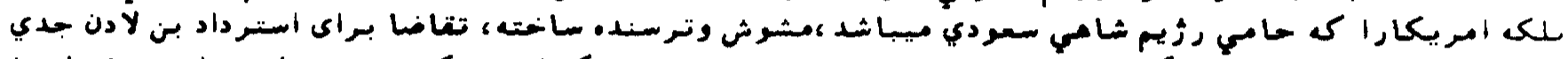

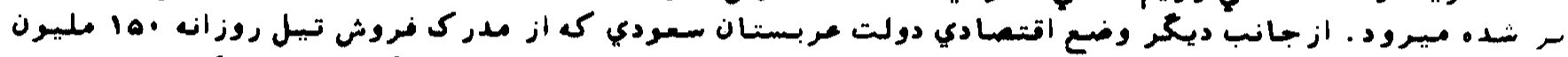

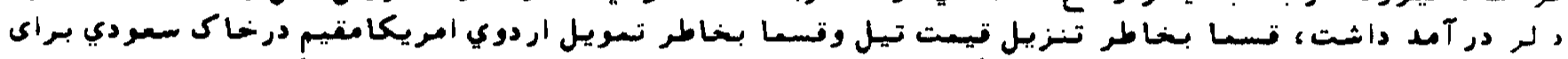

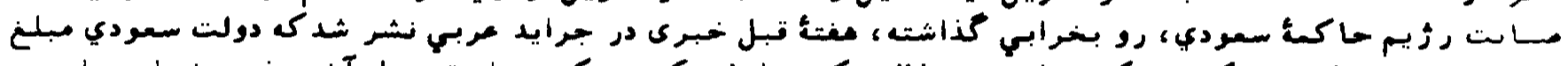

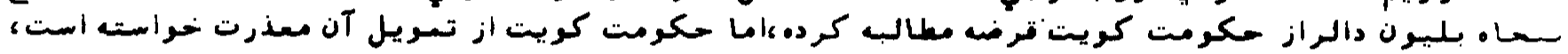

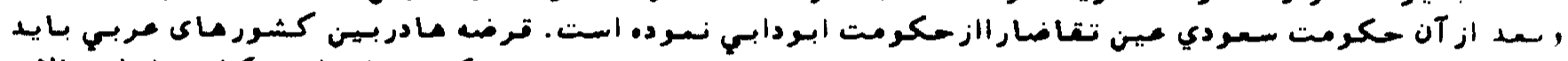

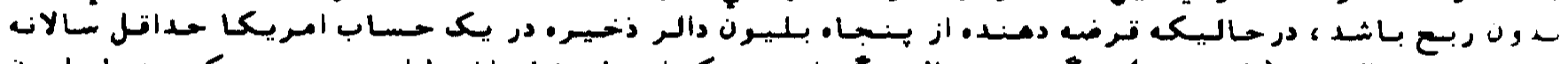

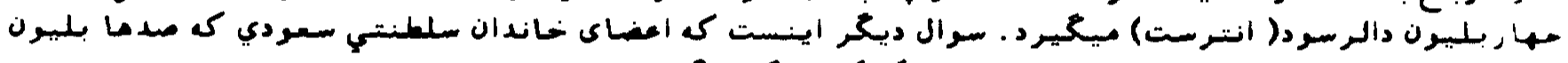

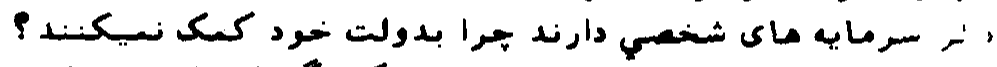

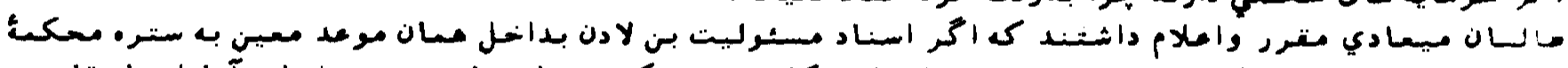

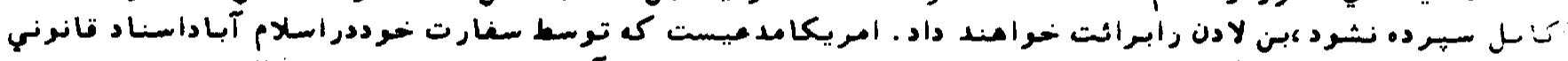

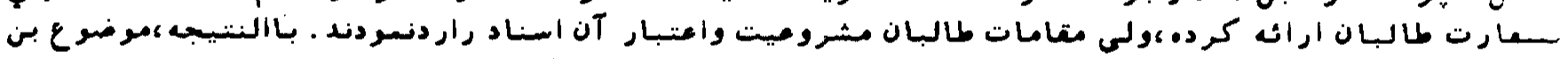

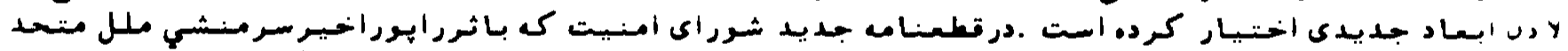

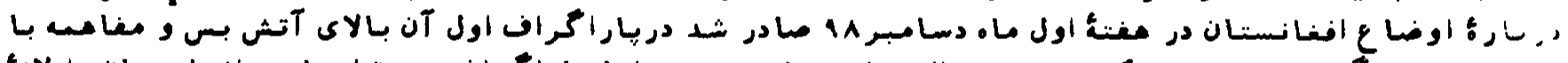

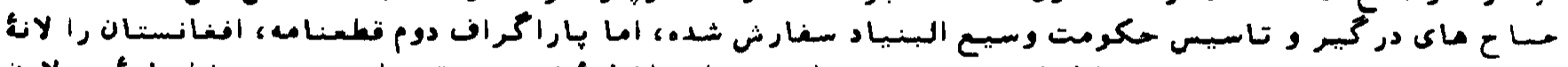

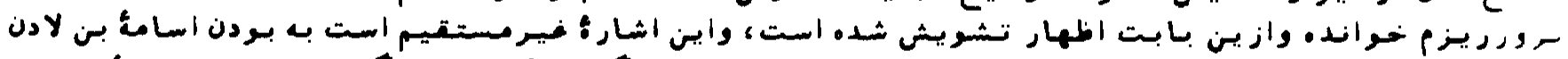

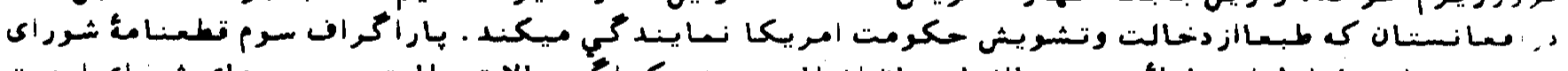

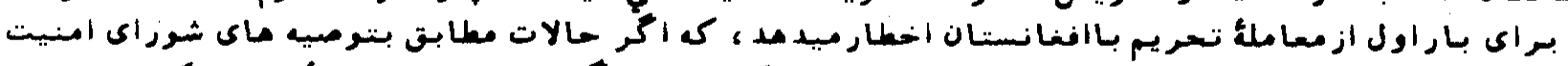

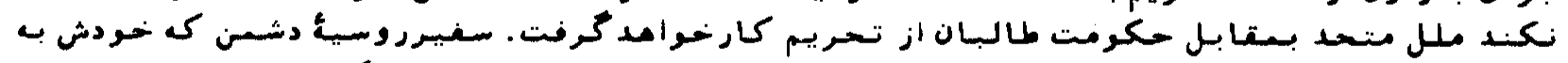

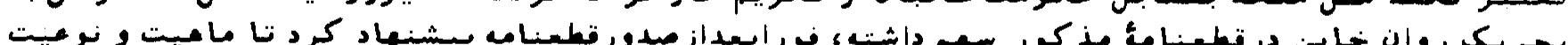

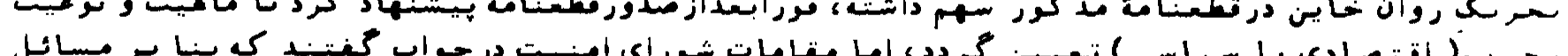

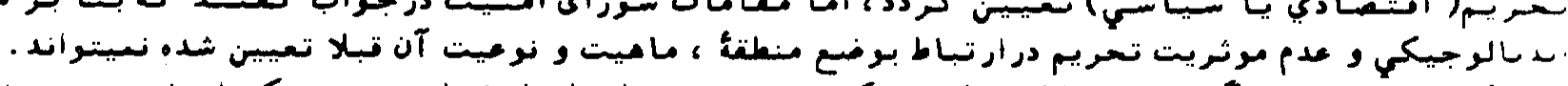

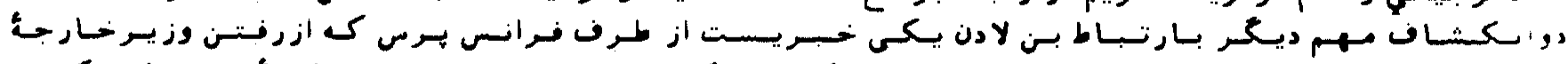

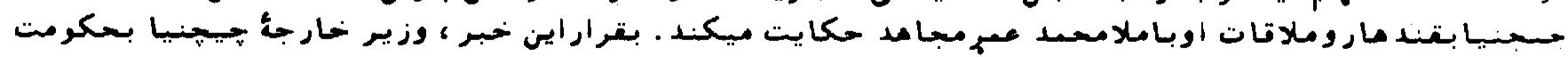

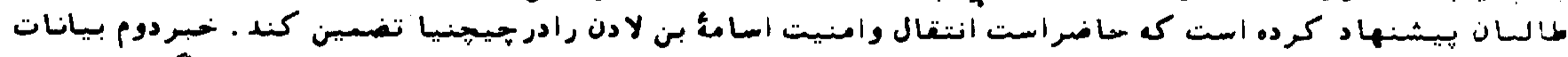

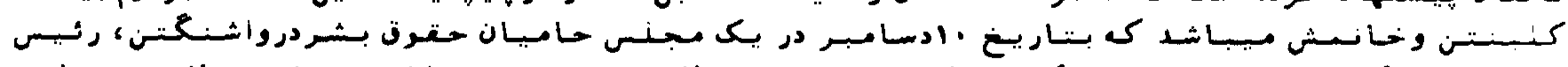

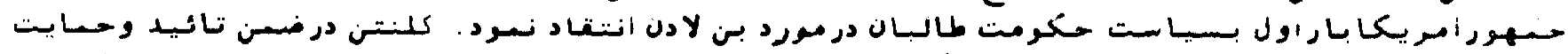

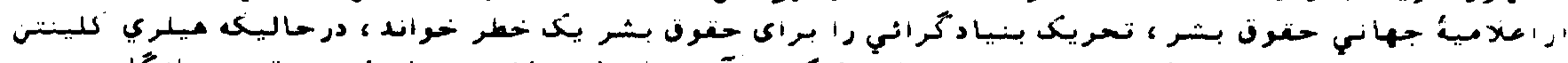

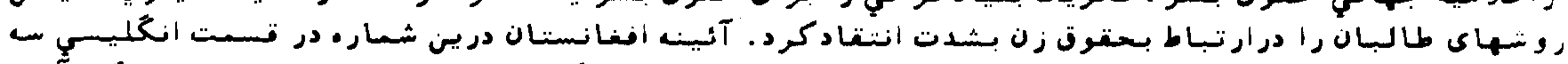

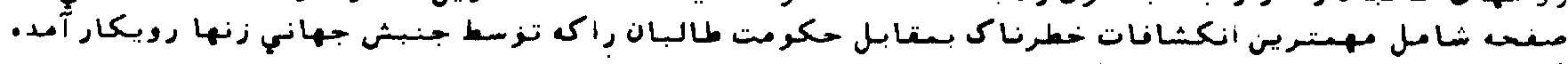

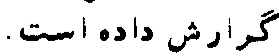

داير ساختس لويس جر كه از تمام اتستار ملت افغان، هعنى انتقال قدرت سمردم رادارد 


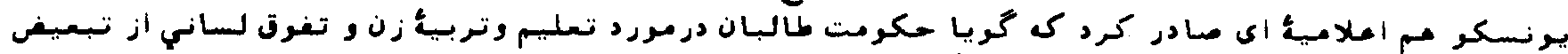

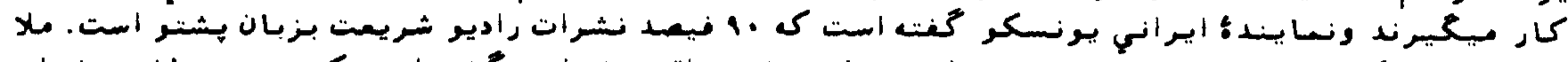

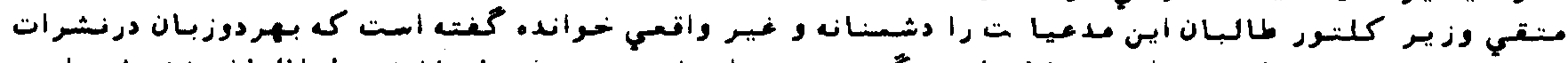

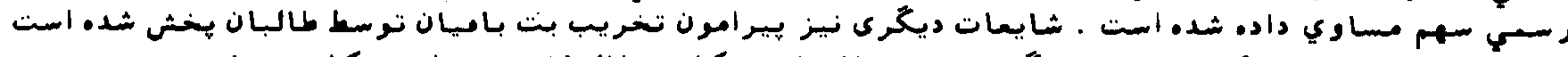

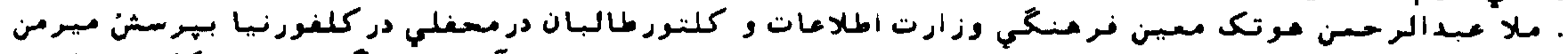

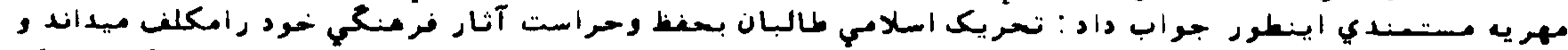

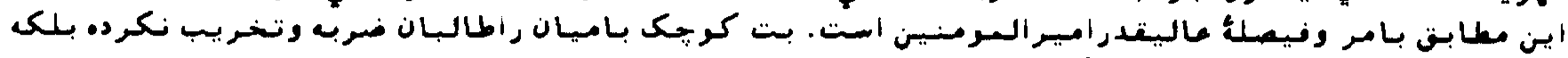

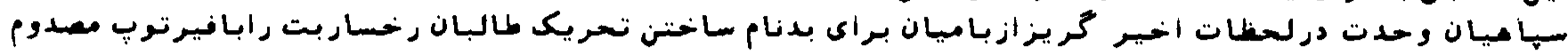

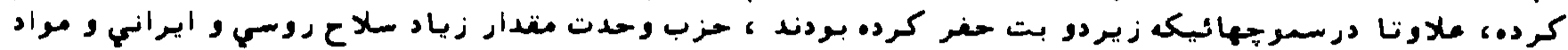

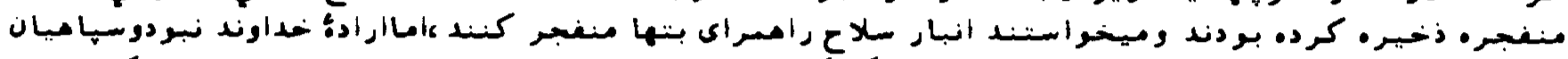

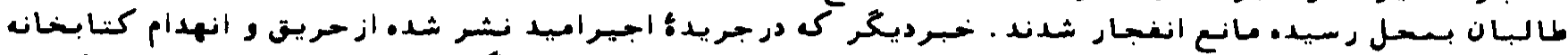

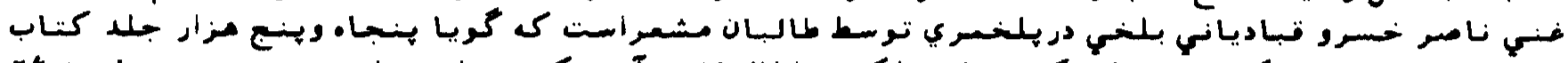

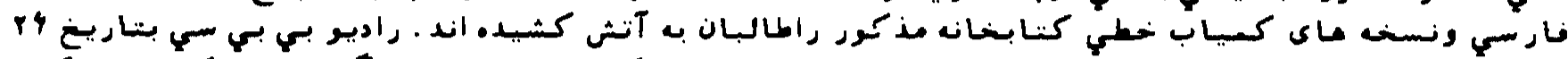

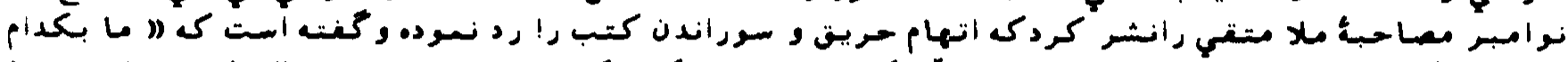

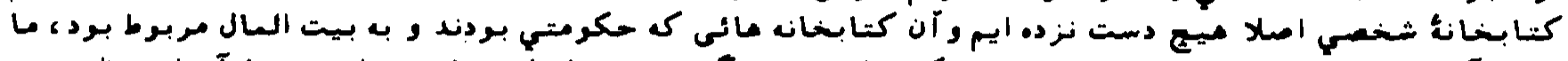

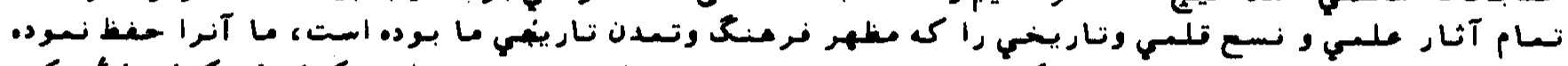

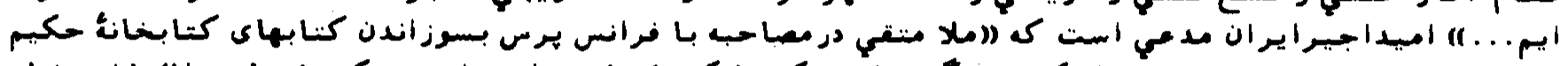

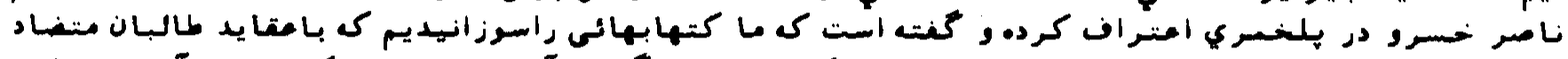

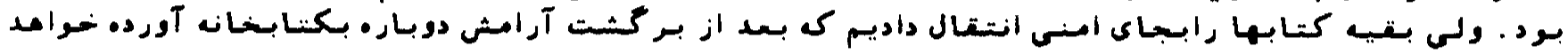

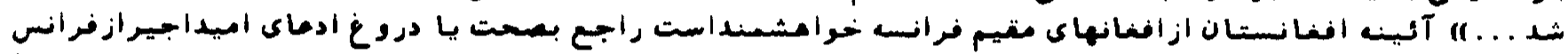

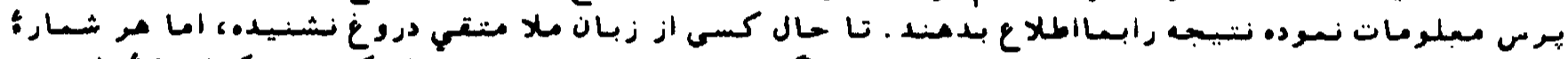

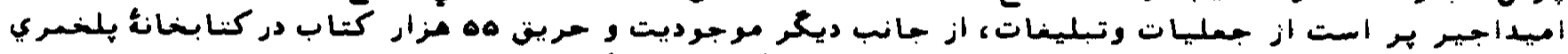

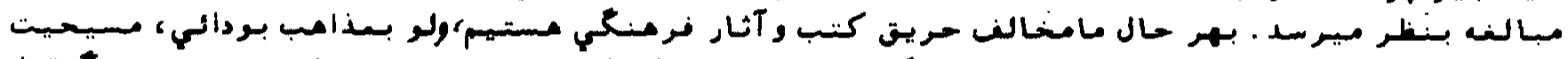

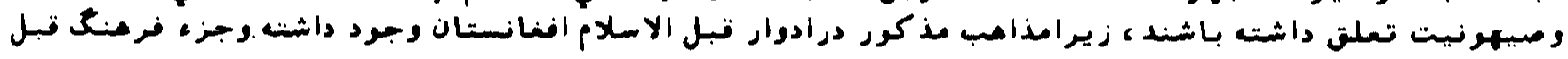

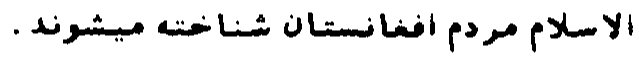

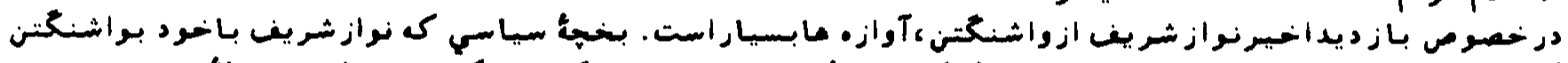

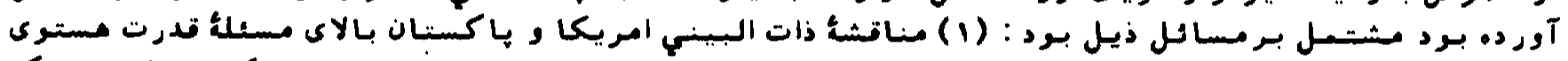

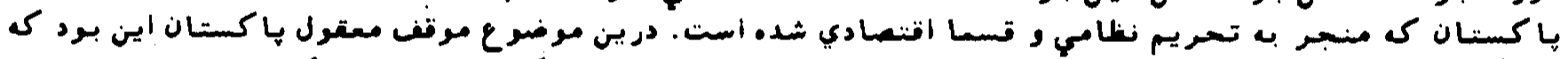

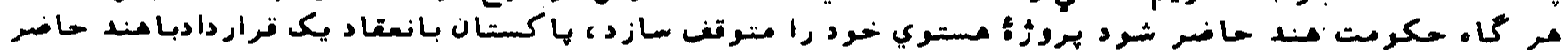

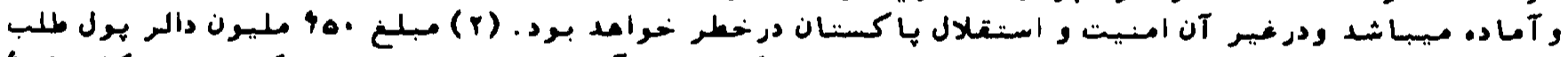

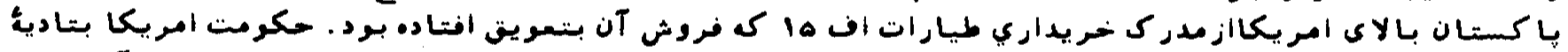

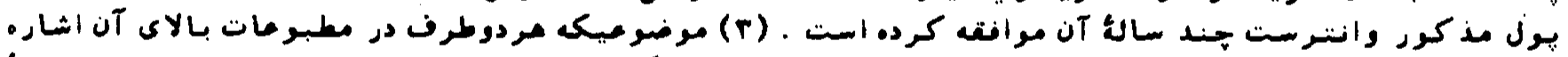

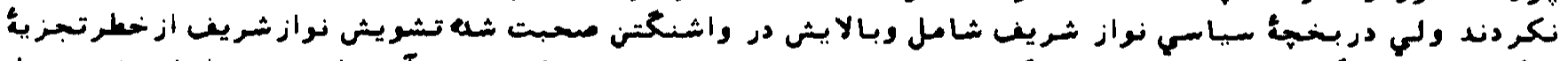

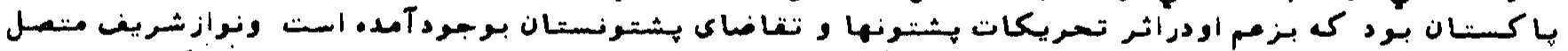

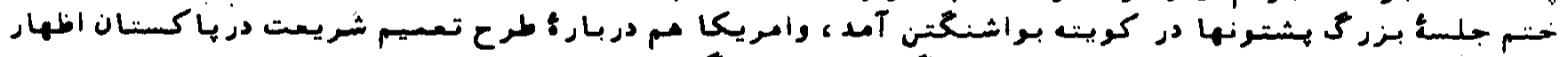

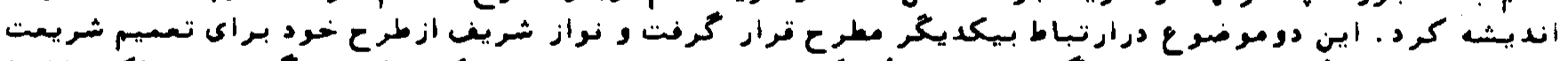

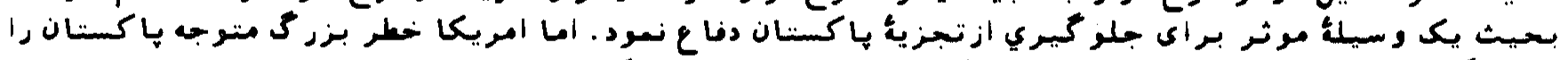

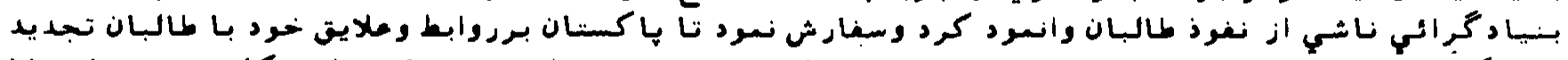

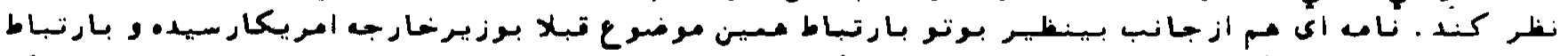

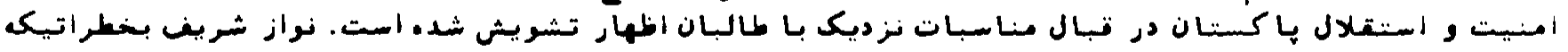

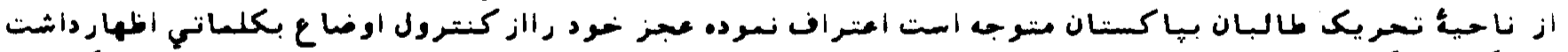

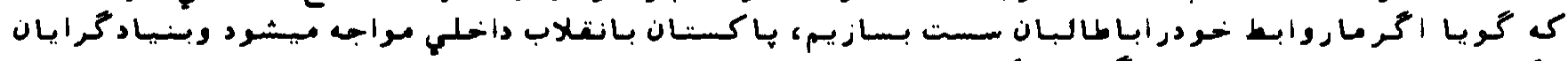

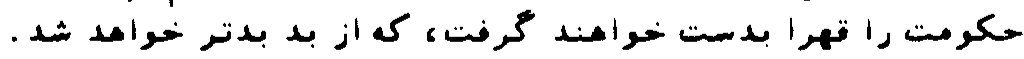

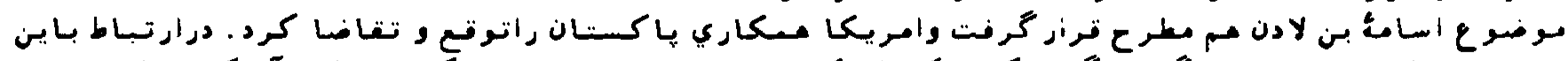

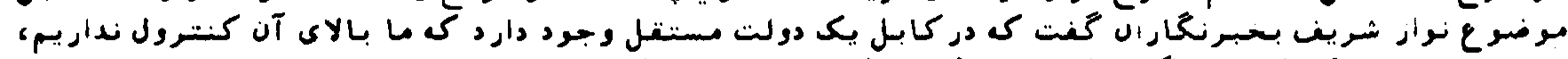

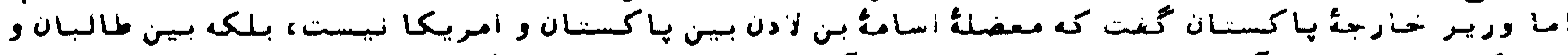

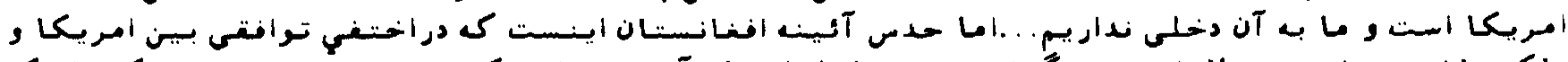

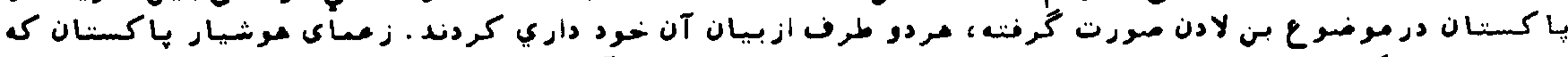

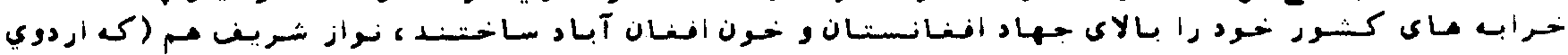
40 بs 


\section{$S / R E S / 1193$}

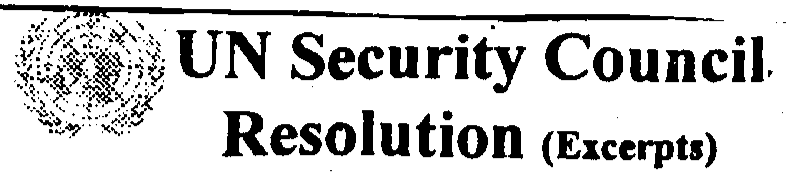

The Secentir Coanct?

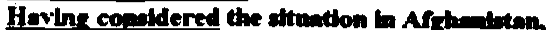

Expresine the ernve concen at the conthued Afghan conflict which has recently sharphy eacolated due to the Trillban force's offenetve in the northera parte of the country, caublag a serfons and growing threat to regtonal and international pence and securtty. as well at extendive husion onfertas. further destroction, rengee fom and other forclble displacement of large numbers of people.

Concerned alop by the increachaty ethaik nature of the connich by reports of ethnic and retidioes-based peraceution, particularty arwinat the Shitien, and by the threat thit powes to the unity of the Afghen State,

Deplortang the fact that deaptie repeated pleas...to halt foreign inferference in Afghaniatan, including the involvement of foreign milltary persomel and the supply of arms and ammunition continues unabated,

Deephy concerned also at the conthuing presence of terroriats in tertion of Afg. and the production and trameldag of drugs.

Remalnting deeply concerned at the conthuing dacrimination against girls and women and other violettons of menan rights,

Deply concerned at the oxtous humanitartan crial in

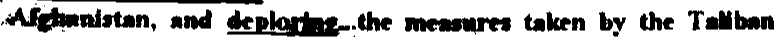

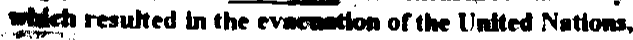

* Relterntes that the Afghan crisis can be settled onty by peaceful mesns...ntmed at achieving a solution accommodating the rights and interests of all Afghans and stresses that the territorial gains through military operations $w i l l$ netther leed to a durable peace in Afghanistan, nor contribute to a comprehenslve settlement of the connict in this multi-culturnl and multi-ethalc country;

"Refterntes once again that any outside interterence in the intermal afrairs of Afg. should cease immediately and calls upon all States to take resolute measures to prohlbit thetr milttary personnel from plenning and participating in military operations in Afghanisten:

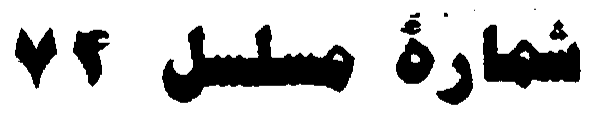

- Condemns the attacka on the Infted Nations perwonnel in the Tuliban-held terrttories:

*Requests the Secretary-General to continue invertigations into alleged mass ldilings of prisoners of war and civilians a well as ethnically-based forced displacement of large zroups of the population and other forms of mass periecution in Afghandoten.

$$
28 \text { August } 1998 \text { - ST1998310 }
$$

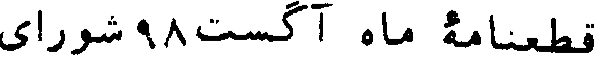

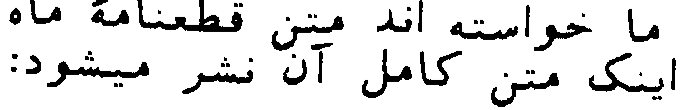

6

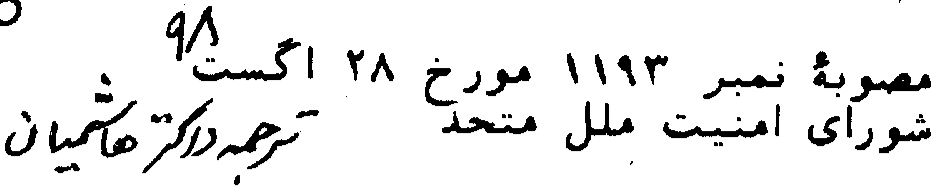

شوراى امنيت بـمد ازغور بـراوضا ع افنانستان

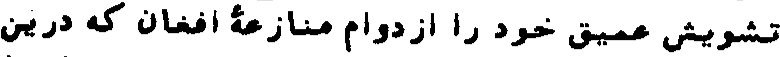

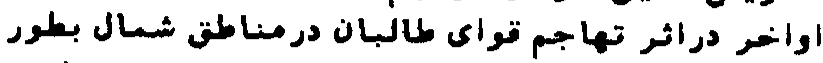

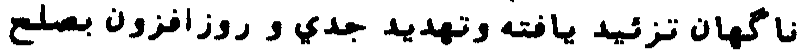

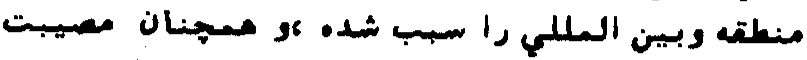

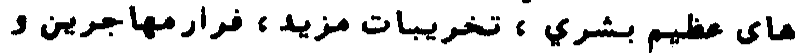

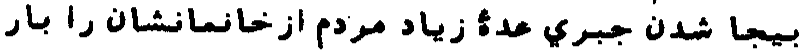

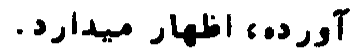

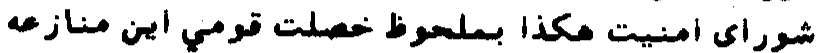

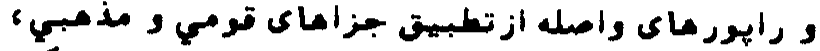

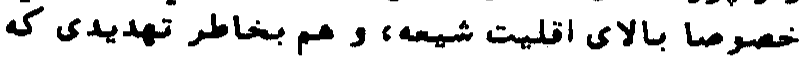

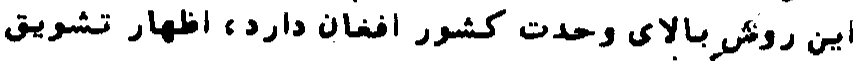

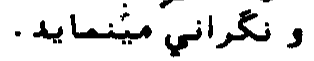

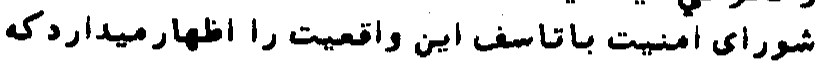

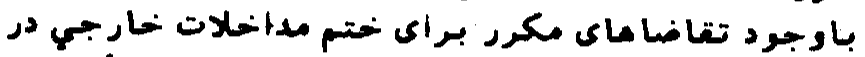

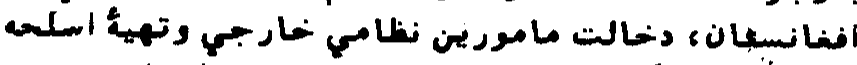

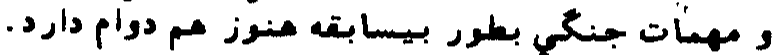

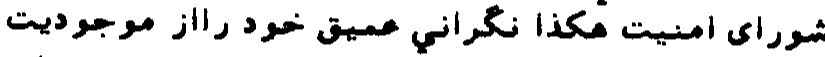

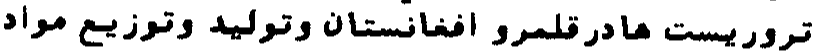

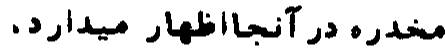

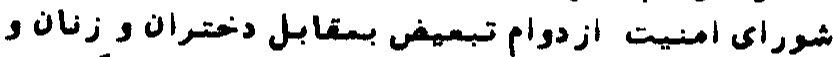

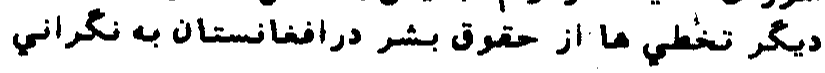

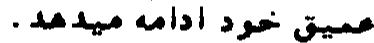

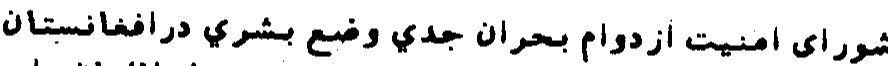

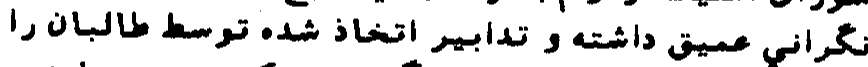

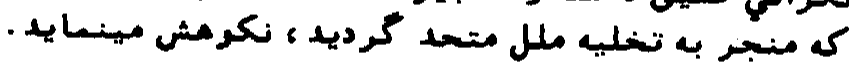

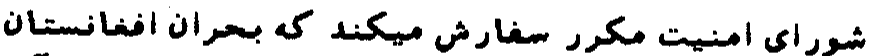

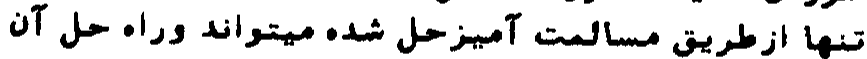

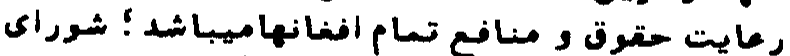

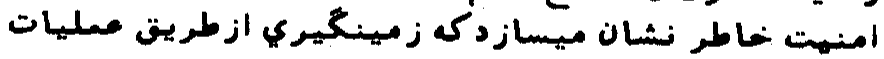

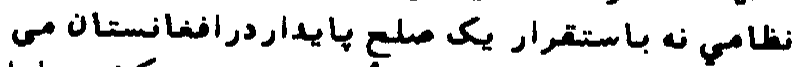

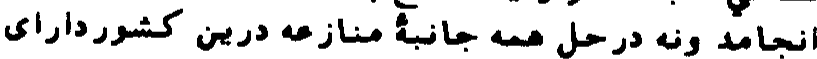

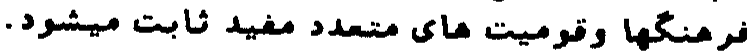

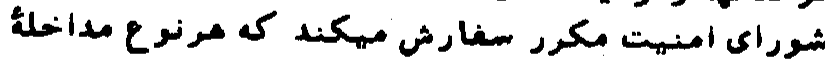

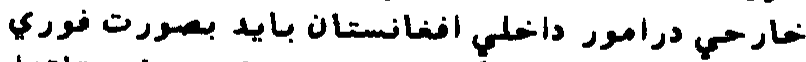

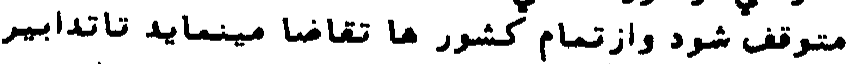

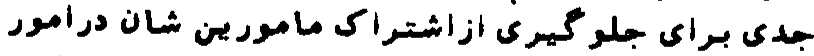

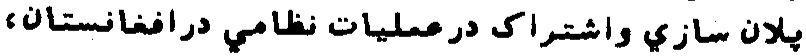

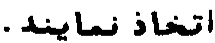

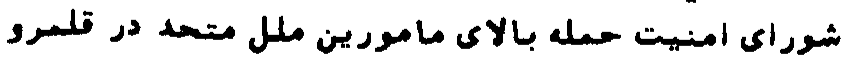

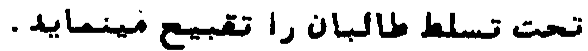

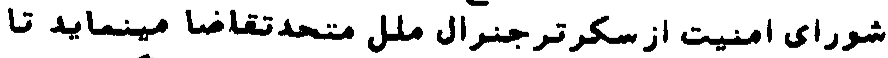

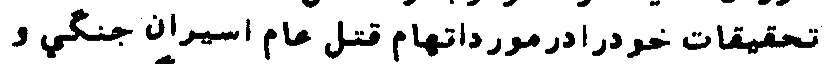

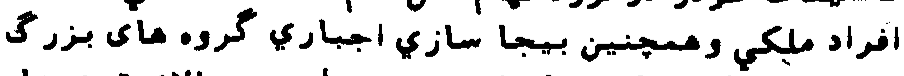

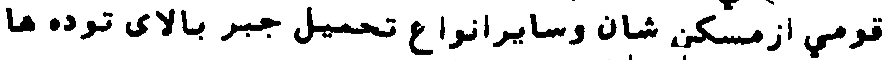

.




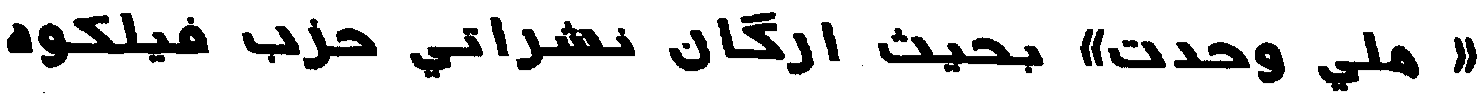

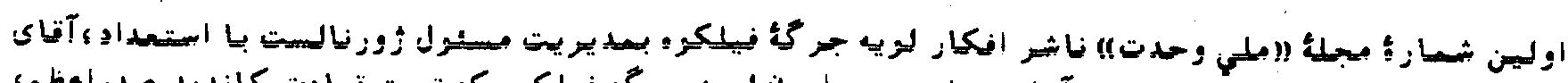

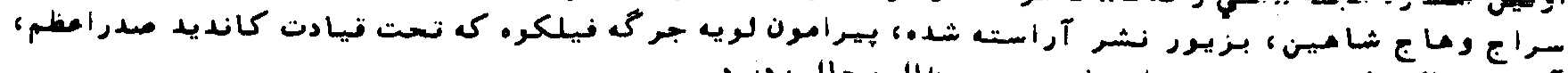

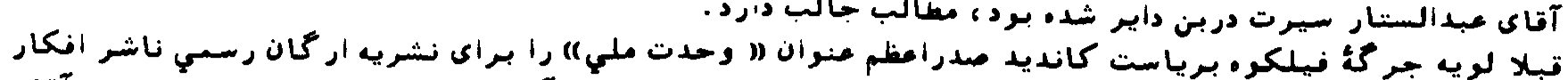

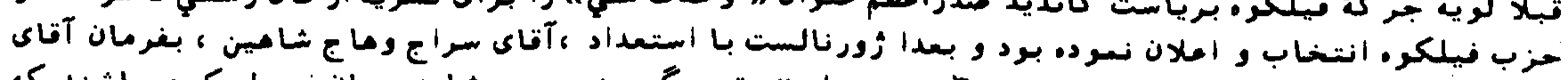

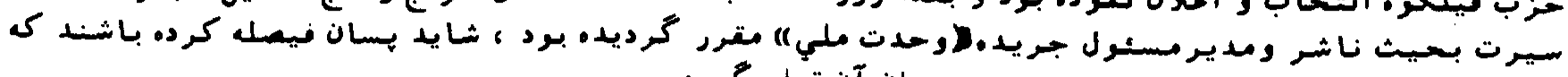

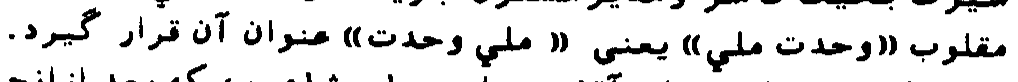

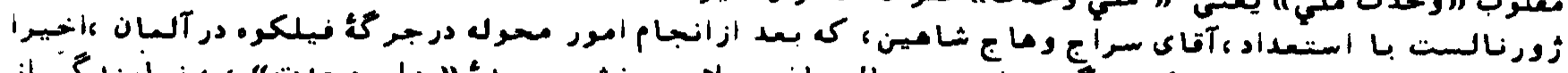

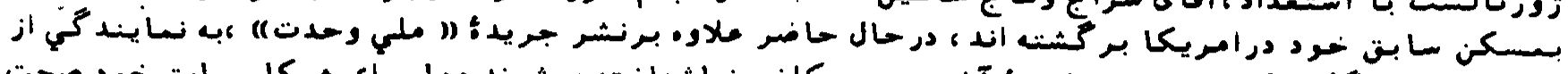

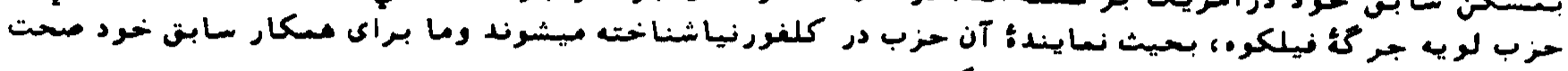

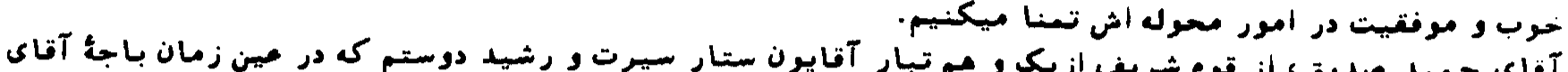

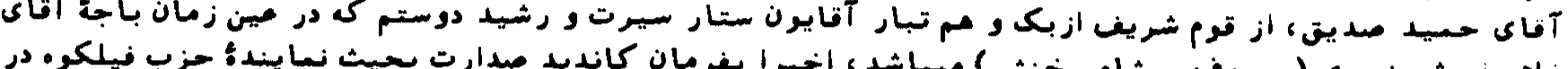

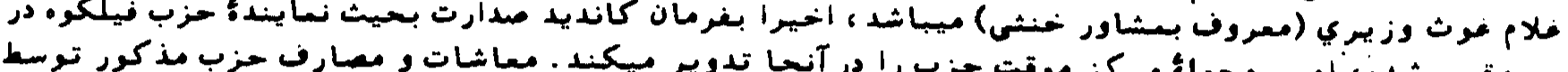

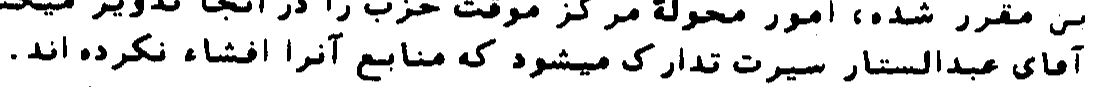

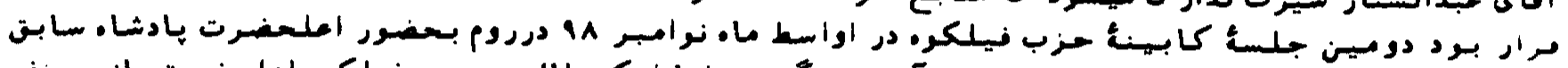

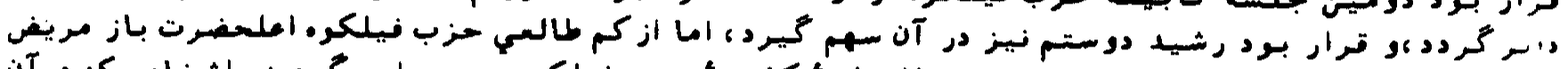

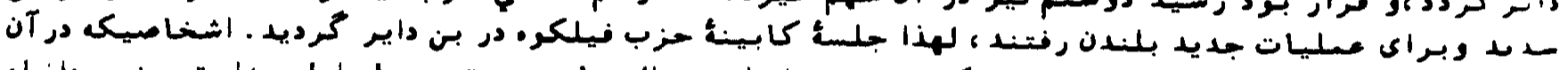

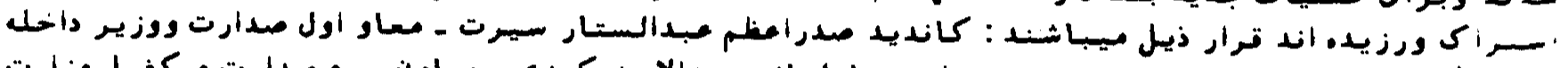

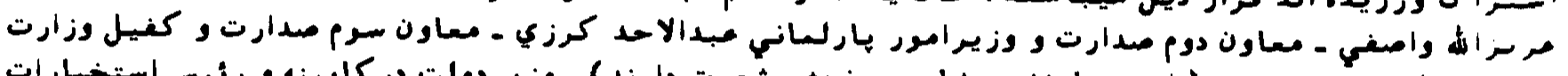

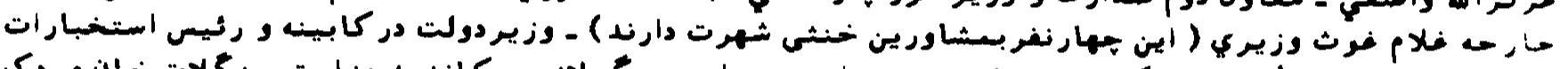

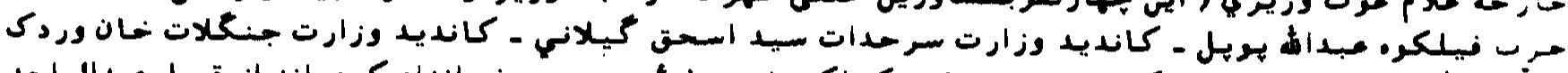

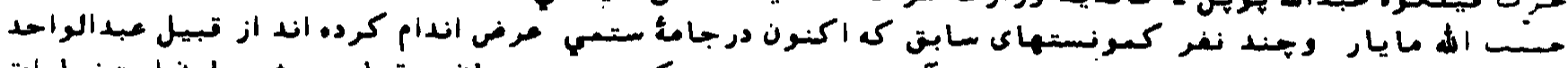

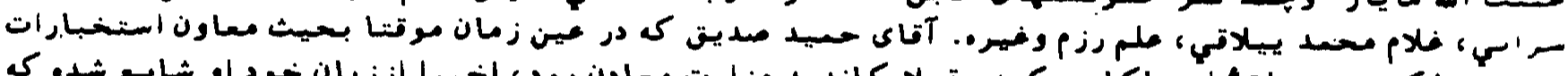

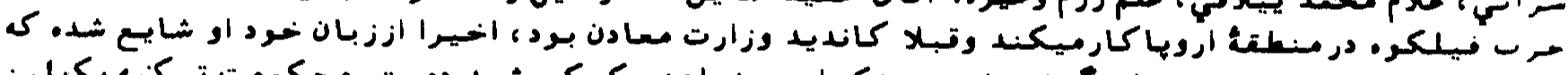

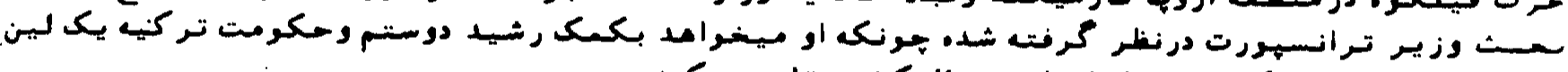

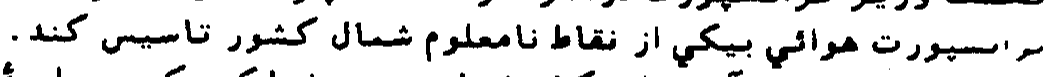

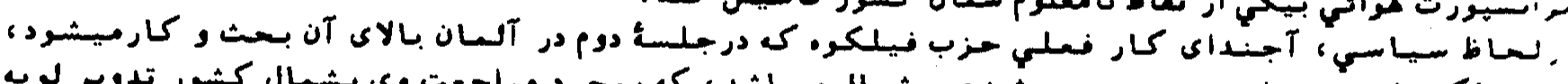

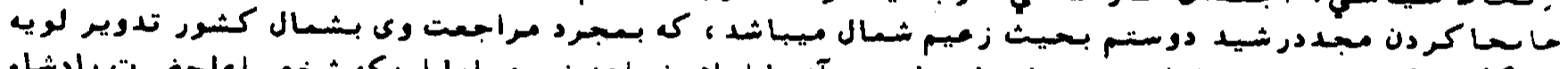

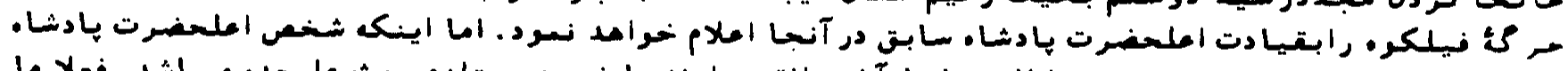

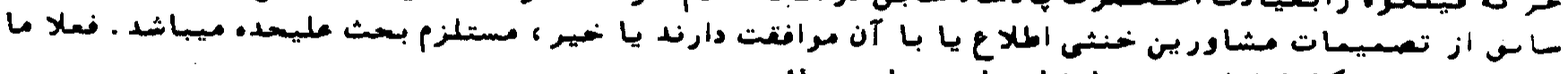

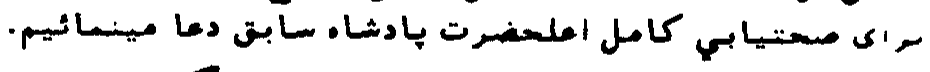

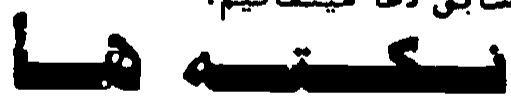

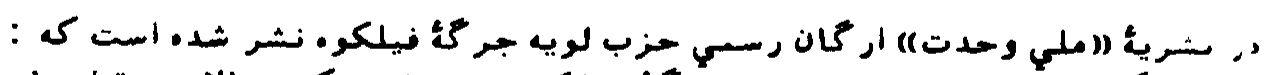

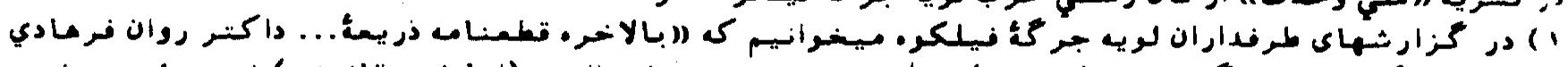

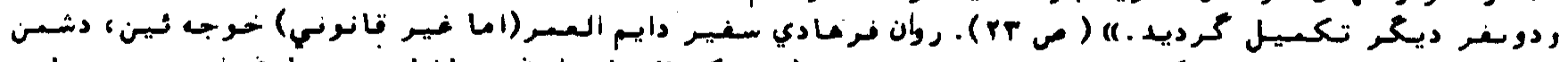

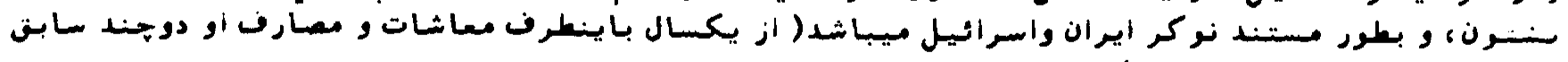

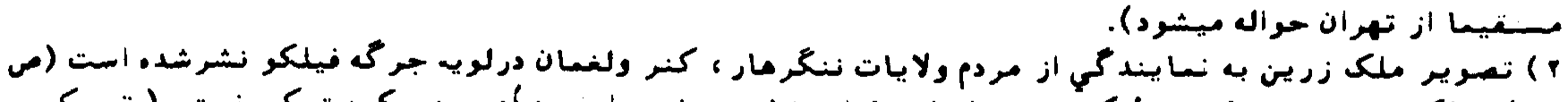

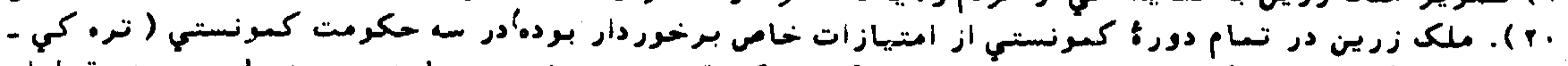

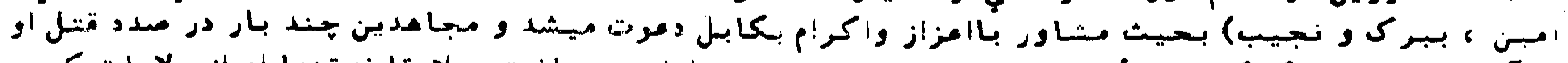

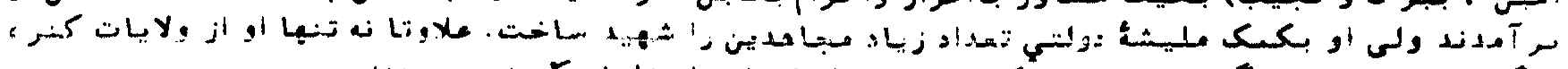

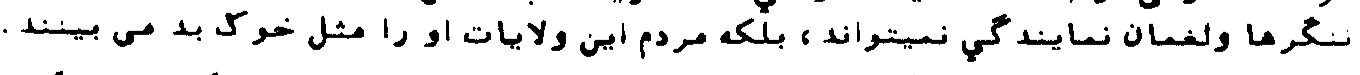

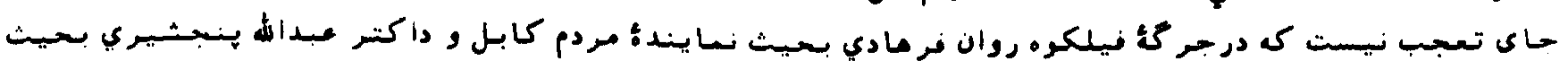

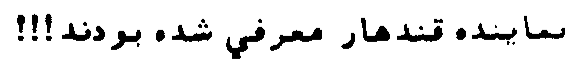

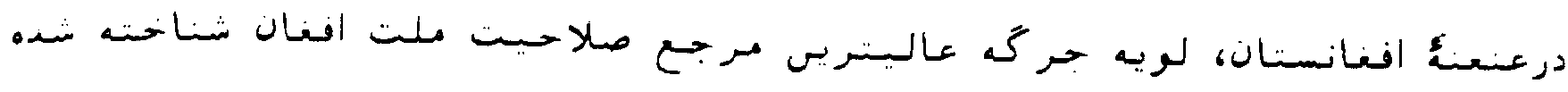




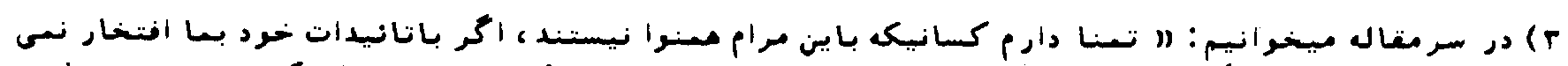

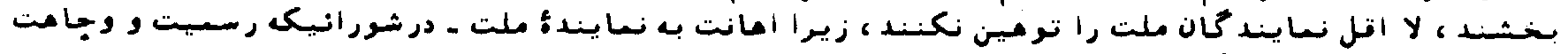

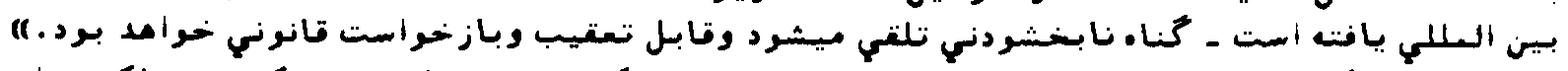

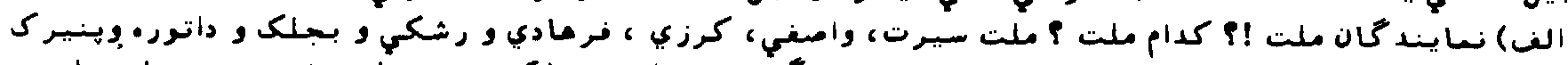

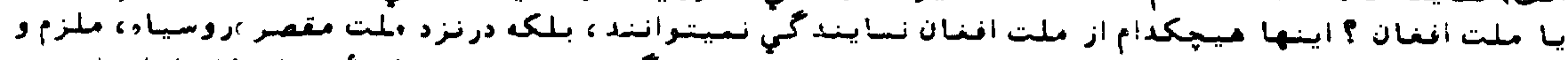

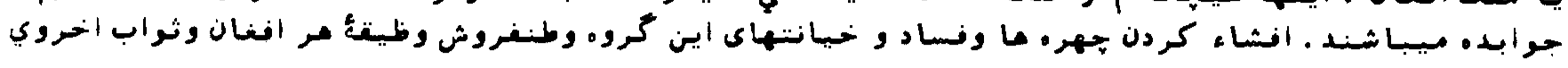

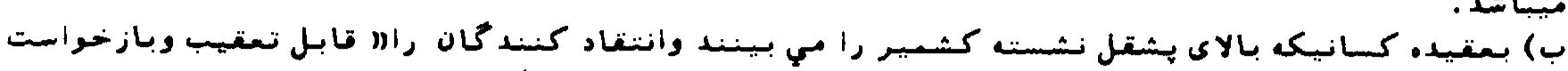

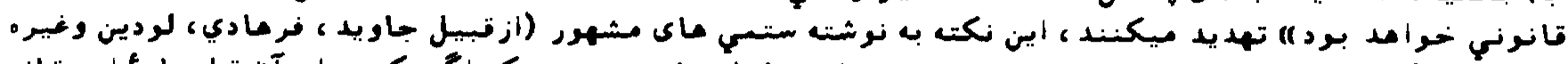

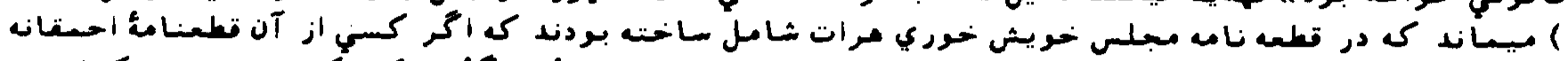

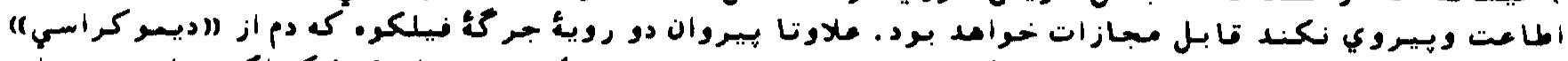

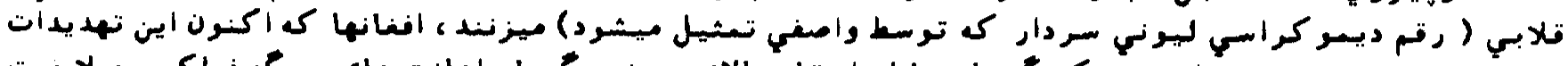

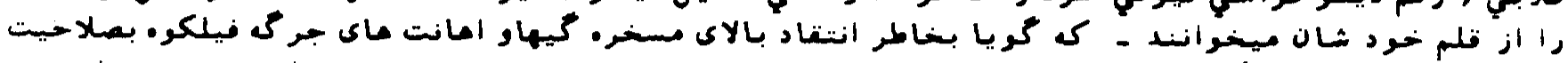

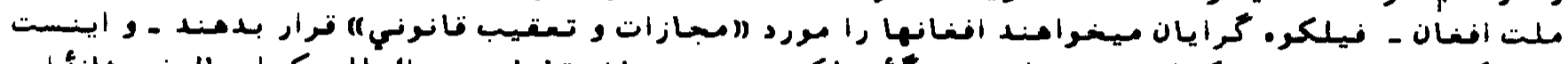

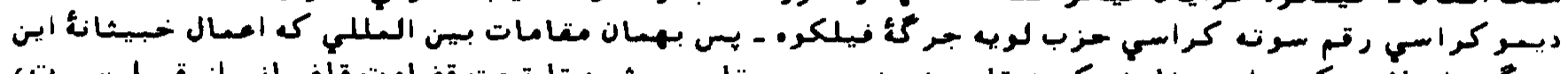

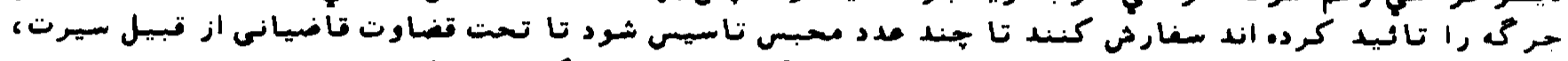

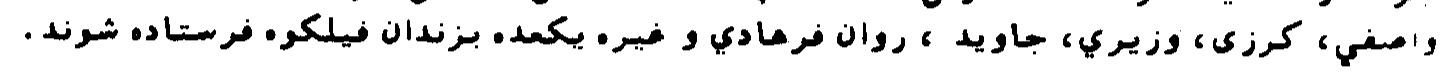

\section{كمنه كار نو نراش}

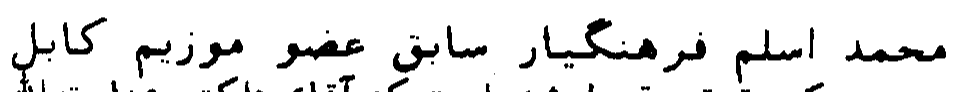

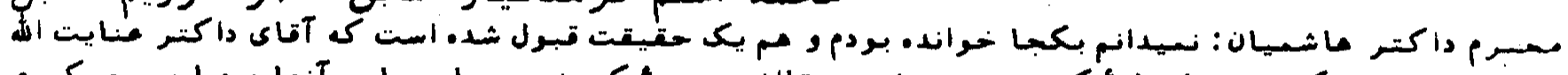

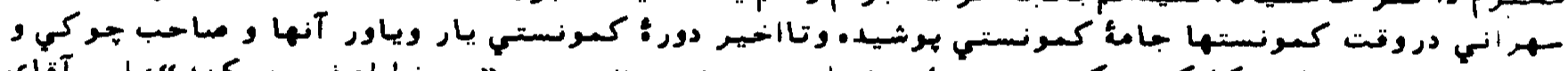

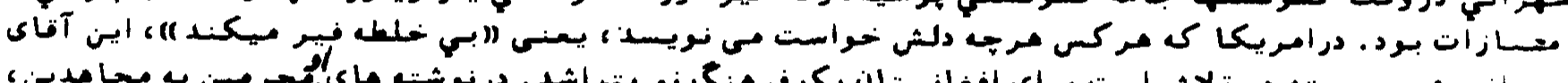

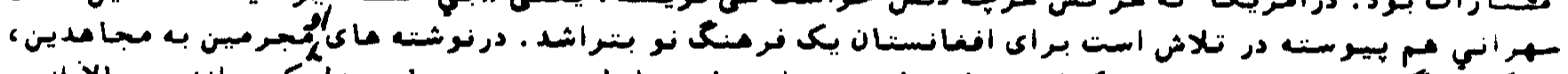

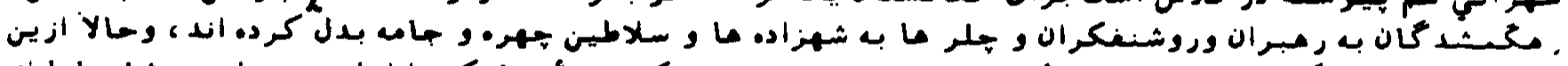

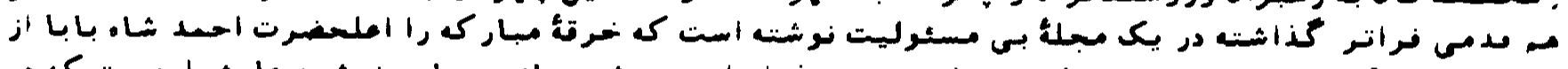

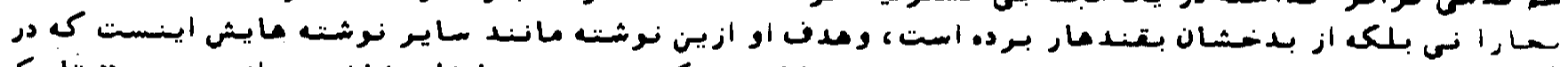

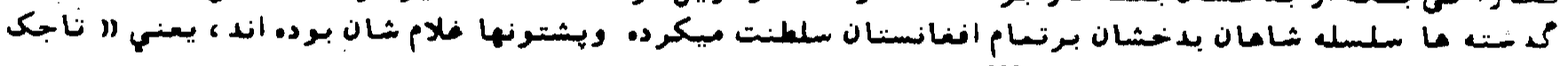

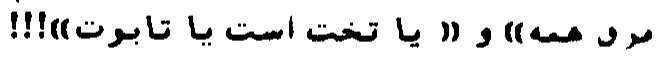

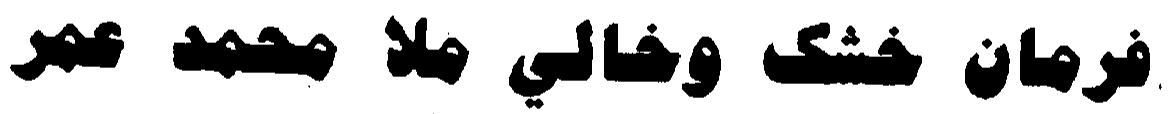

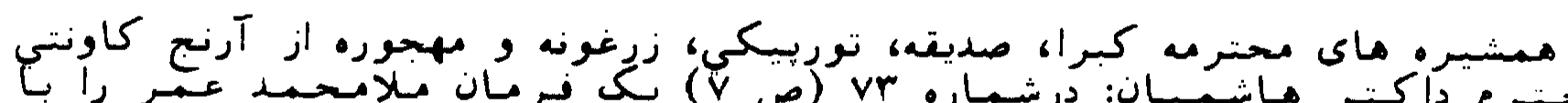

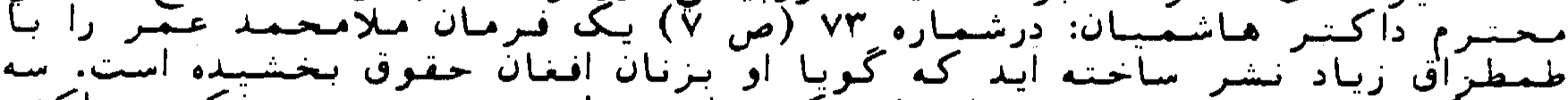

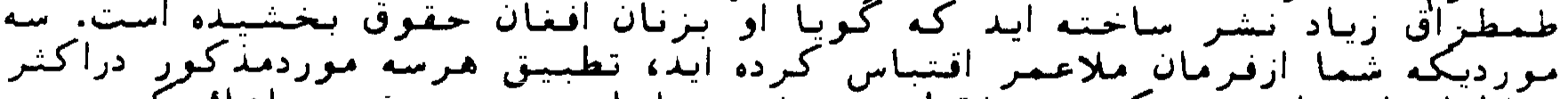

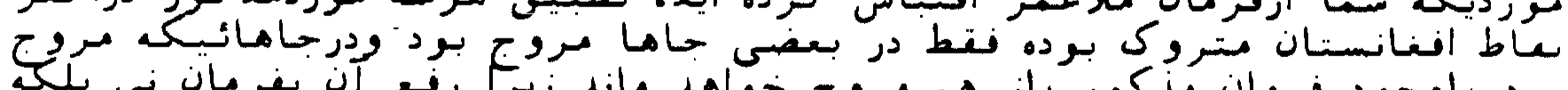

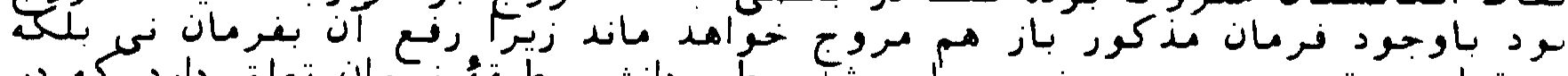

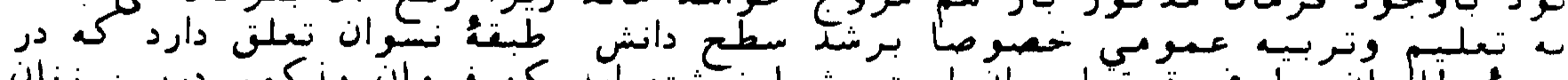

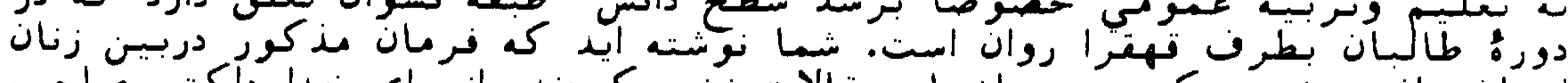

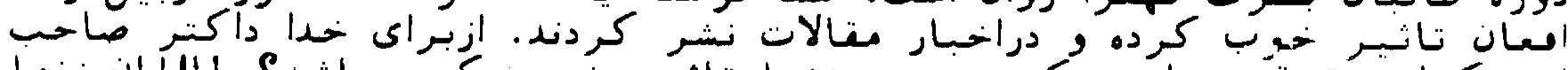

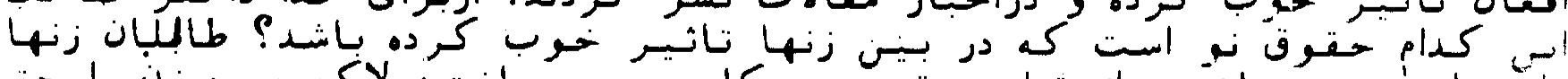

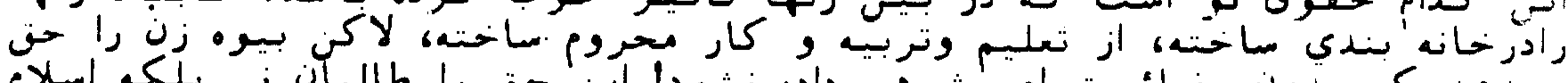

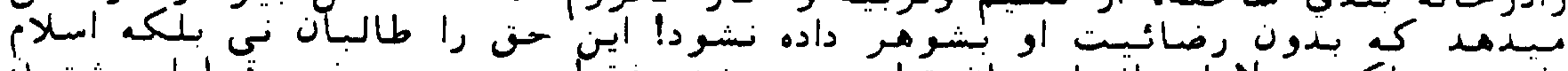

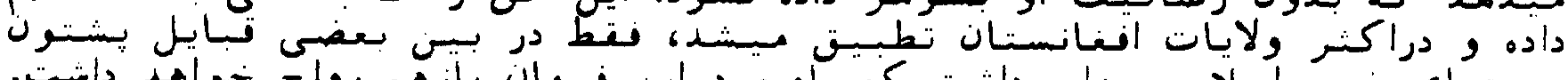

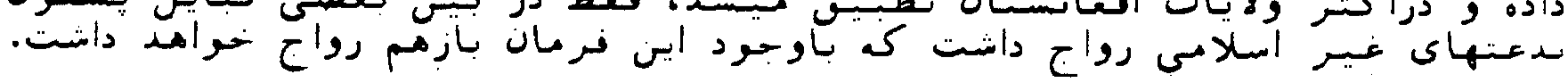




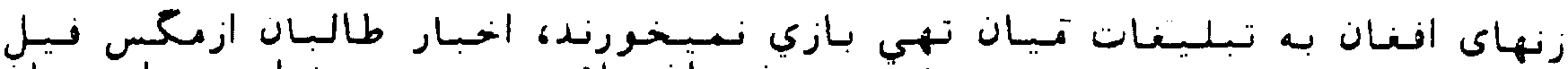

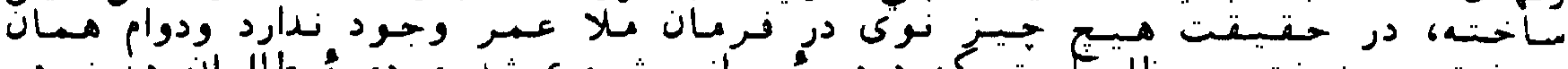

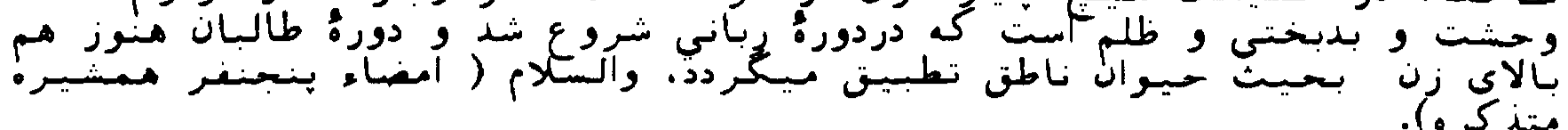

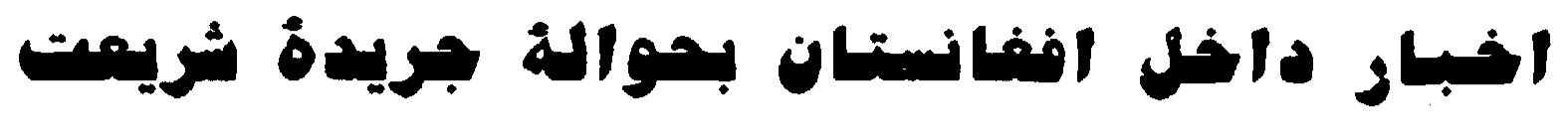

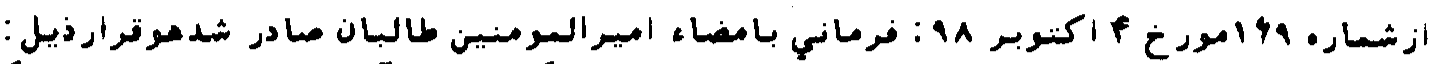

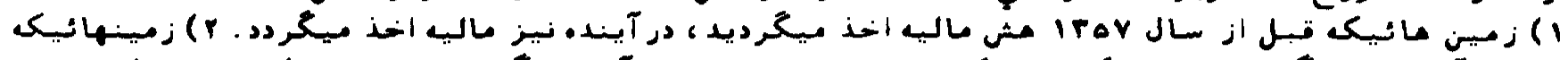

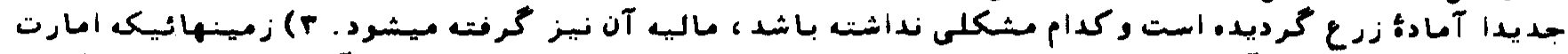

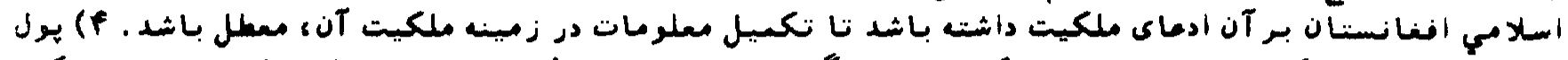

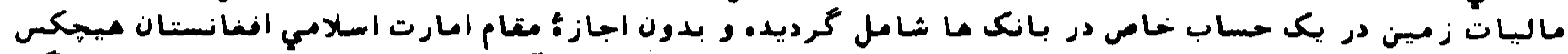

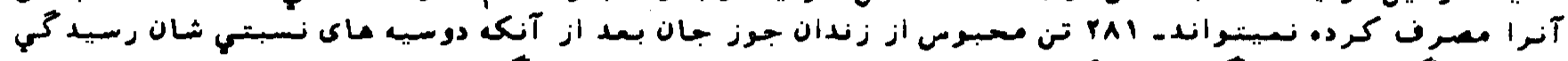

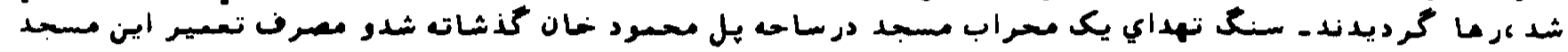

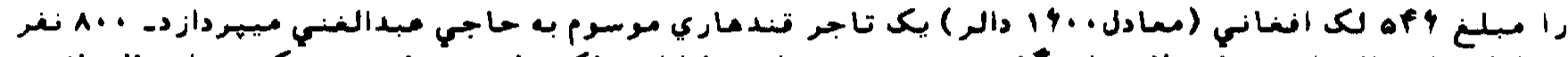

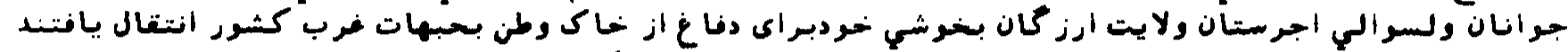

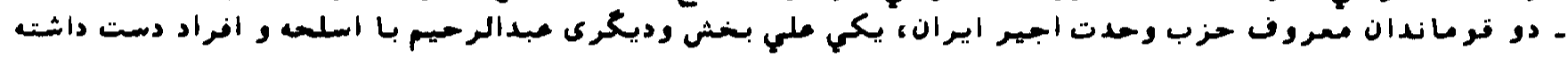

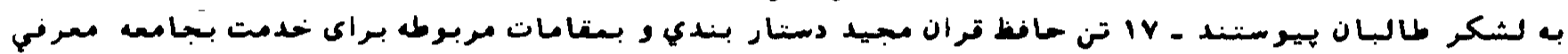

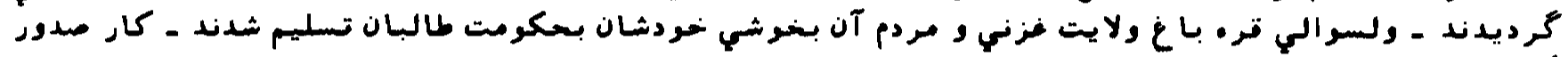

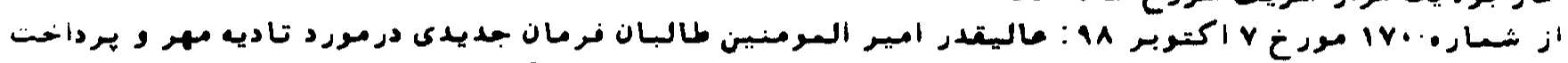

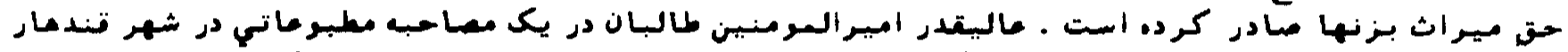

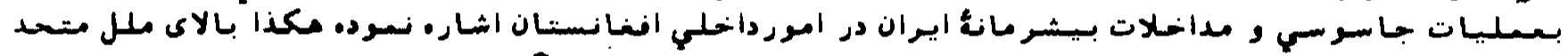

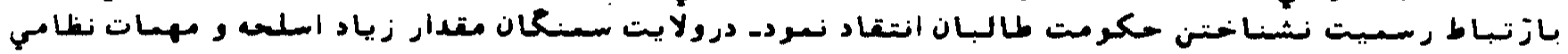

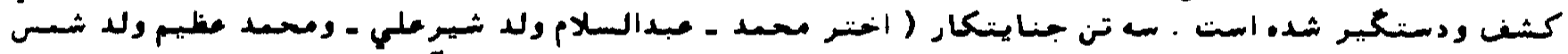

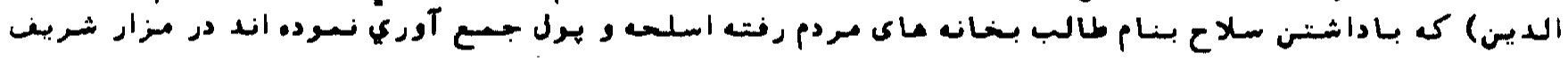

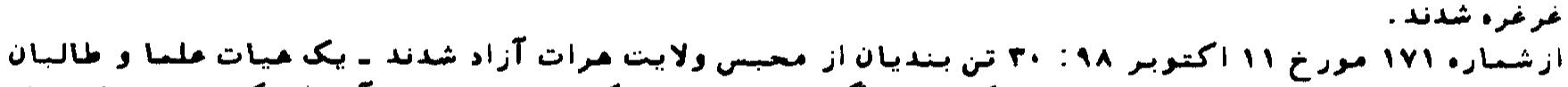

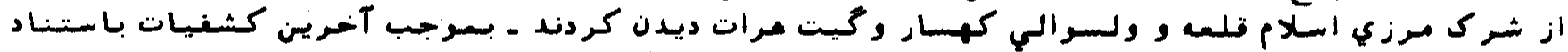

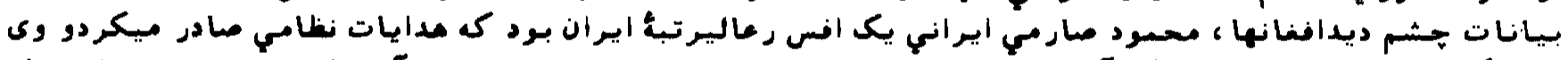

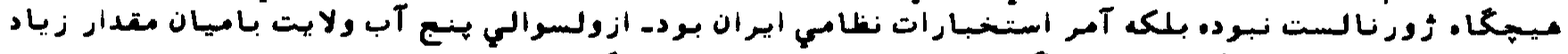

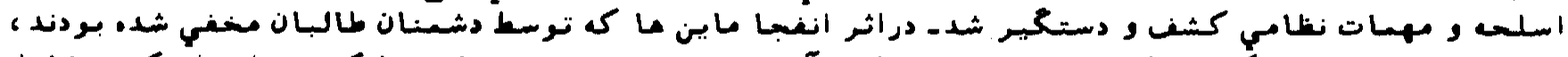

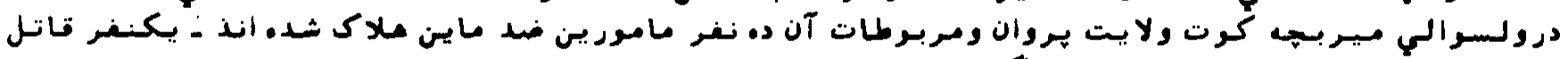

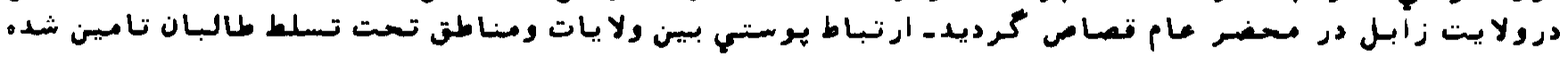

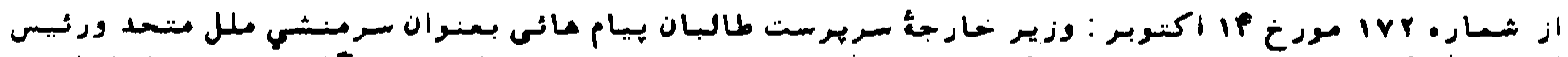

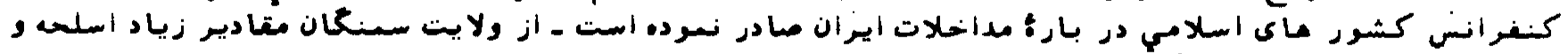

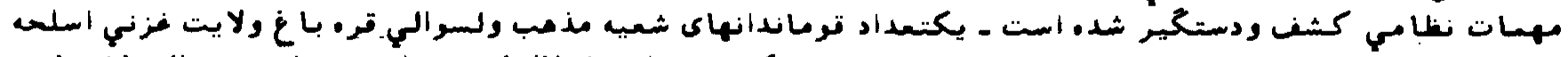

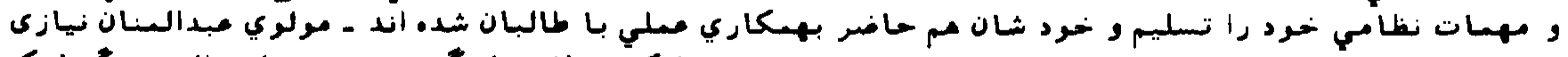

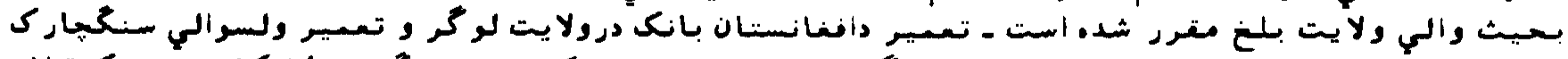

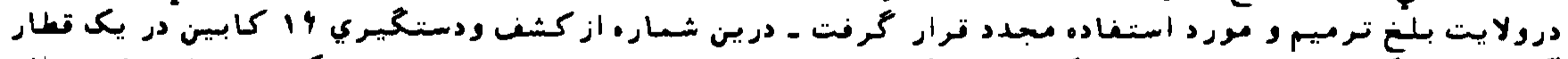

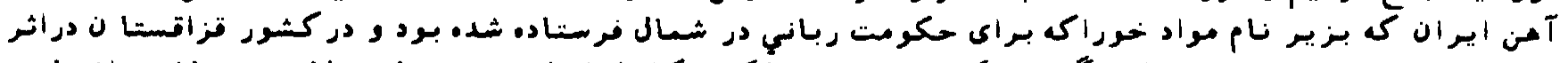

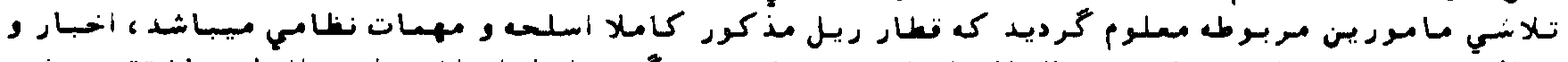

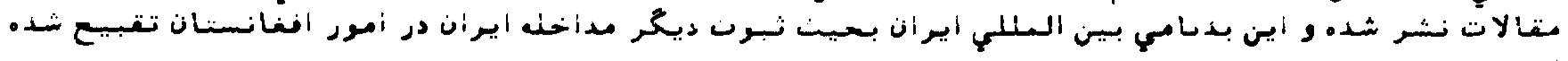

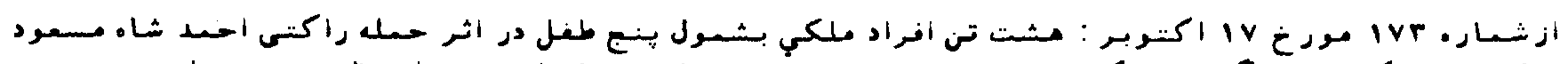

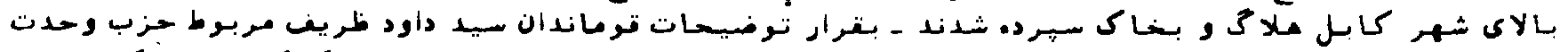

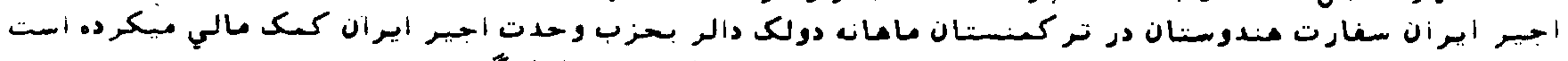

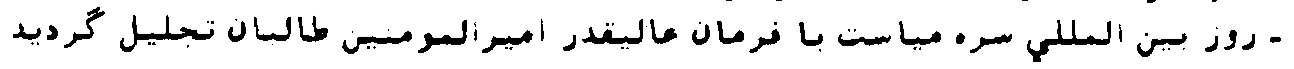




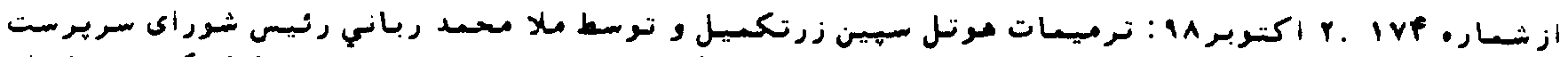

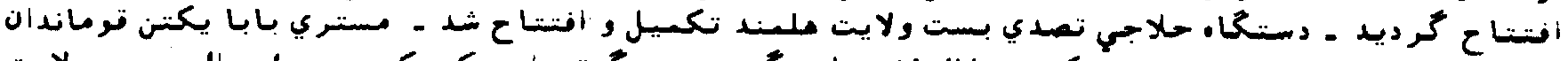

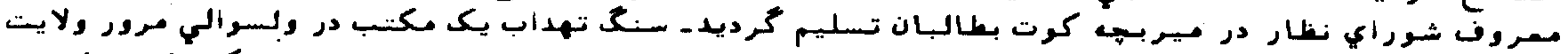

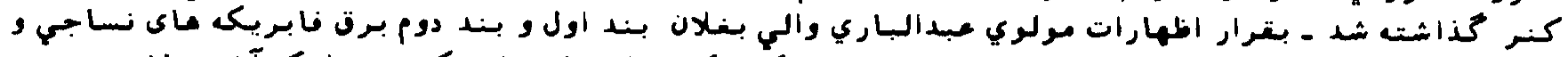

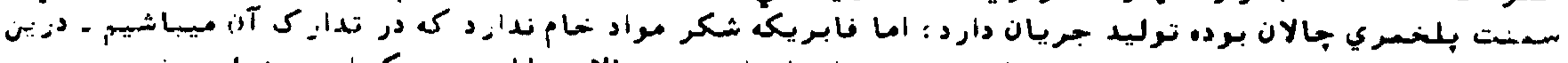

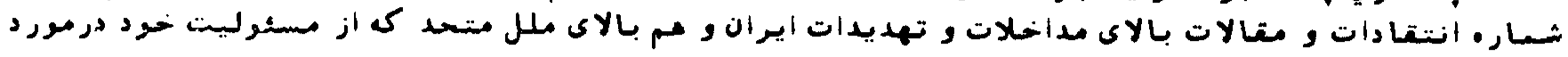

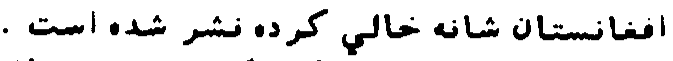

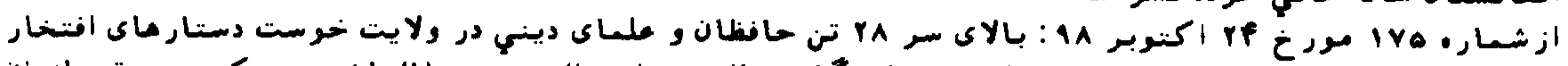

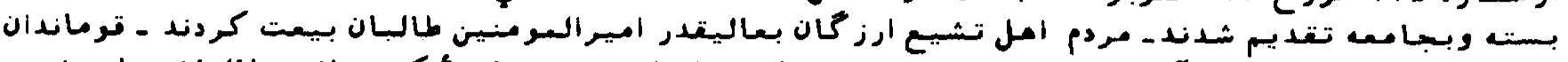

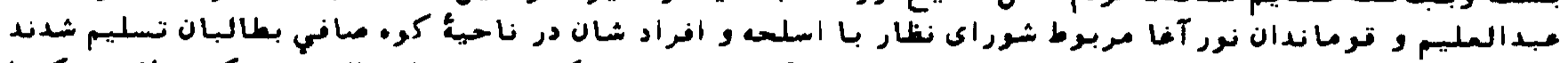

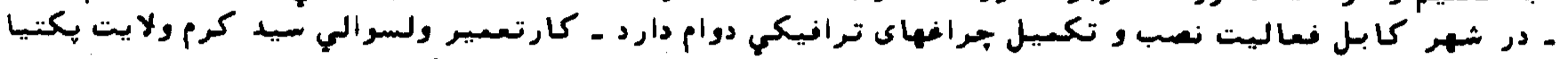

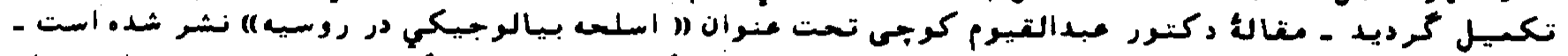

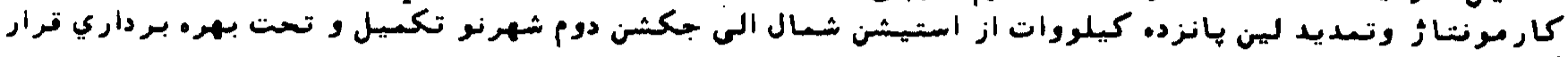

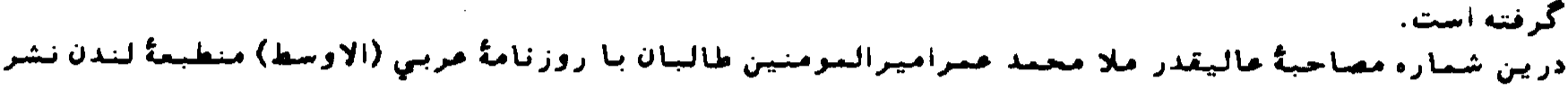

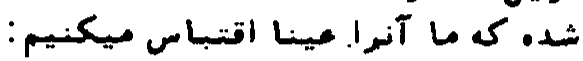

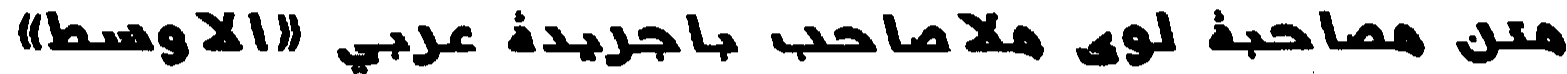

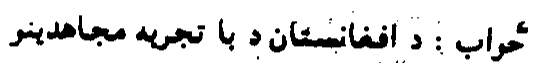

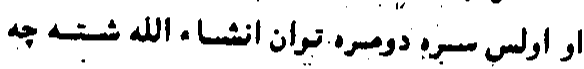
ددخالت بـ خلاذن د. دناع بهاد وركتهي.

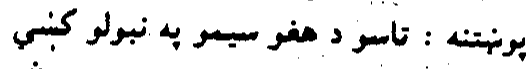
• جه منكي د منغالفبنز به لاس كي وي له ثئ

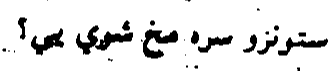

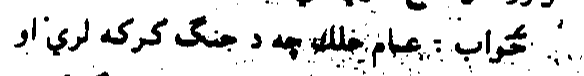

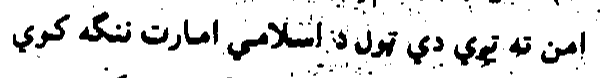

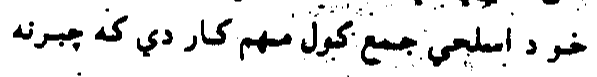

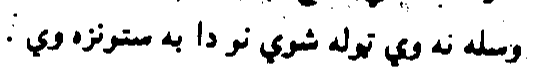
بوبنتنه : ابا ستاسر او د مفالفبنو تر مينغ

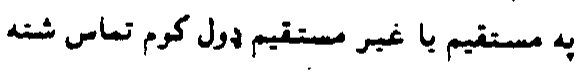

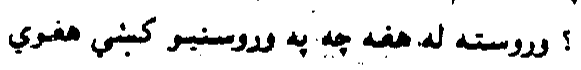

ماثه وخروهن

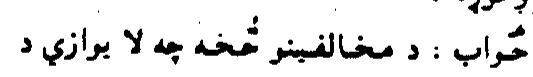

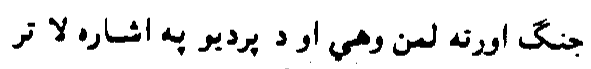

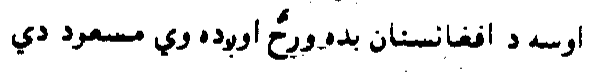

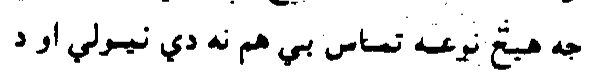

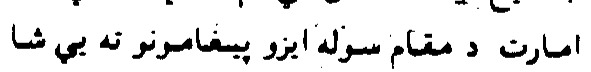

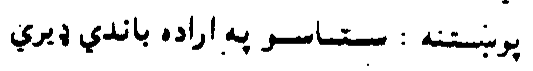

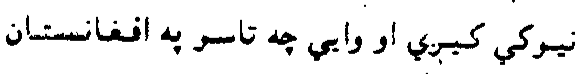

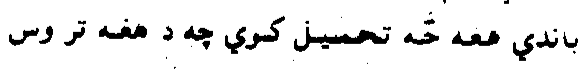

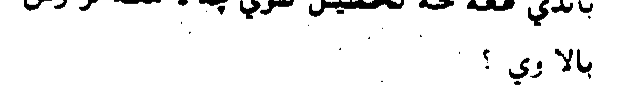

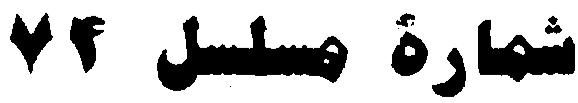

بونيتهن : آبا تاسو به دي عتبده ياست جه

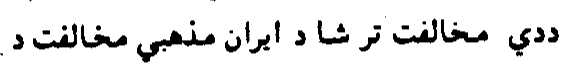

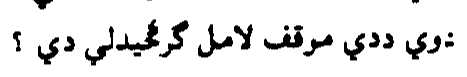

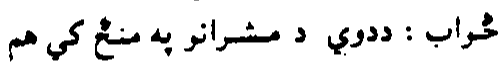

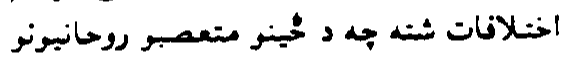

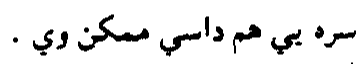

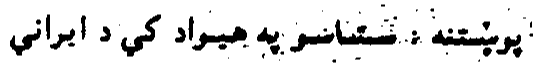

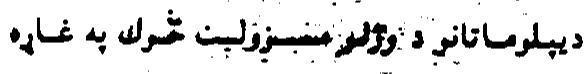

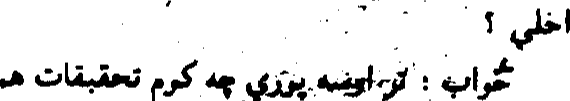

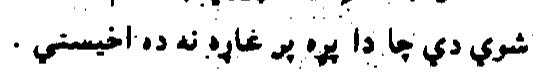

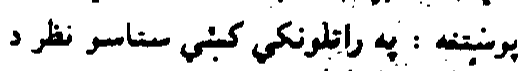

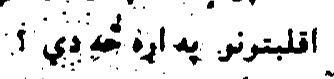

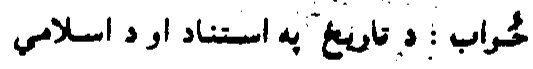

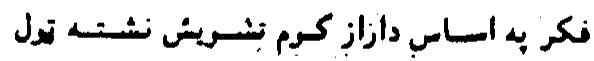

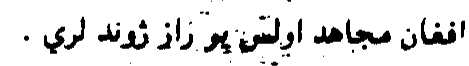

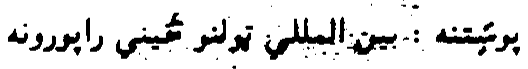

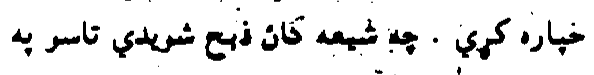

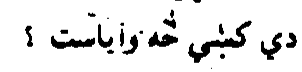

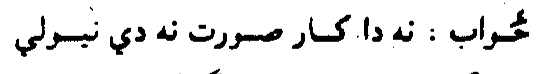

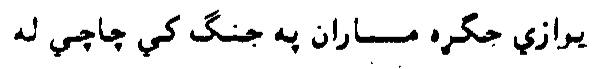

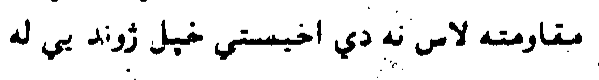

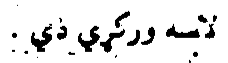

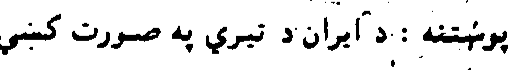

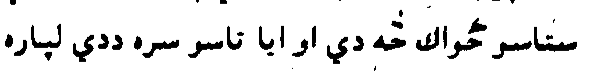

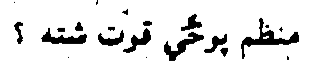

قدرمنر لوسنرنكو ! عاليتدر امير

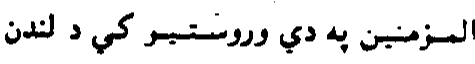

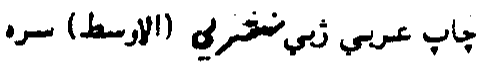

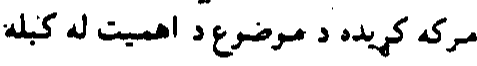

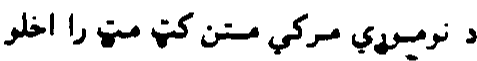

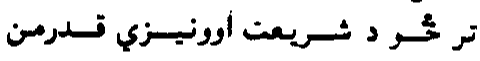
لوسترنكي مم ترينه كثنه بورته كري .

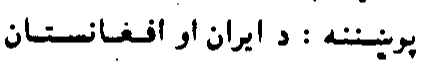

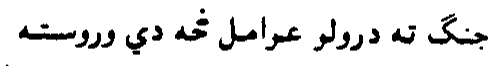

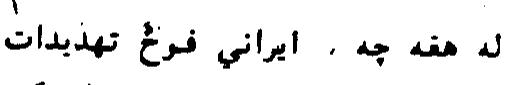

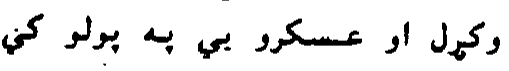
تمرينات بيل كهر او عيكرو بع

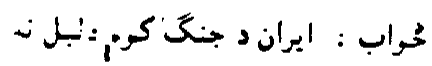

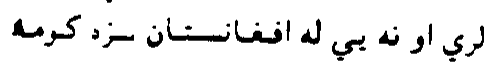

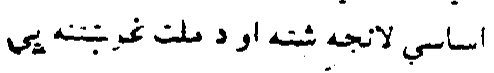

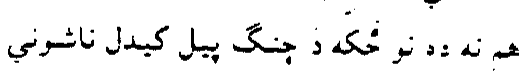

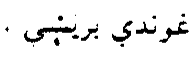

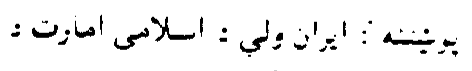

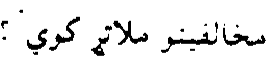

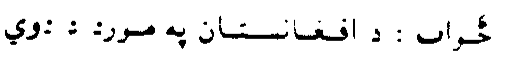

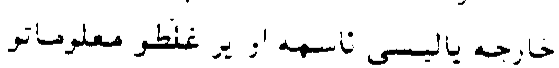

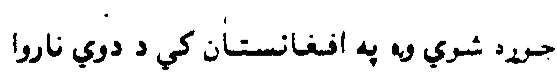

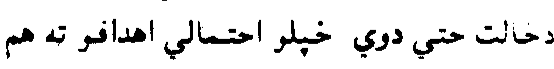

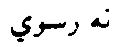




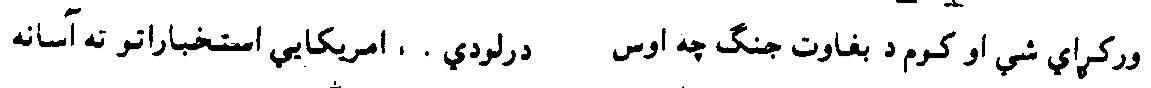

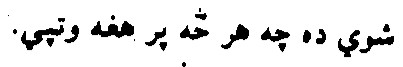

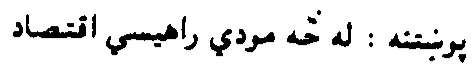

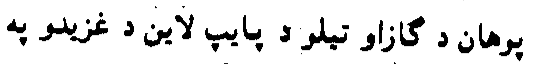

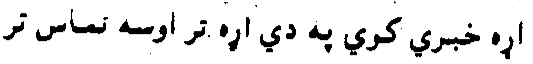

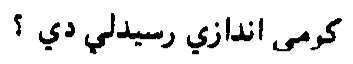
حُرَاب : دأبروزه جه د منطتي لبـار

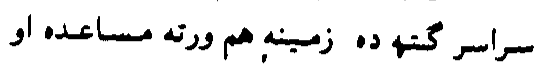

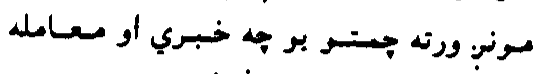

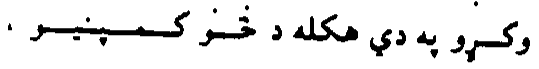

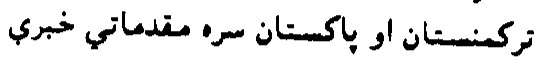

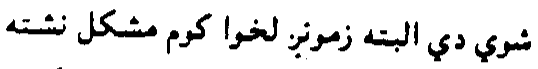

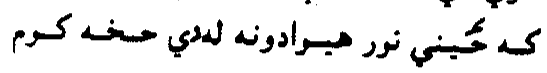
كياسي كار جور نكري اتدام وكري سنه به وي.

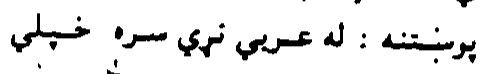

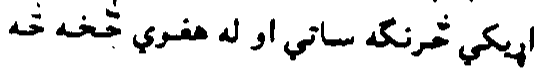
ميلمي لني i

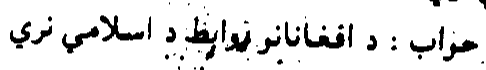
سره د بههاد به دوره كى فبرت المعاده بنه و.

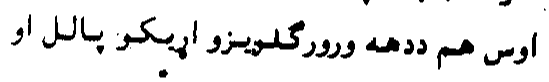

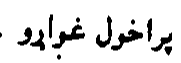

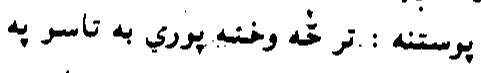

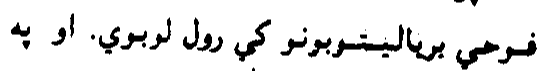

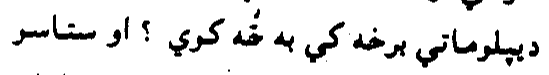
د ميراد سفارنونه اوس مم د مشيالفينر لباره كار كري او د مغري به بإكه كبني دي .

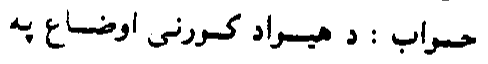
مسيلو سره به خارجي وضعيث هم انشابـ ، الله

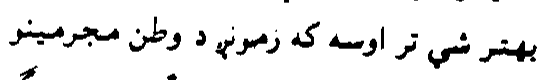

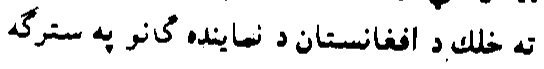
كوري جد دا به له مغوي سره بي غنوري او بي نهي

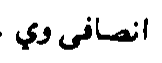

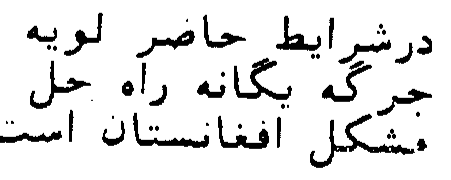

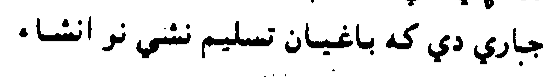

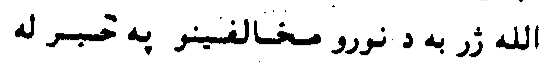
مينحئه ولار نهي.

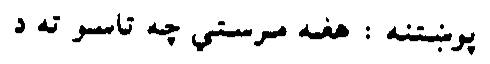

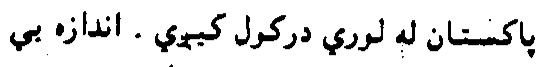

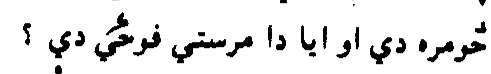

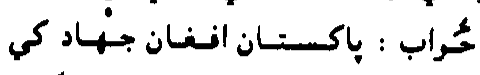

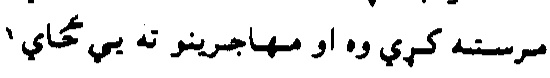

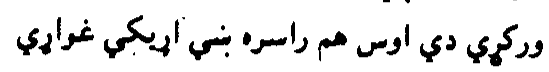

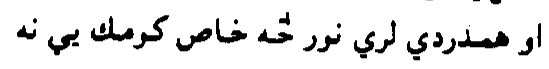
دي كري او عسسكري كسومك ته يم زمسرنب.

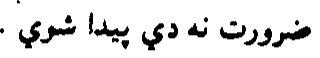

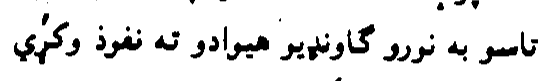

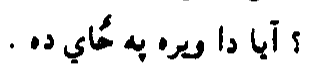

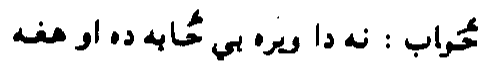
خولك به دا تورونه لكري مغري خبله بالنعل

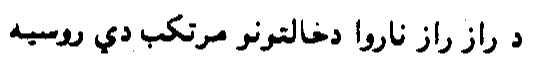
زمرببر سره كوم سرحد نه لري او مرنبر د خهيلو

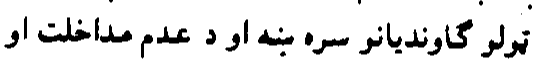

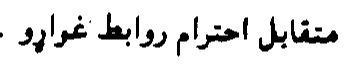

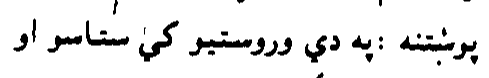

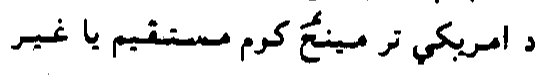

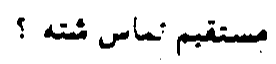
خحواب : د امريكا سكرمت د آفنانستان اسـلامي امـارت به رسسميت نه بيـرني كـوم

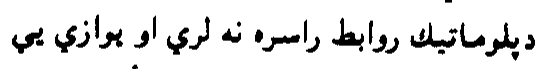

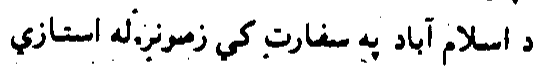

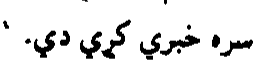

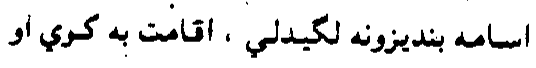

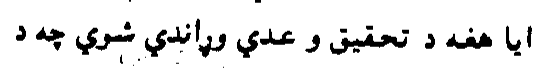

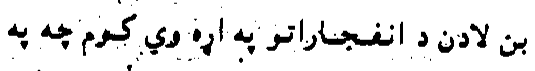

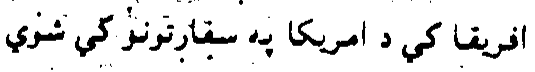

مكراب : اسلامس بن لادن به أنغـانستـان

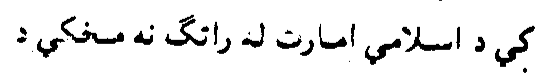

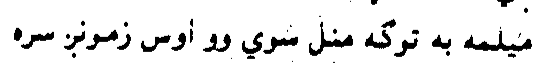

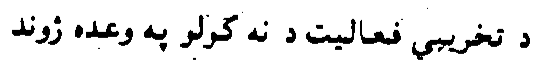
كري او دده به خلاف به تورنو كي باليت راته

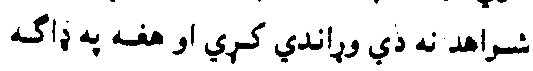

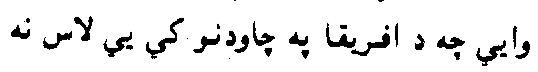

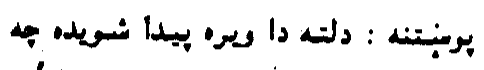

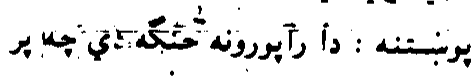

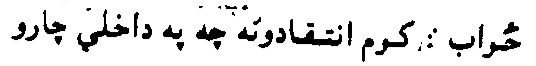

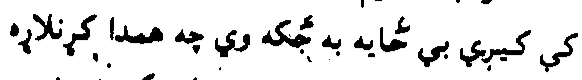

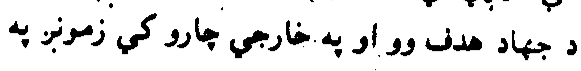

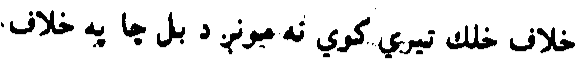

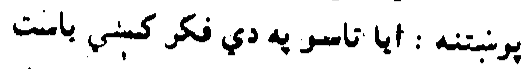

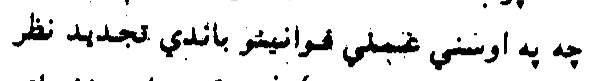

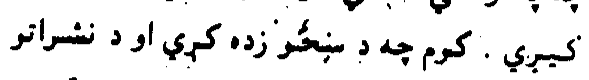

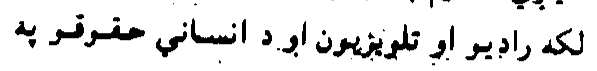

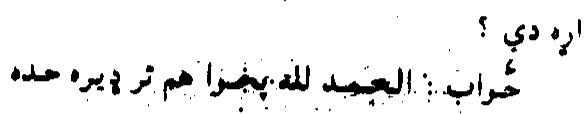

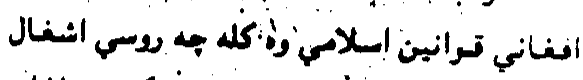

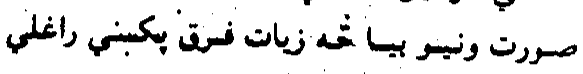

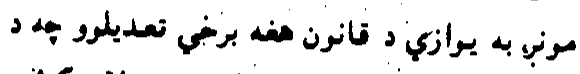

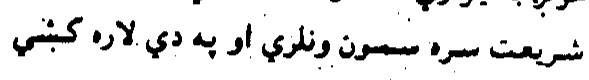

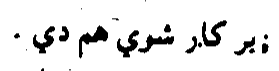

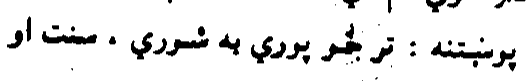

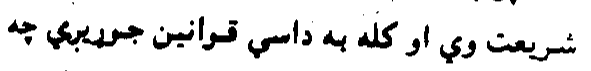

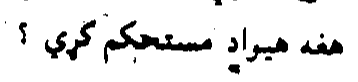

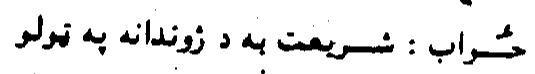

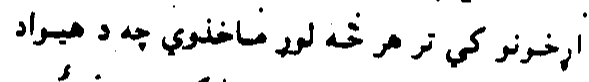

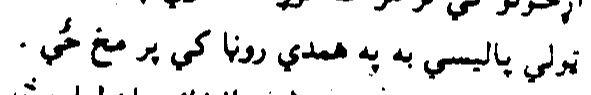

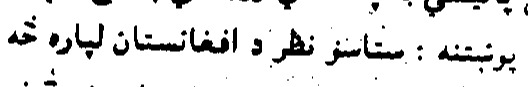

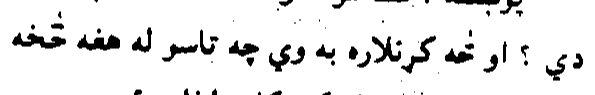

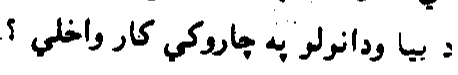

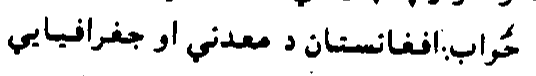

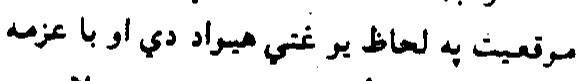

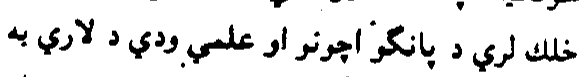

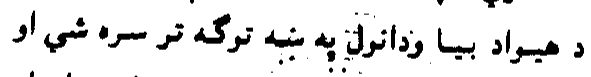

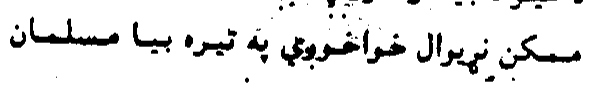
ورونه به خبلمي مرستي ونه سبمري

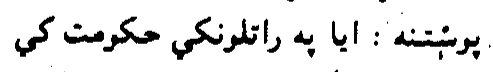

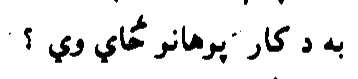

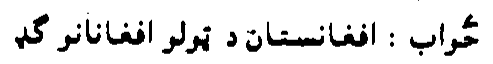

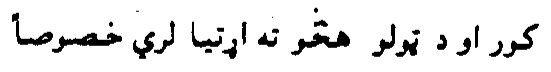

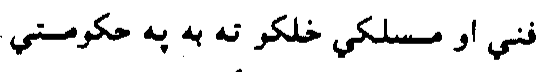

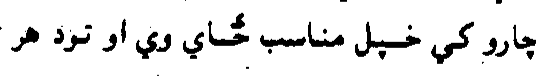

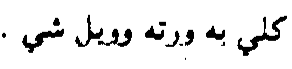

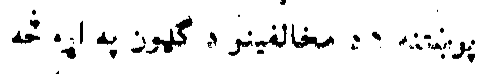
واياست : به راتلرنكي حكرمت كي به حُّاي

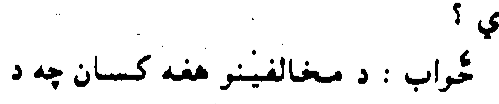
سـرلي دواعبي سـره ير كُاي شهي د خهبل لبانت سره سم به به خـدمت كي نـسـركبت 


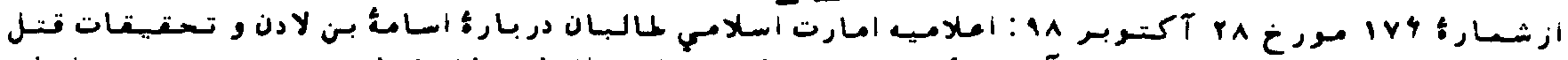

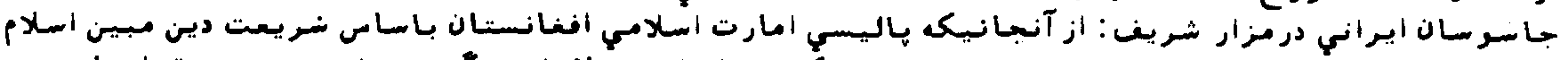

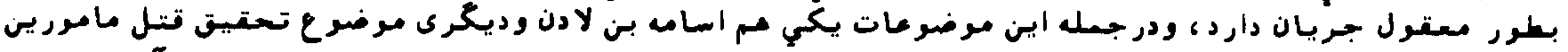

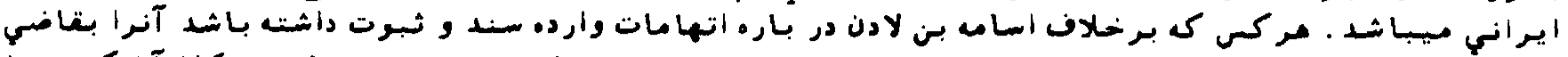

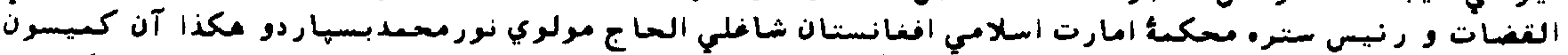

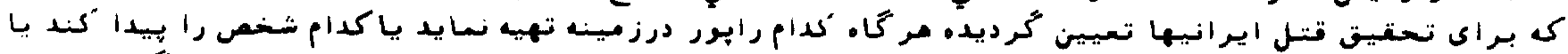

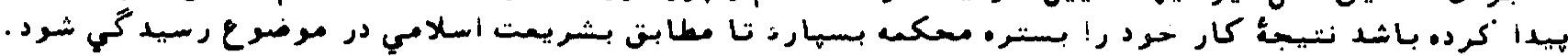

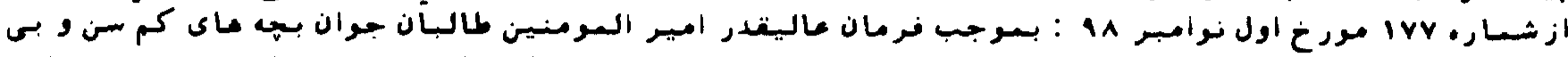

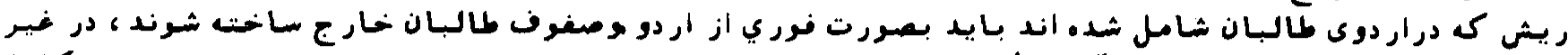

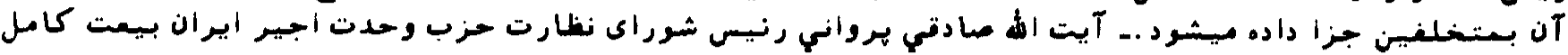

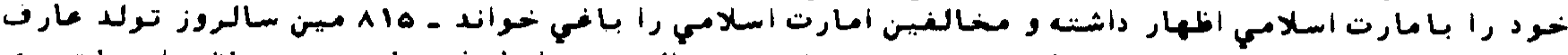

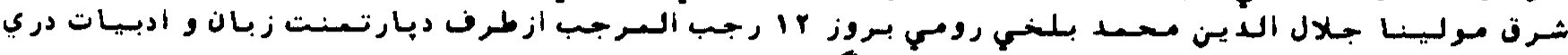

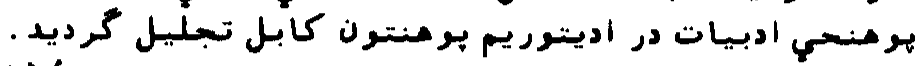

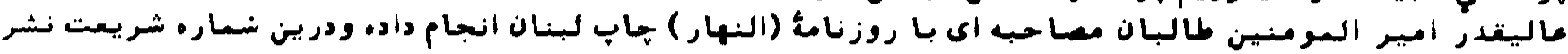

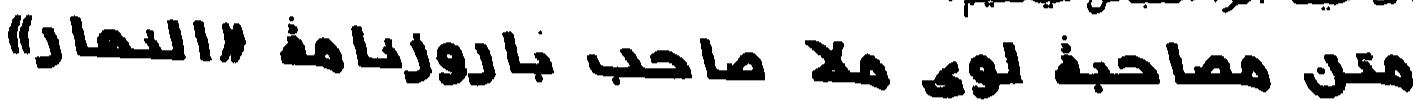

دريخ مزتف ، غربنتني امداف · ورته تشريح

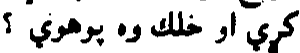

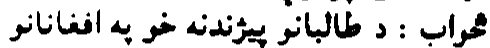

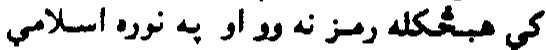

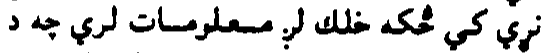

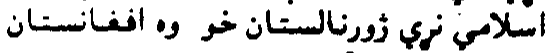

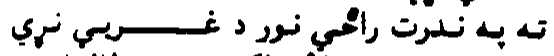

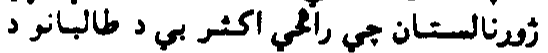

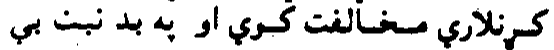

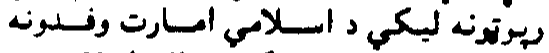

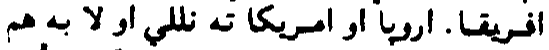

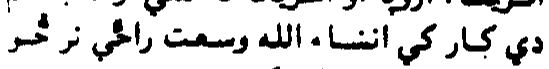

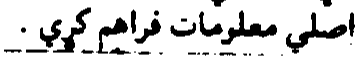

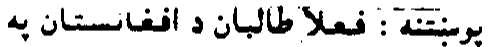

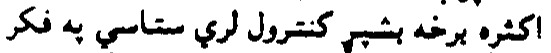

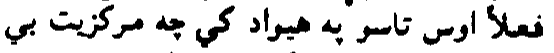

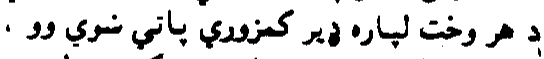

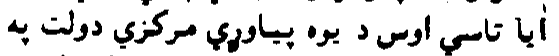

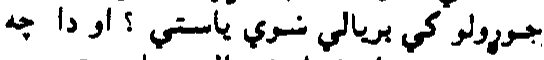

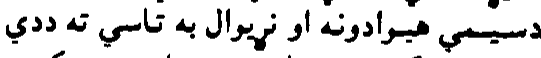

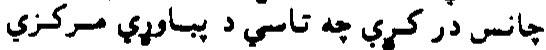

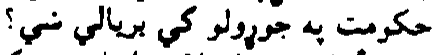

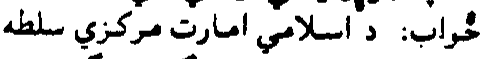

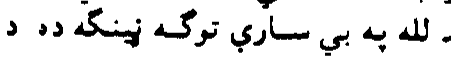

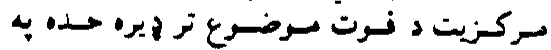

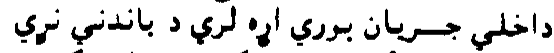

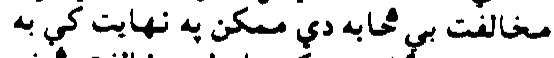

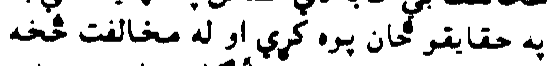

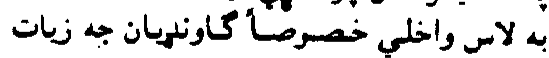
نقن لرلاي ني.

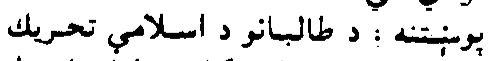

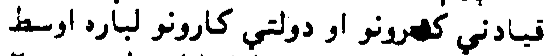

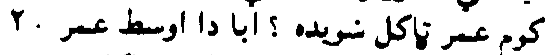

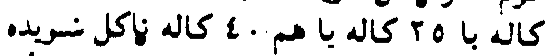

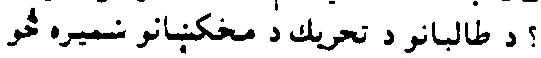

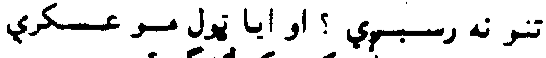

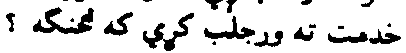

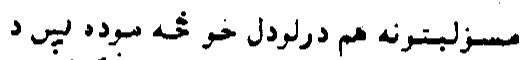

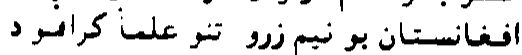

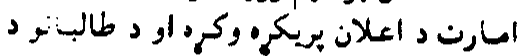

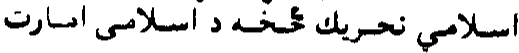

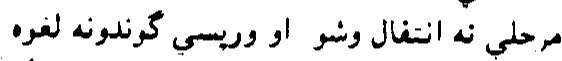

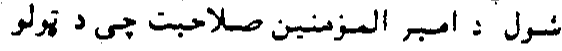

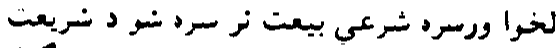

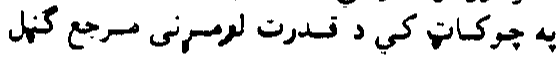

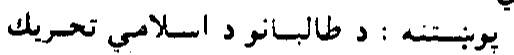

$$
\text { كببي }
$$

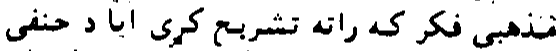

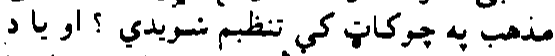

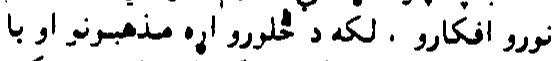

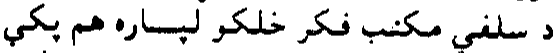

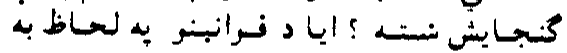

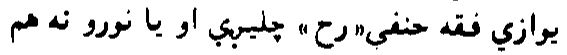
تخه مجال نتسه ؛

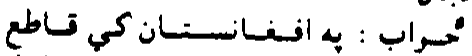

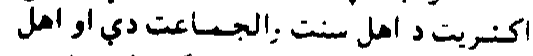

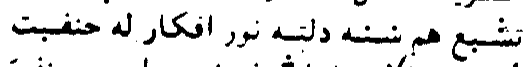

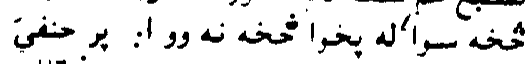

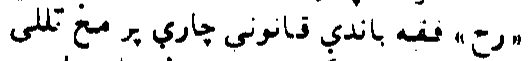

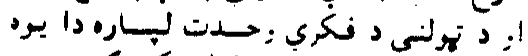

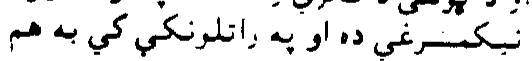

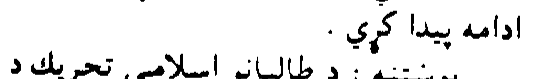
يوبنتسه : د طالبانو اسلامي تحريك د نهري

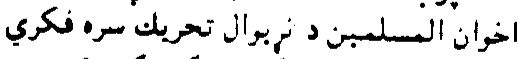

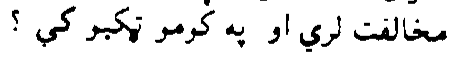

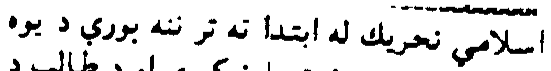

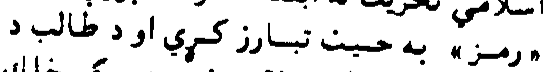

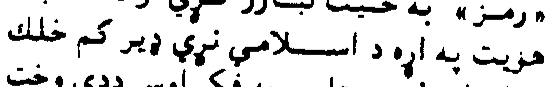

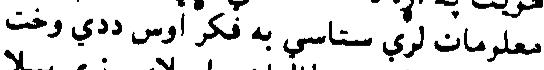

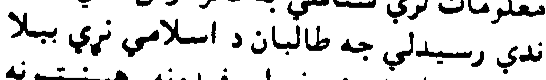

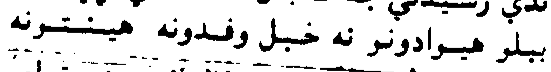

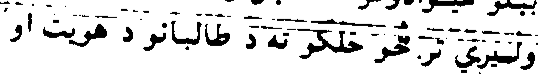

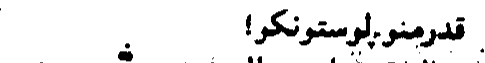

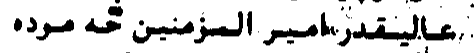

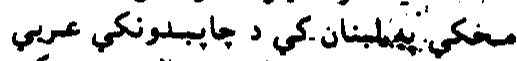

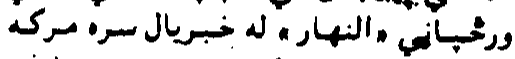

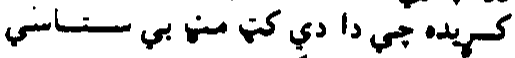

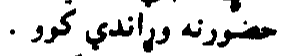

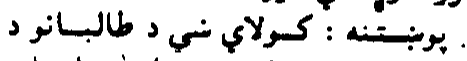

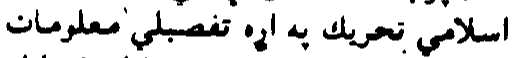

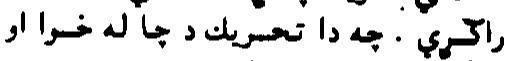

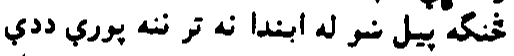

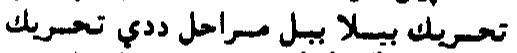

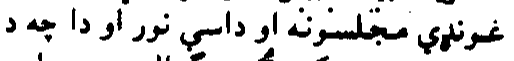

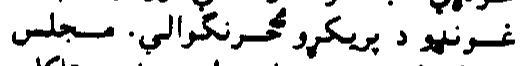

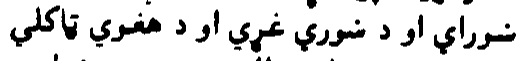

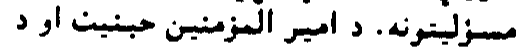

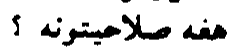

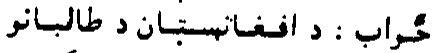

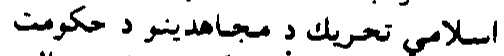

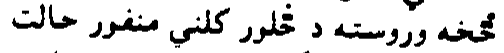

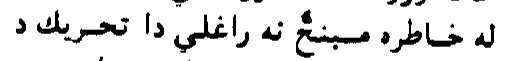

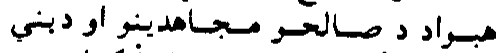

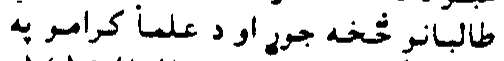

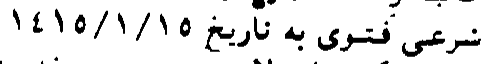

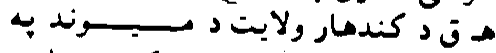
ولسرالي كي به كار شتروع وكره مسرونيت

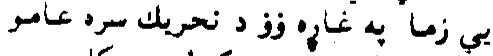

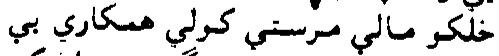

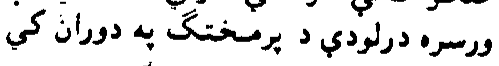

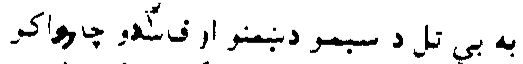

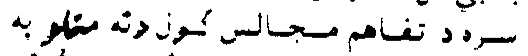

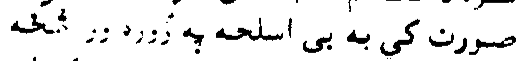

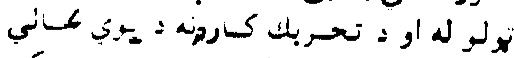

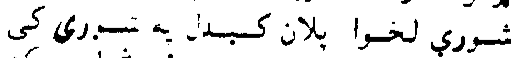

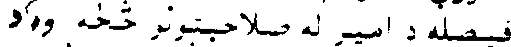

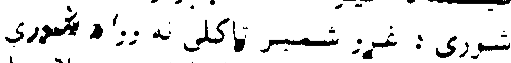

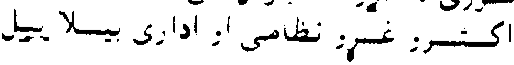


مطلت حرام نه دي مكر د نشي لبـار

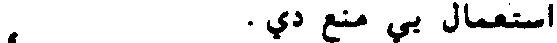

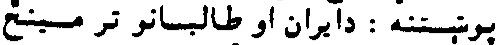

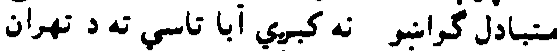

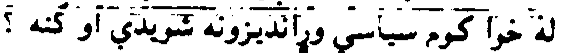

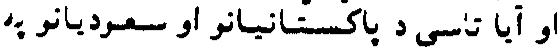

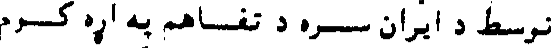

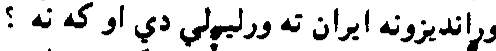

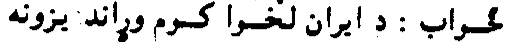

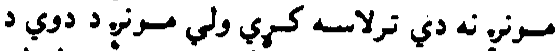

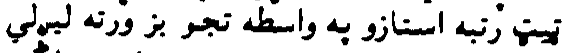

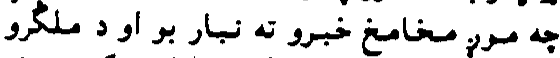

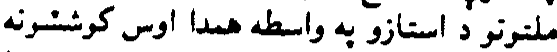

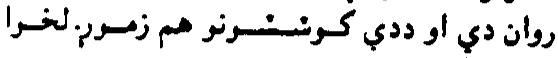

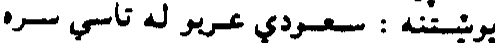

د بيلرماتيكي الهكي تطع كري تاسي دا ويري

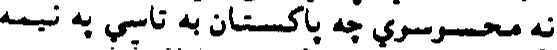

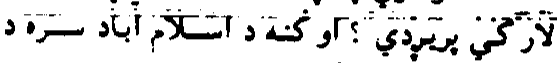

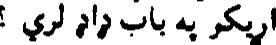

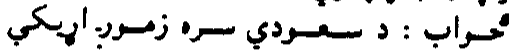

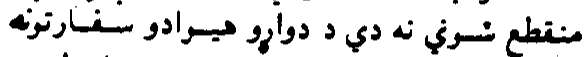

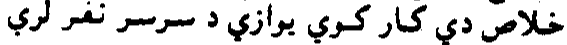

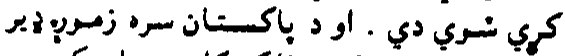

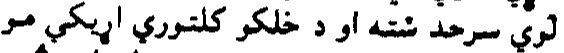

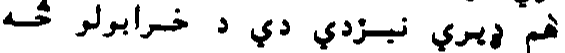

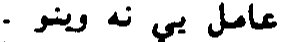
بوبتنته : لحه نكر كوي به دو سعردي عريو

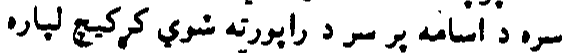

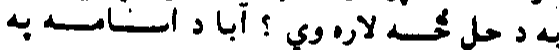

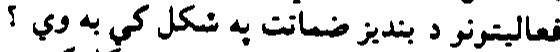

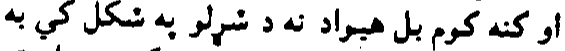

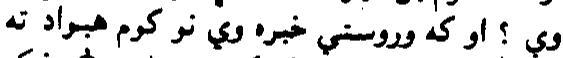

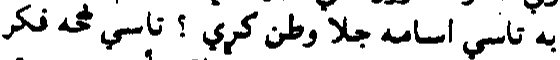

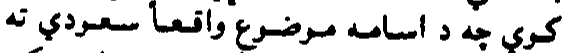

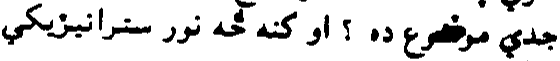

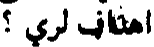

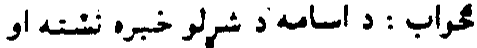

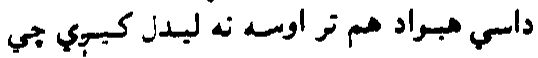

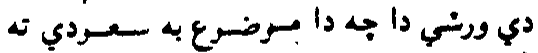

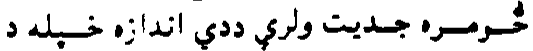

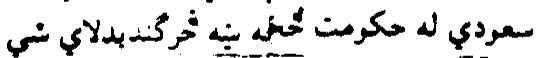

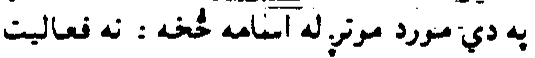

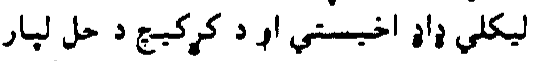

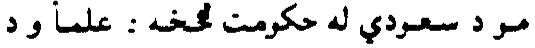

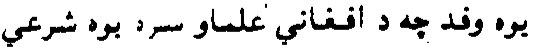
حل ته ورسمجي ورانديز كري دي

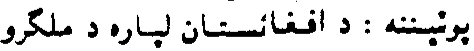
ملتو خام امتـازي لهندر براميسي ايران او

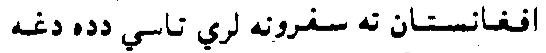

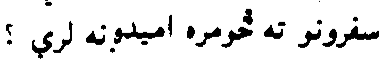

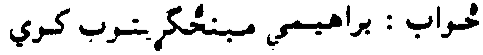

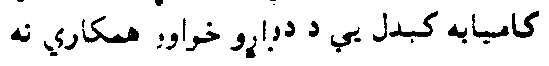

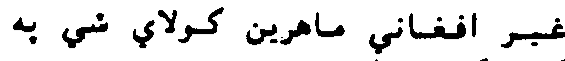
كارو كي دخيل ني :

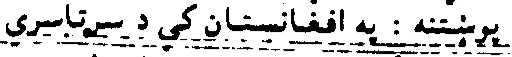

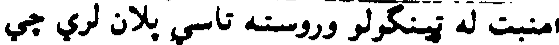

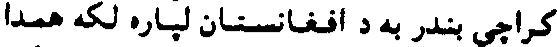

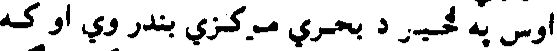

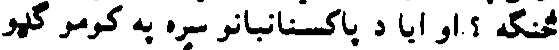

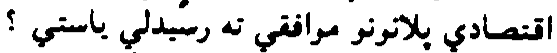

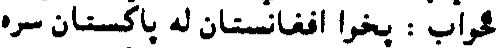

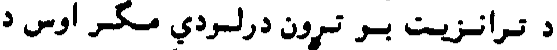

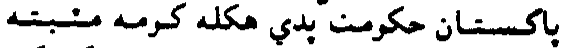

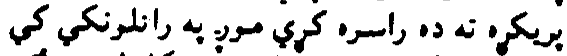

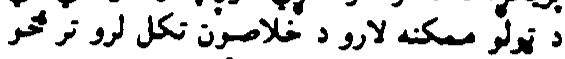
تمارت وده وكري مي ل

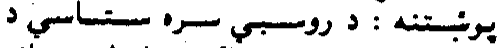

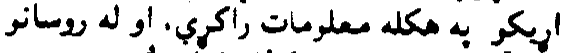

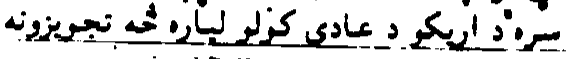

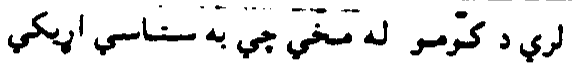

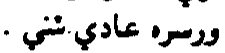

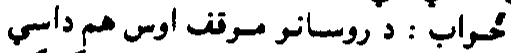

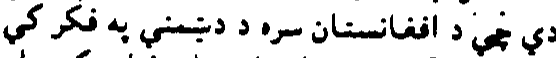

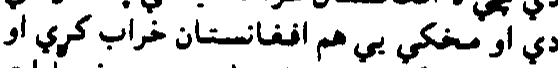

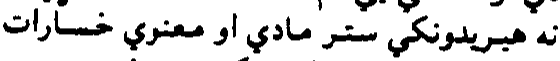

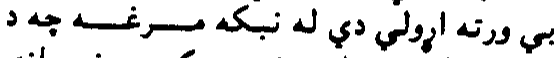

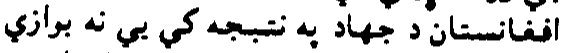

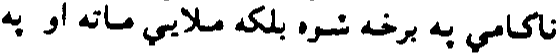

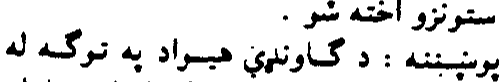

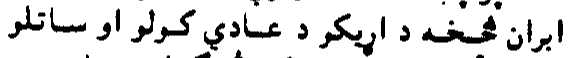

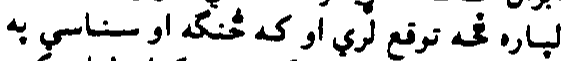

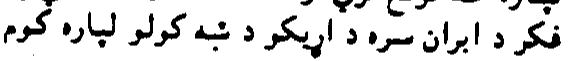

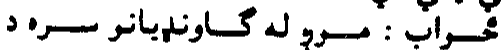

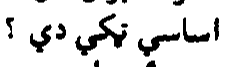

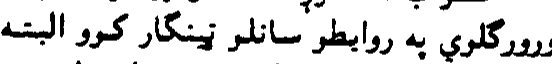

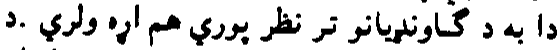

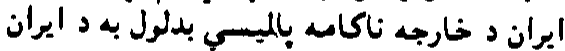

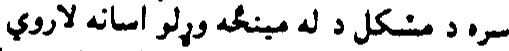

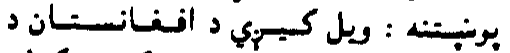

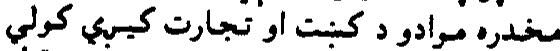

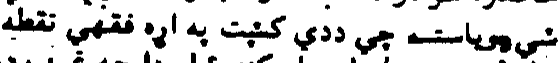

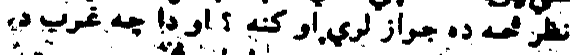

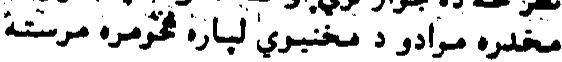

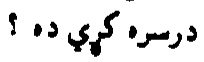

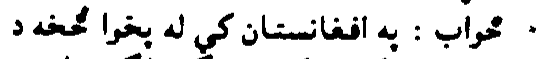

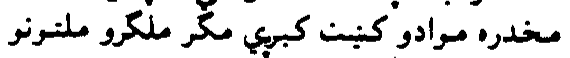

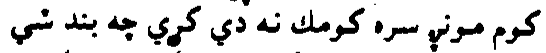

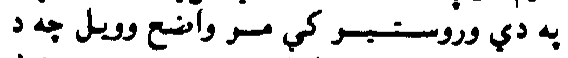

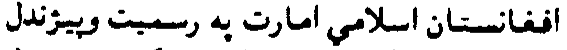

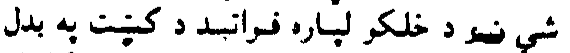

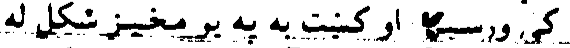

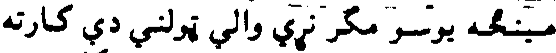

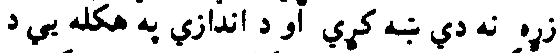

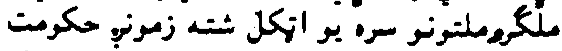

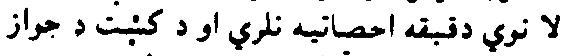

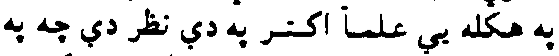

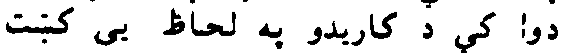

معاب :د بلوغ غنمه برته تر مغن ونته

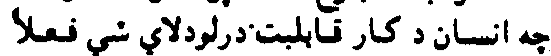

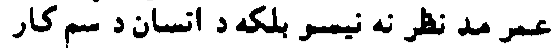

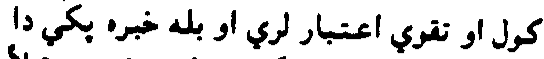

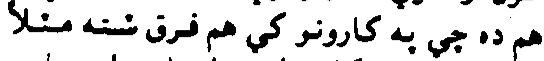

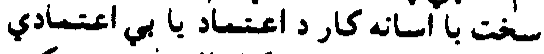

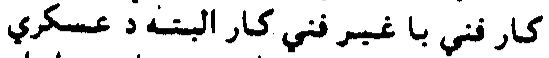

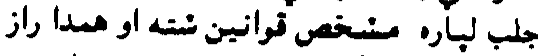

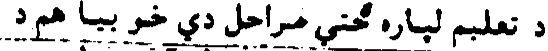

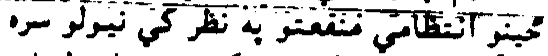

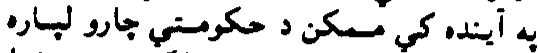

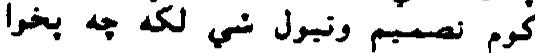

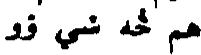

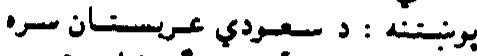

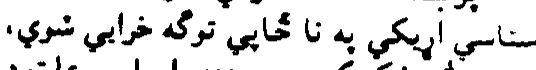

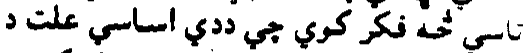

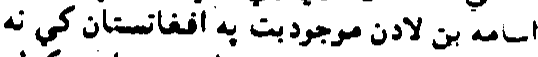

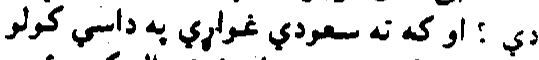

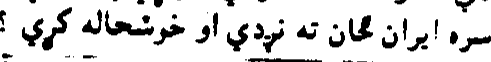

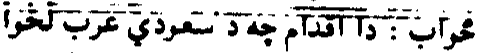

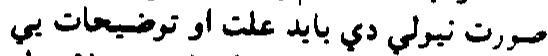

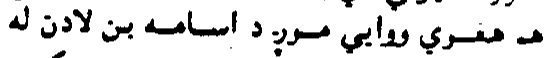

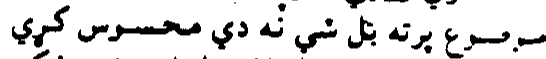

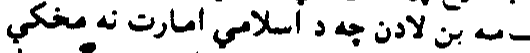

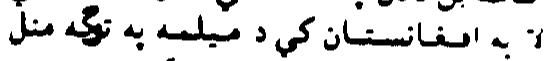

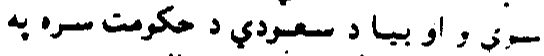

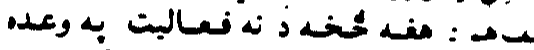

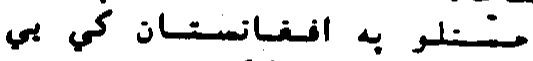

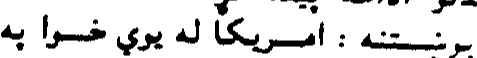

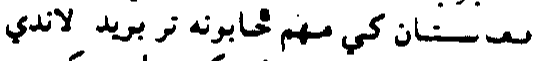

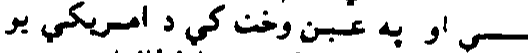

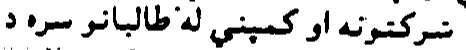

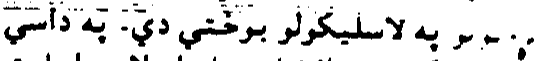

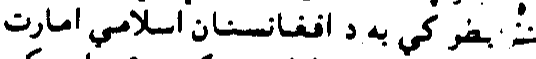

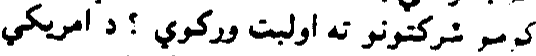

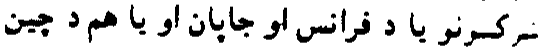

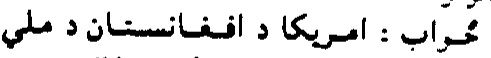

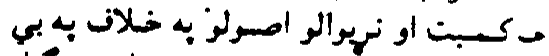

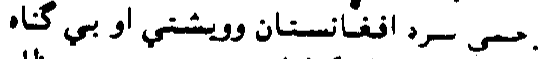

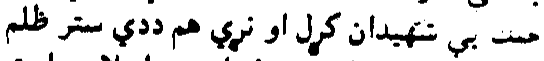

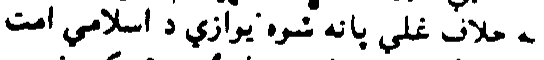

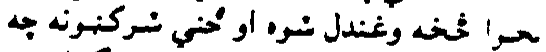

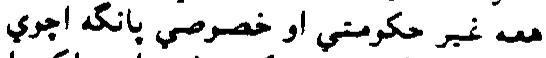

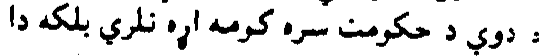

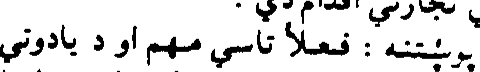

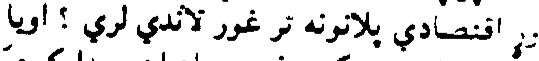

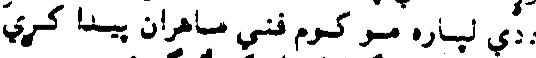

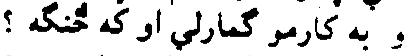

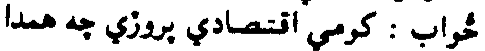

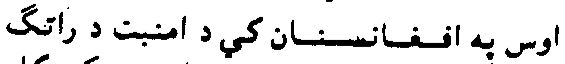

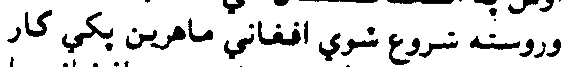

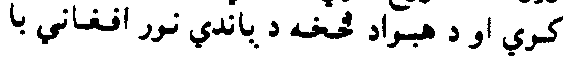




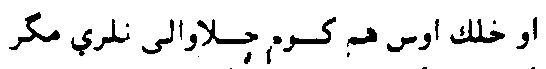

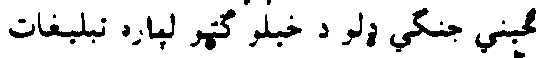

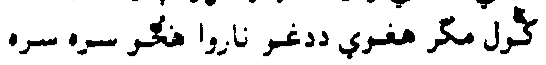

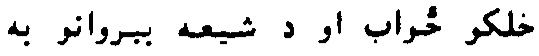

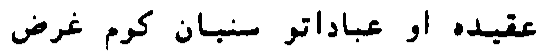

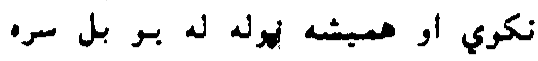

$$
\text { نبه روند تيروي مئد }
$$

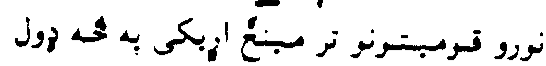

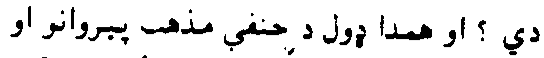

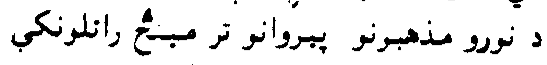

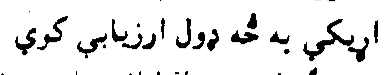

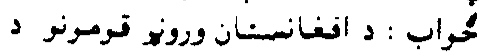

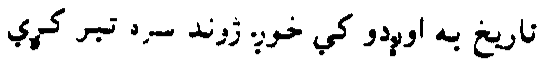

ضرورت لري زمونز مسبوط مسكاني له مغوي

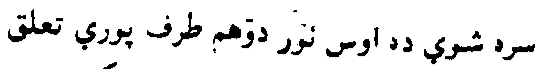

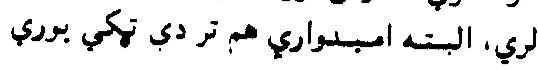
مورتونه

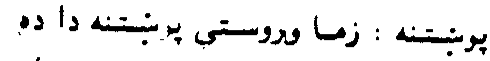

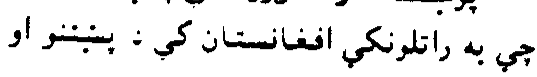

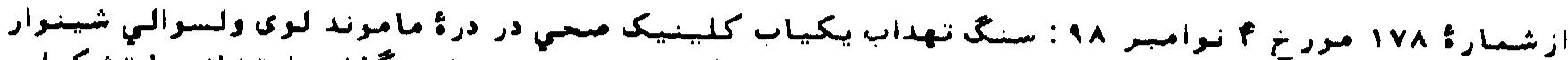

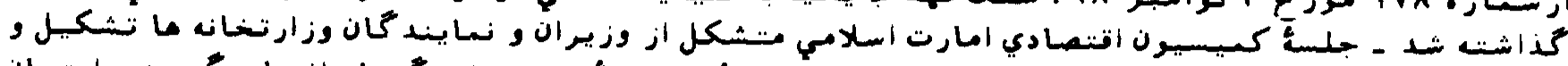

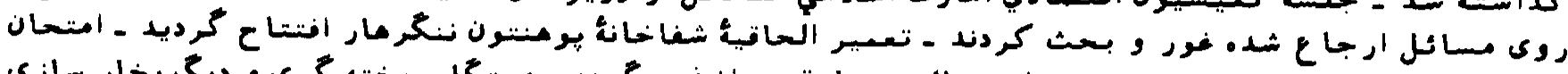

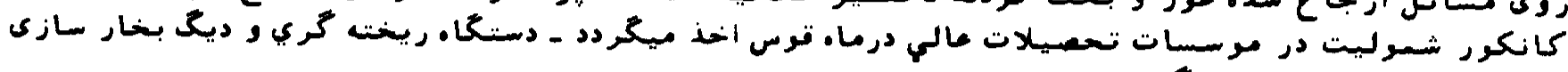

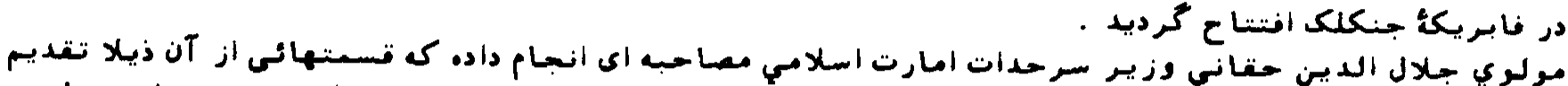

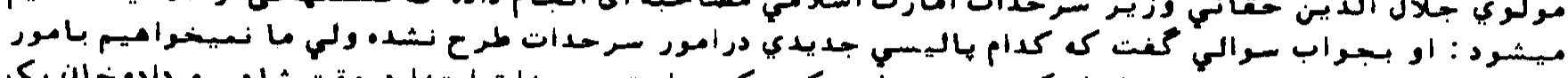

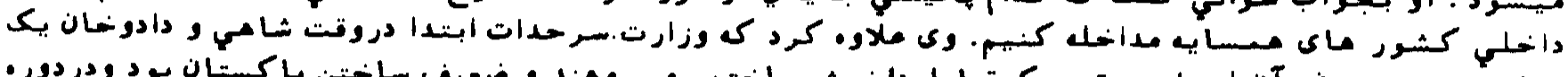

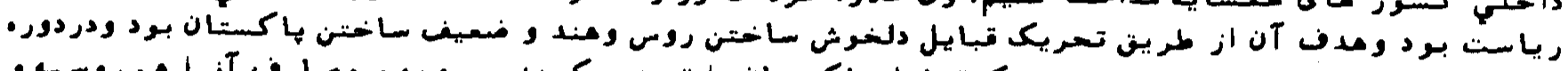

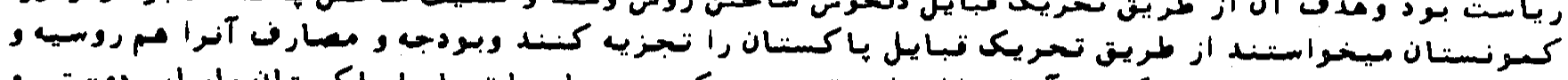

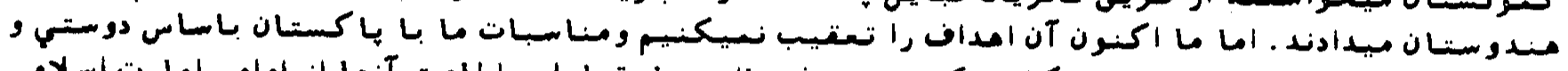

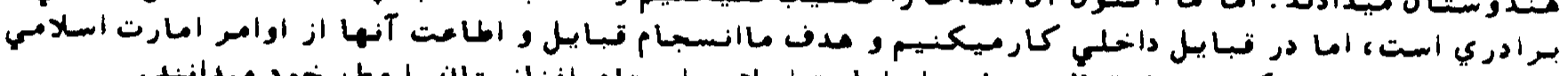

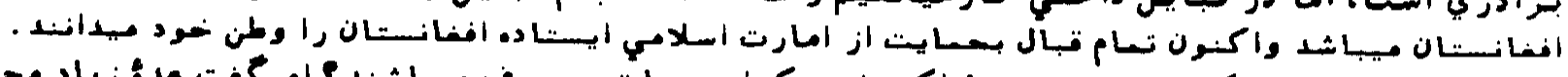

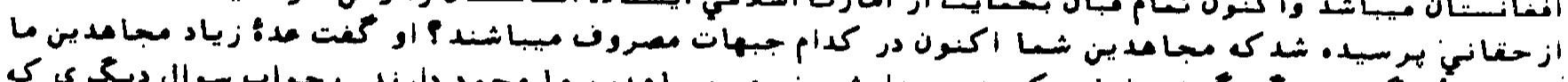

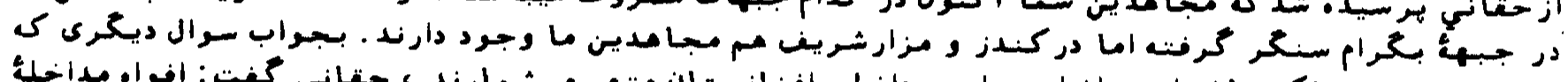

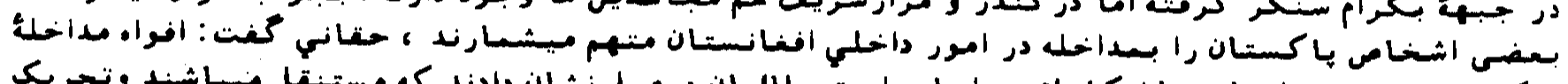

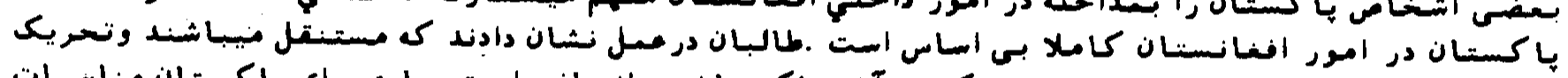

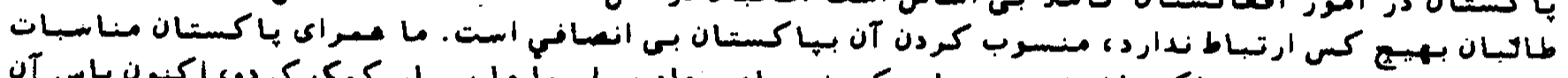

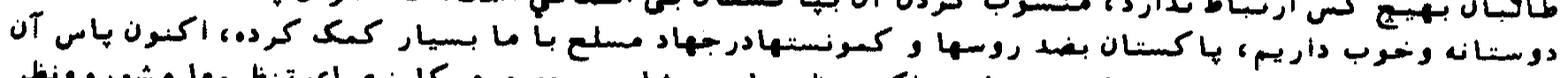

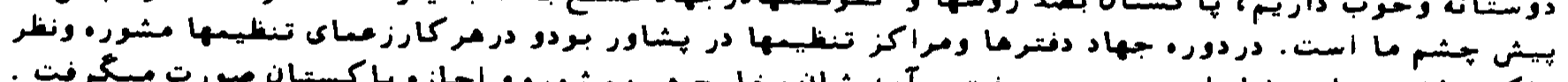

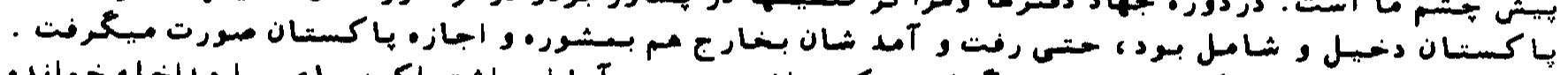

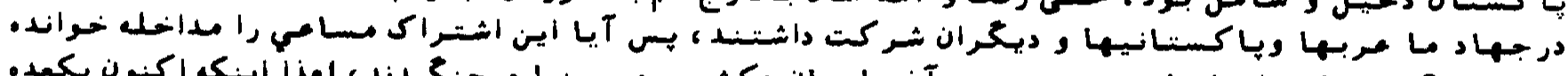

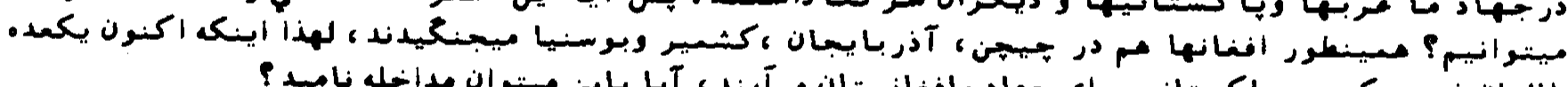

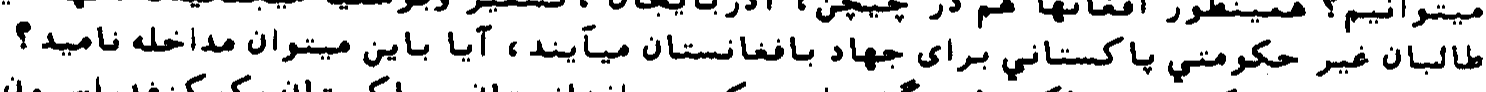

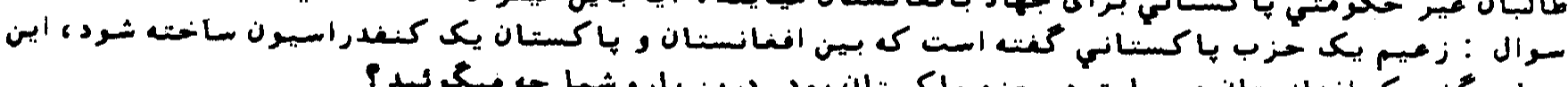

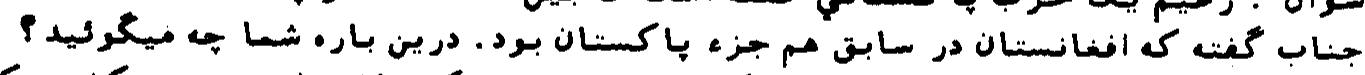

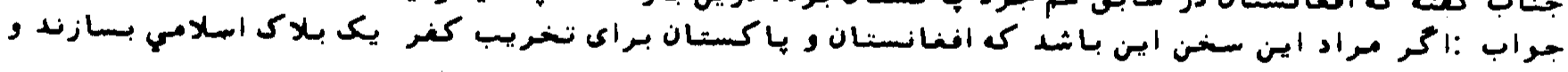

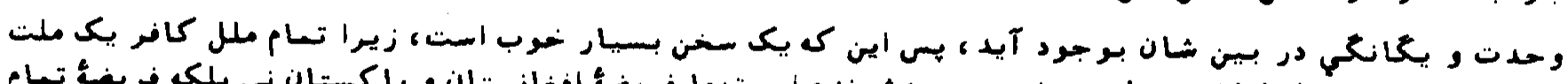

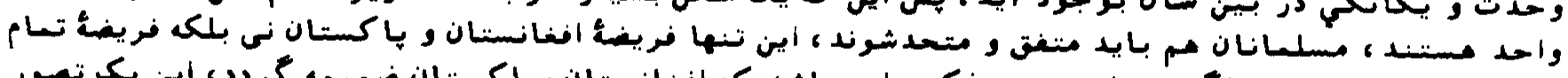

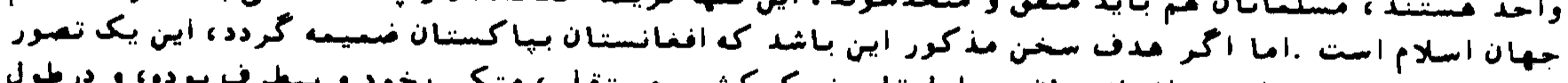

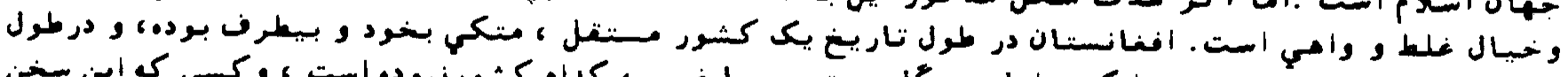

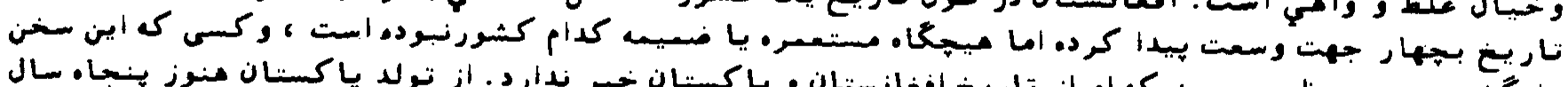

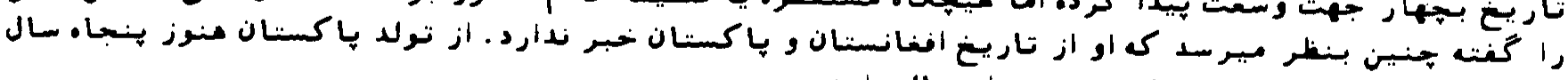

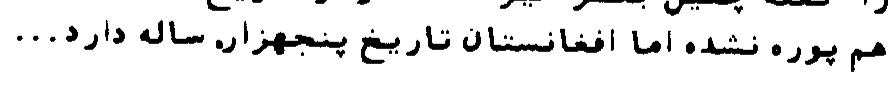

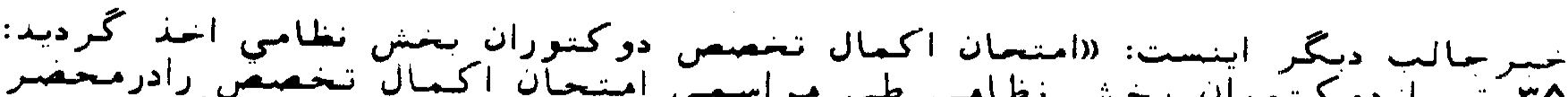

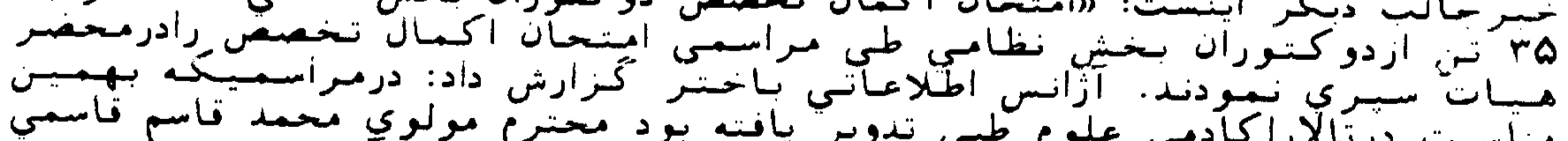

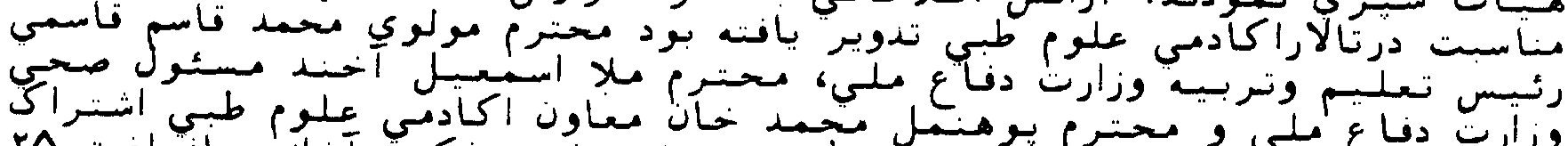

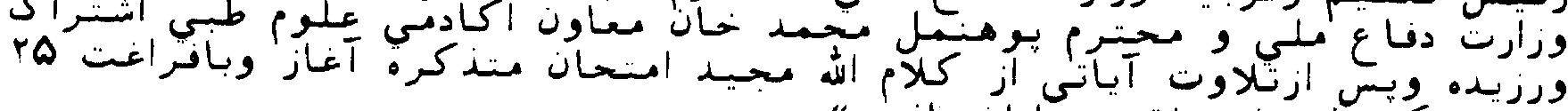

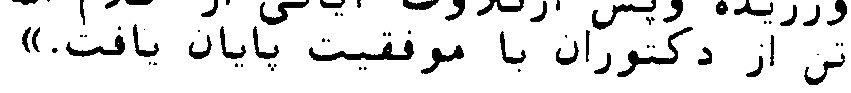




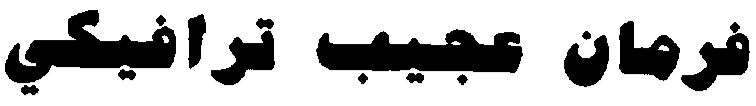

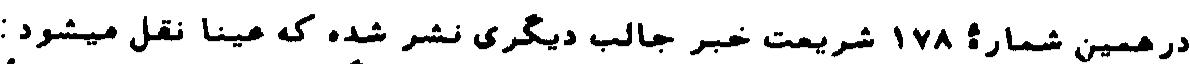

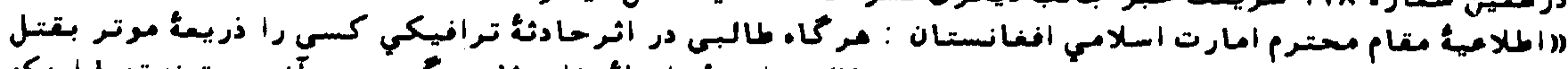

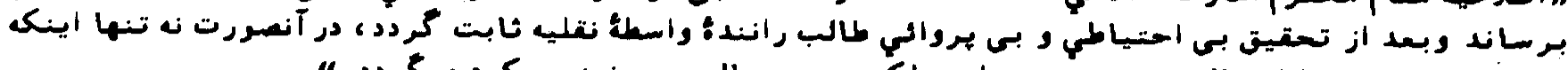

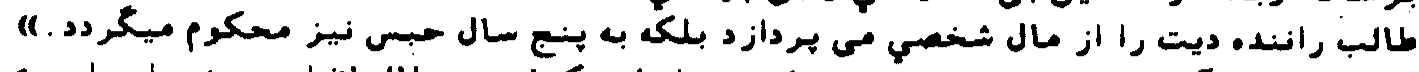

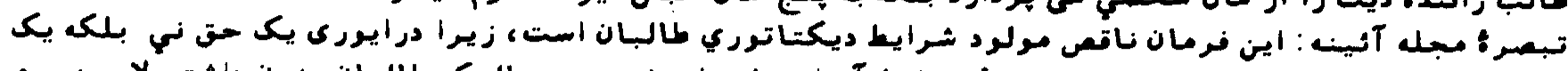

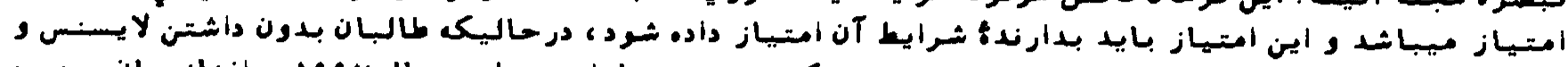

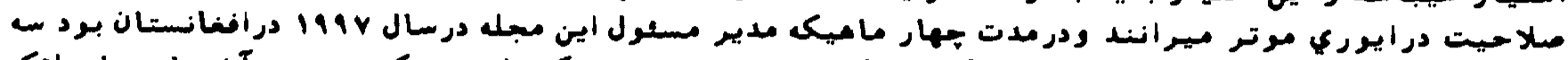

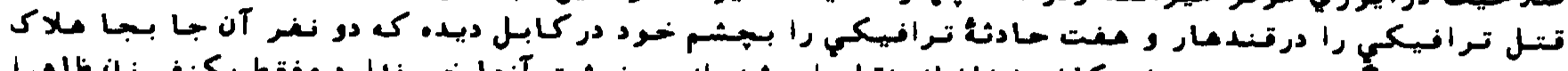

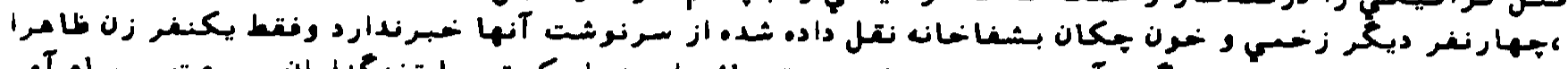

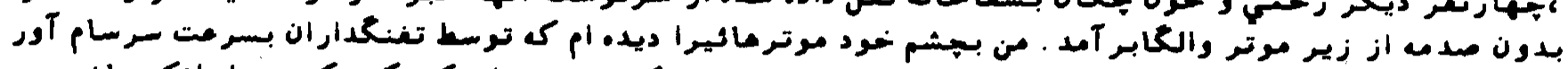

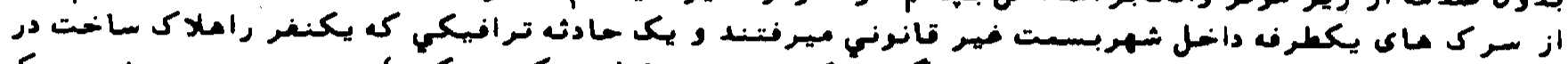

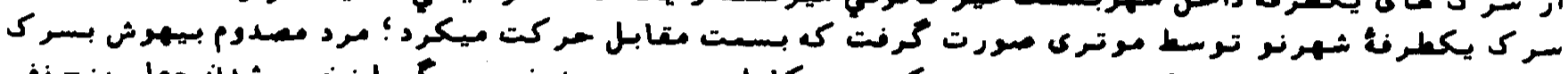

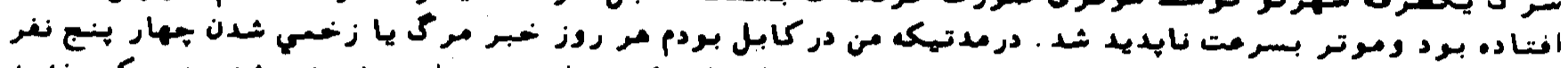

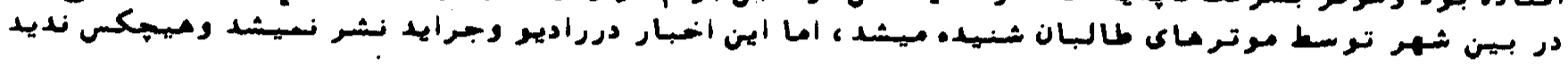

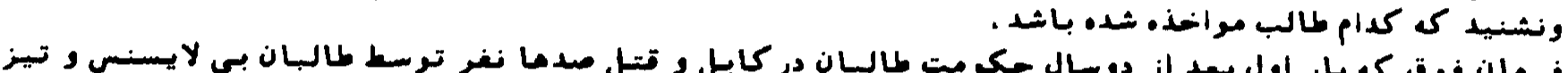

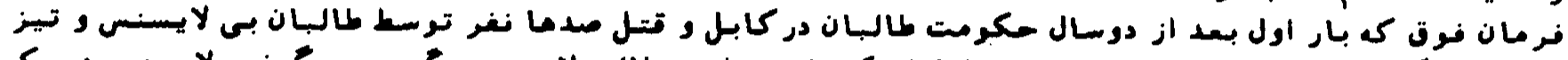

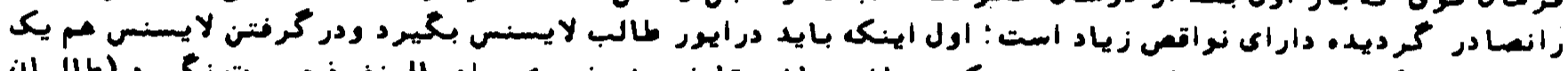

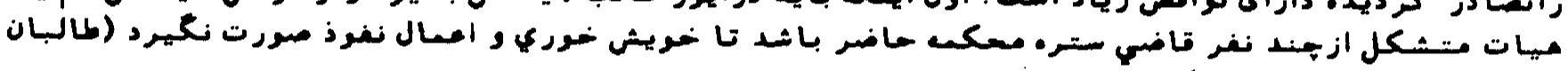

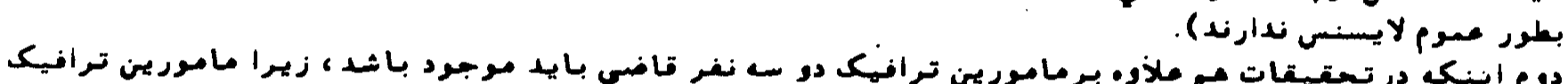

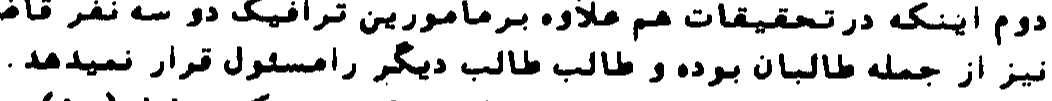

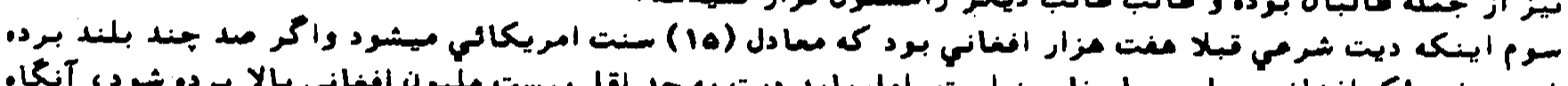

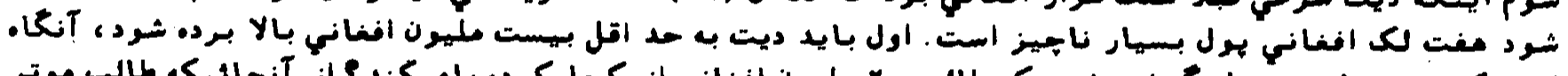

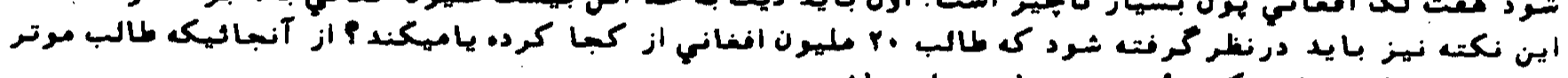

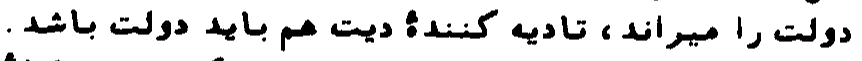

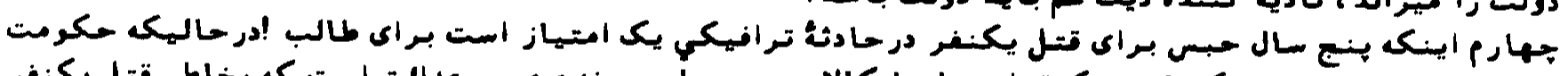

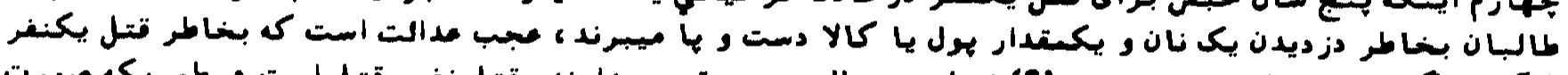

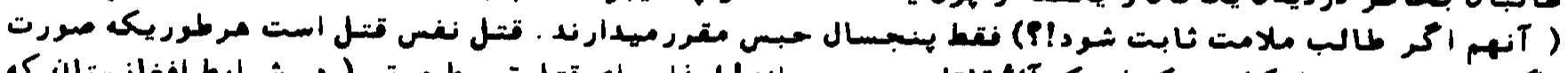

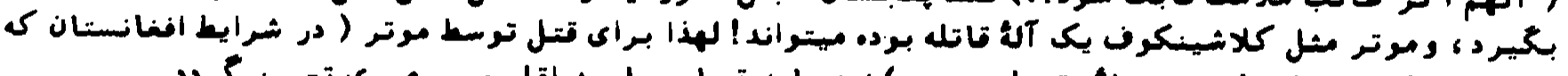

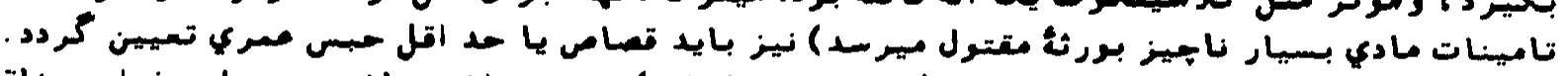

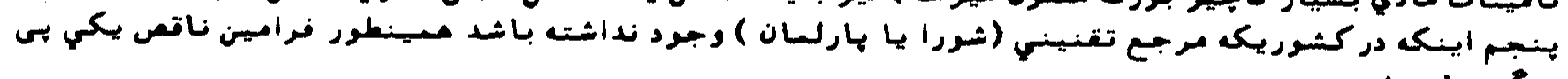

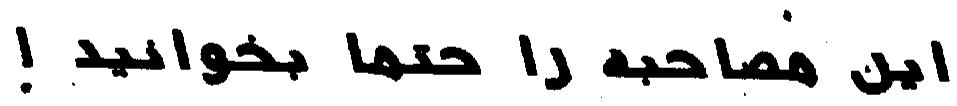

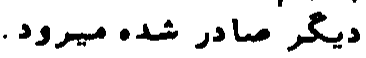

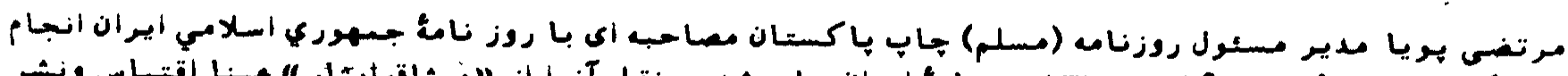

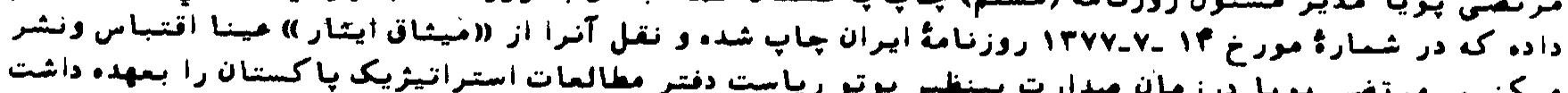

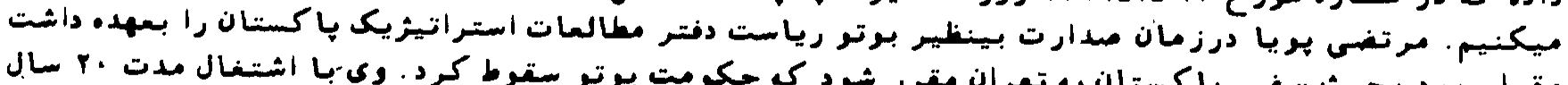

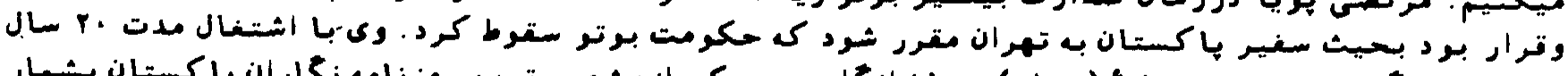

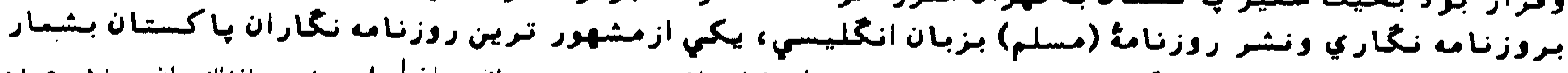

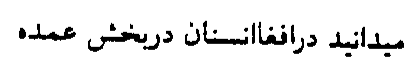

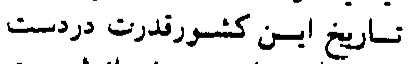

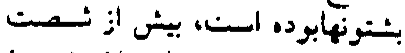

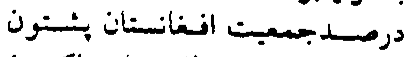

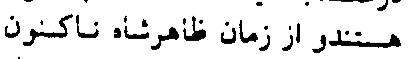

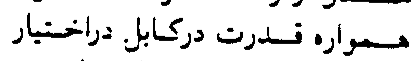

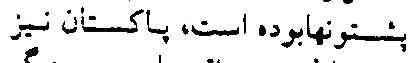

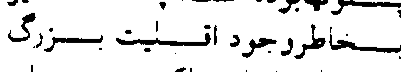

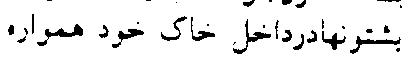

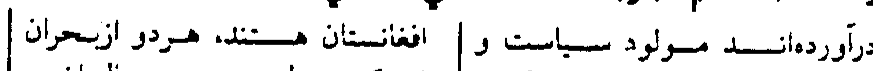

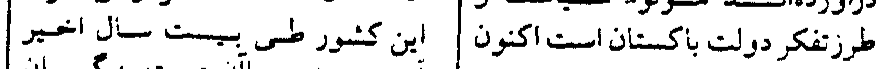

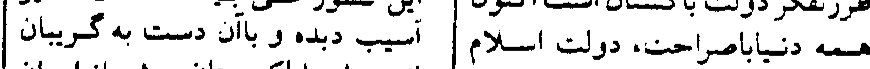

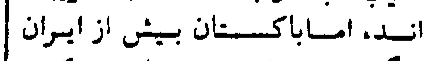

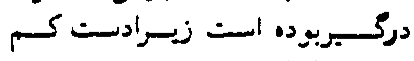

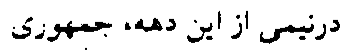

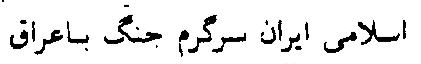

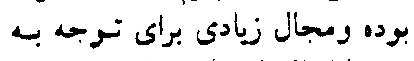

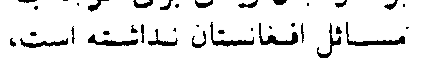

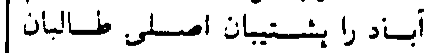

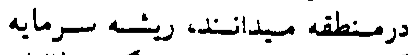
كذارى باكسنان بر روى كرز، طألبان به بودs أسـت بكان

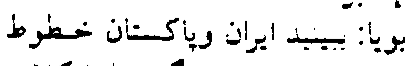

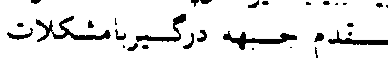

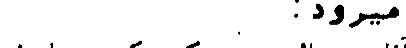
أفاى بوبا! نصورميكنبد كد سـوادث

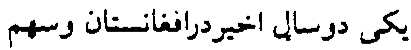

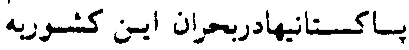

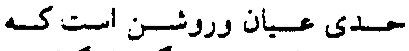
مقأمأت باكسنانى ديكرمثل عحندينه

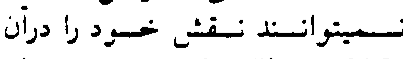

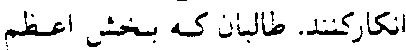

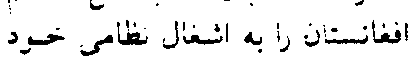




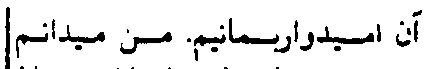

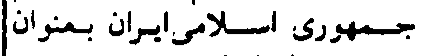

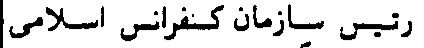

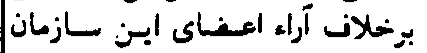

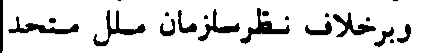

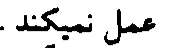

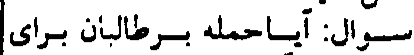

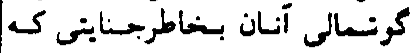

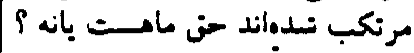

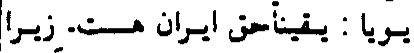

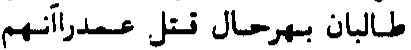

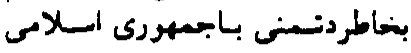

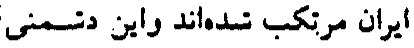

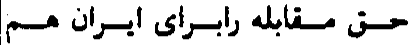

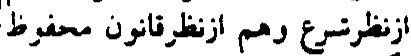

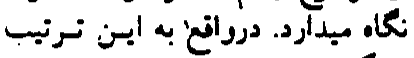

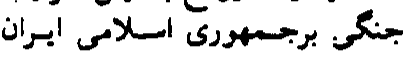

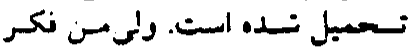

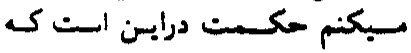

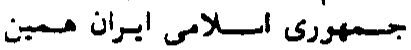

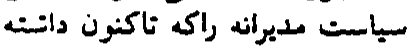

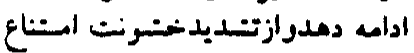

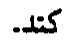
سوالى: جراجينين اعنقادى راداريد ؟

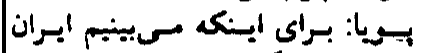

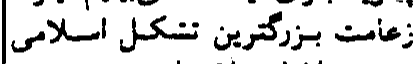

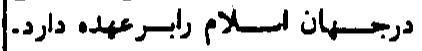

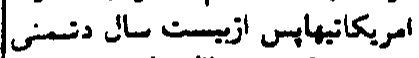

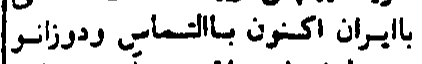

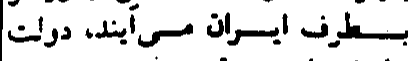

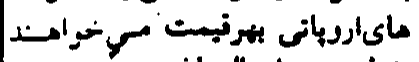

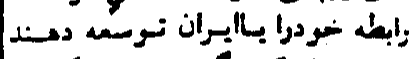

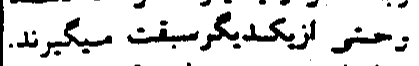

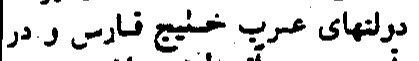

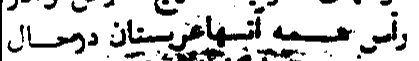

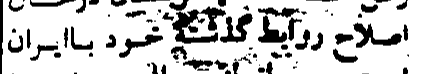
إنـا

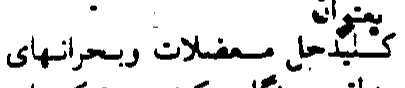

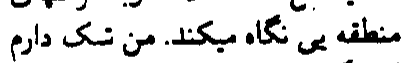

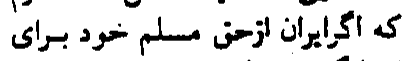

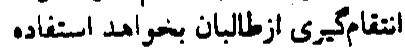

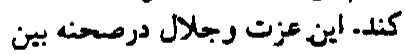

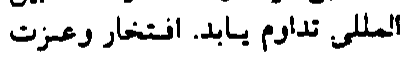

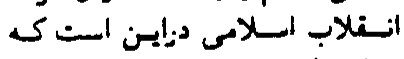

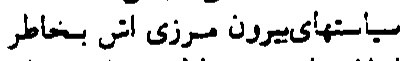

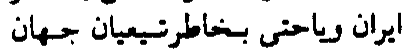

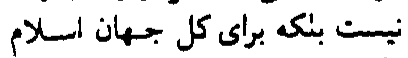

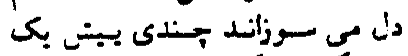

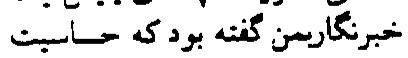

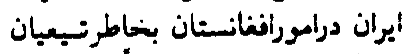

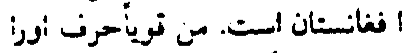

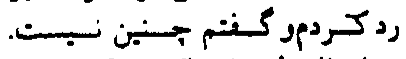

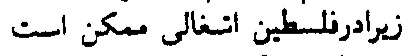

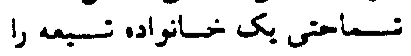

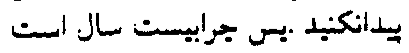

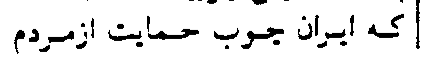

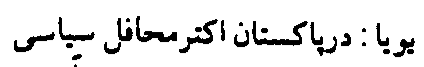

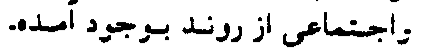

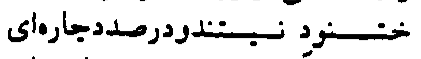

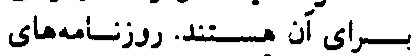

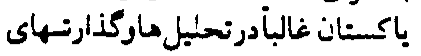
خودة. بـ نغع بمهورى أسلامى أبران

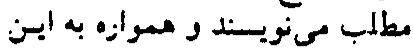

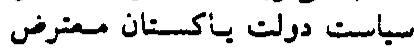

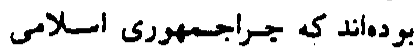

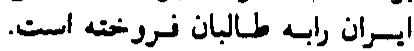

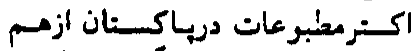
يـيانى دولت اسـلام آبـاد بـاطالبان

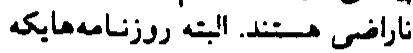

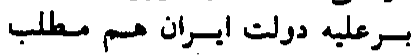

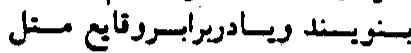

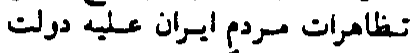

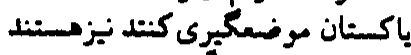

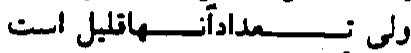

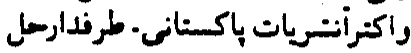

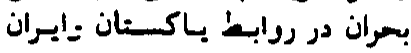

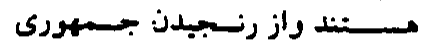

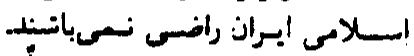

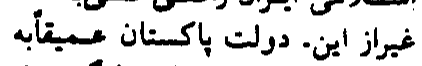

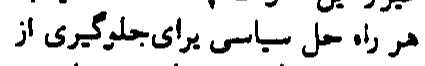

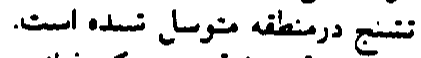

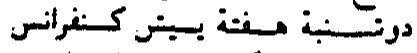

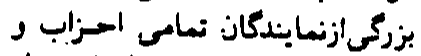

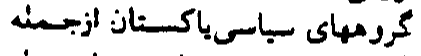

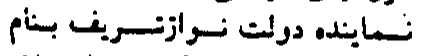

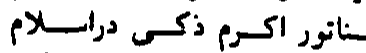

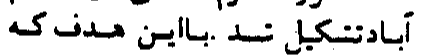

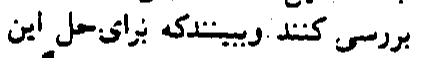

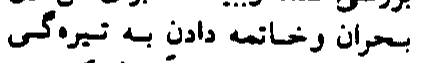

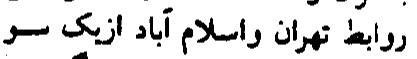

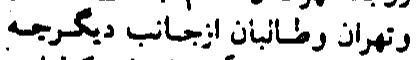

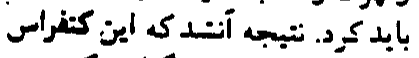

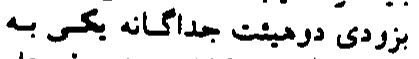

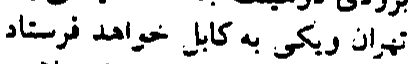

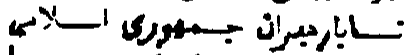

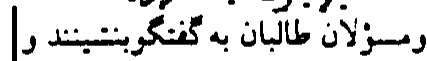

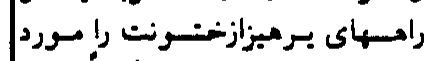

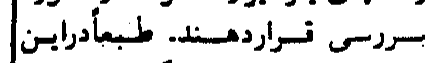

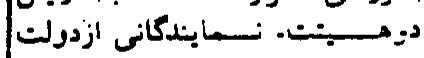

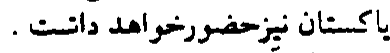

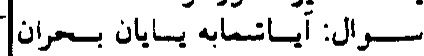

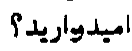

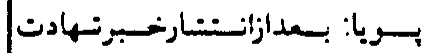
دييلماتهاىايرانى درمزارنسريف كي

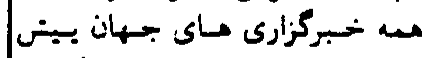
بـبنى كـرده بـودندأيسران بـ طـ طـالبان

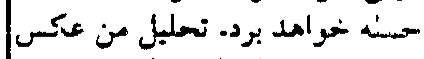

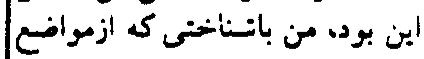

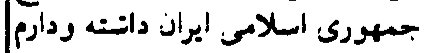

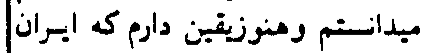

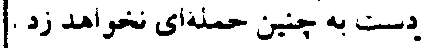

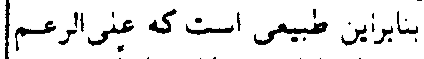

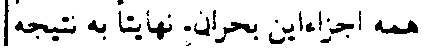

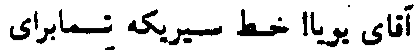

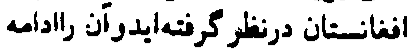

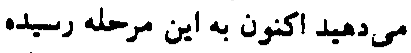

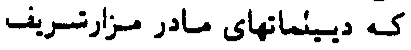

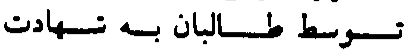
رسبدماند.سفيروكاركنان موترسينارت

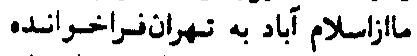

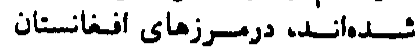

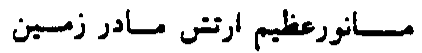

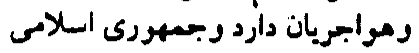

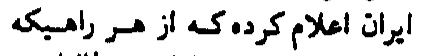

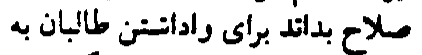

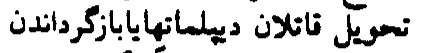

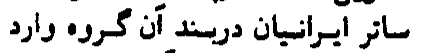

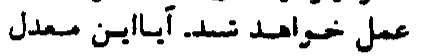

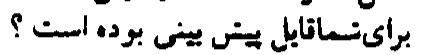

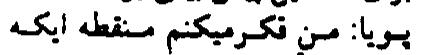
مرونسمادرأن نـراركرفثهايسم درحسال

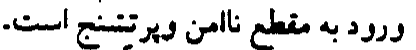

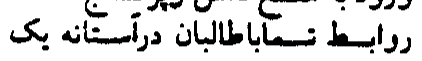

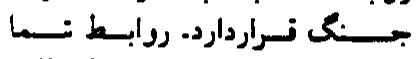

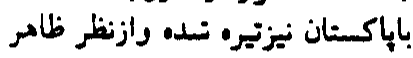

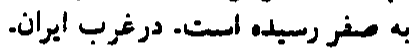

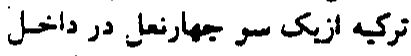

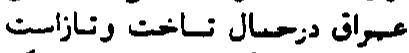

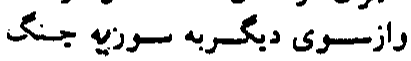

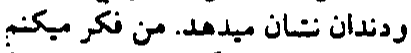

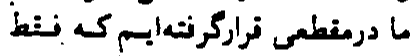

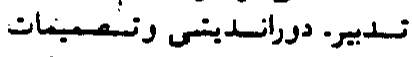

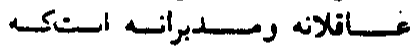

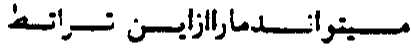

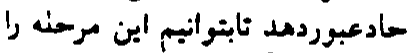

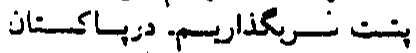

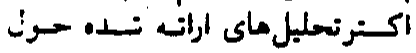

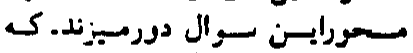

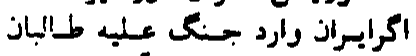

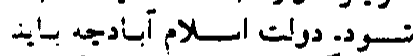

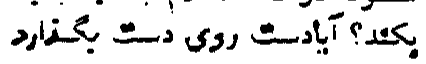

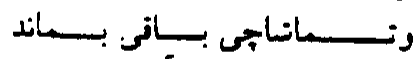

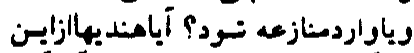

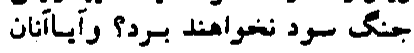

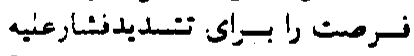

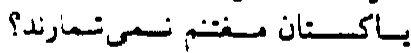

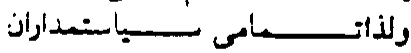

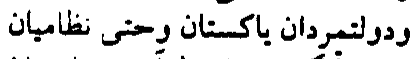

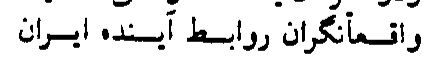

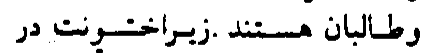

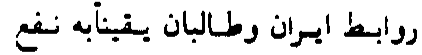

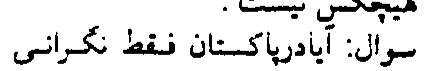

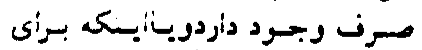

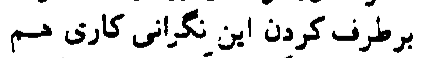

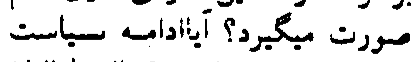

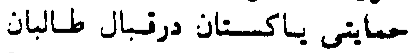
بردامنه بحران نسى افزابد ودرنتيجني

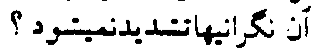

سعى درحسبابت ازيتــنونها دأتسنه

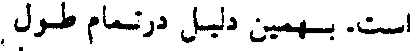

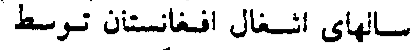

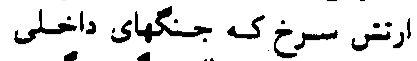

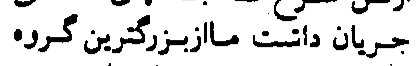

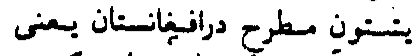

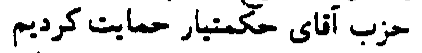

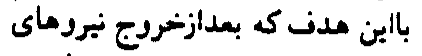

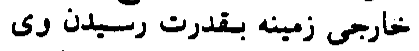

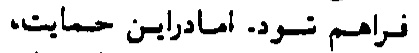

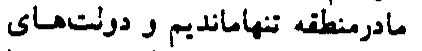

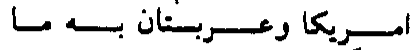

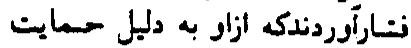

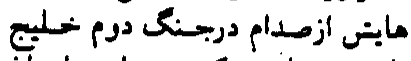

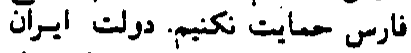
مـم حساظـربه حسبابت ازمكسيتبار

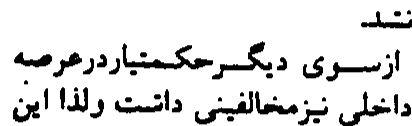

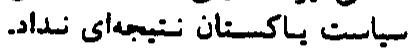

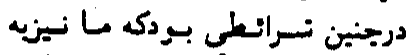

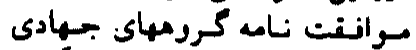

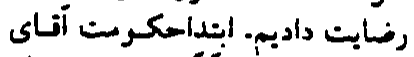

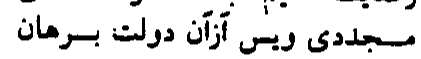

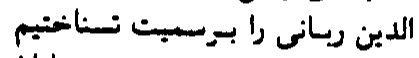

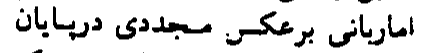

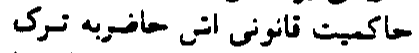

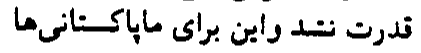

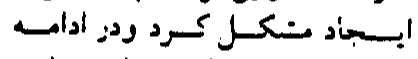

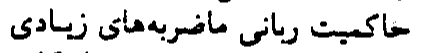

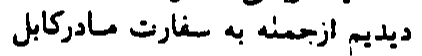

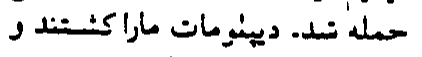

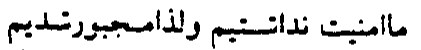

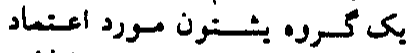

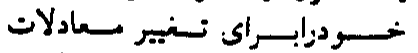

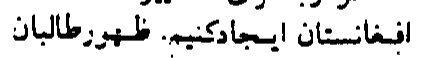

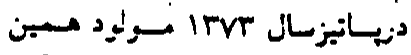

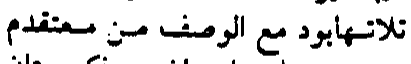

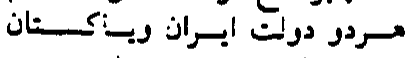

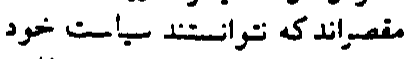

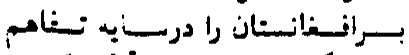

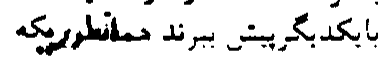
مردر دولت تربانى سروادت دردئن

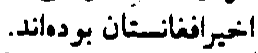

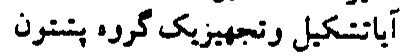

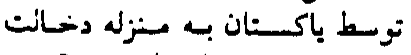

درامور داشخلى افغانســان نبود

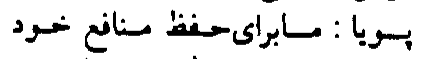

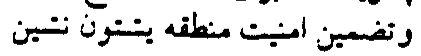

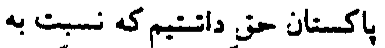

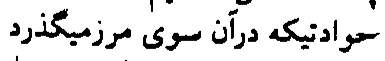

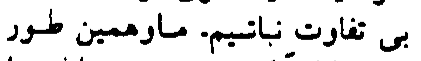

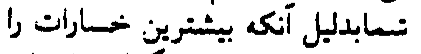

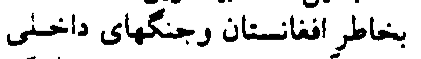

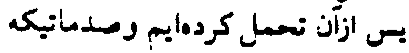

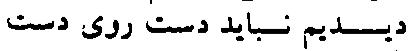

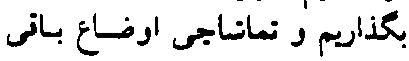




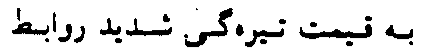

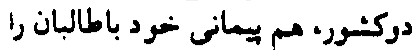

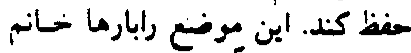

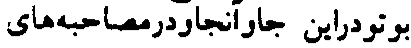

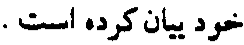

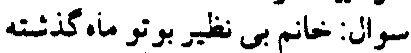
درمعاسبه بأروزنامه انكنيسى زئن زيان

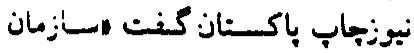

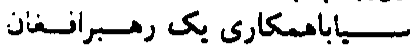

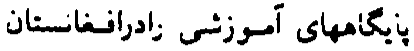

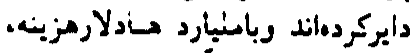

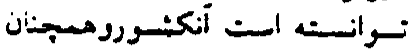

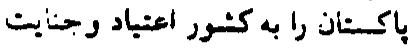

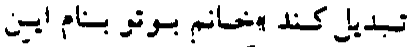

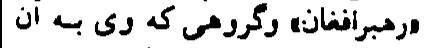

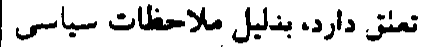

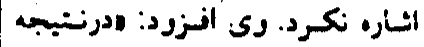

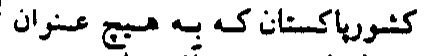

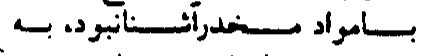

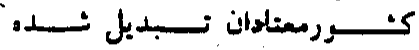

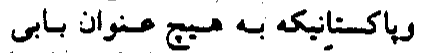

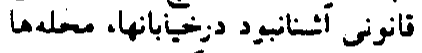

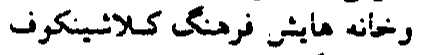

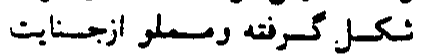

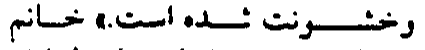

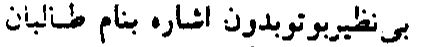

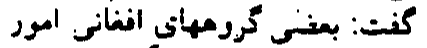

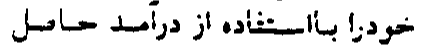

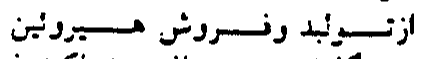

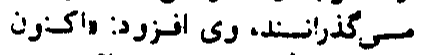

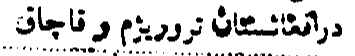

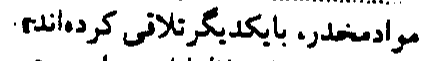
نحليل نسمالزاين. اظظهاراث خحانم بونو

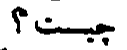

بويا: فرمنك كلانيسنكون دترويج

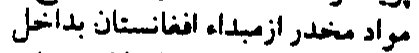

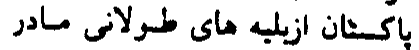

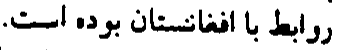
مستهل ابـن بـلا در دوران طـالبان

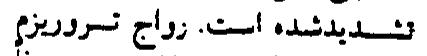

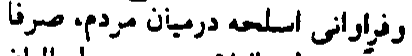

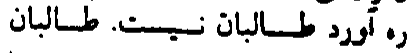

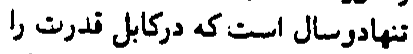

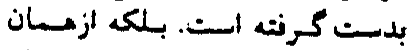

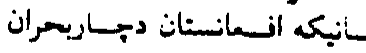

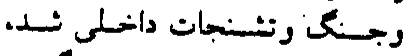

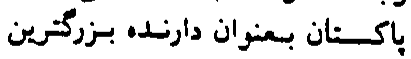

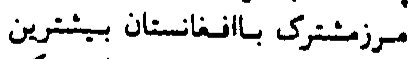

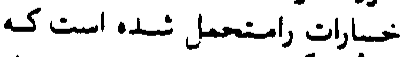

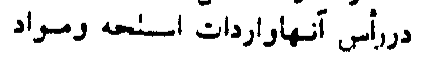
سوال :بنظريسماجرا عربستان إنسان روابط

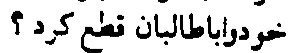

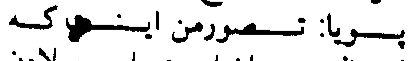

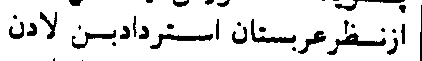
|نوسط طالبان بك خردرنت أساس لادرن

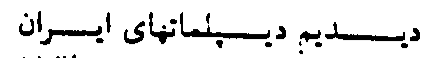

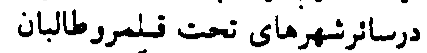

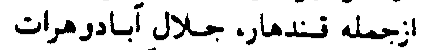

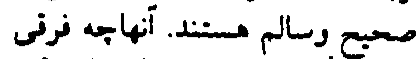

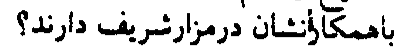

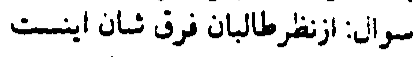

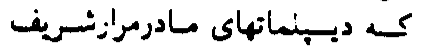

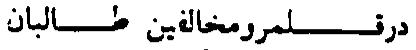

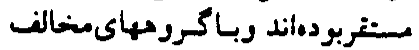
طابِن كازمبكردند.

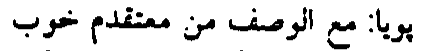

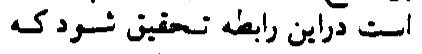

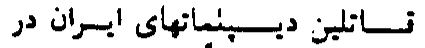

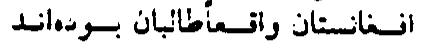

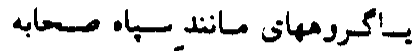

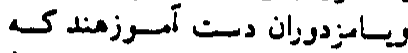

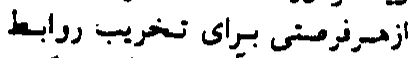

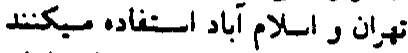

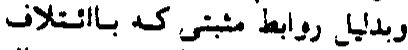

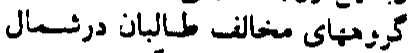

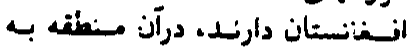

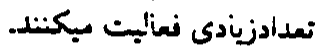

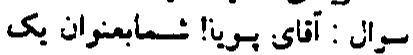

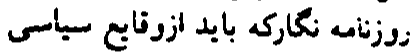

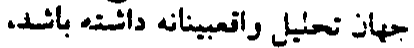

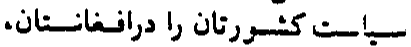

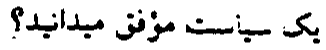

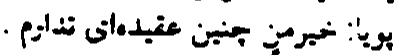

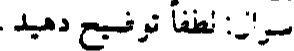

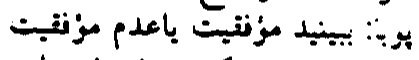

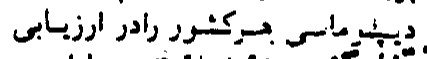
i

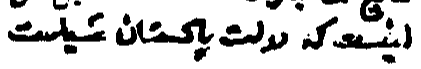

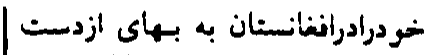

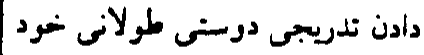

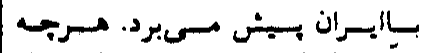

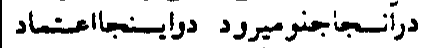

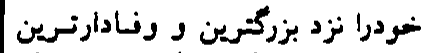

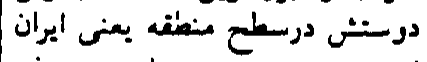

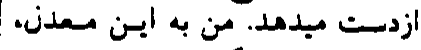

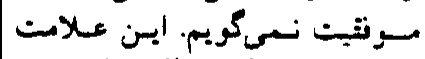

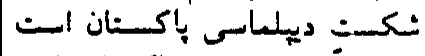

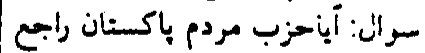

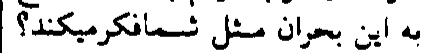

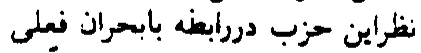

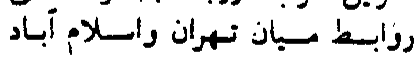

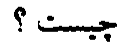
بويا: حـزب مردم بـ بـ رمبرى، بس

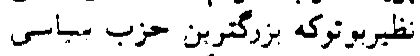

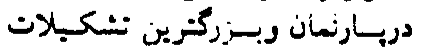

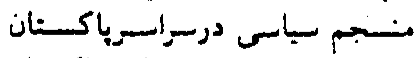

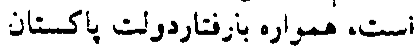
درنبال ايراني وطابان مخانفت كـرده

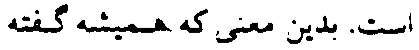

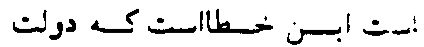

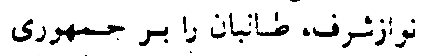
إسلامى ايران نرجبح دمد بطوريك
بكسىنتف حسريم انفغانستان، دوم

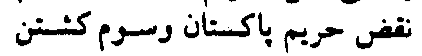

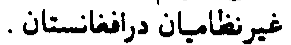

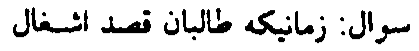

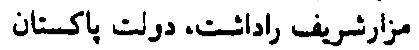

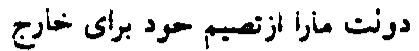

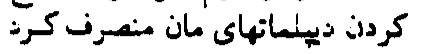

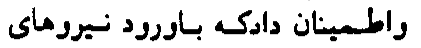

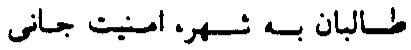

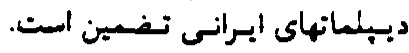

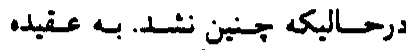

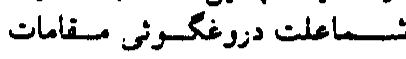

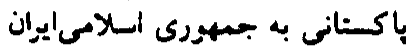
جبه بود؟

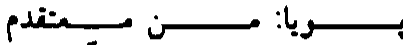

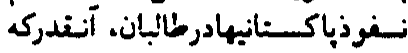

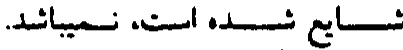

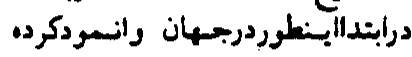

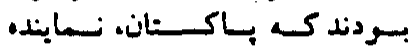

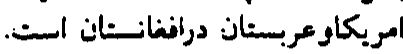
اماحمله مـونسكى المـريكابه نـلمرد

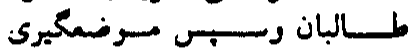

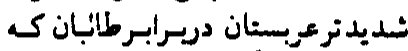

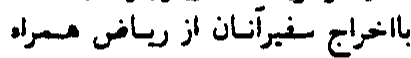

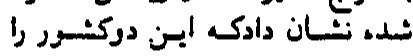

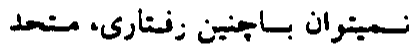

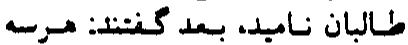

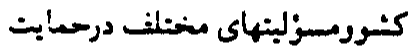

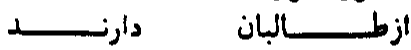

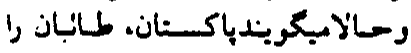

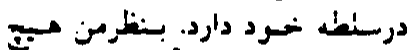

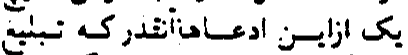

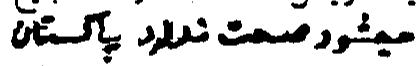

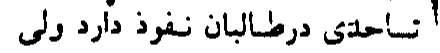

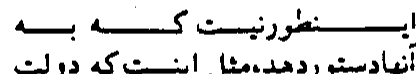

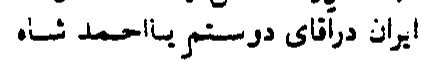

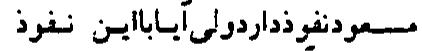

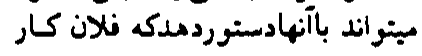

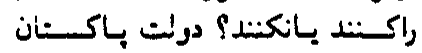

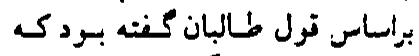

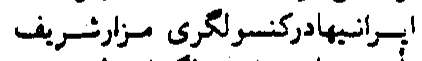

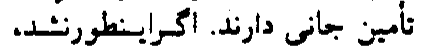

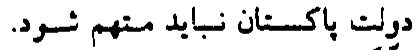

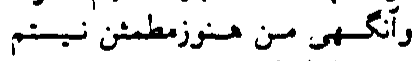

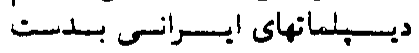

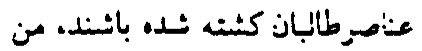

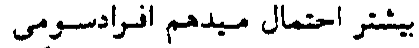

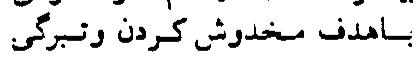

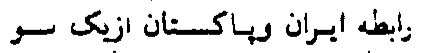

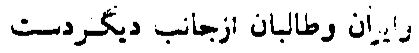

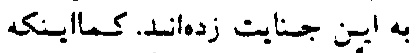

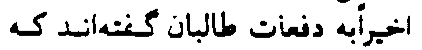

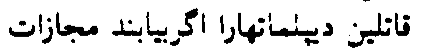

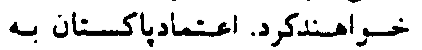

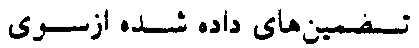

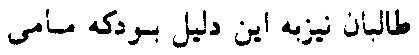

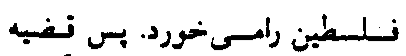

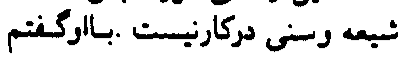

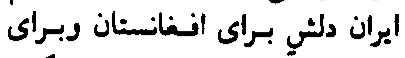

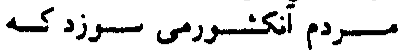

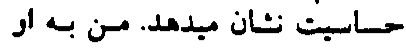

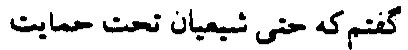

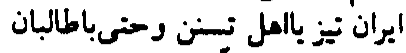

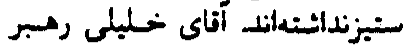

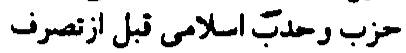

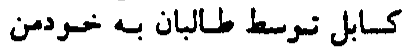

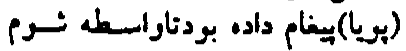

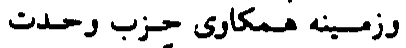

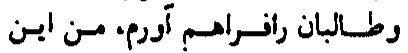

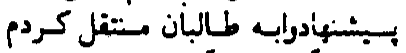

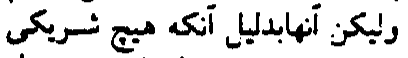

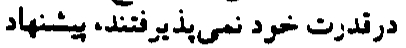

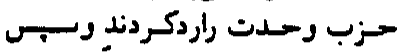

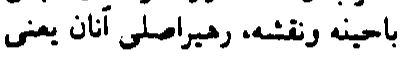

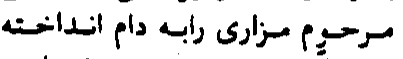

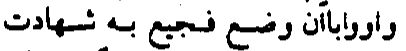

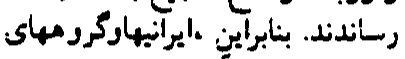

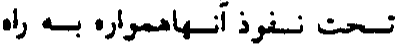

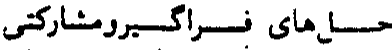

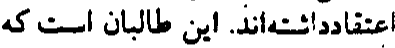
مشاركت درنظام رمبرى انـغانستان

$$
\text { را نسم بذيرد }
$$

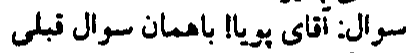

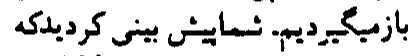

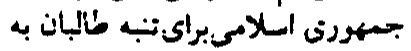

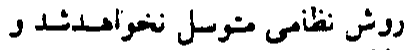

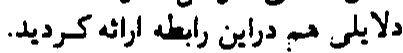

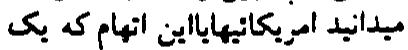

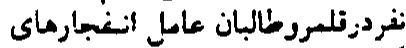

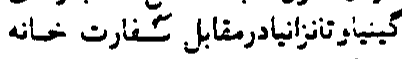

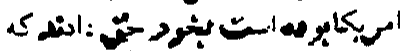

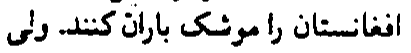

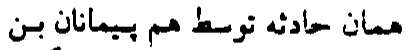

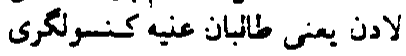

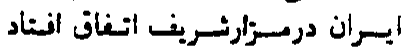

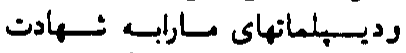

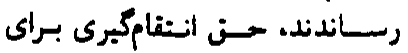

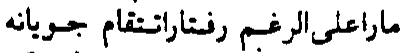

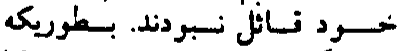

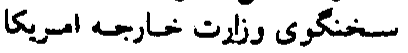

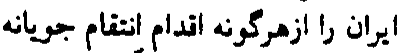

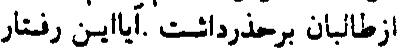

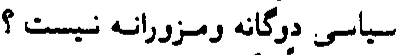

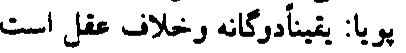

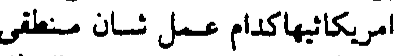

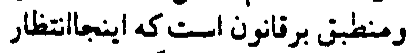

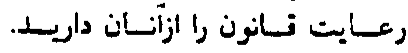

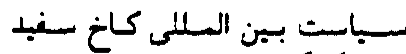

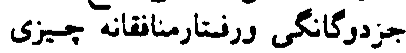

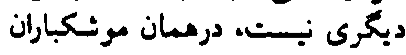

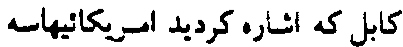

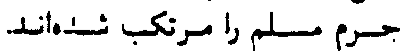




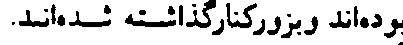

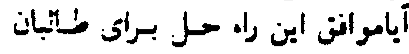

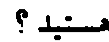

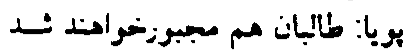

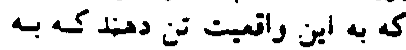

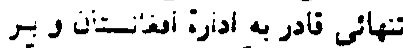

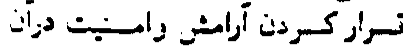

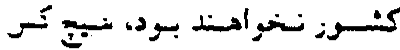

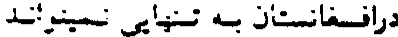

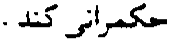

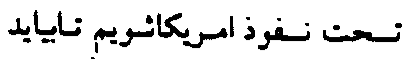

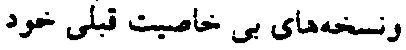

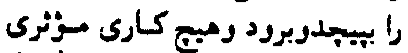

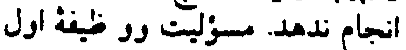

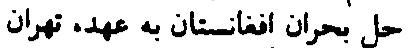

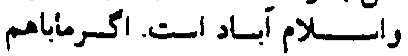

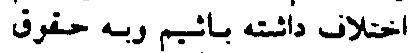

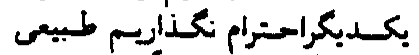

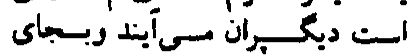

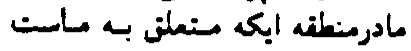

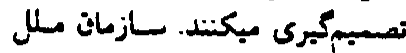

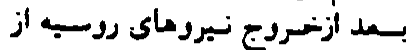

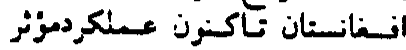

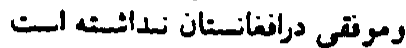

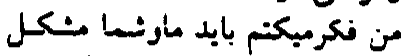

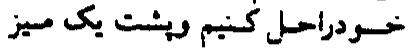

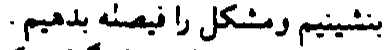

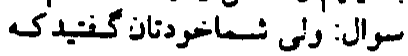

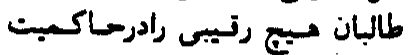

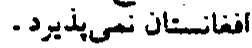

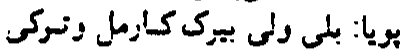

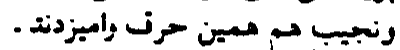

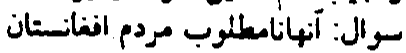

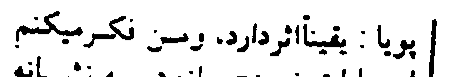

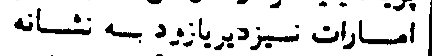

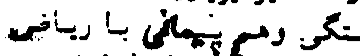

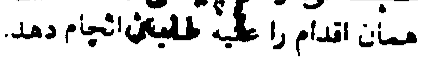

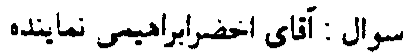

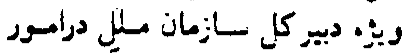

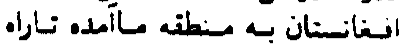

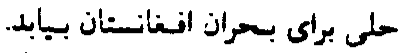

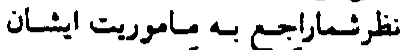

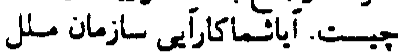

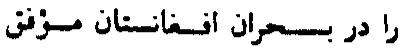
مبدانبد؟

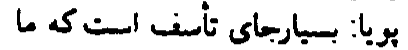

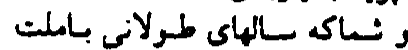

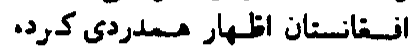

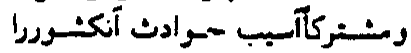

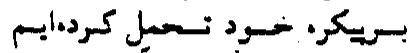

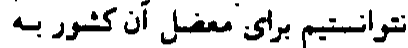
بكى نورمول منــرك يرسبم. درواتع

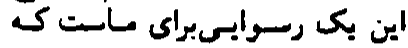

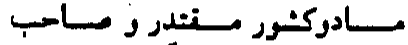

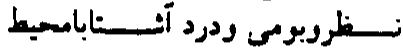

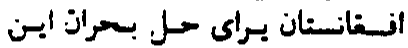

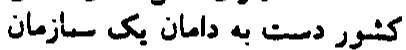

رأست. دولت عسربستان بـمصوم

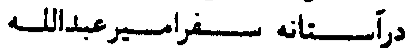

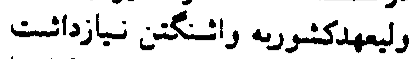

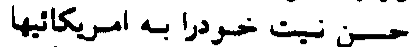

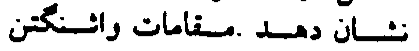

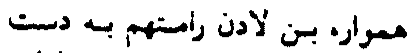

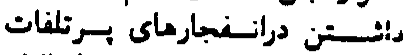

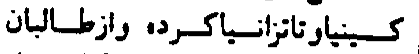

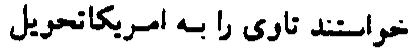

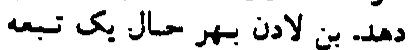

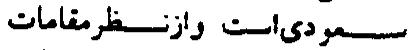

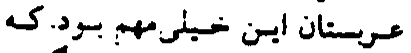

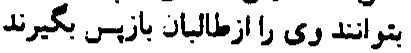

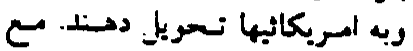

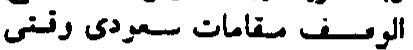

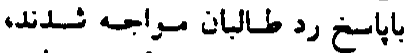

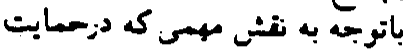

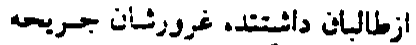

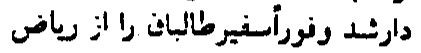

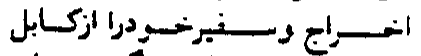

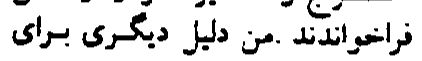

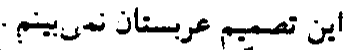

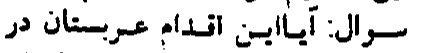

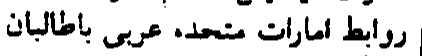

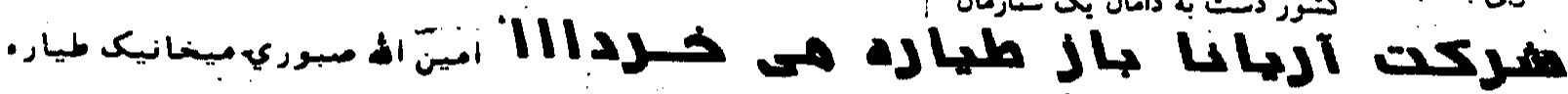

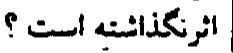

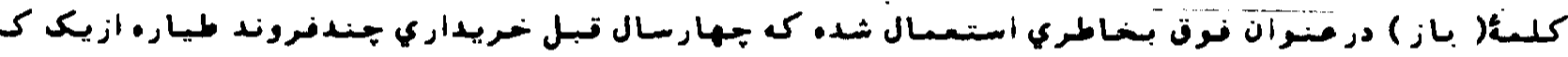

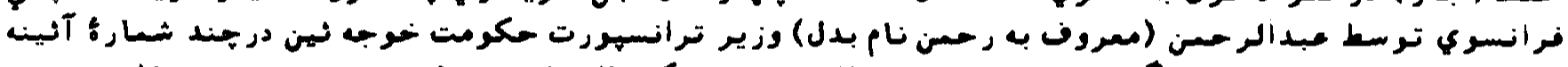

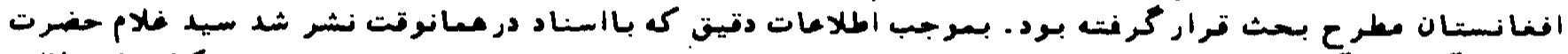

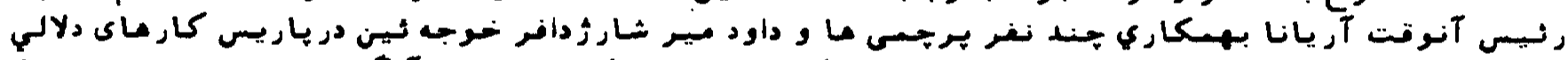

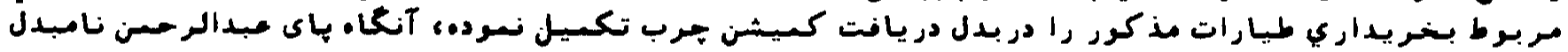

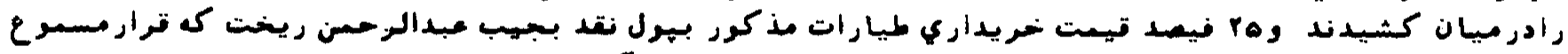

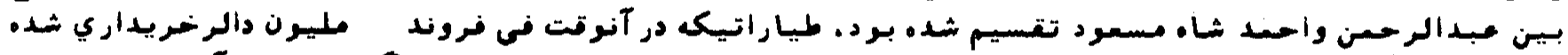

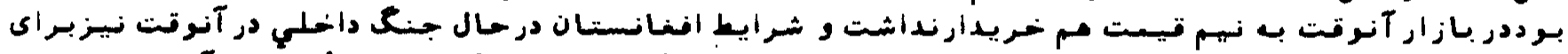

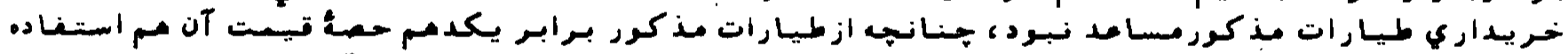

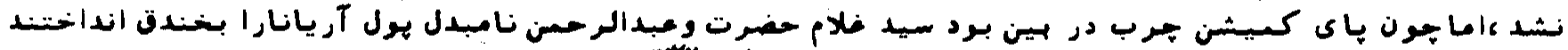

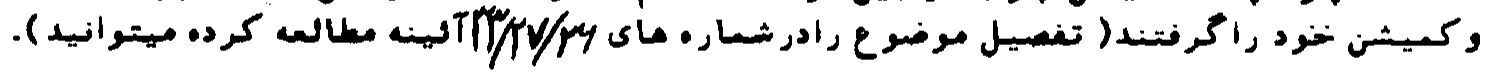

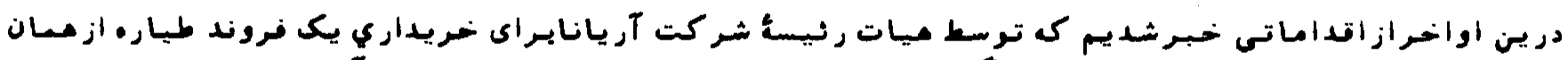

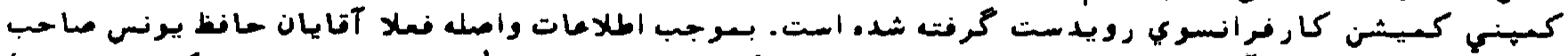

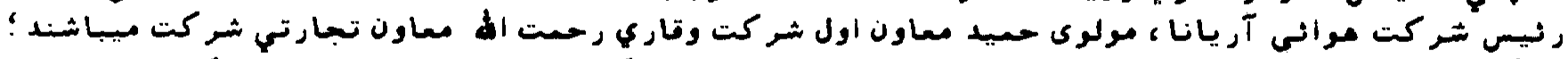

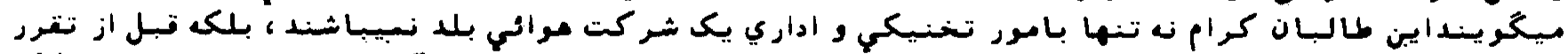

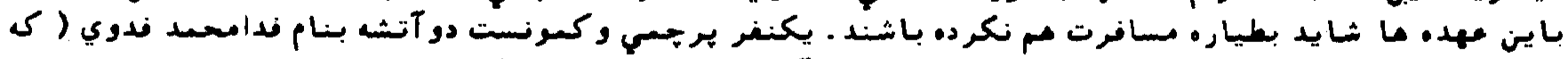

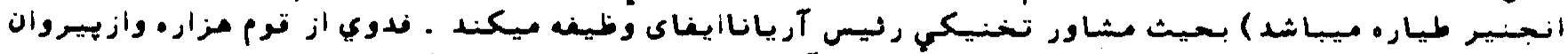

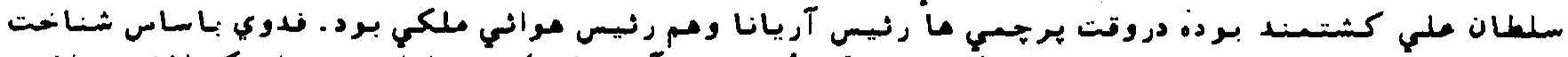

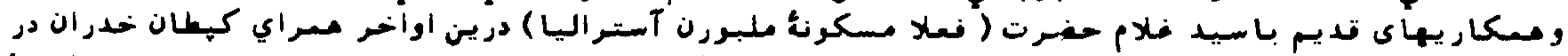

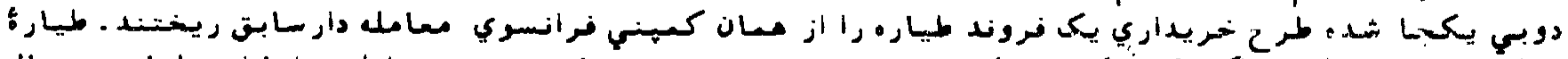

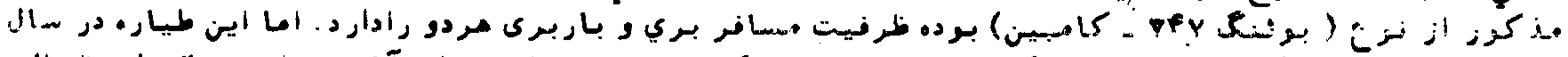

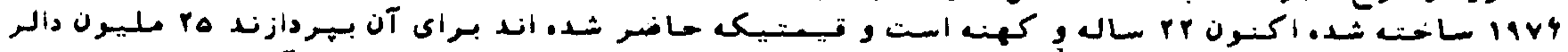

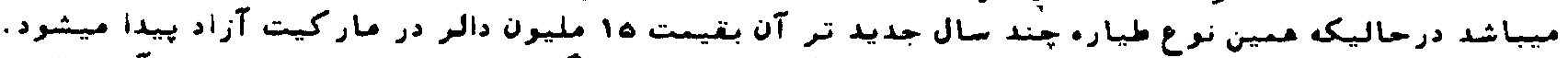

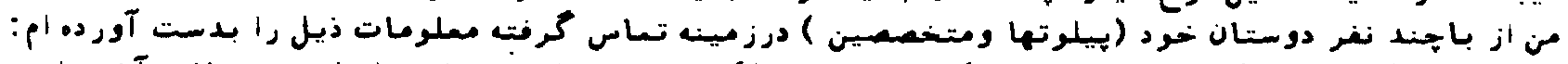

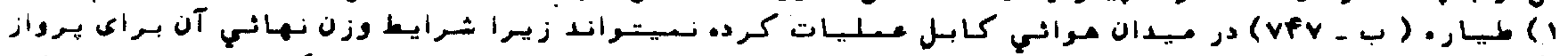

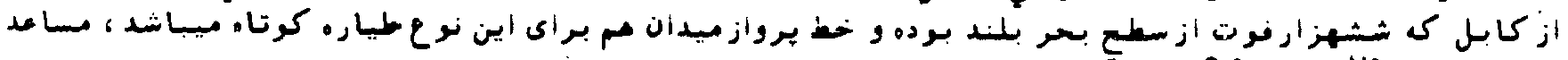




\section{المدها وصموق بشل 19}

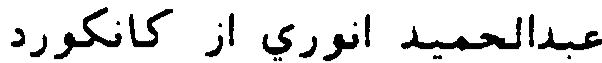

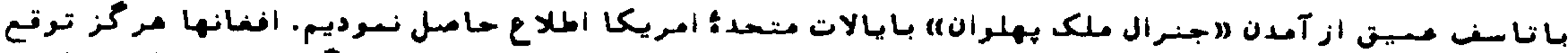

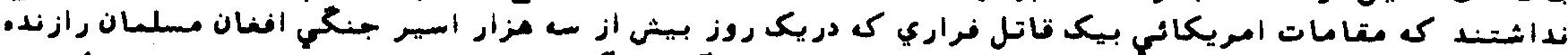

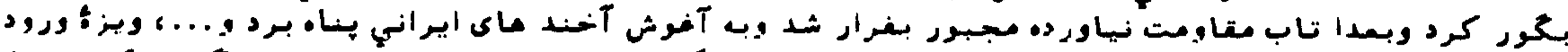

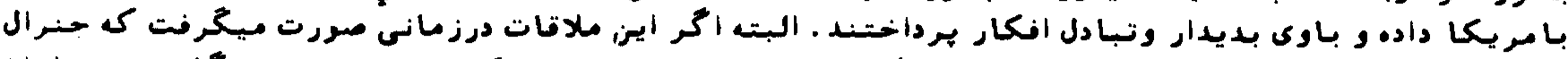

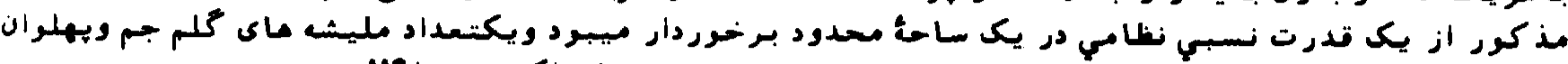

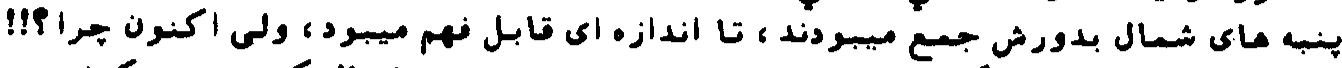

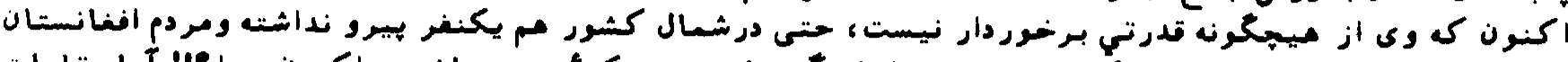

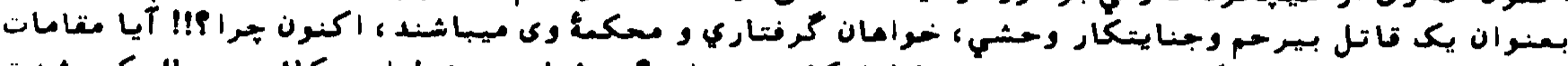

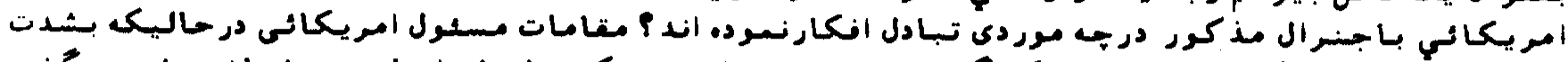

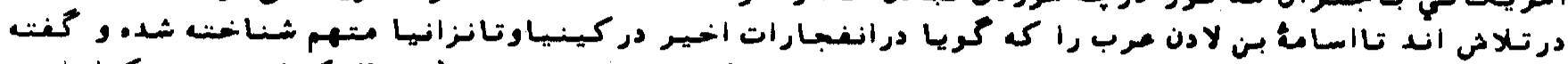

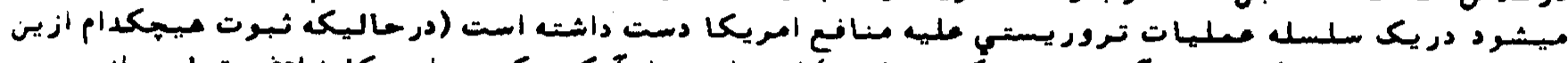

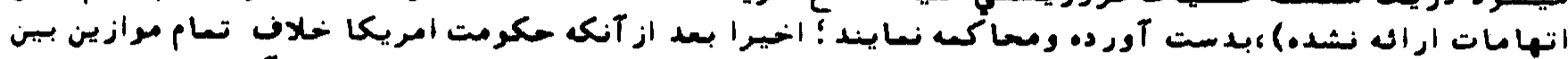

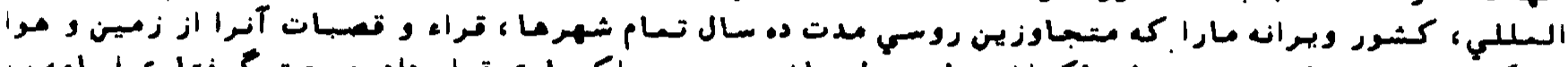

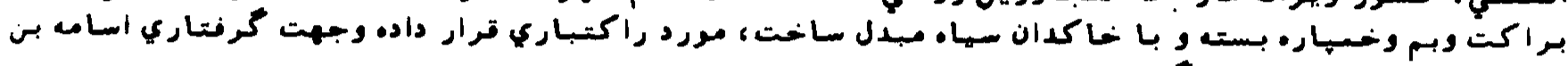

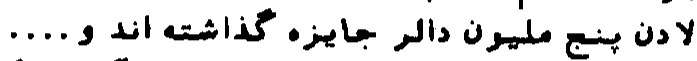

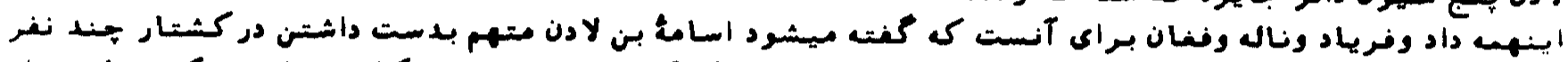

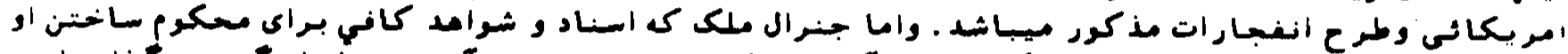

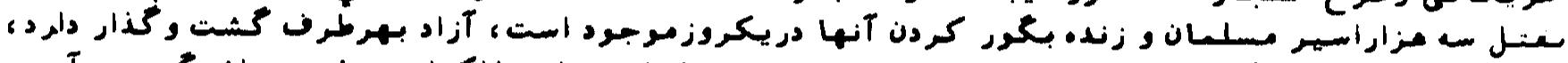

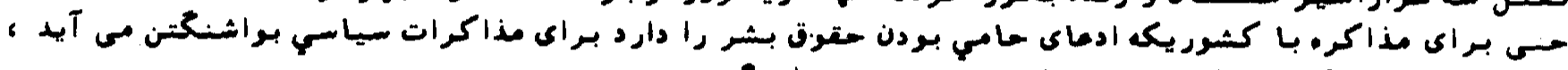

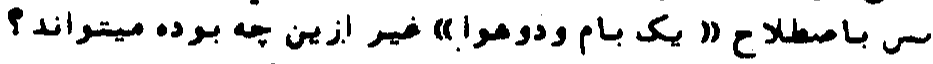

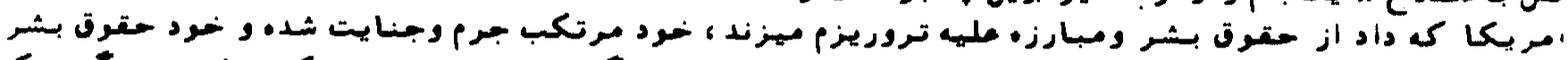

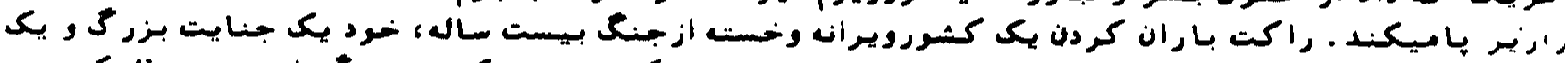

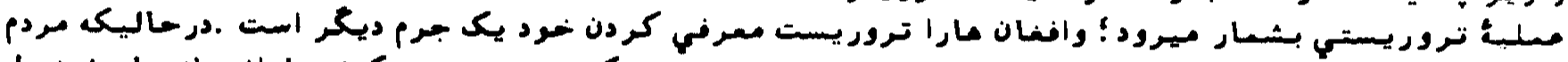

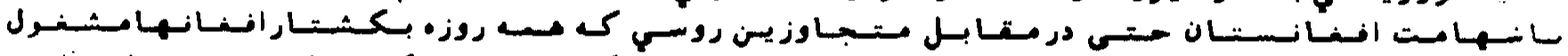

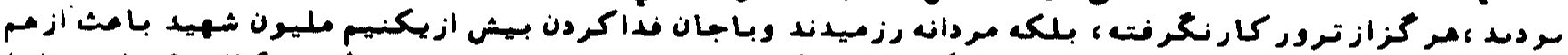

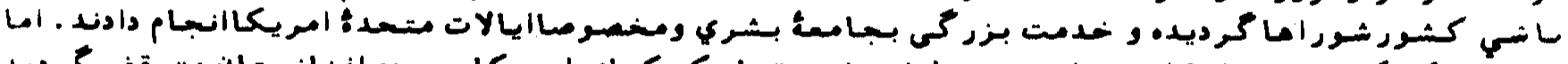

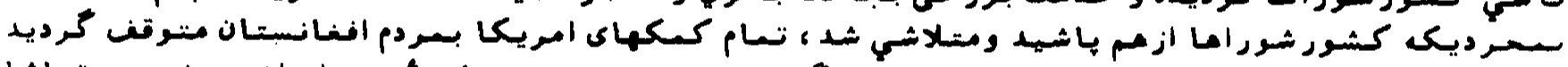

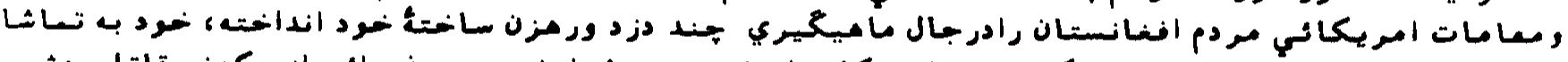

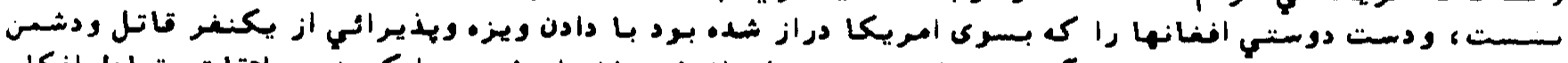

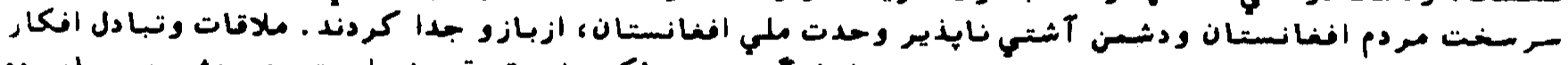

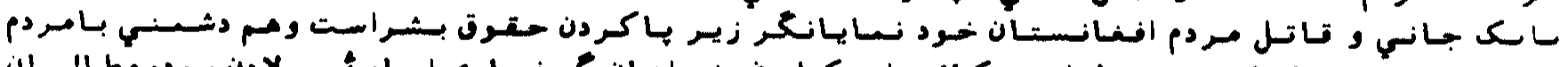

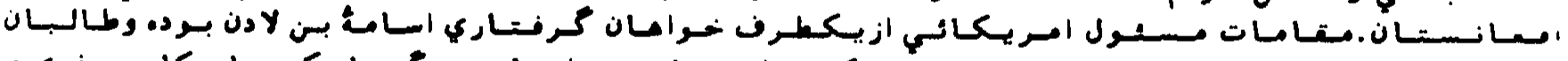

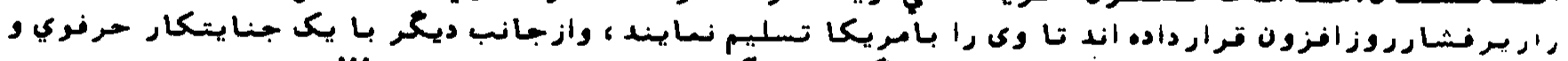

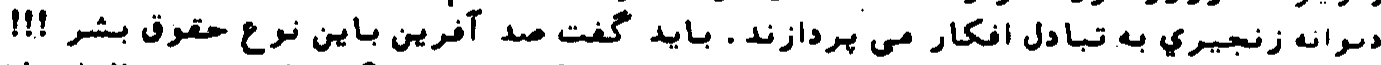

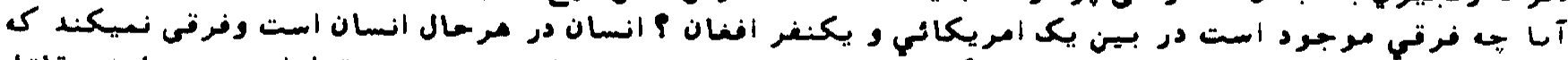

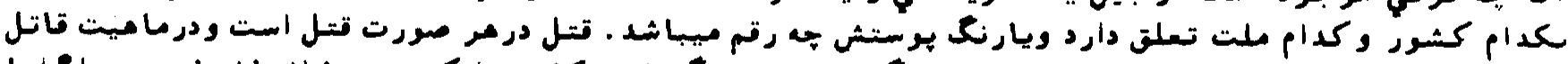

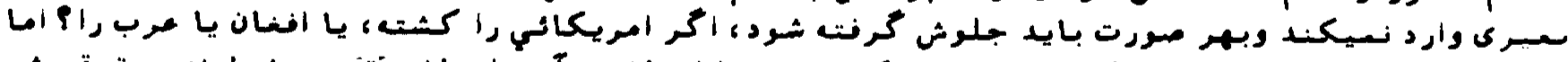

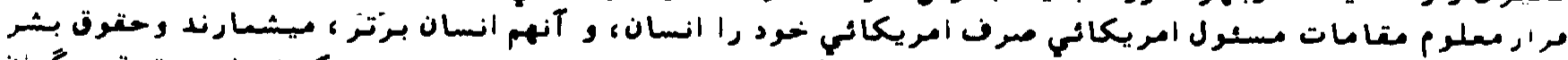

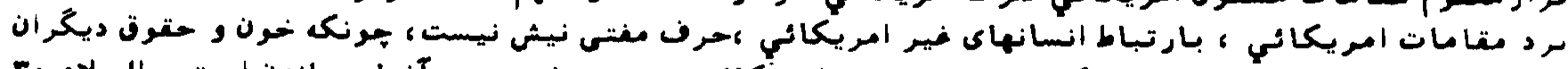

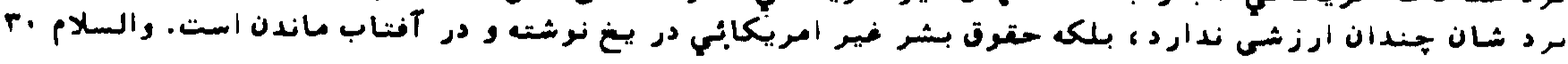

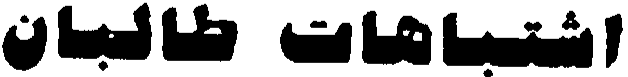

مرامبر 1911

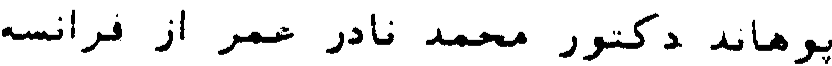

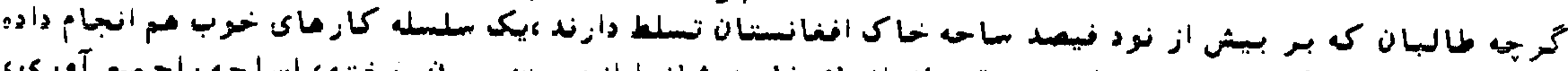

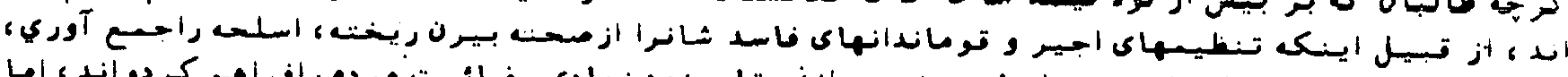

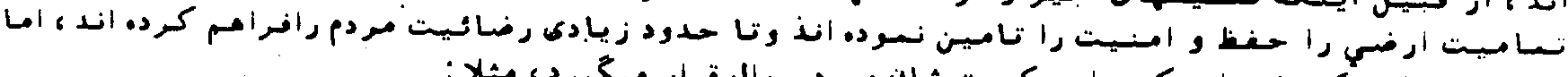

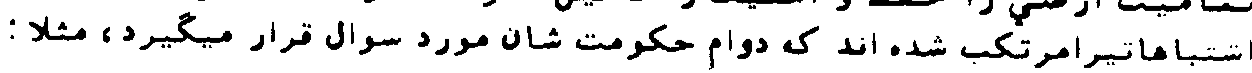




\section{0}

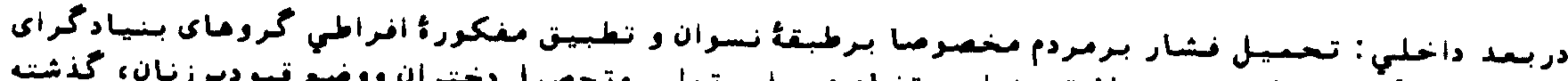

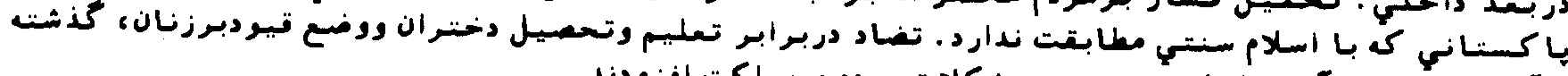

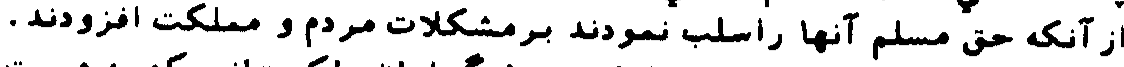

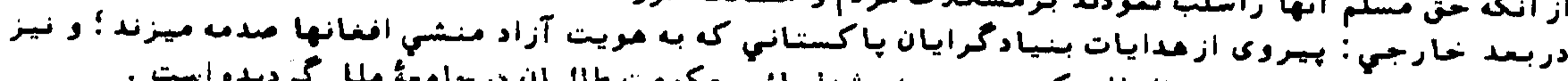

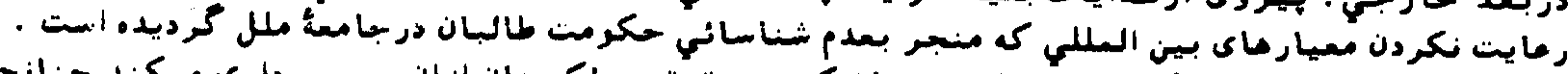

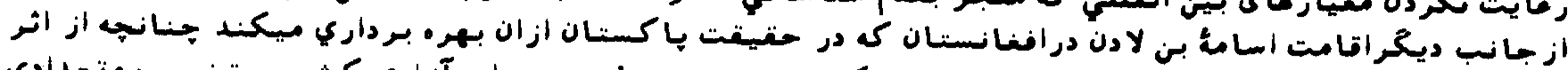

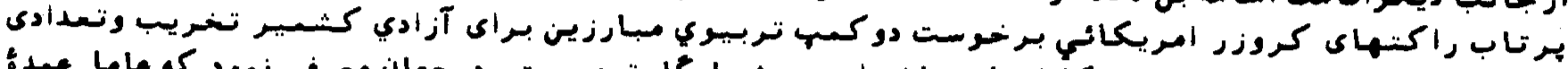

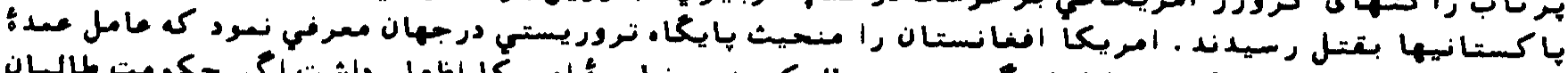

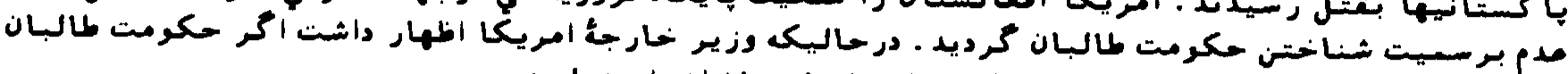

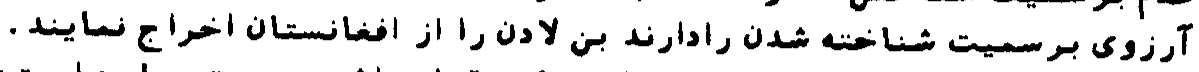

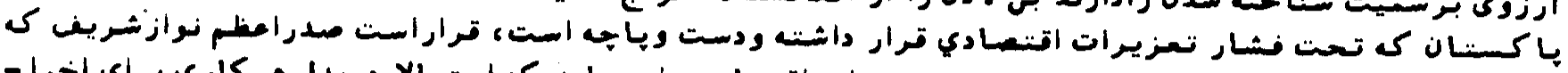

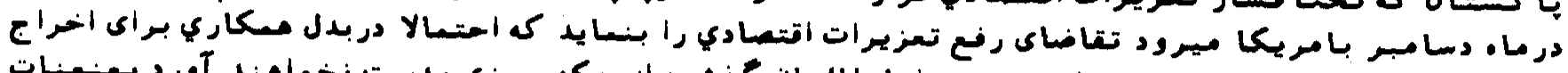

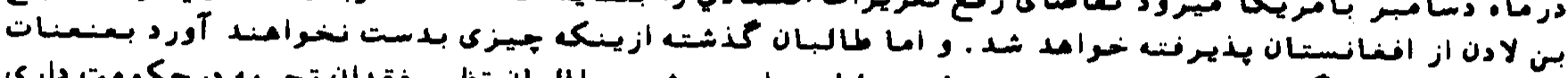

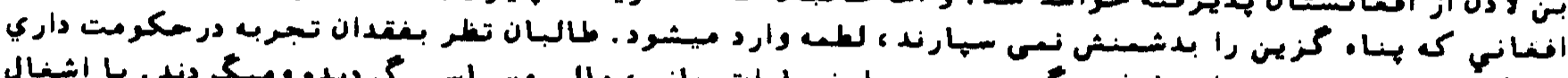

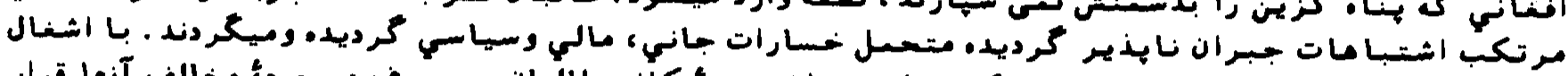

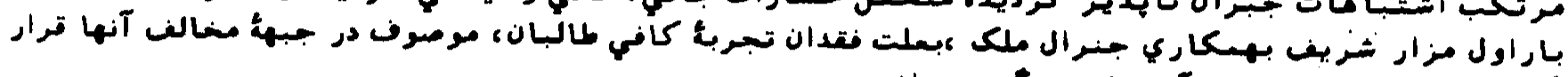

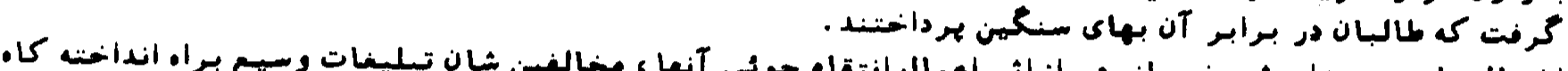

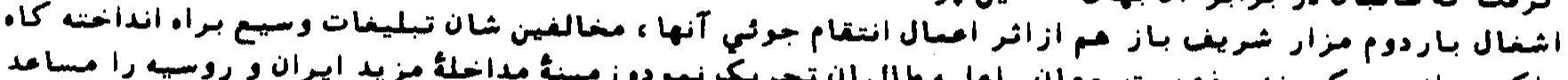

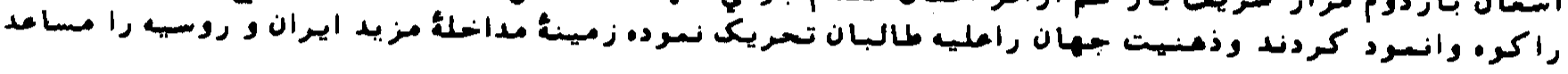

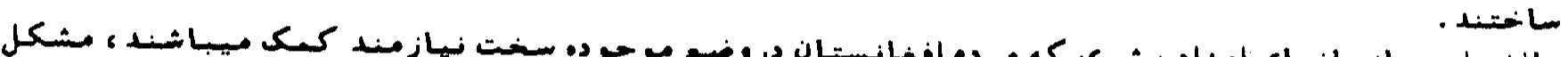

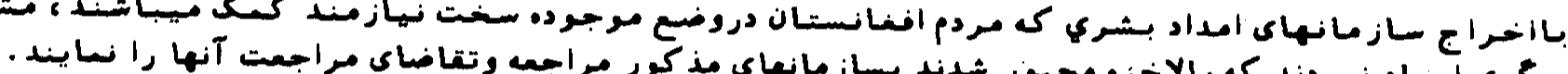

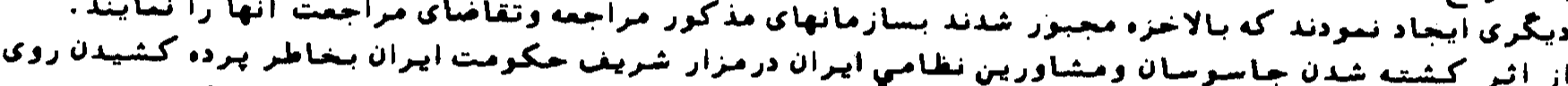

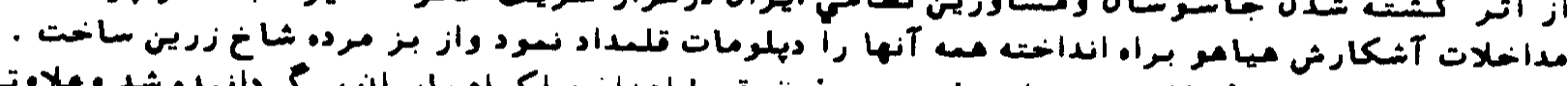

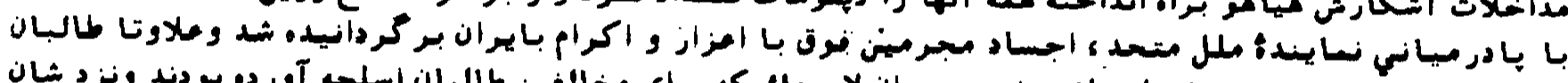

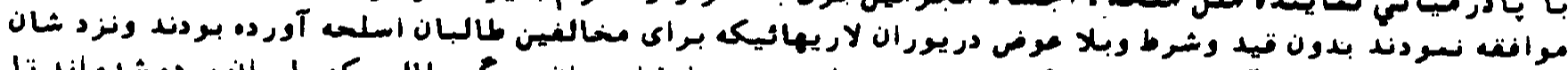

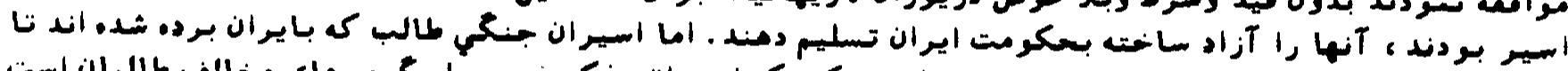

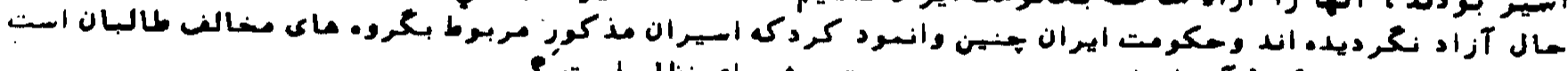

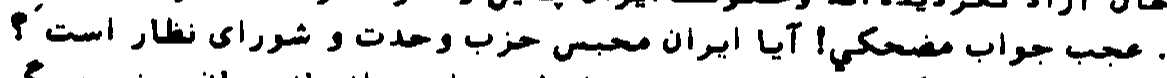

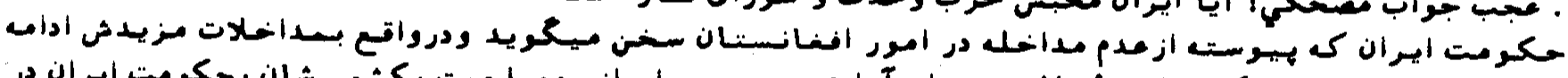

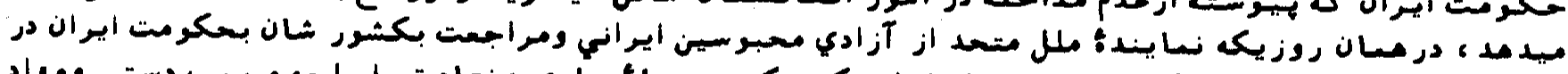

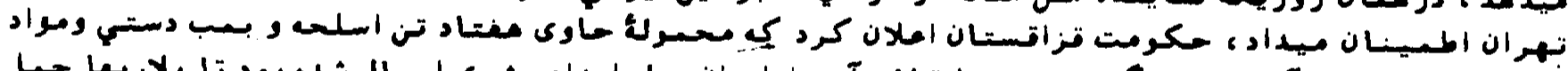

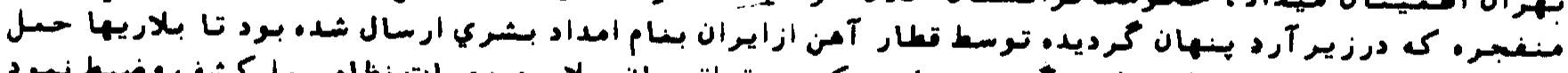

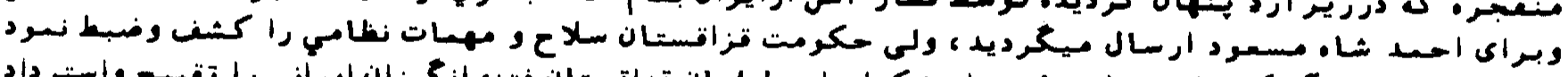

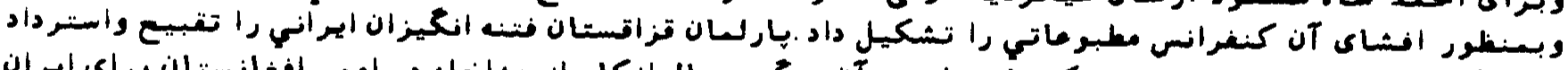

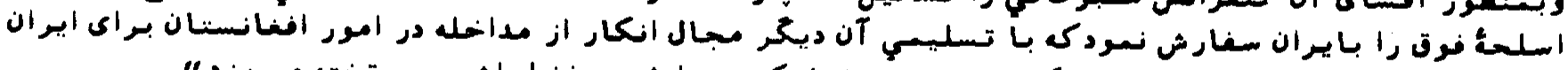

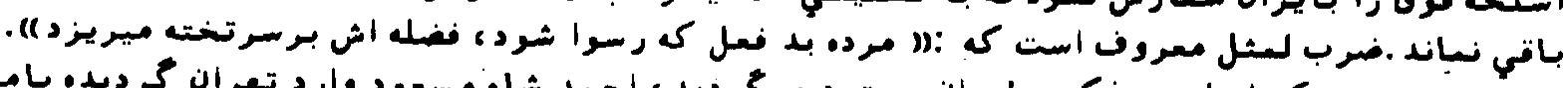

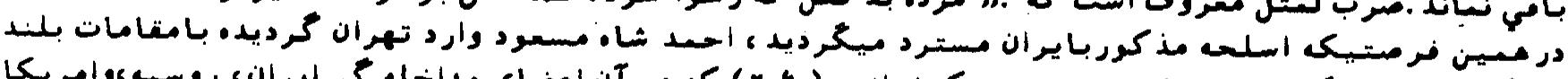

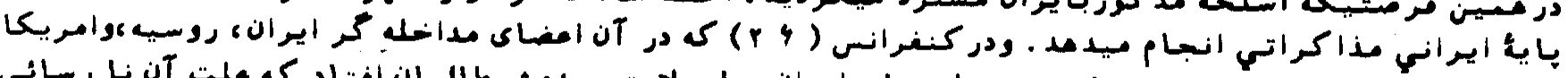

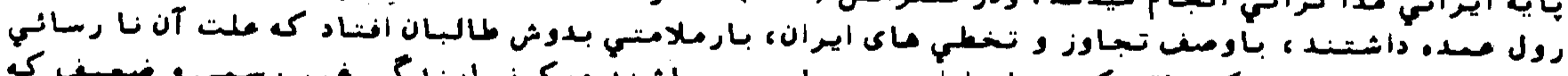

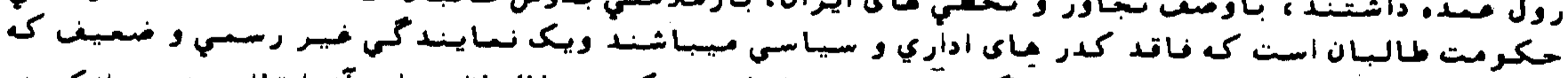

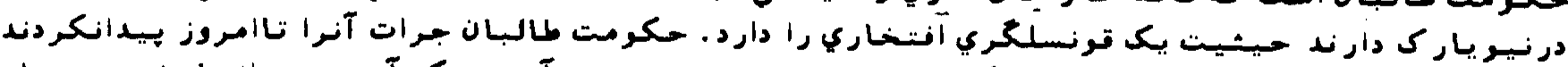

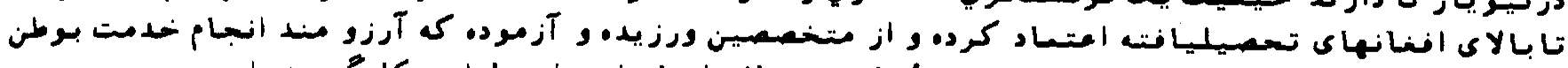

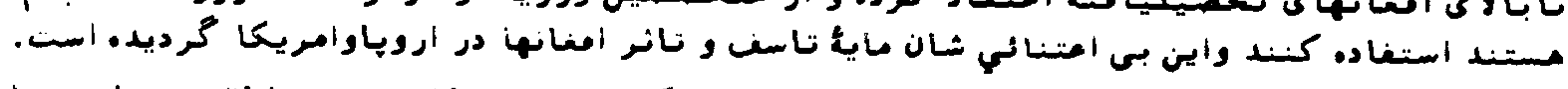

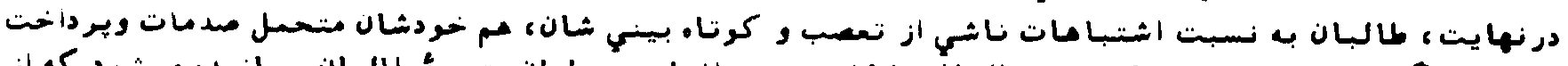

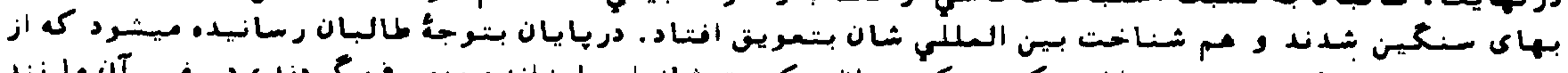

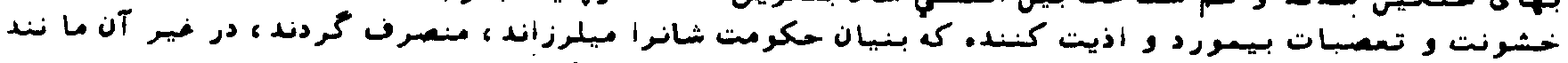

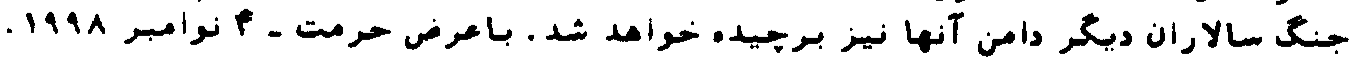




\section{1

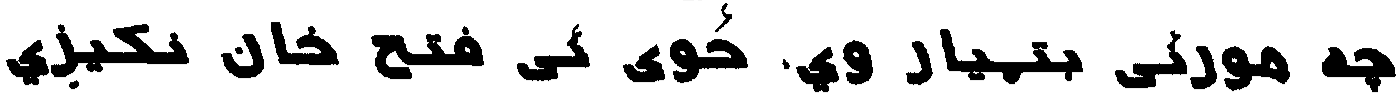

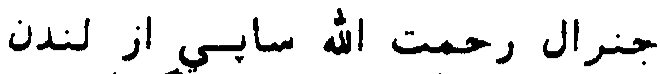

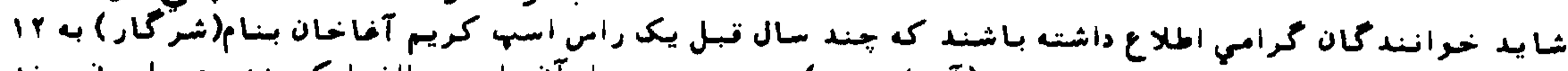

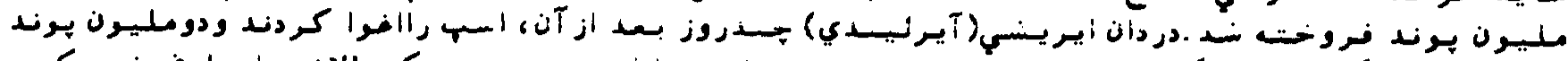

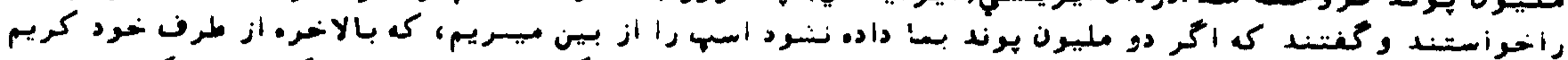

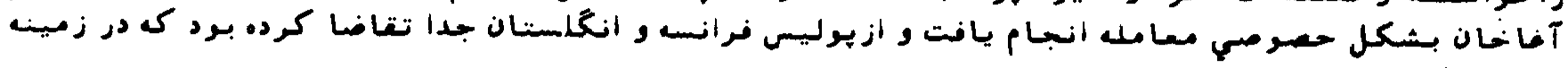

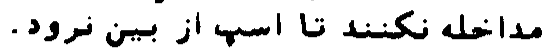

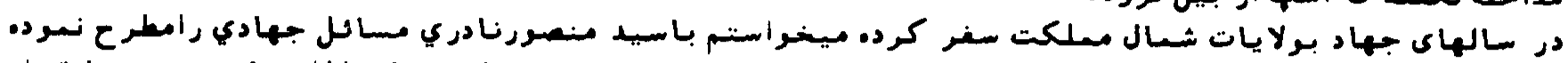

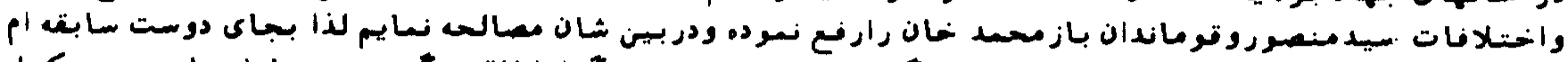

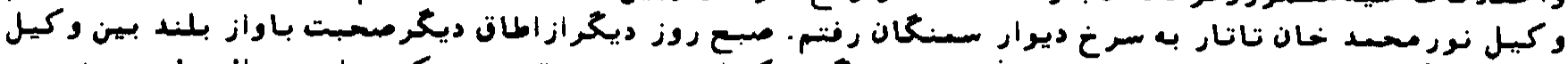

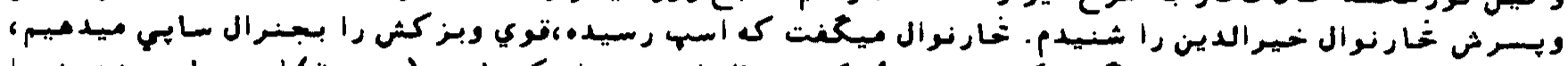

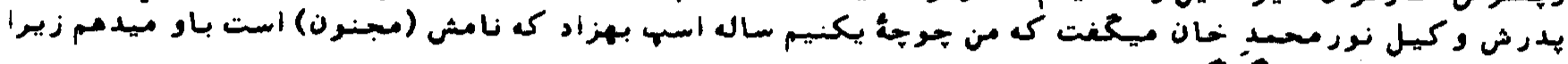

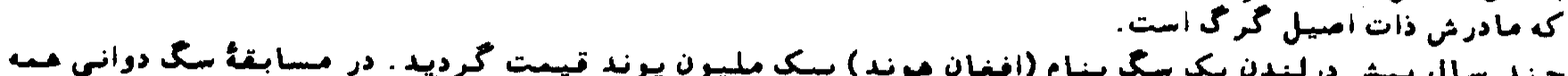

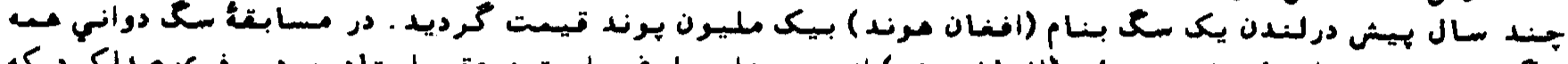

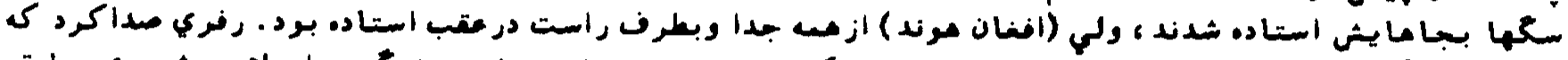
")

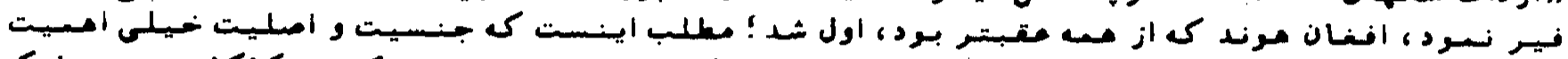

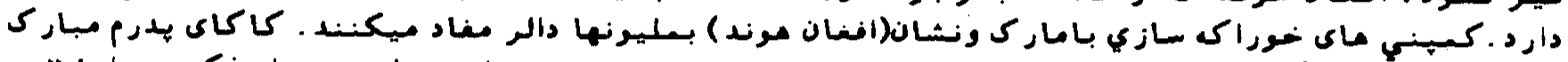

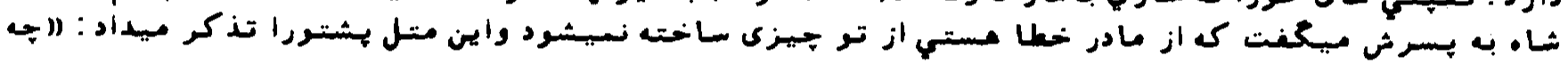

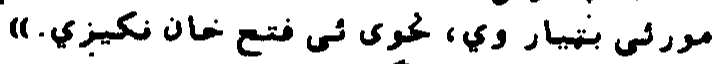

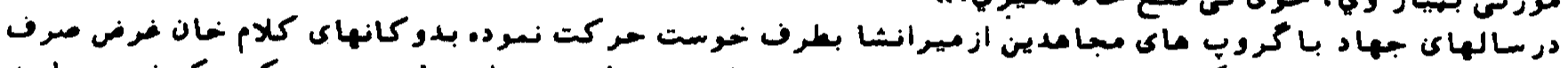

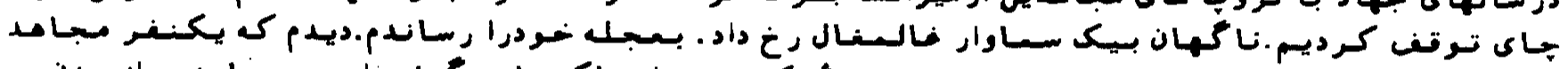

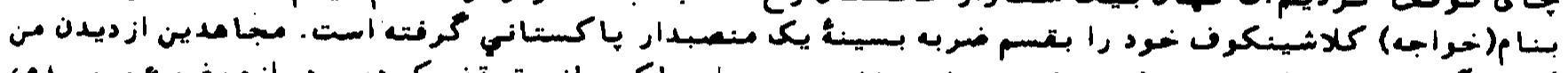

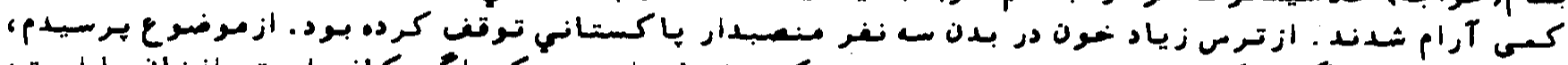

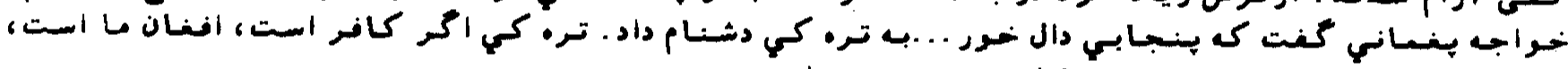

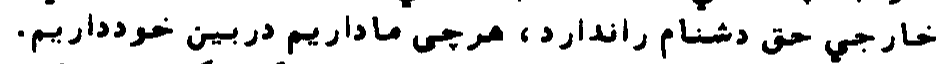

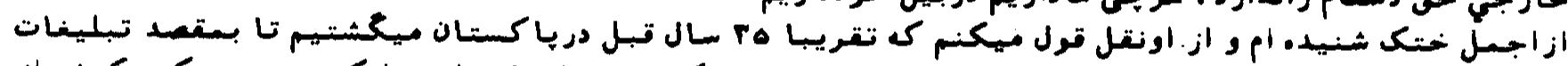

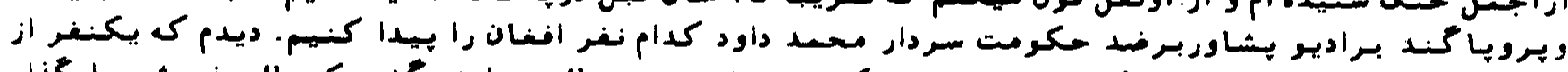

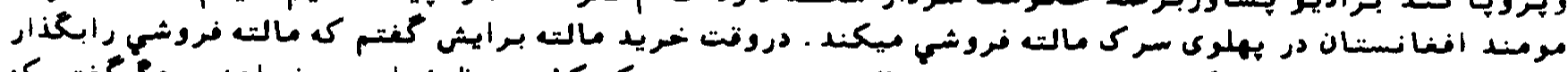

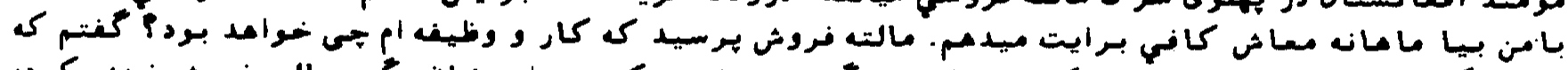

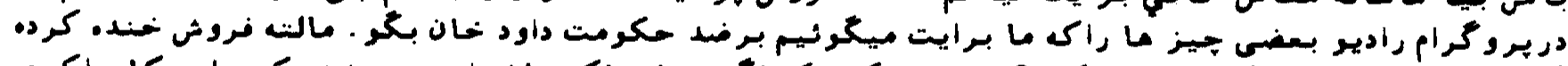

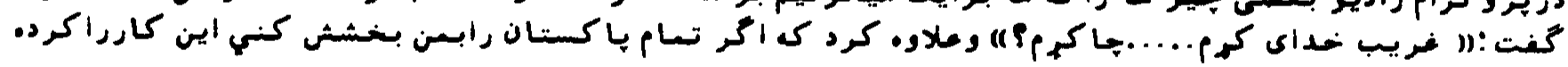

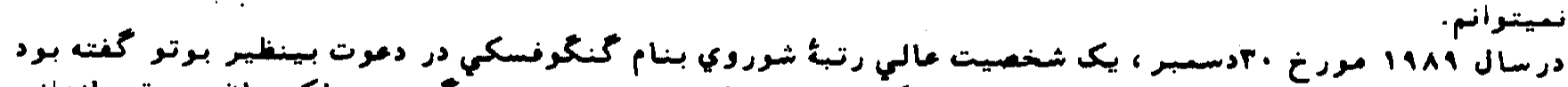

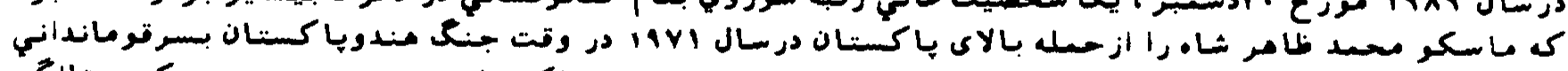

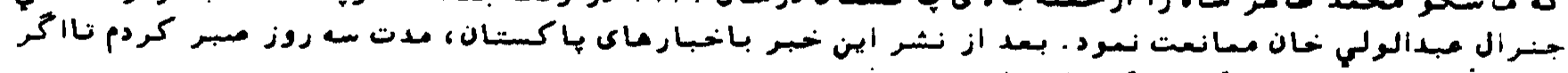

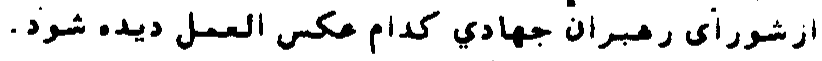

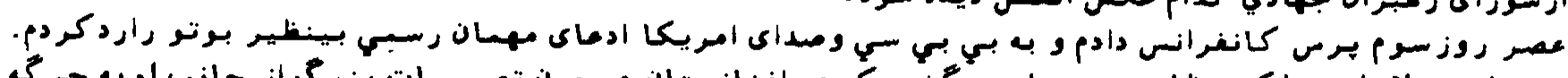

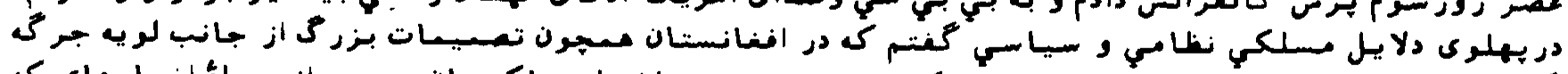

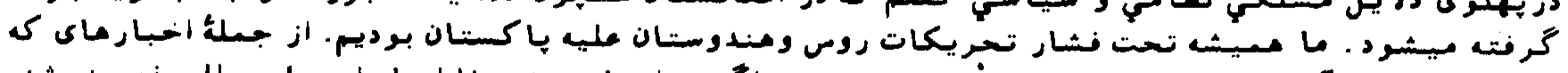

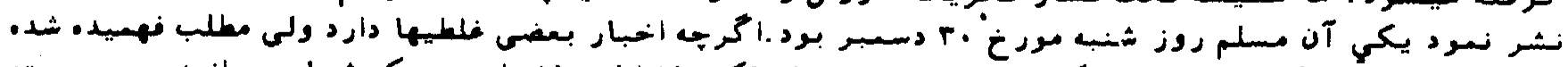

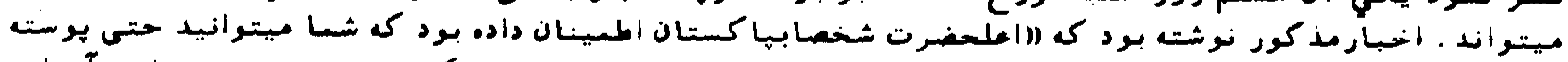

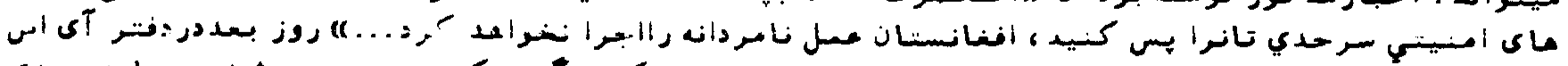

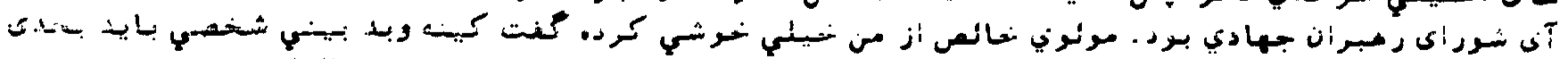

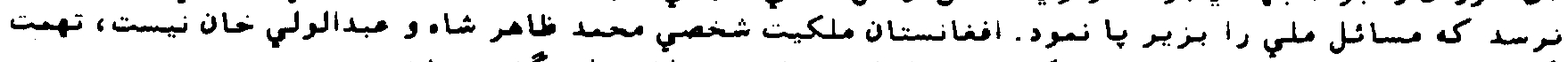

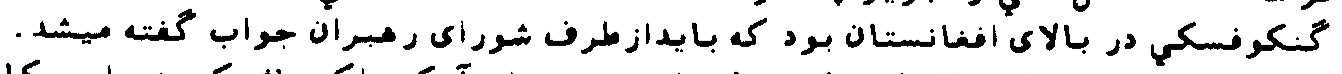

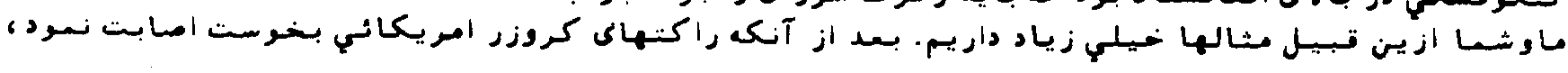

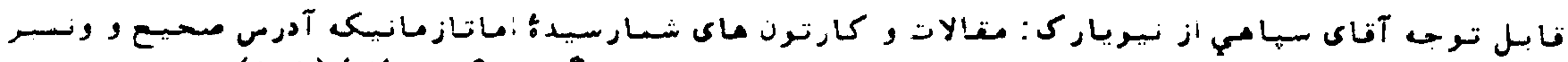

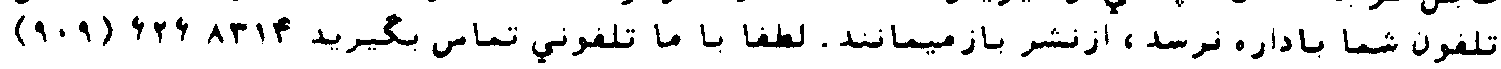




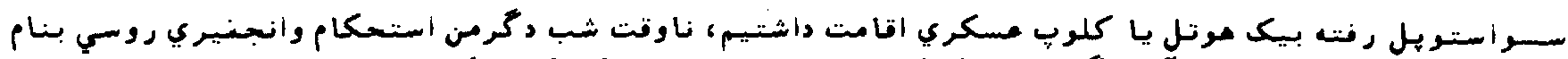

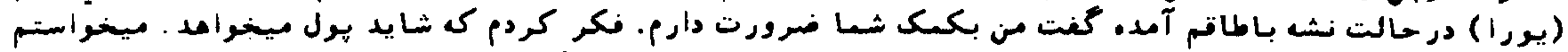

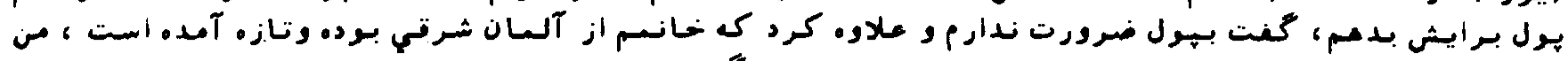

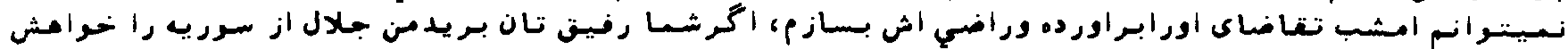

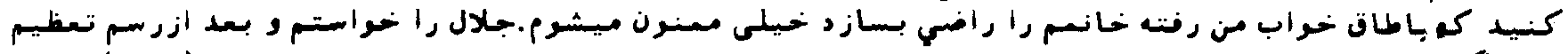

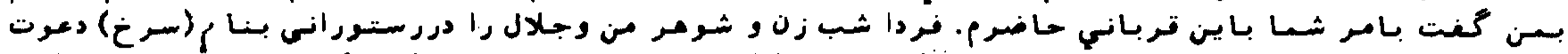

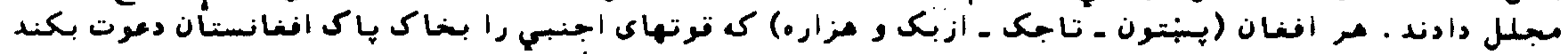

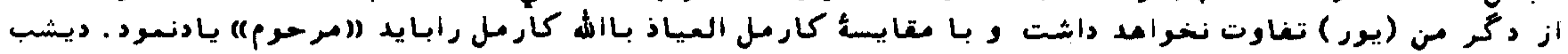

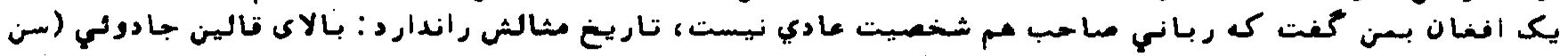

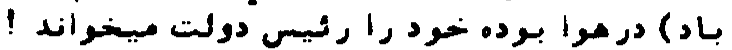

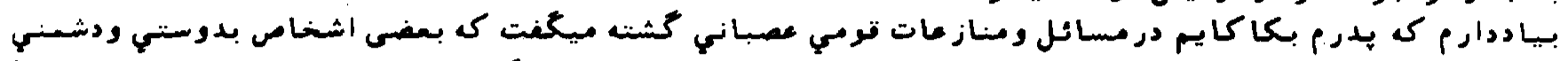

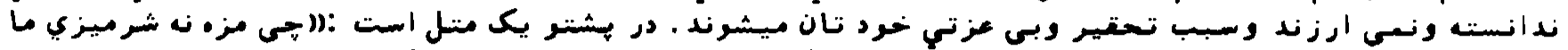

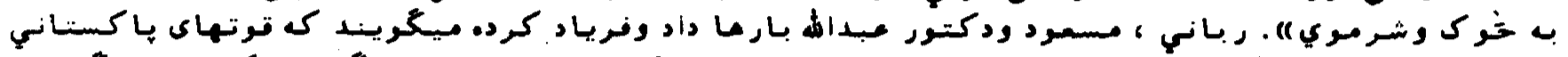

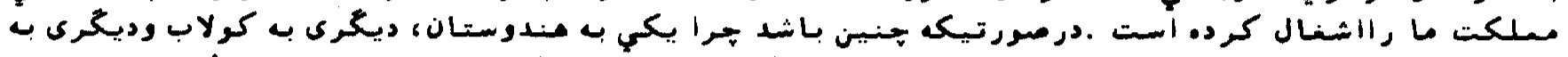

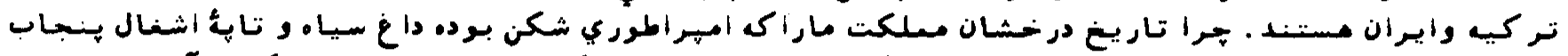

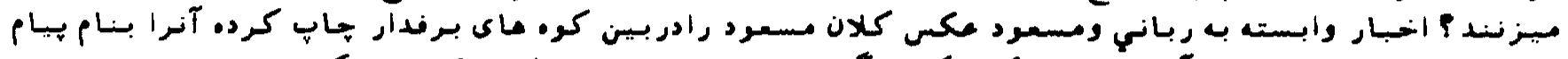

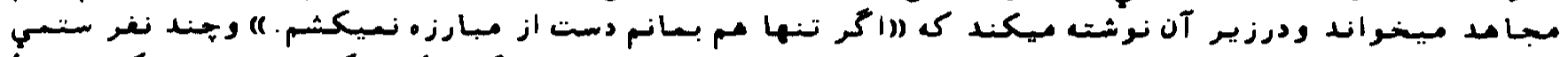

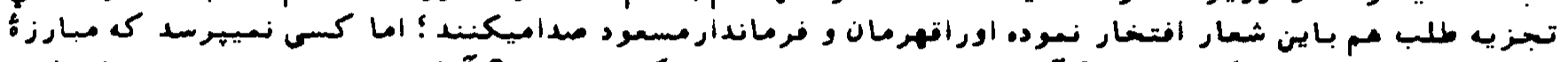

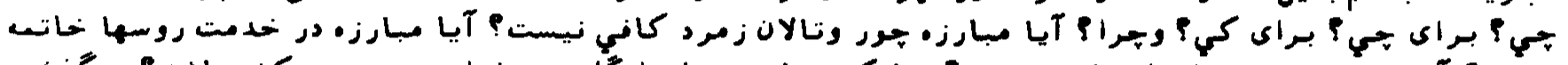

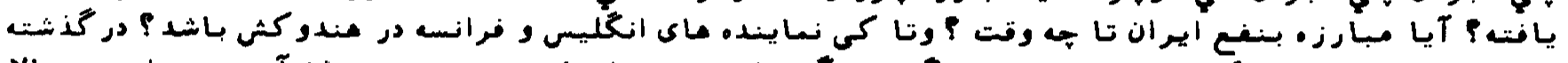

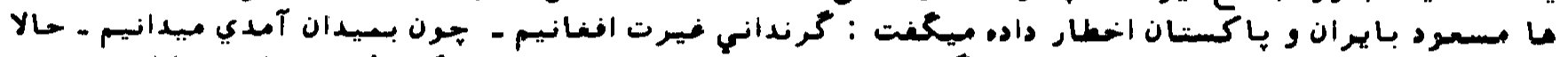

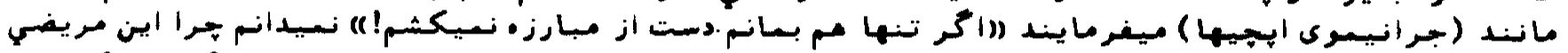

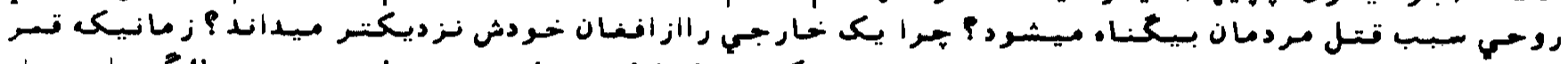

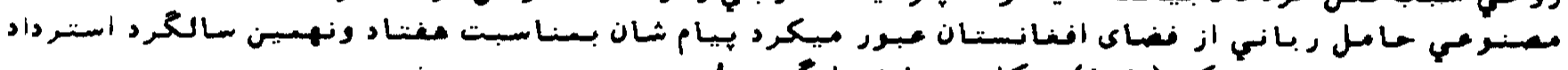

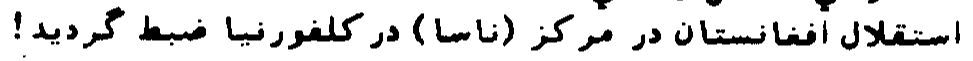

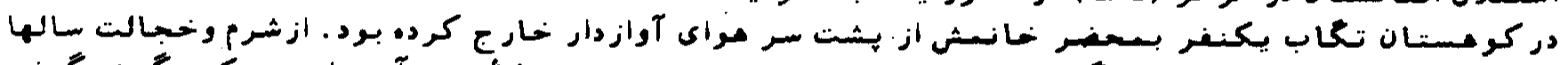

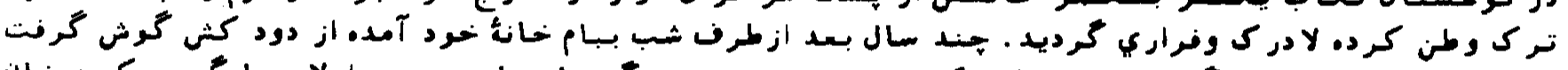

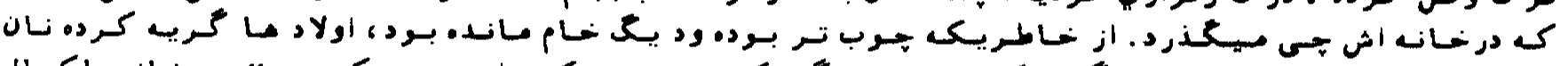

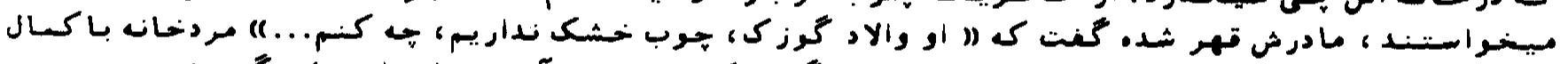

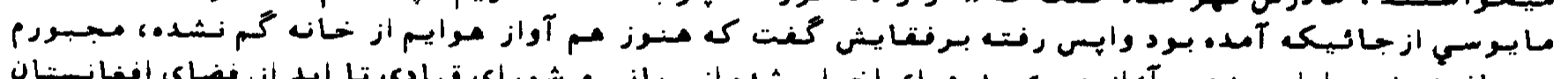

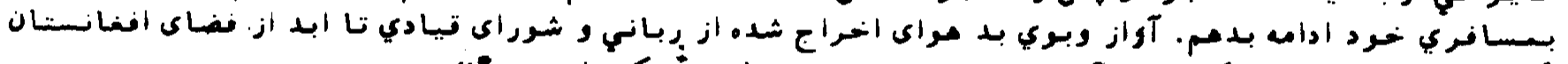

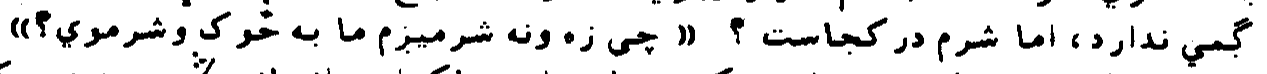

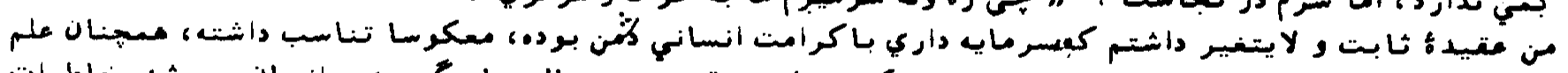

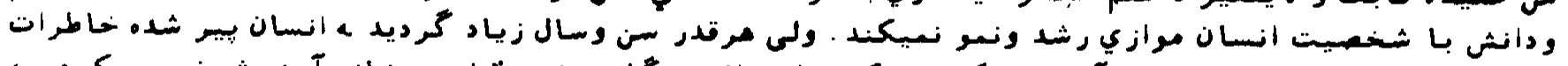

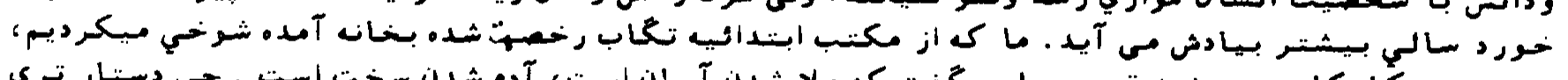

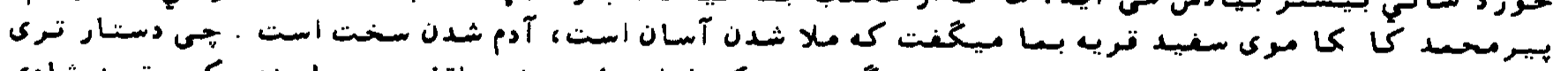

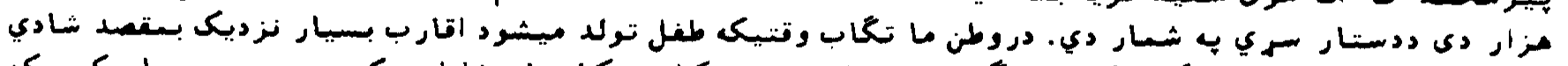

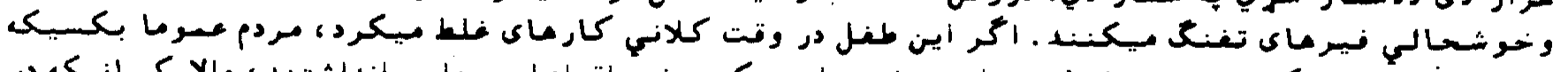

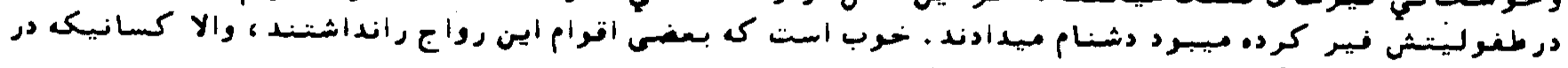

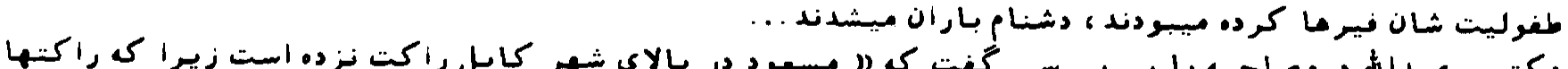

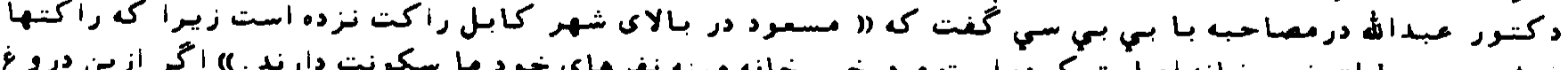

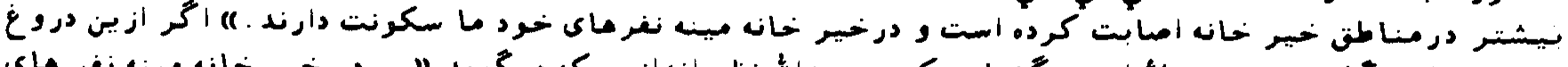

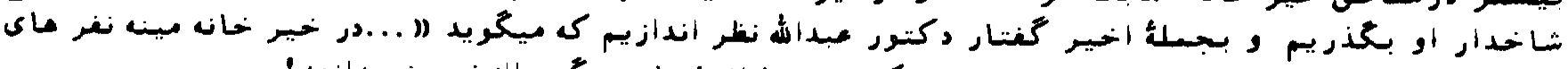

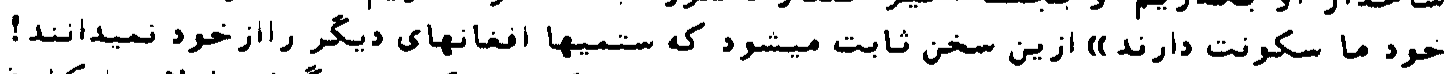

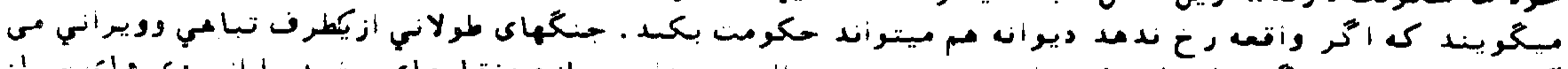

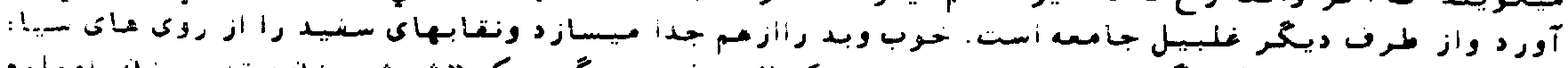

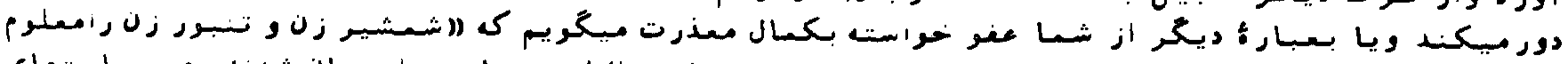

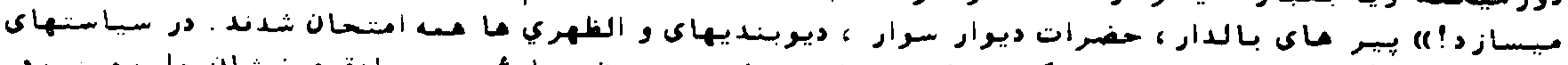

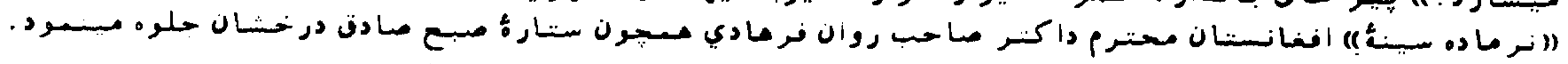




\section{4}

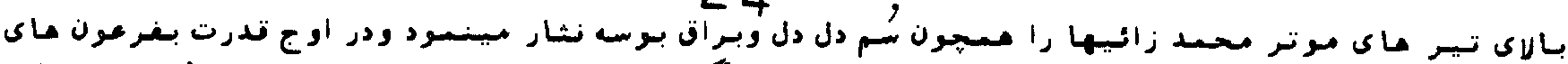

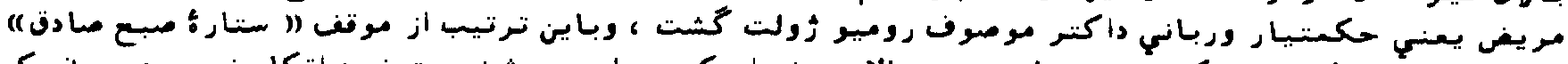

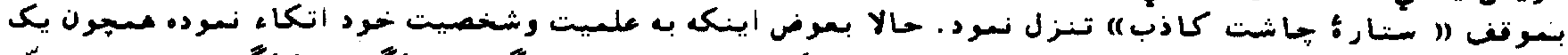

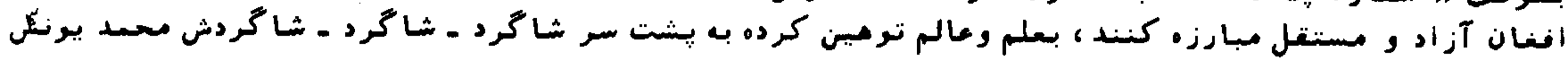

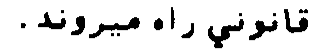

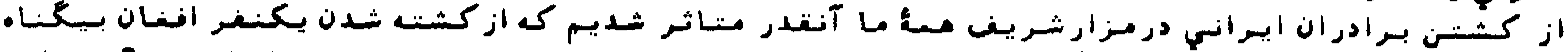

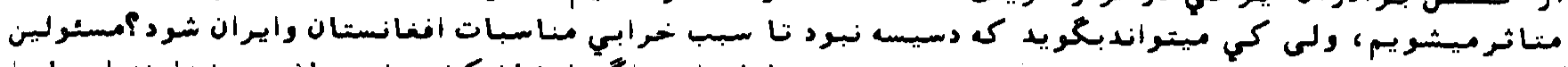

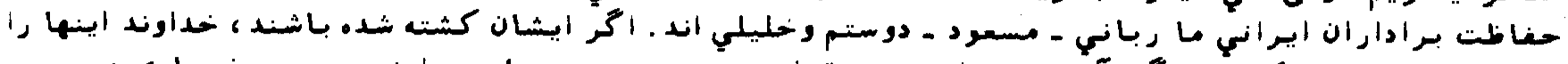

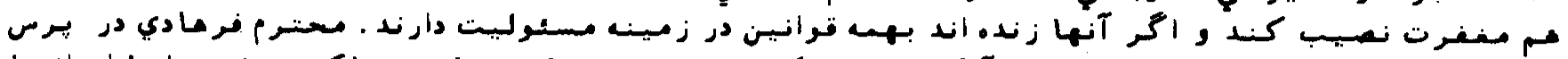

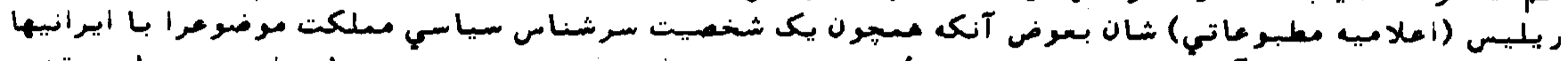

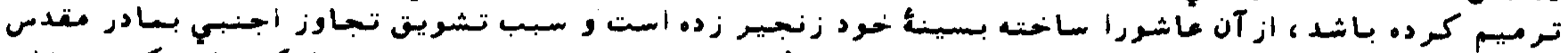

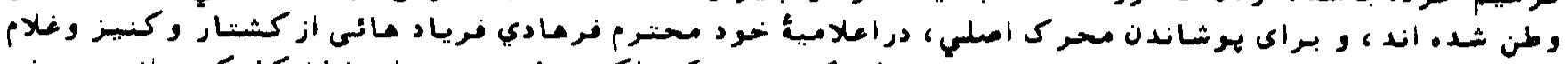

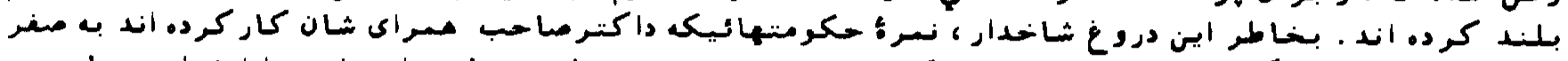

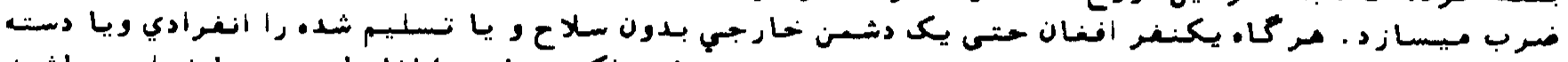

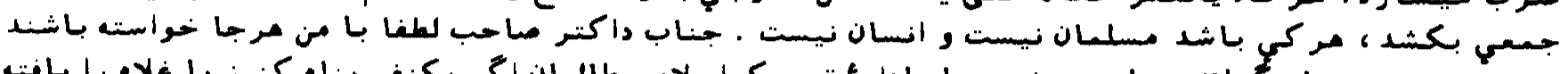

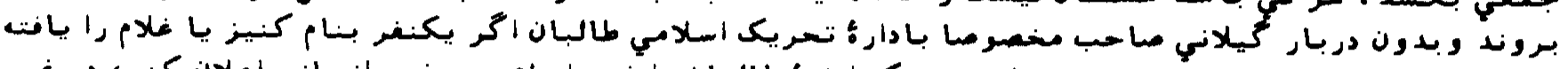

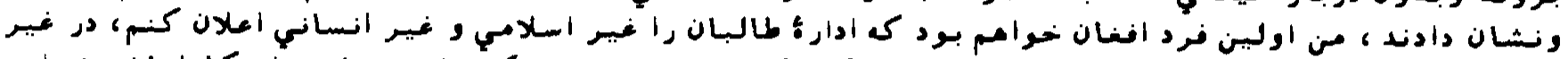

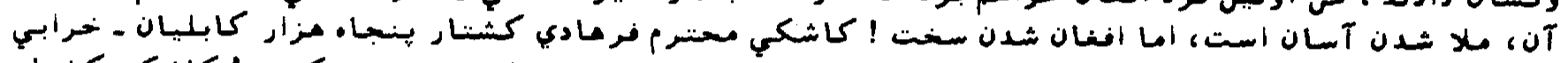

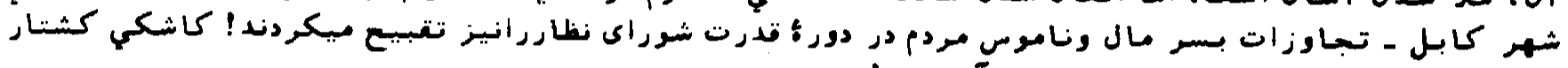

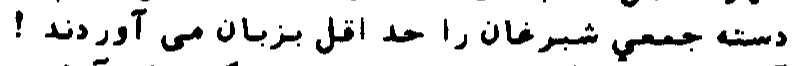

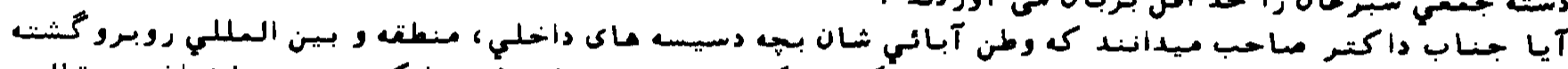

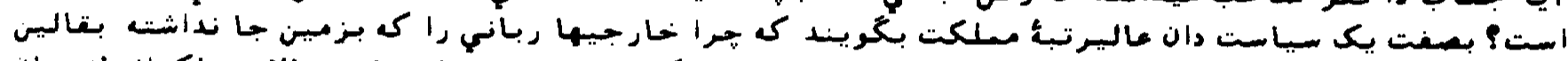

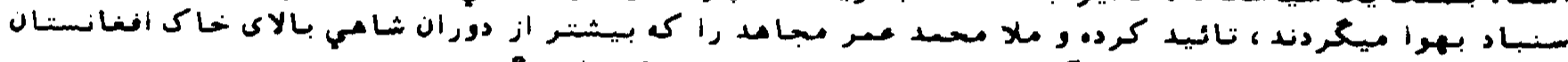

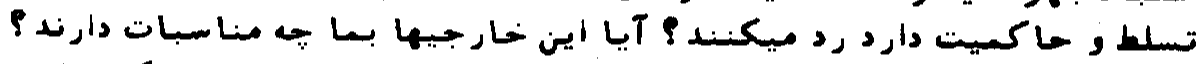

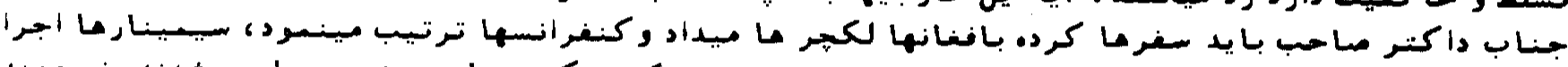

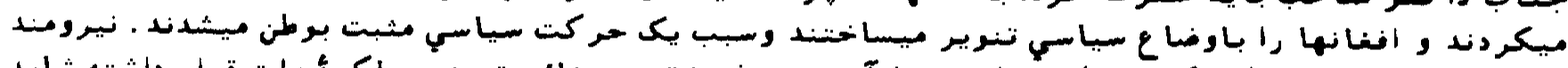

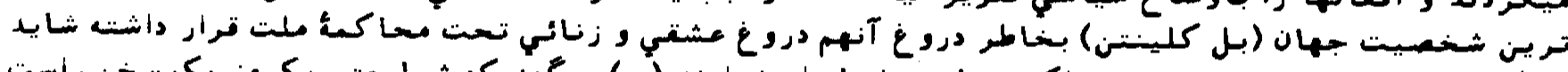

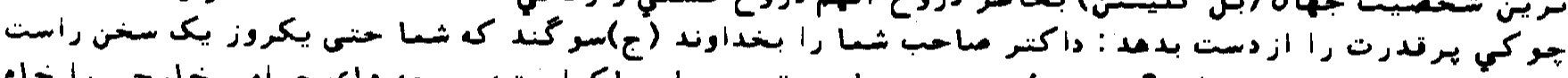

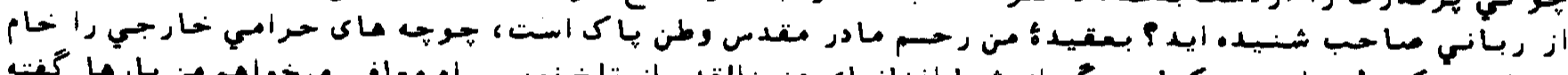

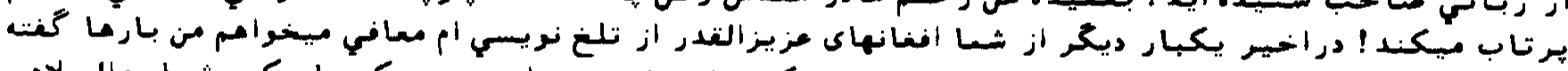

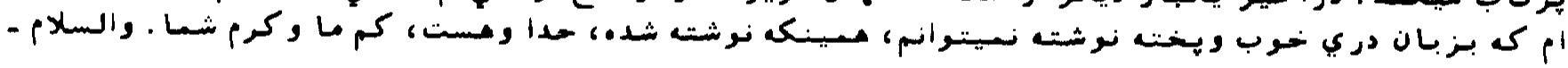

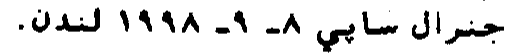

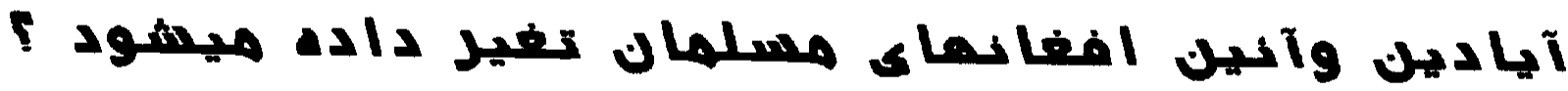

عبدالكريم حكيسمي ازدالاس - تكراس

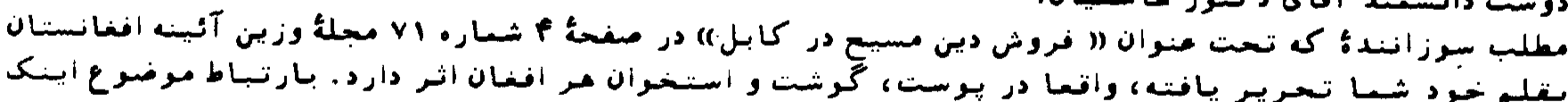

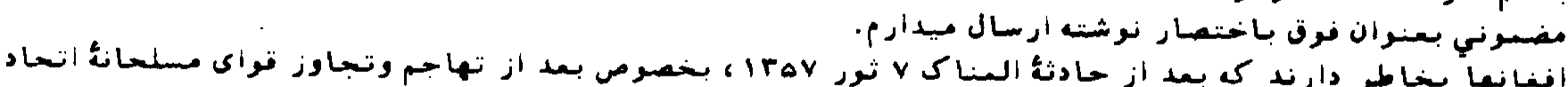

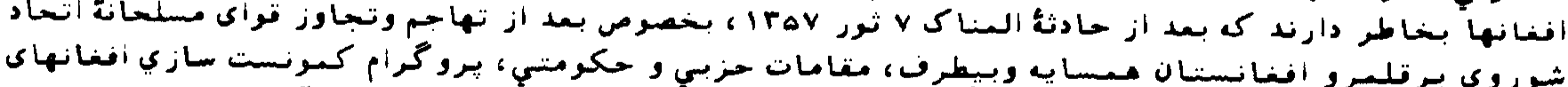

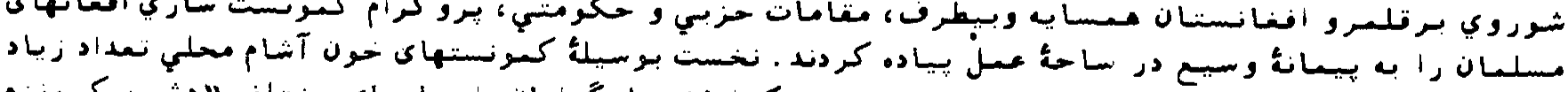

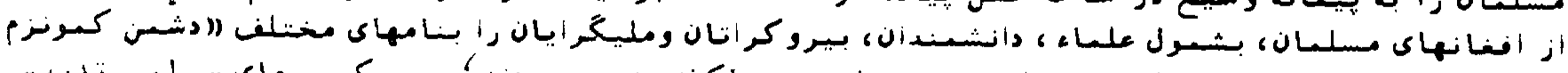

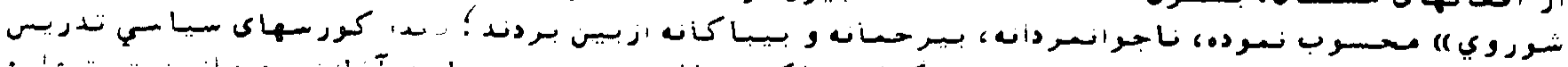

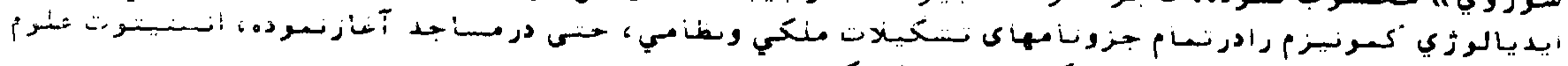

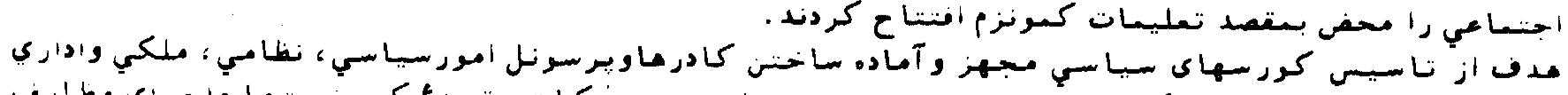

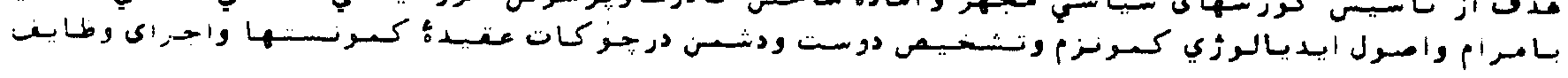




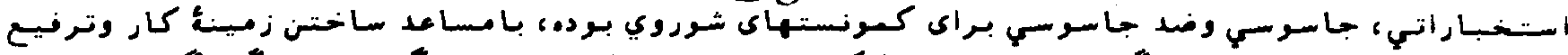

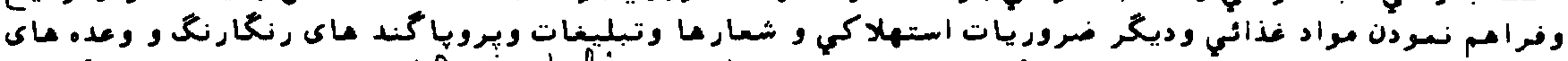

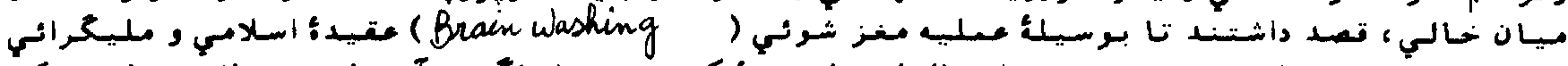

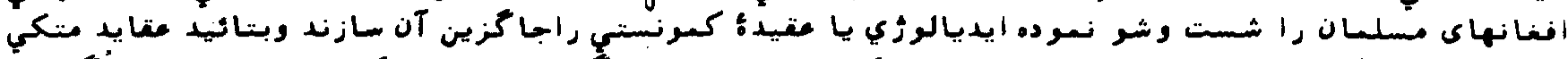

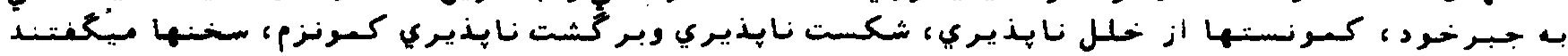

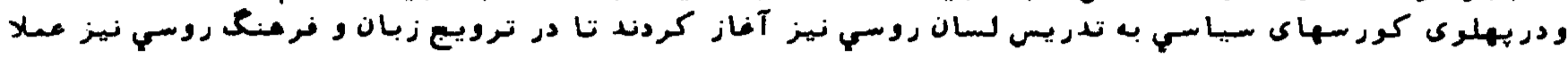

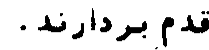

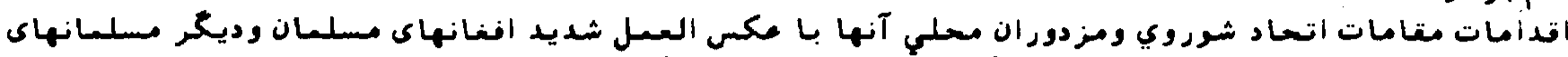

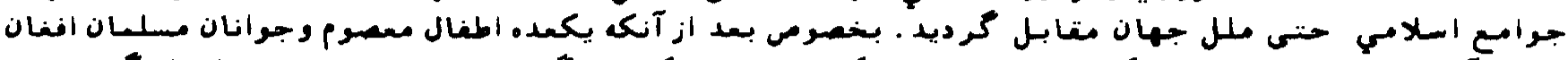

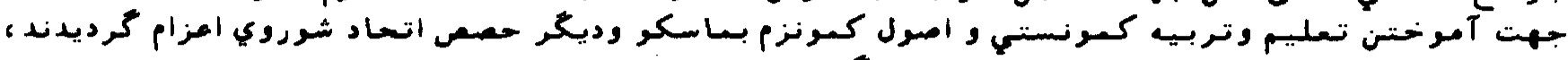

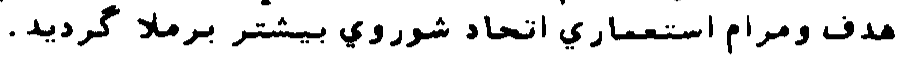

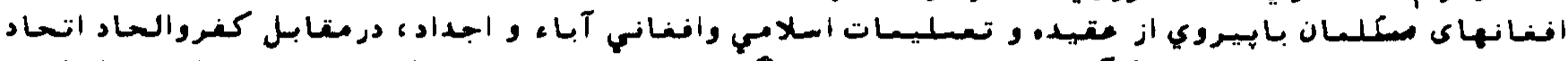

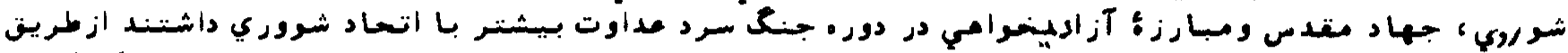

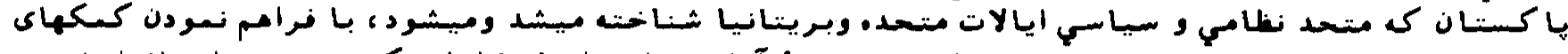

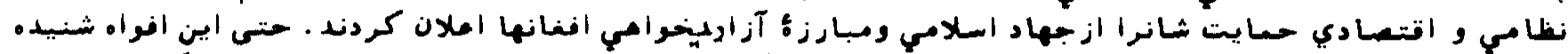

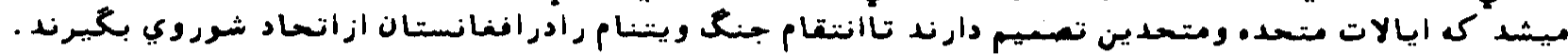

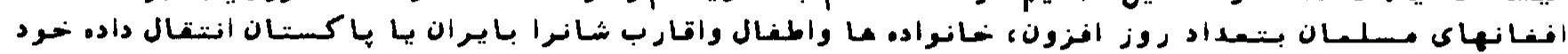

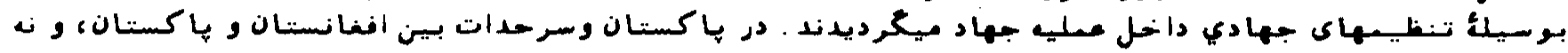

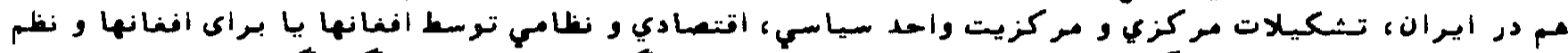

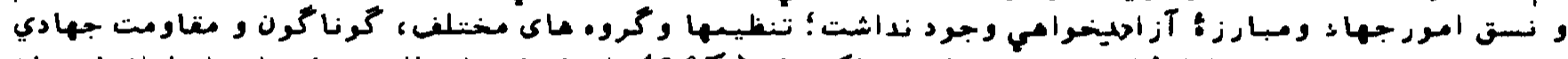

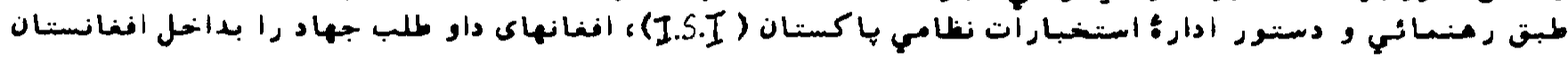

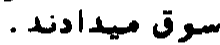

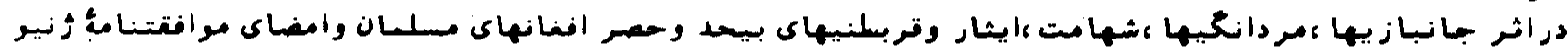

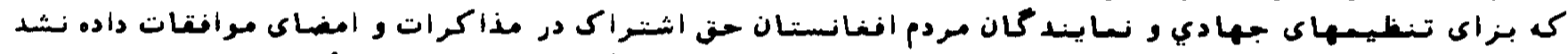

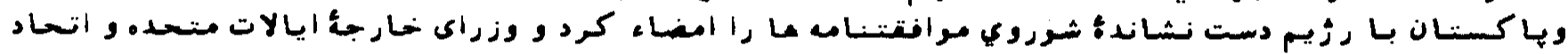

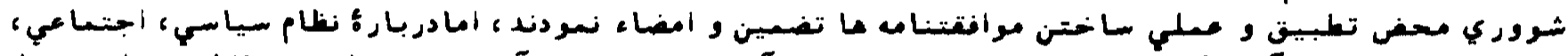

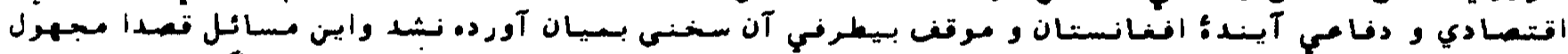

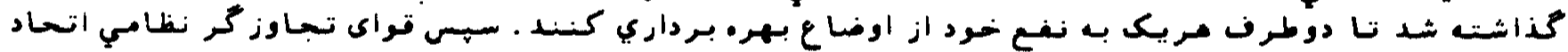

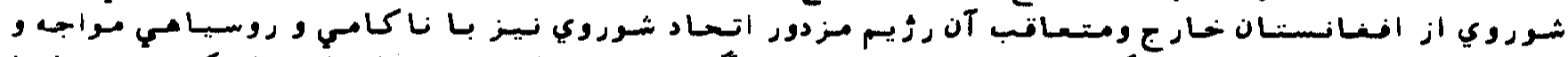

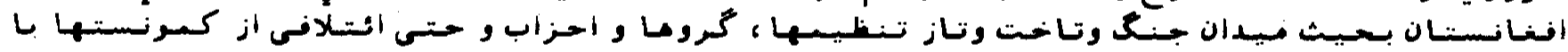

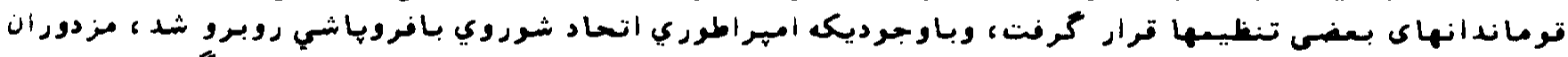

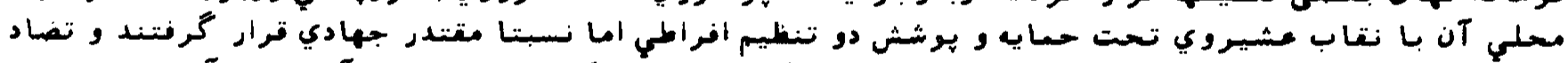

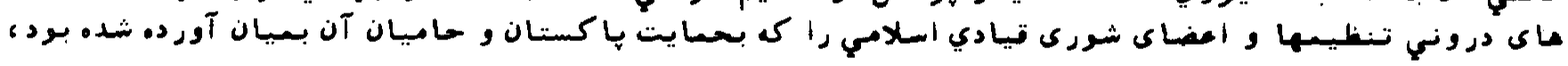

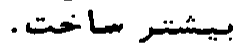

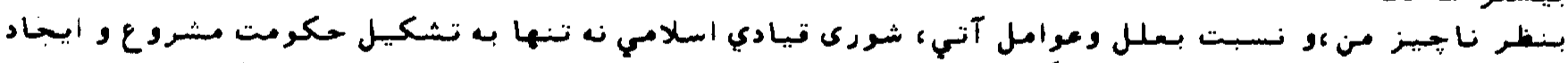

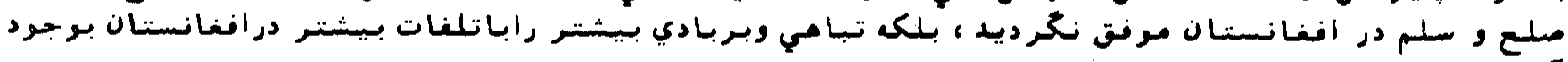
آوردي

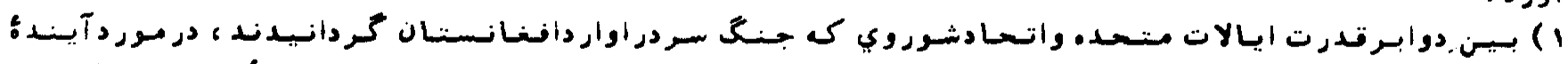

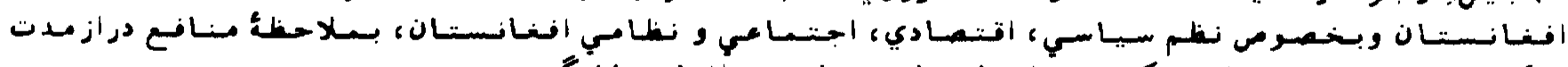

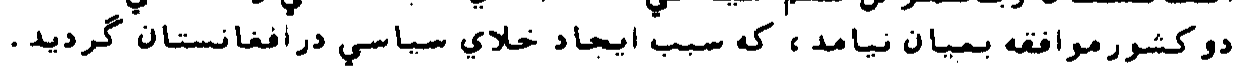

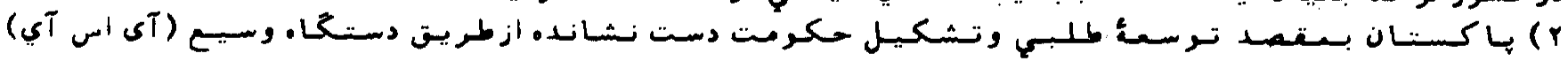

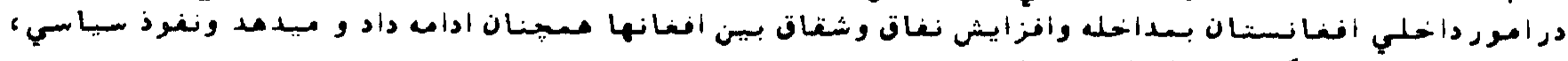

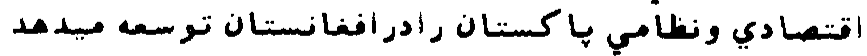

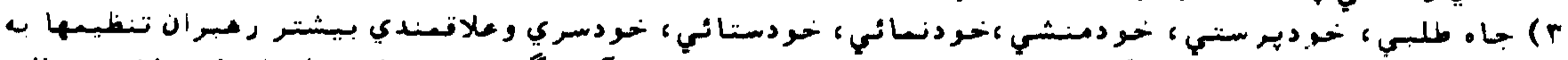

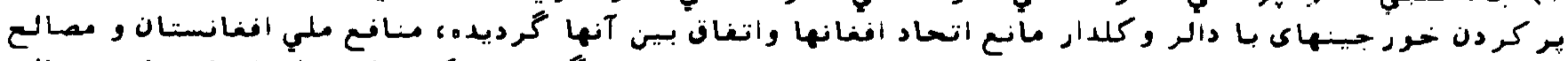

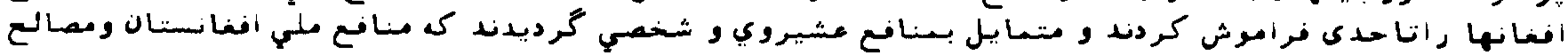

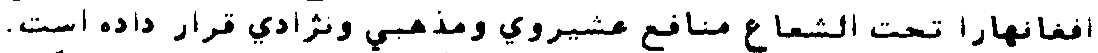

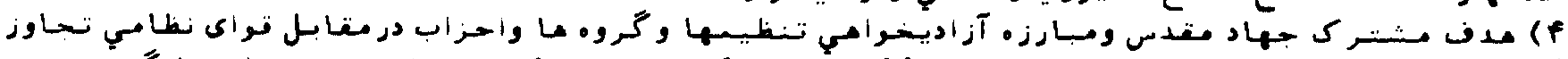

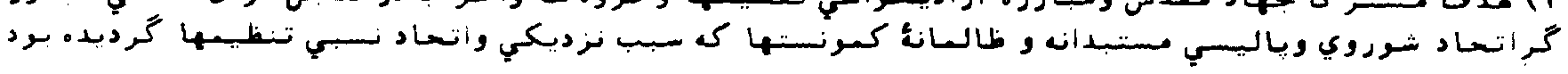

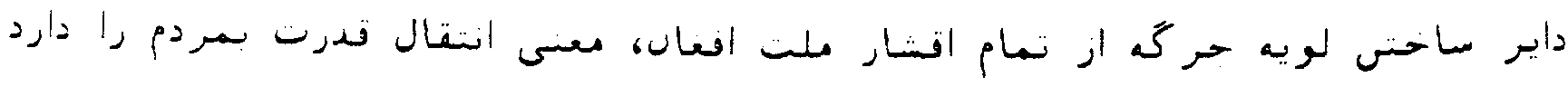




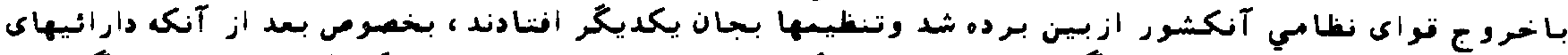

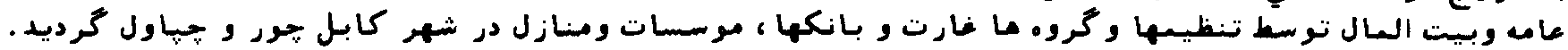

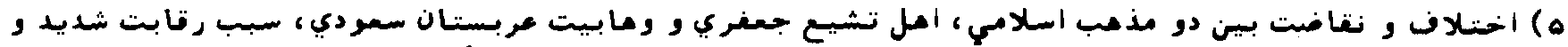

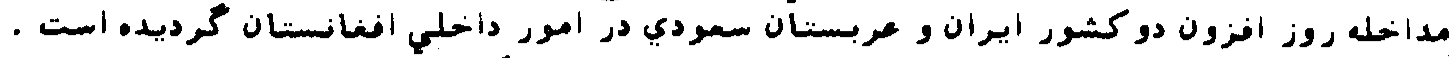

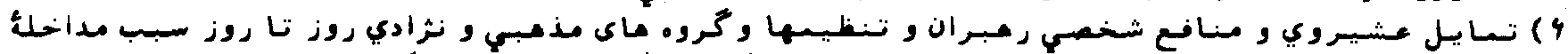

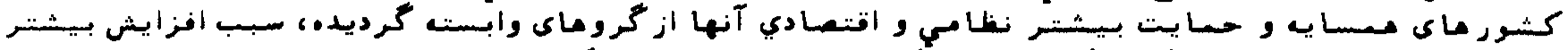

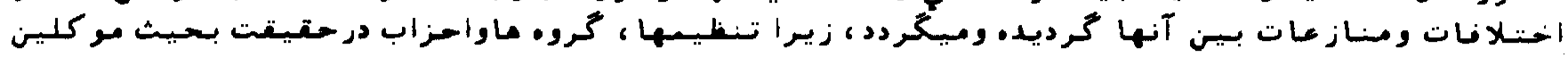

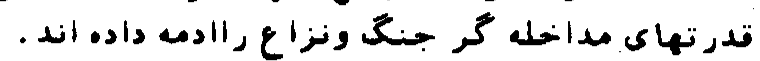

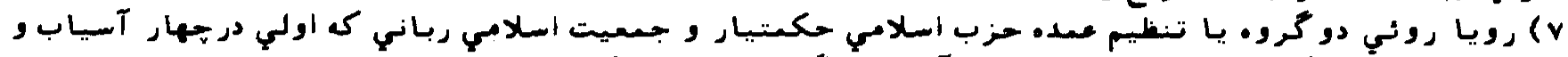

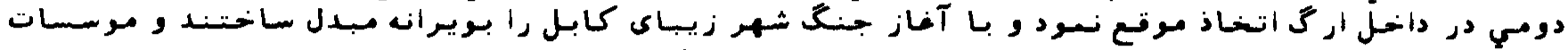

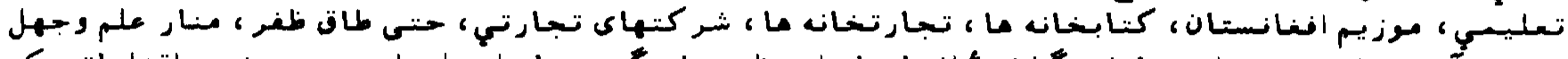

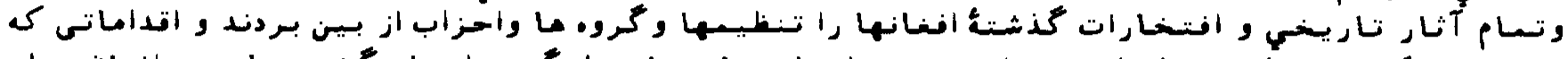

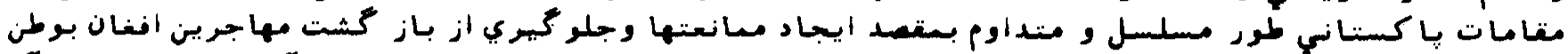

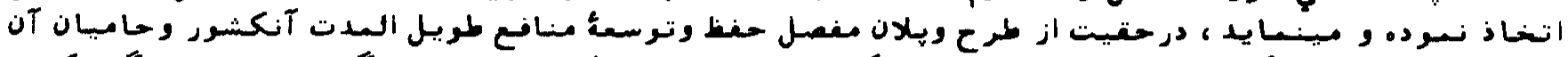

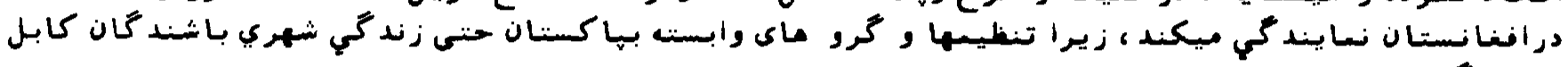

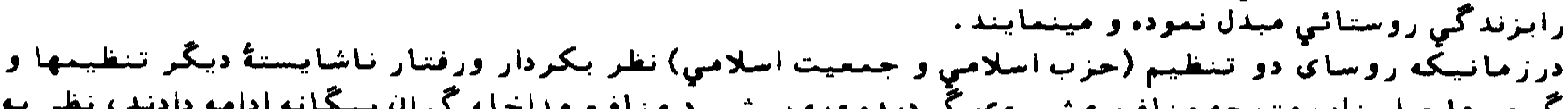

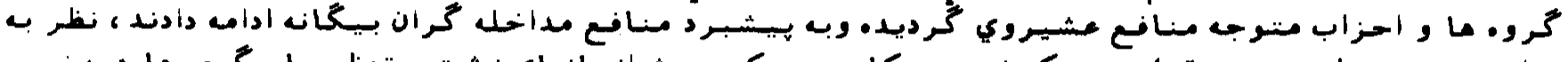

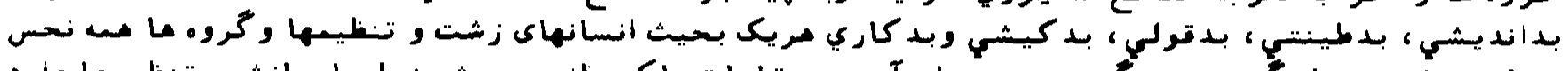

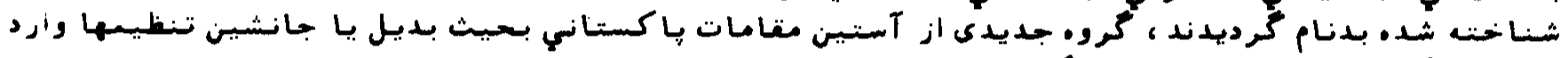

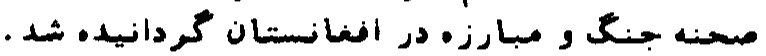

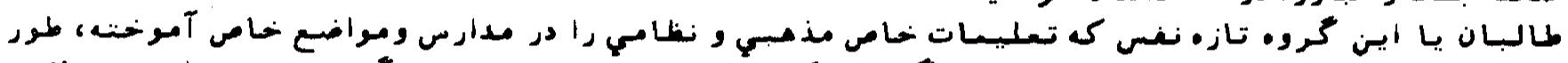

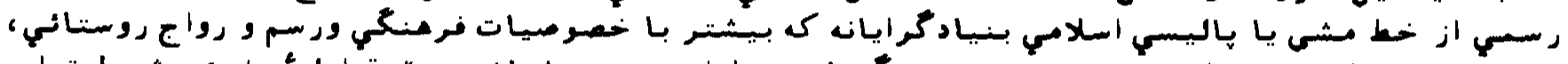

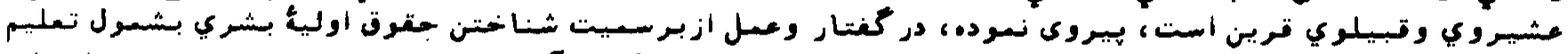

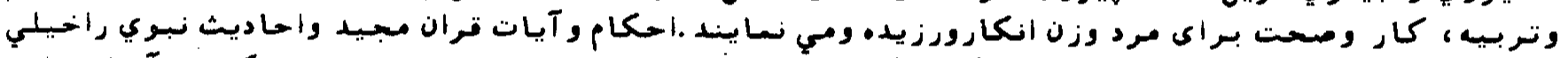

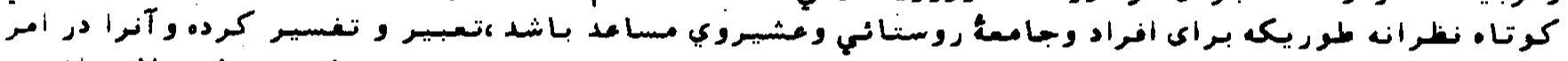

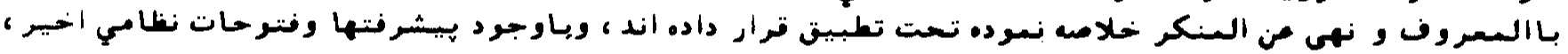

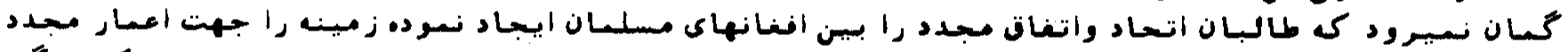

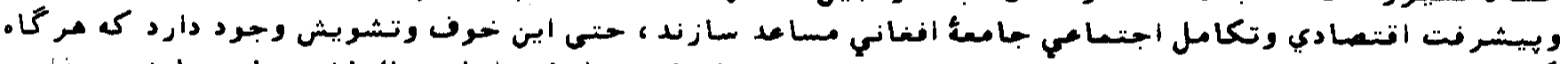

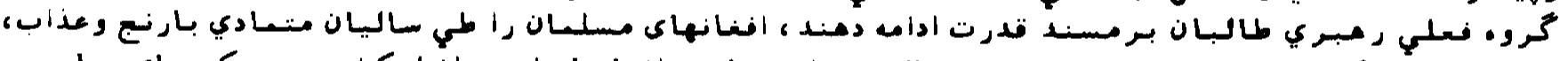

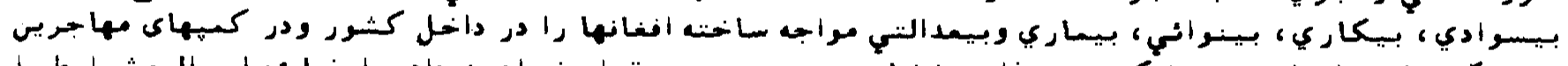

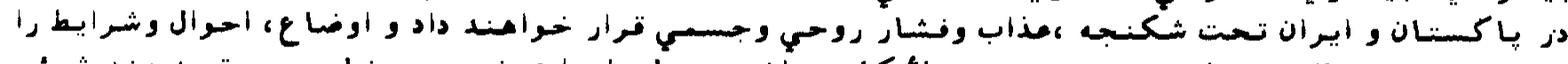

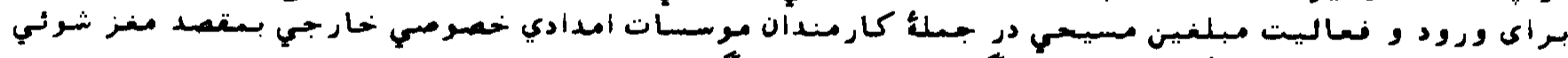

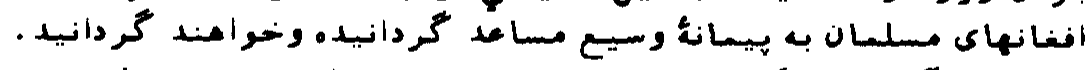

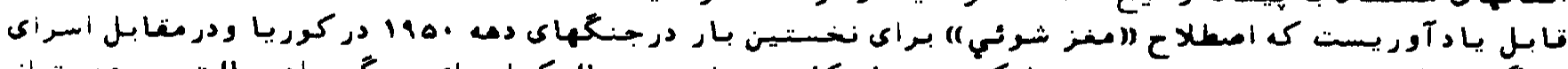

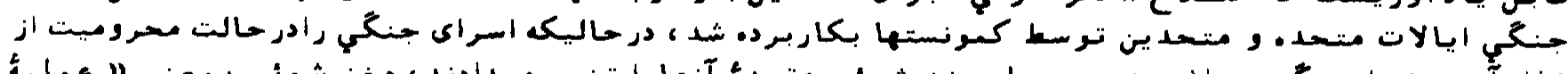

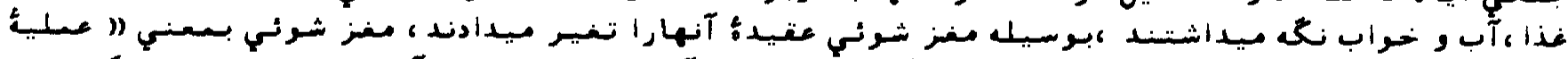

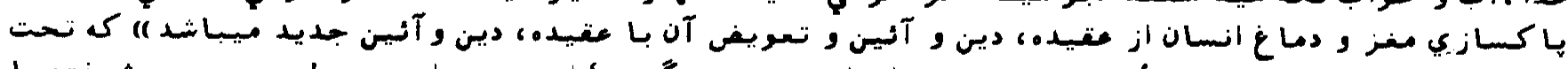

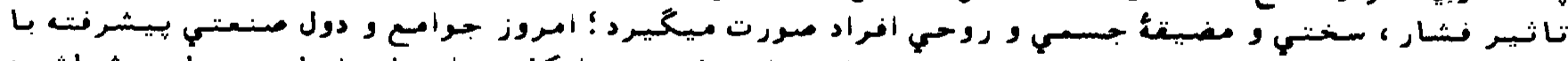

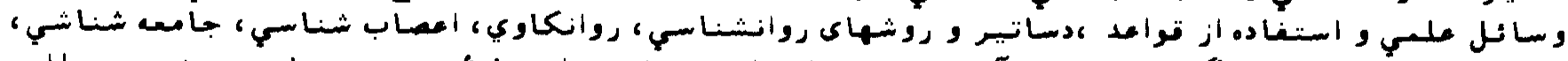

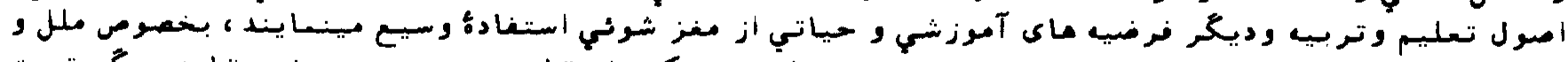

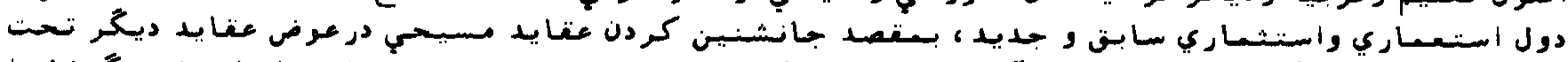

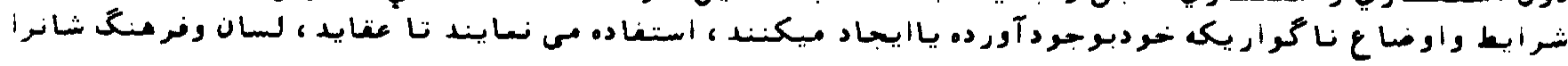

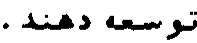

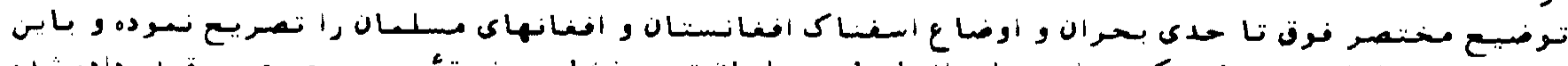

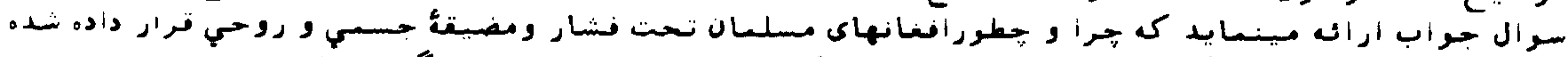

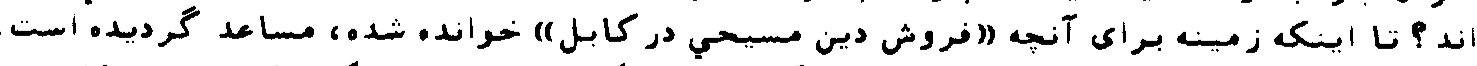

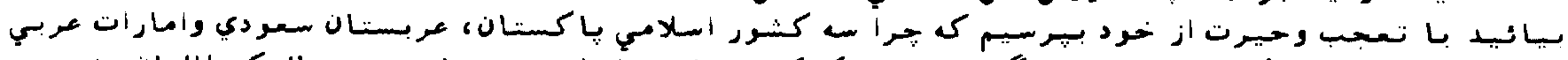

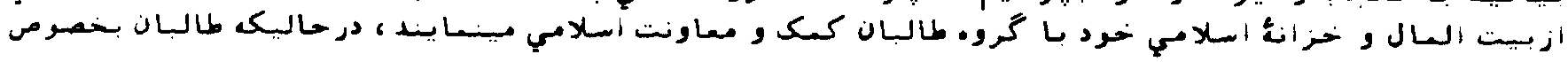




\section{7}

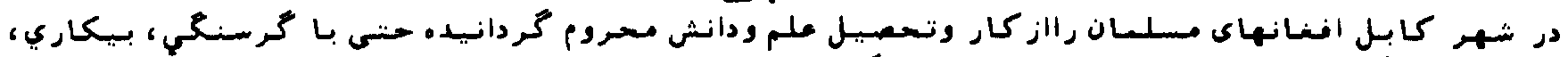

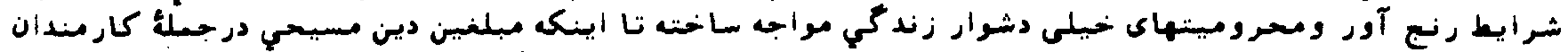

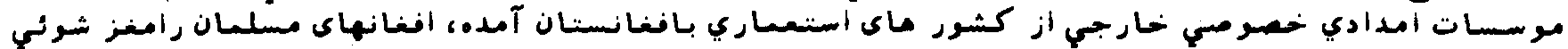

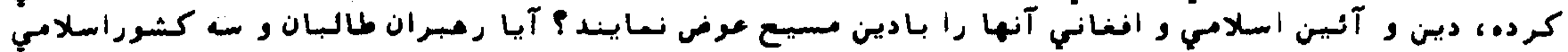

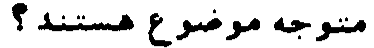

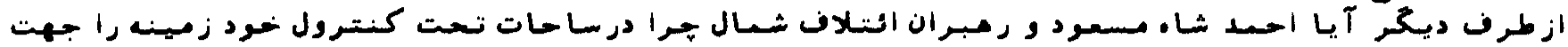

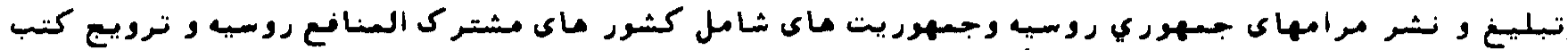

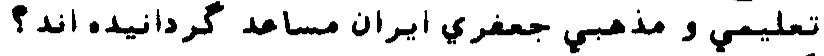

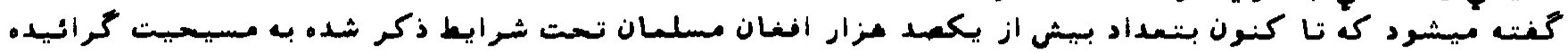

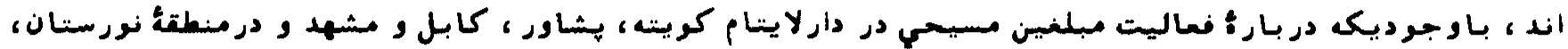

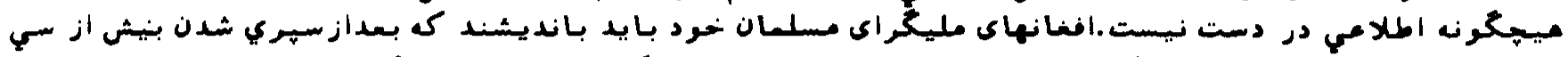

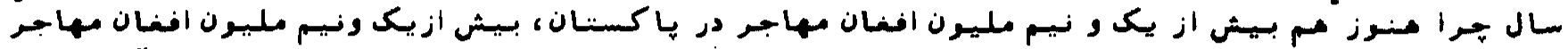

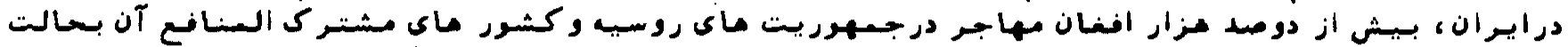

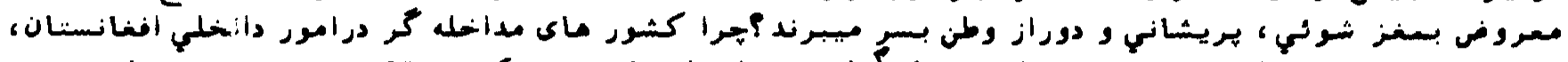

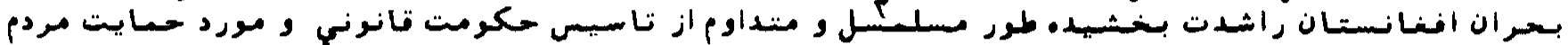

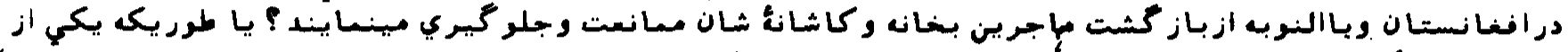

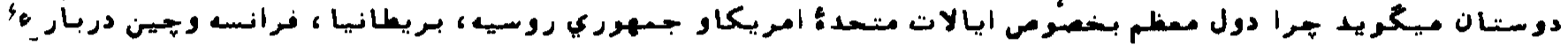

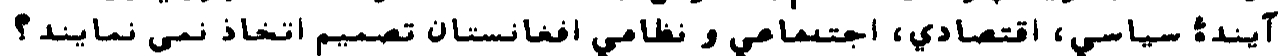

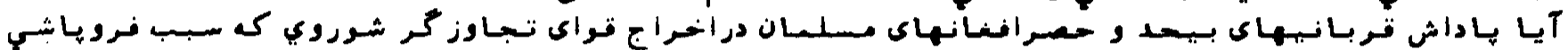

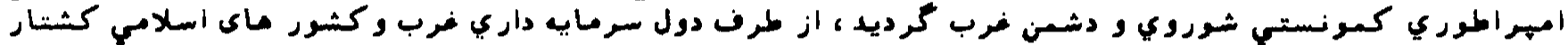

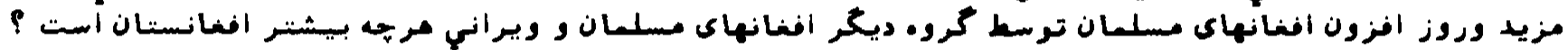

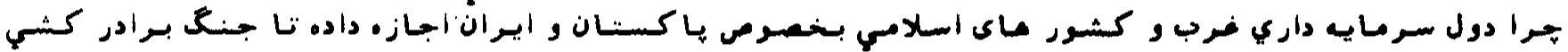

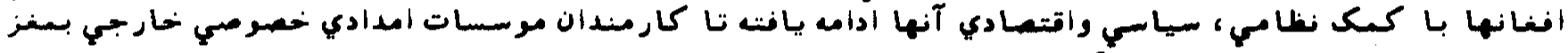

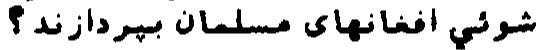

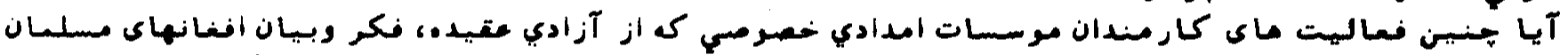

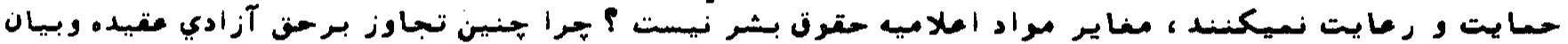

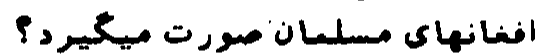

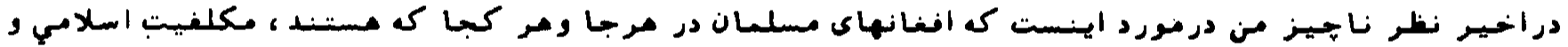

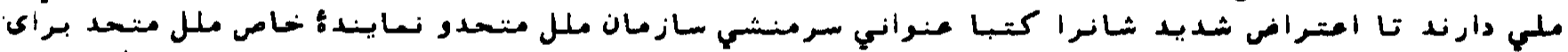

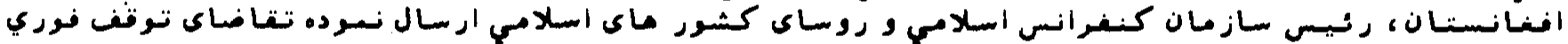

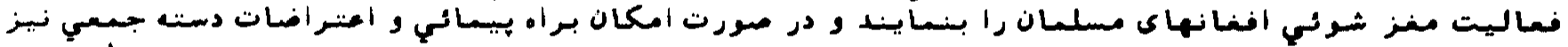

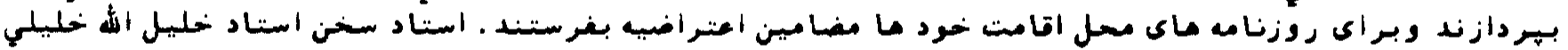

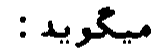

ز فرش زمين تا ثريا مباد

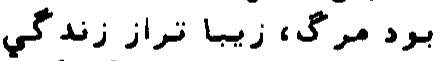

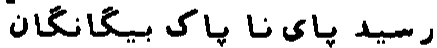

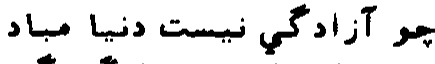

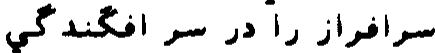

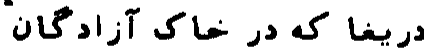

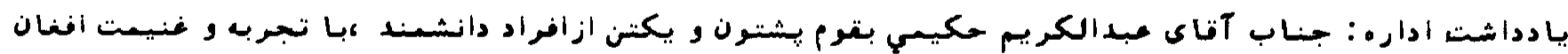

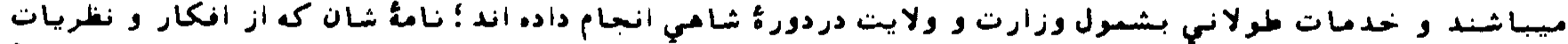

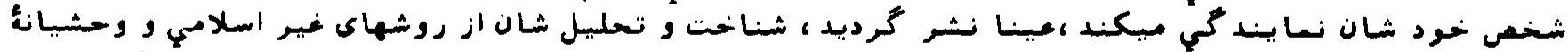

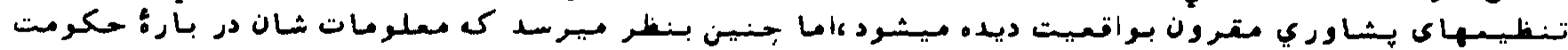

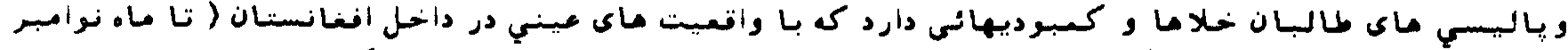

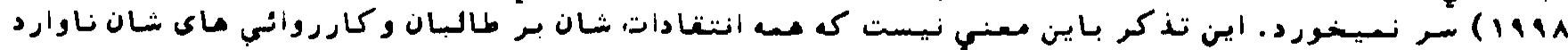

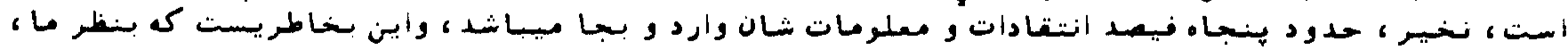

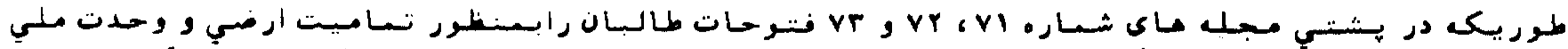

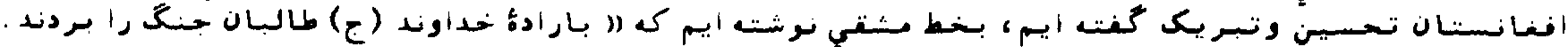

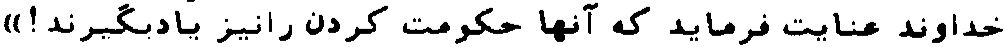

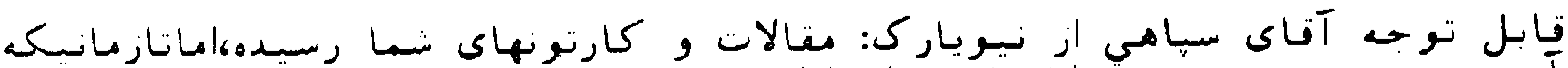

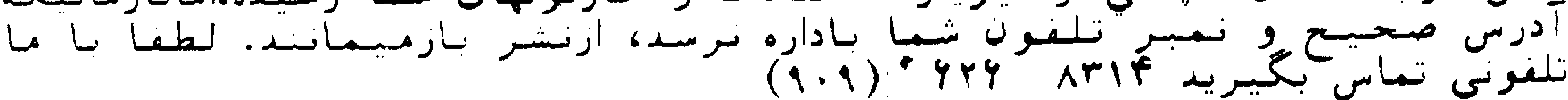




\section{8 كورخود وبهناي ديكران

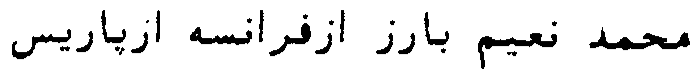

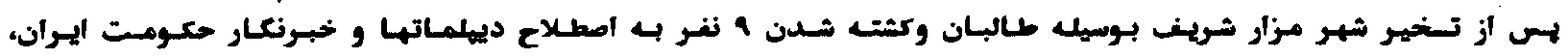

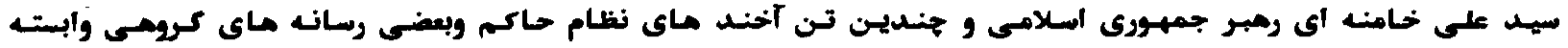

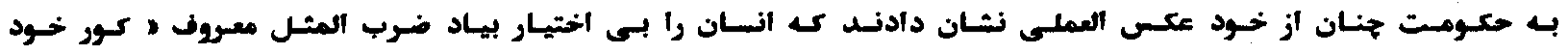

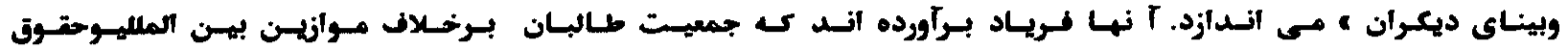

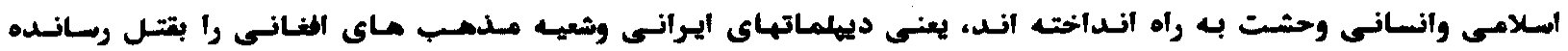

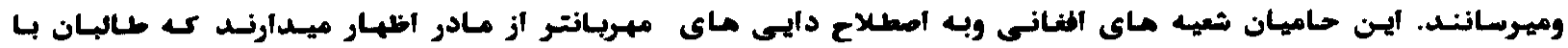

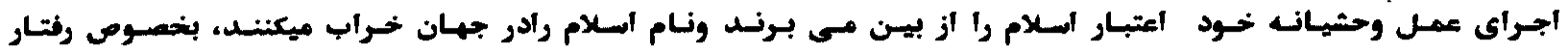

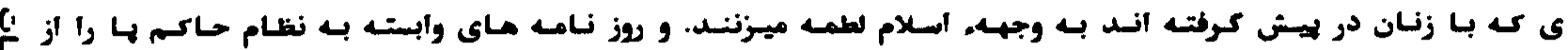

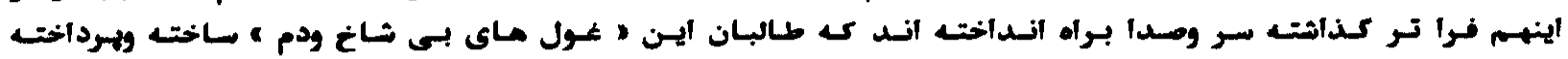

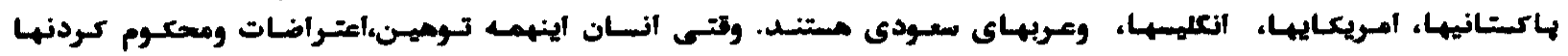

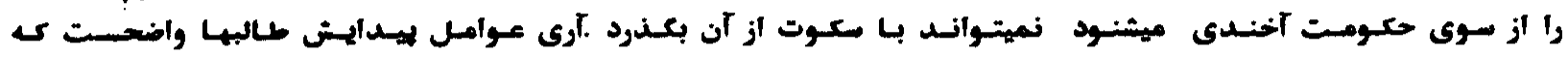

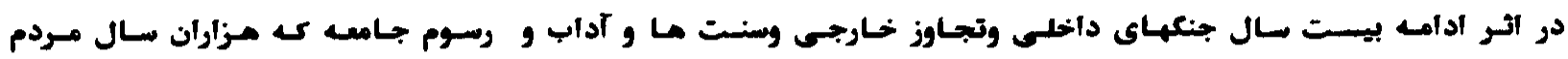

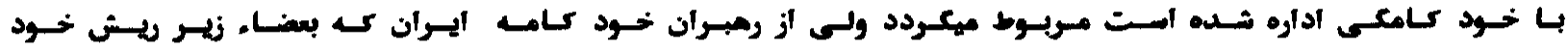

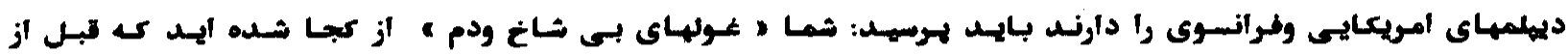

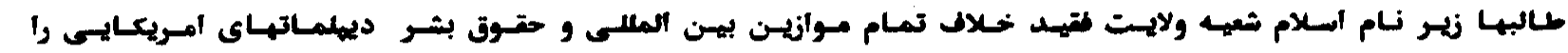

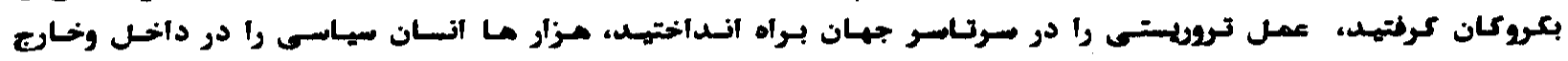

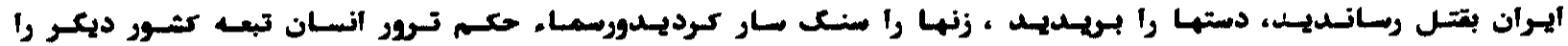

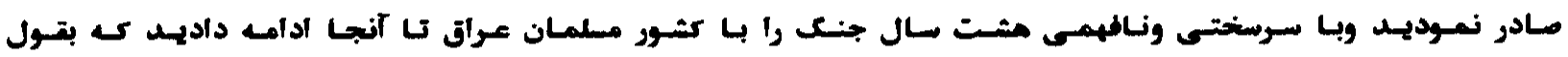

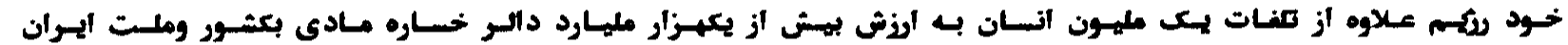

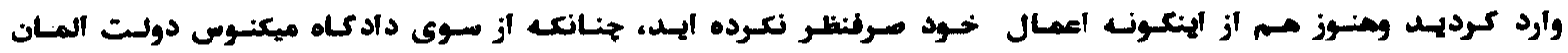

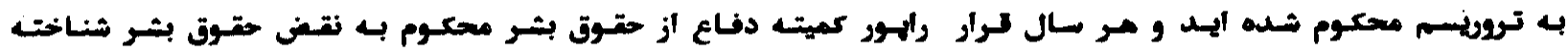

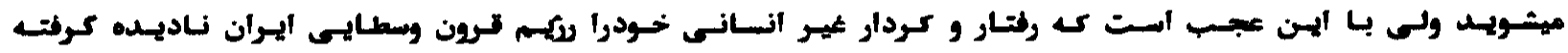

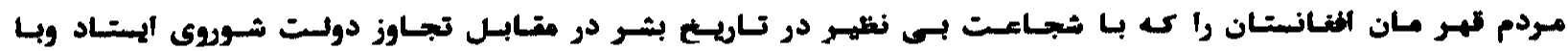

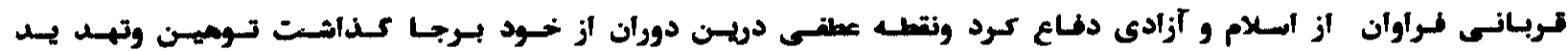
ميكند.

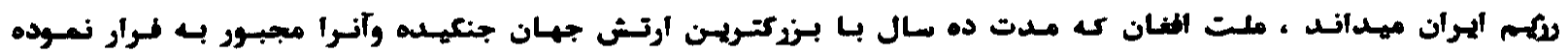

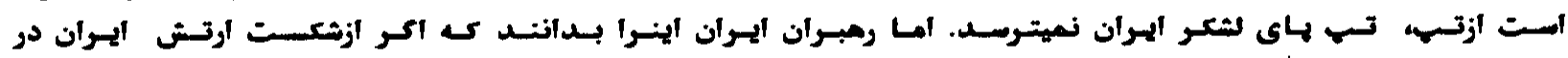

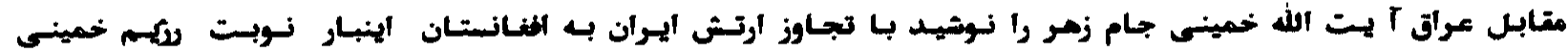

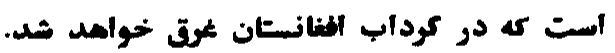

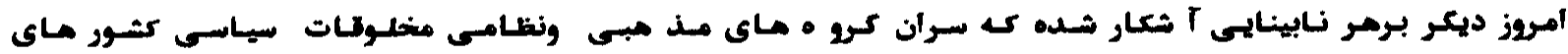

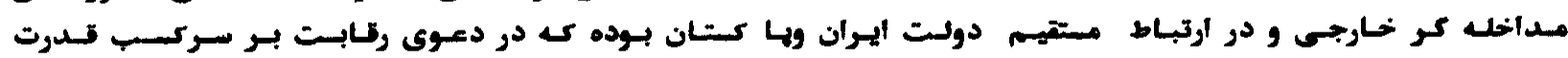

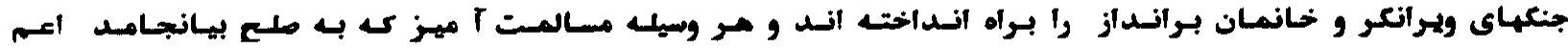

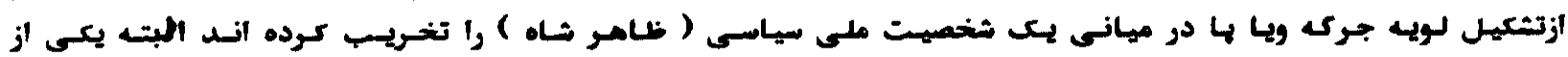

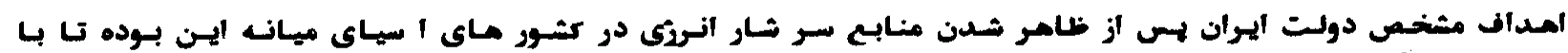

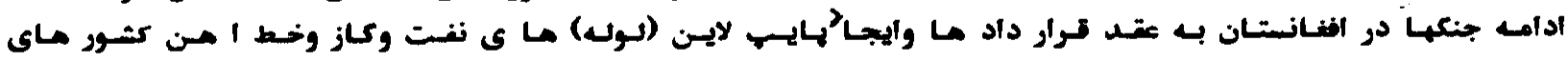

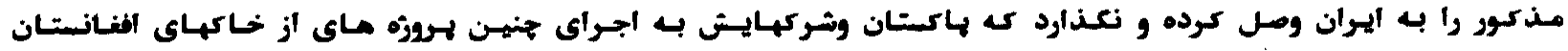

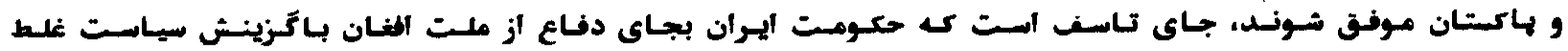

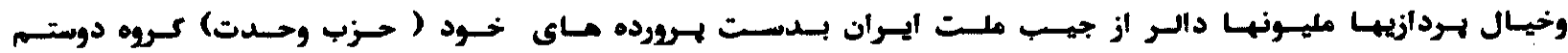

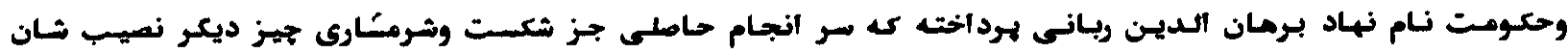

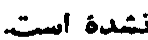

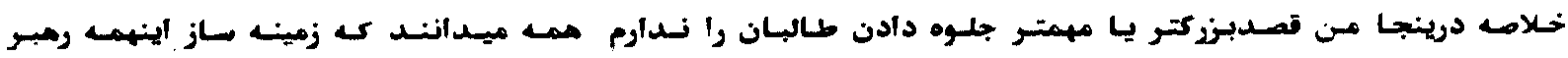

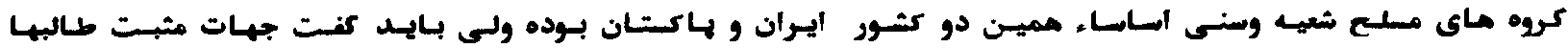

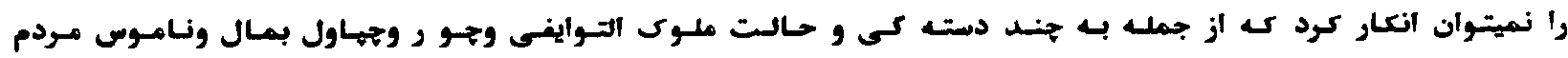

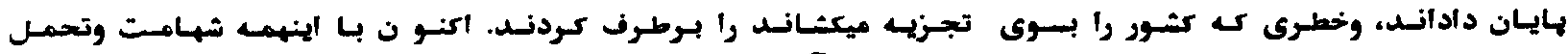

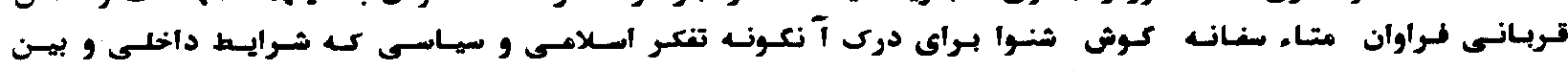

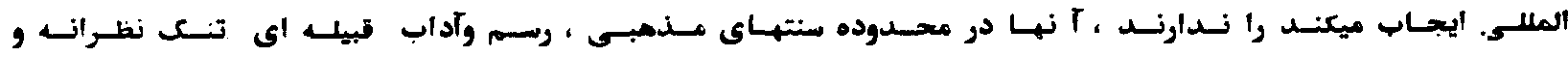

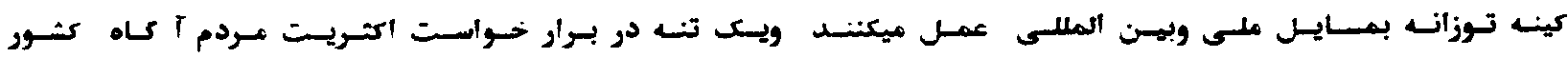




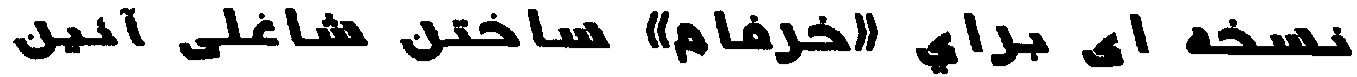

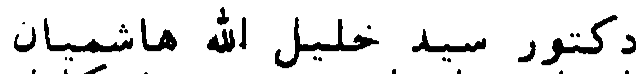

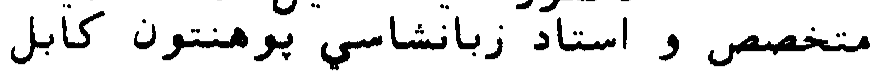

تسمت دوم

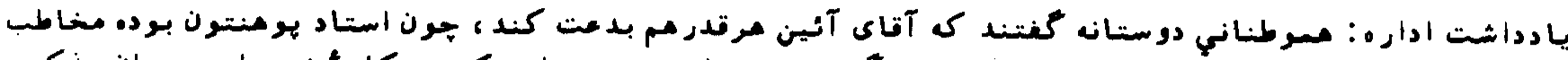

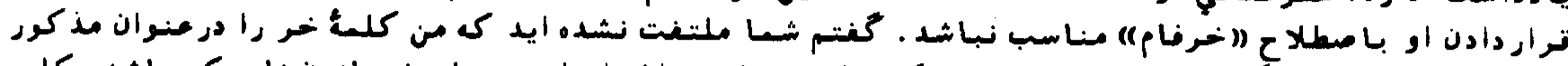

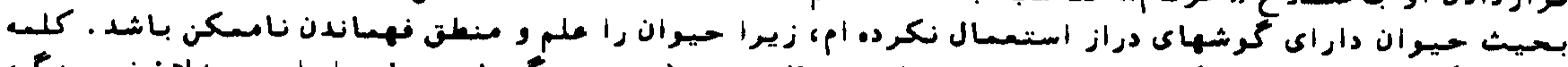

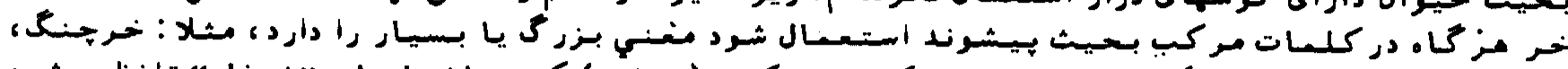

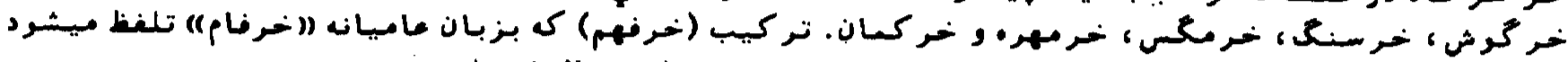

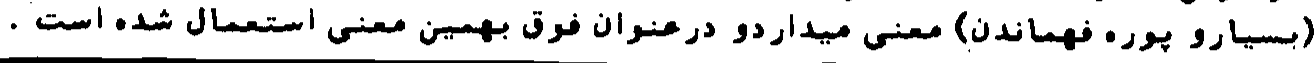

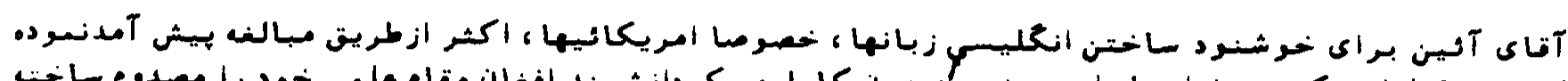

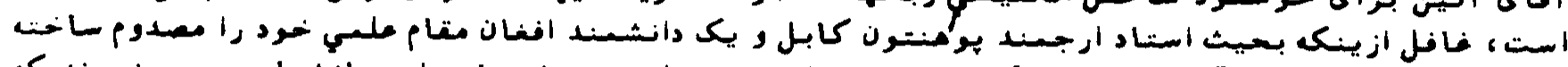

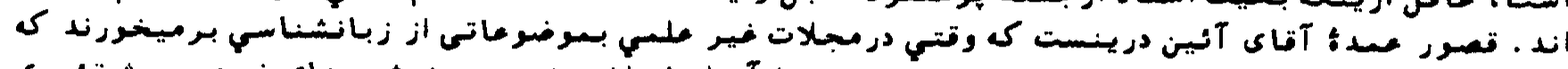

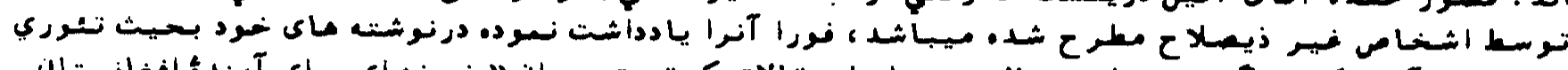

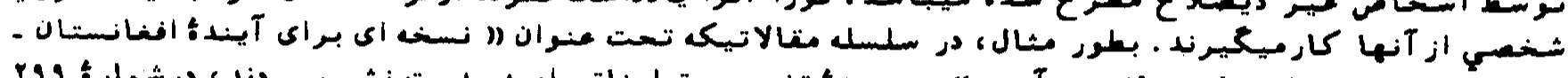

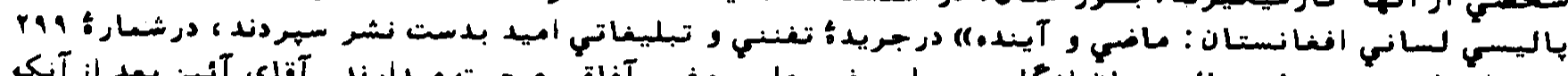

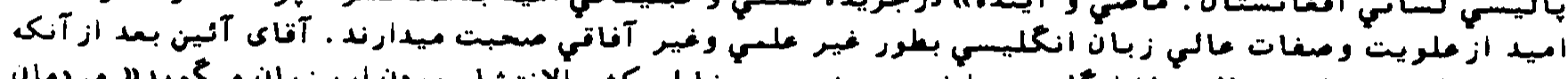

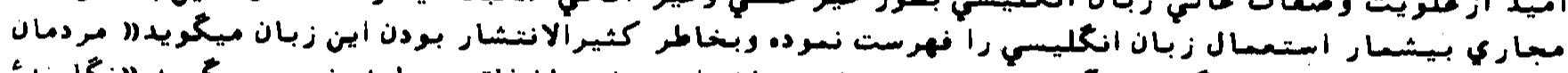

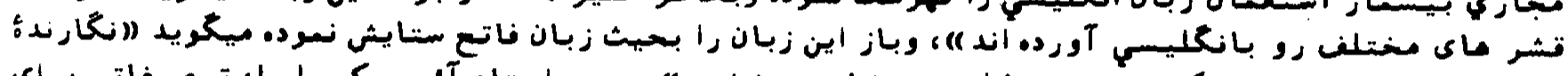

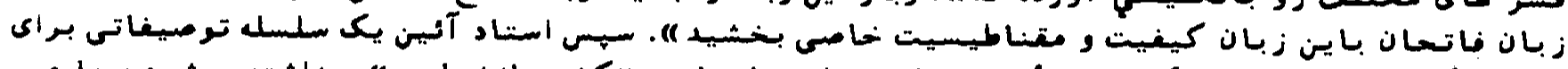

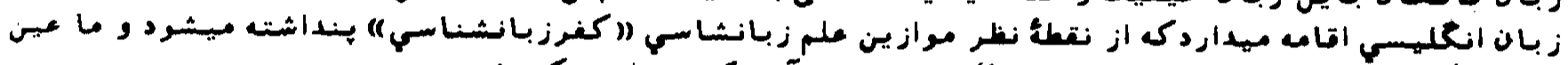

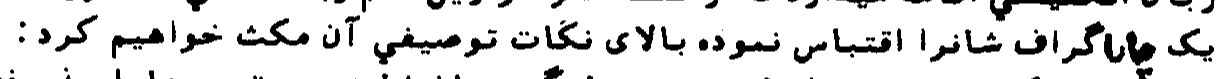

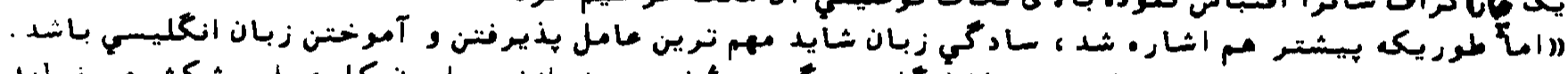

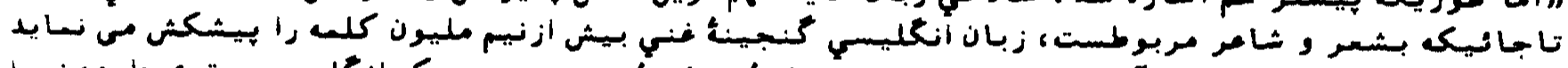

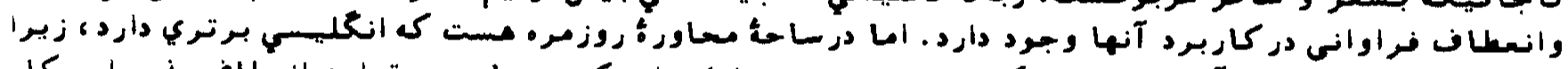

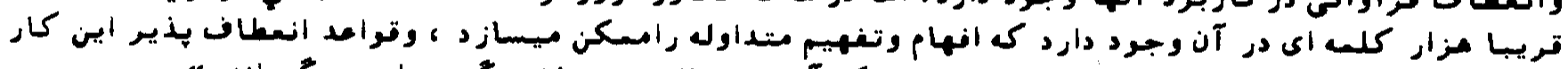

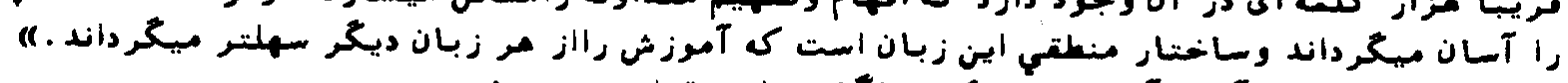

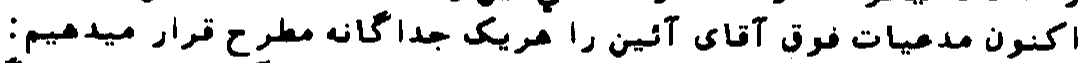

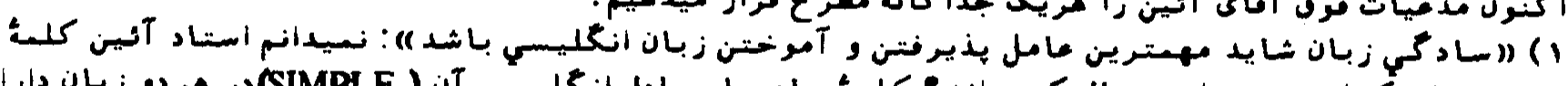

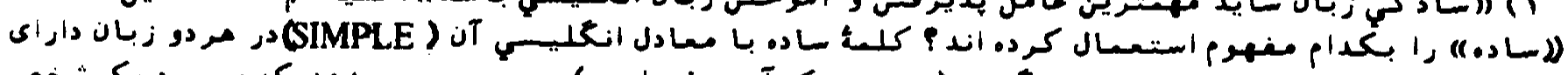

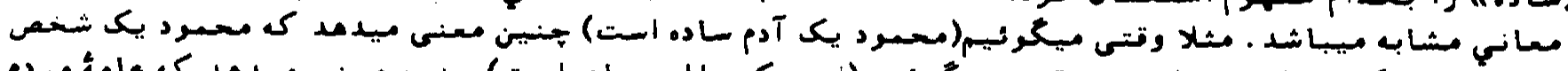

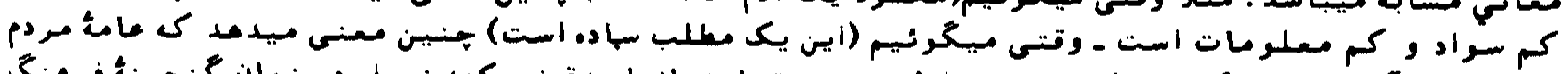

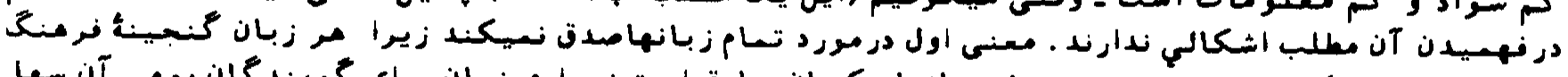

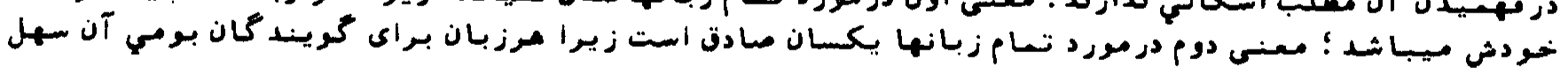

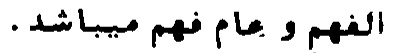

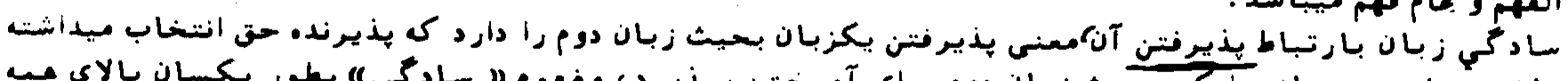

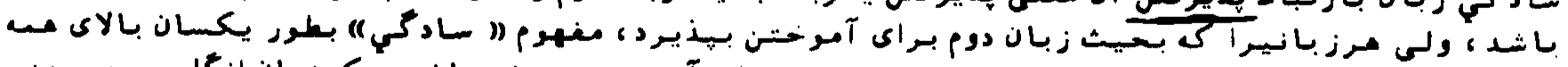

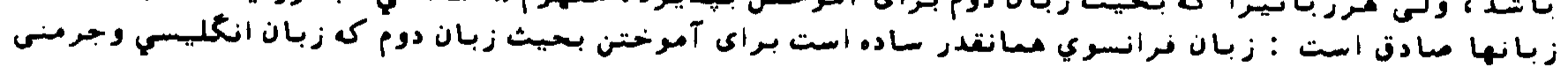

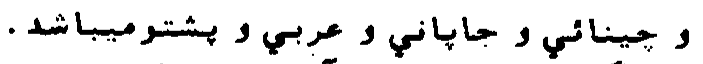

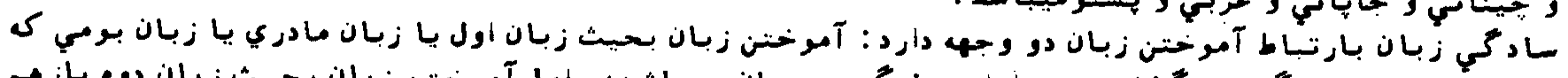

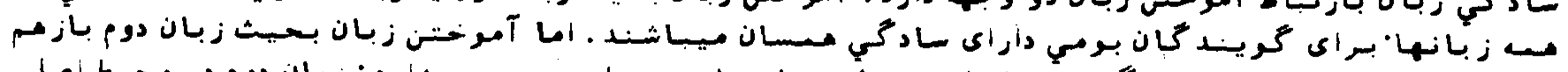

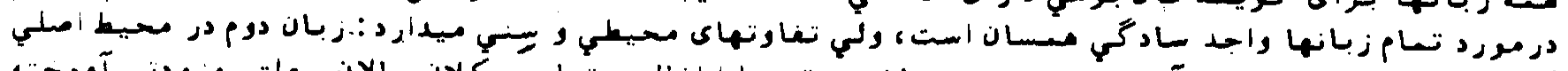

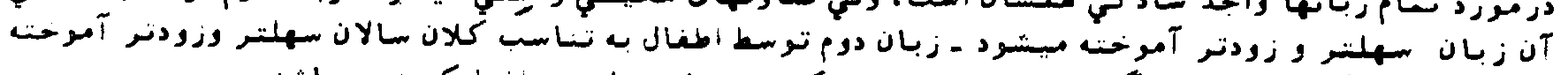

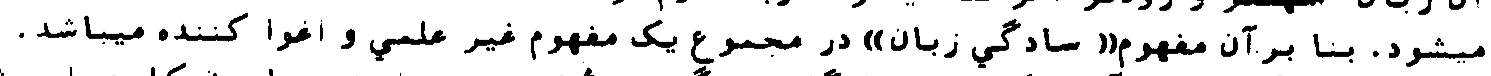

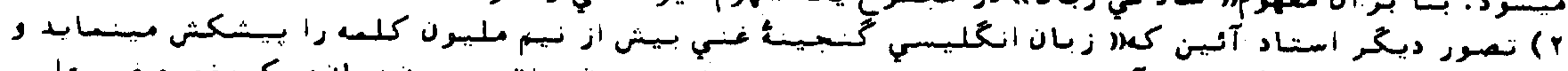

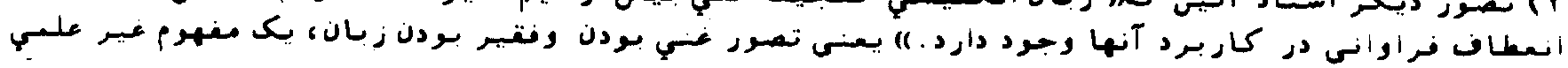




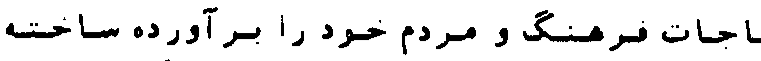

$-125$

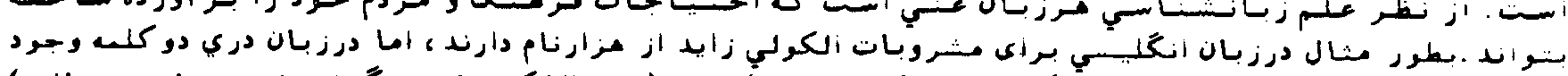

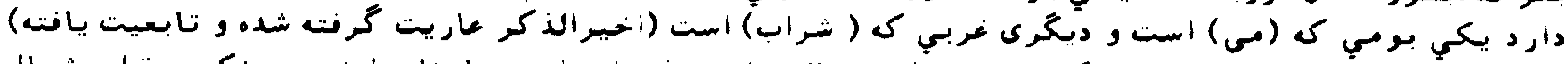

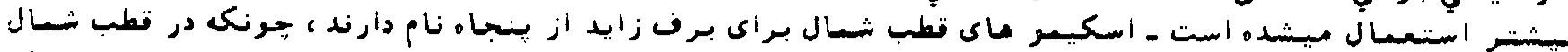

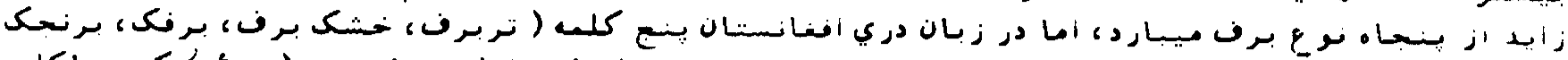

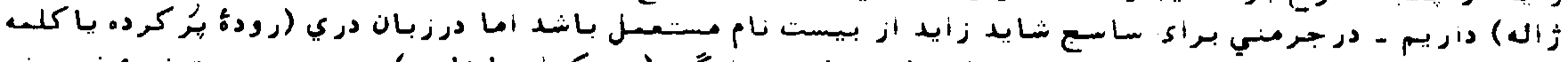

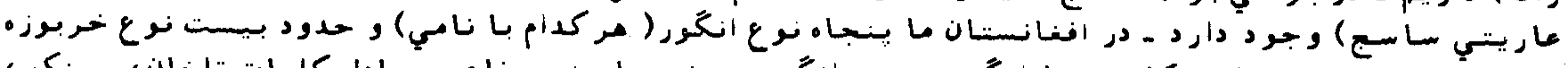

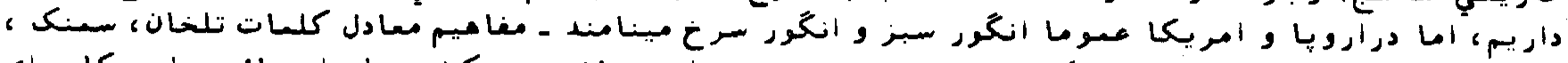

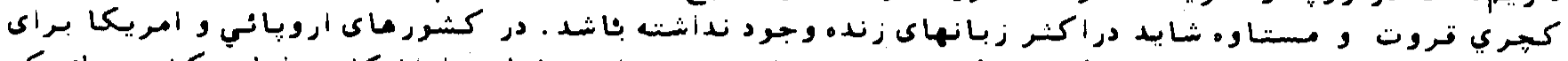

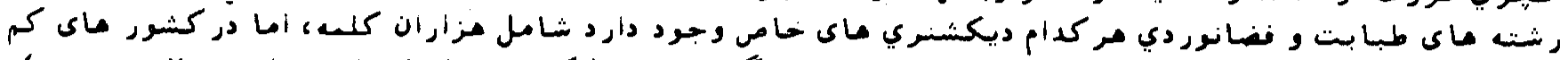

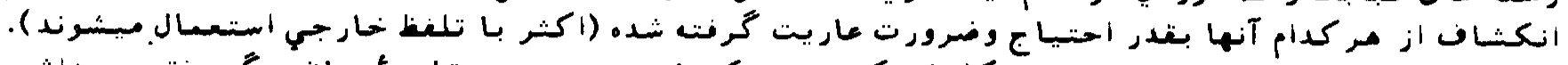

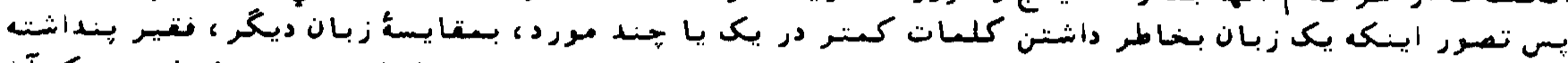

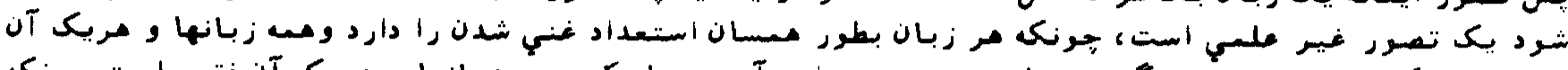

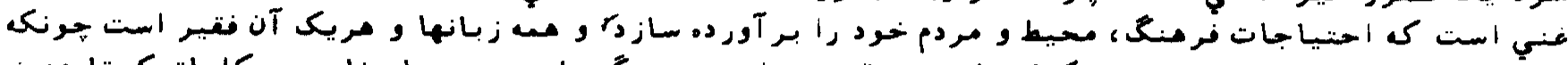

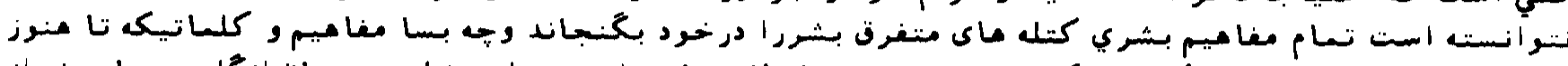

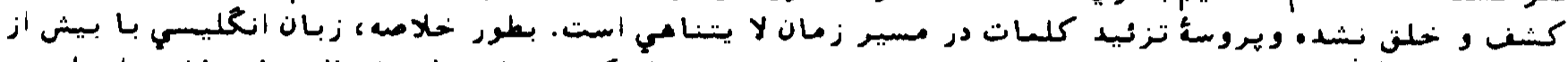

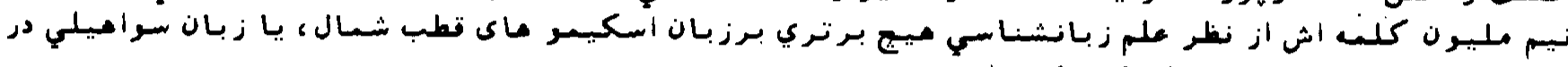

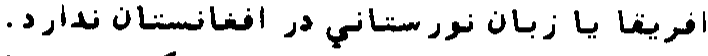

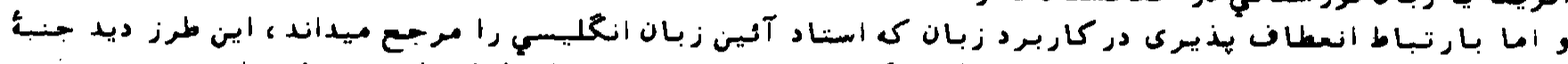

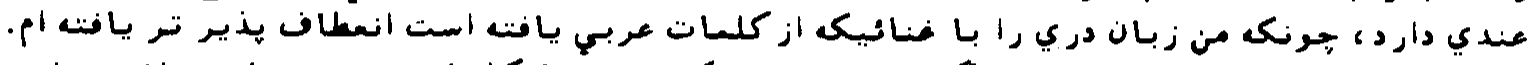

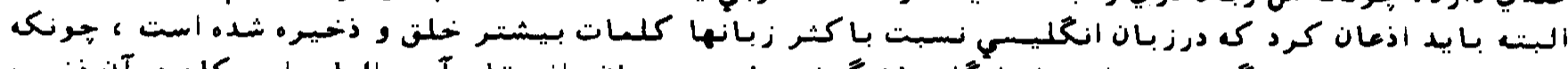

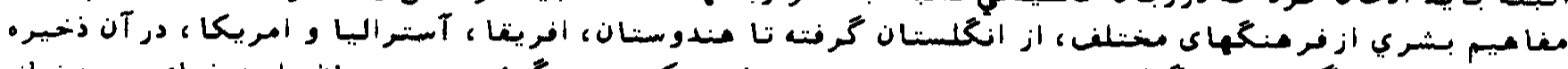

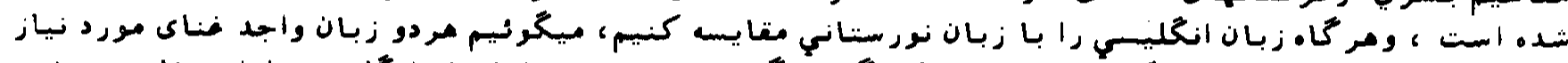

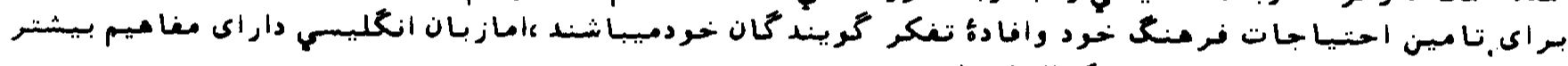

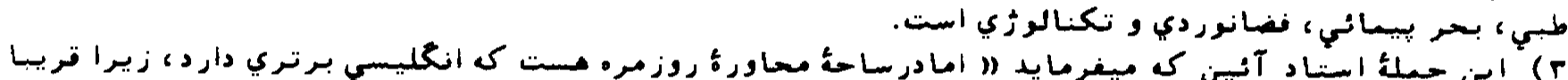

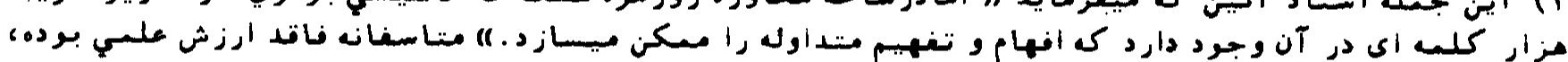

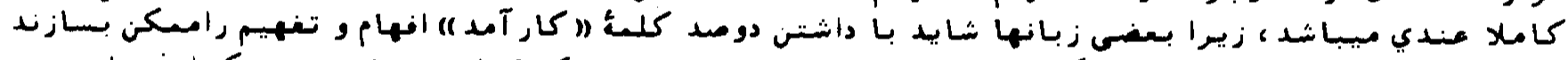

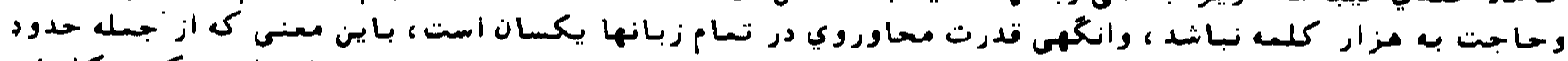

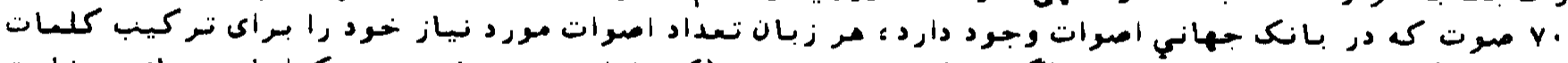

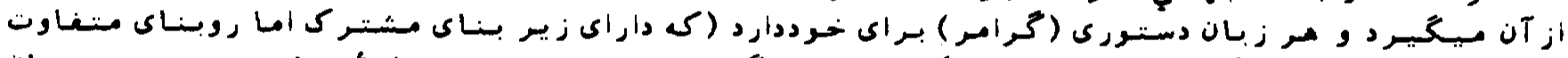

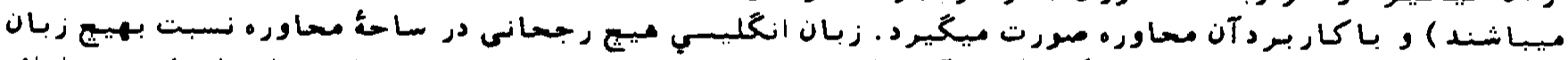

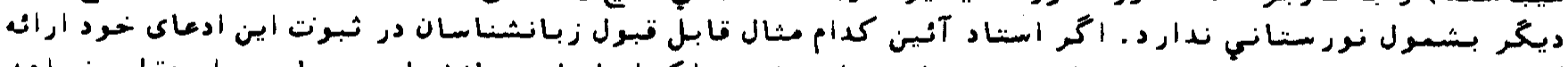

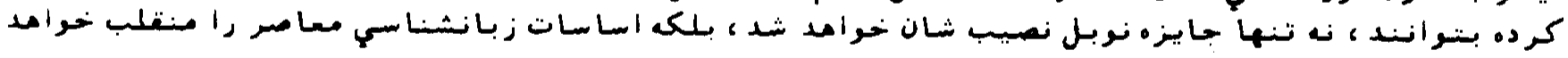

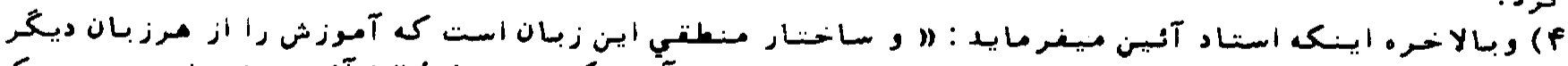

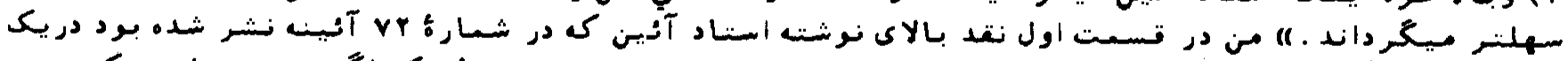

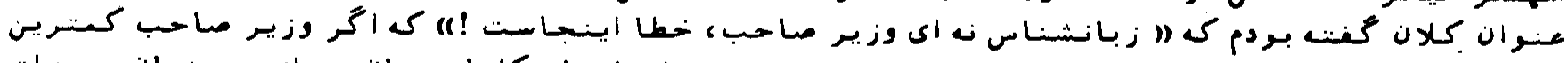

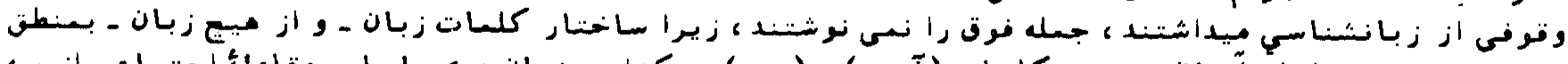

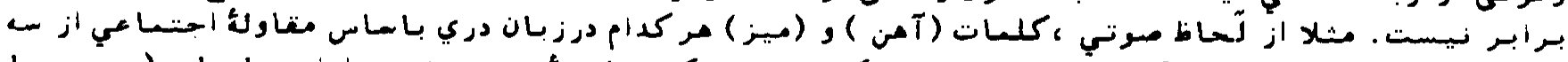

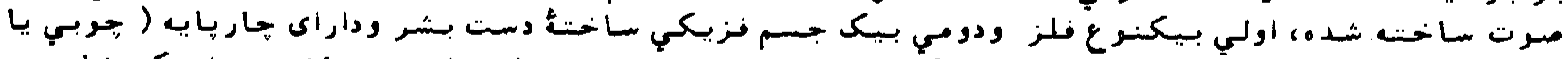

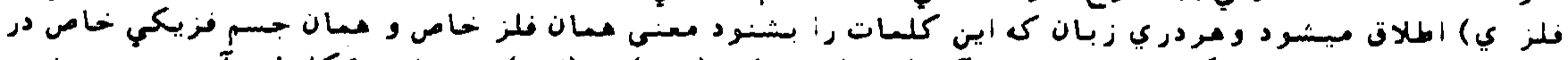

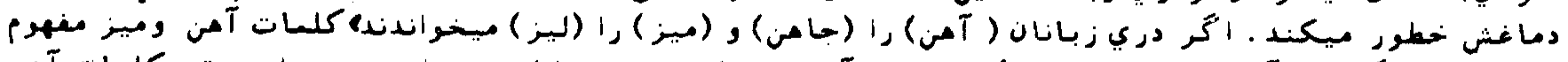

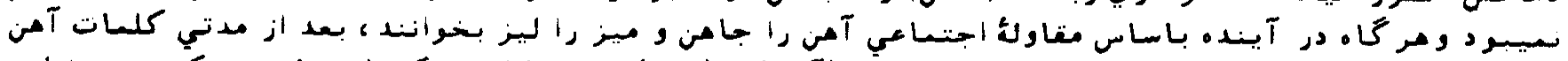

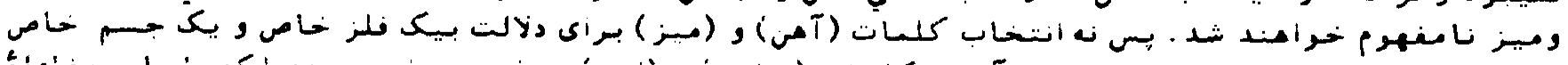

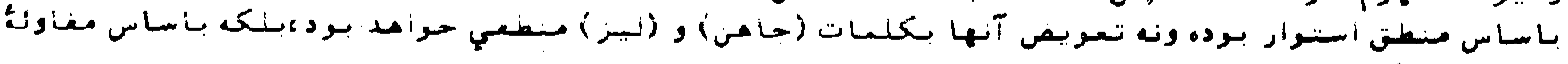

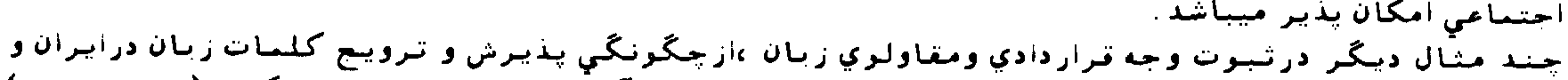

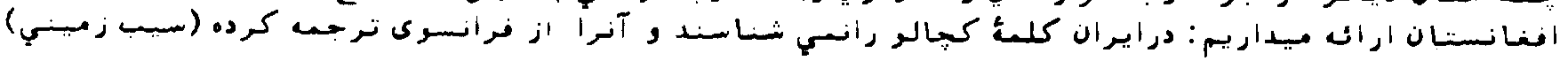




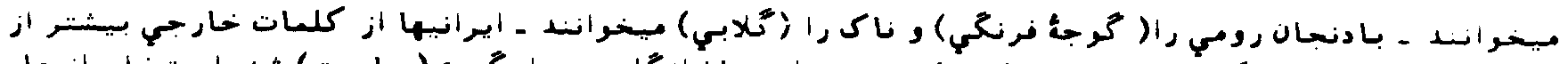

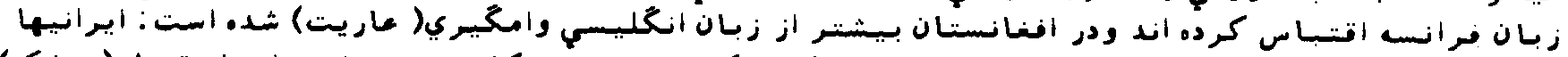

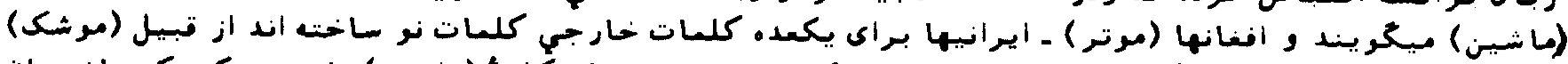

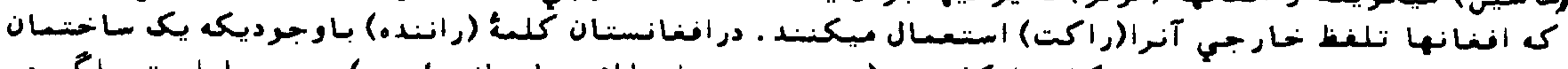

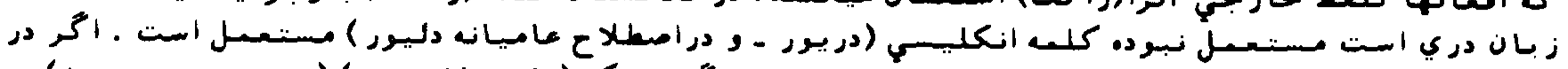

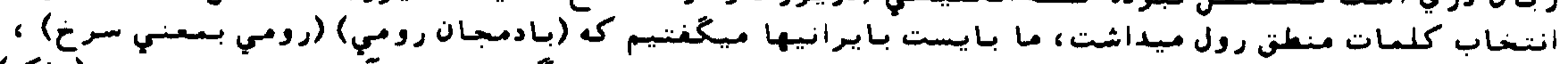

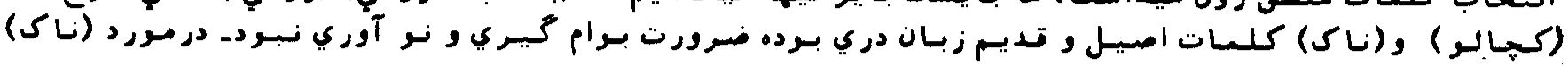

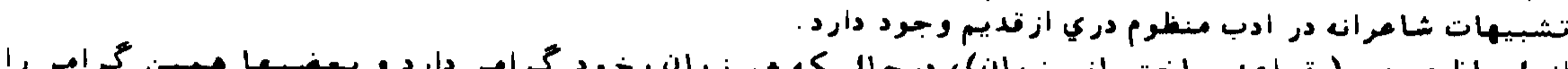

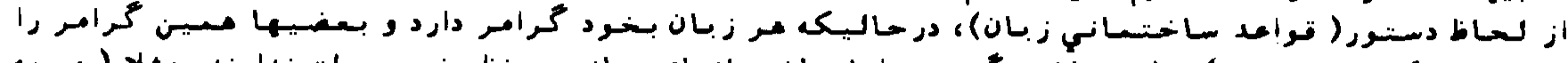

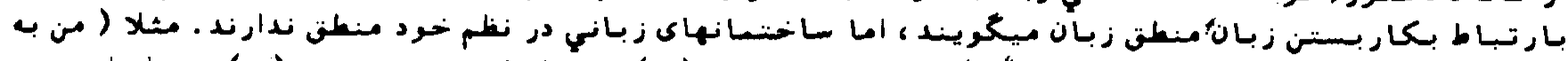

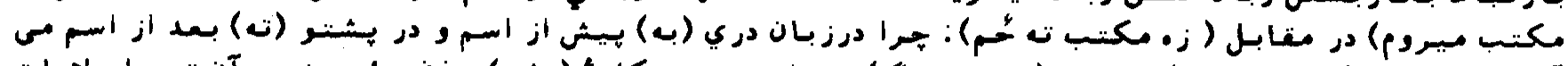

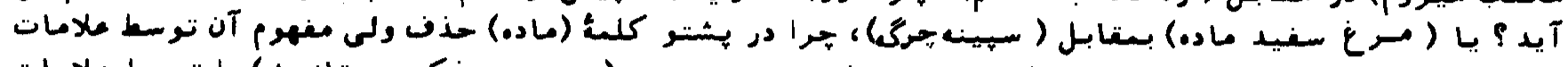

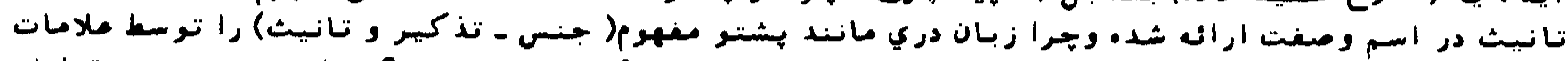

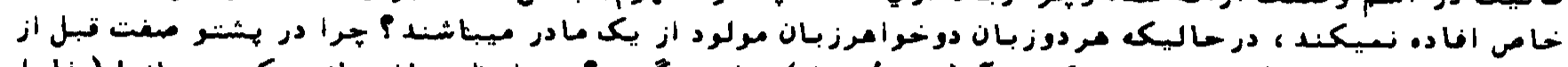

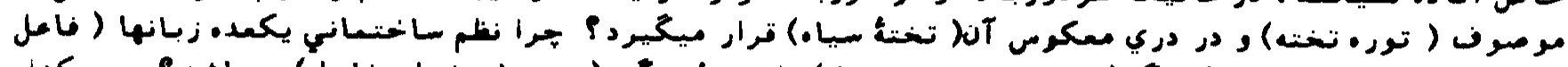

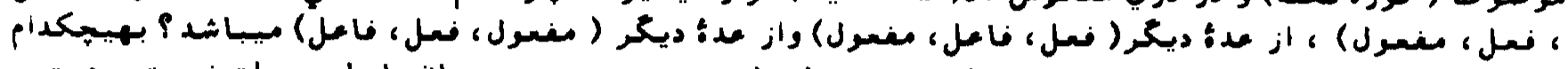

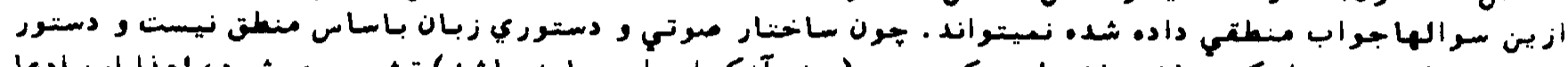

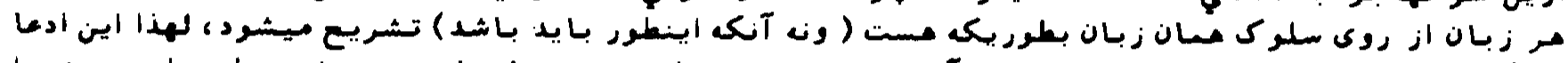

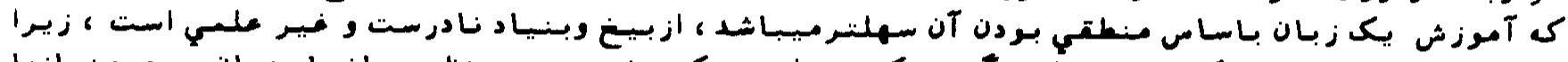

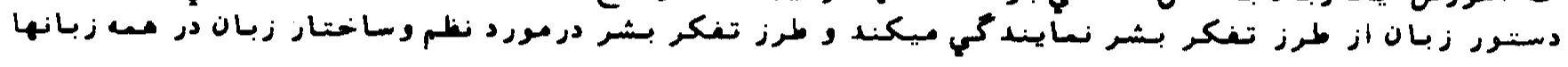

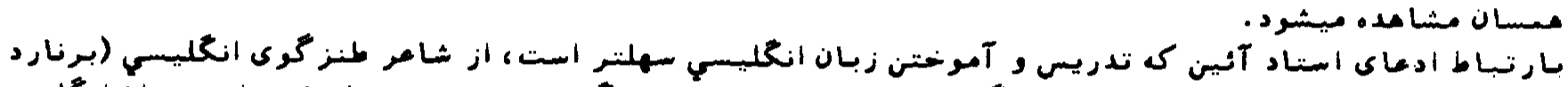

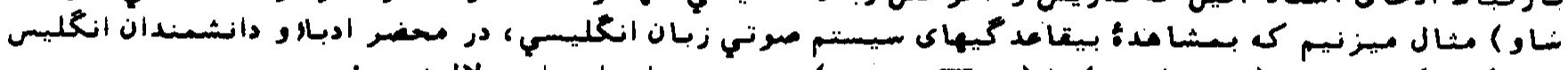

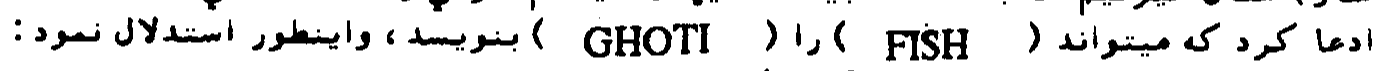

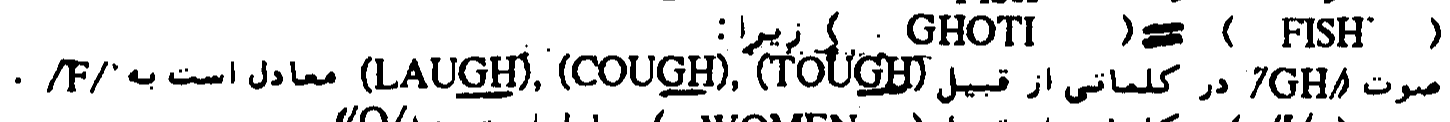

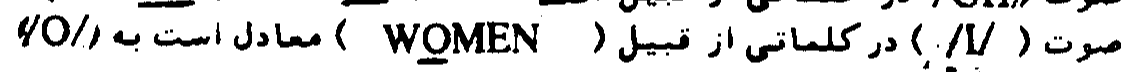

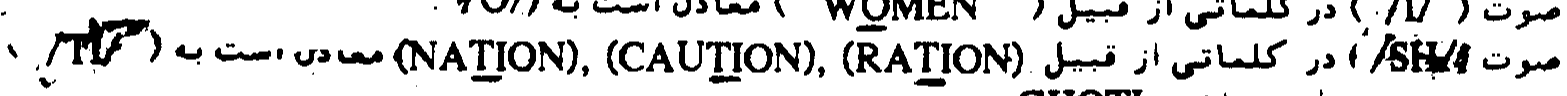

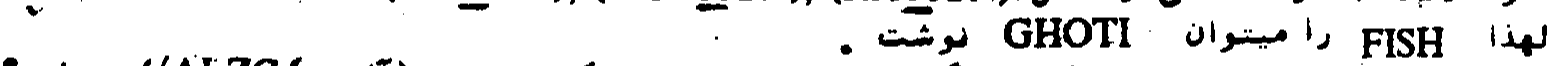

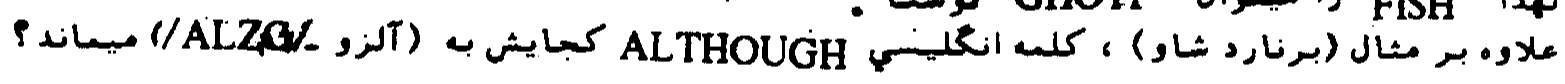

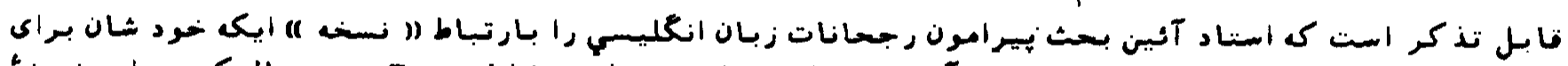

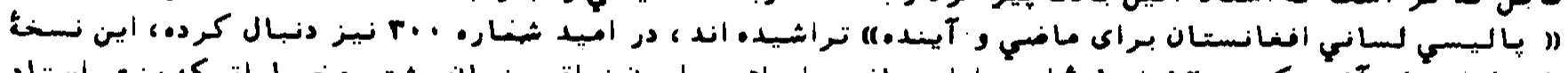

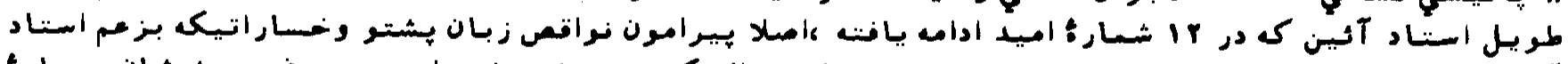

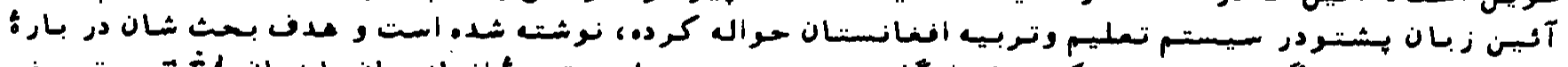

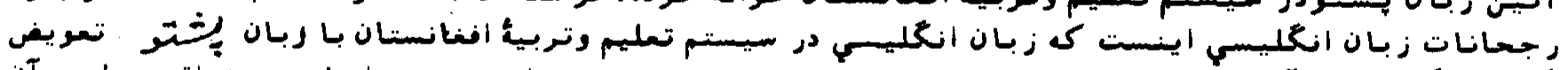

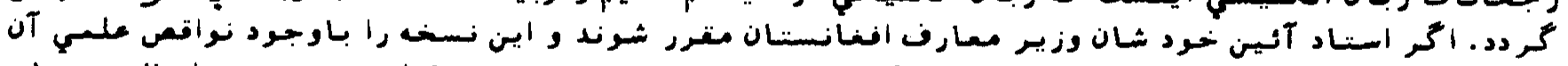

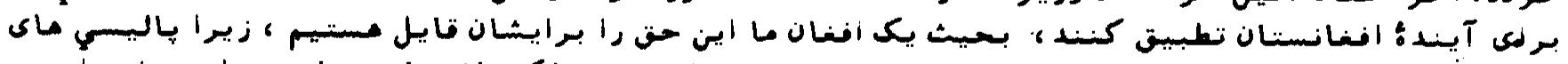

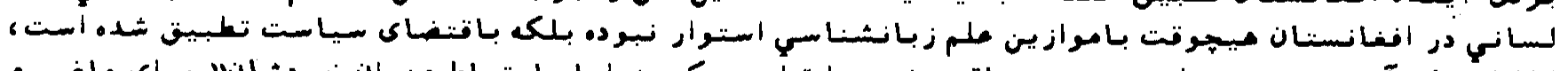

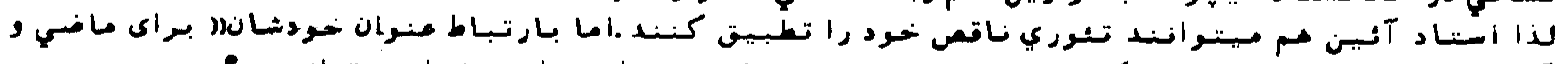

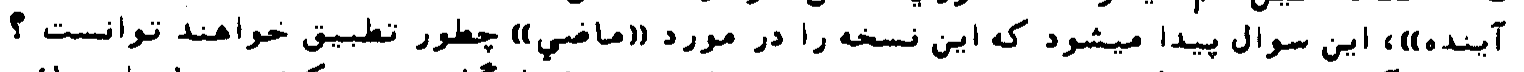

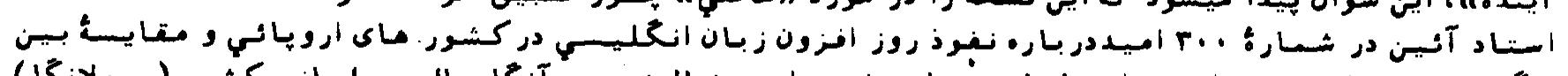

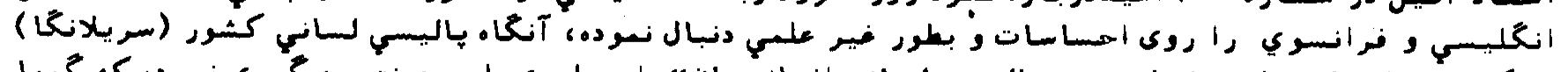

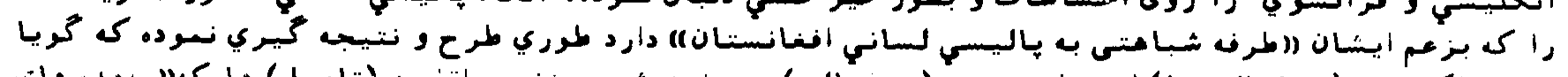

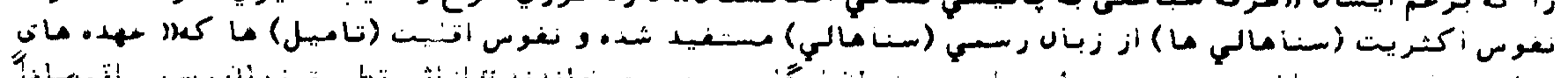

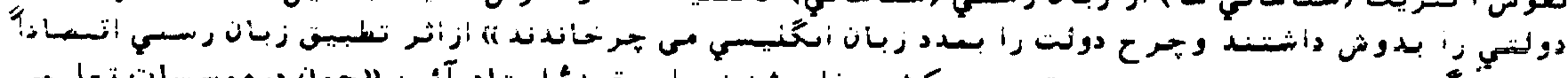

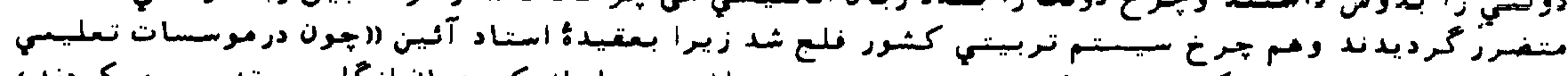

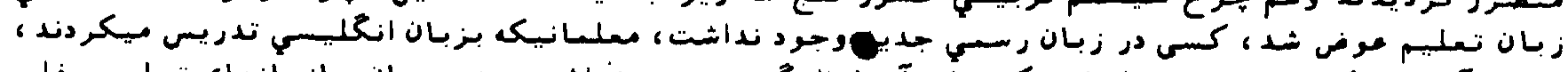

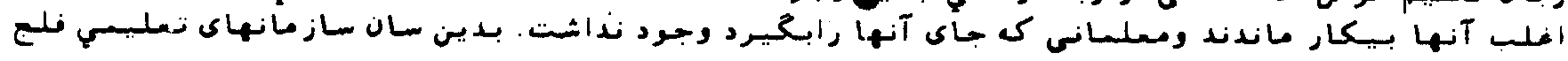




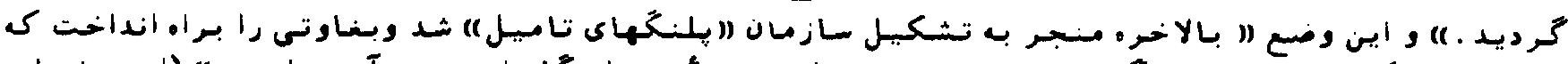

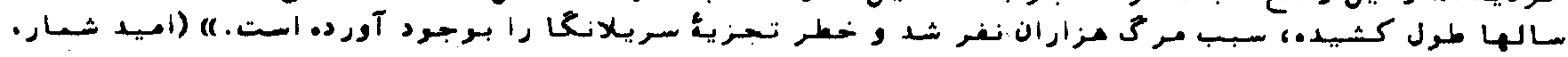
. ( $r$. .

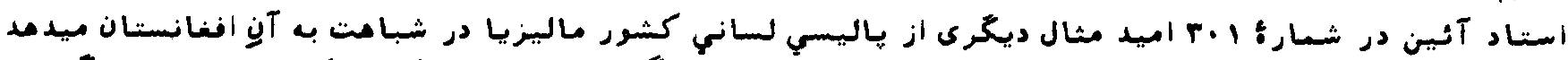

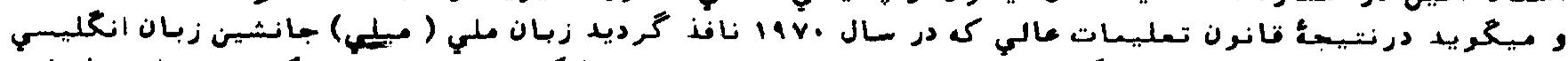

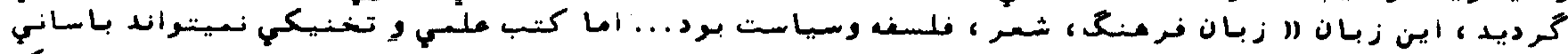

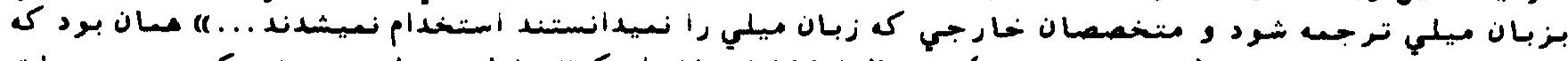

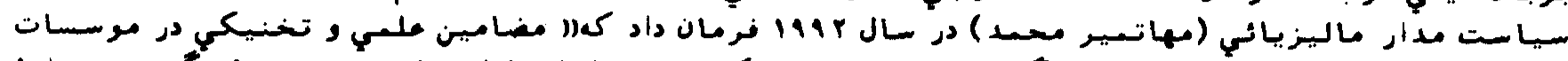

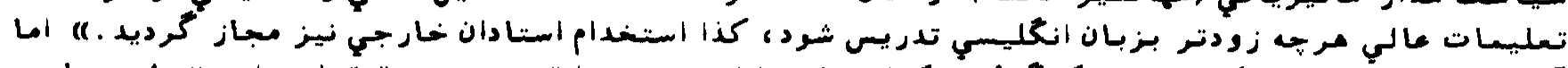

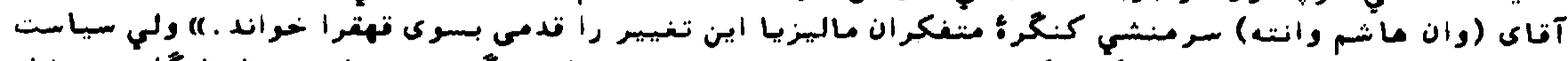

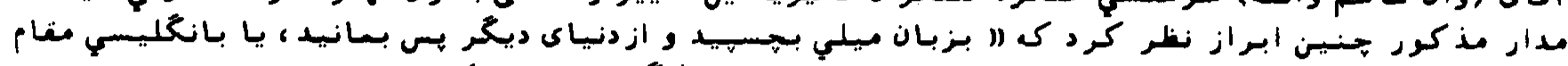

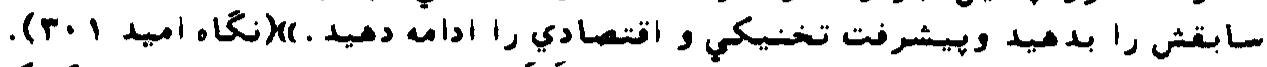

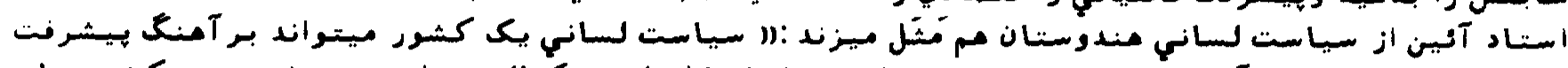

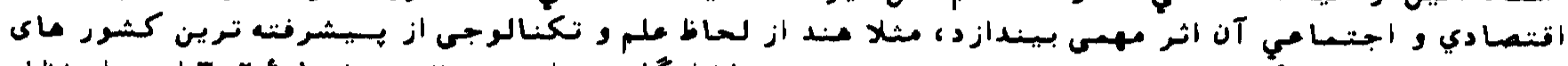

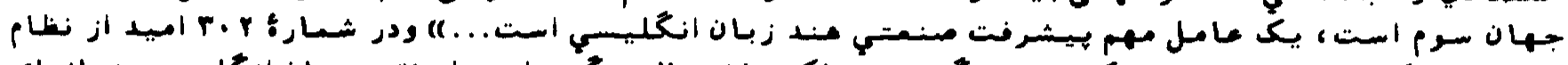

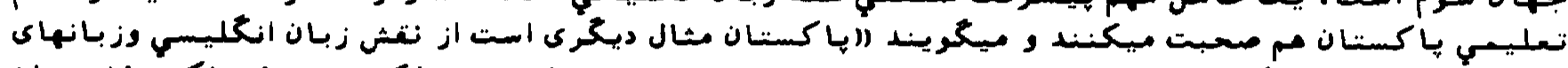

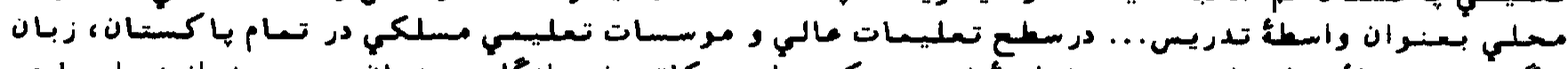

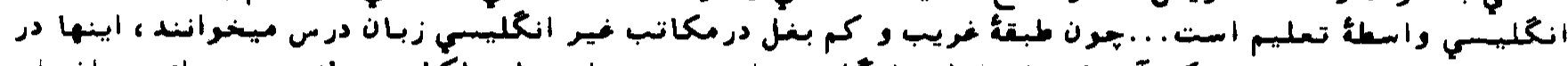

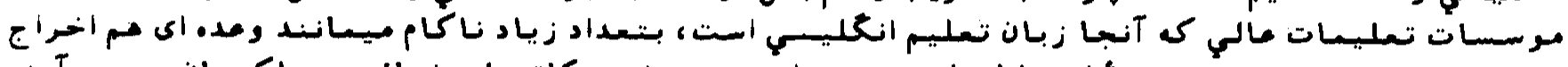

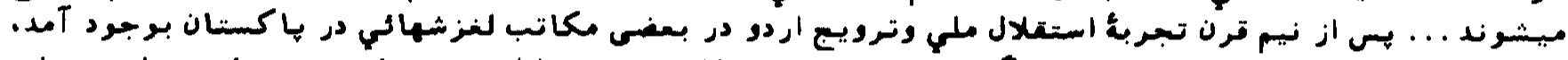

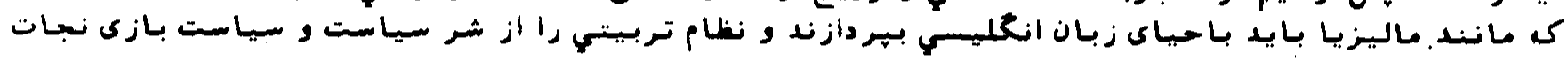
دمند.... (I)

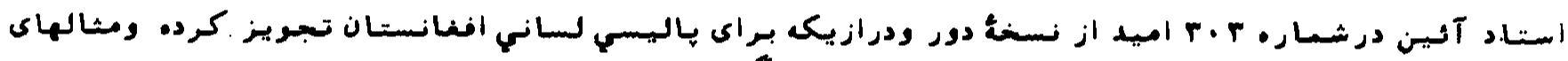

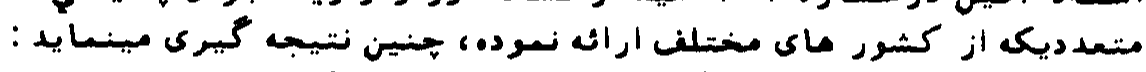

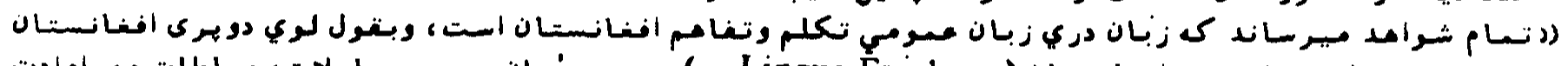

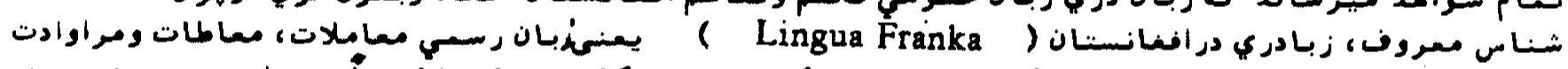

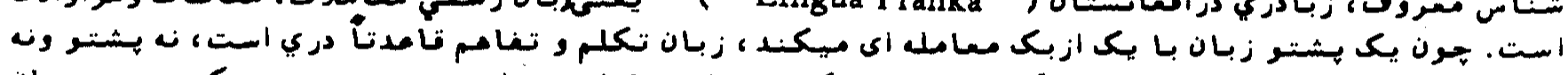

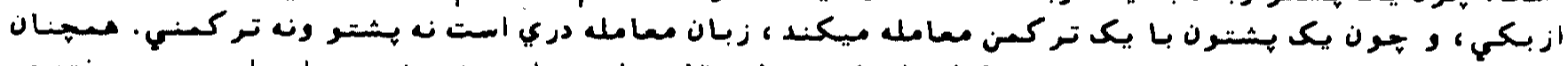

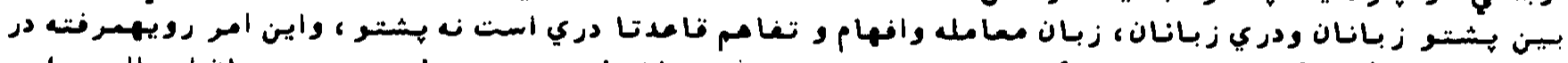

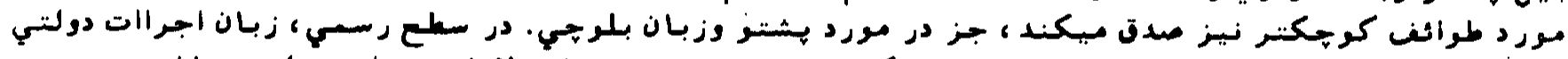

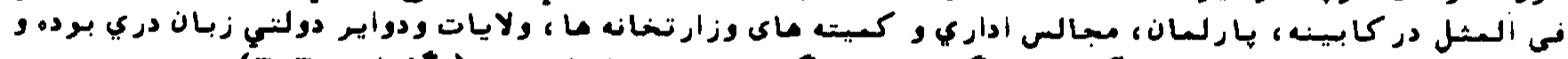

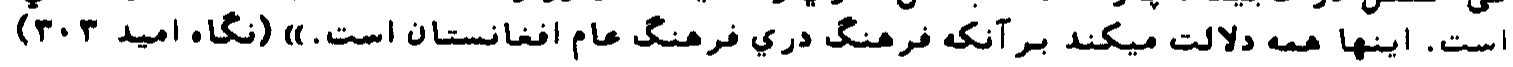

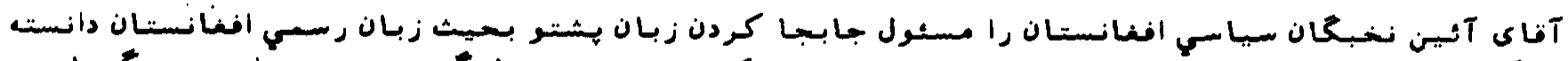

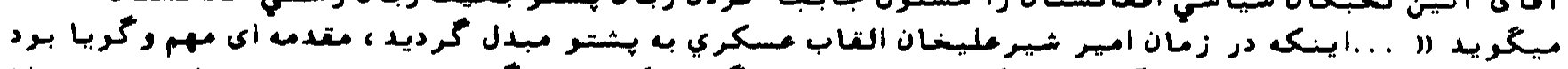

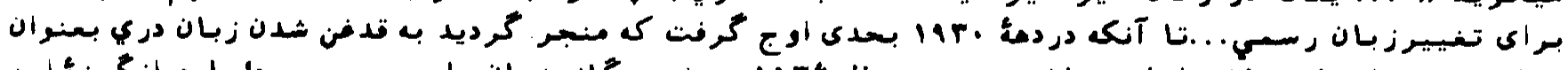

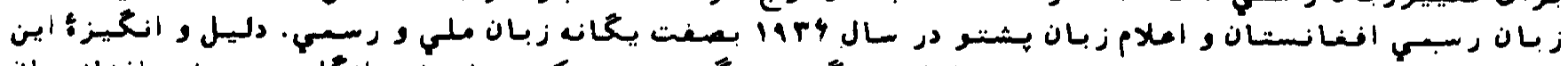

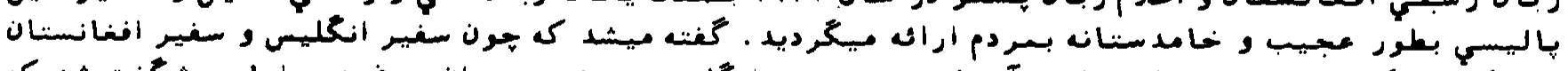

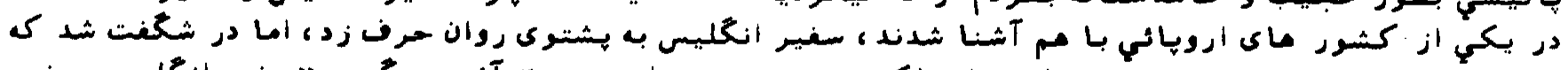

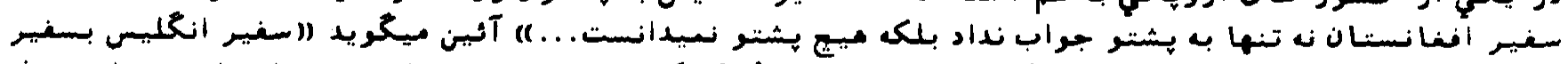

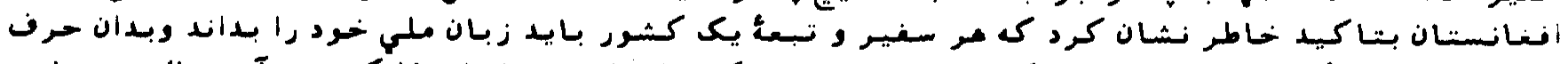

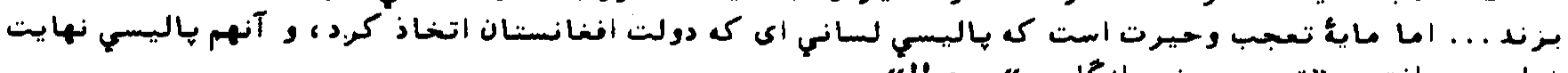

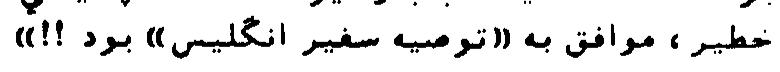

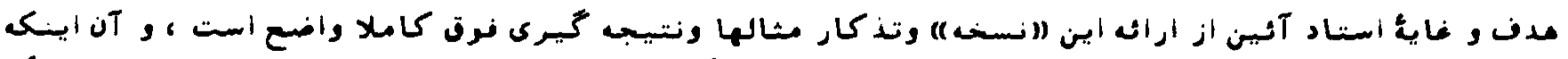

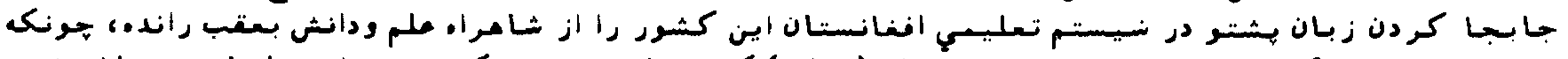

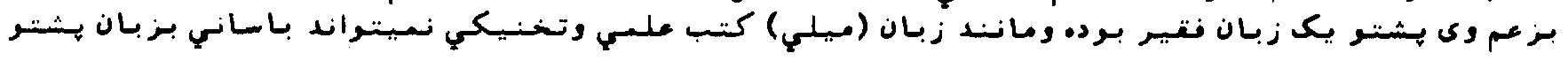




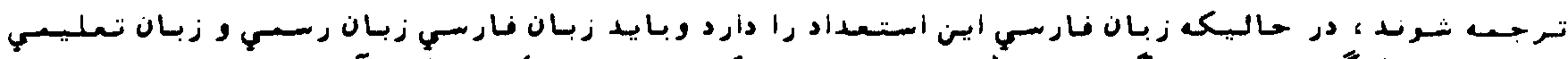

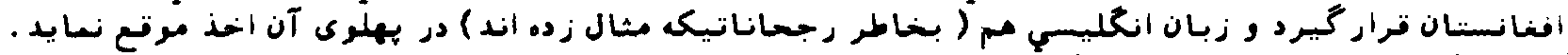

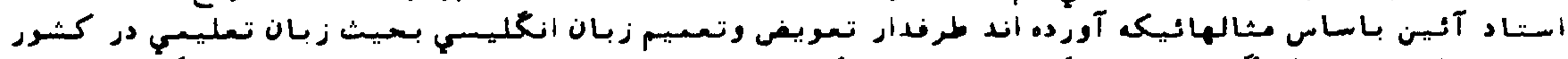

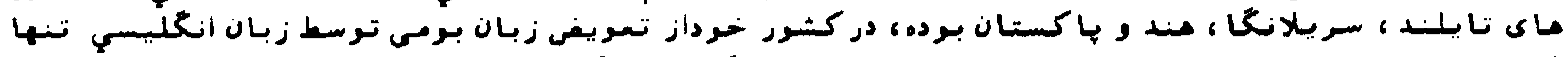

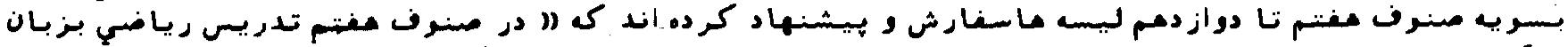

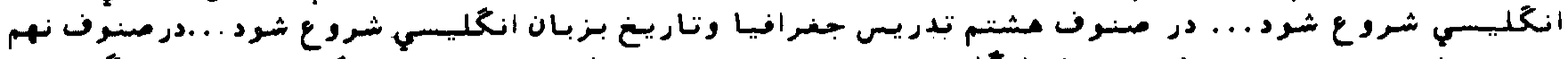

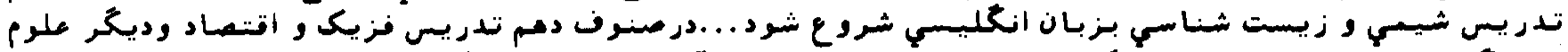

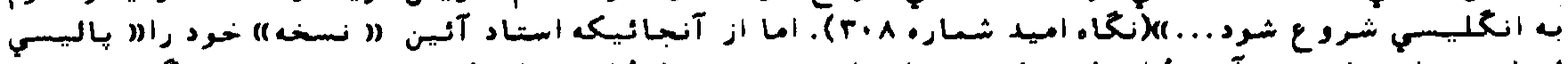

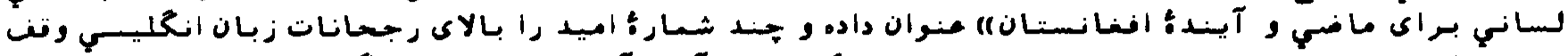

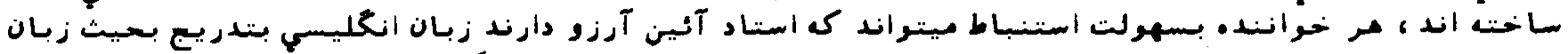

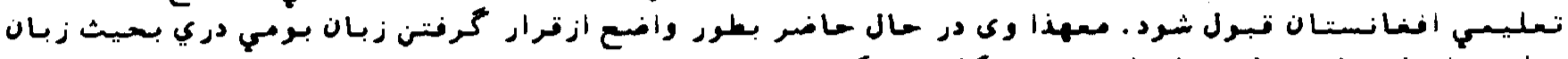

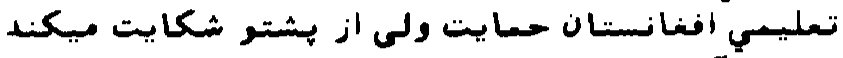

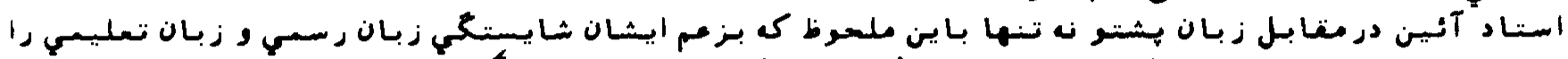

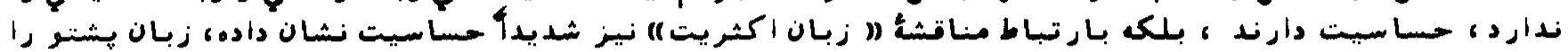

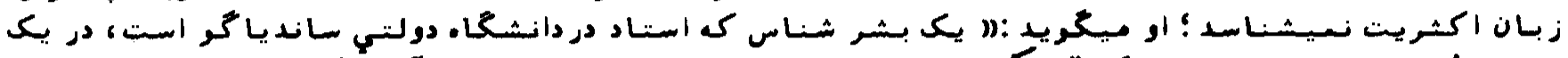

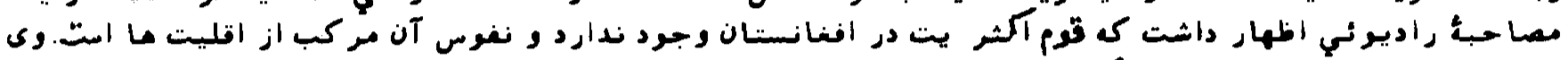

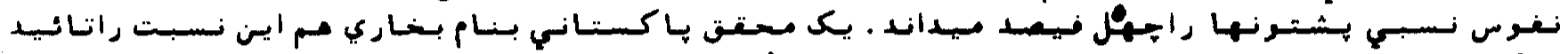

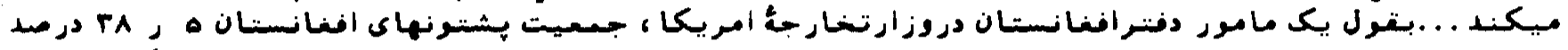

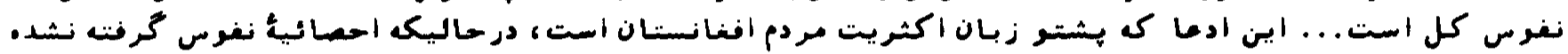

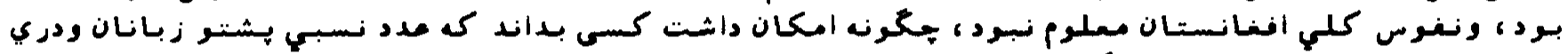

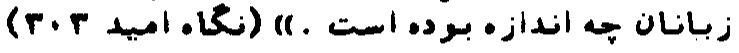

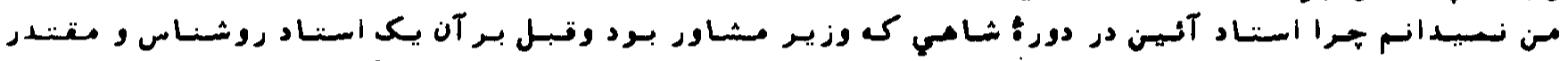

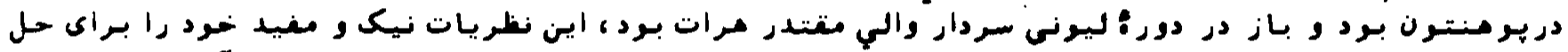

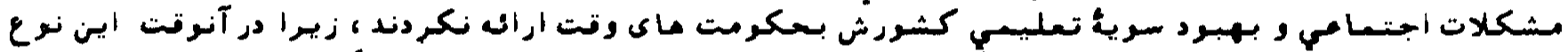

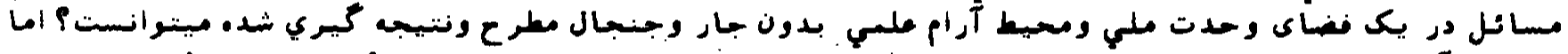

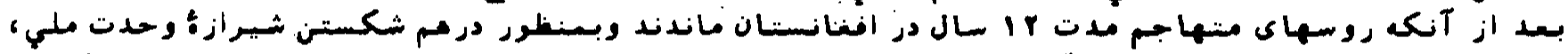

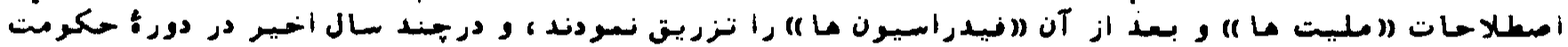

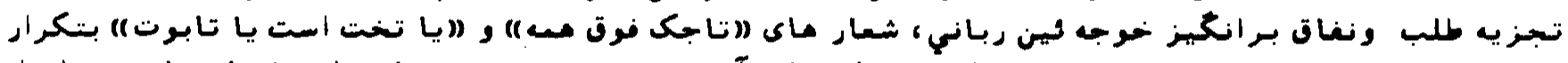

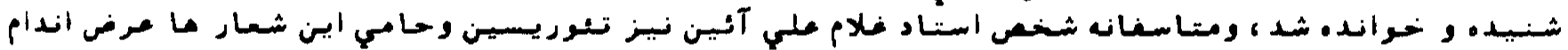

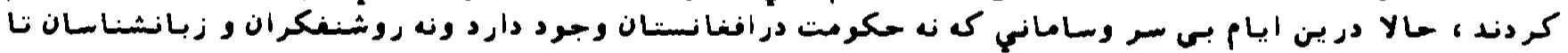

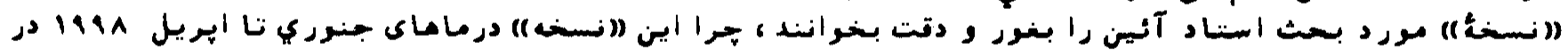

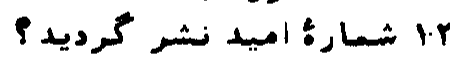

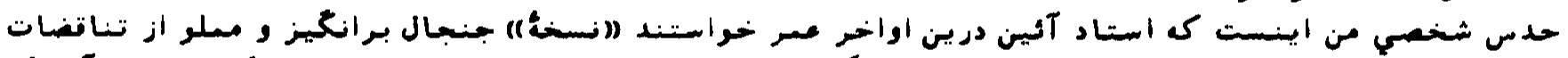

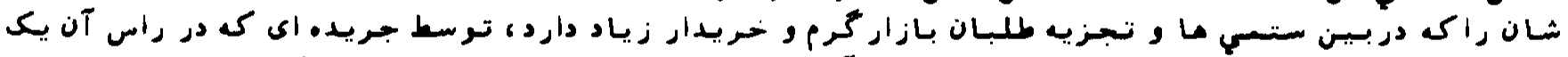

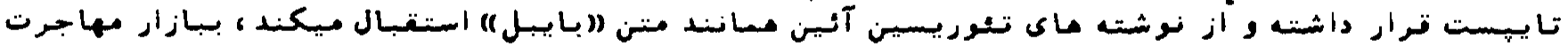

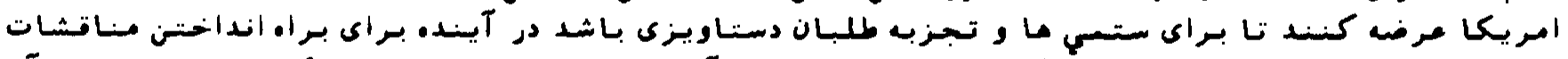

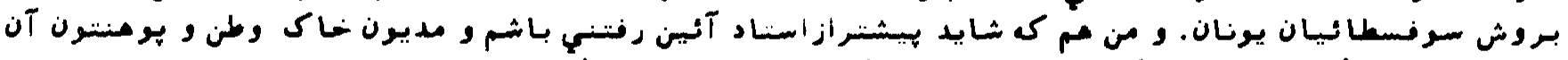

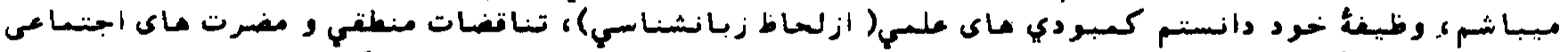

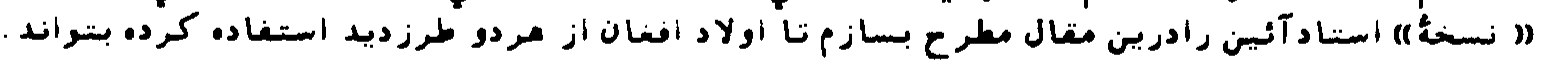

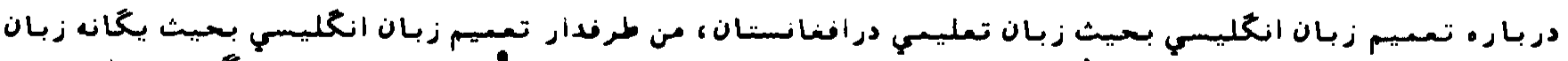

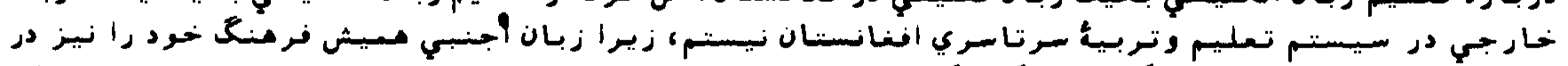

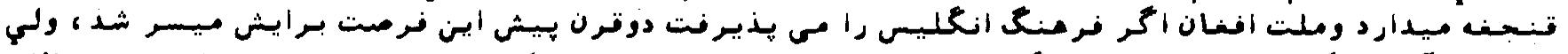

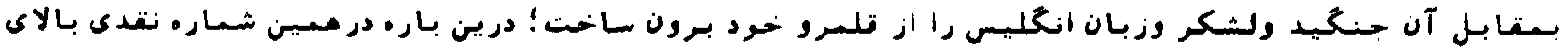

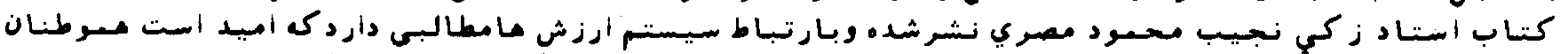

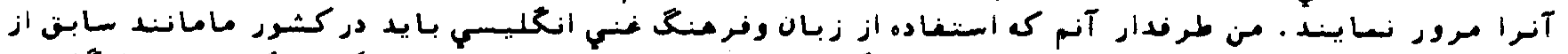

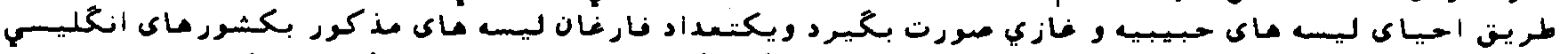

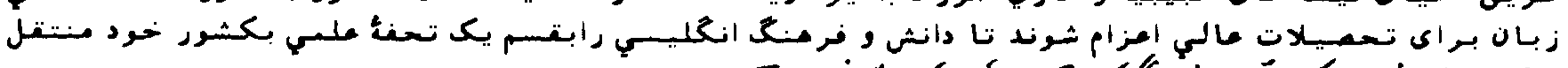

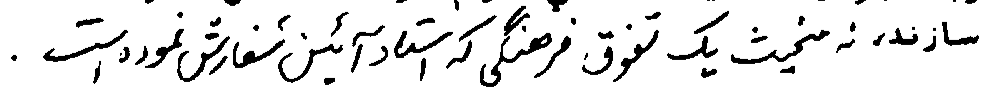




\section{4}

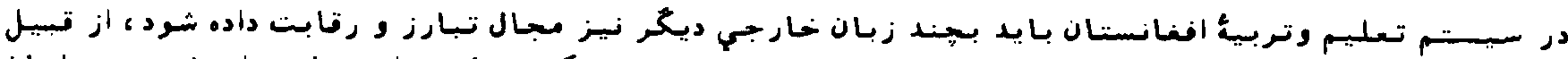

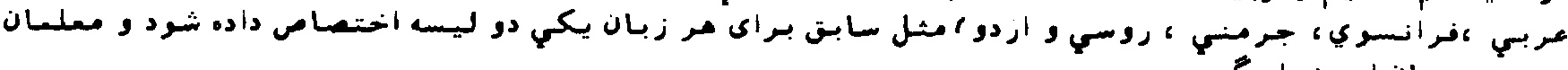

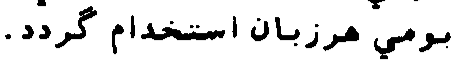

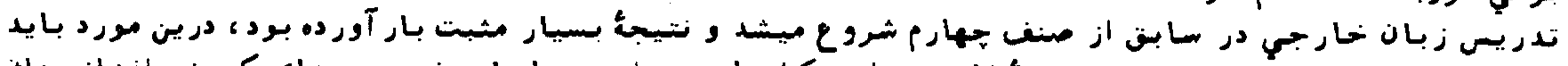

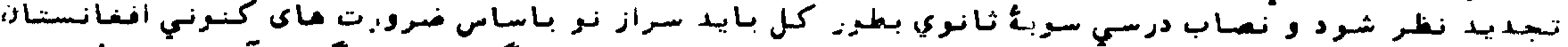

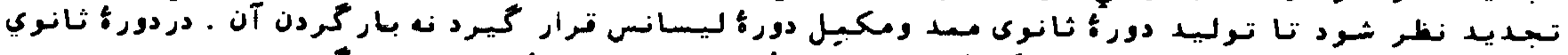

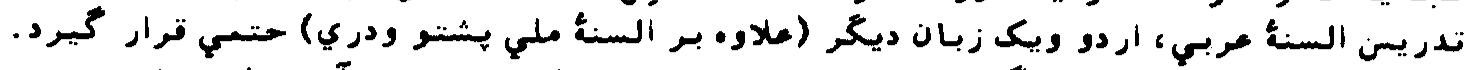

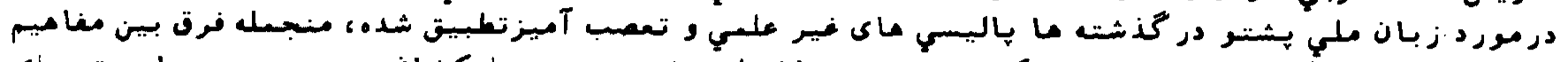

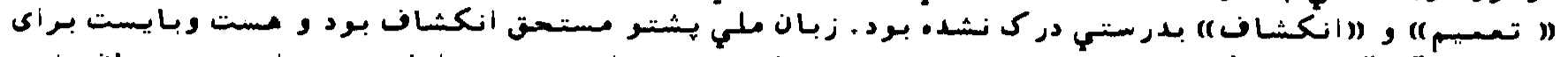

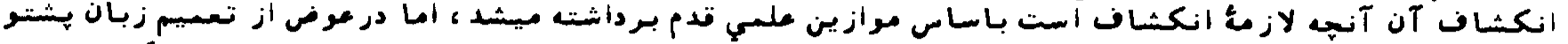

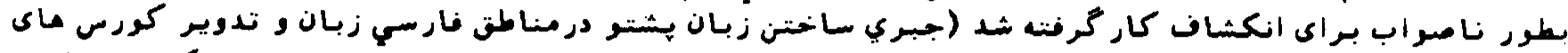

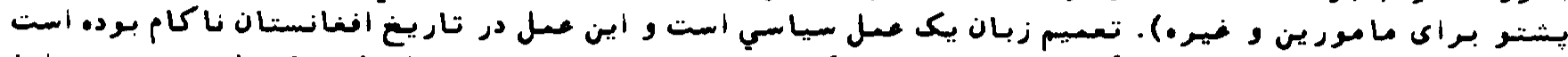

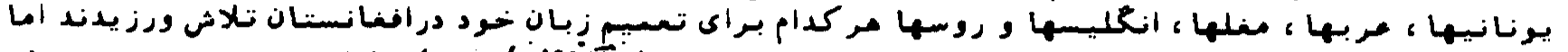

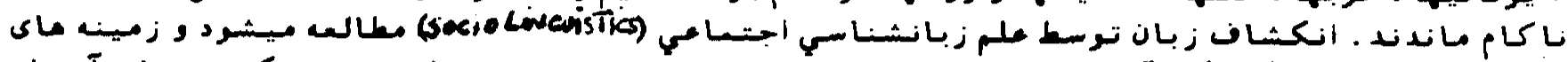

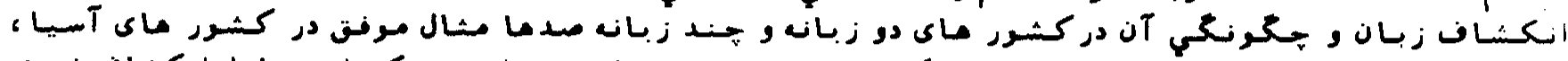

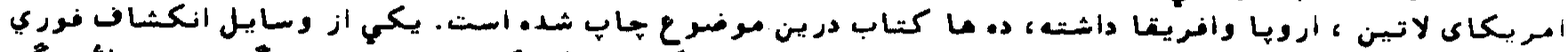

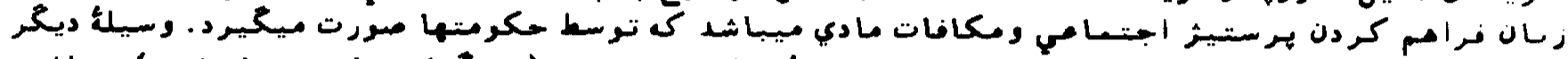

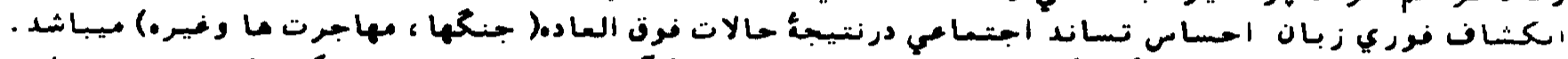

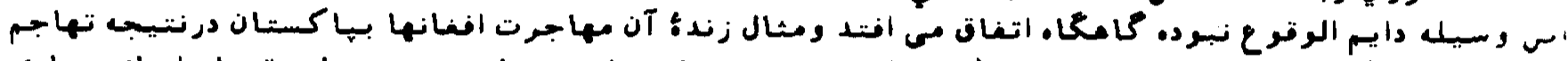

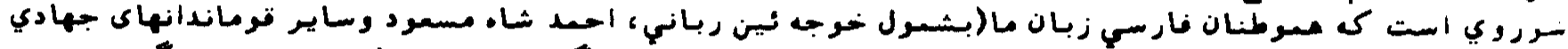

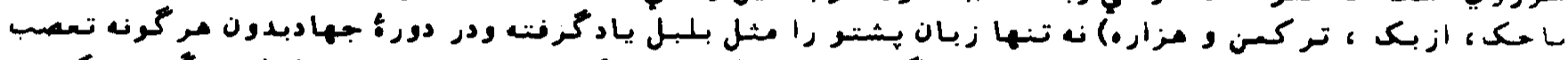

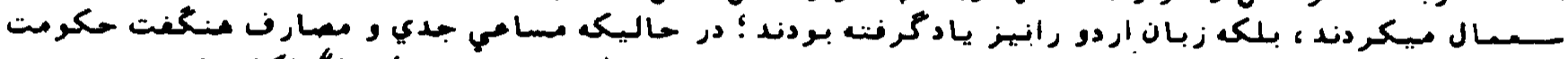

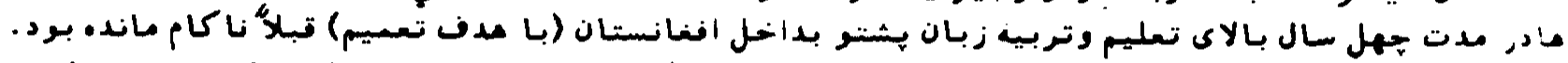

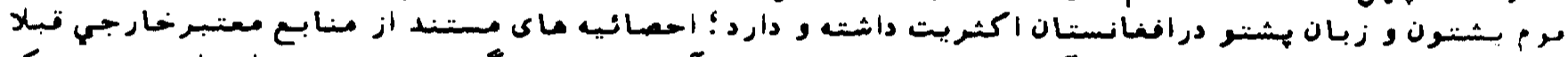

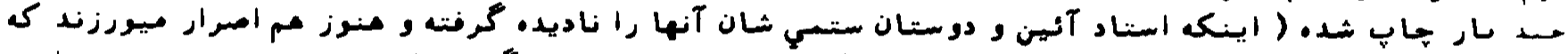

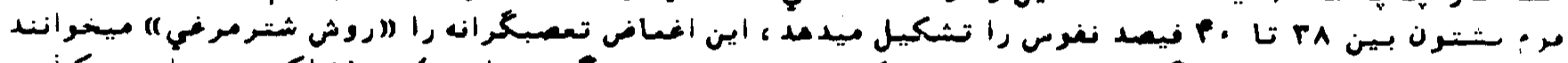

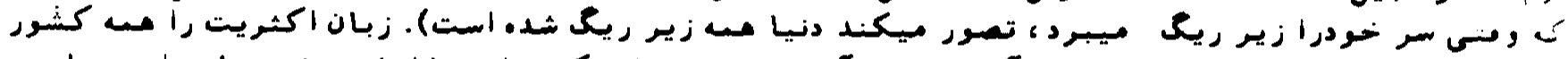

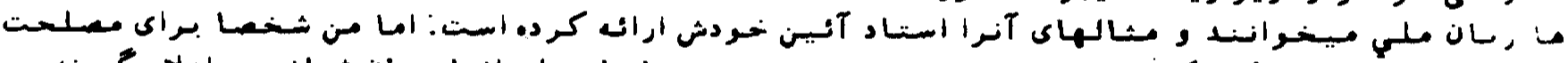

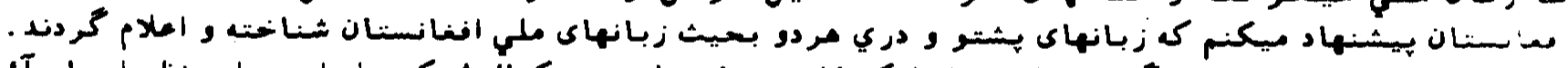

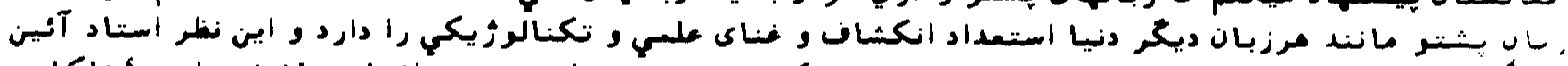

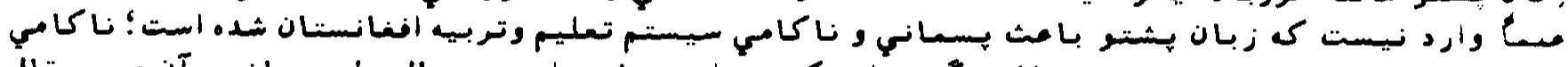

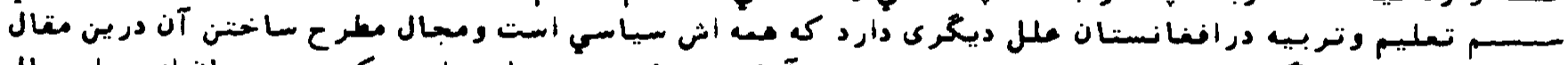

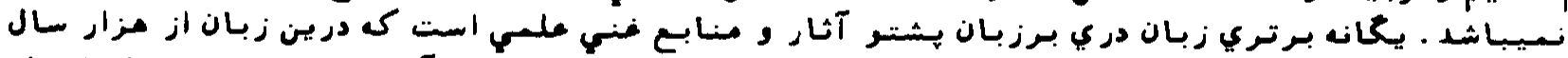

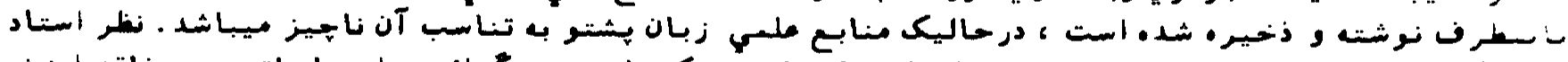

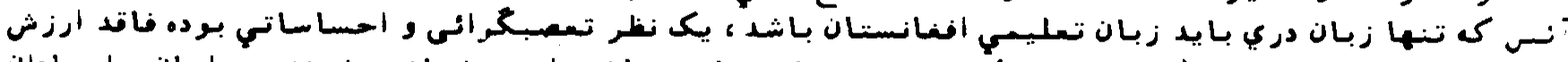

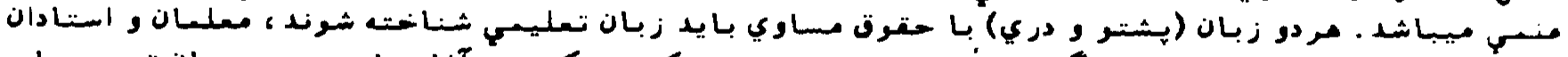

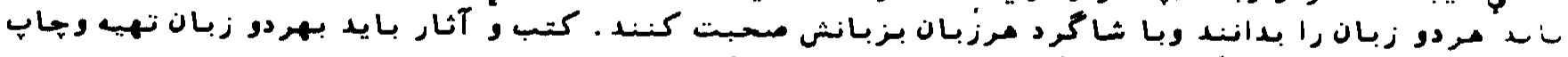

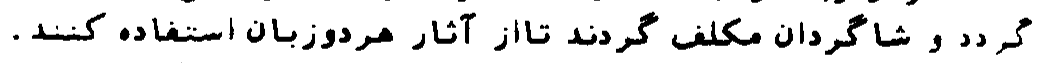

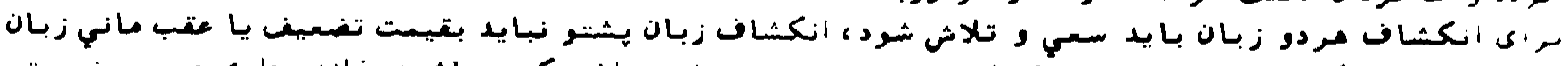

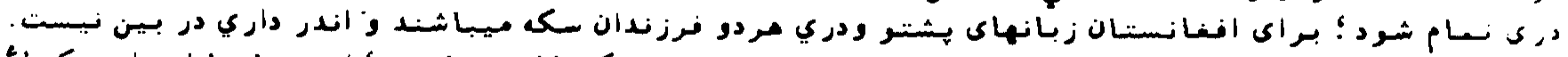

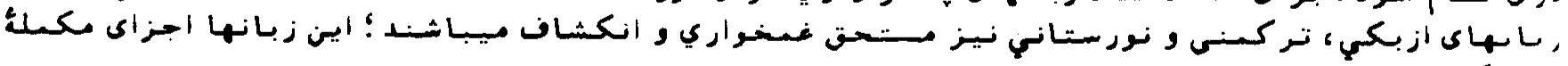

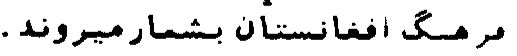

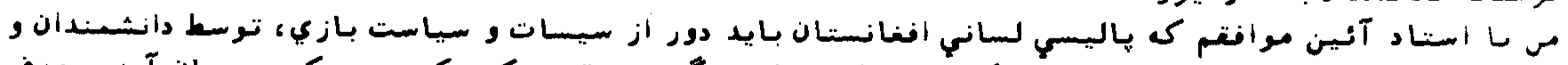

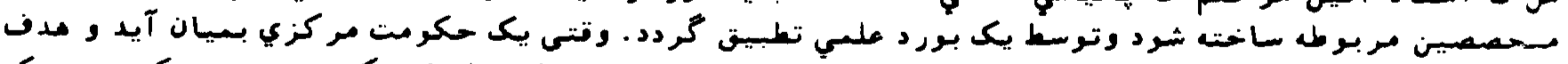

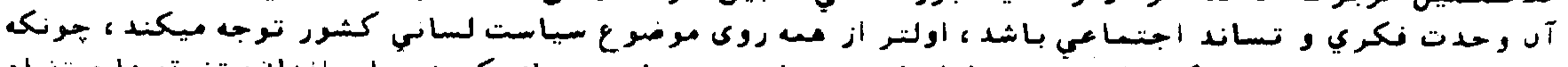

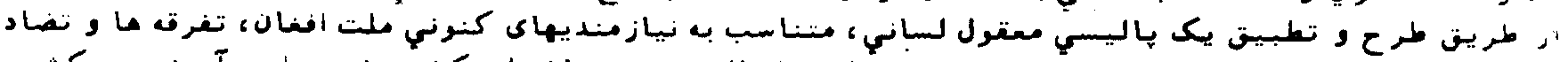

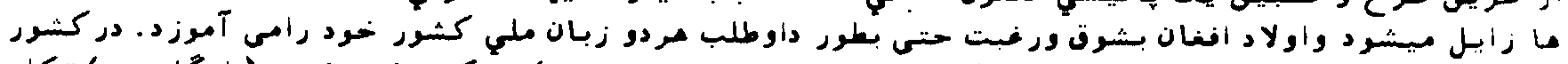

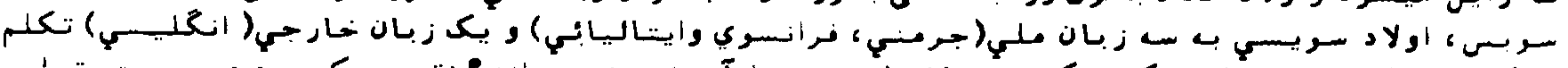

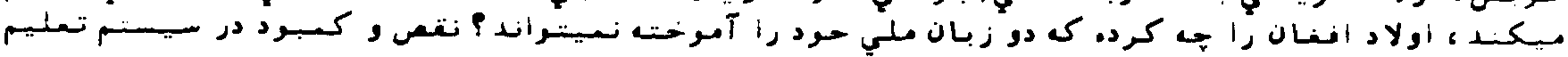




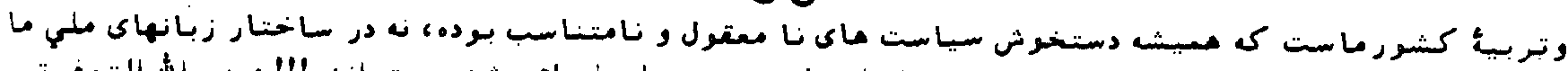

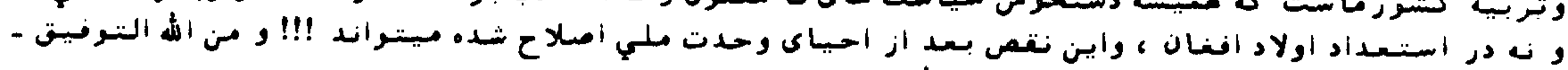

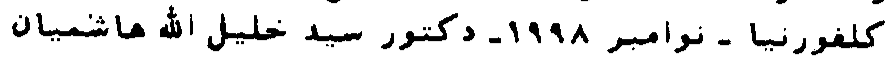

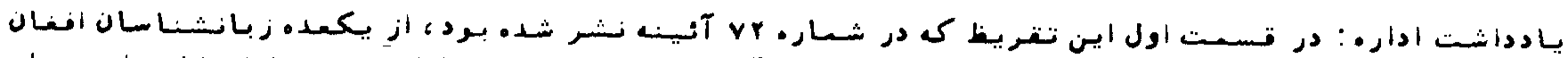

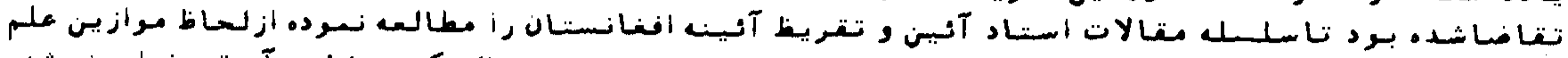

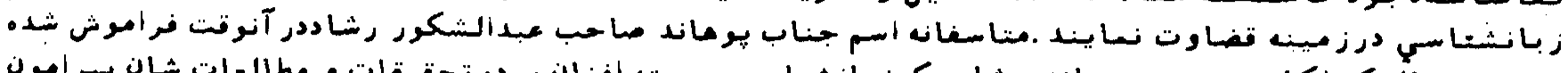

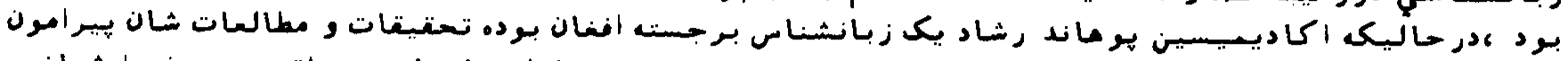

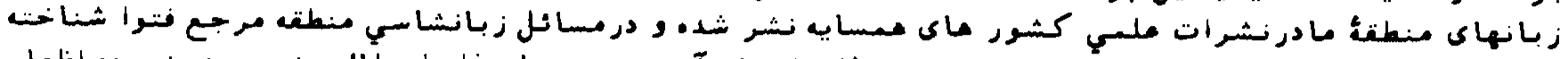

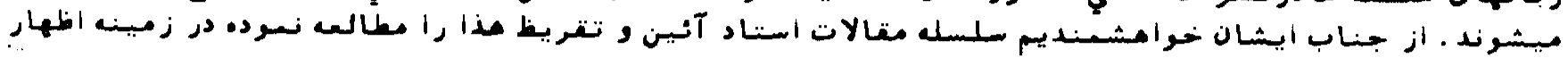

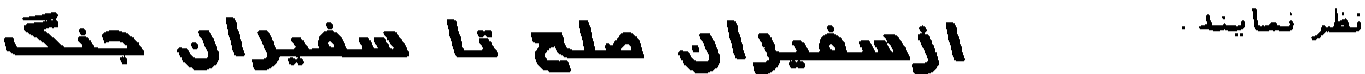

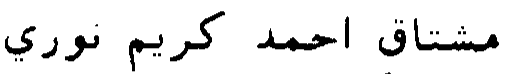

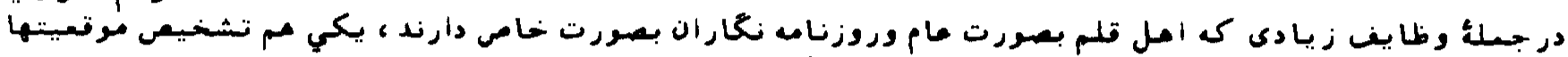

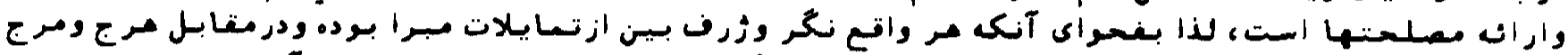

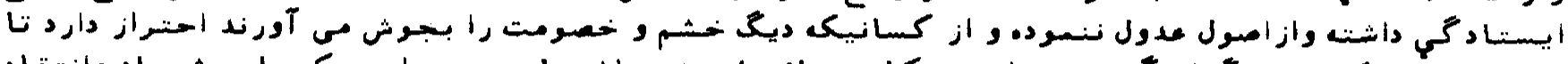

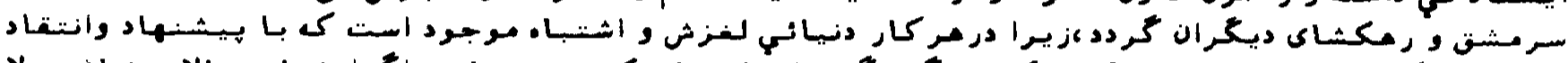

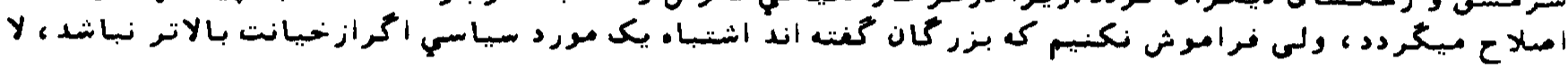

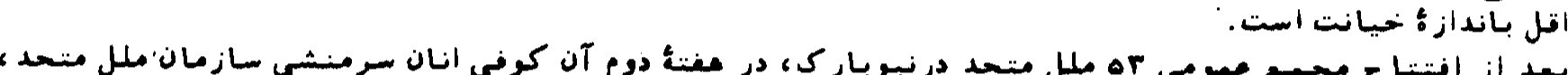

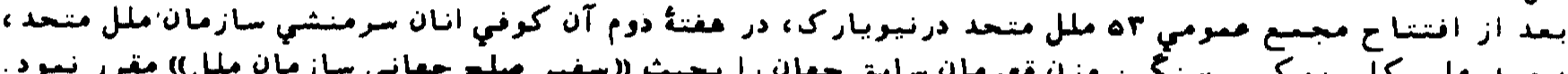

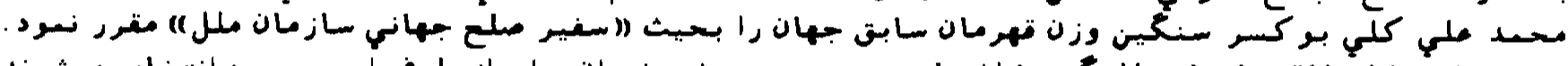

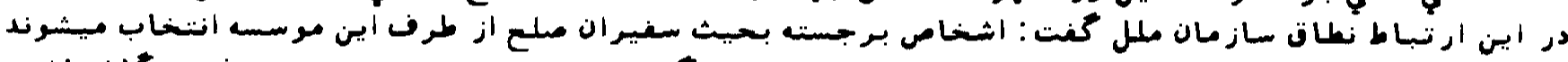

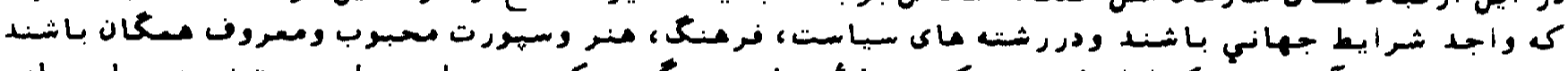

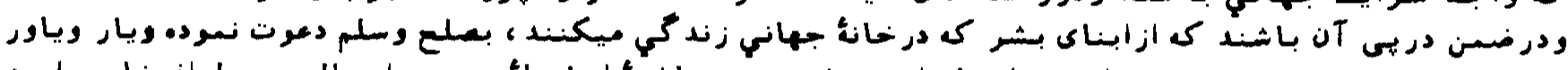

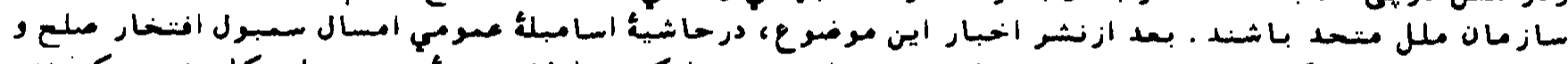

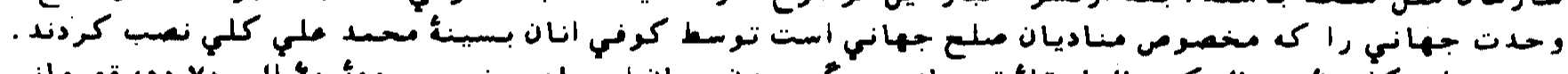

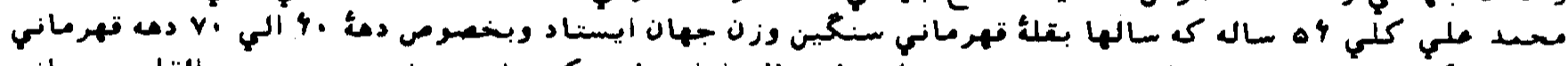

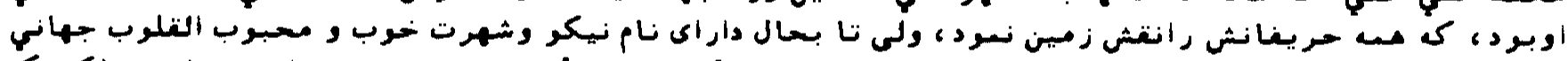

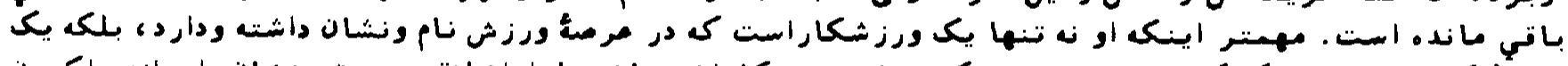

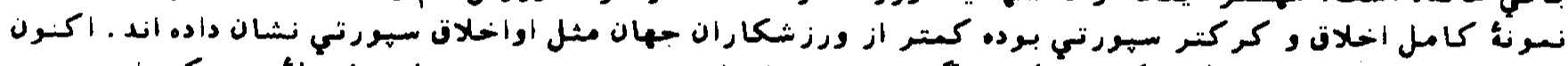

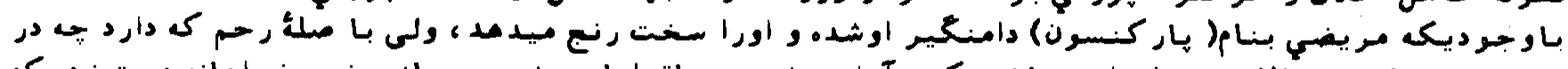

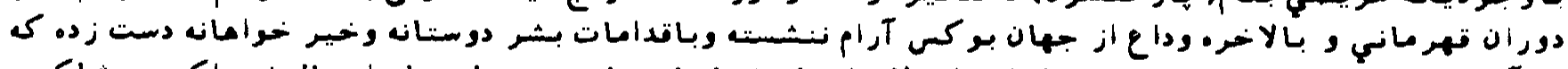

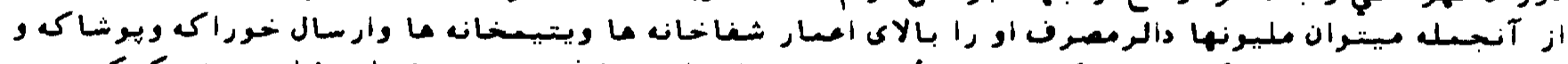

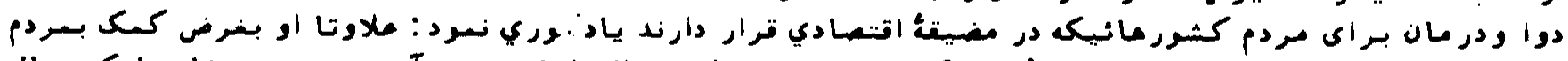

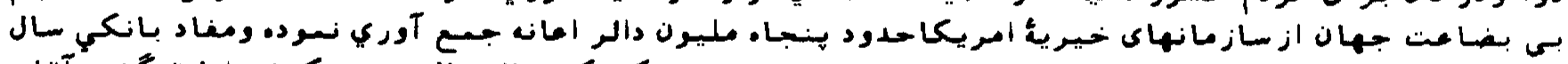

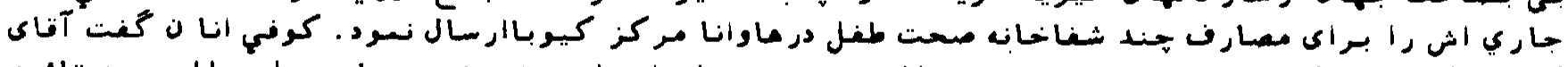

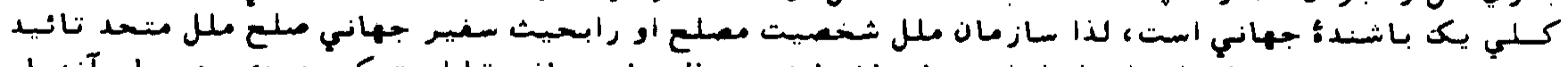

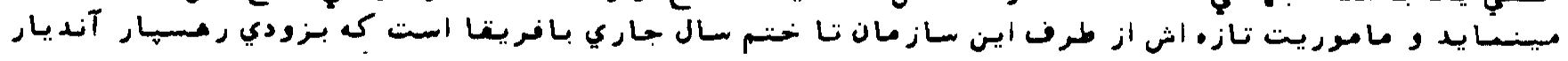

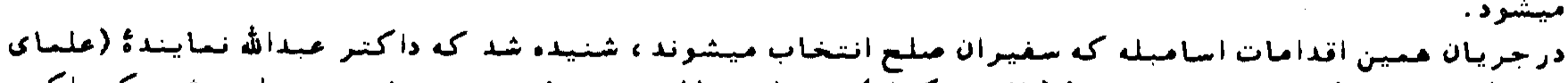

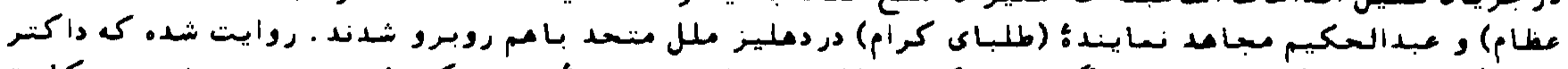

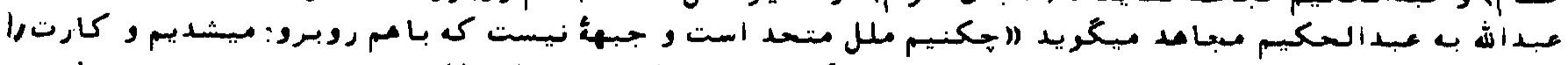

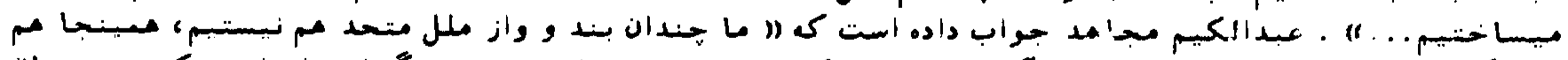

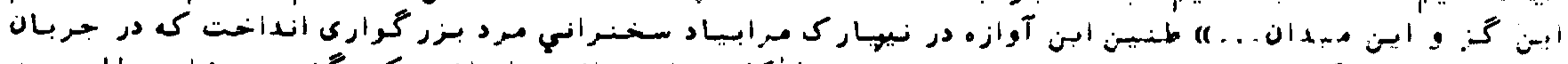

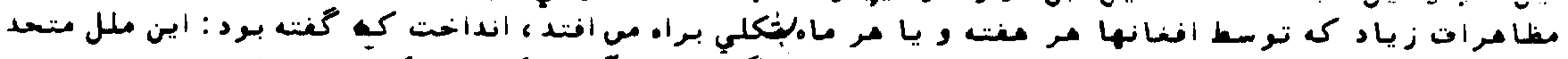

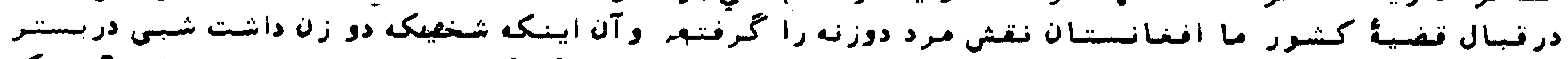

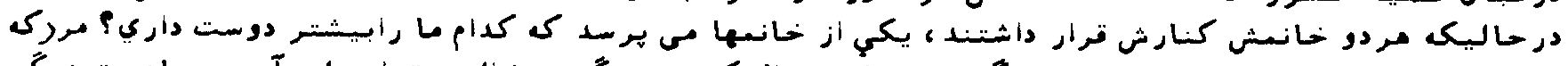

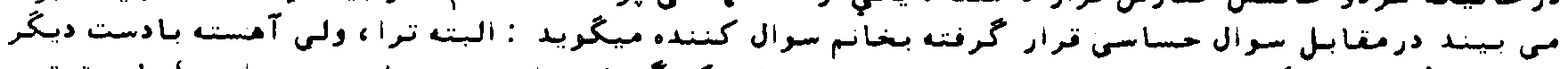

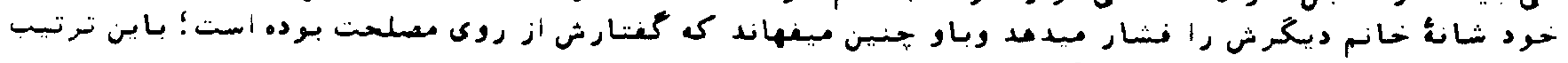




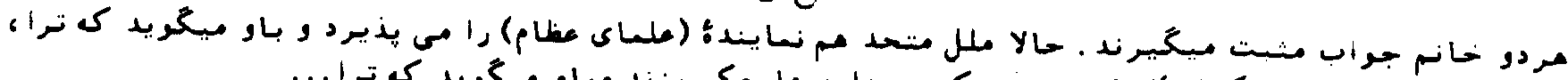

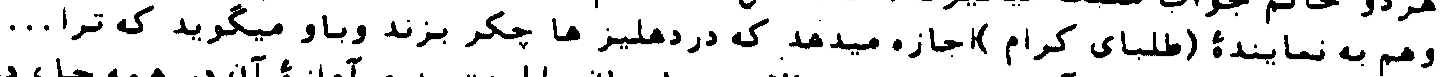

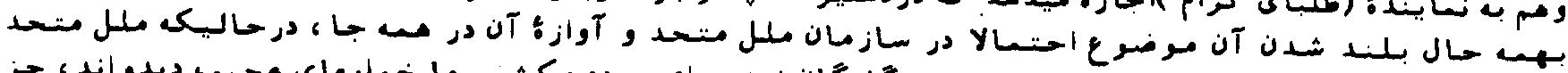

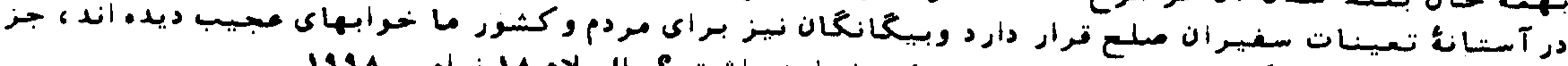

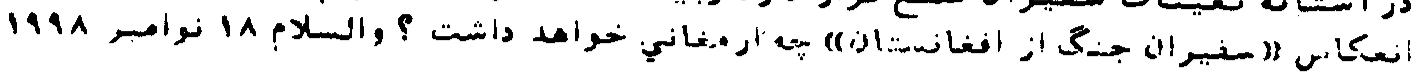

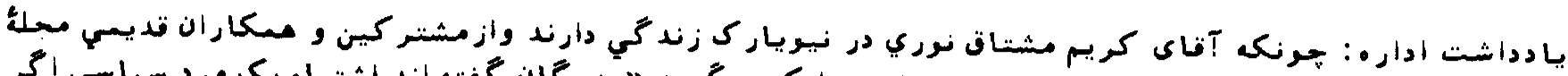

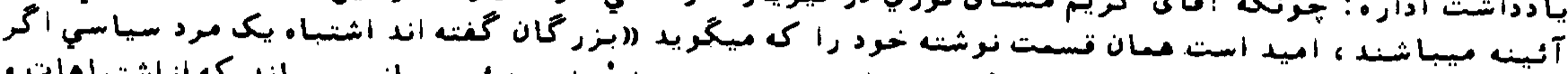

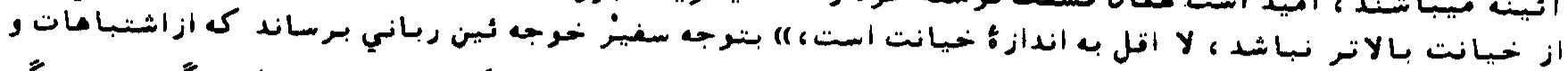

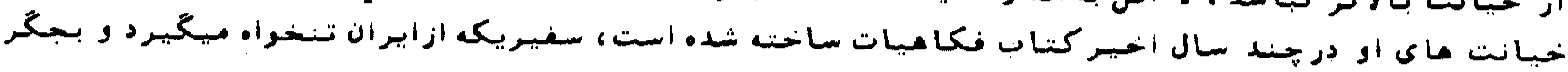

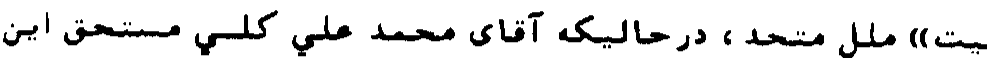

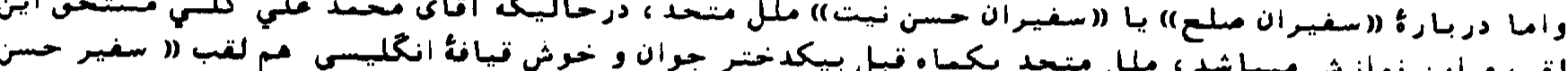

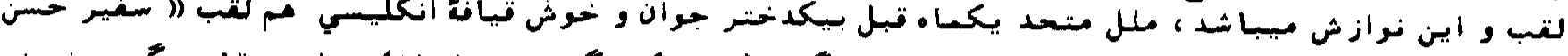

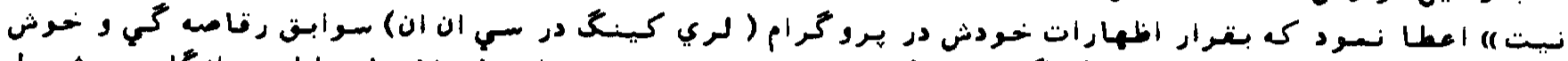

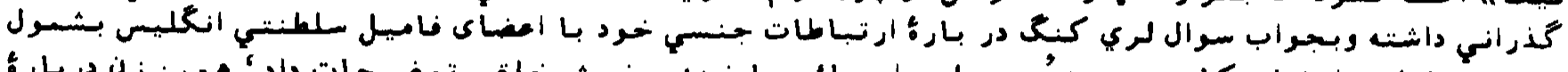

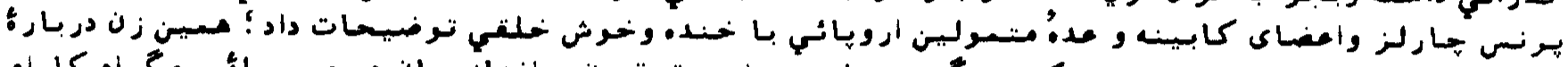

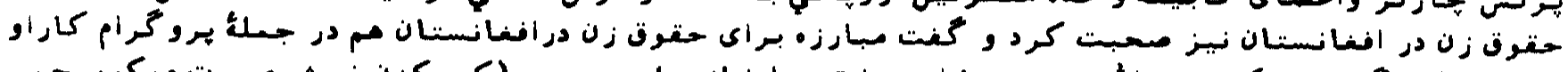

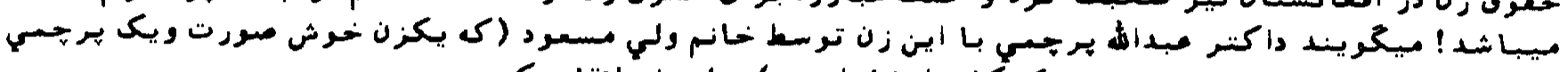

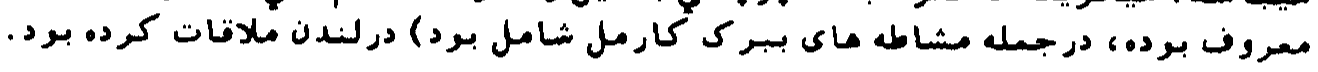

\section{SGL ثIدL}

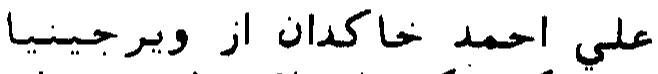

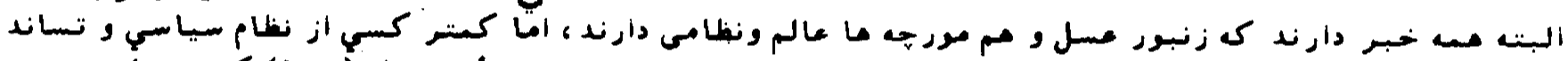

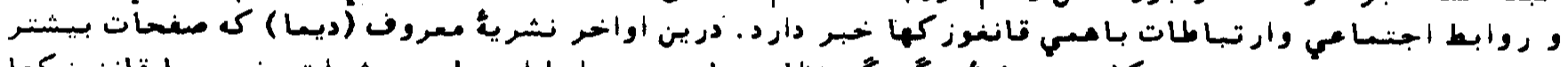

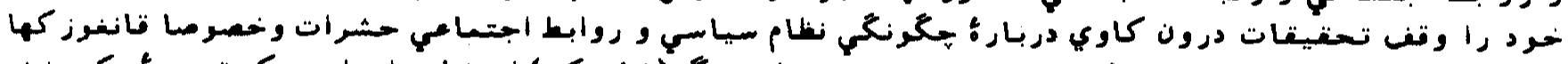

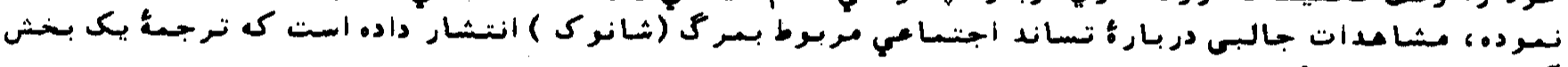

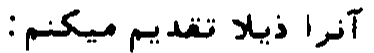

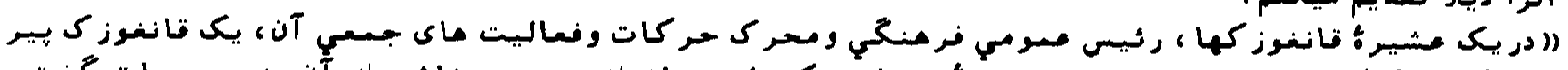

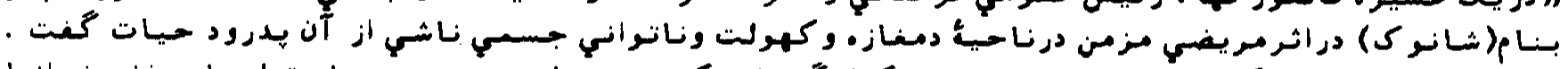

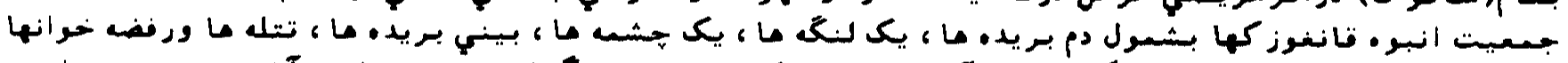

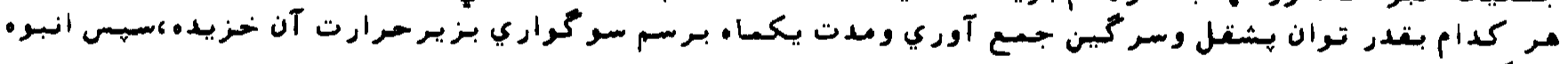

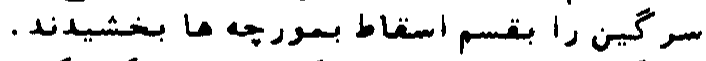

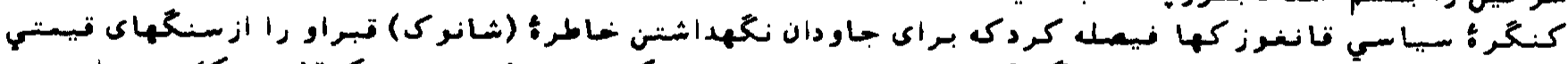

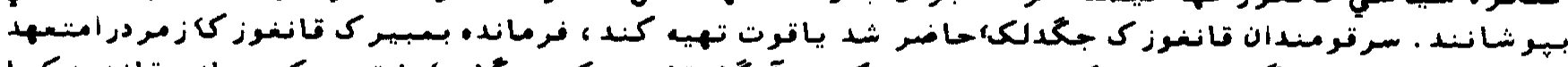

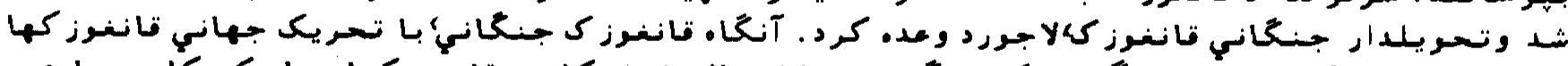

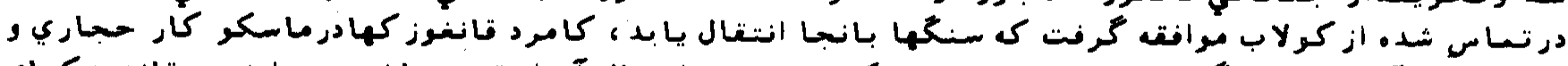

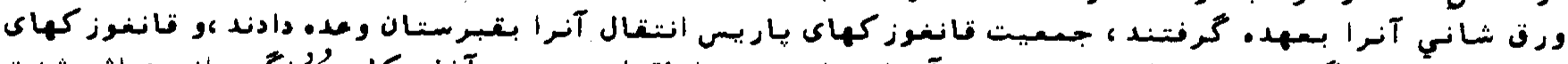

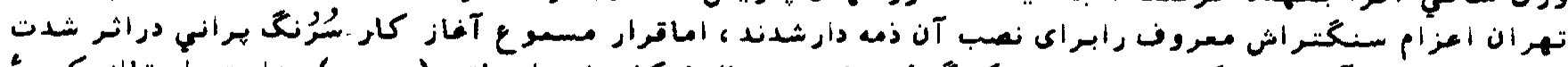

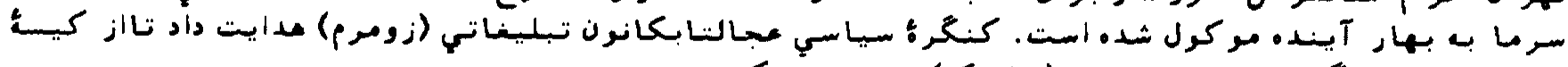

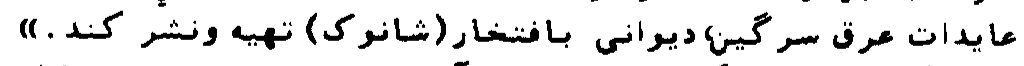

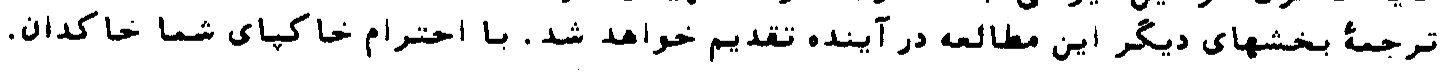

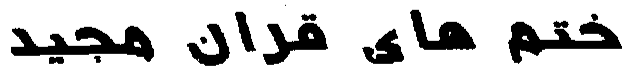

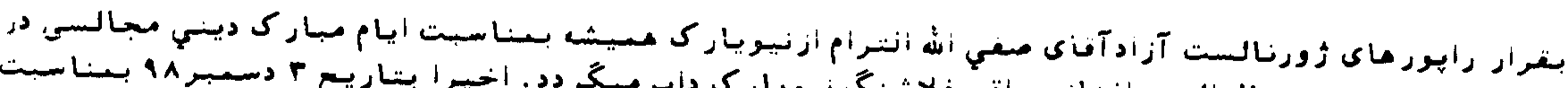

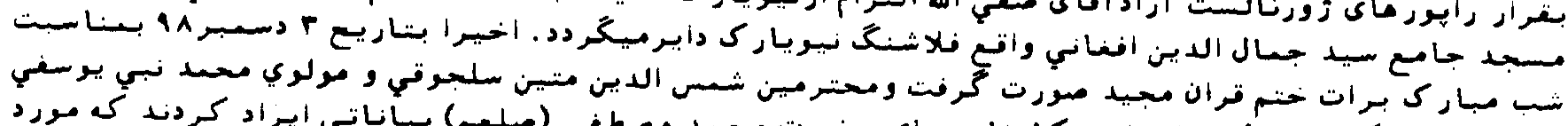

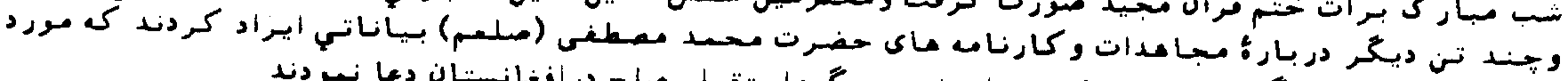

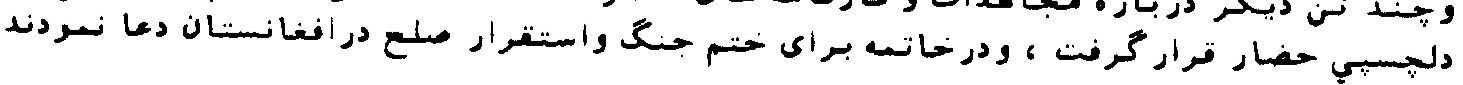




\section{899 (2)}

عبدالصمد وهابزاده (فريار )

بسلسلة كَذشته - قسمت سوم و اخيسر

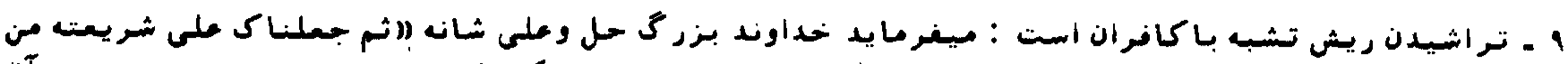

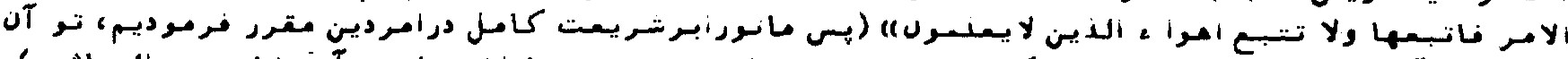

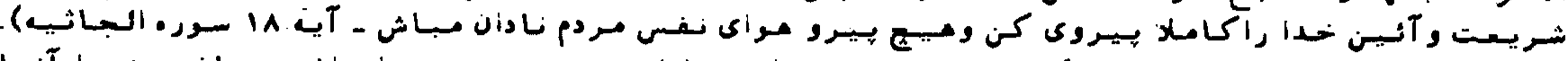

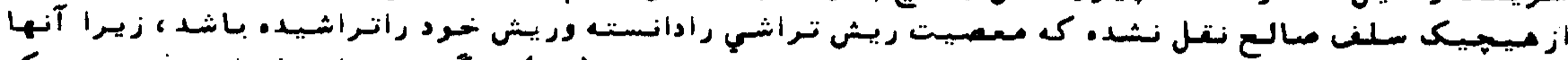

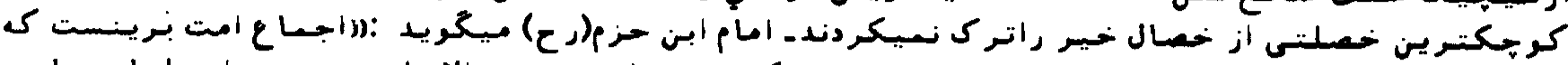

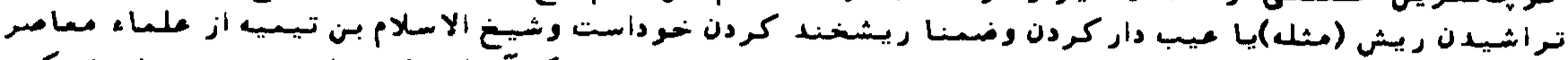

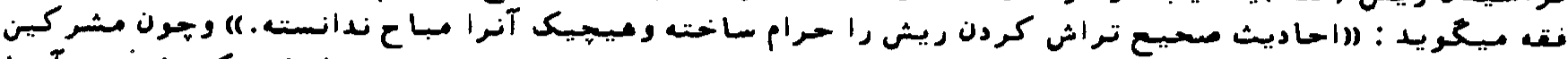

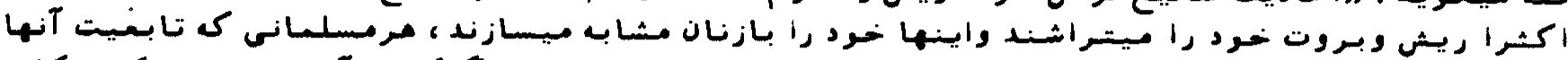

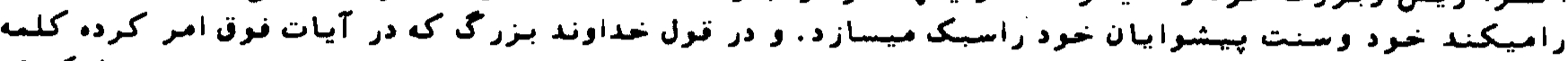

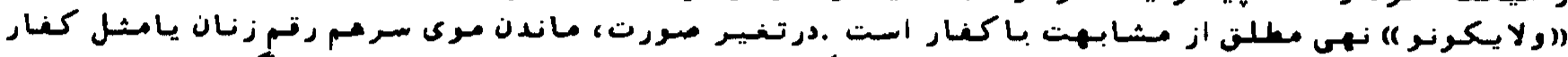

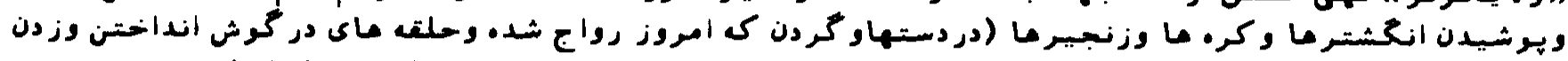

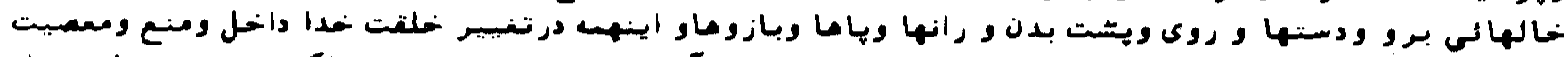

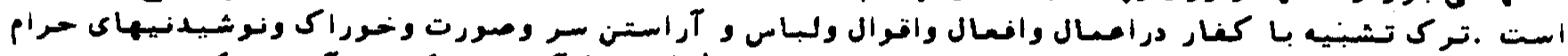

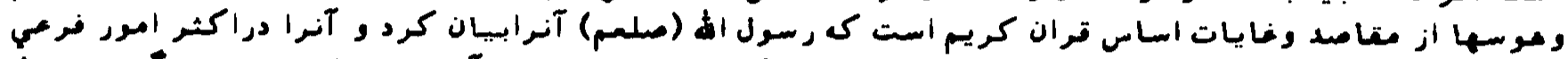

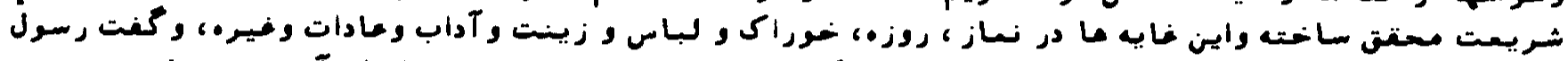

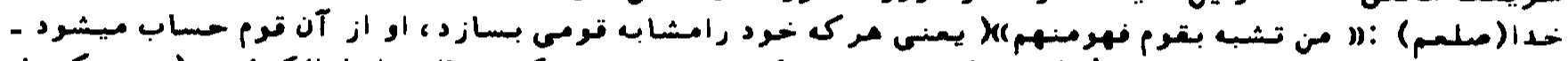

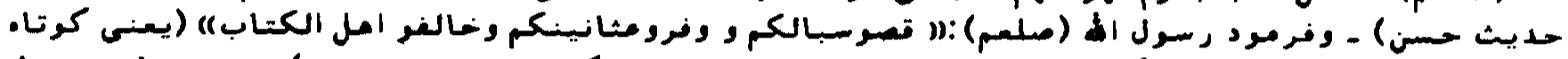

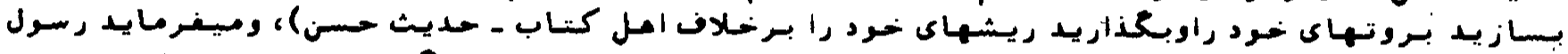

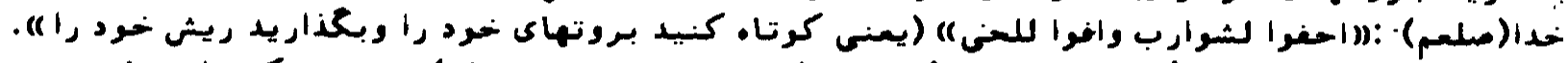

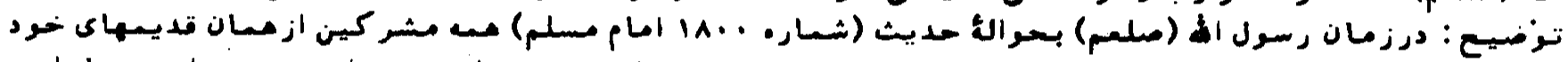

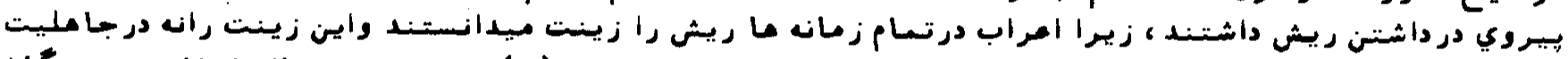

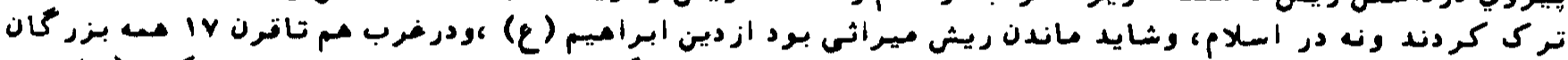

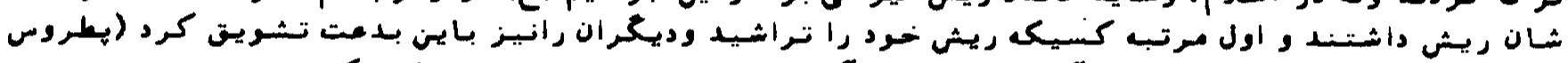

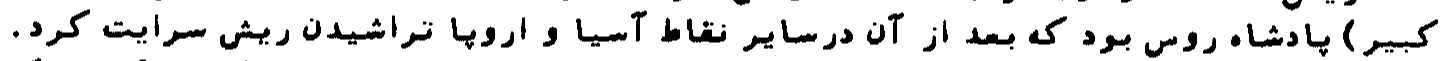

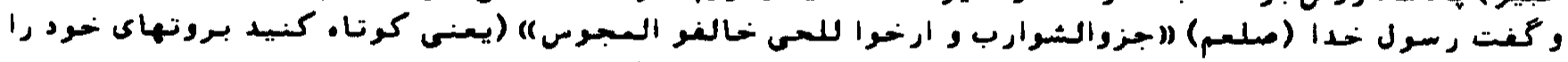

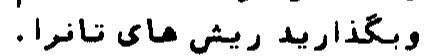

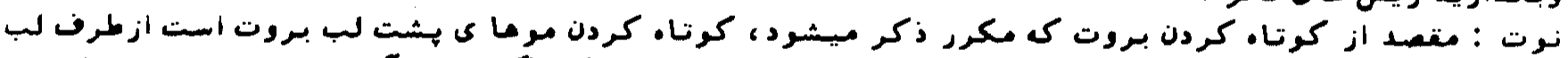

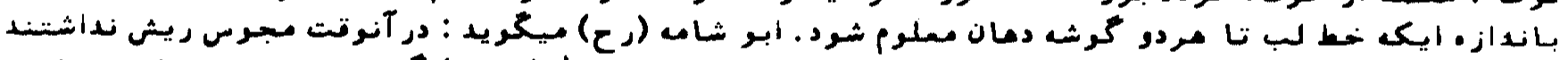

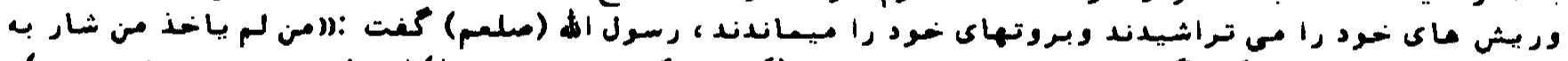

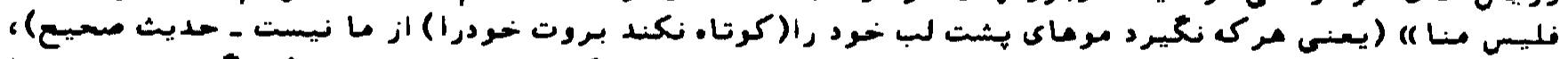

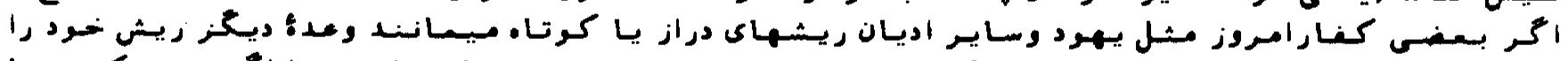

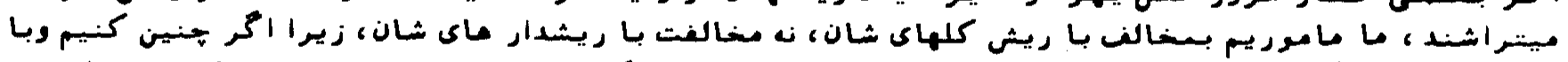

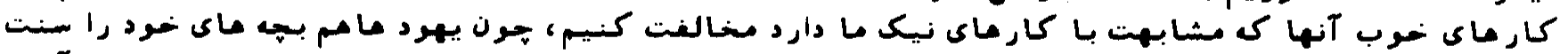

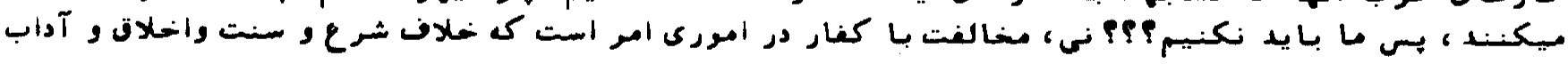

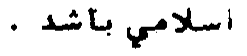

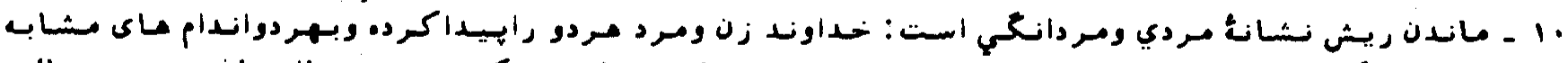

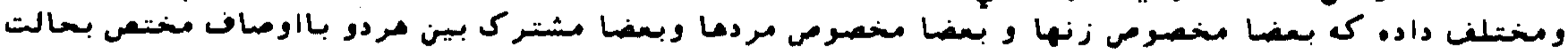

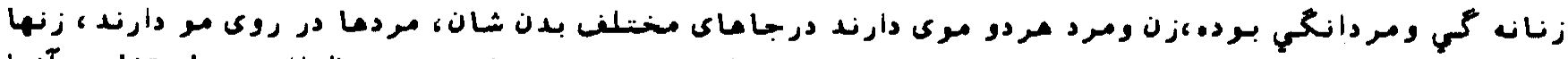

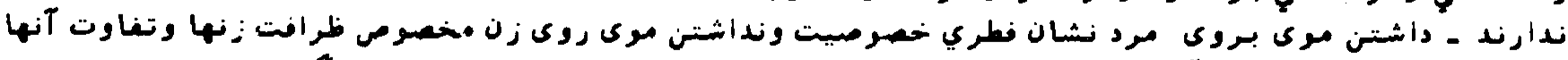

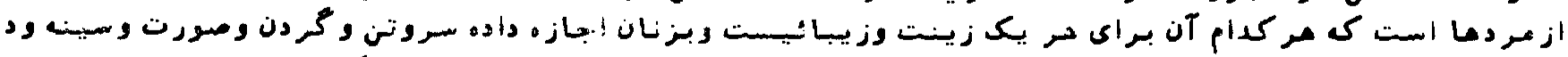

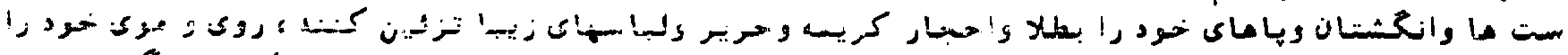

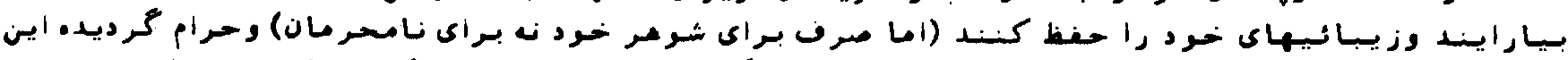

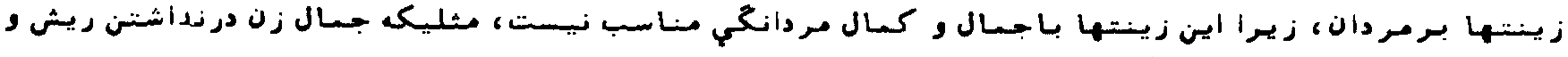

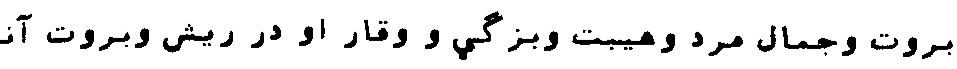




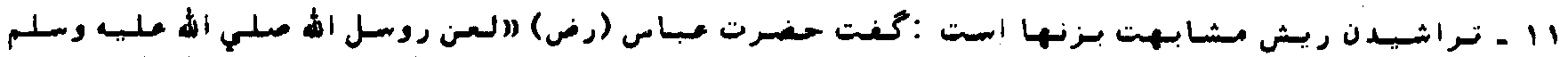

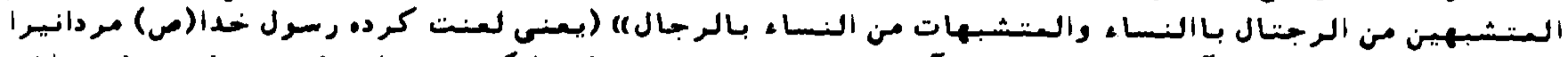

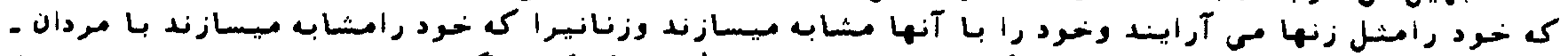

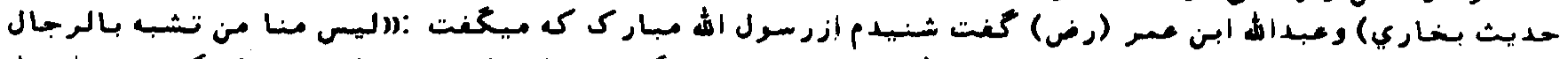

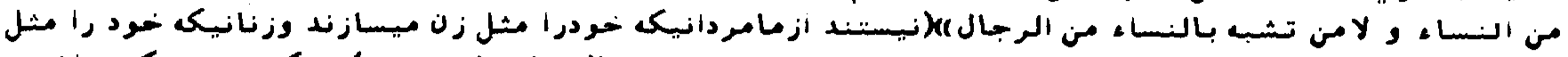

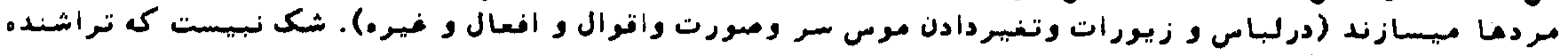

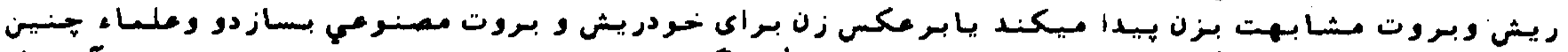

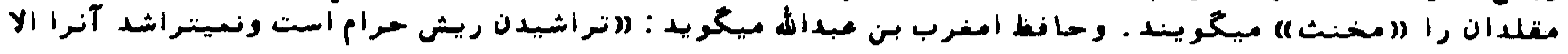

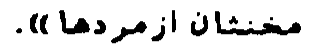

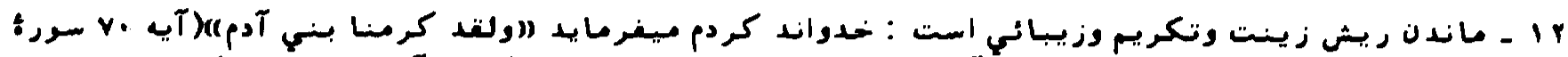

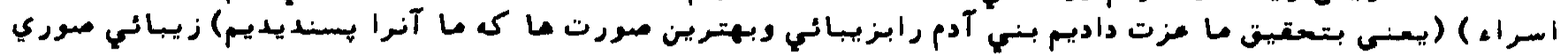

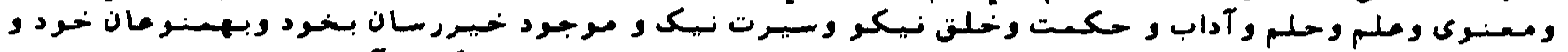

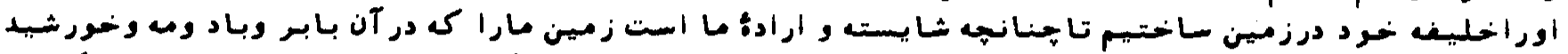

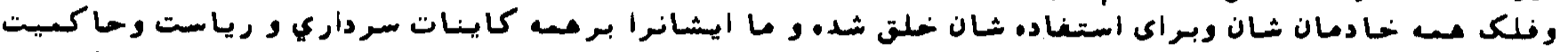

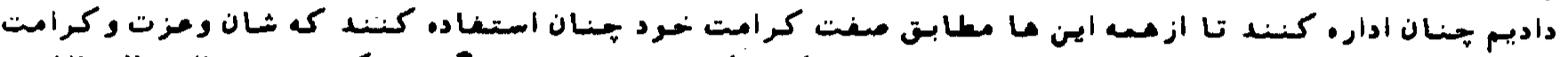

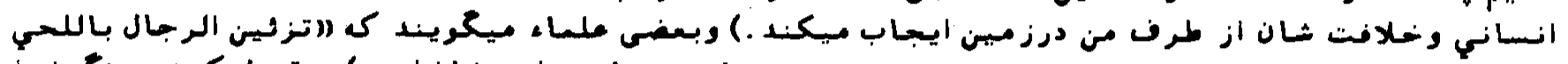

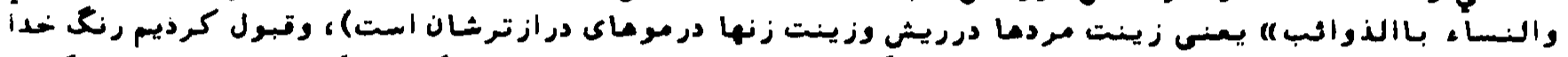

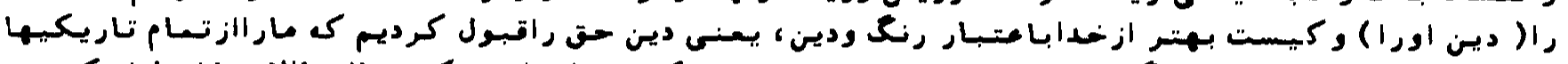

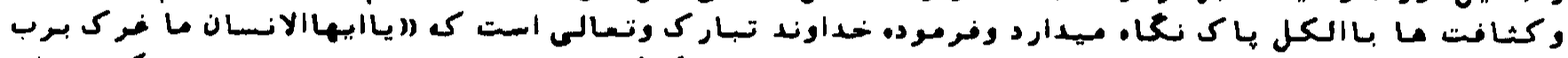

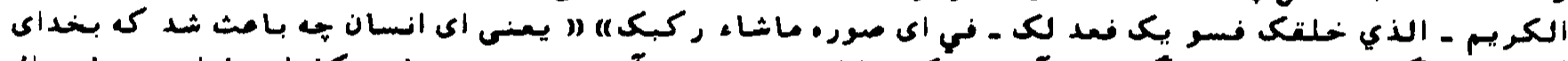

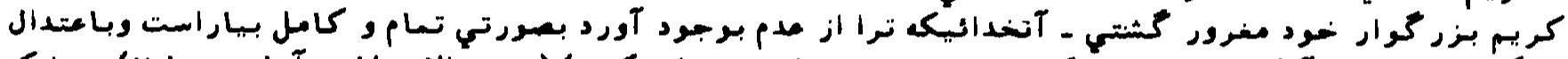

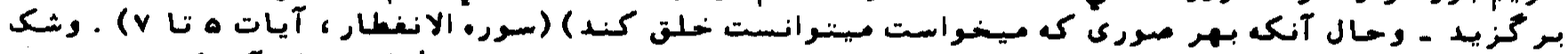

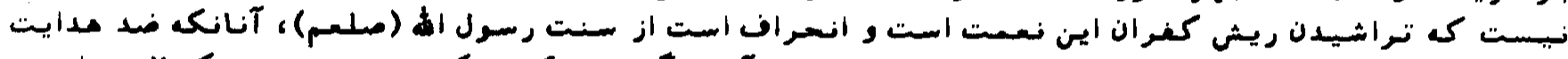

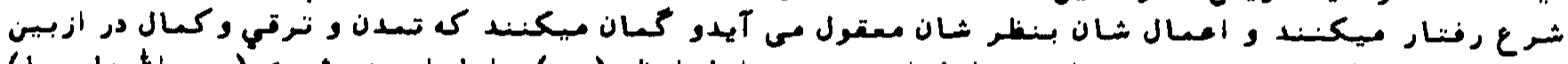

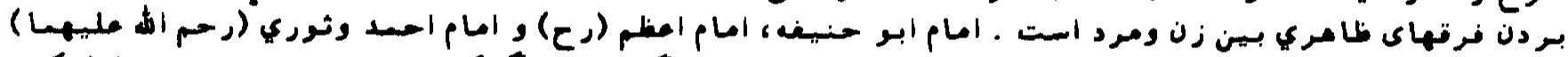

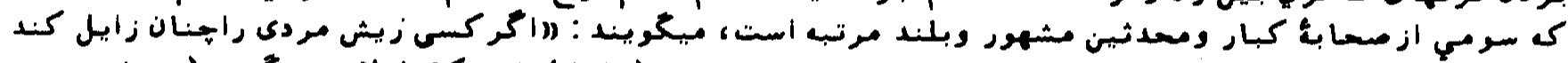

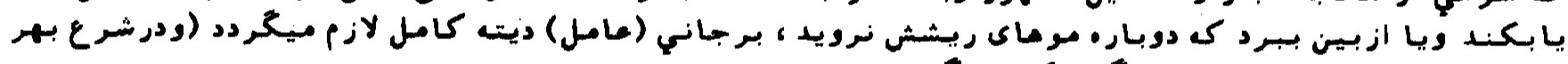

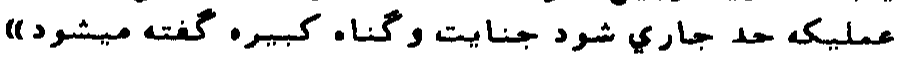

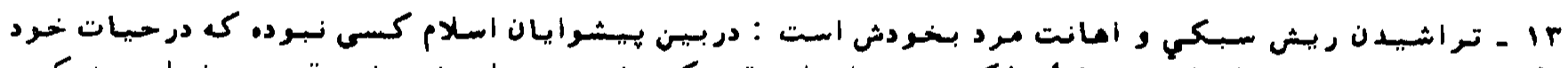

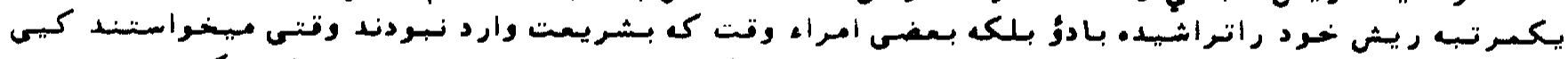

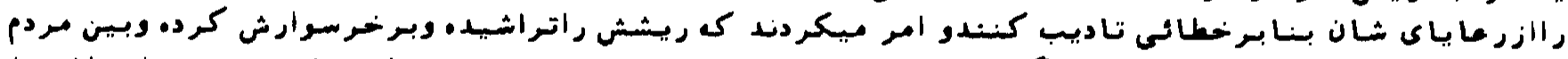

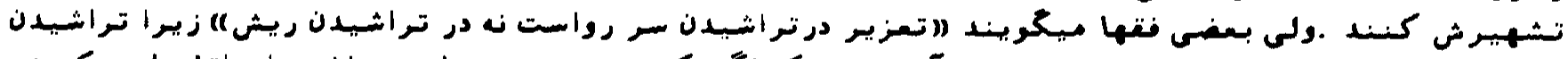

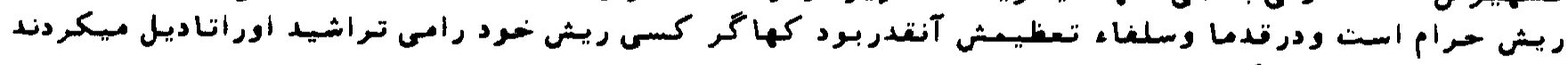

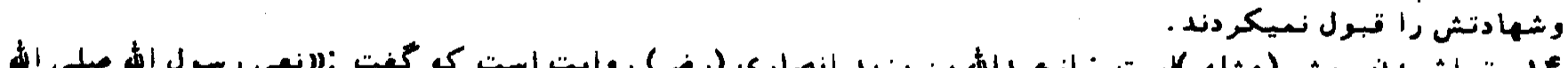

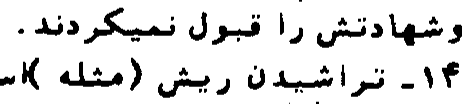

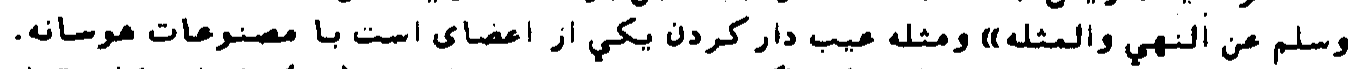

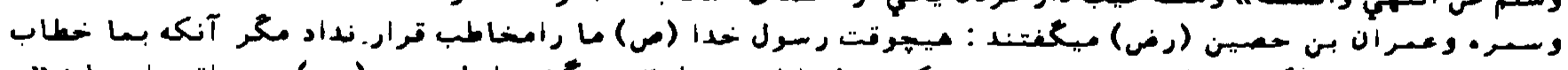

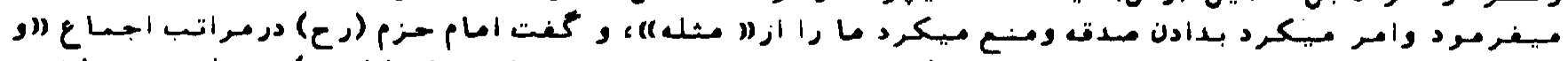

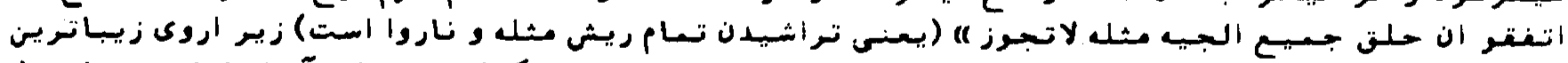

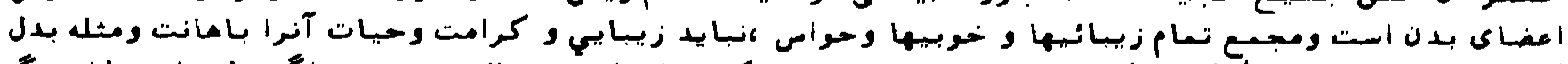

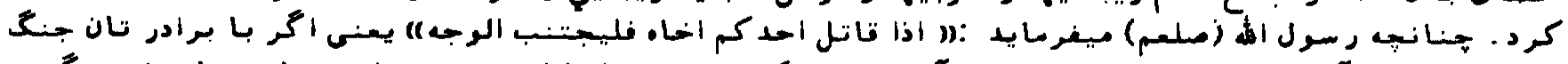

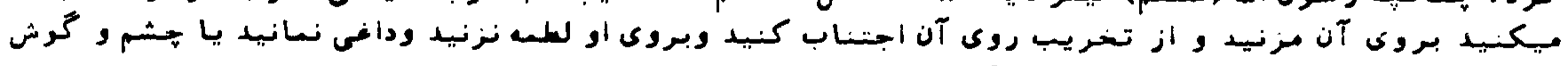

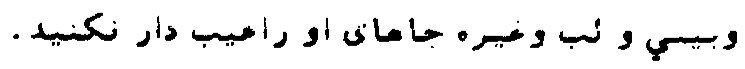

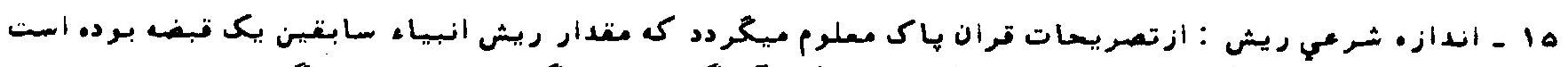

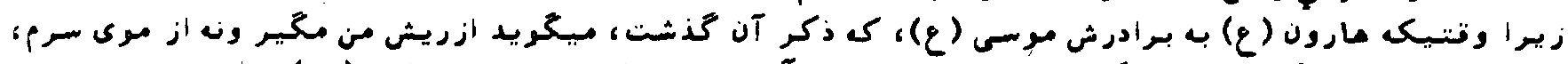

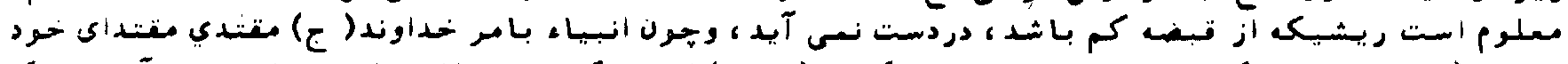

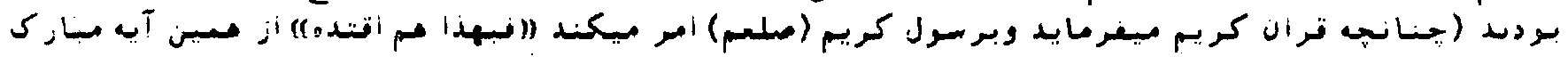




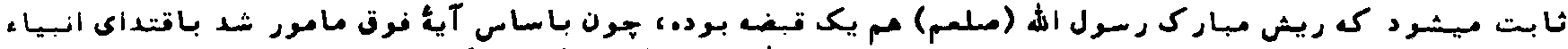

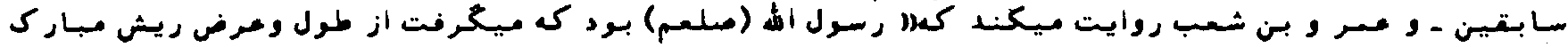

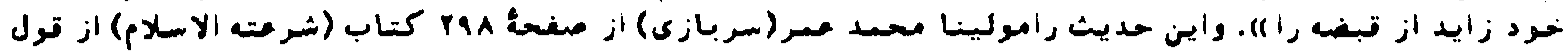

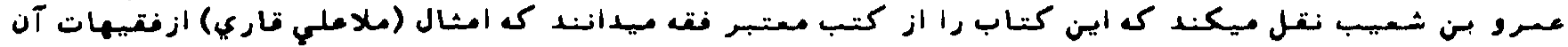

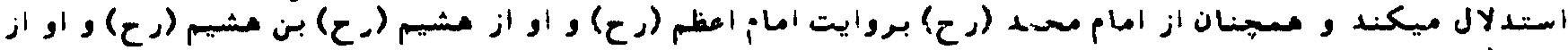

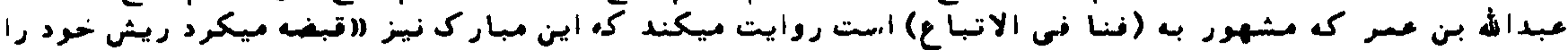

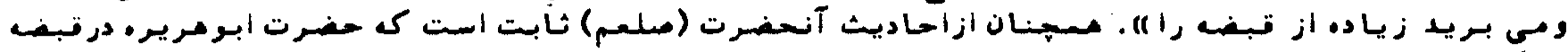

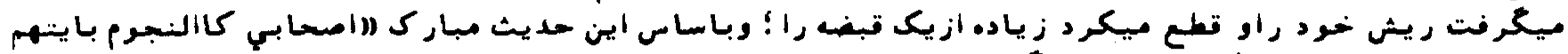

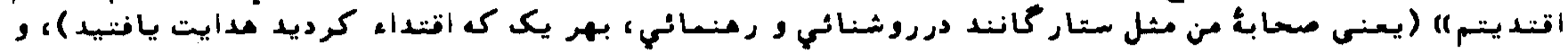

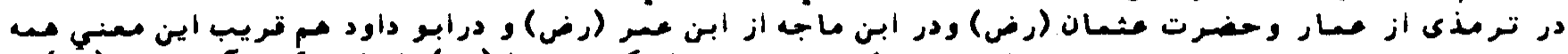

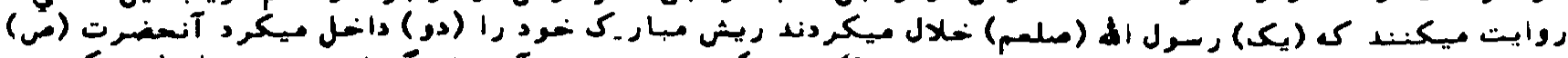

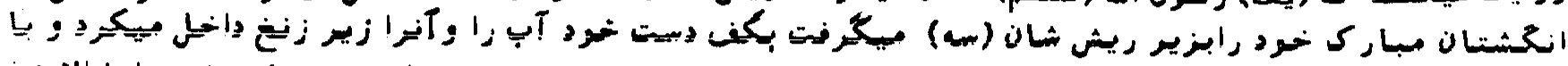

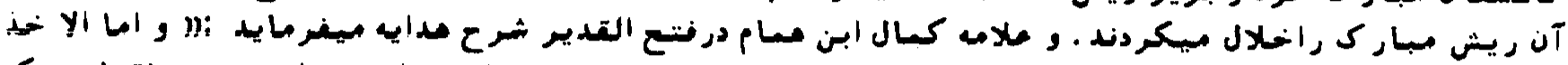

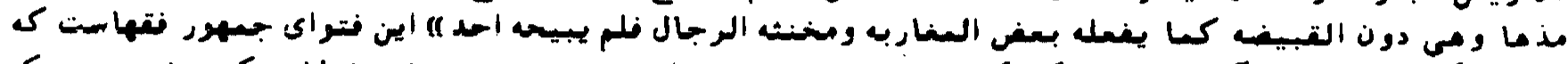

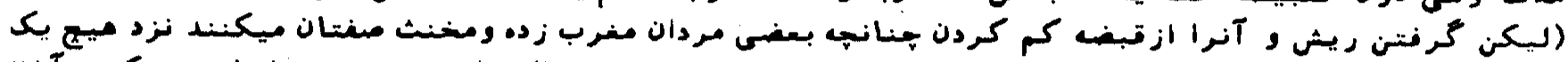

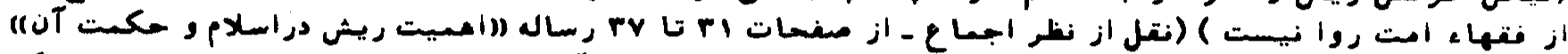

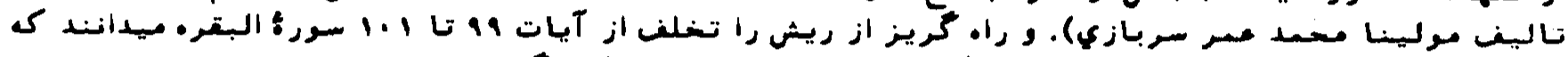

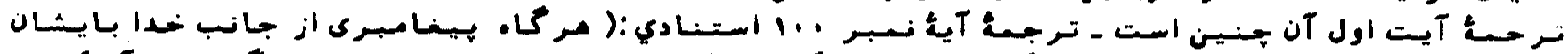

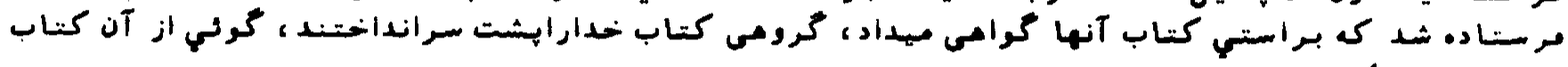
مسع نسيدانند).

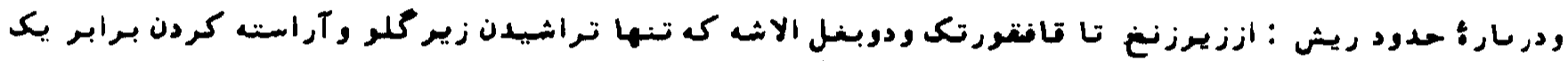

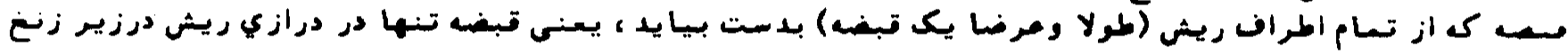

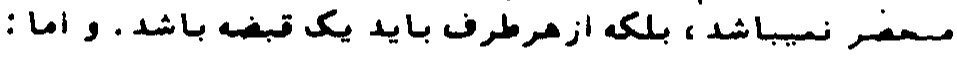

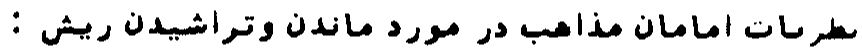

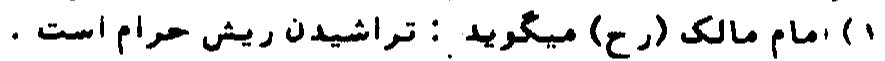

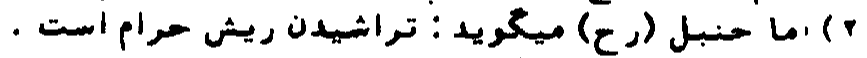

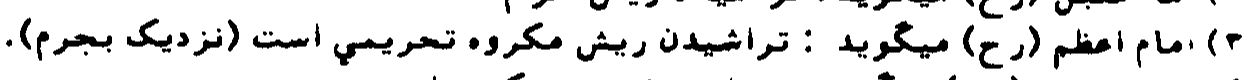

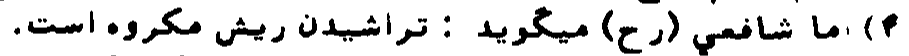

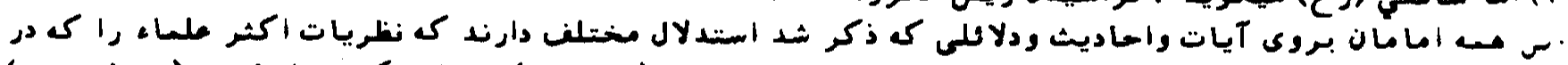

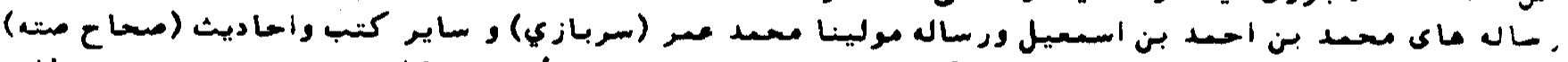

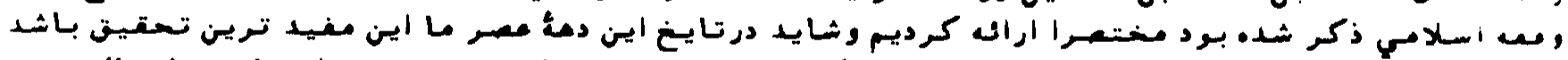

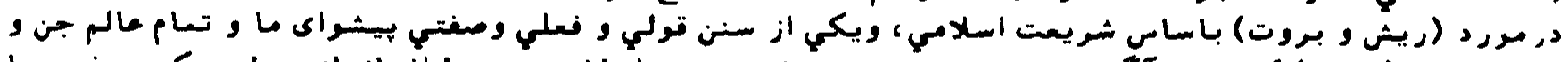

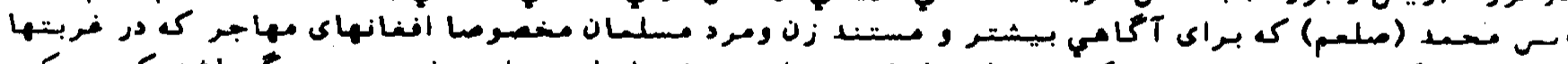

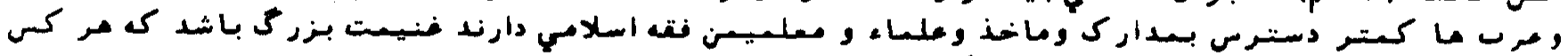

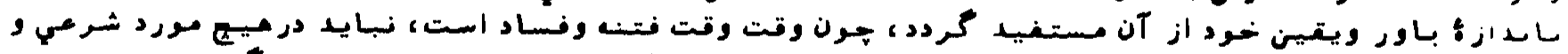

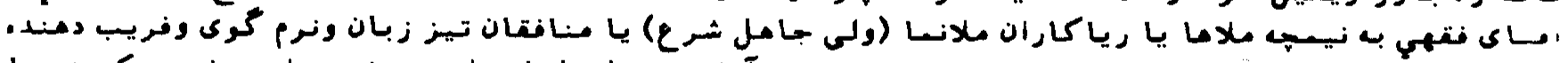

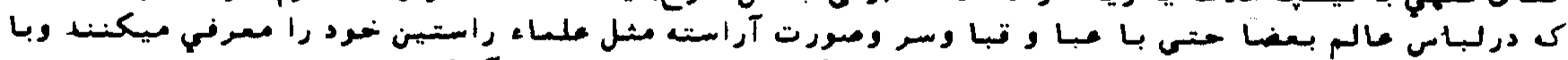

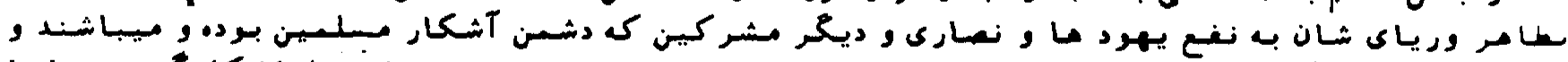

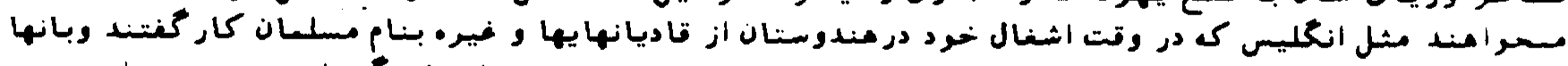

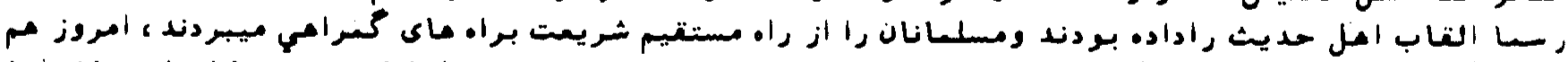

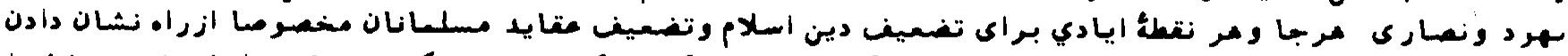

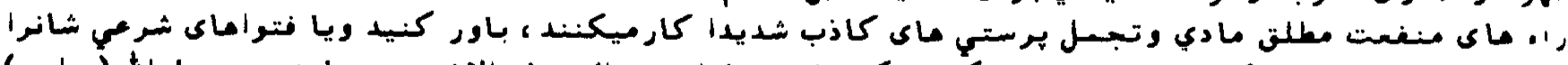

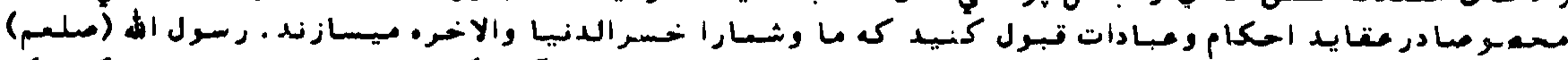

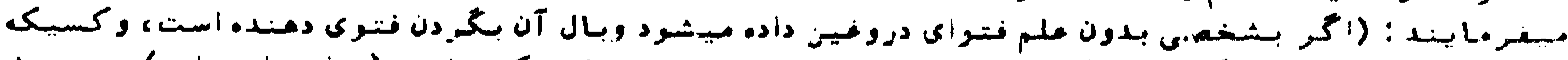

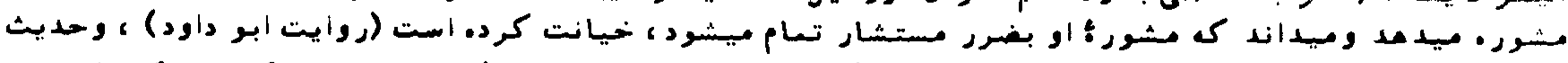

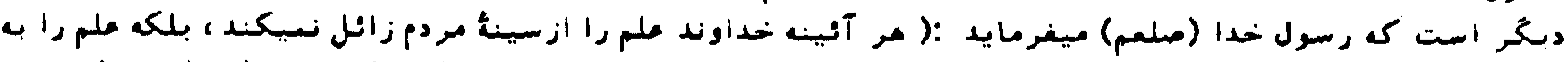

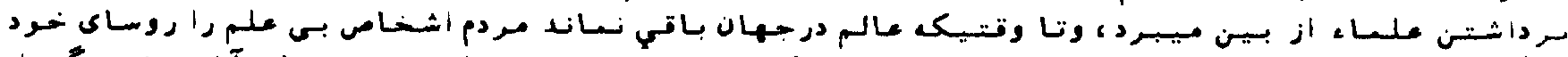

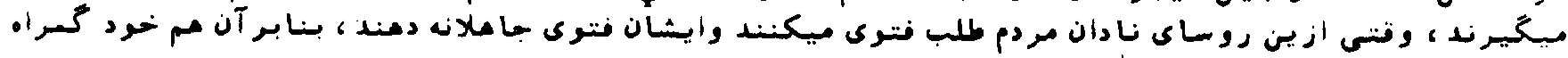

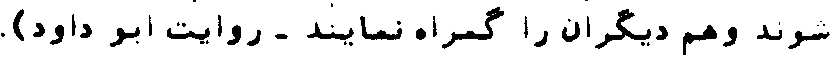




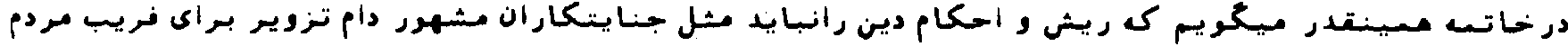

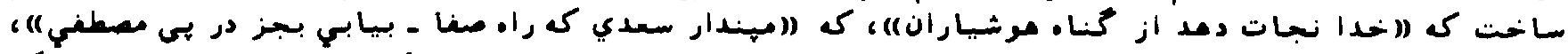

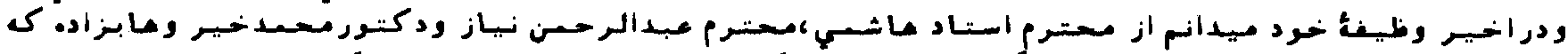

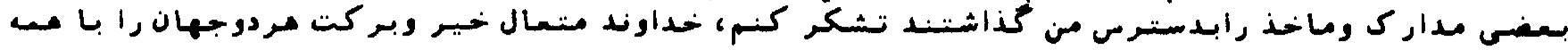

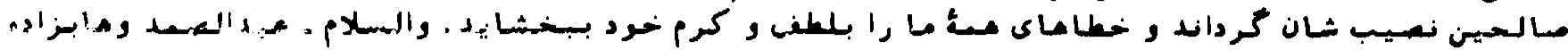

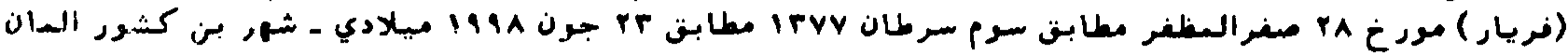

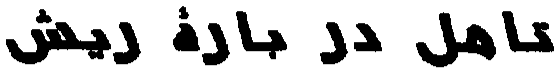

محمدسرورنورافشان از انكلستـان

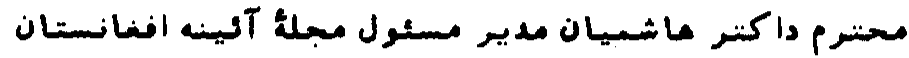

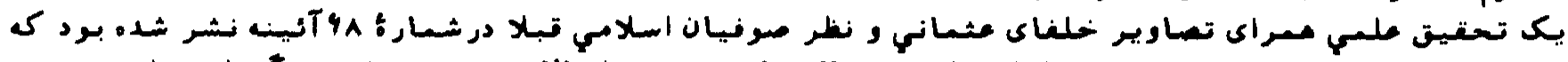

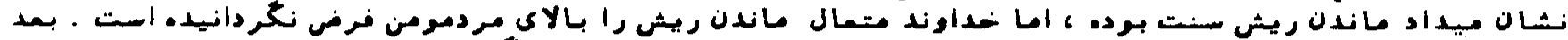

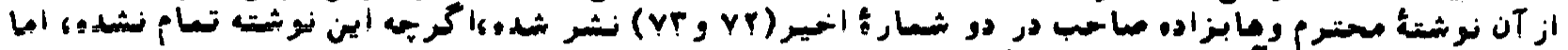

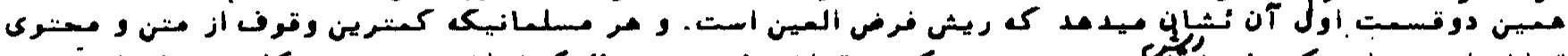

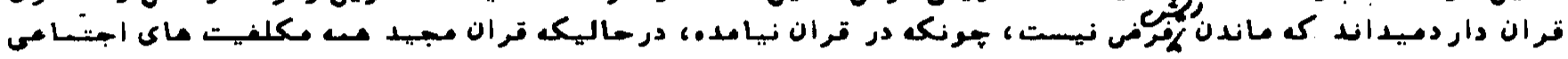

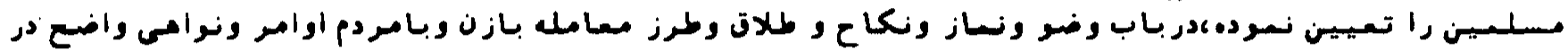

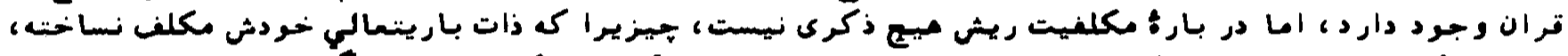

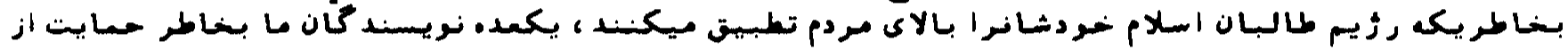

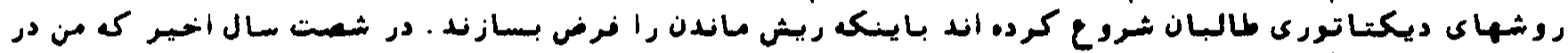

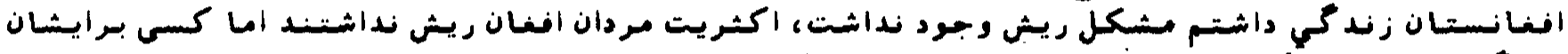

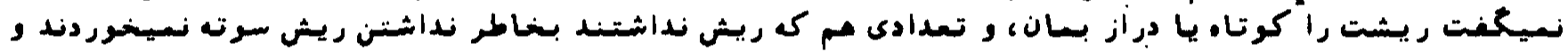

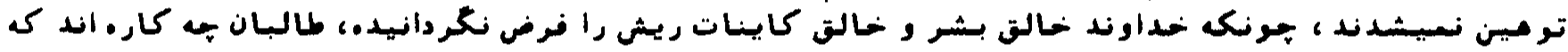

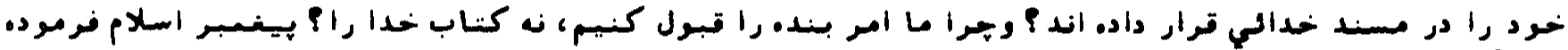

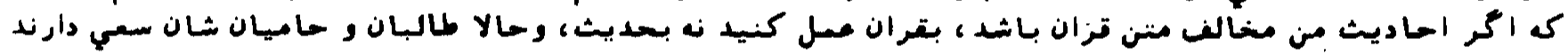

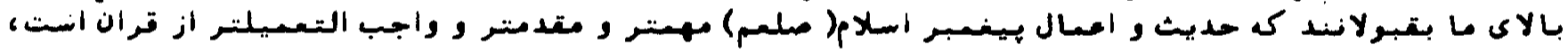

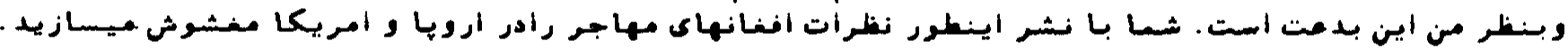

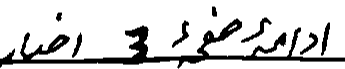

والسلام. ومن

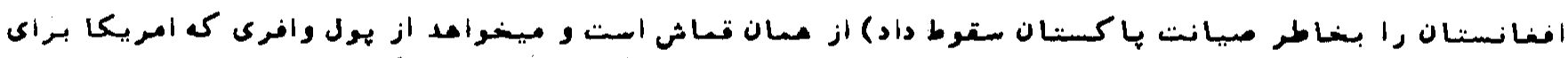

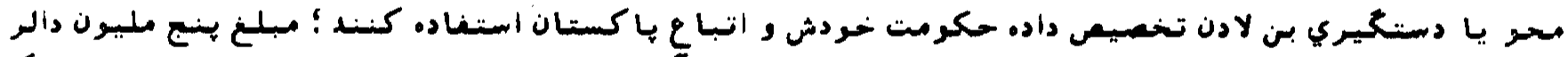

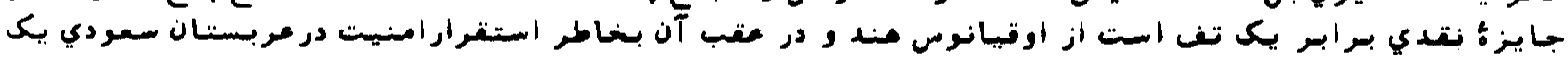

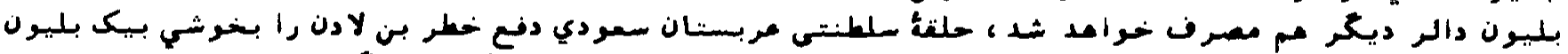

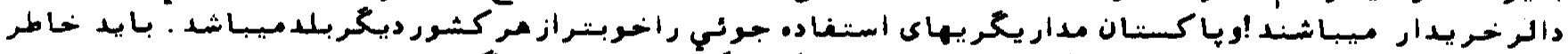

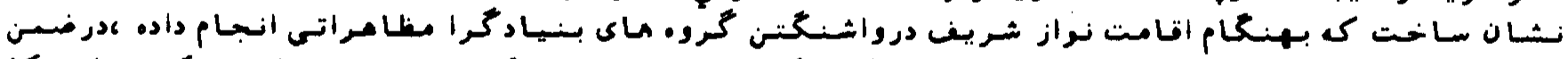

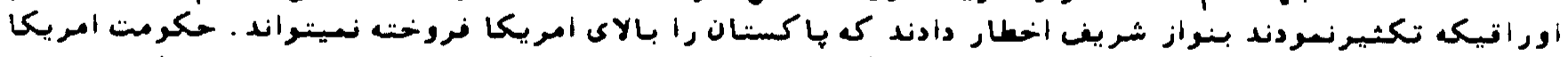

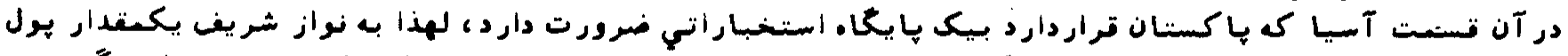

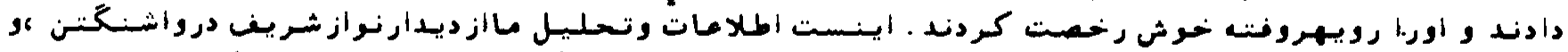

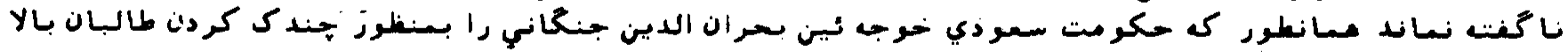

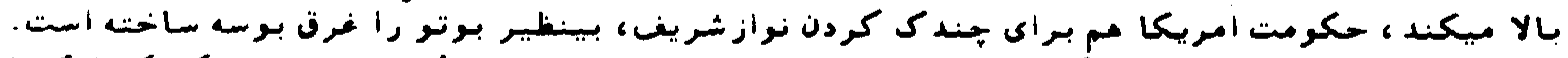

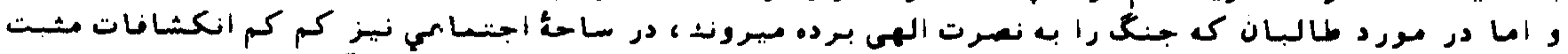

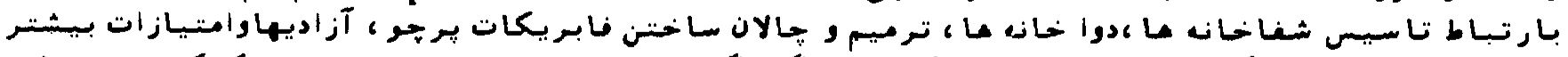

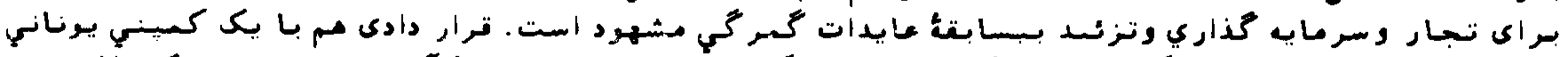

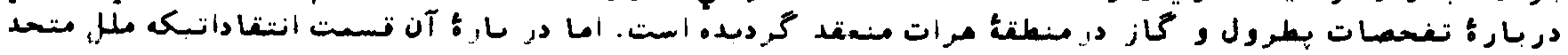

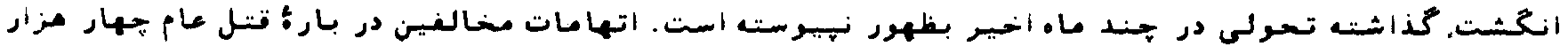

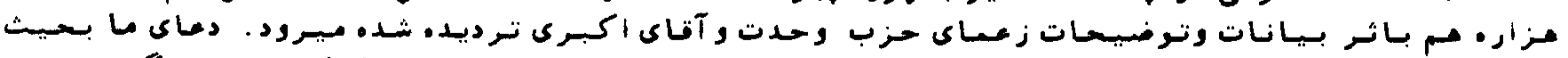

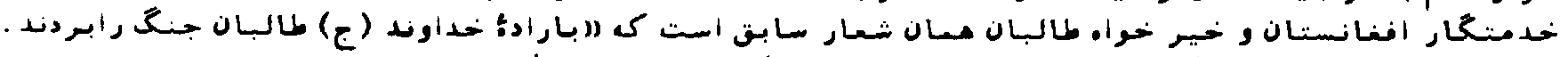

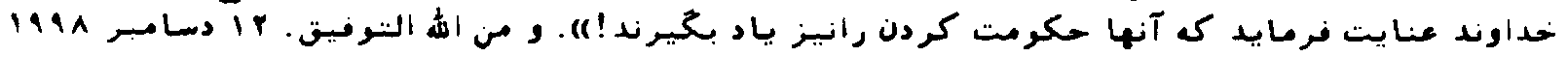




\section{1

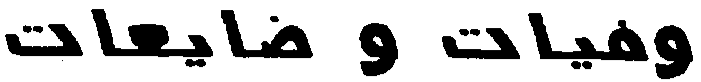

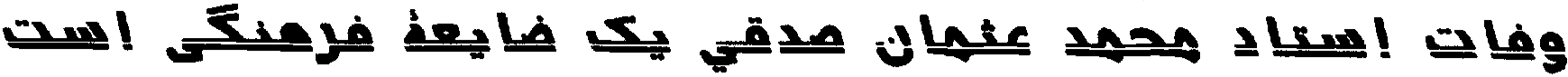

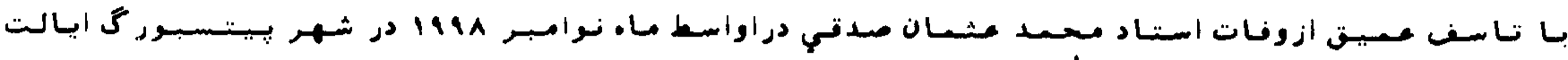

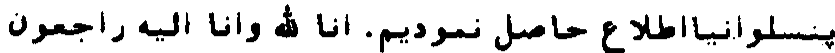

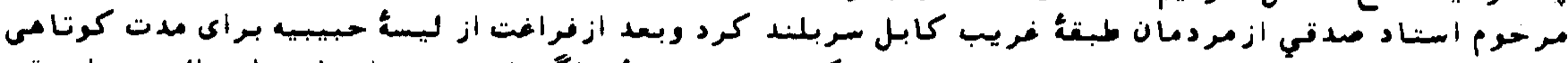

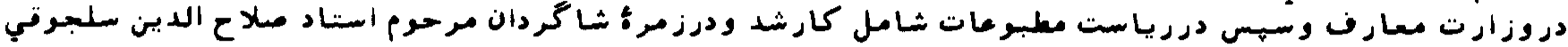

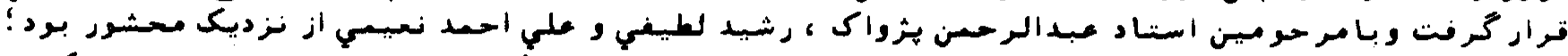

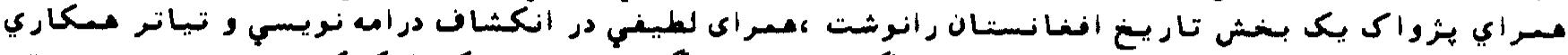

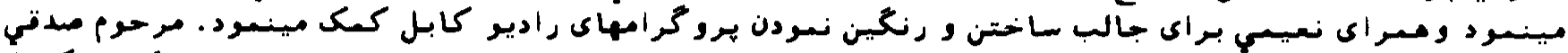

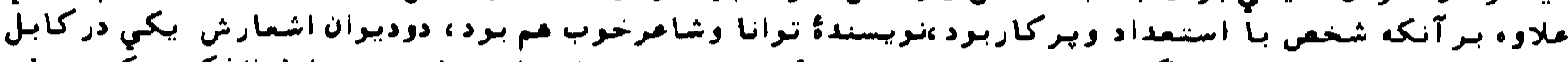

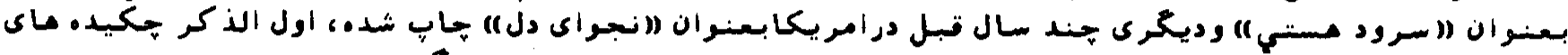

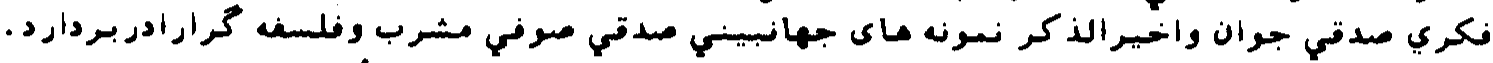

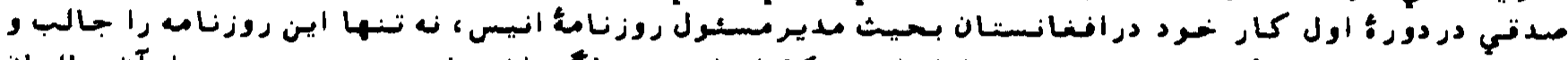

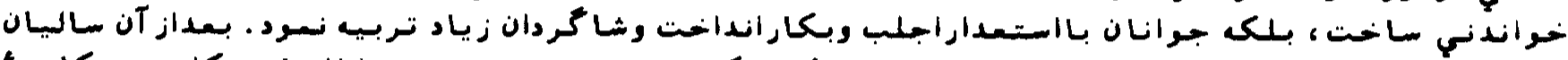

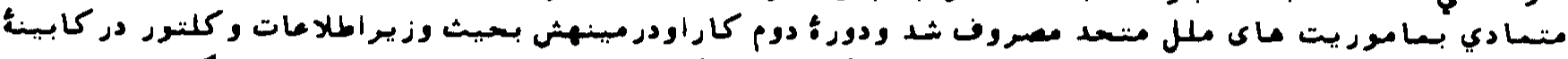

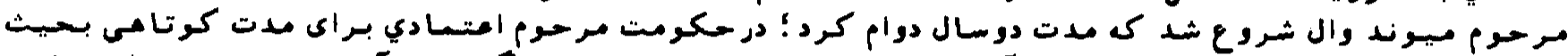

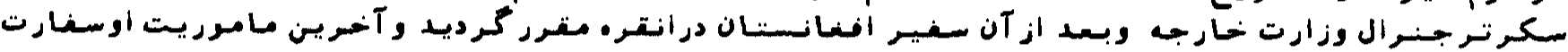

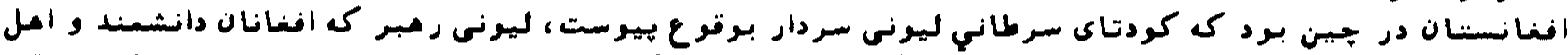

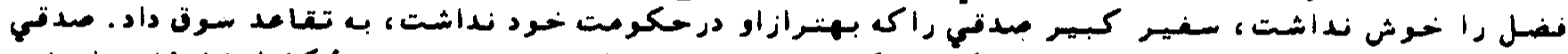

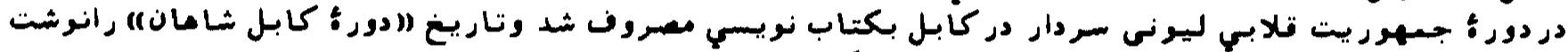

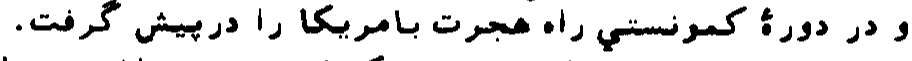

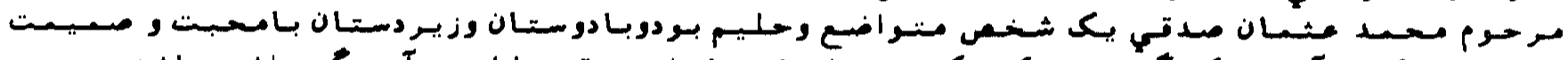

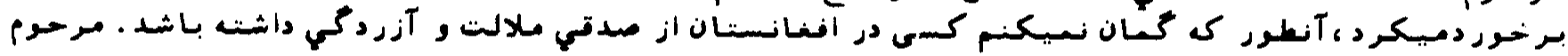

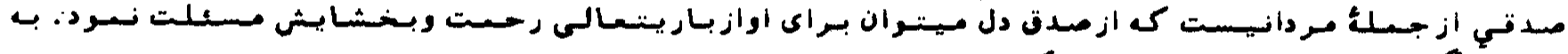

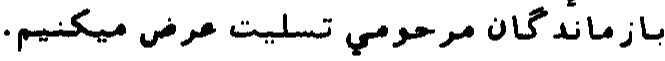

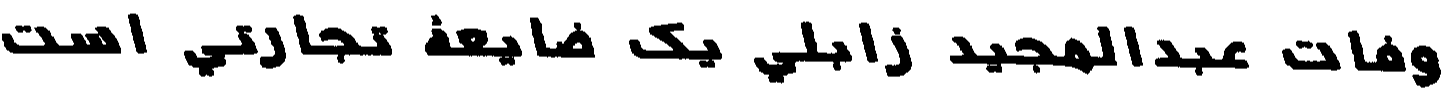

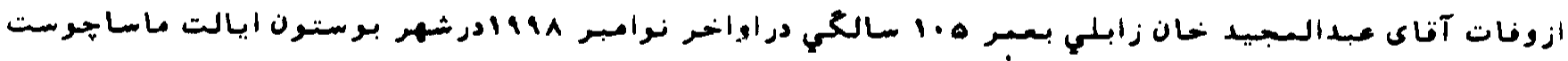

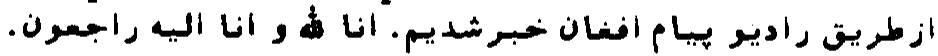

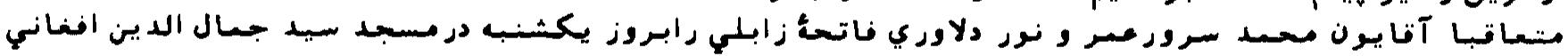

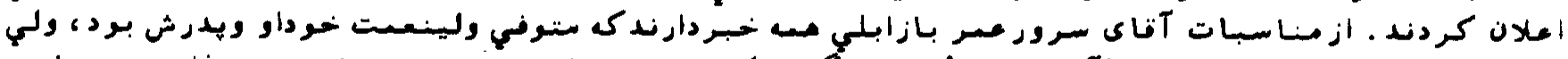

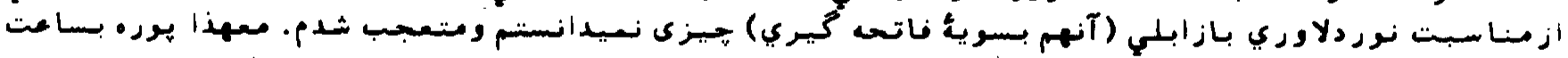

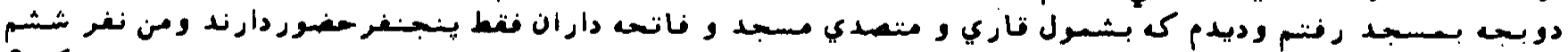

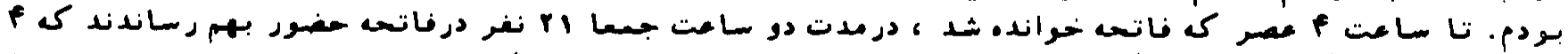

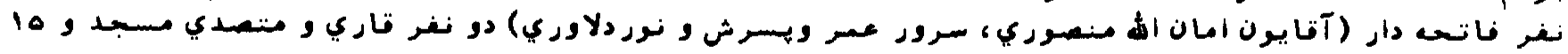

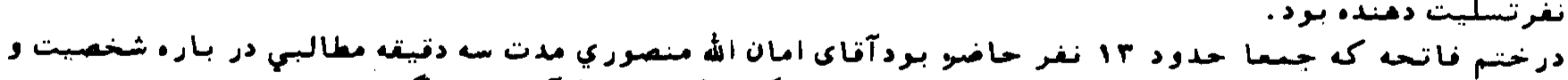

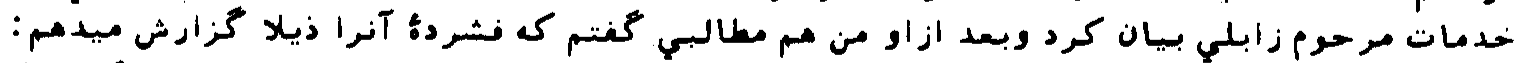

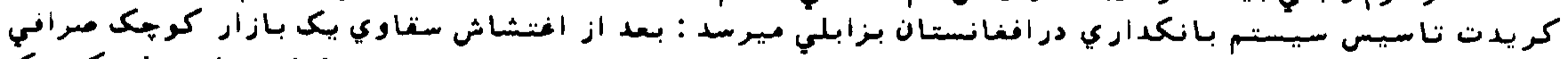

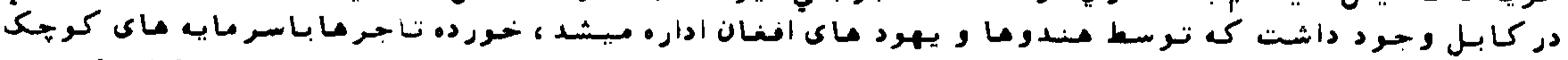

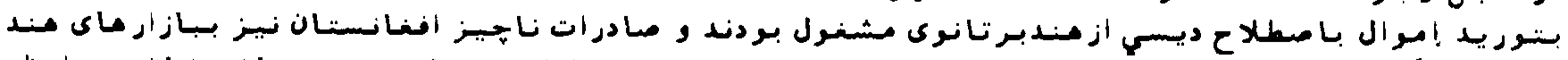

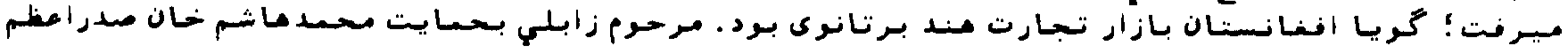

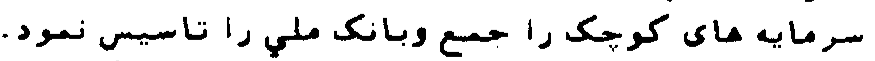

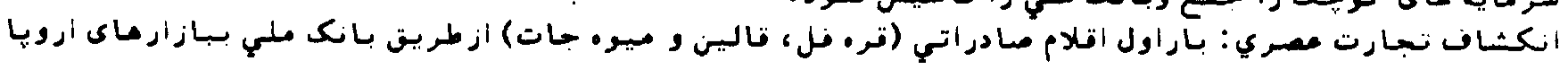




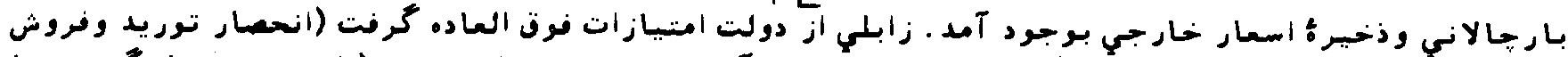

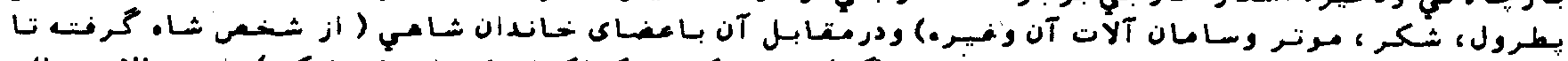

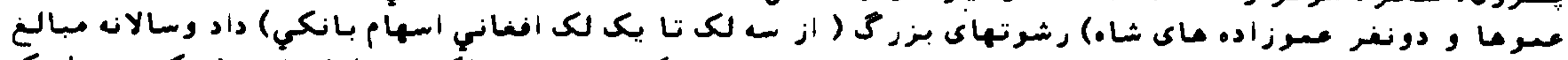

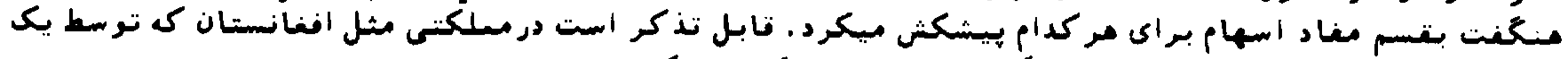

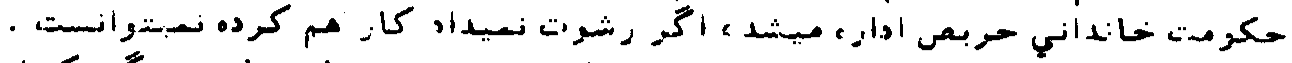

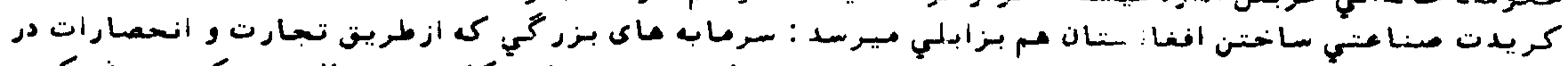

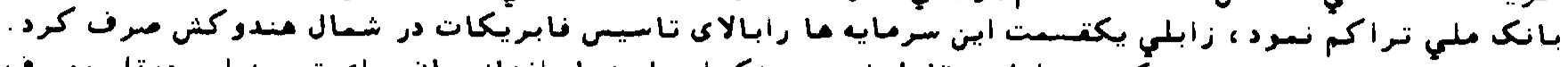

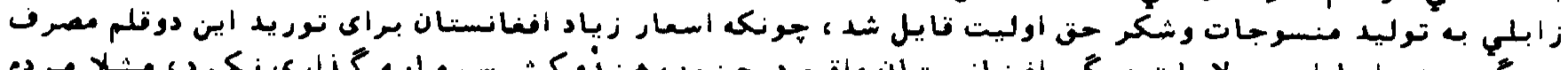

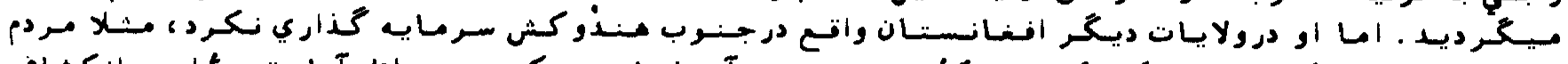

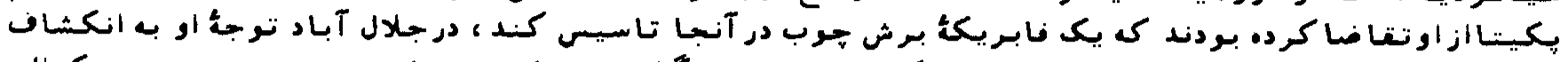

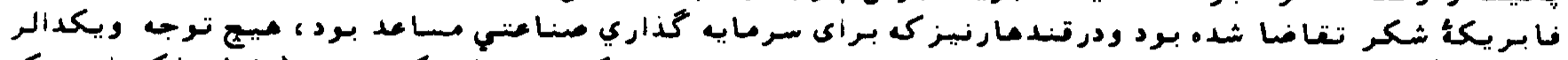

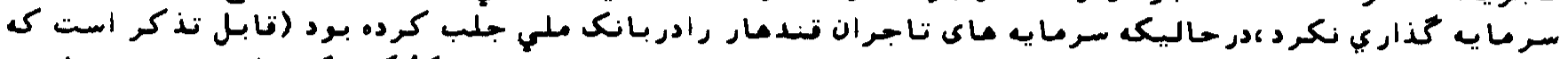

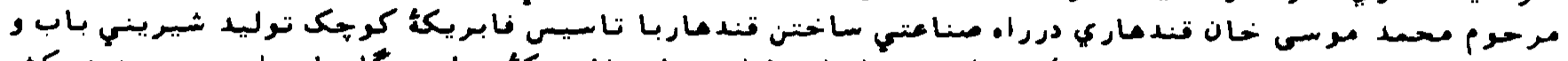

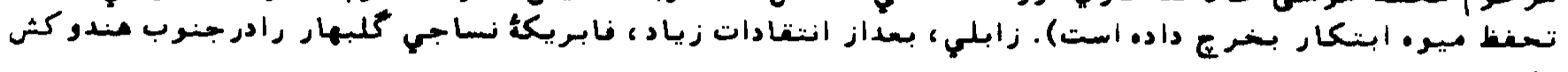

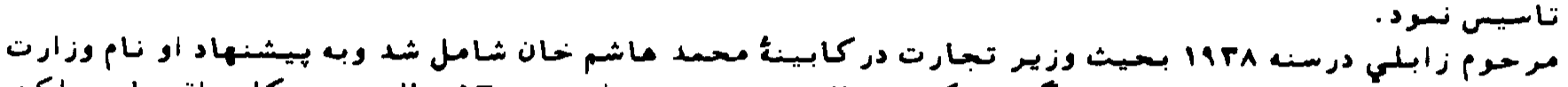

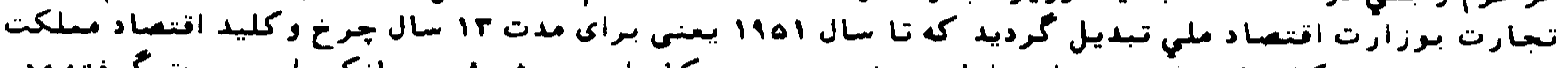

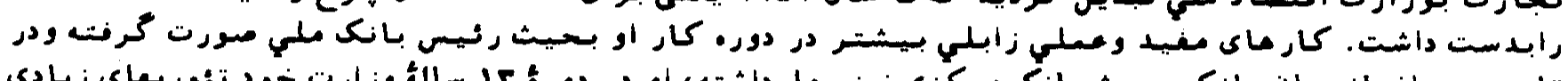

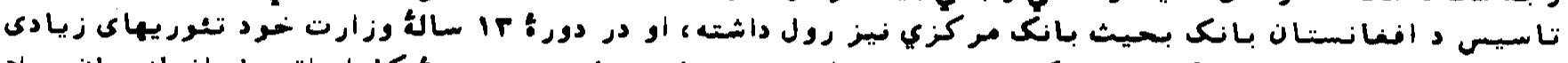

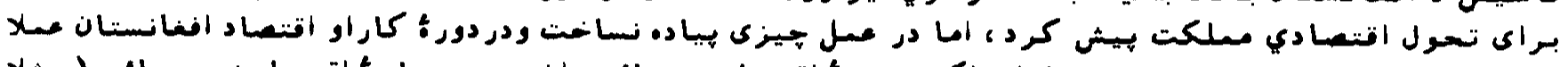

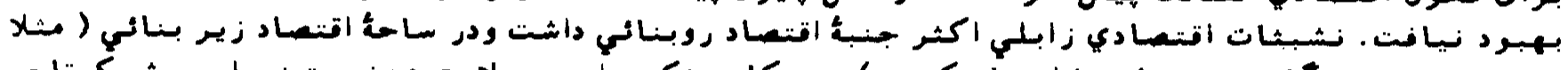

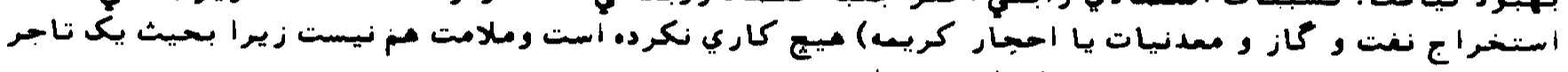

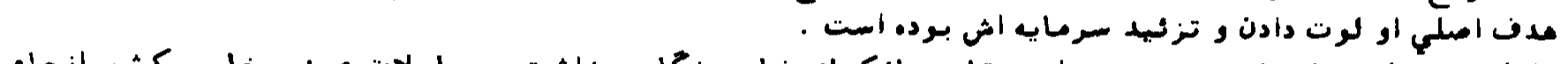

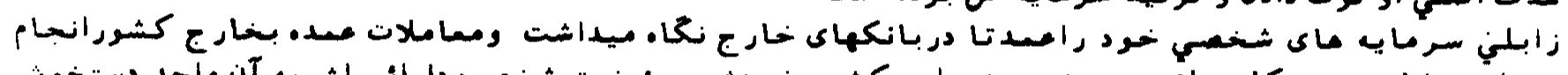

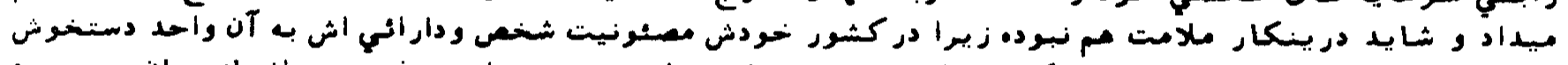

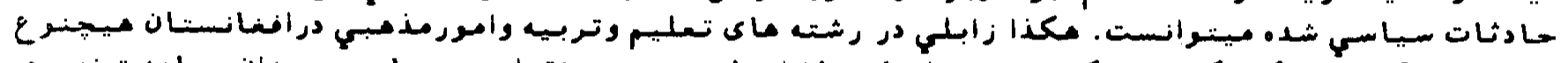

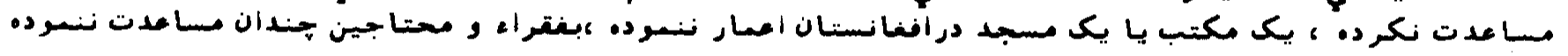

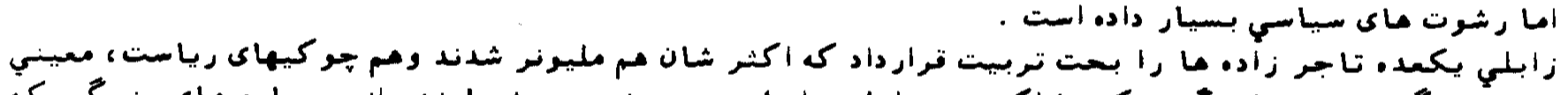

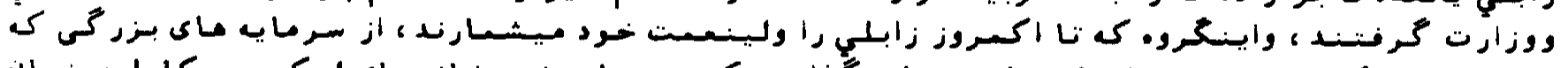

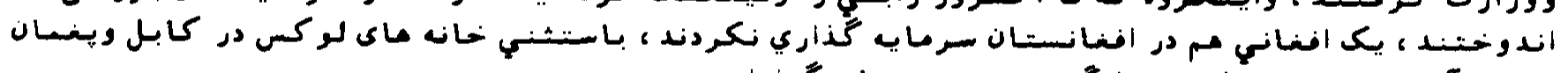

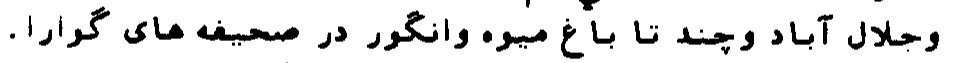

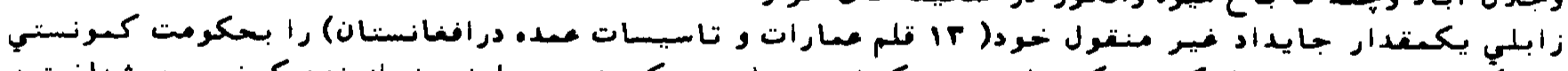

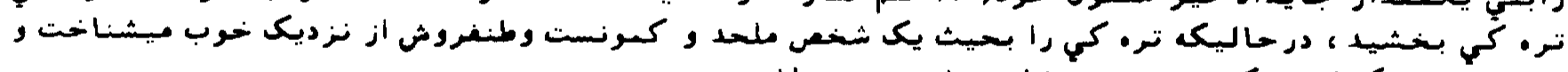

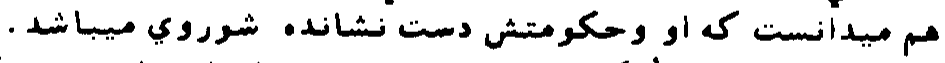

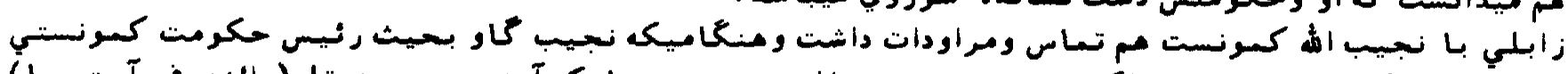

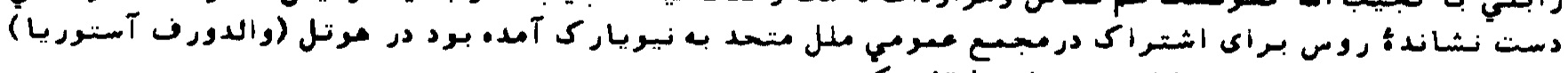

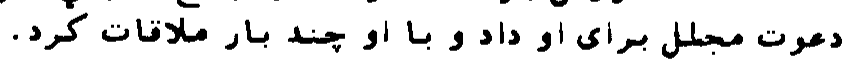

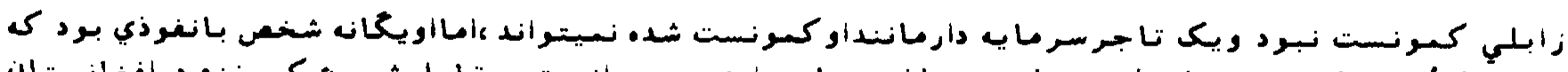

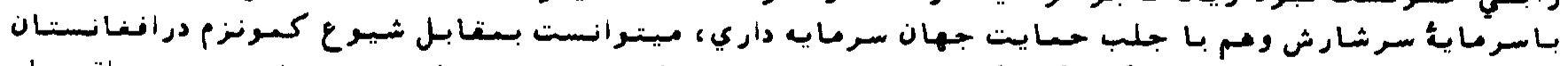

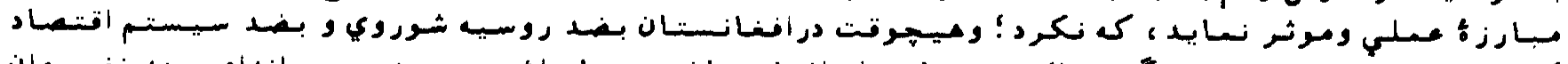

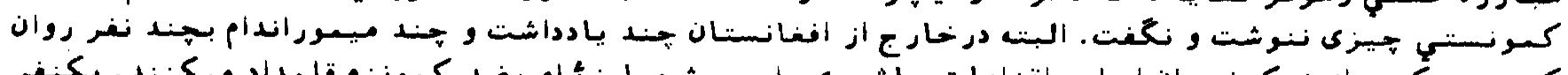

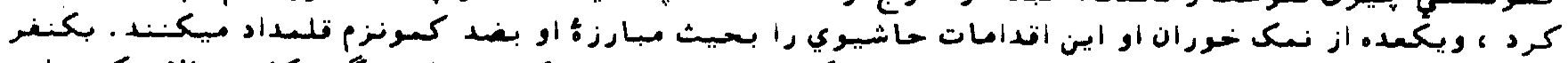

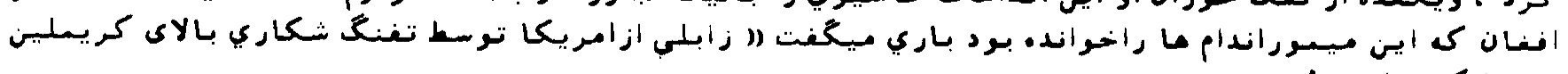

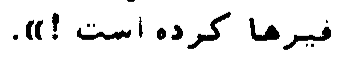

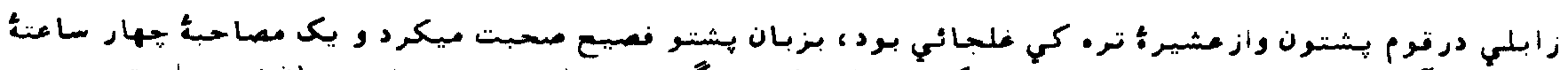

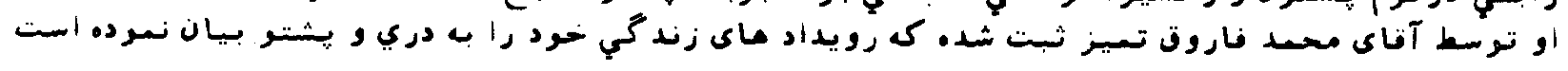




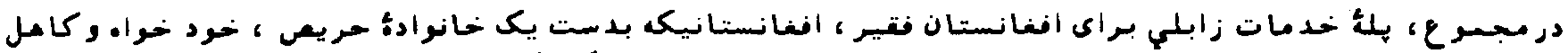

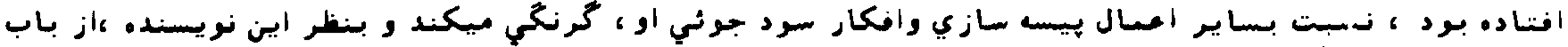

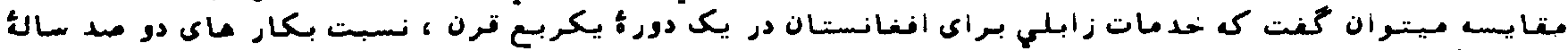

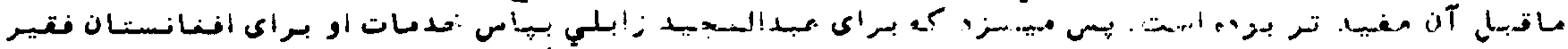

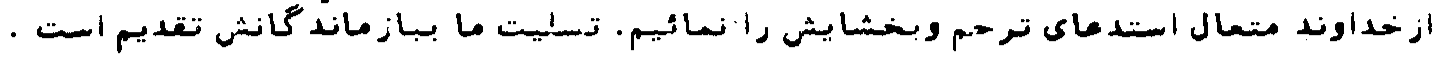

\section{وفات الهناك يك هادر جوال افغلاد}

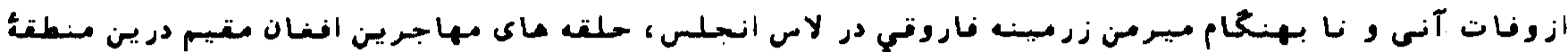

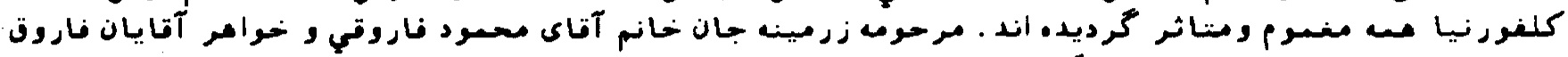

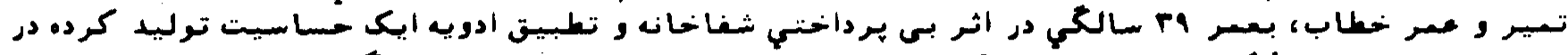

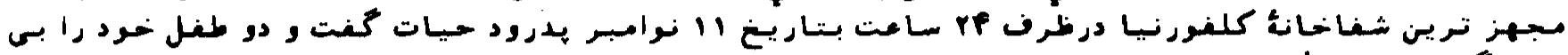

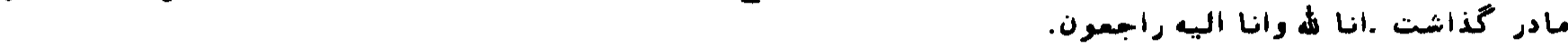

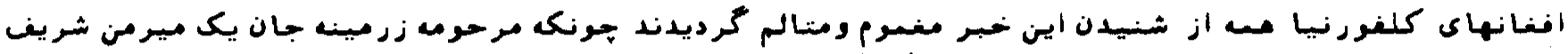

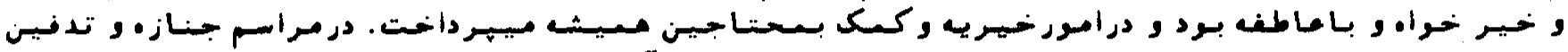

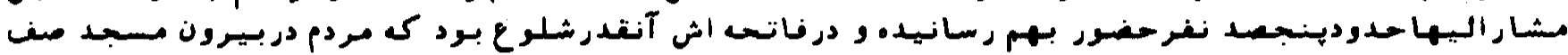

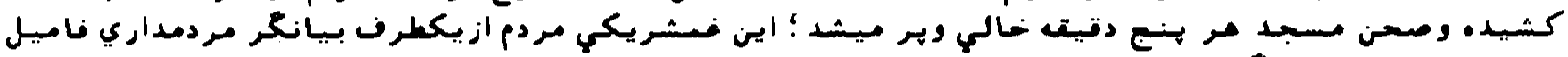

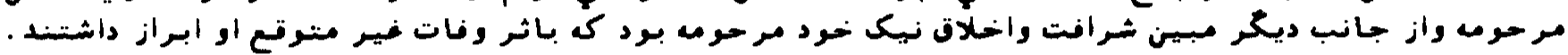

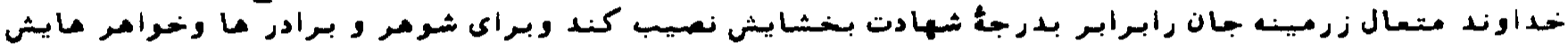

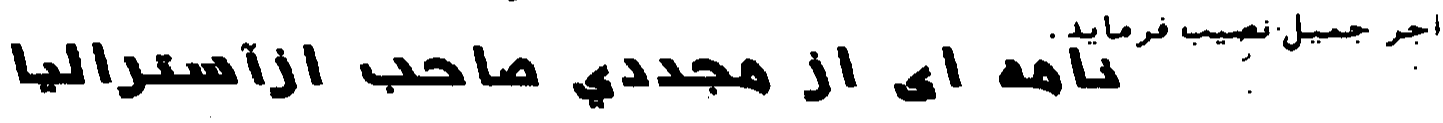

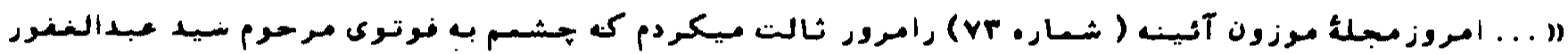

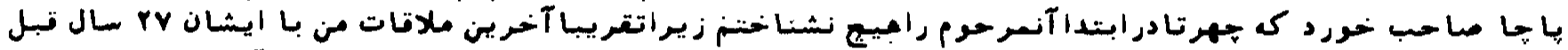

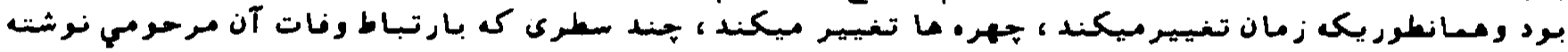

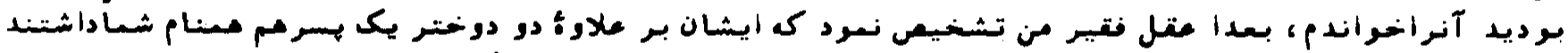

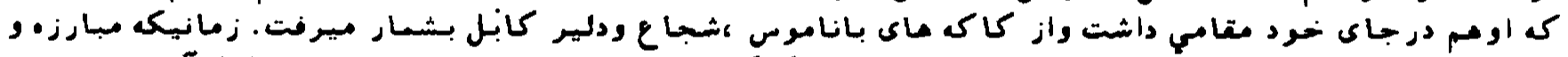

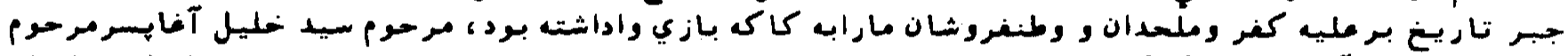

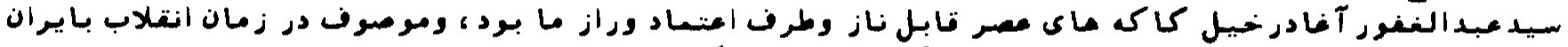

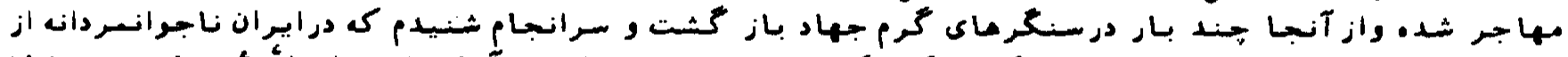

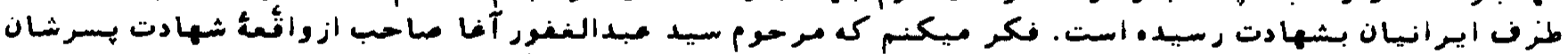

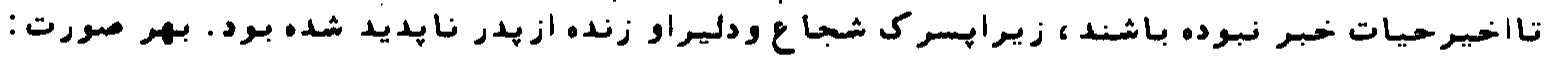

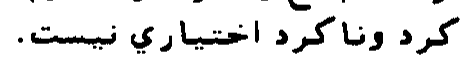

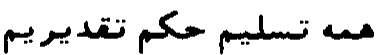

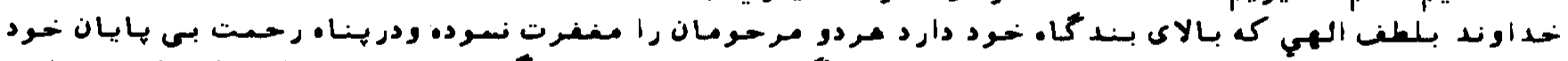

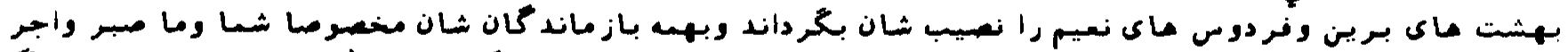

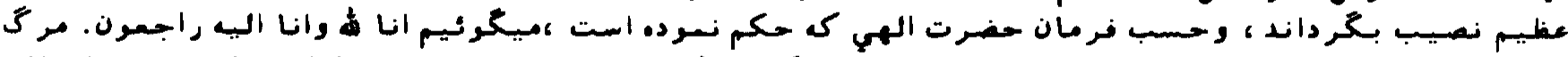

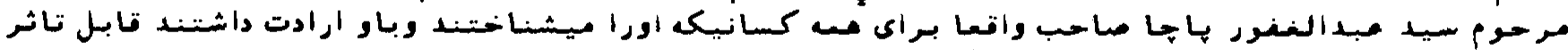

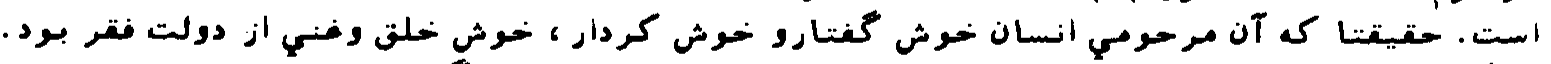

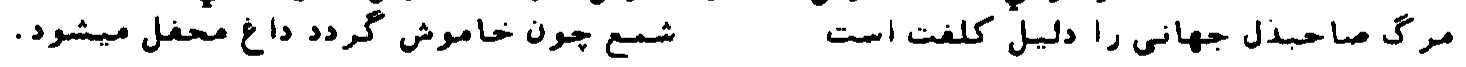

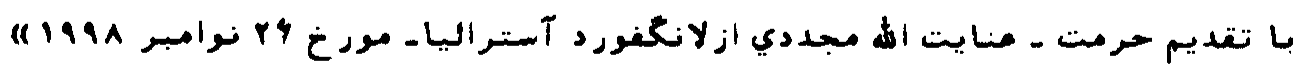

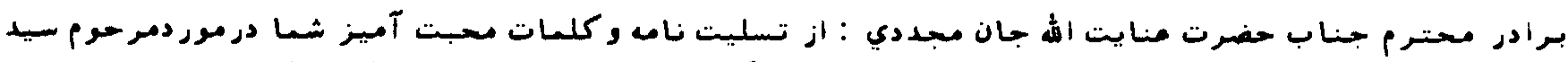

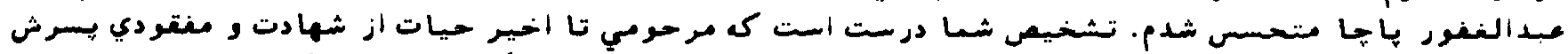

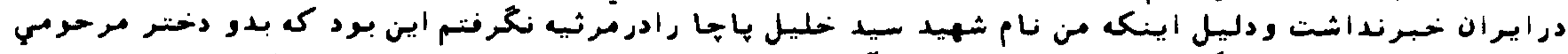

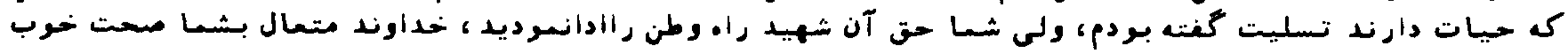

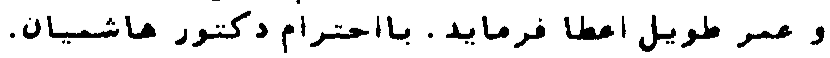

\section{شFمارة}




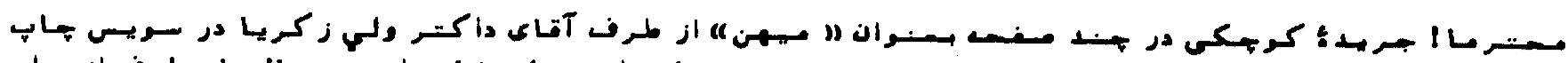

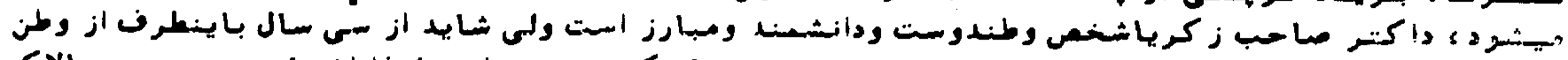

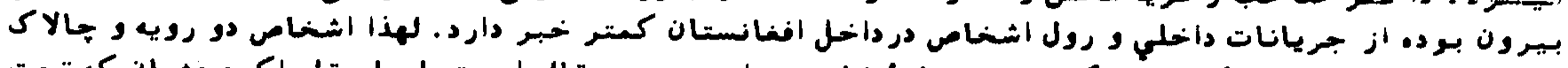

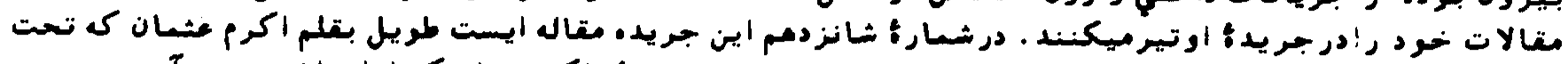

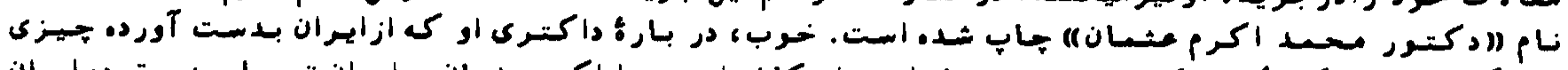

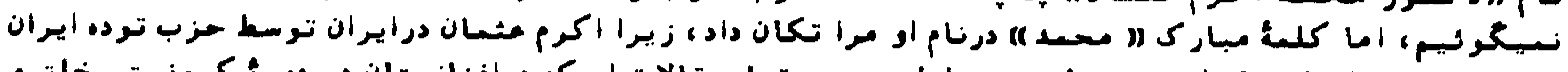

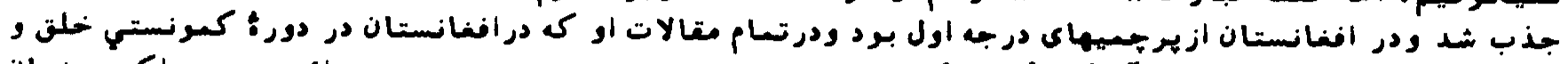

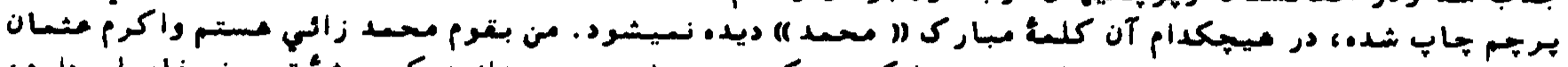

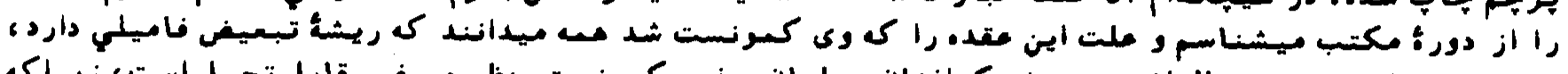

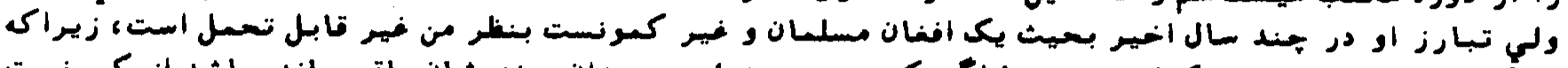

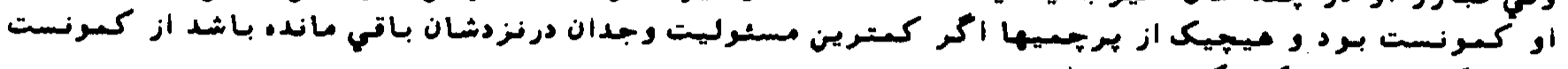

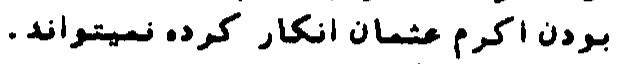

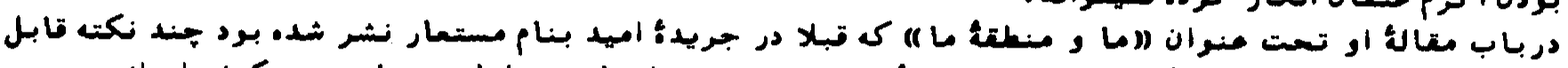

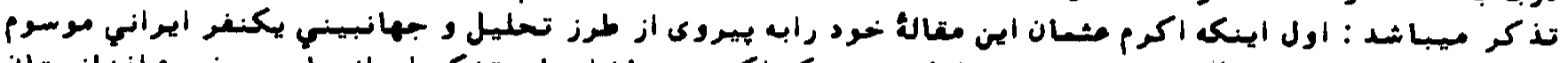

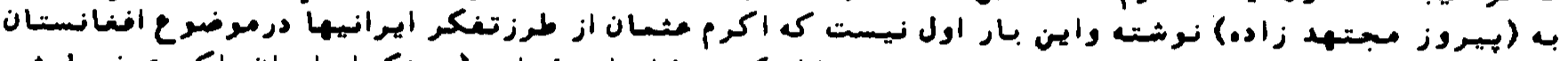

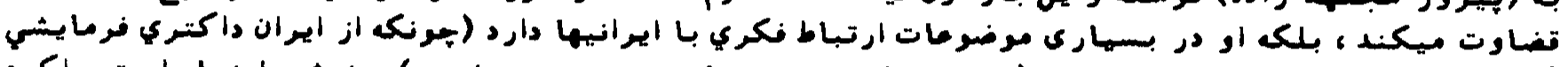

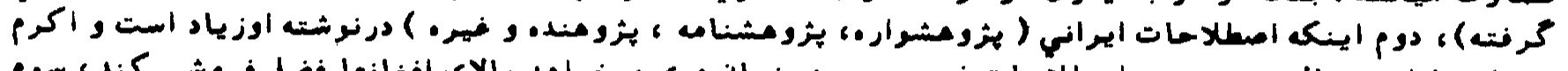

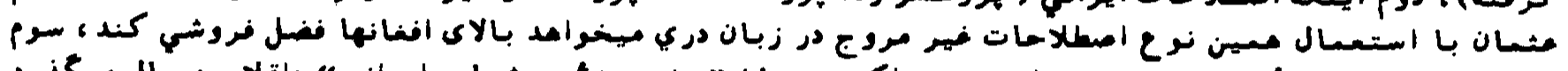

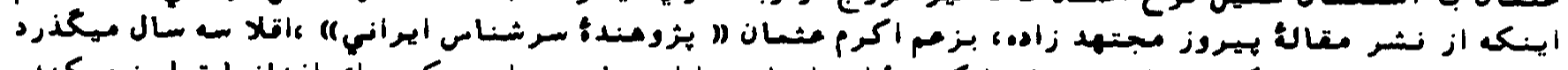

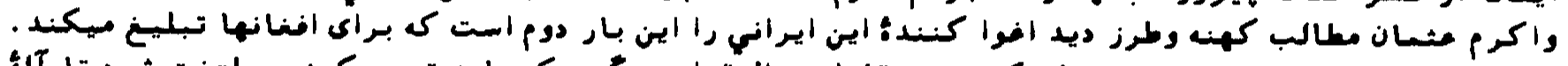

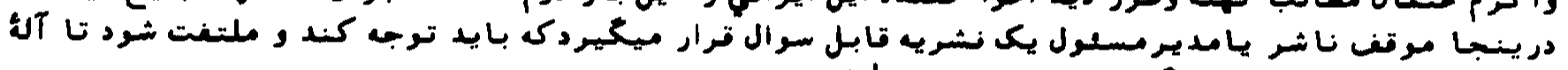

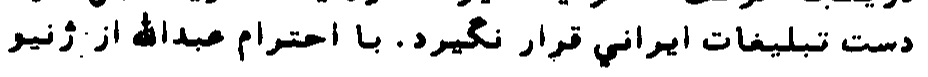

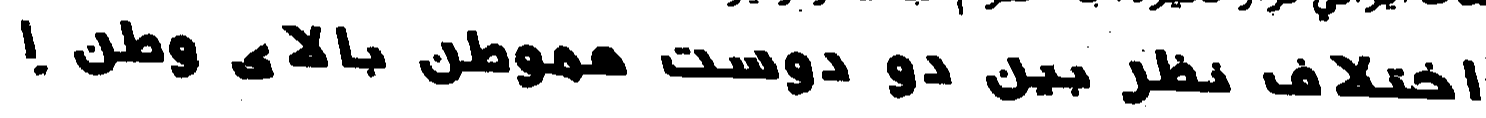

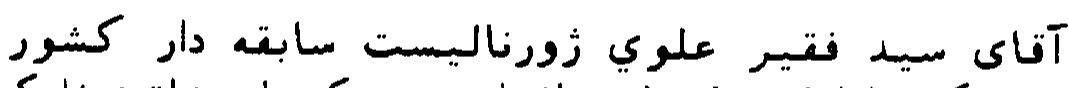

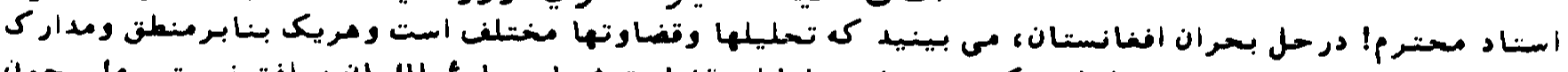

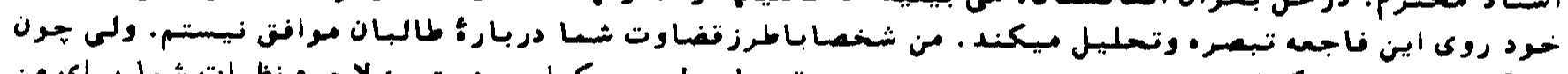

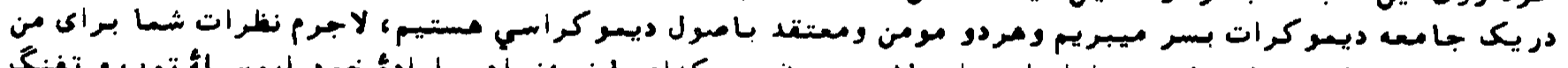

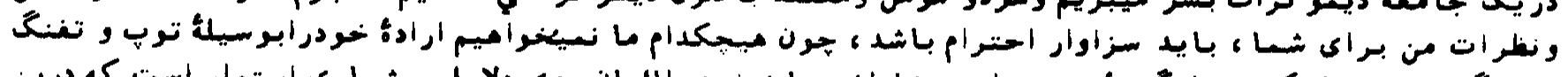

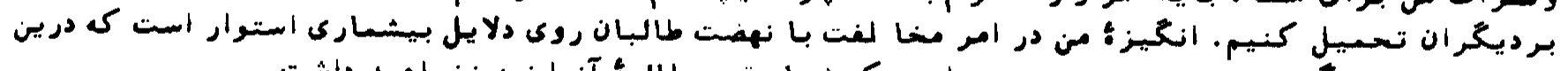

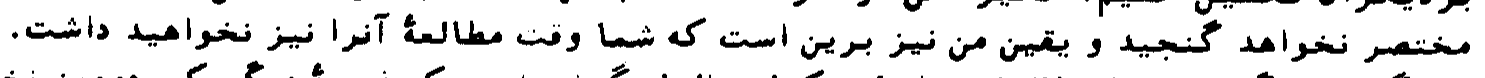

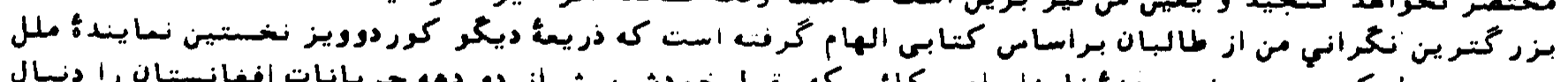

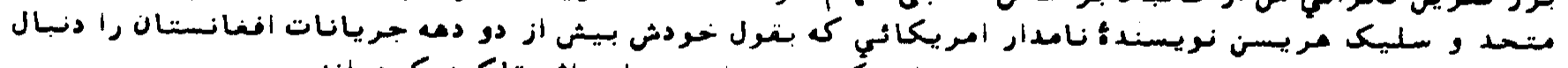

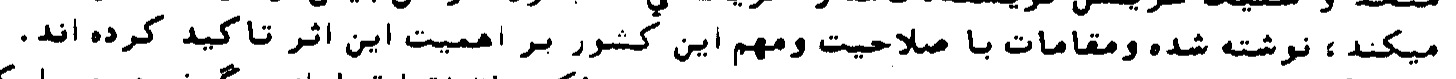

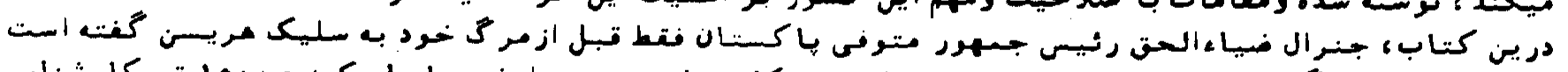

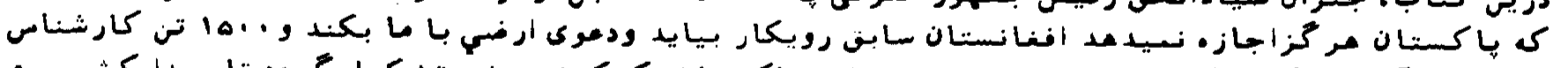

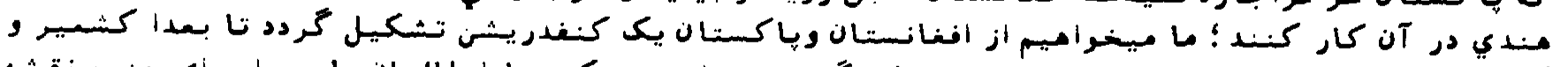

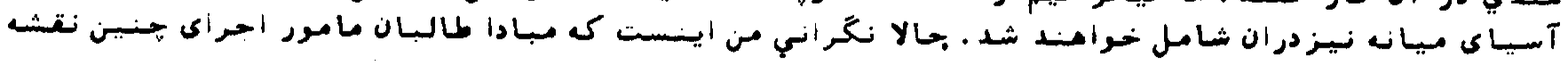

خاينانه نباشثند ميان

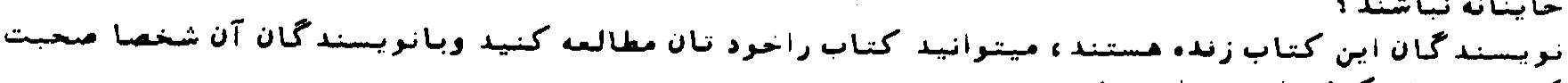

Gut of Aighanistan The Inside Story of the Soviet Withdrawal

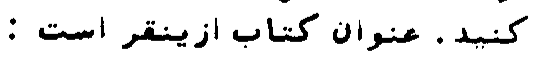
by Diego Cordovez, Seliq S. Harrisson

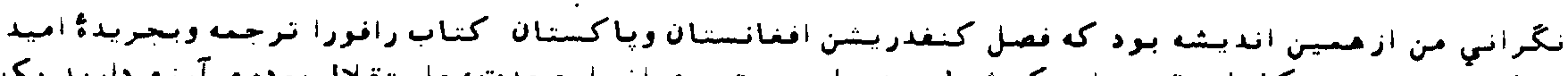

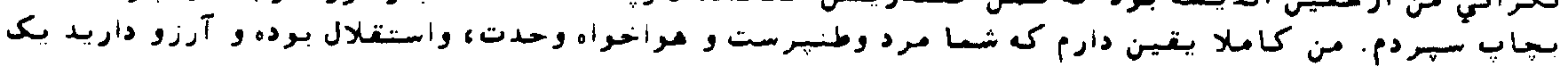




\section{5}

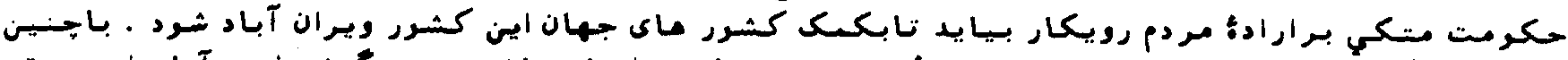

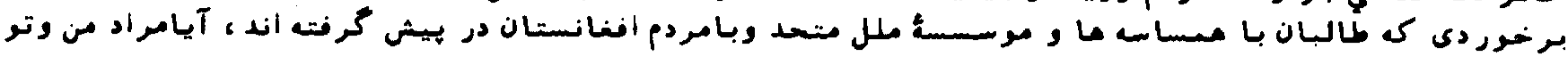

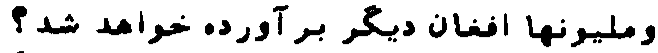

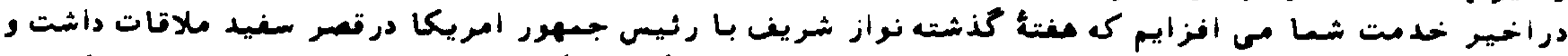

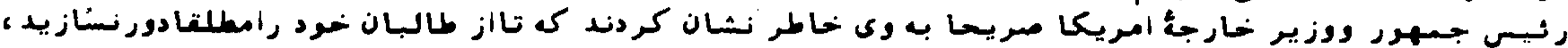

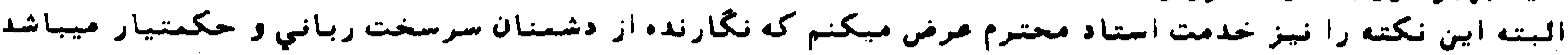

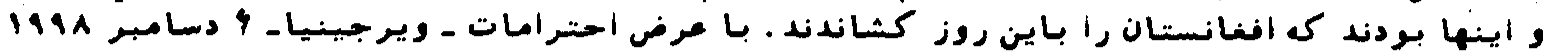

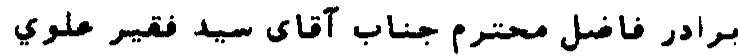

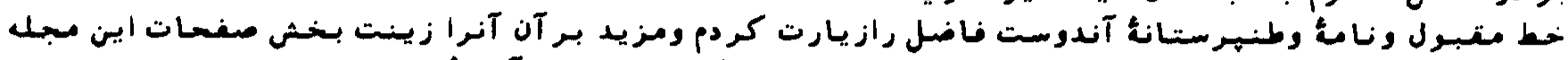

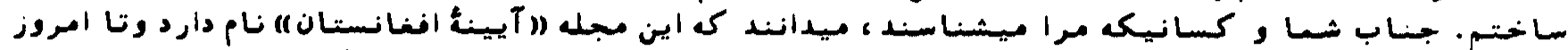

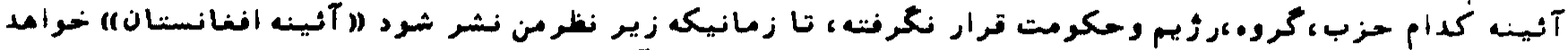

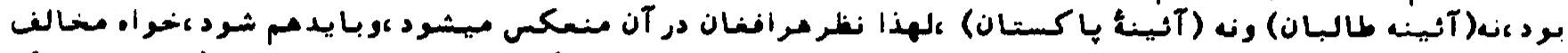

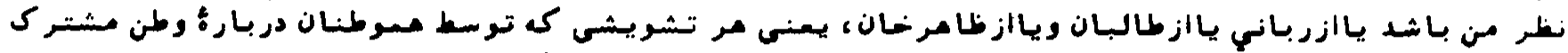

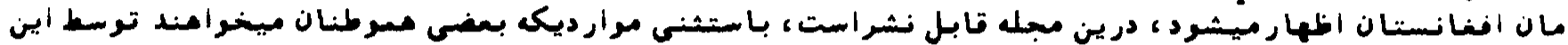

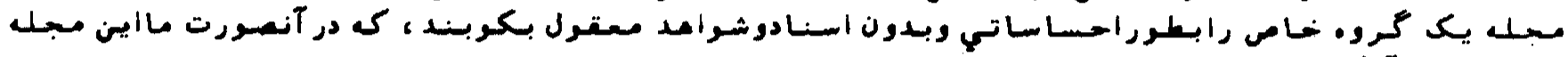

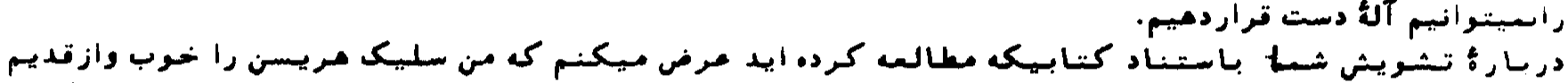

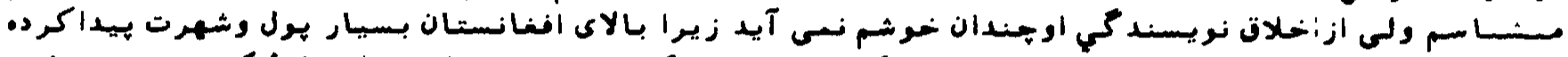

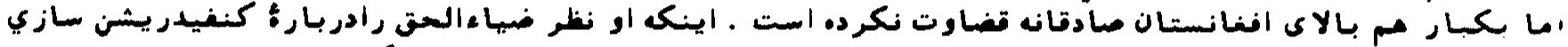

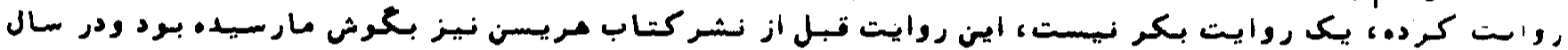

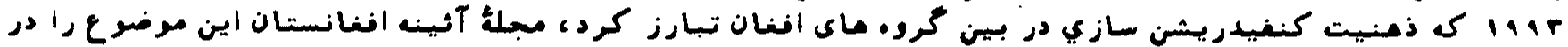

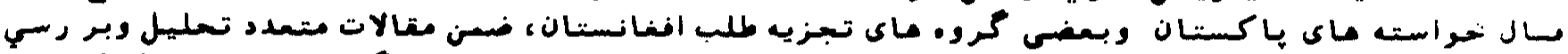

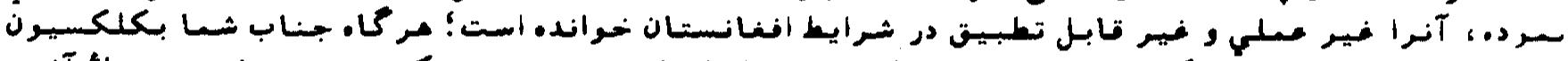

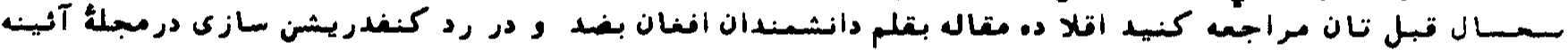

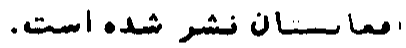

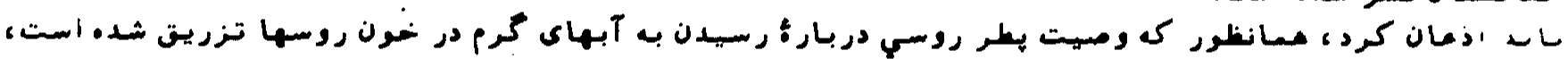

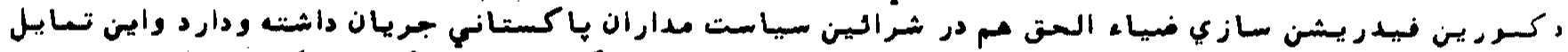

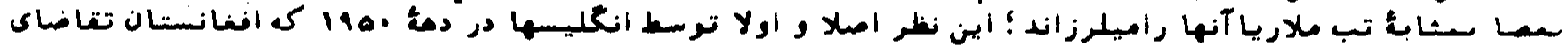

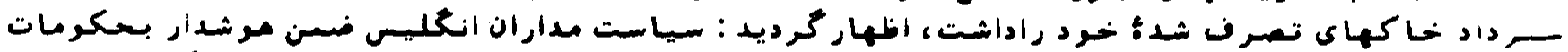

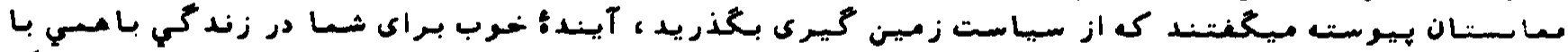

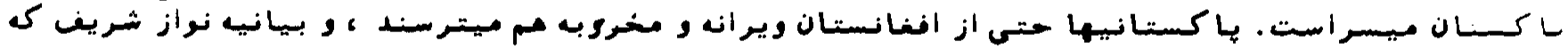

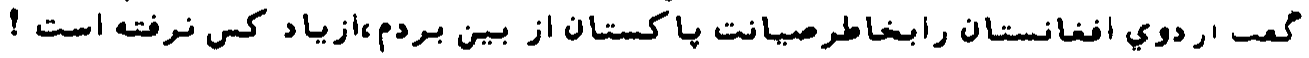

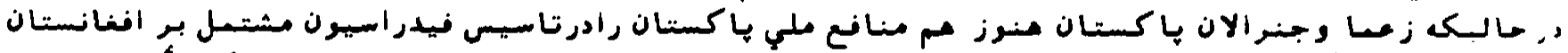

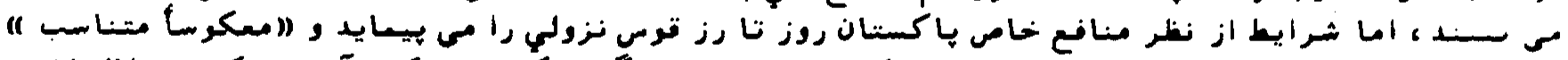

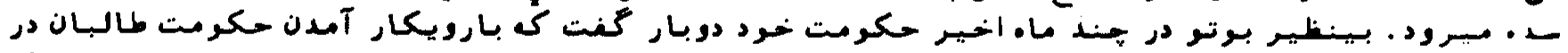

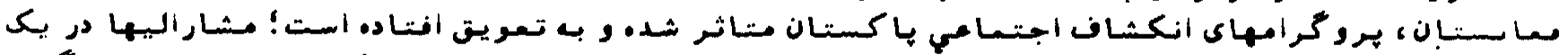

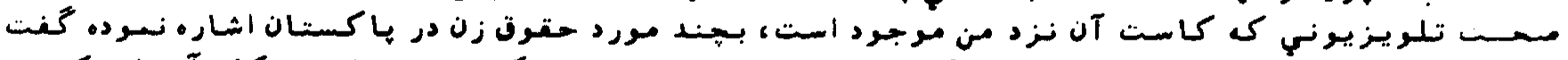

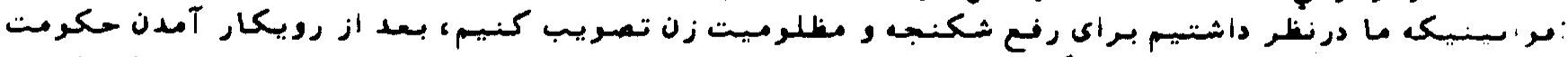

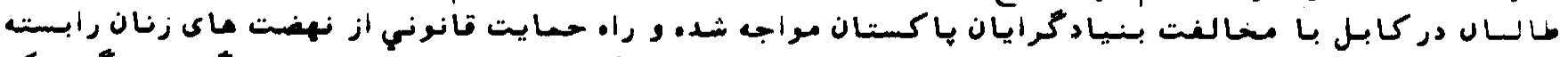

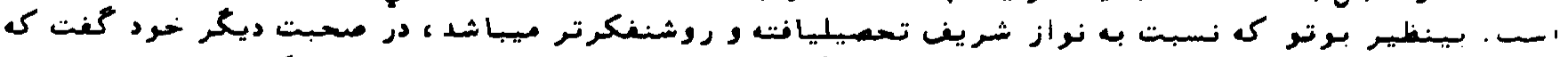

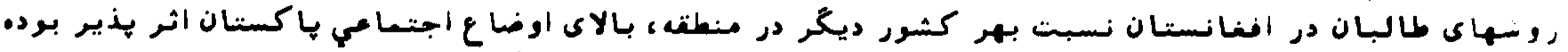

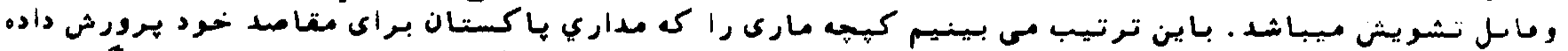

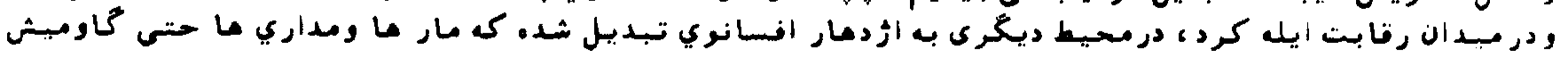

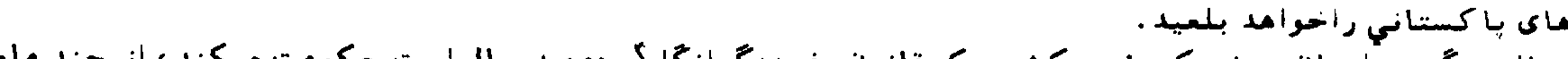

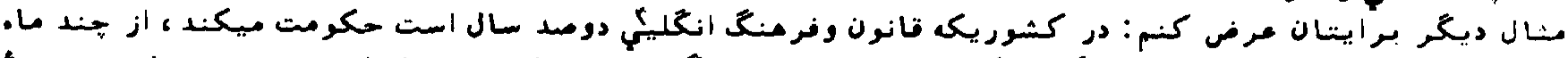

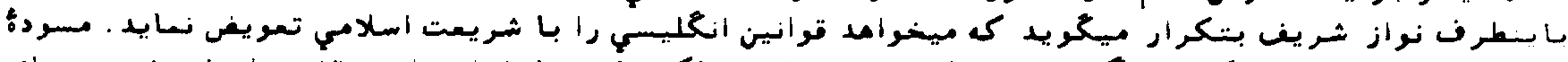

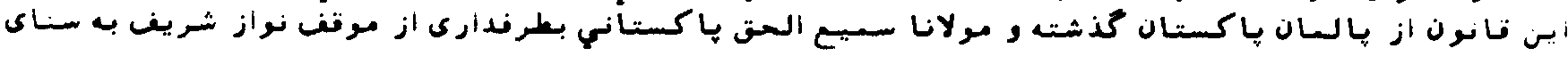




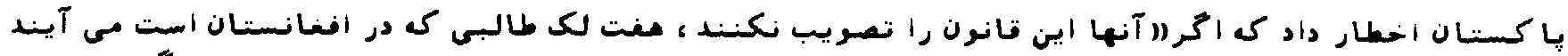

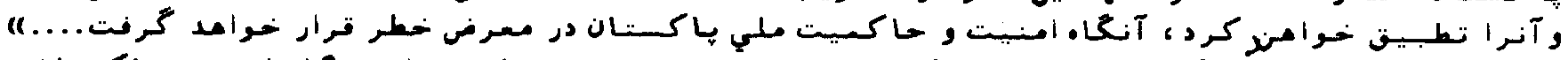

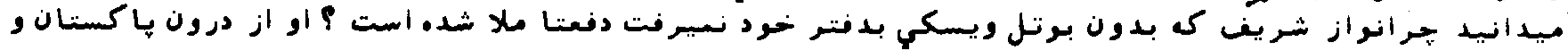

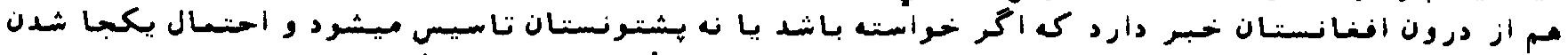

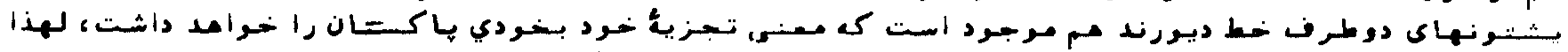

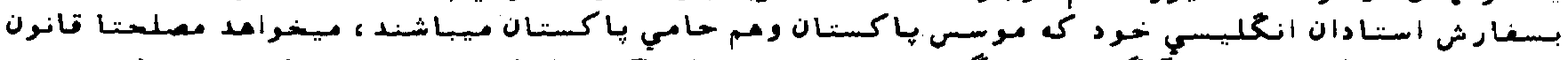

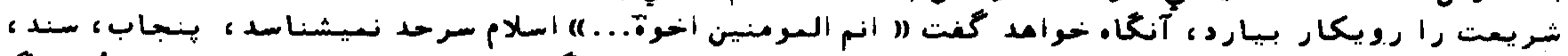

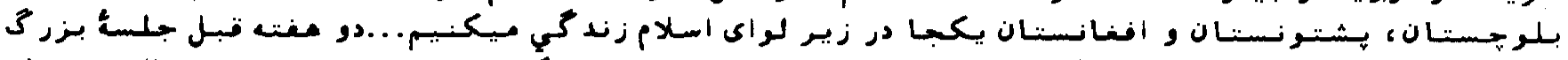

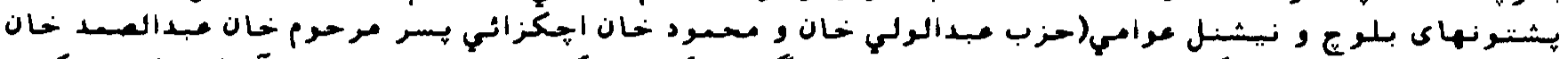

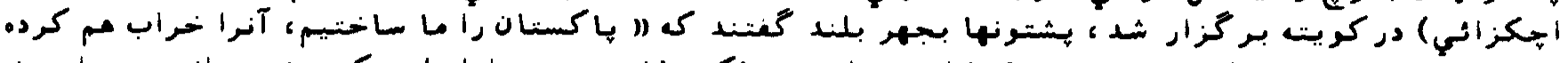

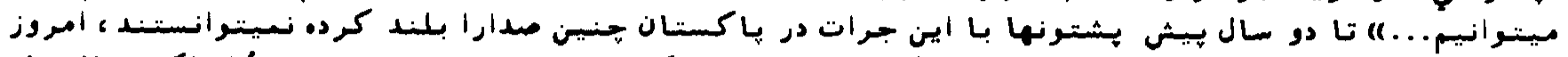

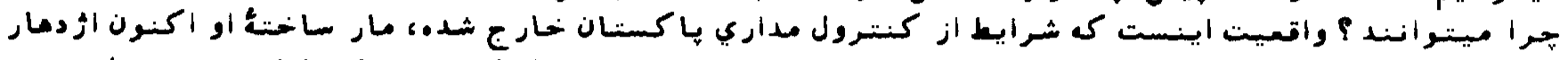

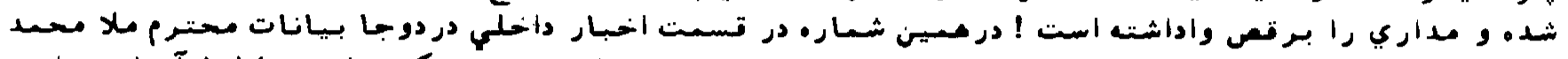

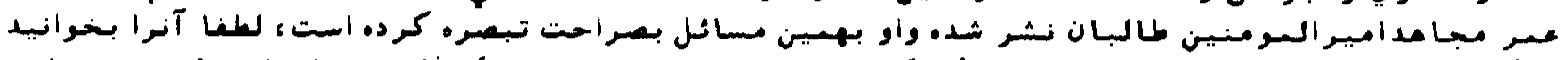

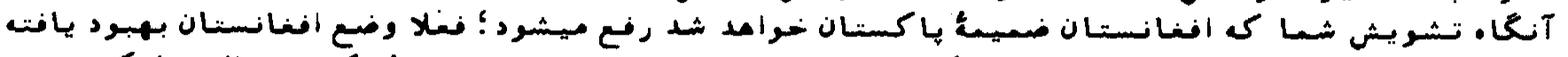

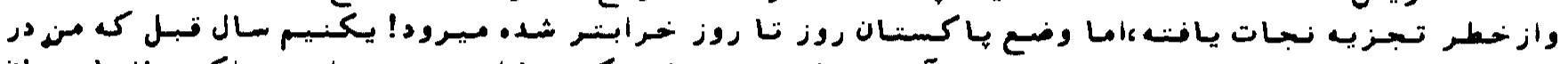

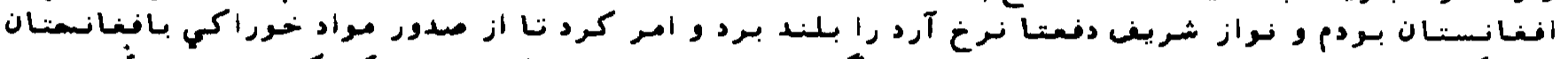

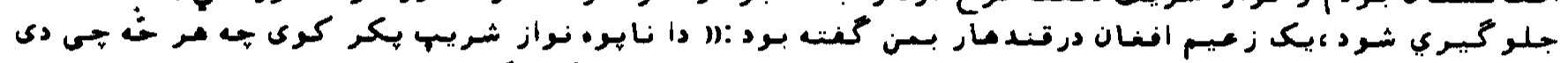

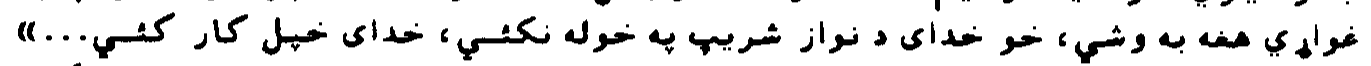

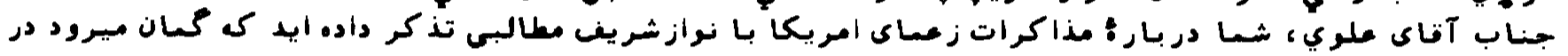

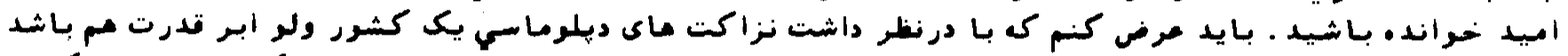

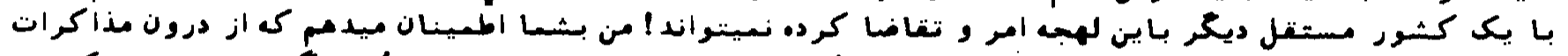

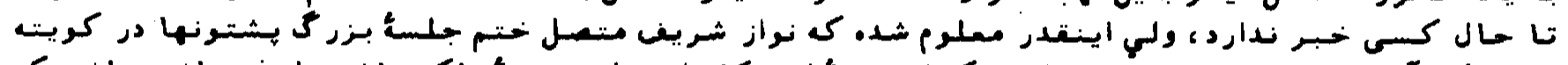

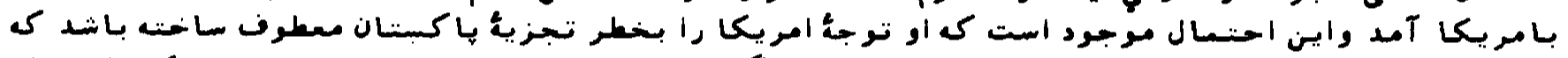

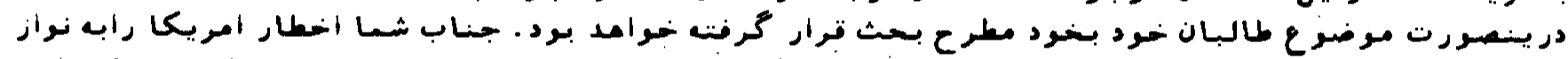

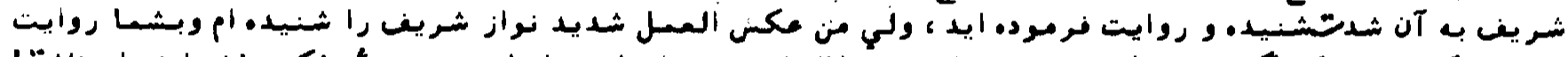

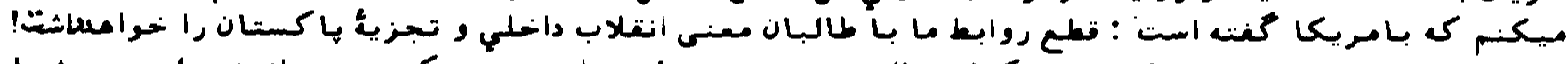

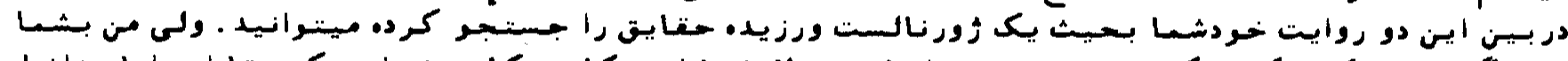

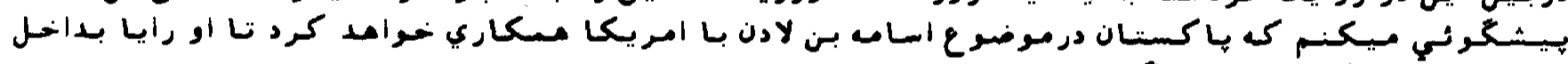

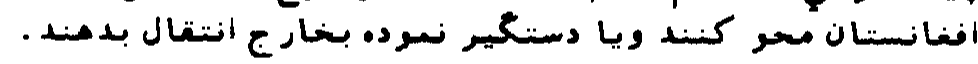

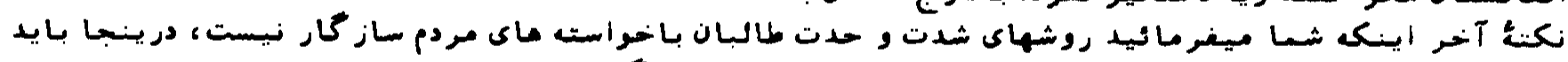

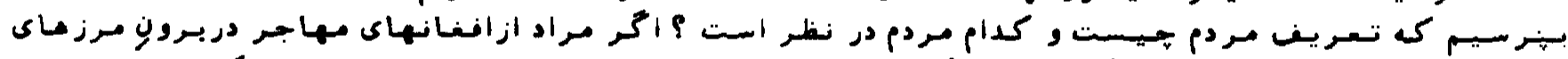

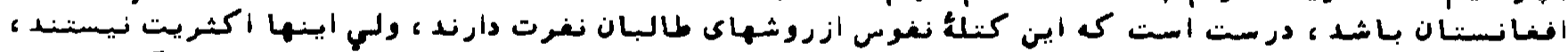

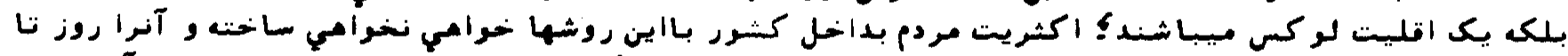

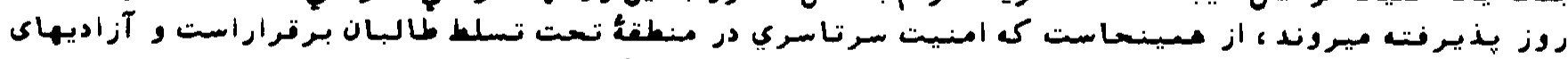

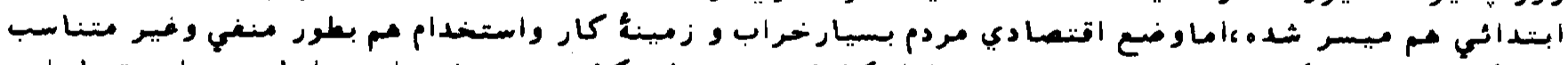

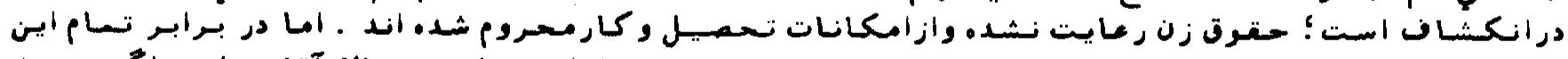

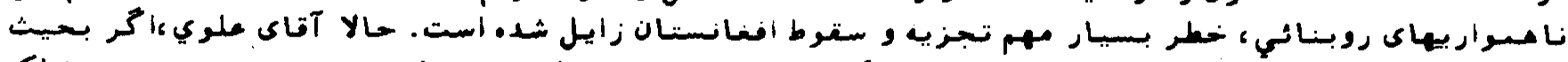

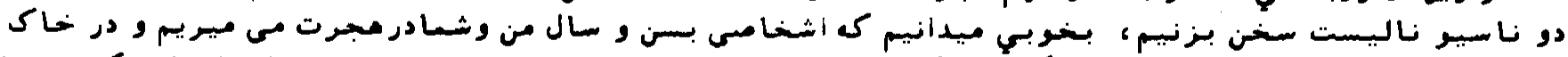

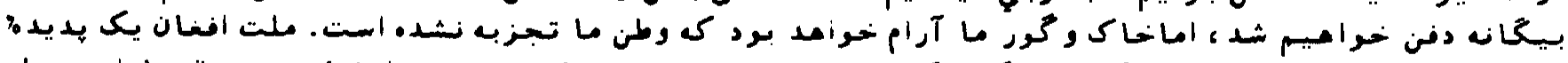

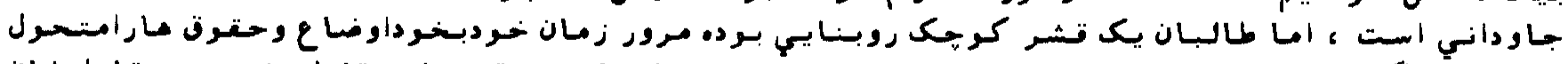

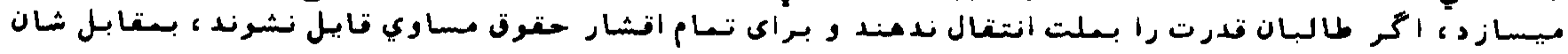

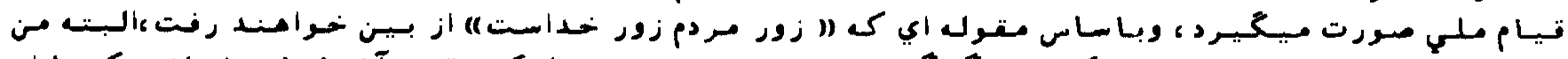

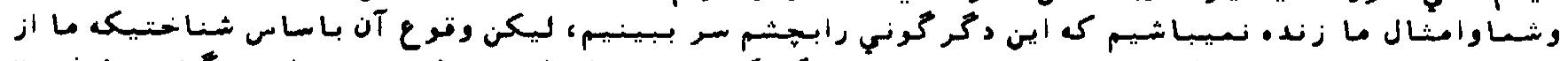

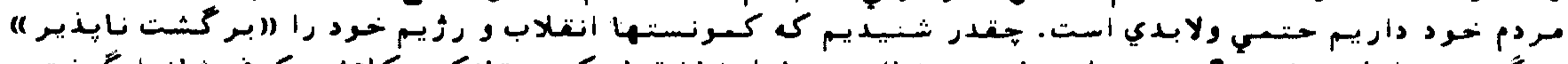

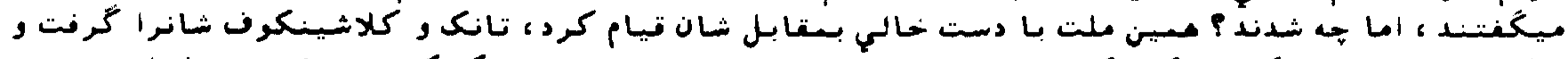

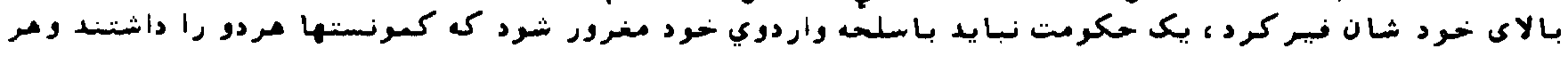

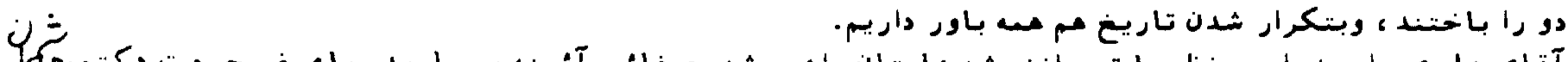

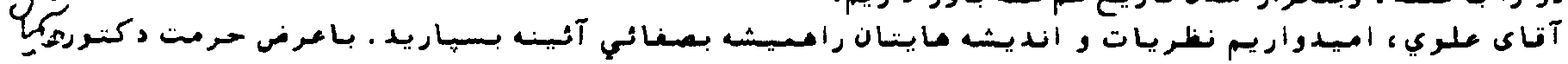




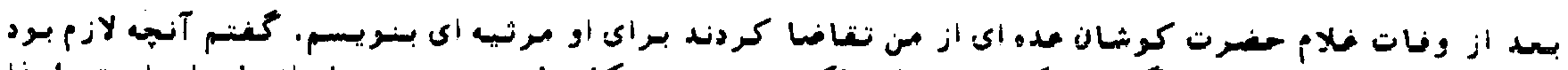

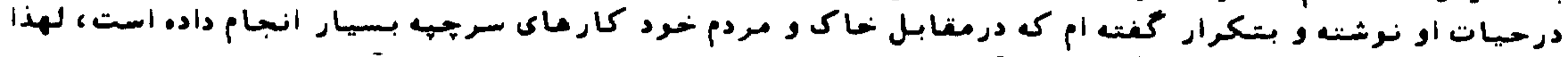

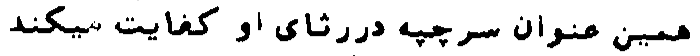

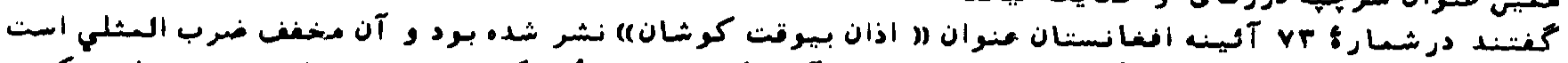

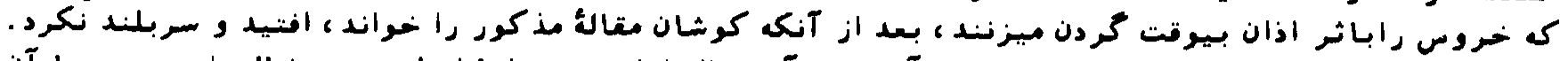

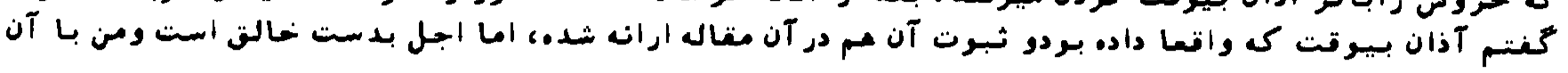

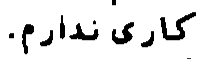

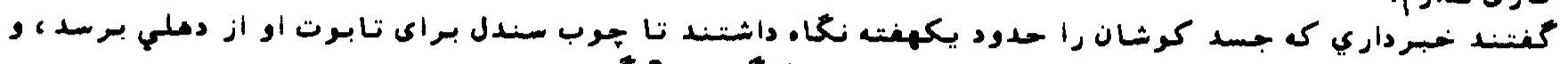

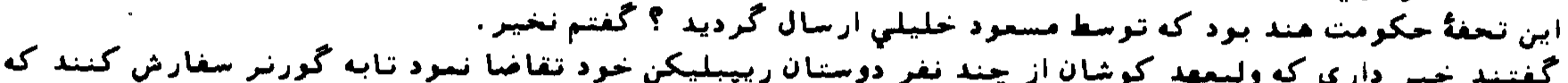

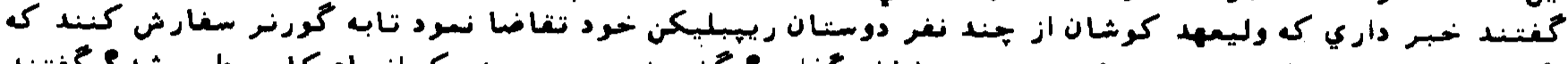

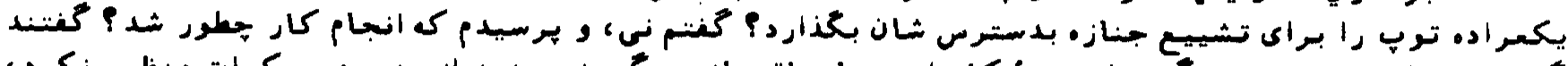

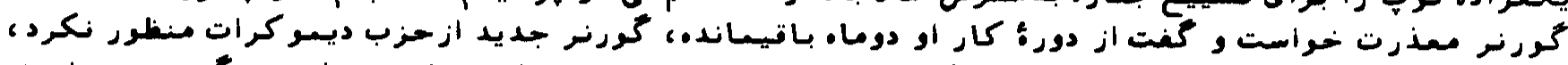

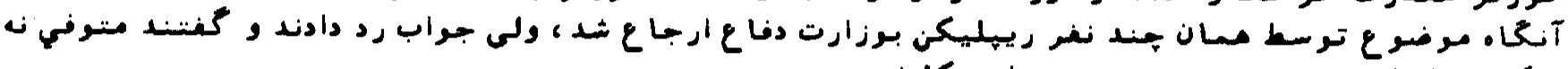

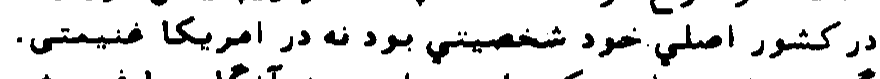

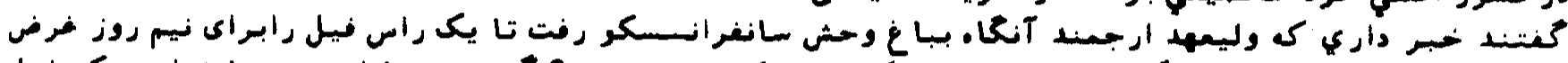

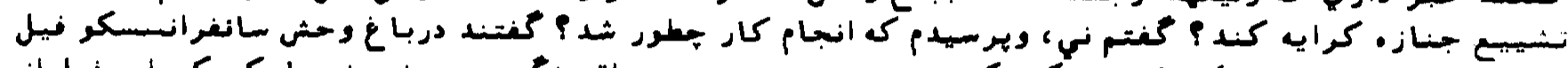

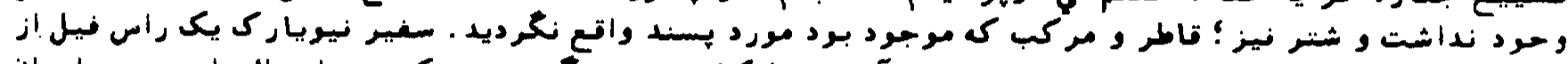

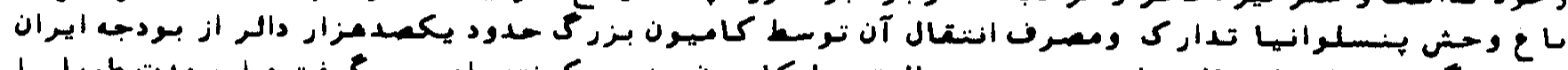

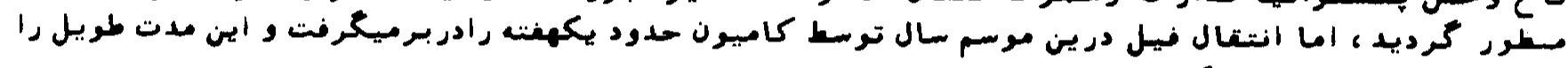

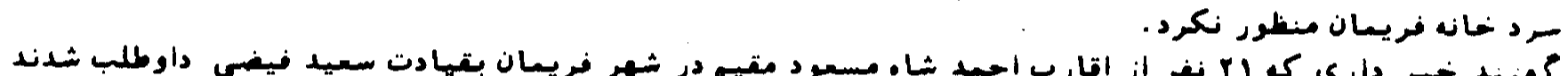

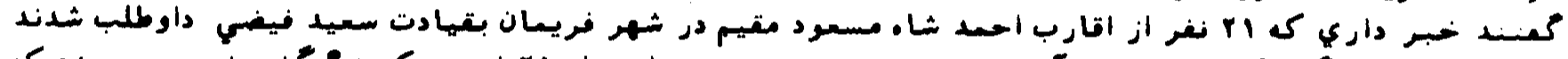

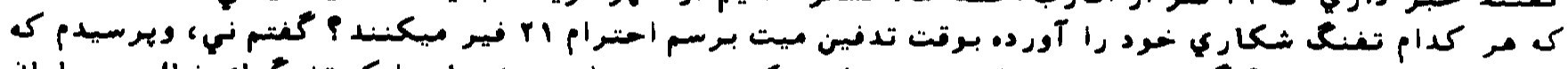

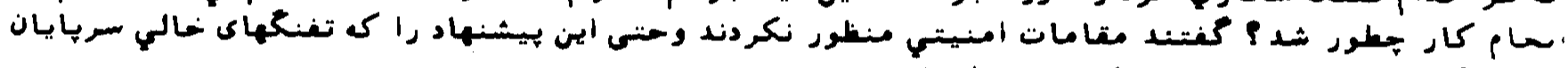

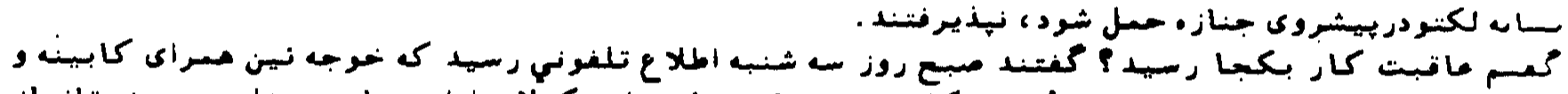

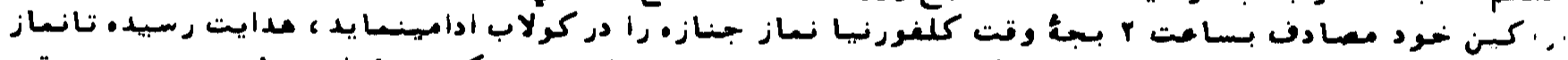

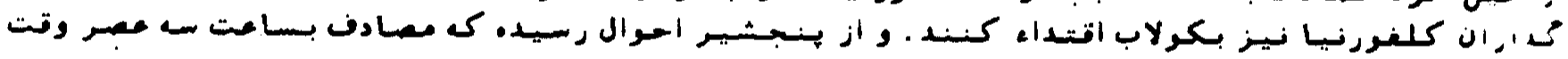

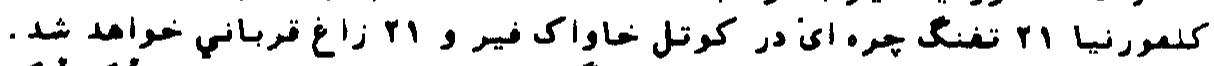

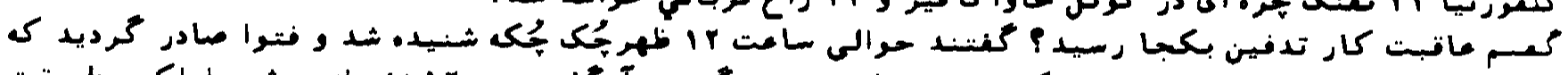

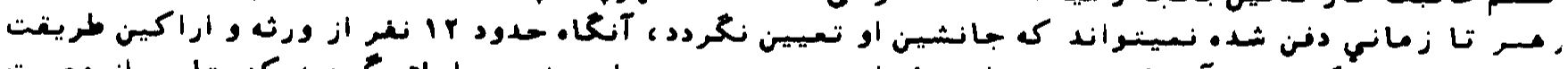

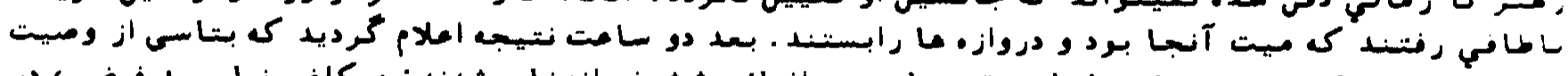

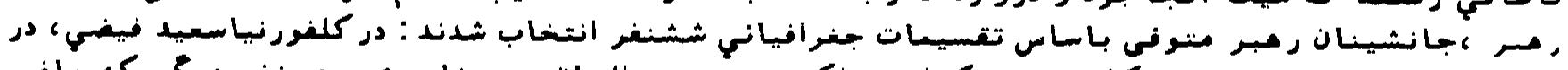

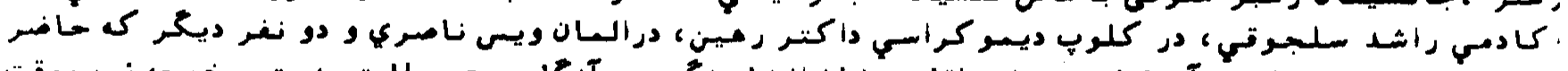

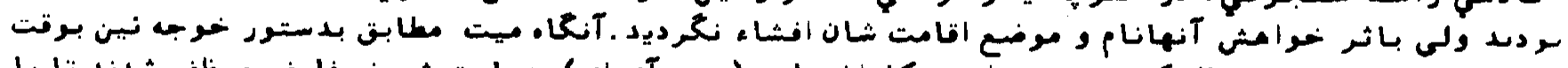

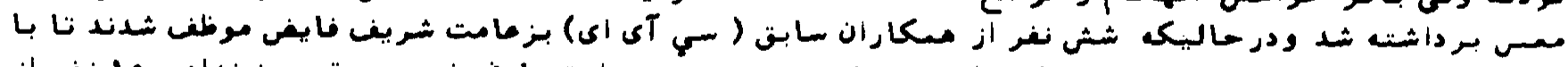

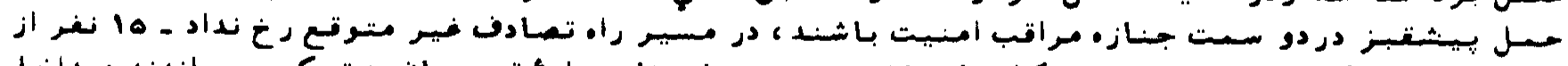

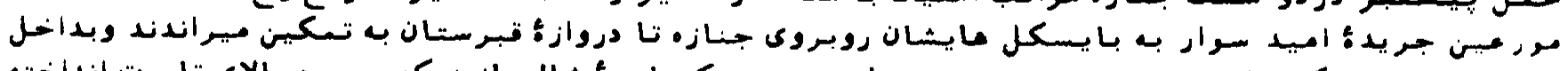

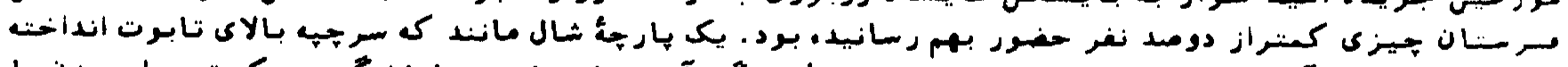

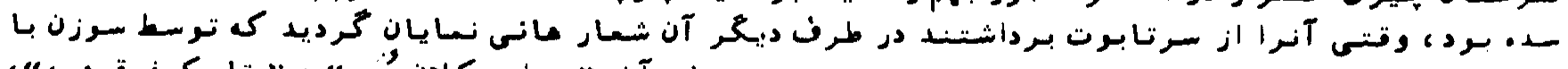

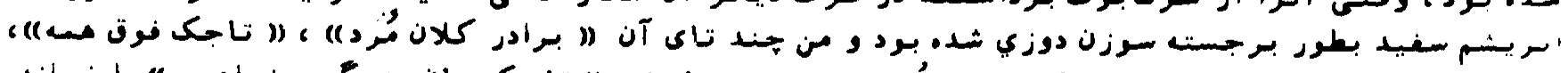

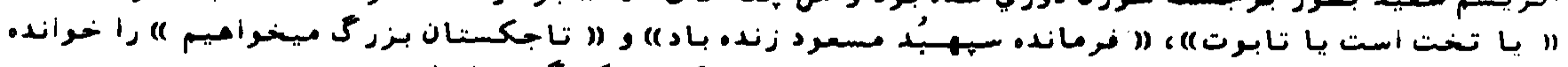

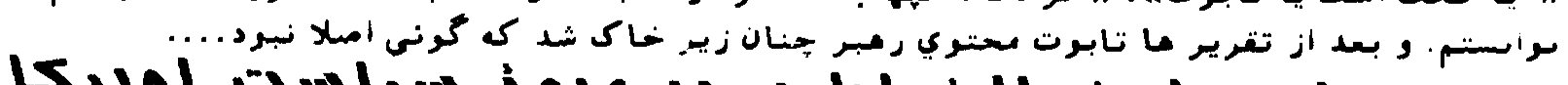

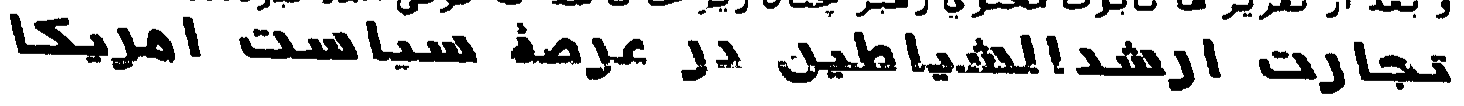

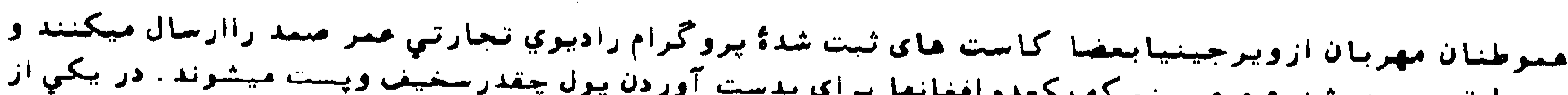

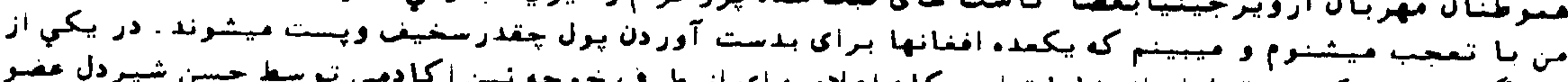

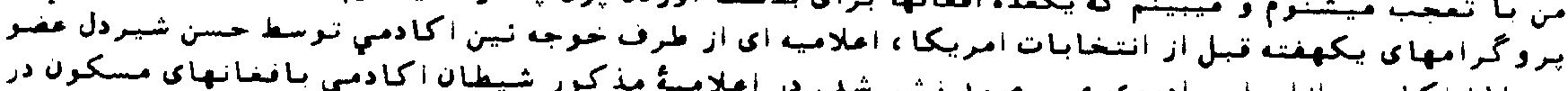

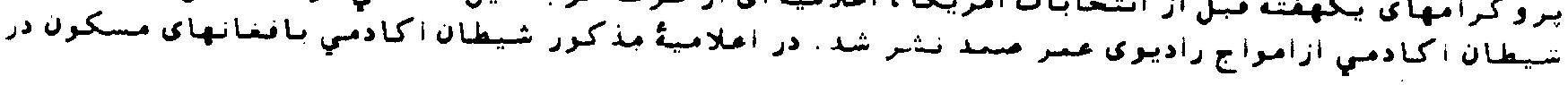




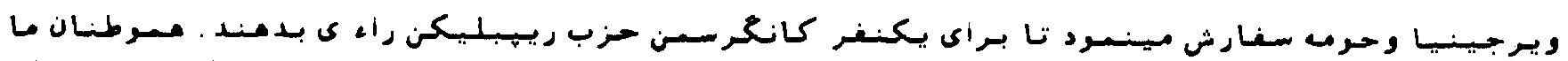

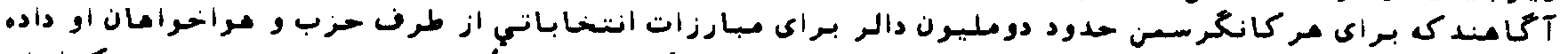

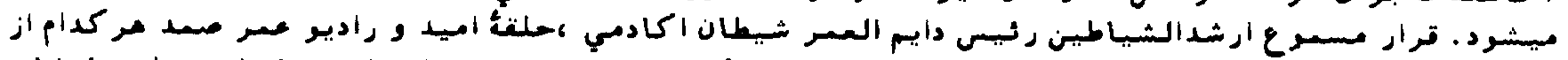

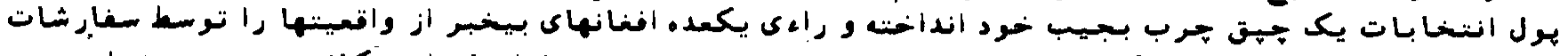

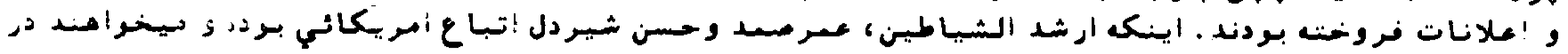

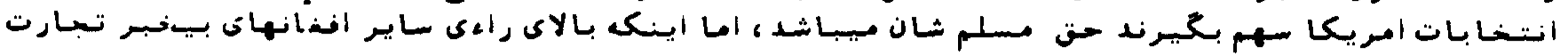

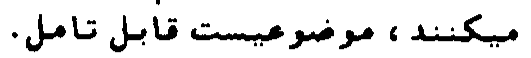

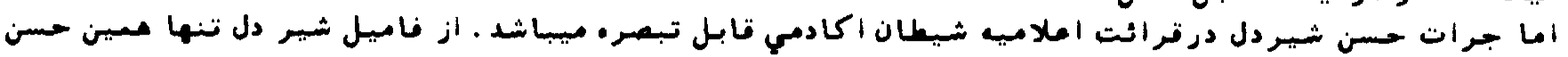

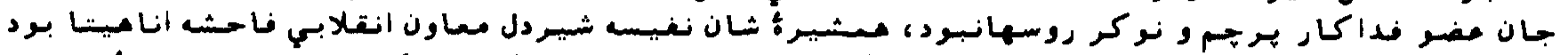

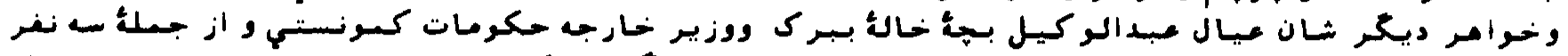

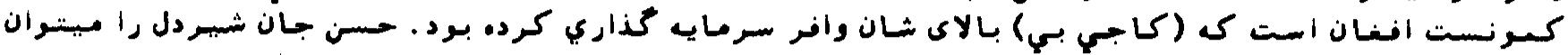

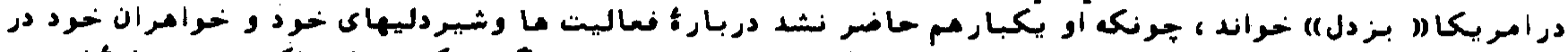

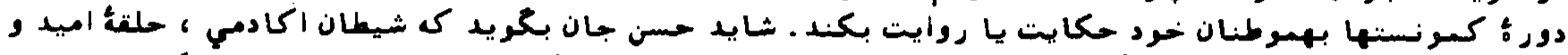

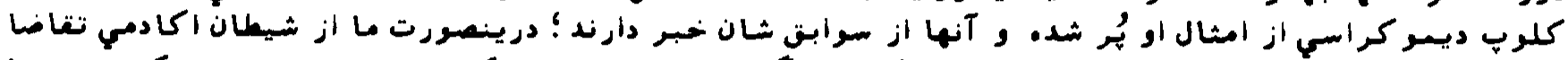

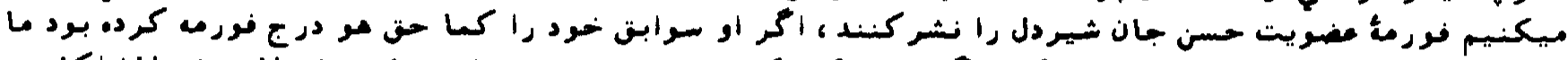

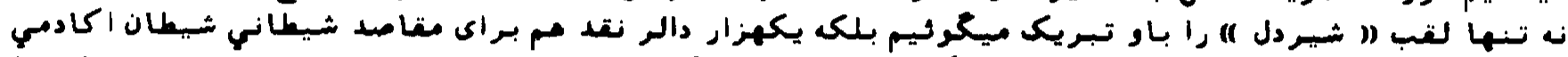

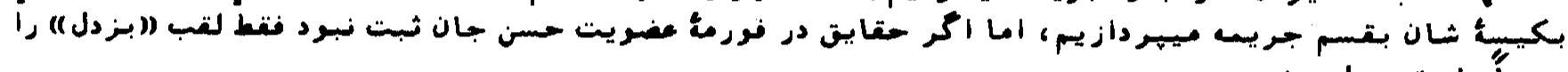

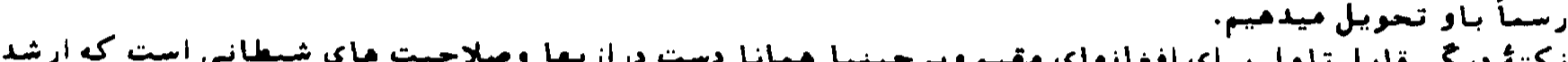

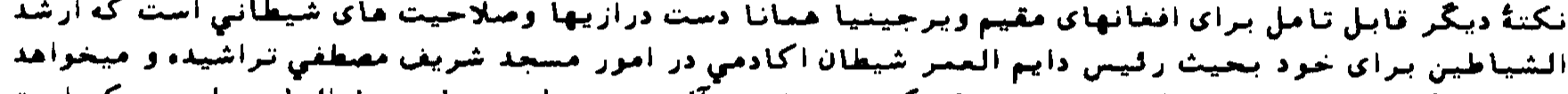

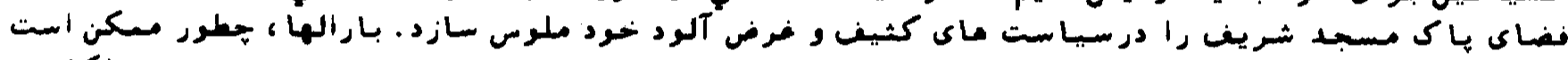

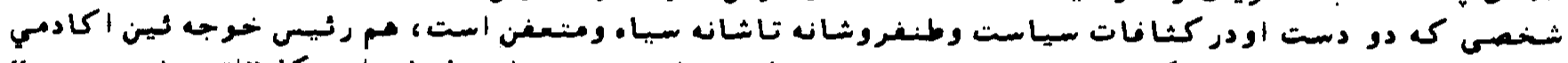

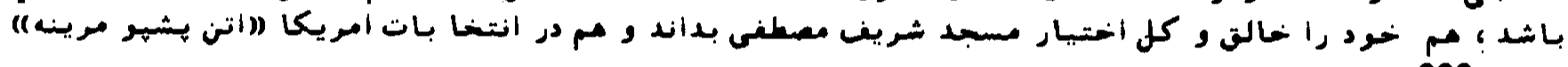

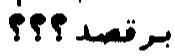

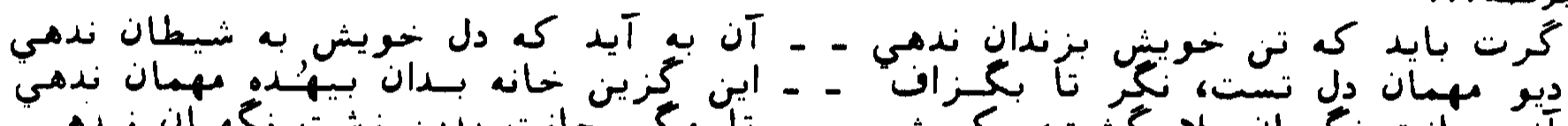

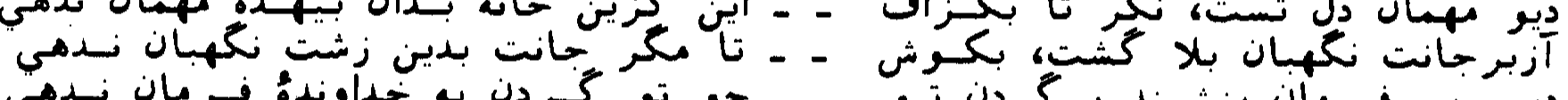

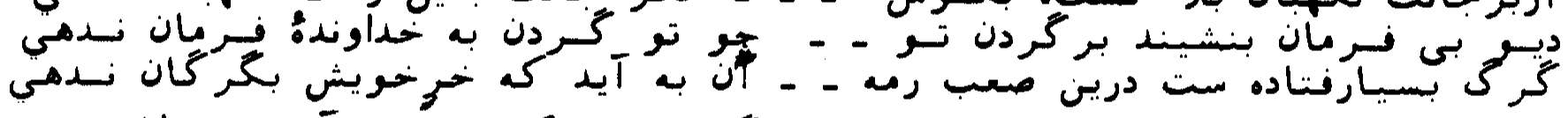

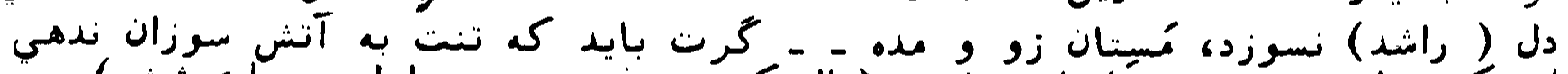

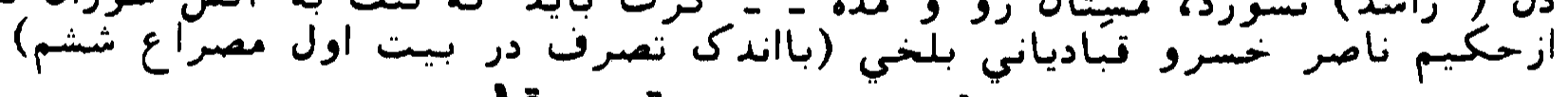

\section{مئم}

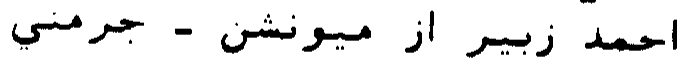

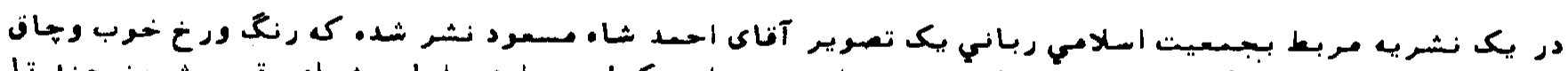

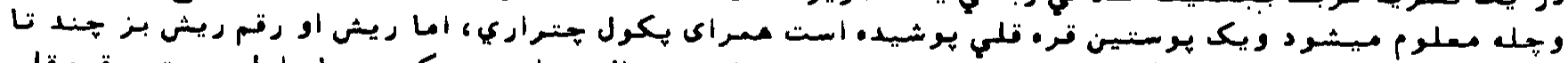

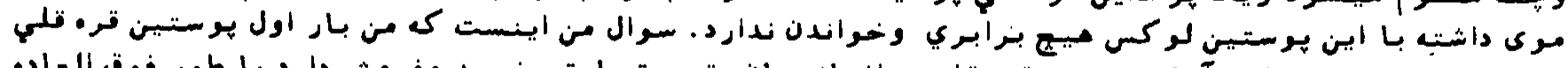

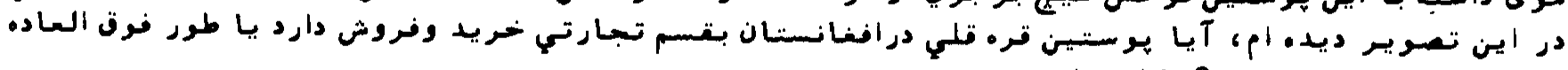

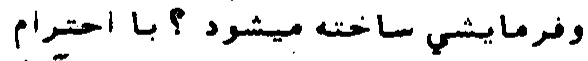

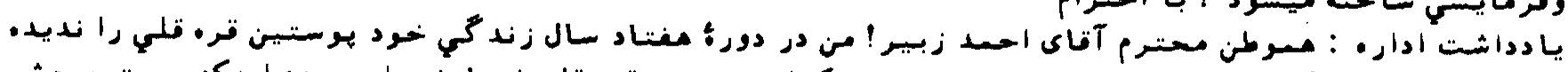

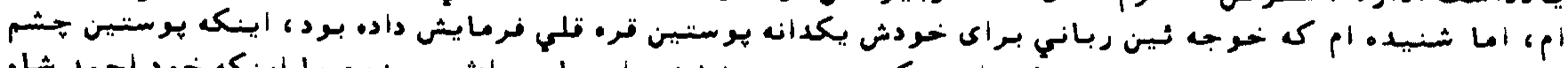

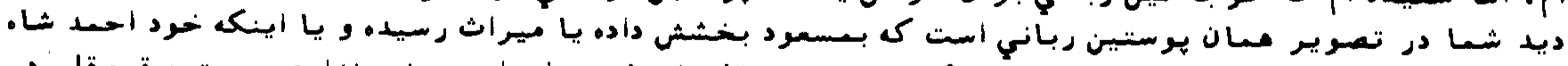

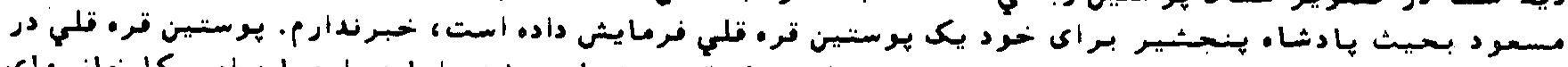

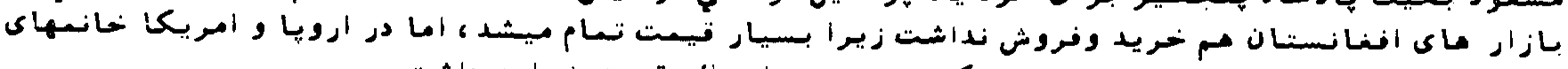

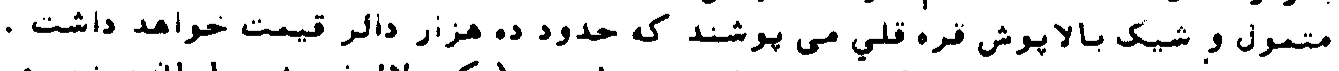

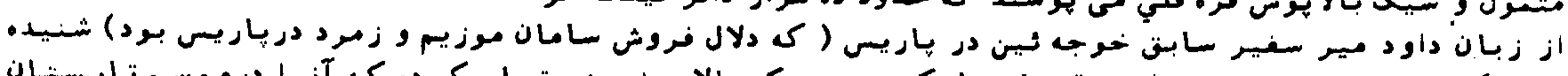

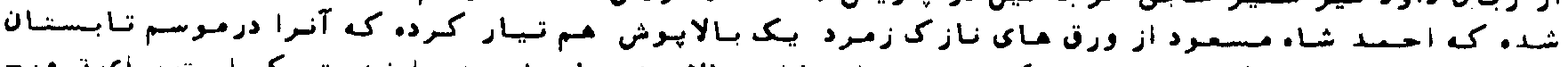

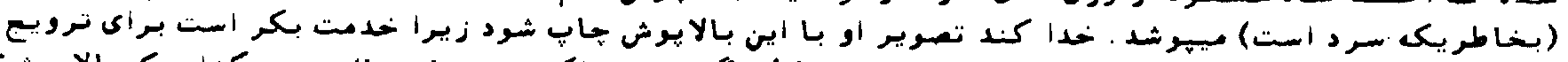

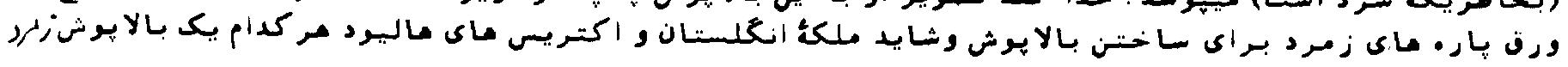



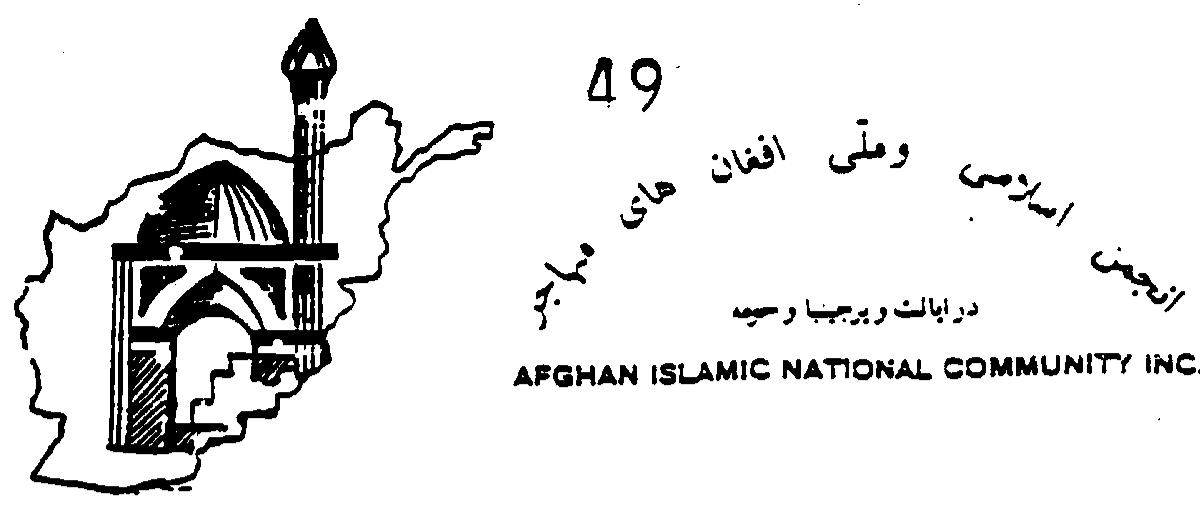

AFGHAN ISLAMIC NATTONAL COMMUNITI INC.

-P.O.908 613

Springleld. Va. 22150

Tele

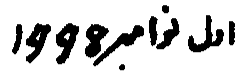

اطلاعيهُ انجمن اسلامي وملي افغانان مهاجر درايالت ويرجينيا وحرمه

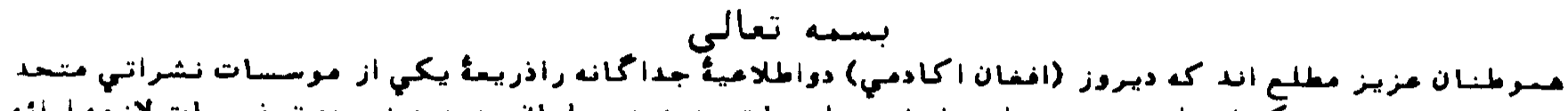

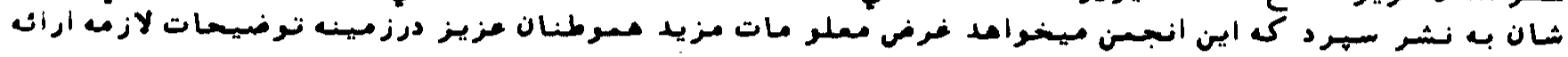

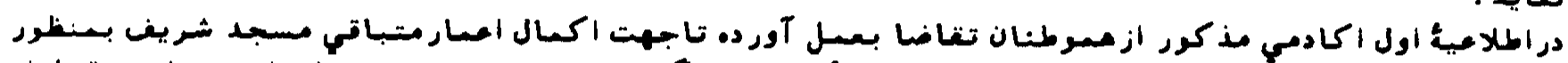

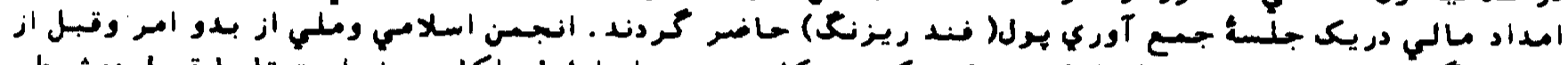

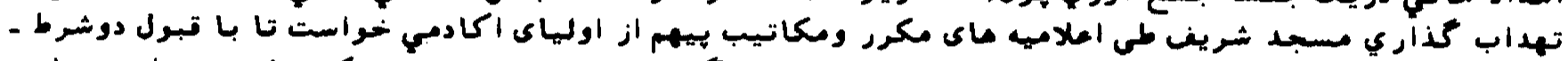

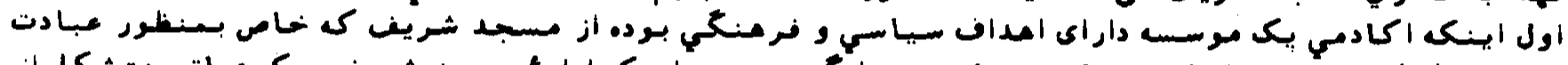

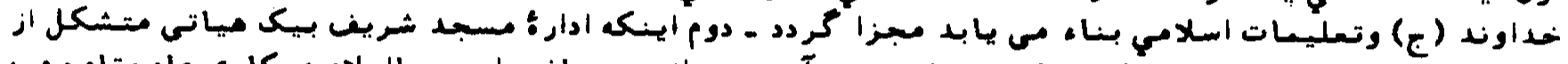

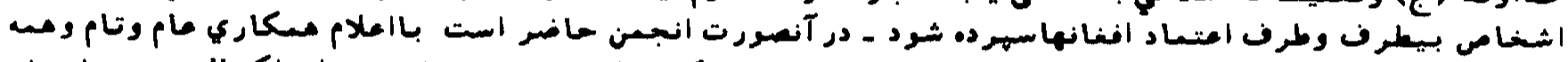

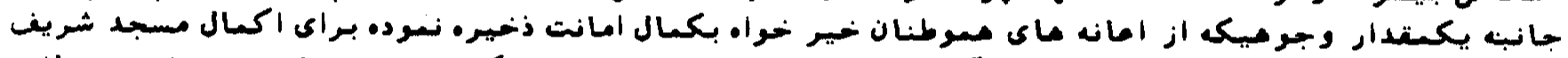

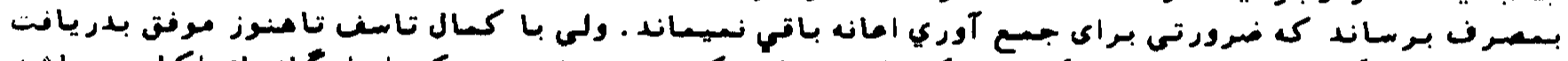

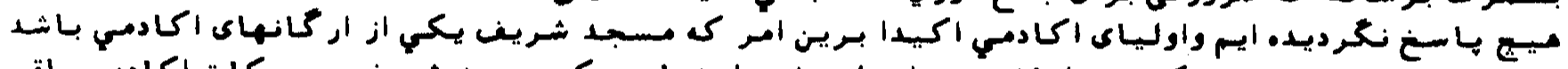

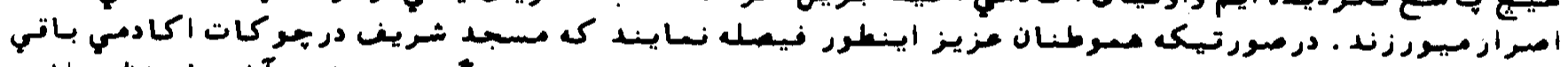

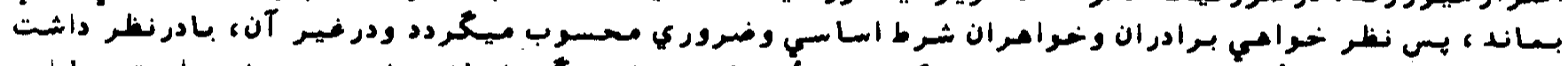

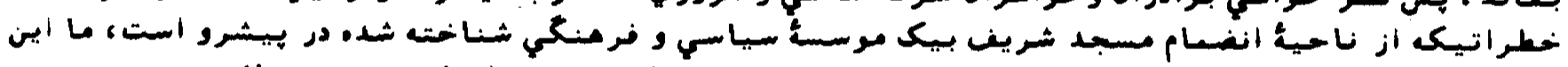

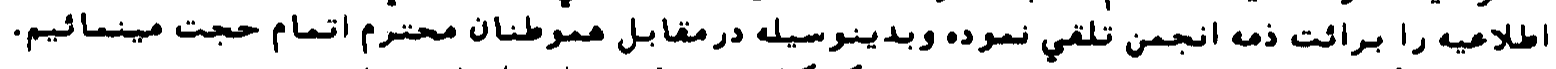

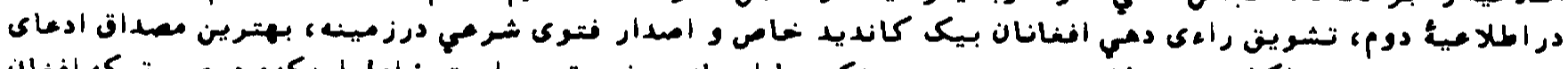

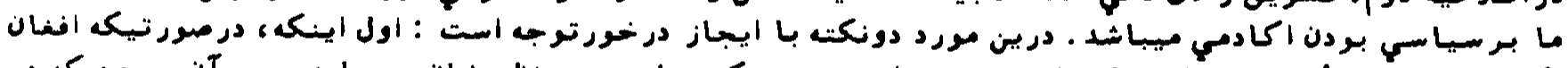

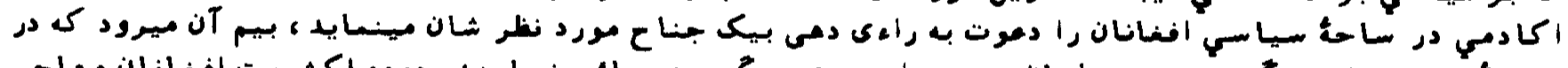

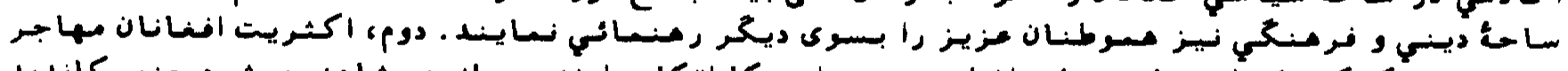

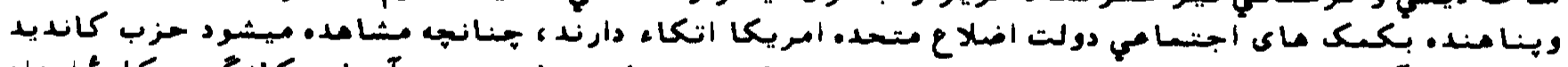

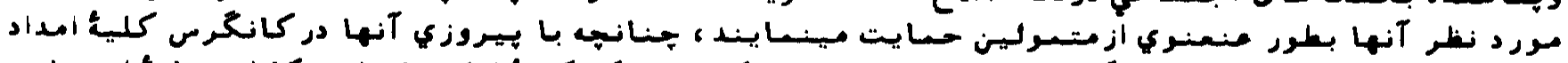

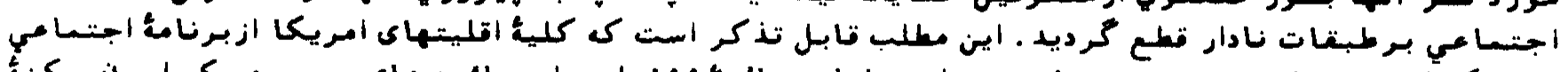

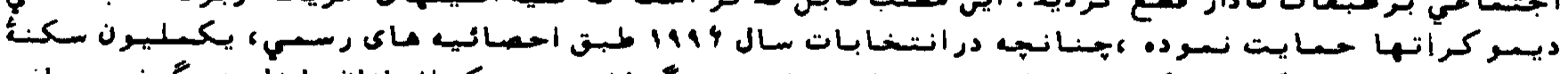

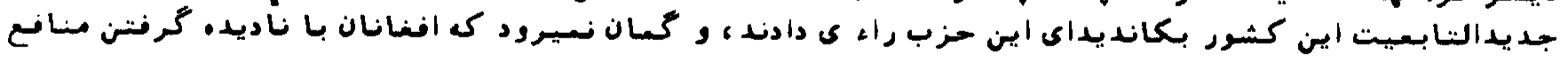

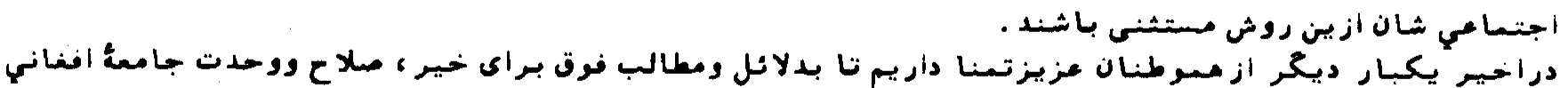

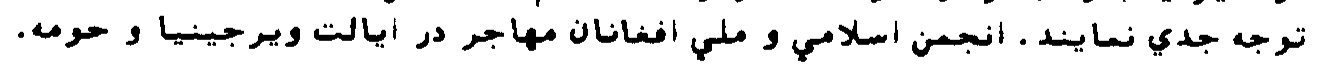

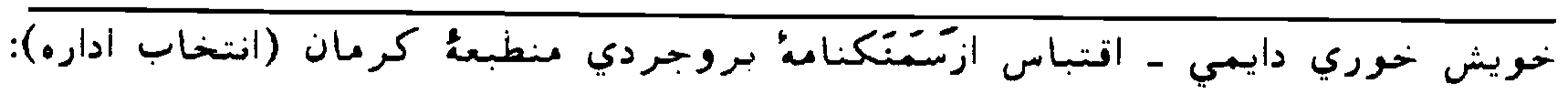

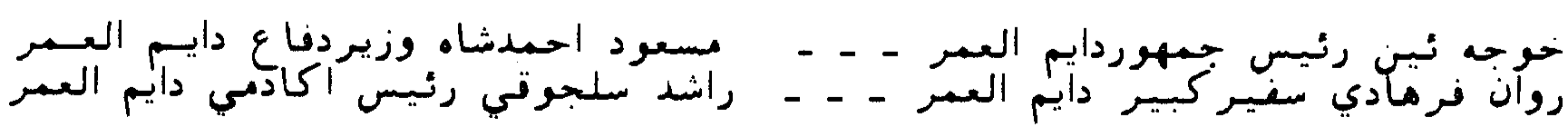

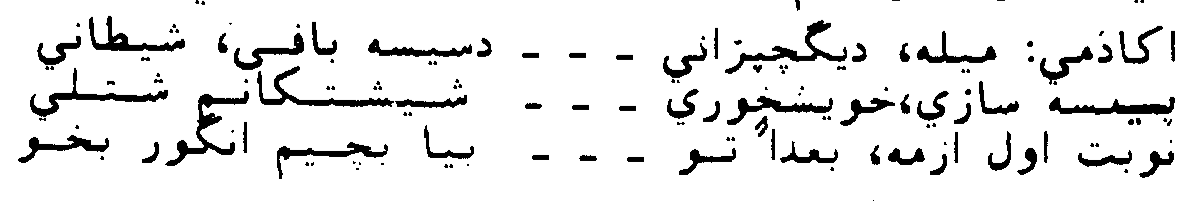




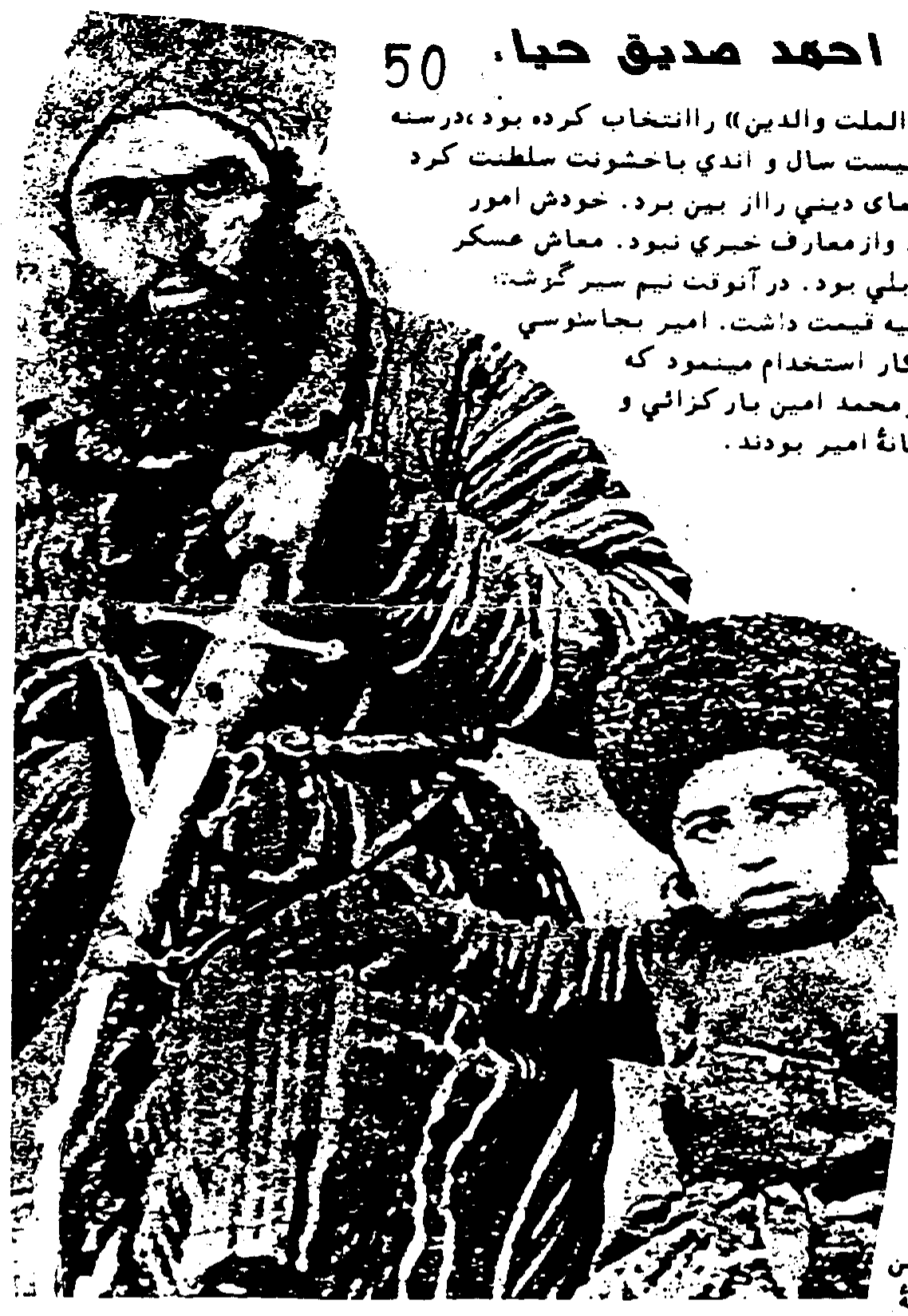

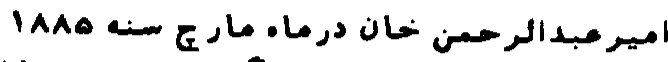

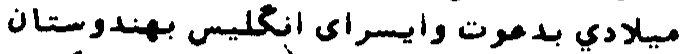

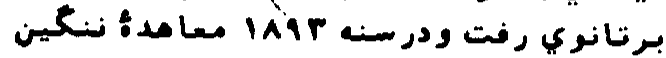

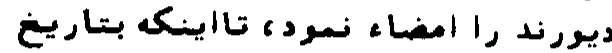

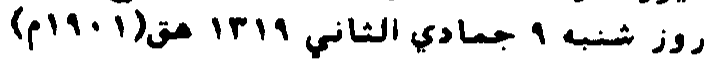

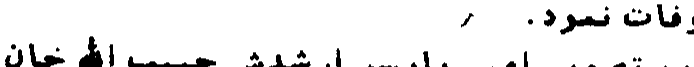

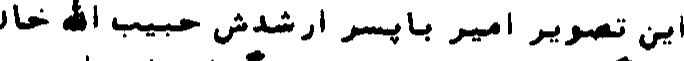

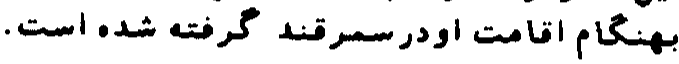
امير مبدابلرحمن خان بسد كافي انبخليس

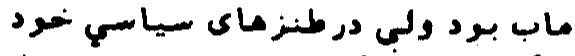

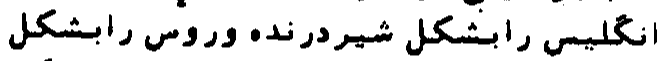

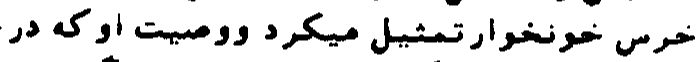

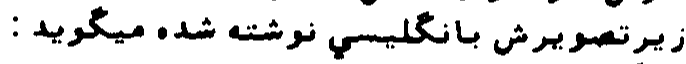

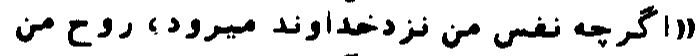

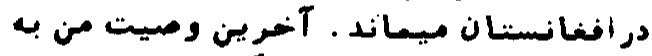

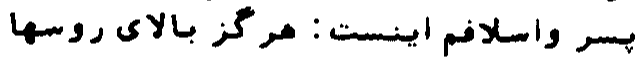

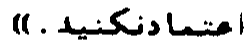

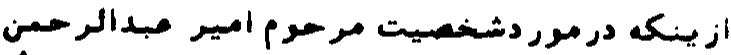

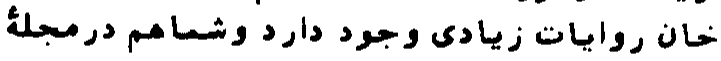
Abdur Rahman Khan and son, Habibullah, while exiled in Russia.

"Though my soul will go to God, my spirit will remain in Afghanistan. My last words to you my son and successors are: Never trust the Russians."

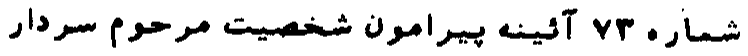

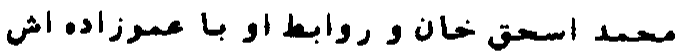

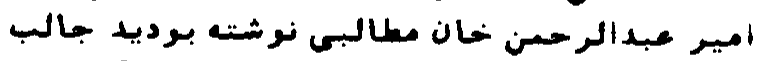

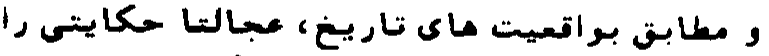

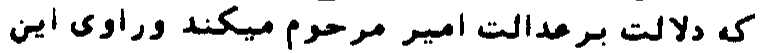

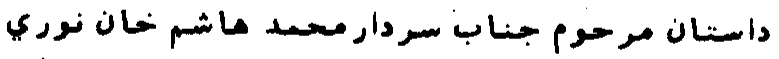

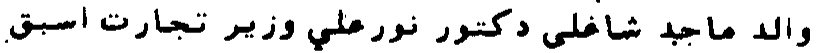

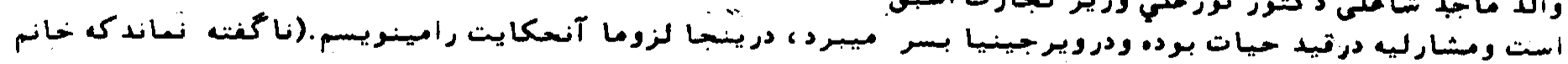

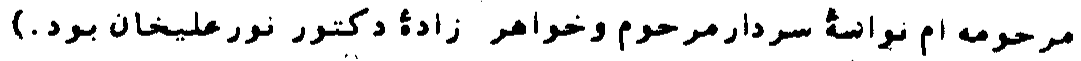

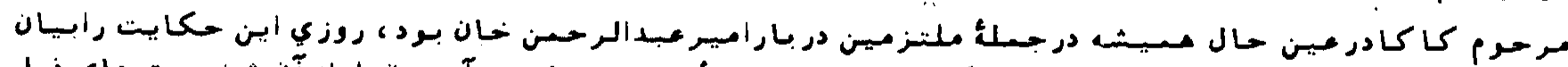

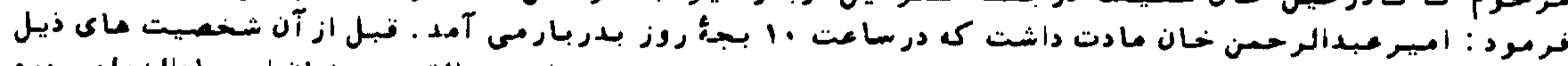

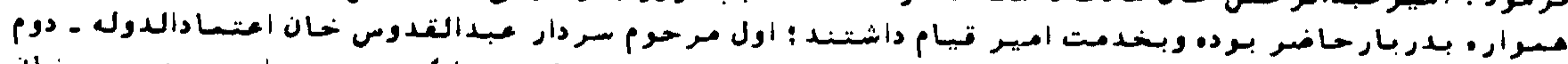

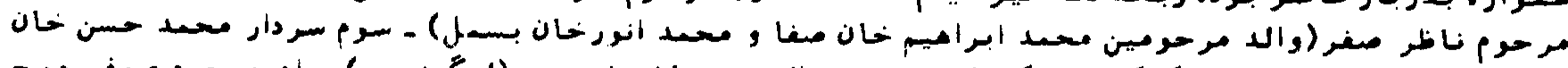

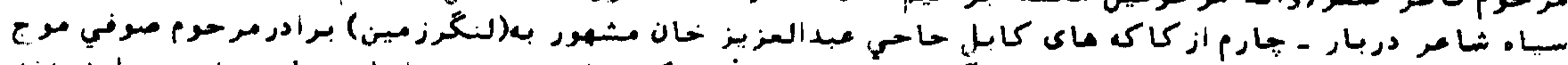

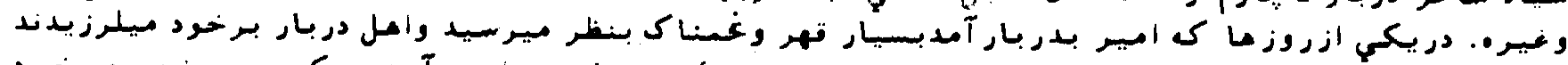

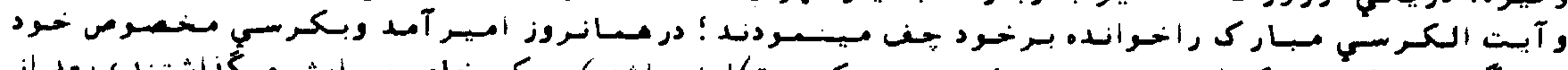

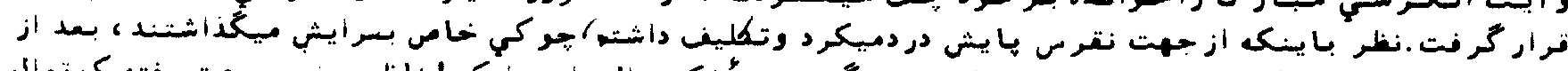

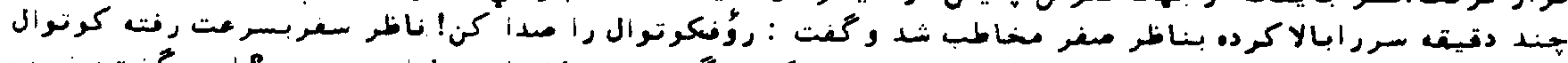

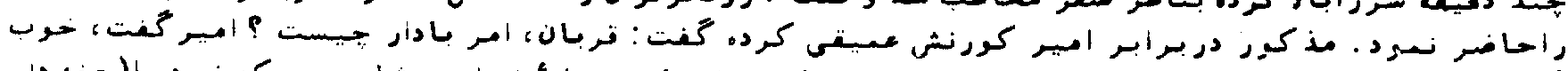

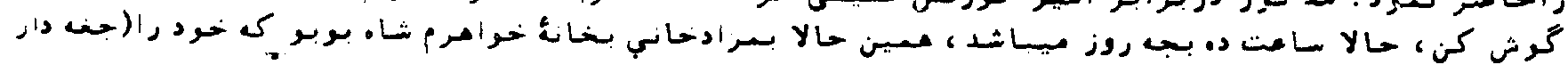




\section{1}

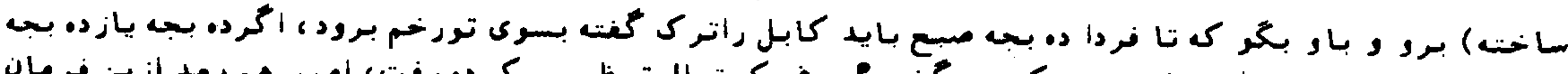

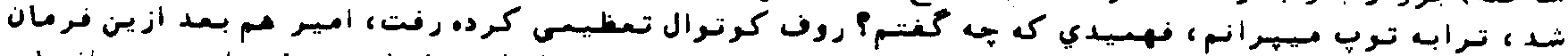

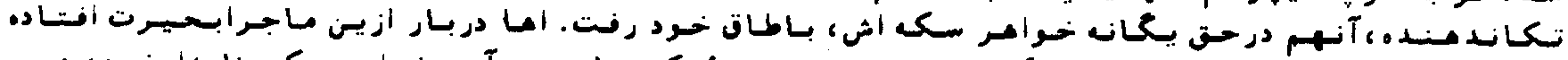

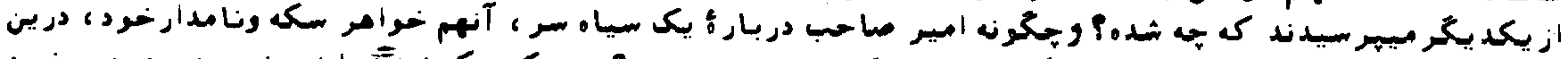

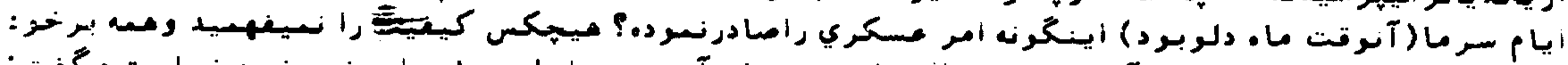

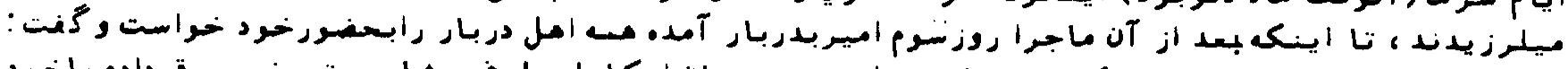

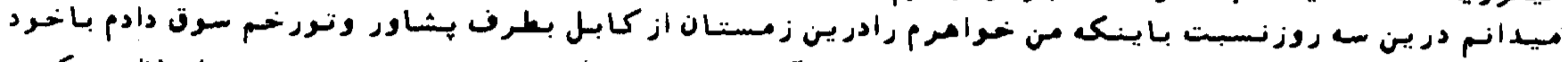

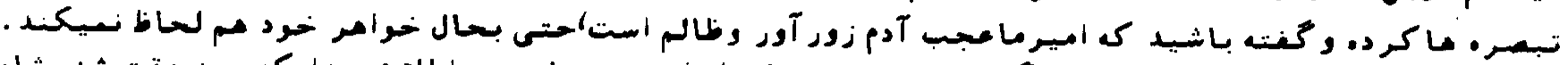

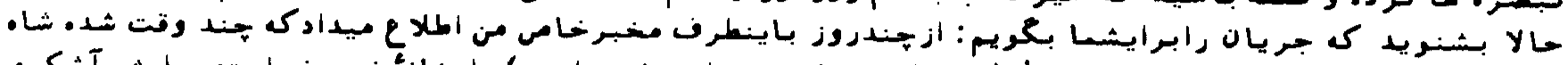

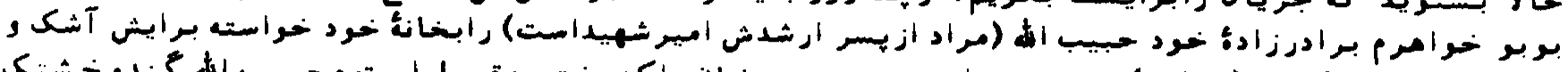

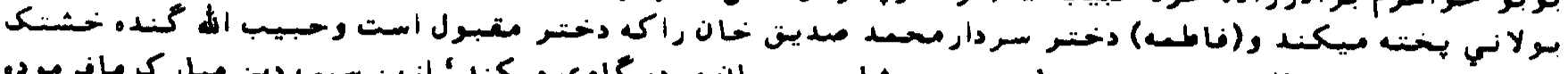

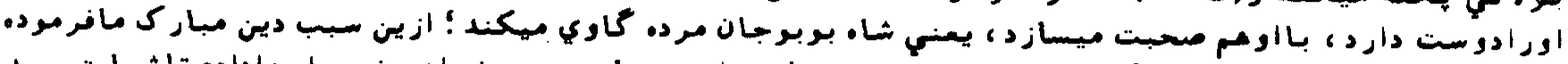

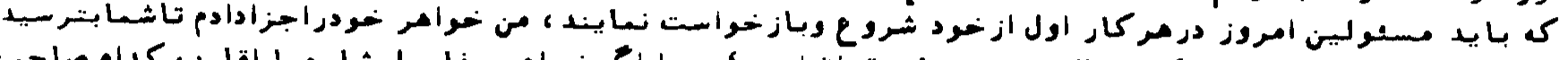

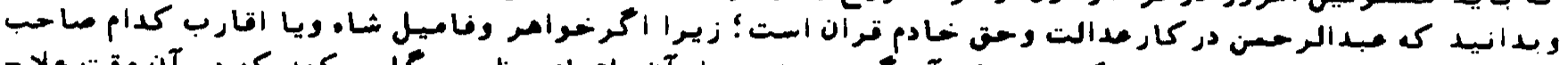

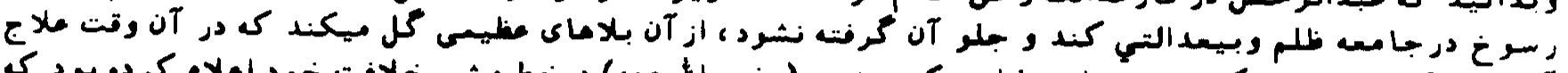

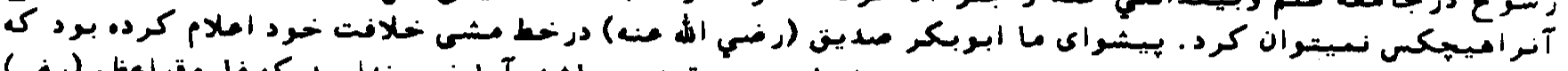

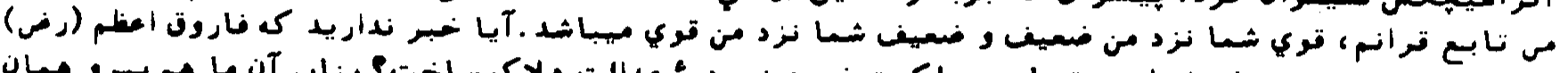

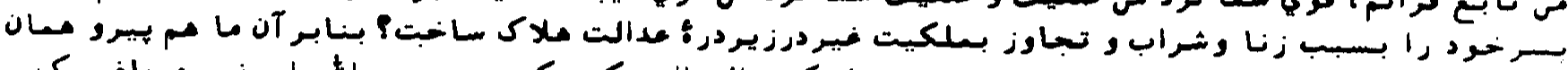

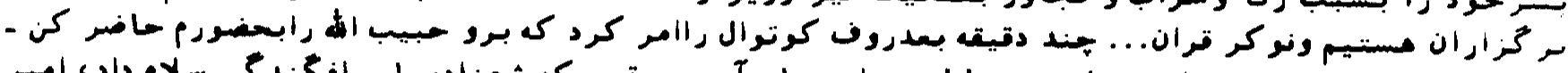

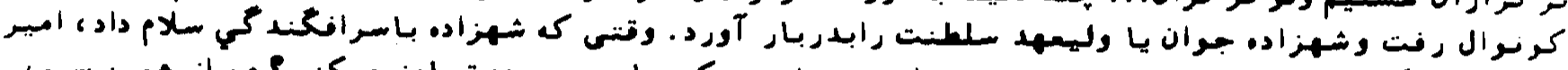

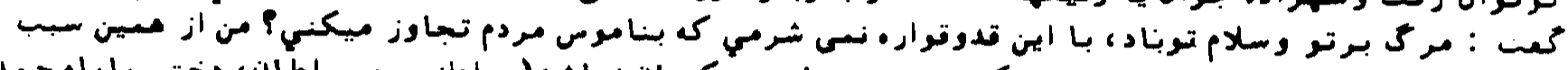

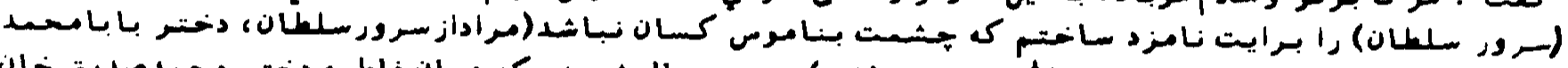

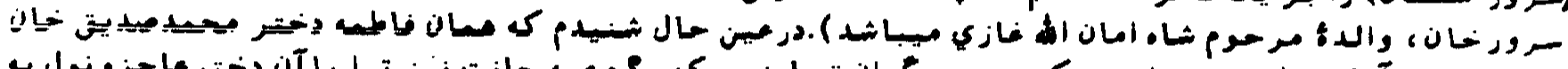

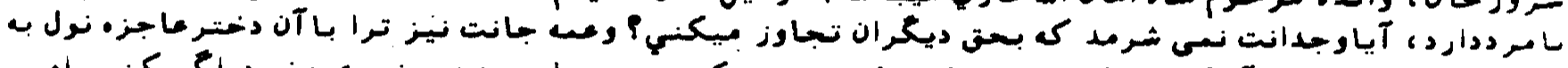

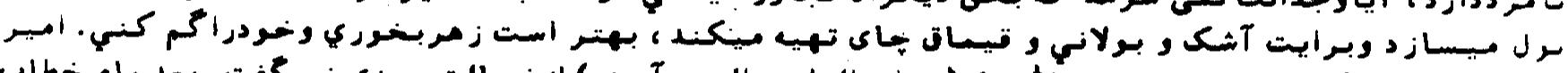

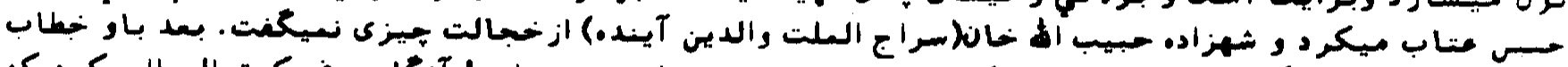

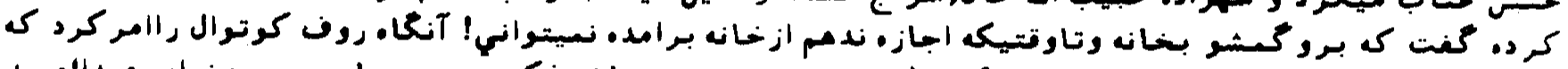

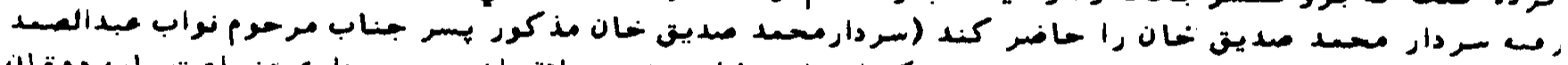

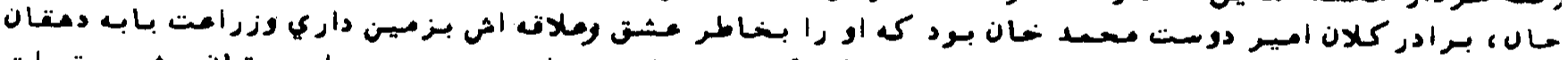

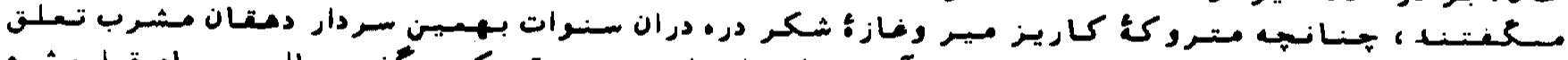

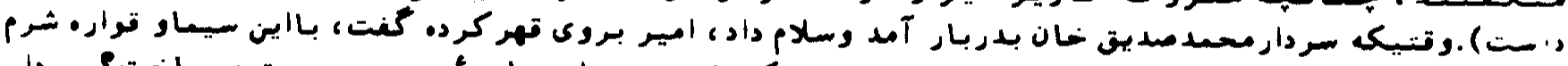

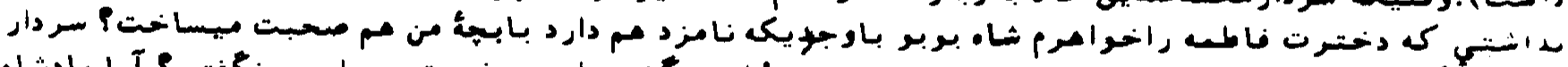

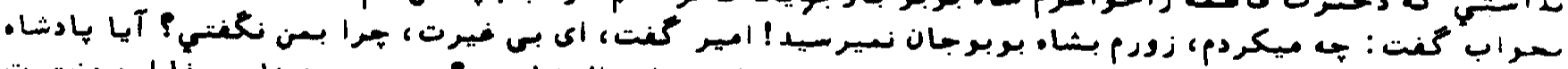

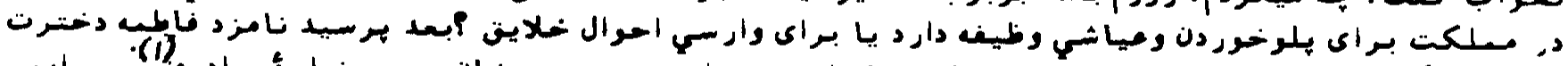

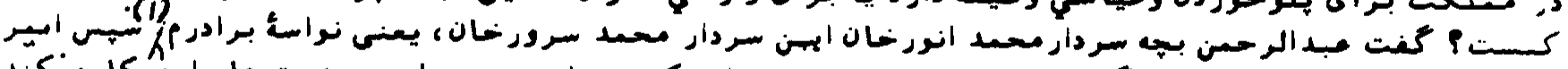

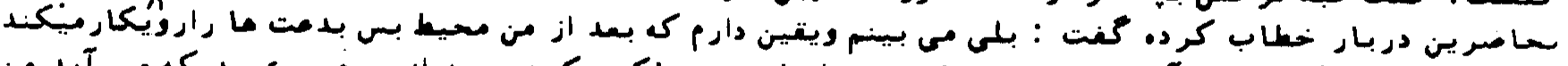

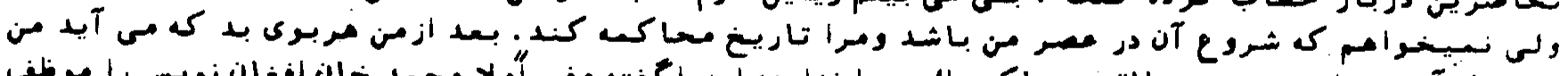

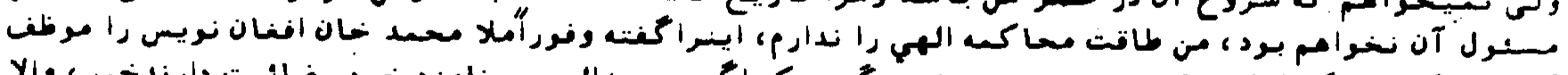

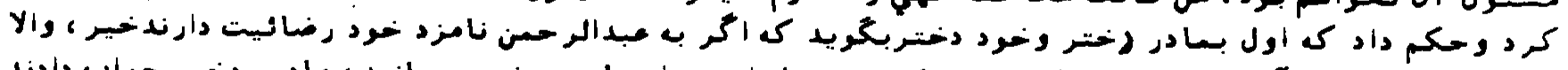

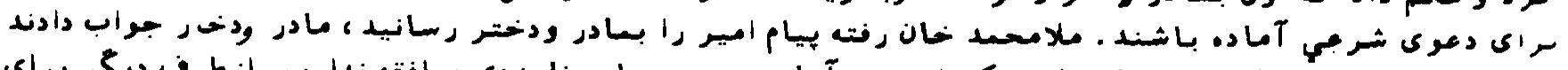

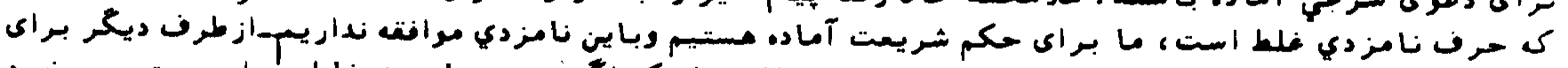

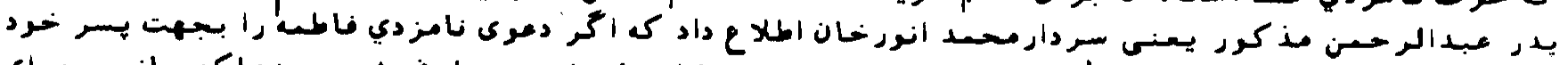

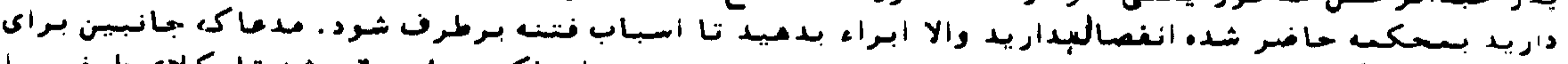

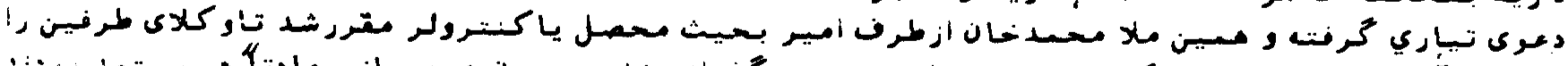

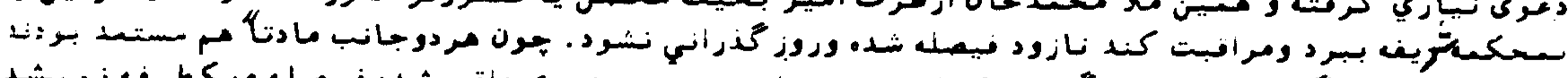

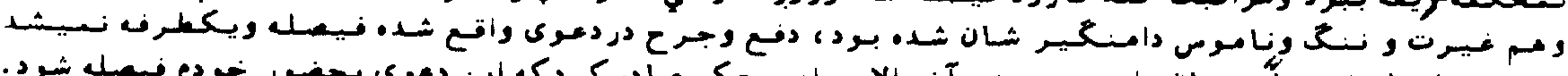

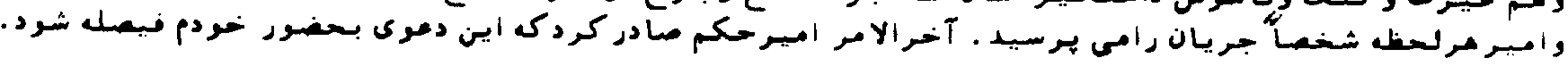

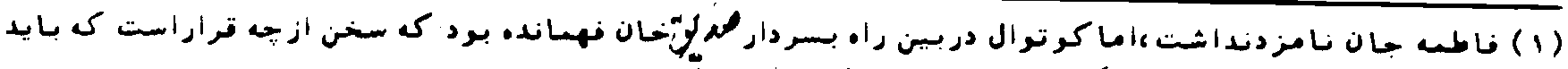

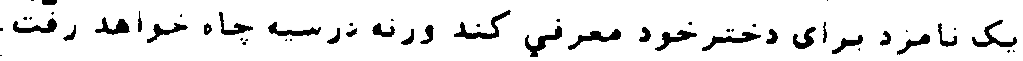




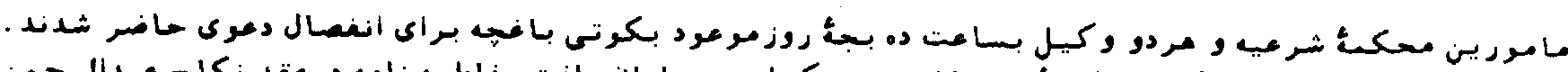

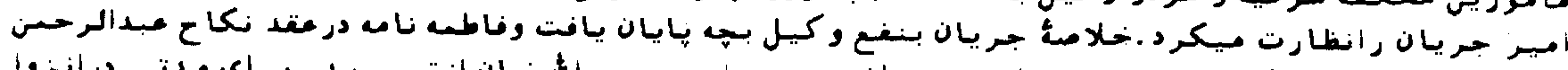

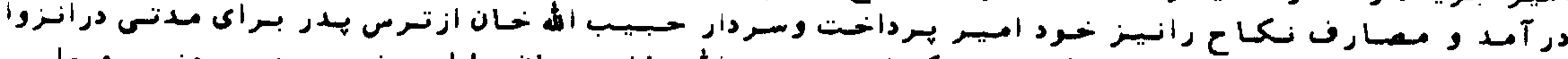

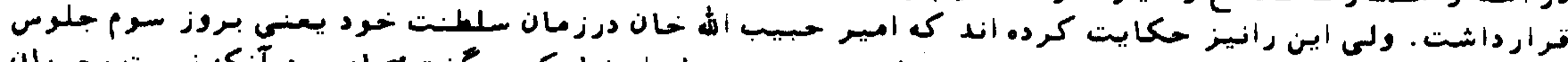

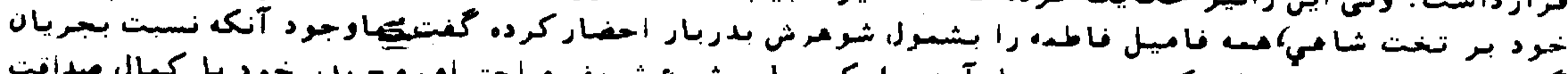

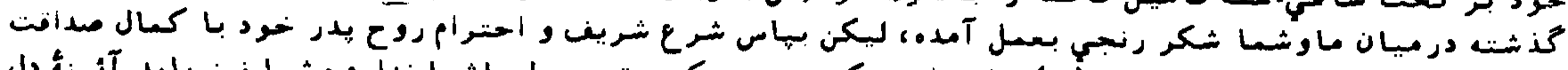

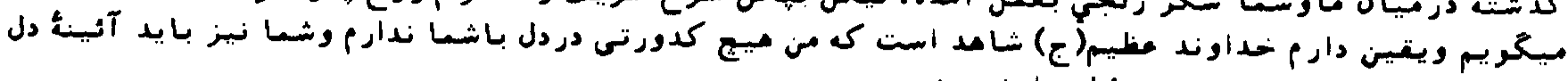

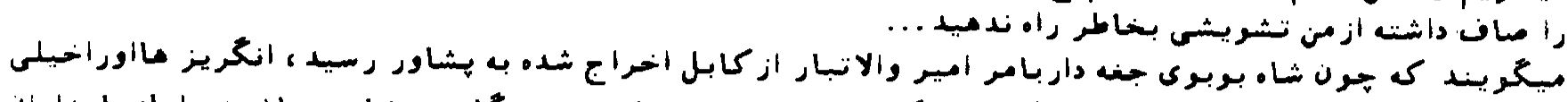

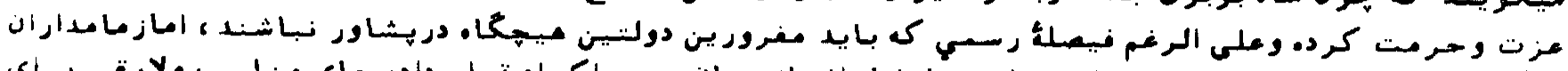

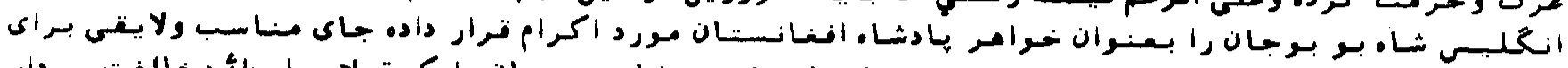

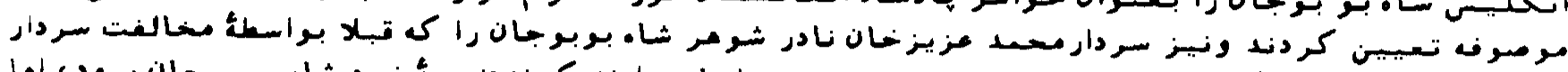

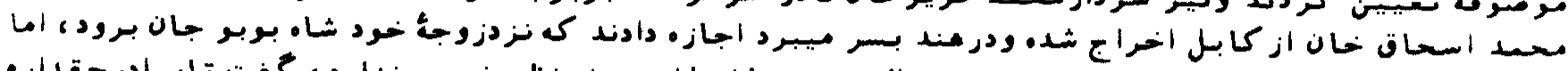

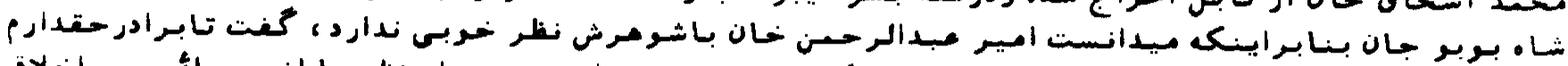

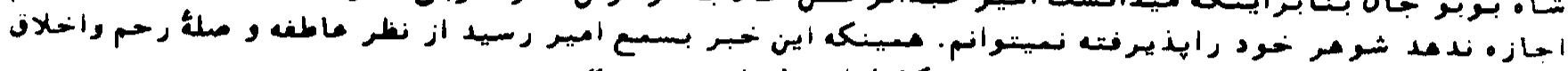

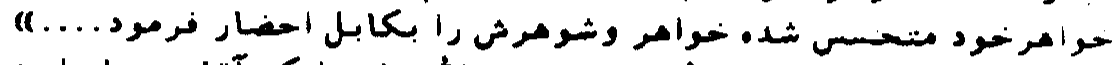

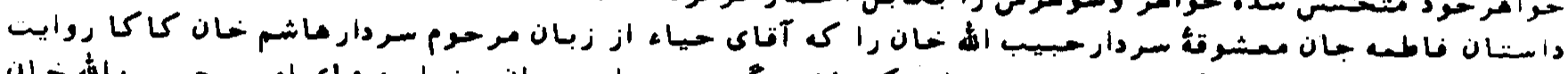

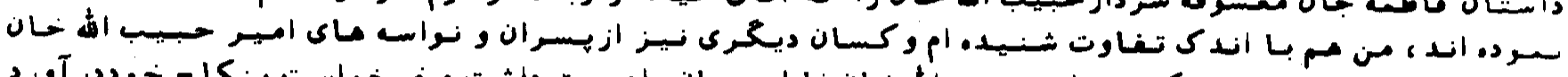

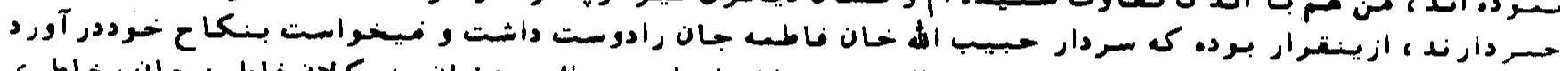

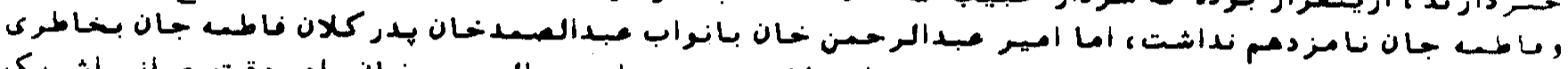

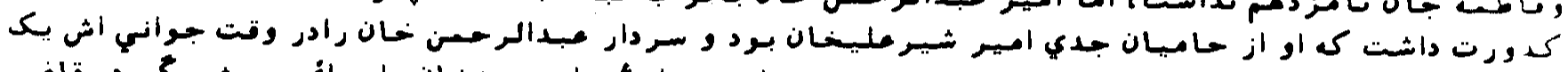

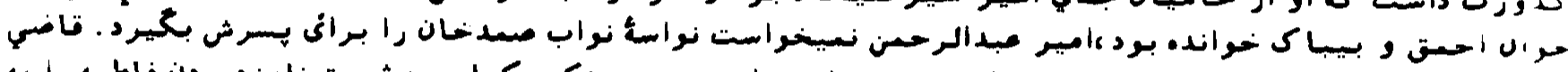

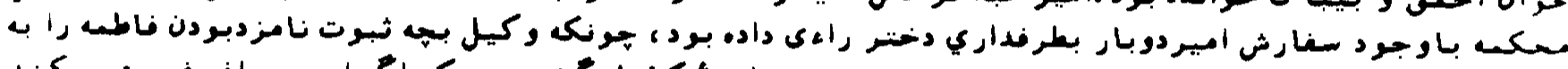

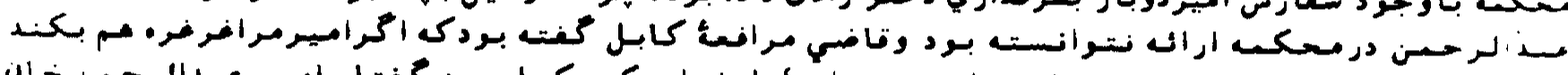

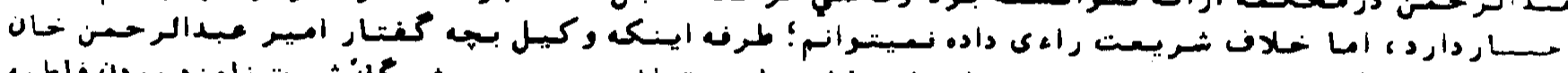

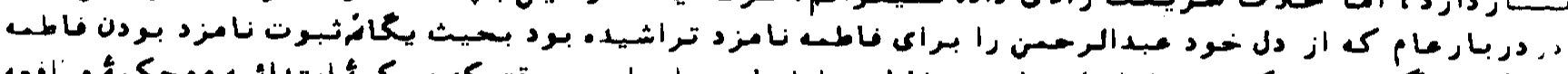

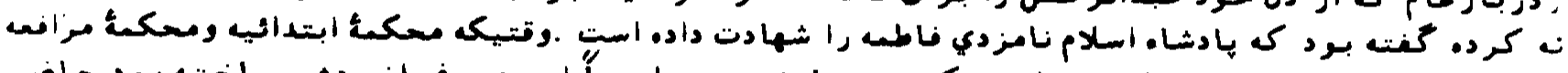

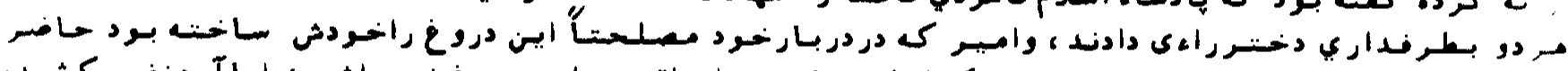

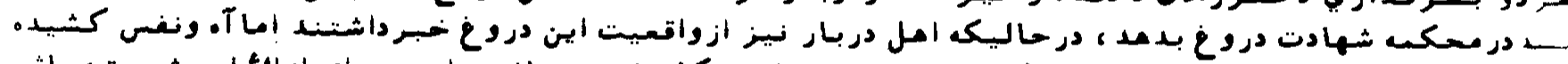

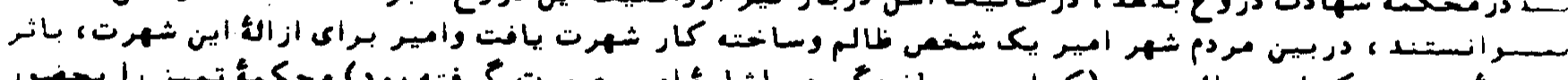

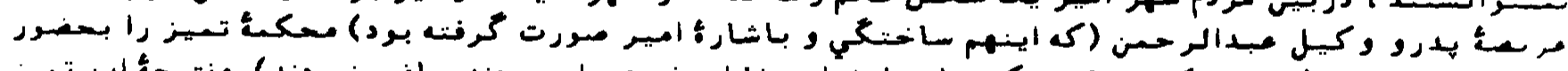

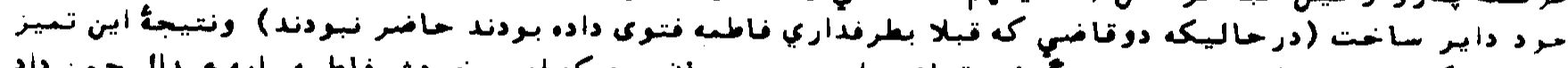

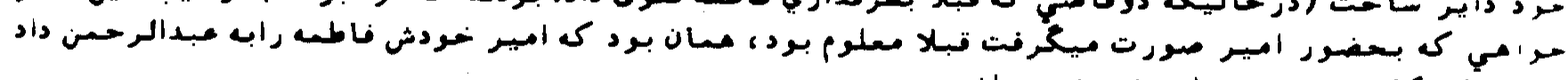

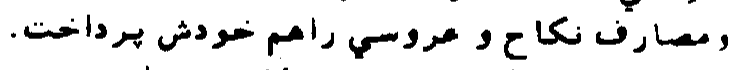

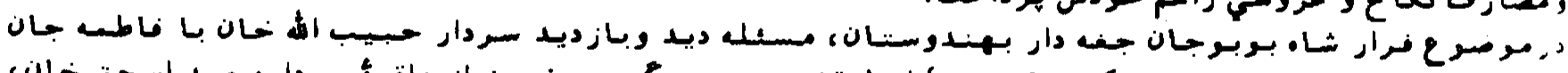

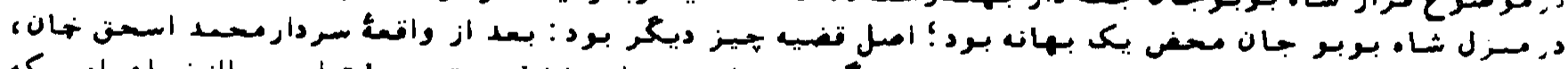

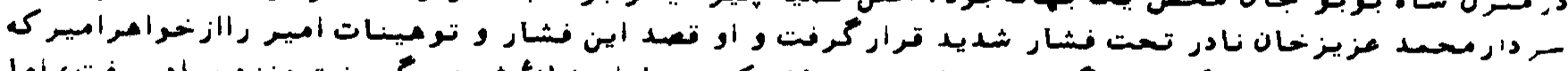

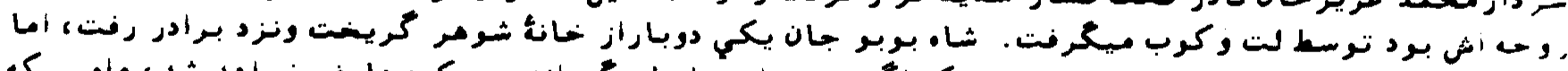

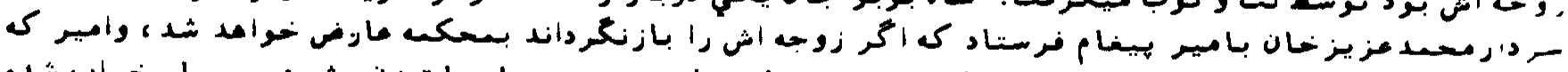

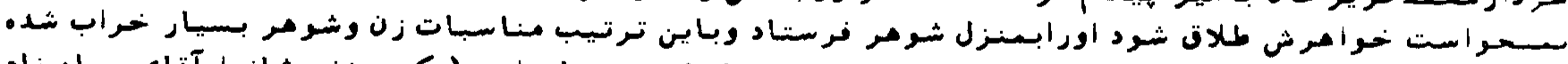

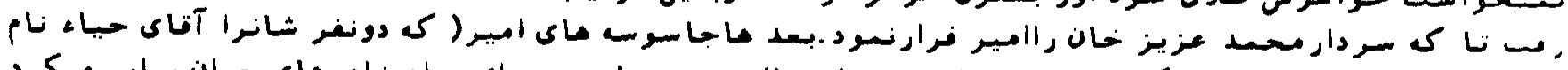

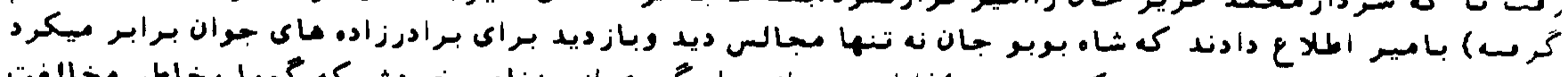

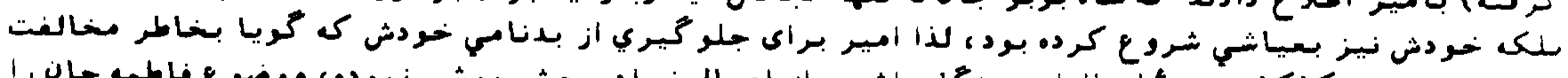

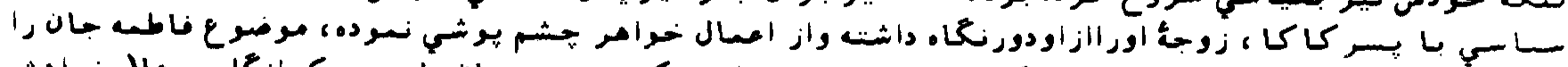

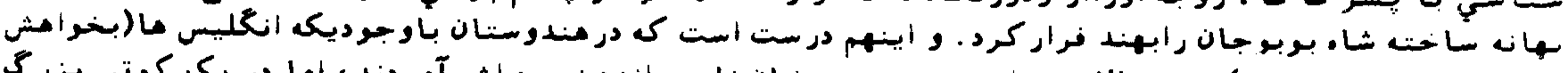

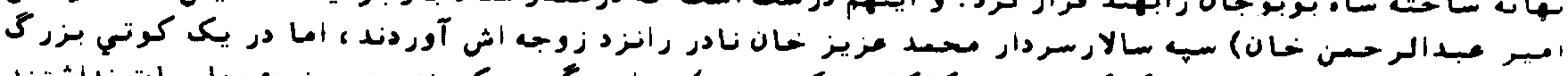

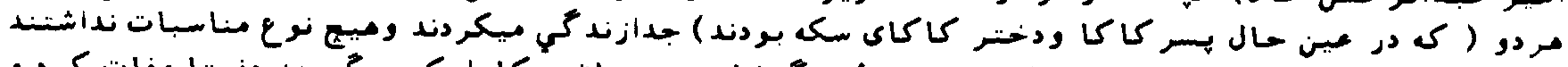

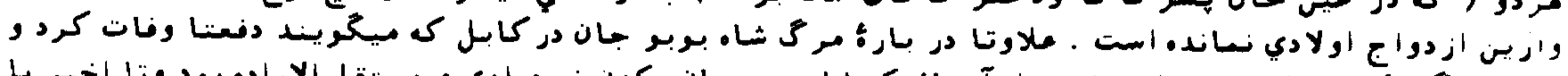

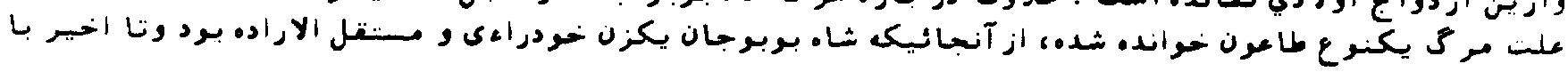




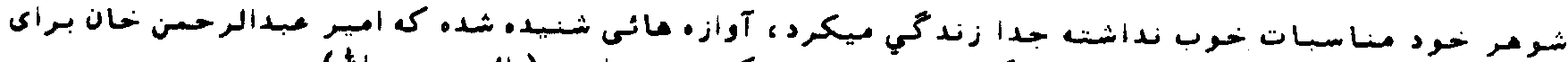

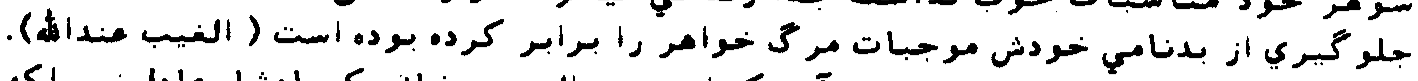

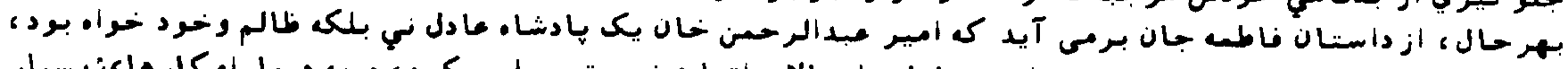

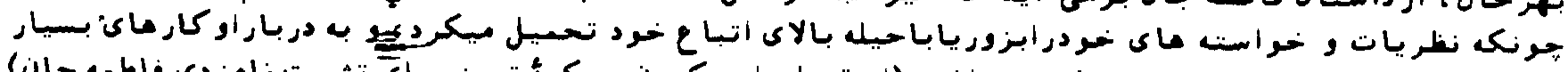

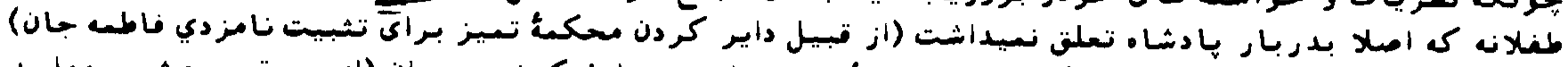

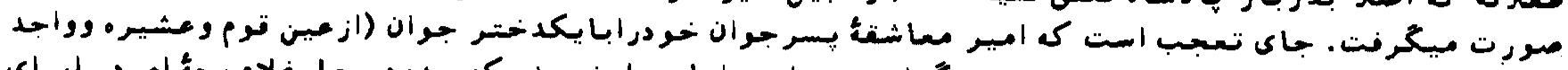

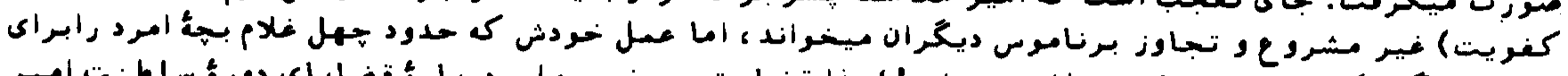

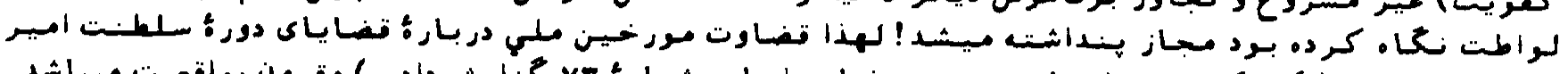

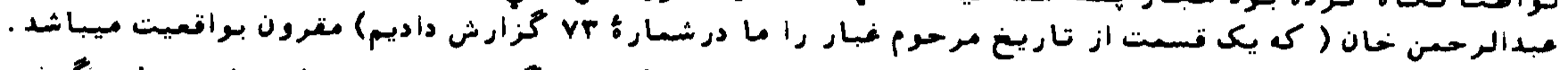

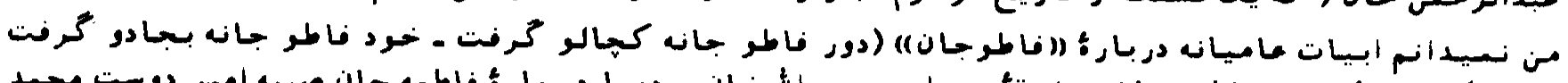

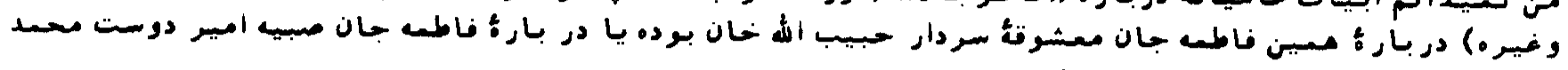

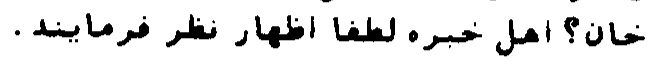

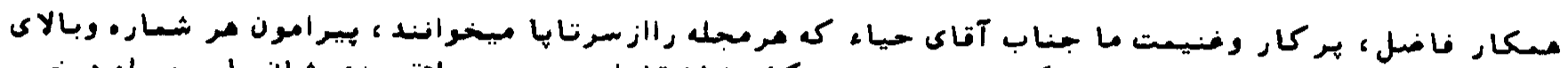

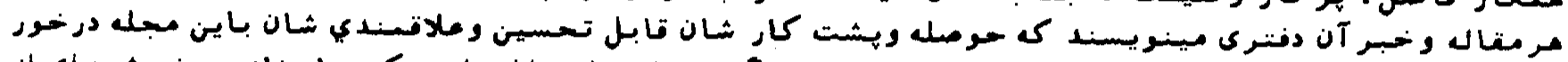

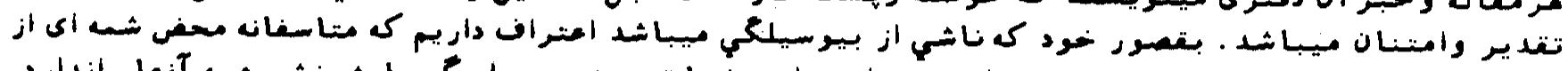

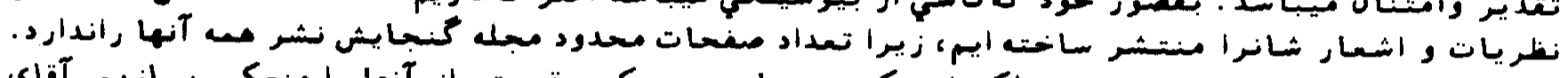

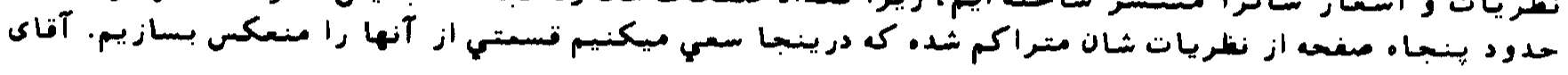

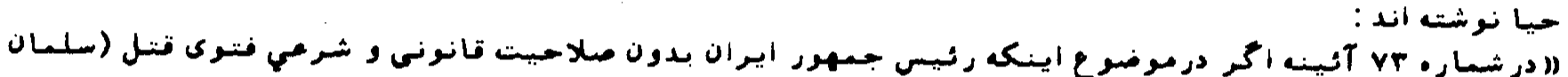

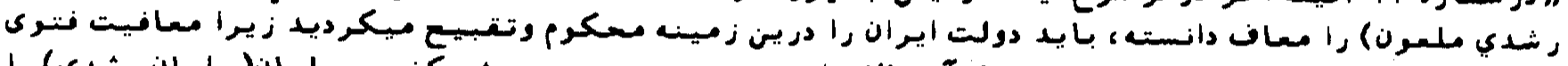

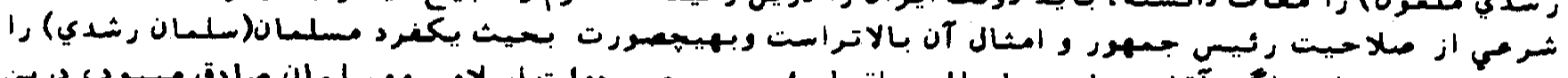

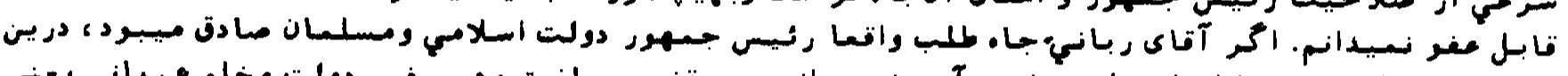

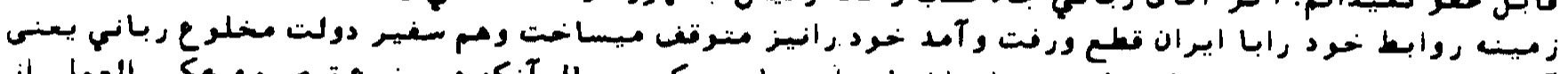

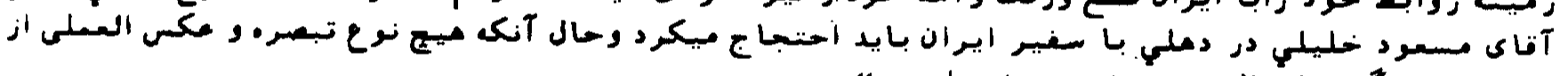

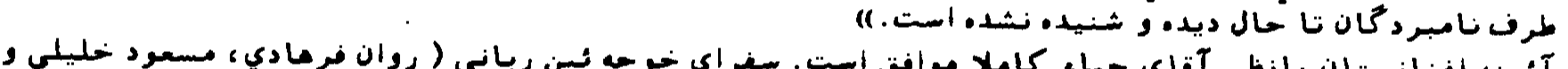

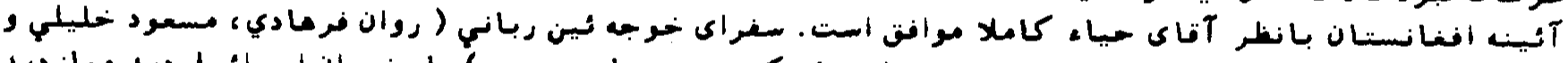

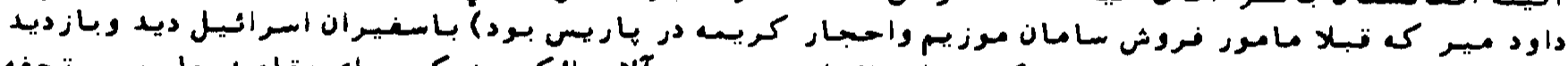

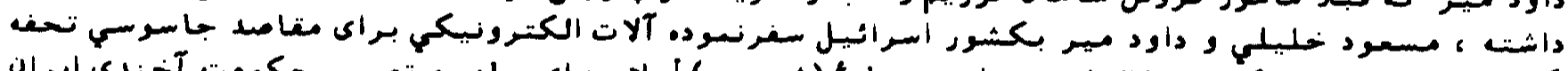

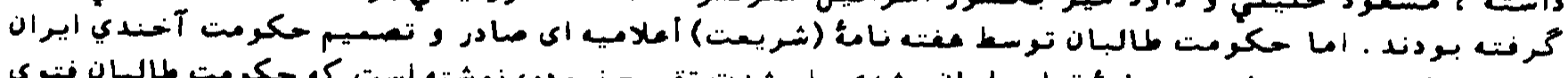

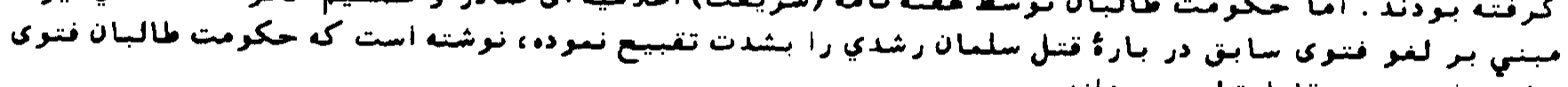

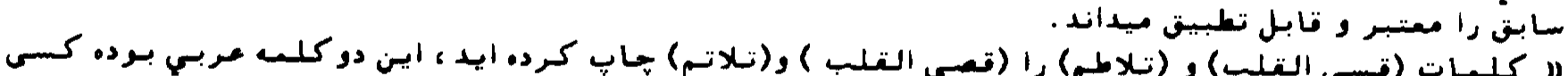

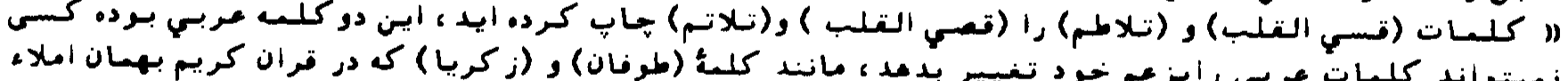

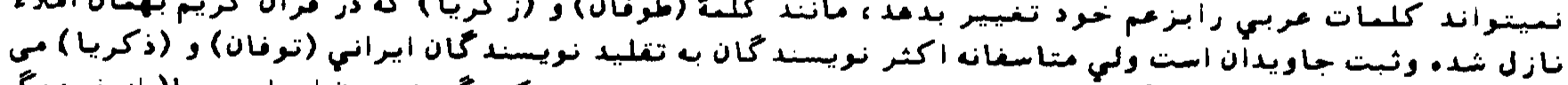

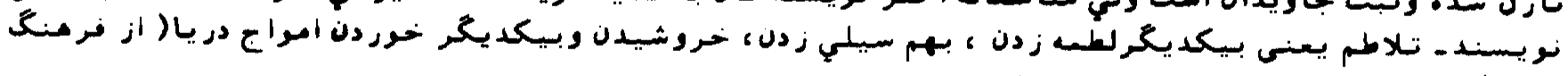

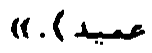

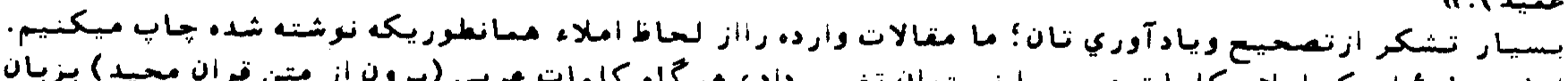

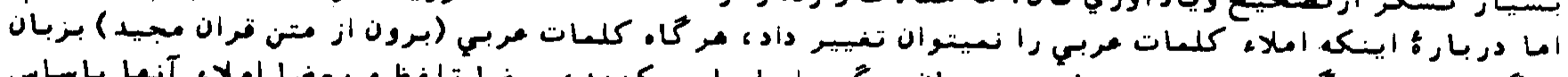

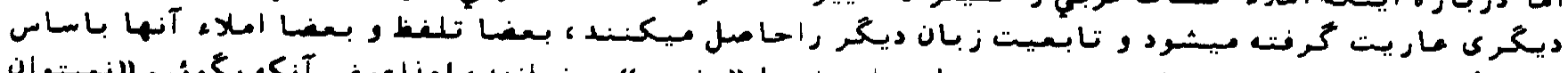

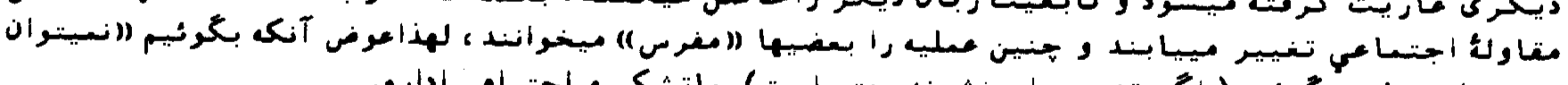

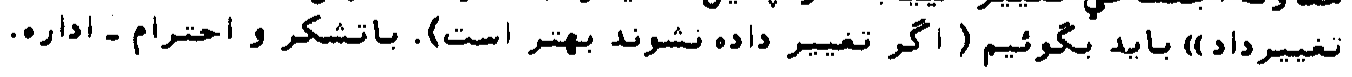

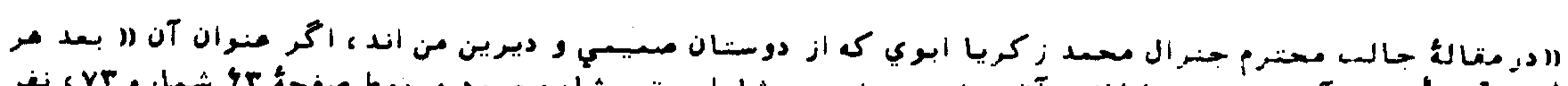

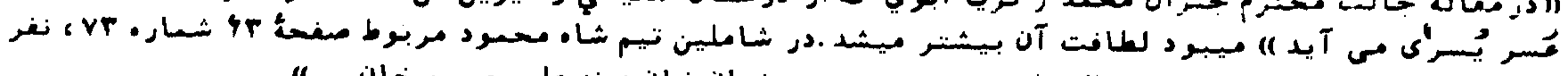

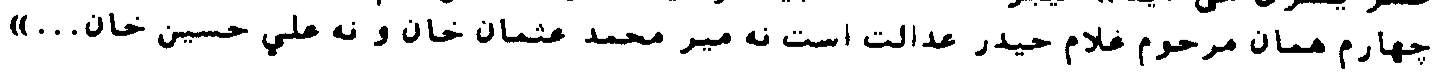




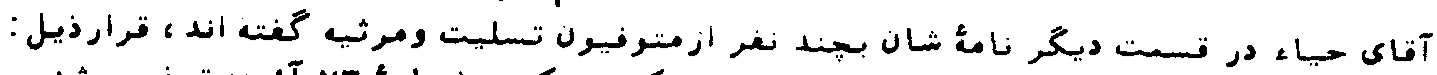

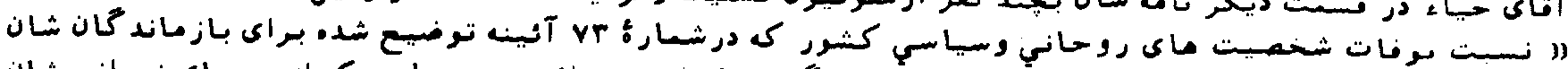

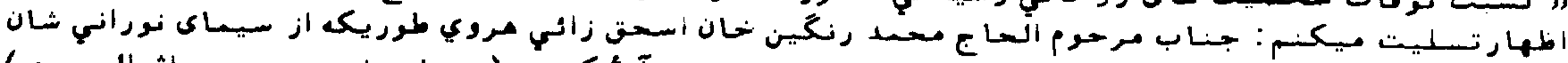

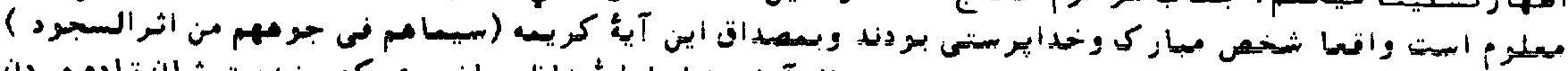

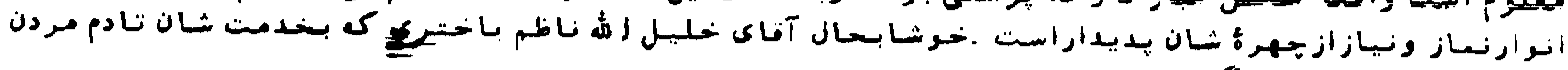

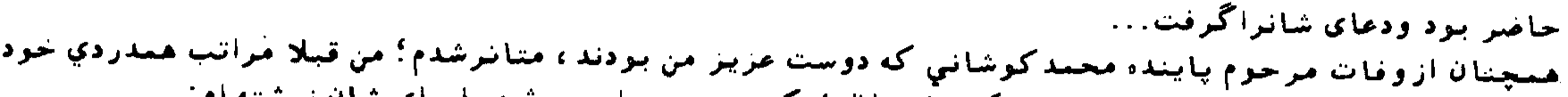

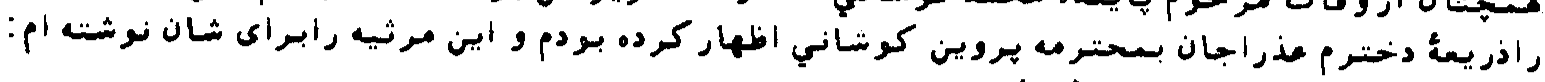

مسبوري يميشه كن دايم دوا كن كن

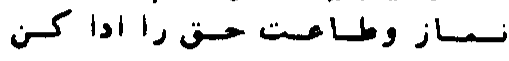

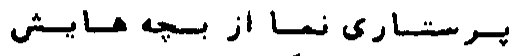

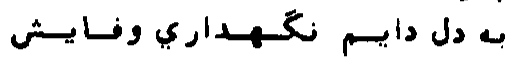

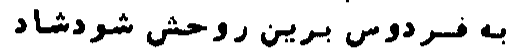

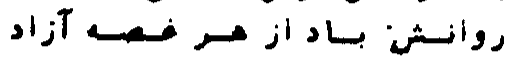

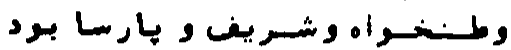

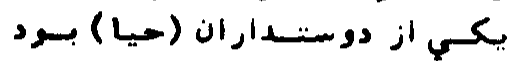

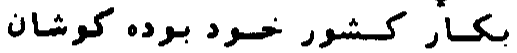

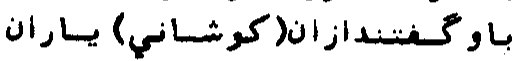

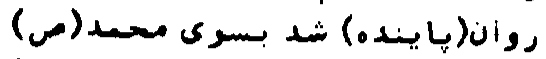

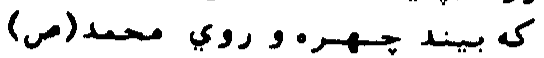

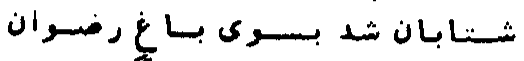

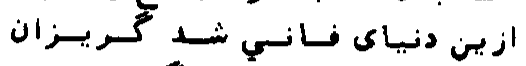

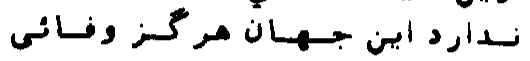

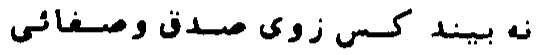

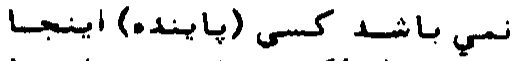

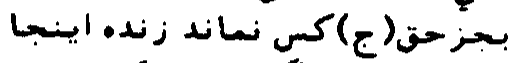

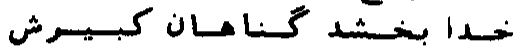

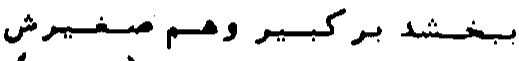

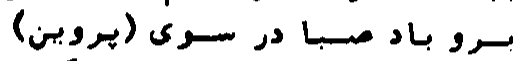

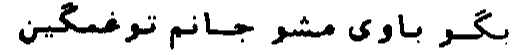

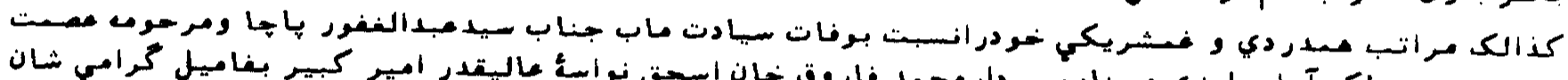

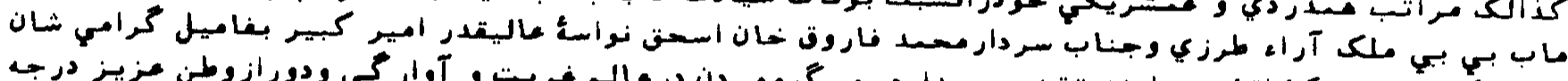

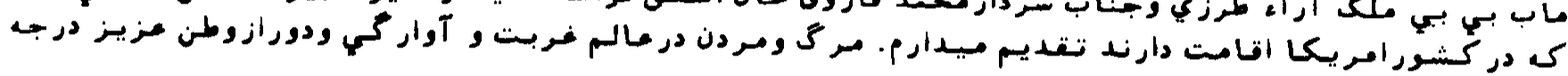

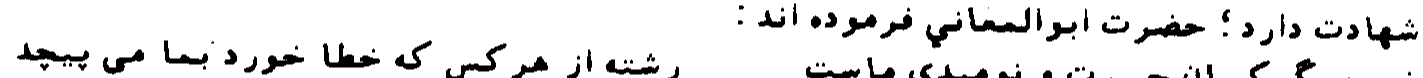

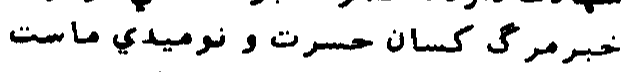

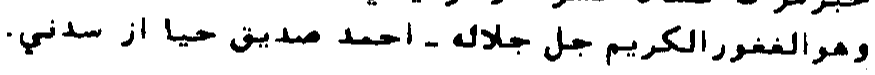

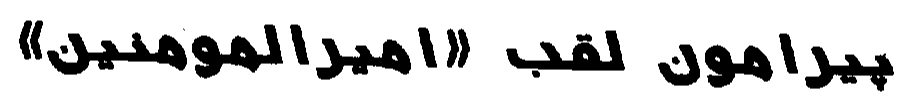

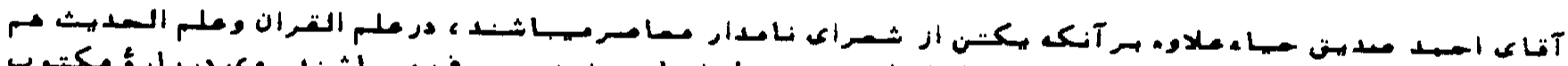

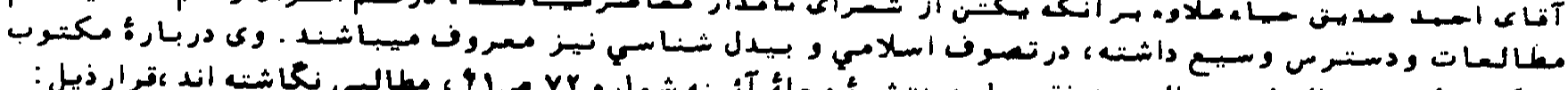

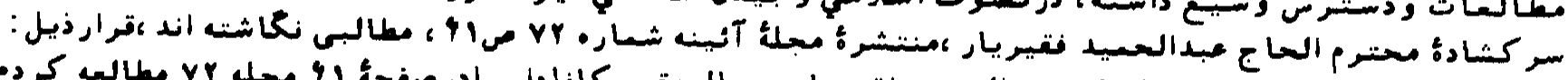

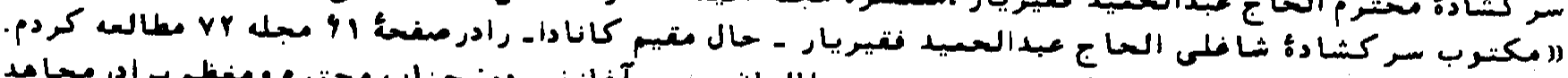

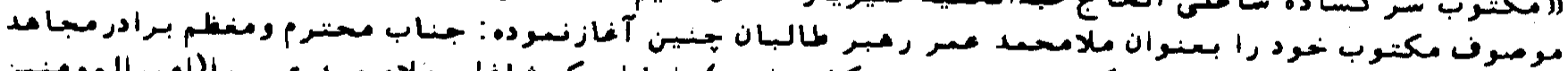

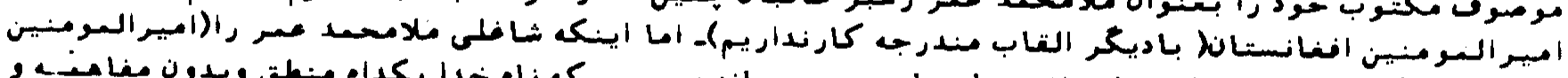

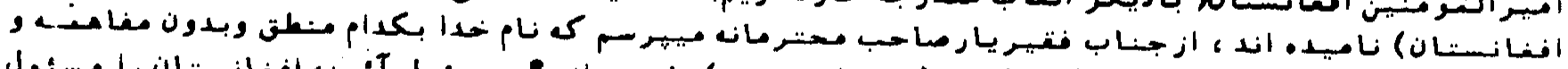

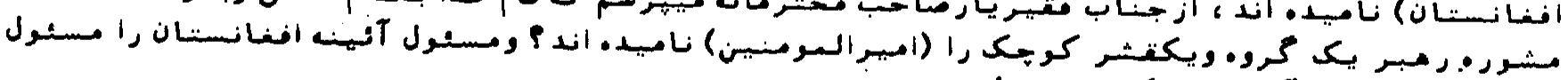

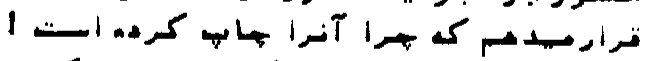

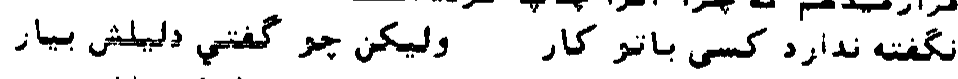

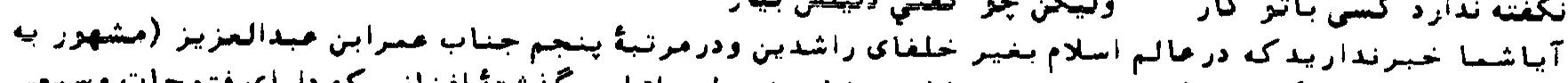

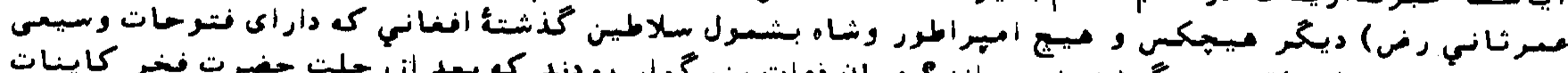

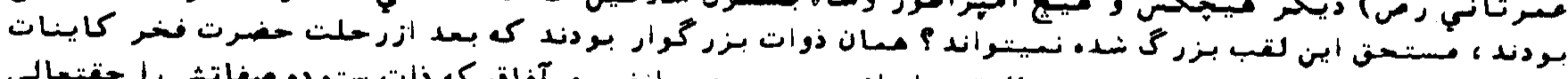

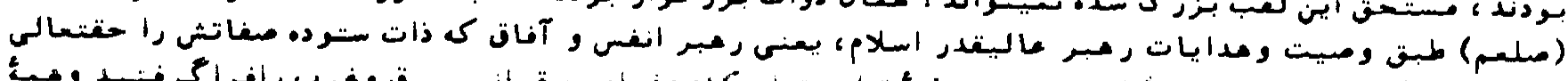

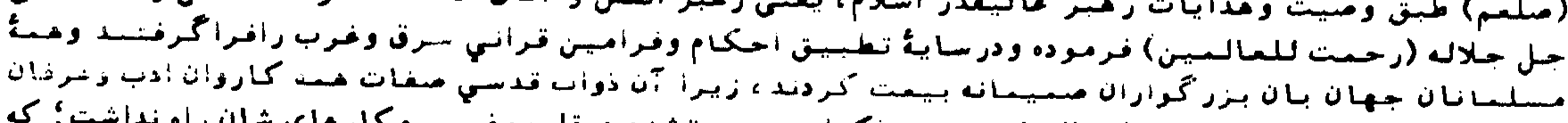

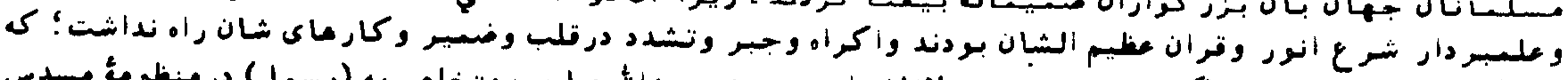

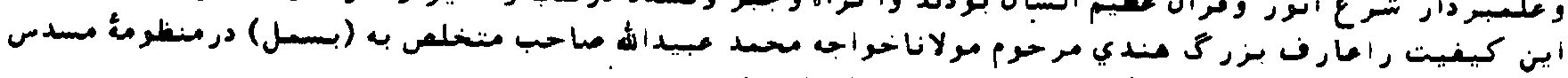

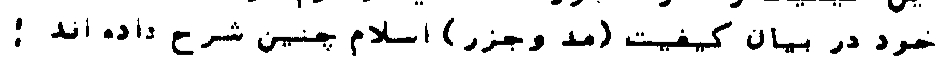


كساروانس دردو كالم بيث ايسزد سرفراز

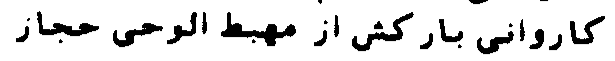

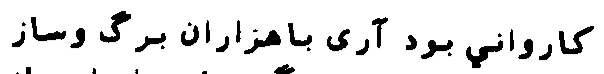

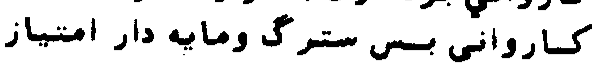

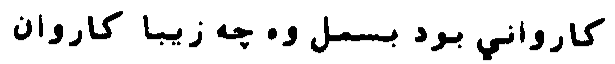

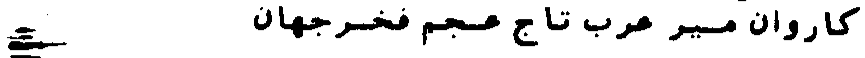

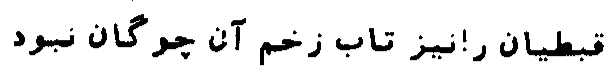

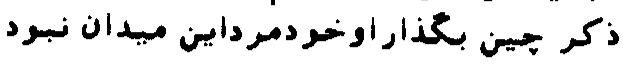

روميان راهيش أيشان طاتت جولان نبرد

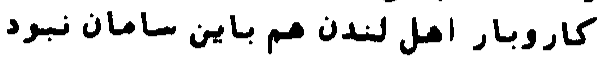

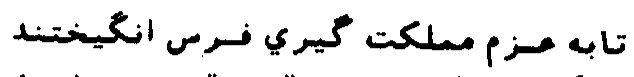

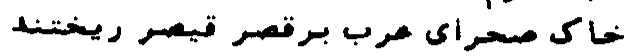

درركاب نمسرت ثـان مقل دور انديش بـود

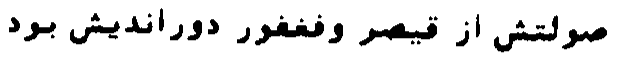

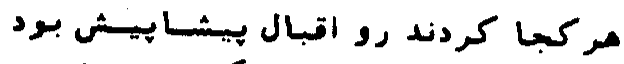

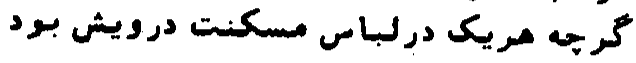

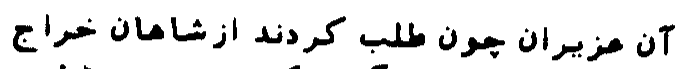

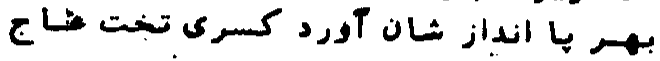

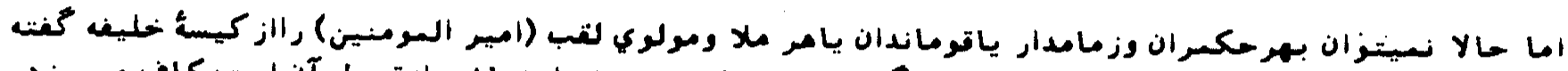

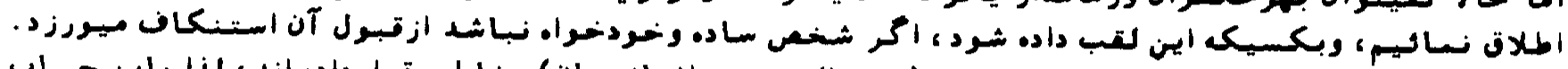

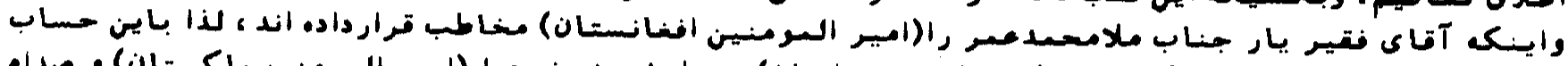

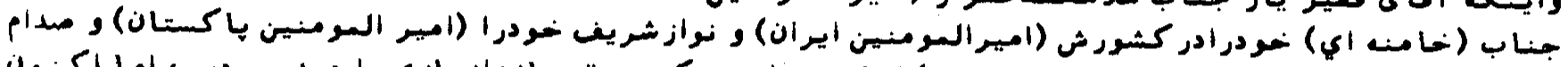

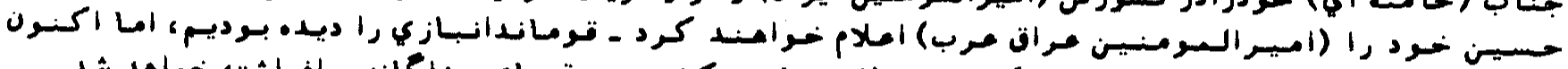

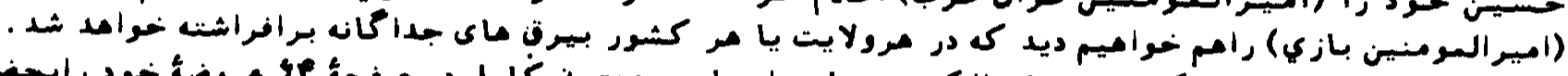

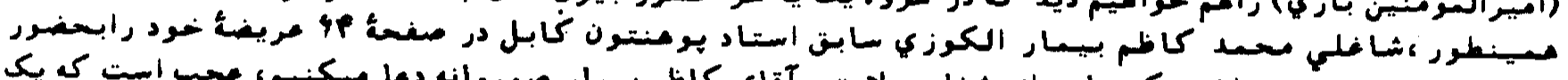

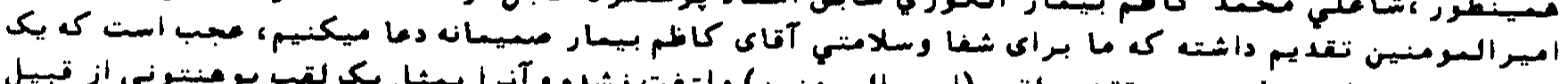

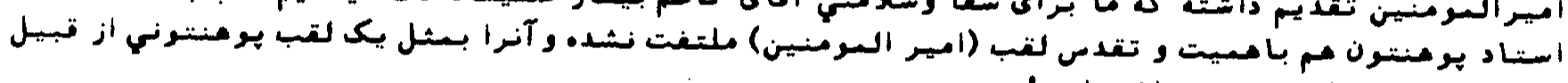

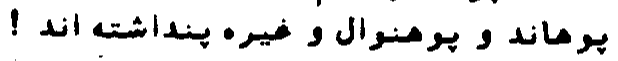

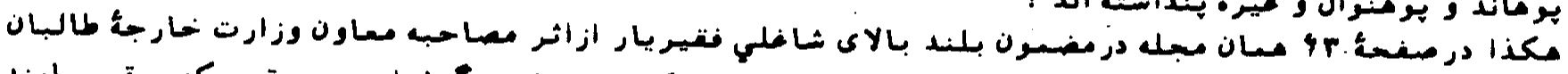

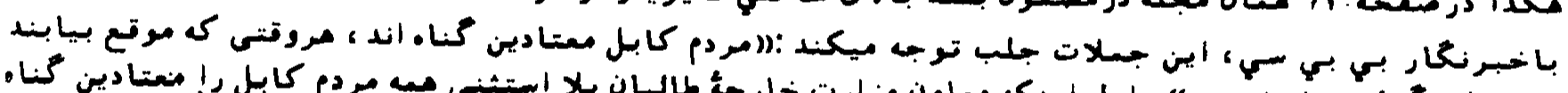

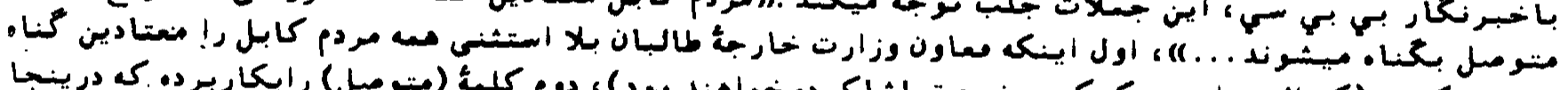

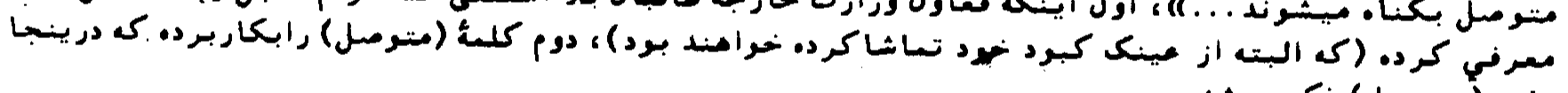

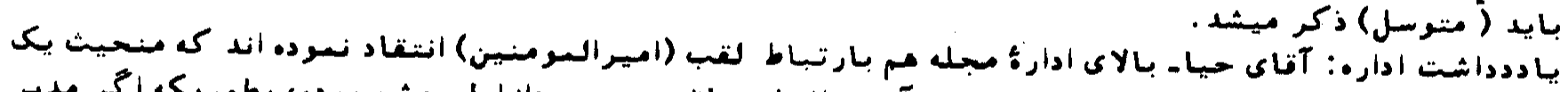

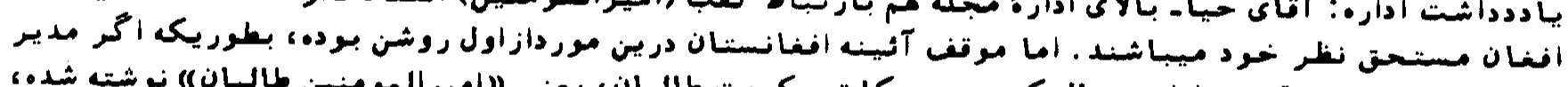

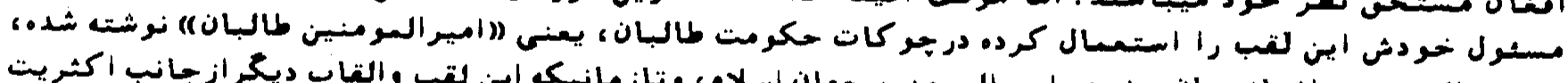

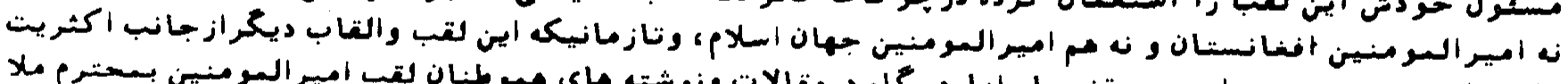

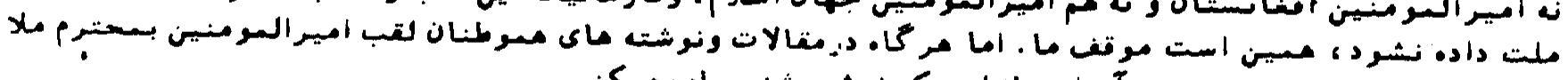

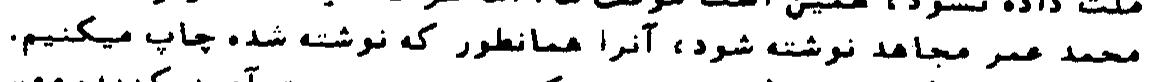

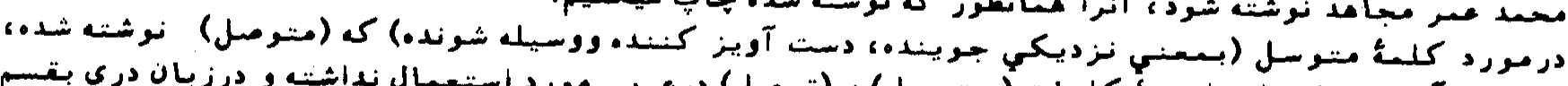

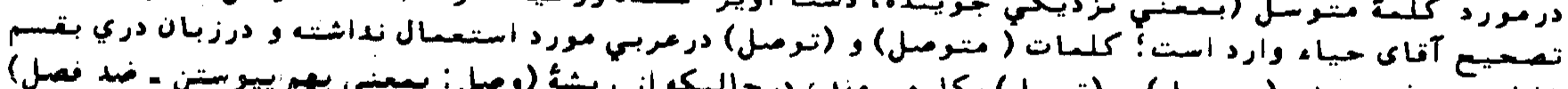

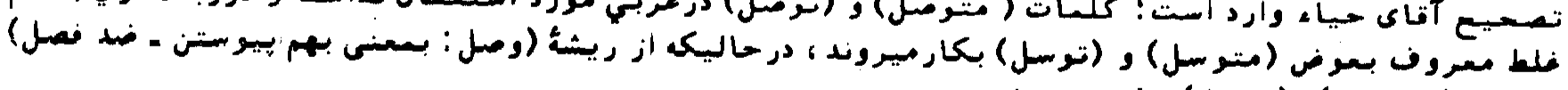

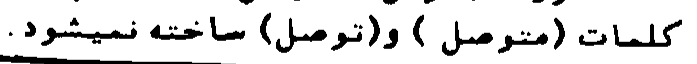

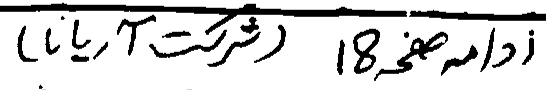

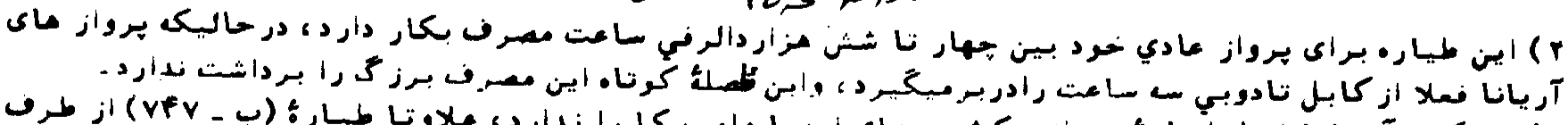

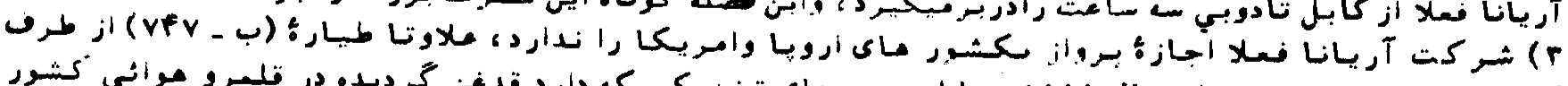

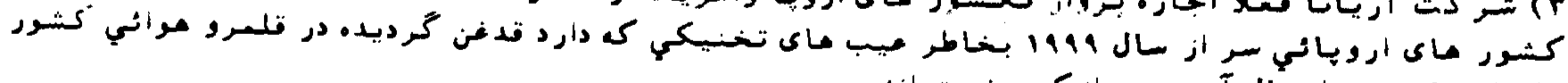

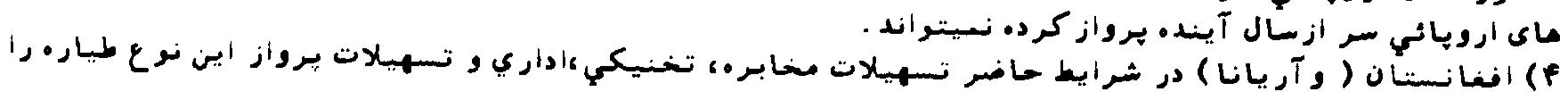

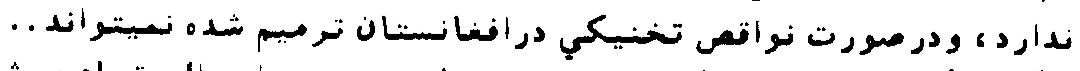

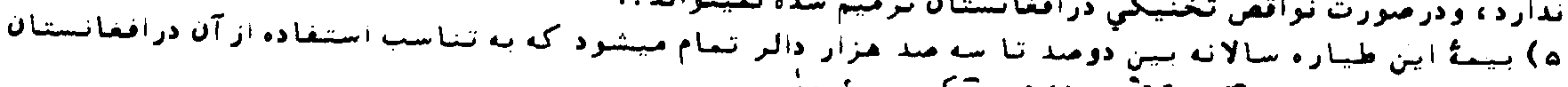

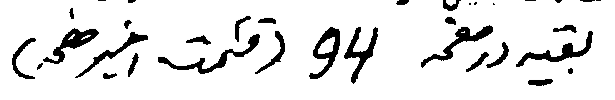




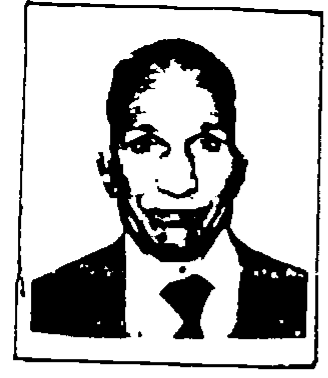

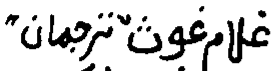

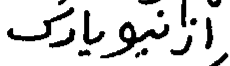
الكتوبر 1991

ازناشامر منباب تستربيق

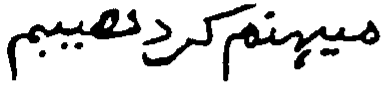

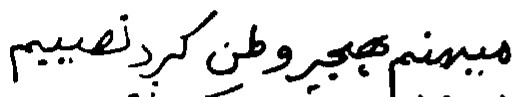

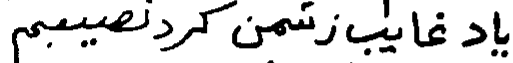

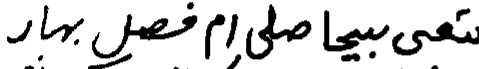

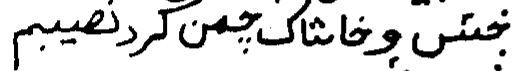

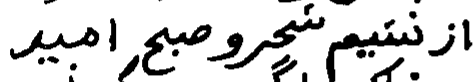

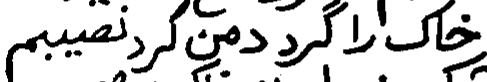

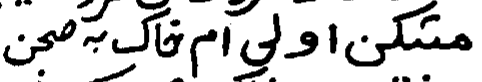

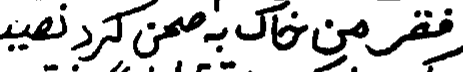

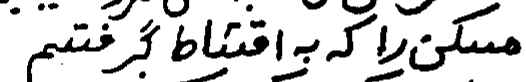

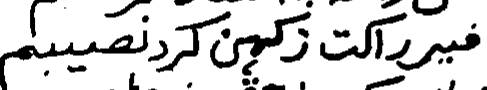

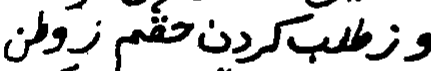

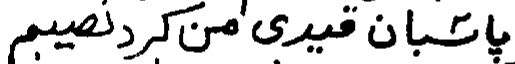

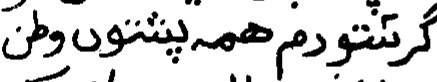

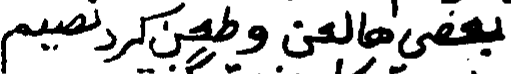

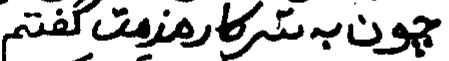

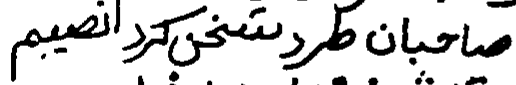

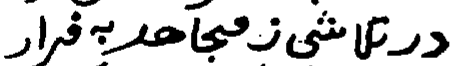

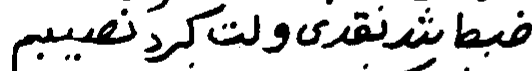

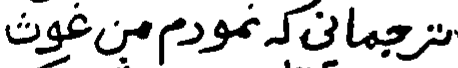

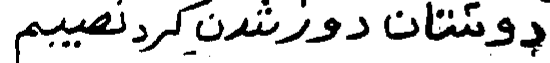

\section{Ghulam Ghaus}

1141 Tiffany Strest Apts 27

Bronx. New York. jo459

Pno.ie (716) 617.4737

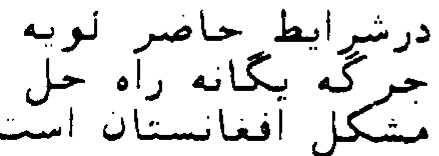

\section{7 نكومشن الفمر وفاعران}

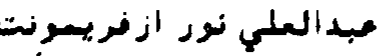

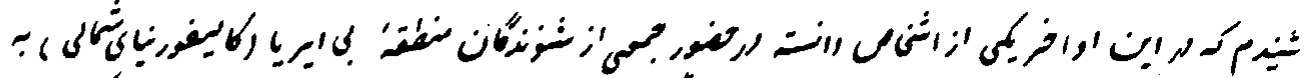

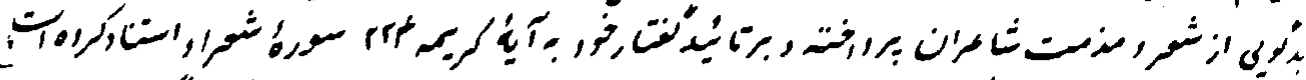

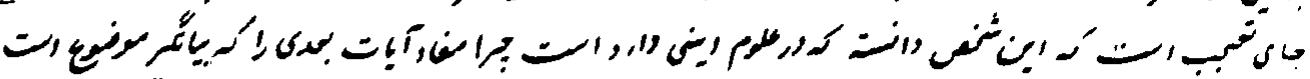

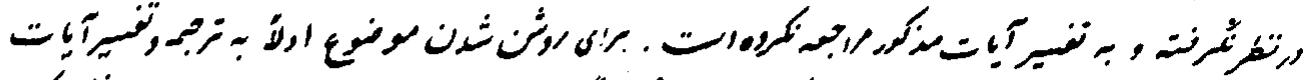

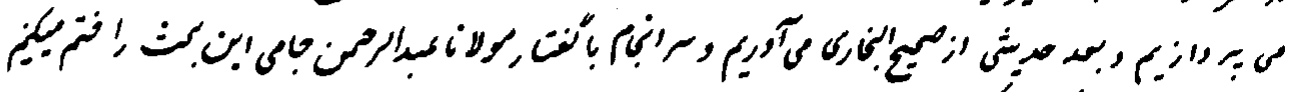

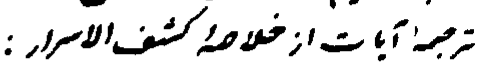

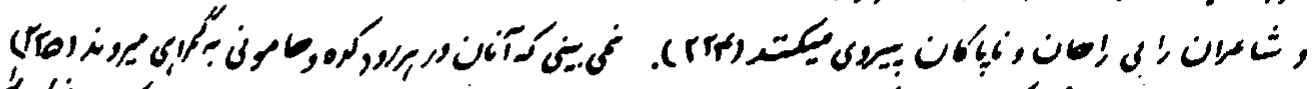

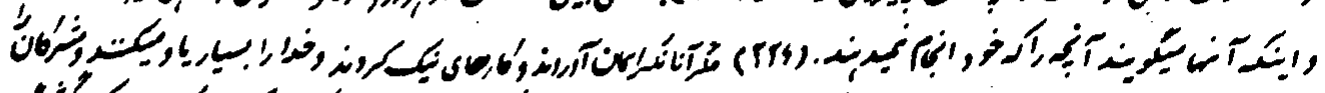

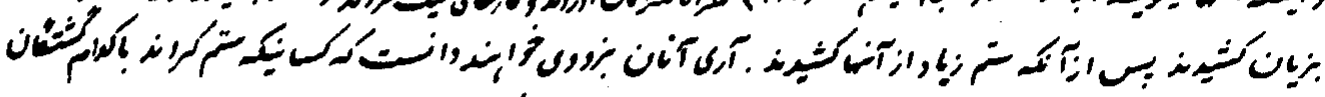

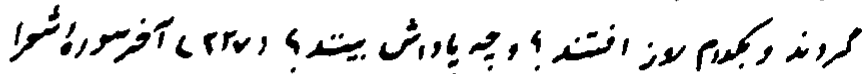

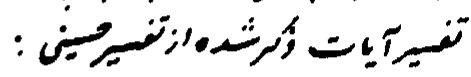

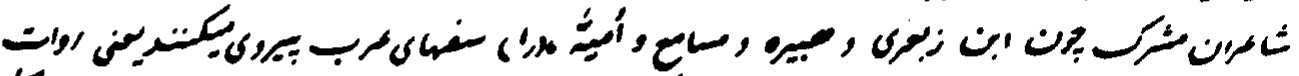

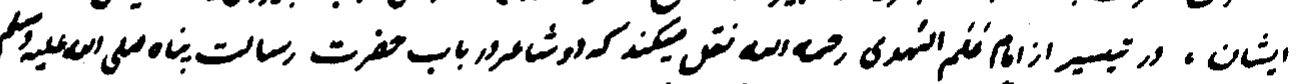

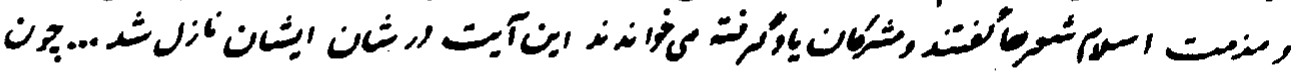

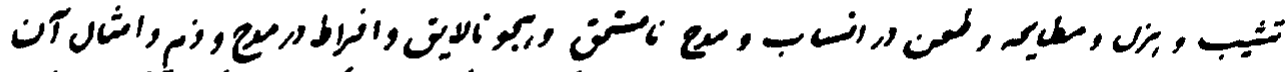

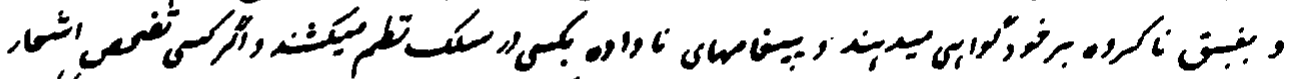

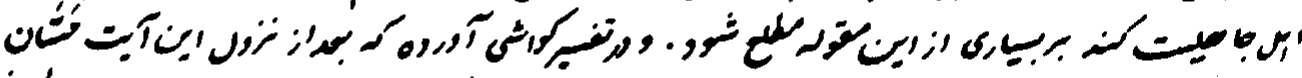

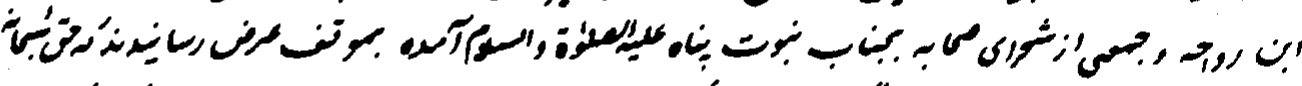

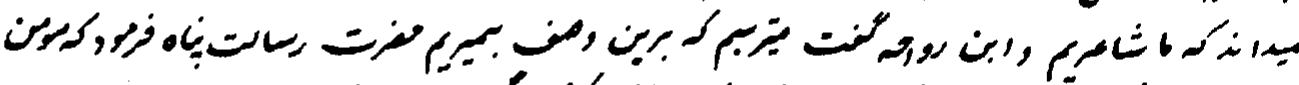

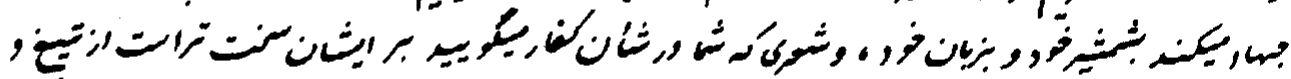

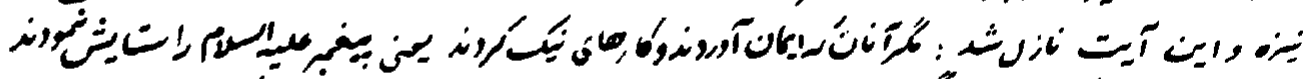

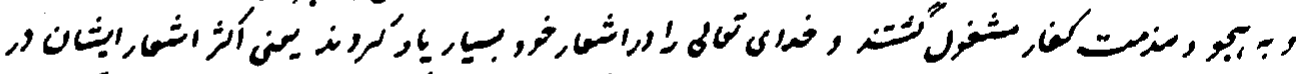

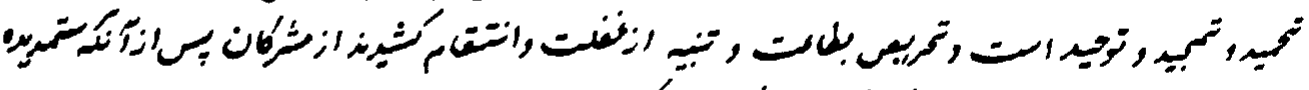

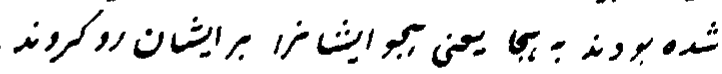

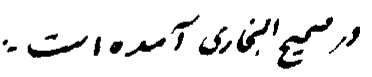

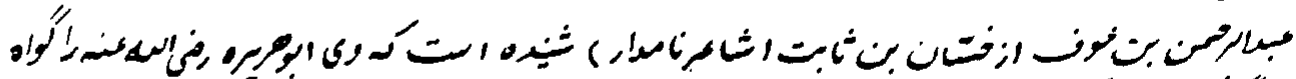

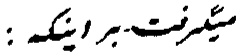

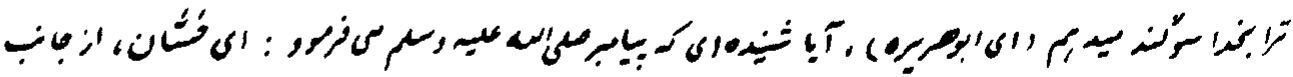

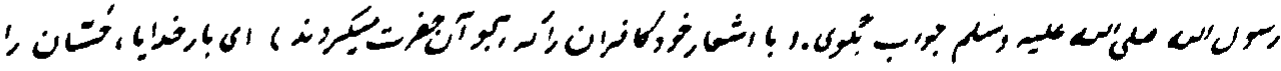

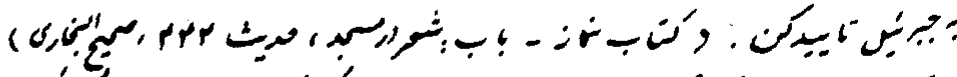

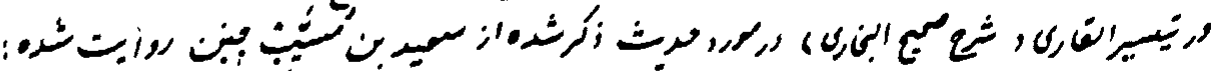

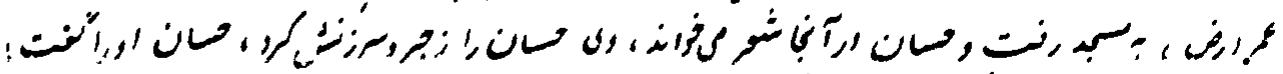

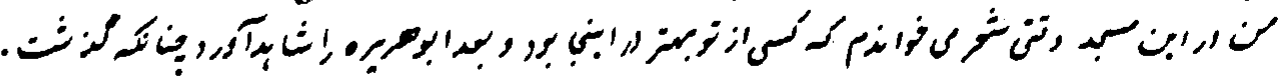

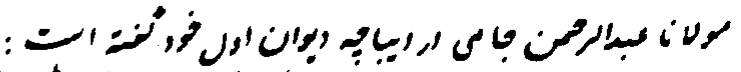

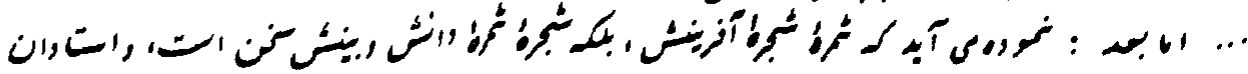

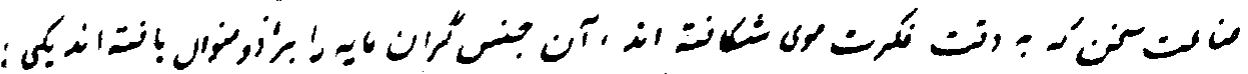


हो दूa .

- द. $=\xi$

:

3.

र. हो

- 19 . c.

$\rightarrow c$

: :

.

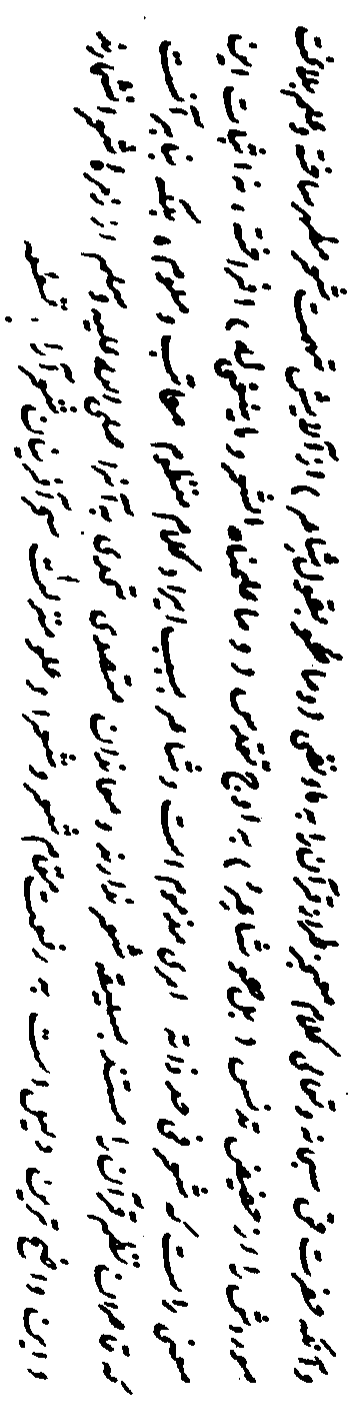

5

5

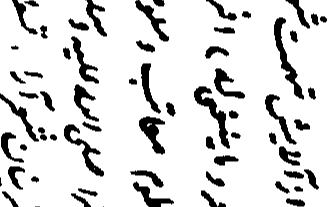

$\therefore$ F:

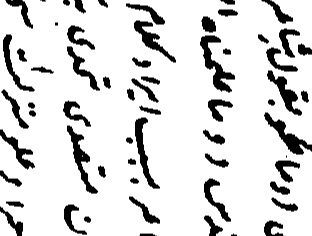

प. द.

1.5. $1 \div$

눈

if 5

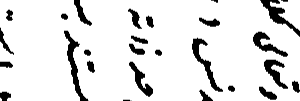

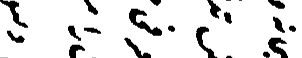

5 E.

3.

$\Leftrightarrow$ i 5

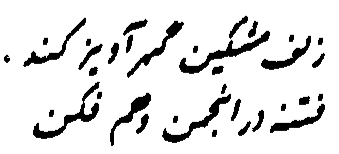

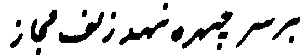

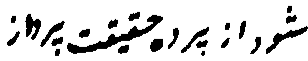

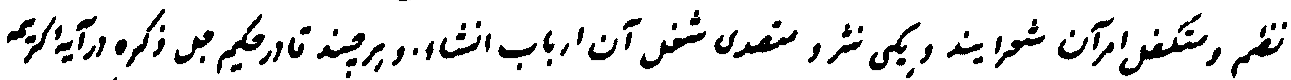

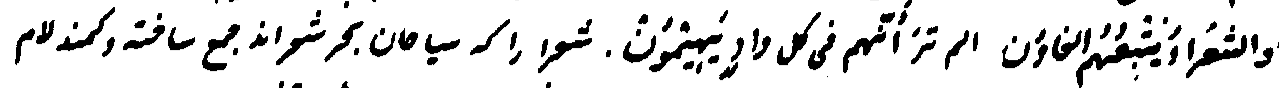

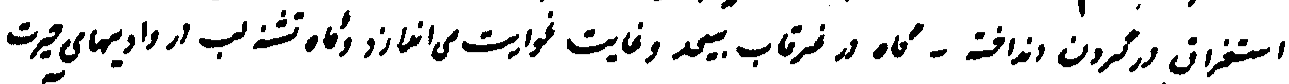

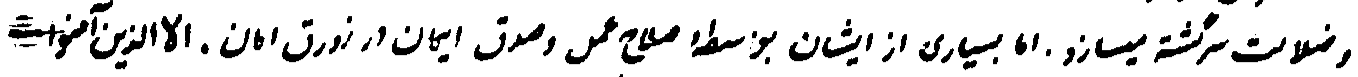

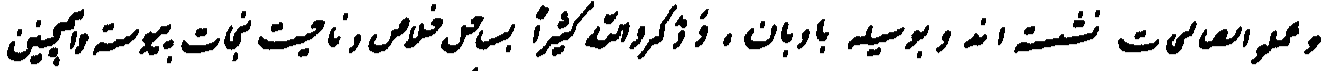

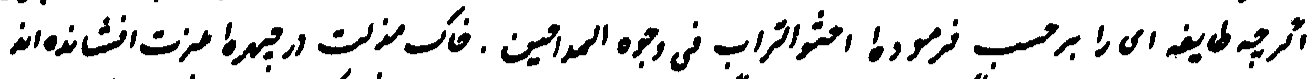

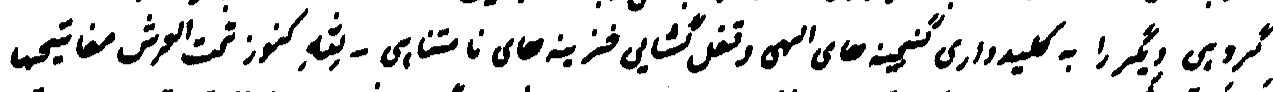

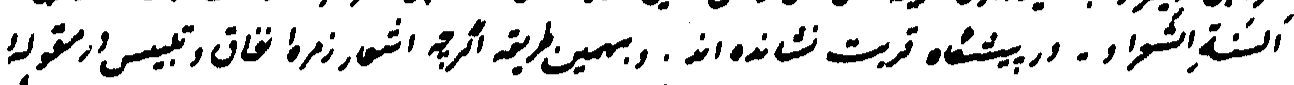

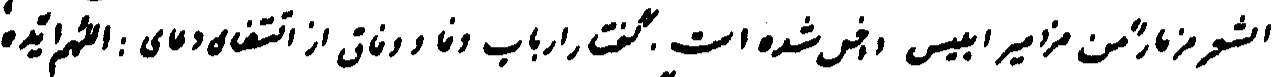

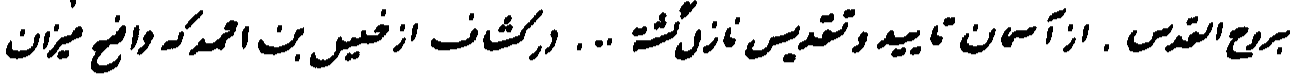

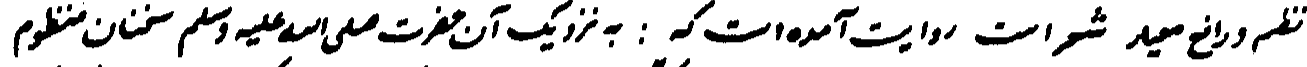

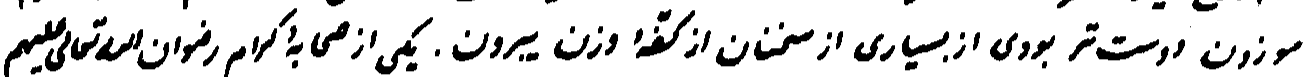

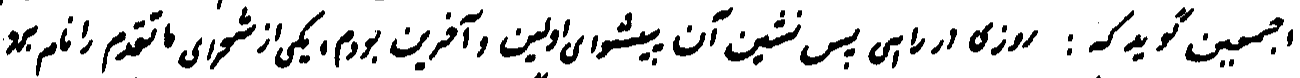

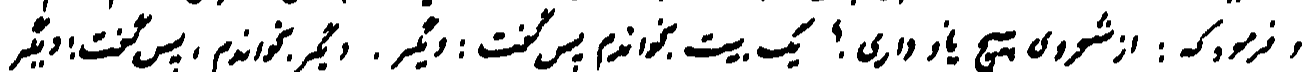

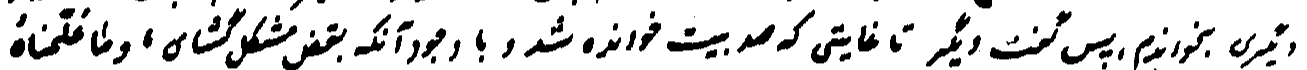

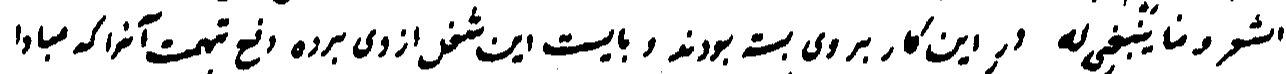

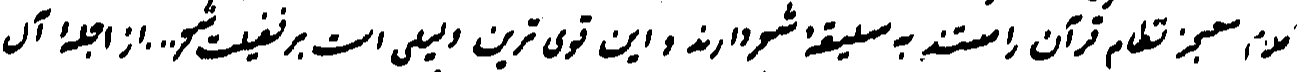

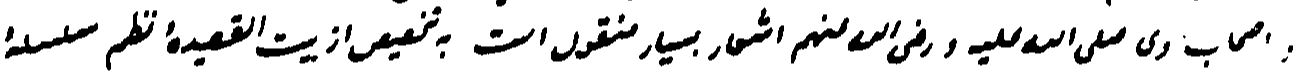

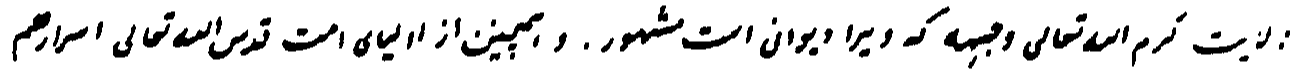

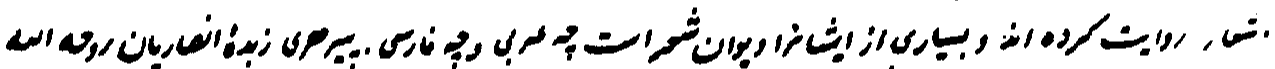

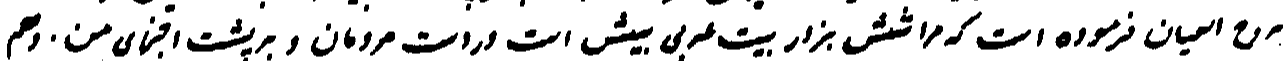

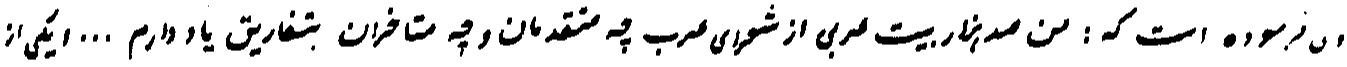

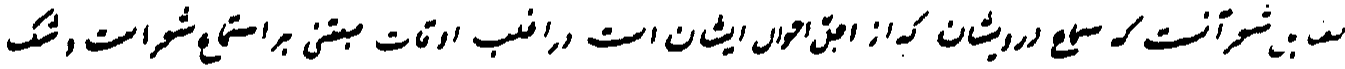

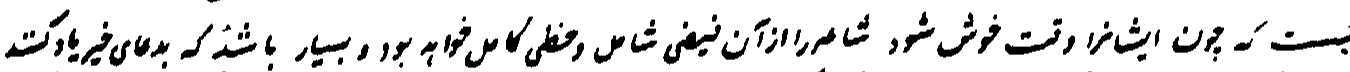

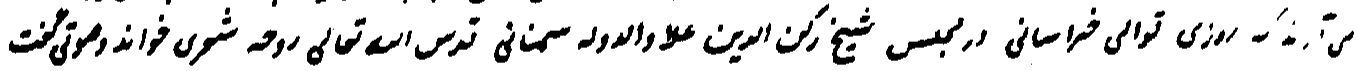

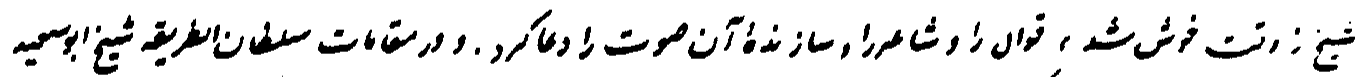

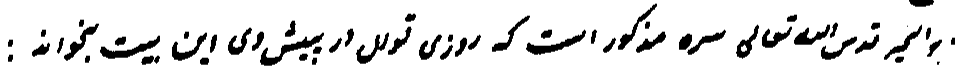

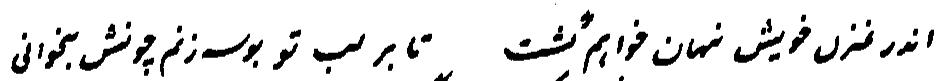

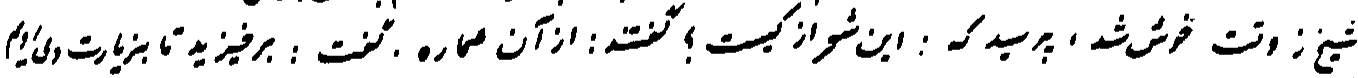

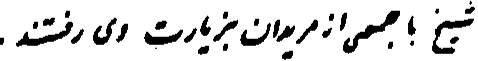

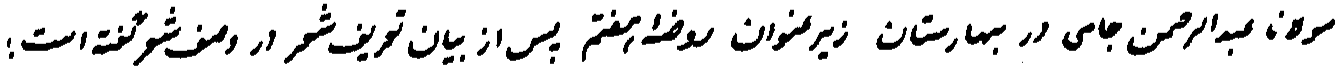

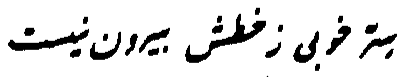

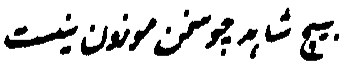

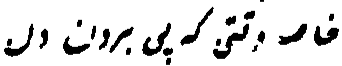

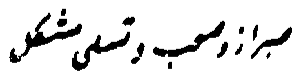

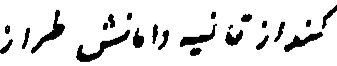

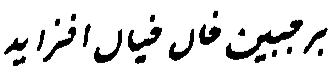

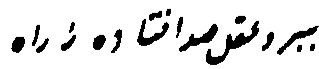

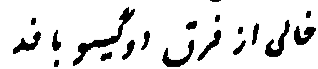

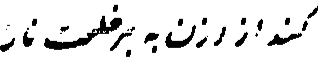

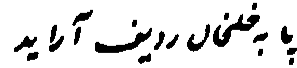

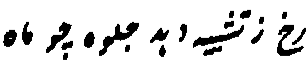

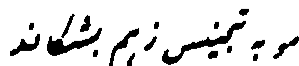


59

حمن كريهي

هملغ الميد

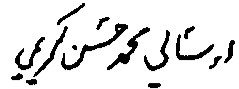

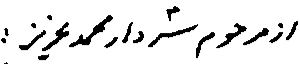

ازحفيز سمل

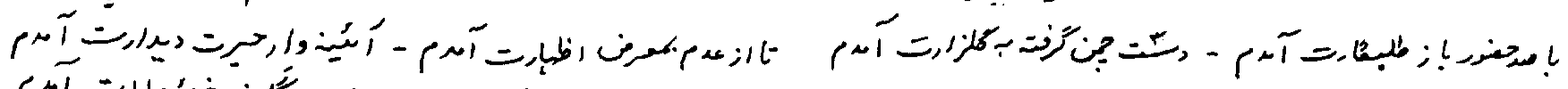

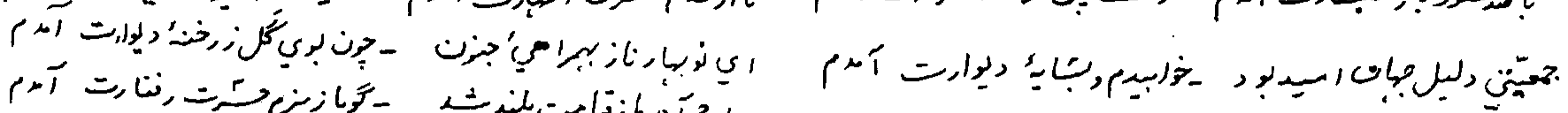
p

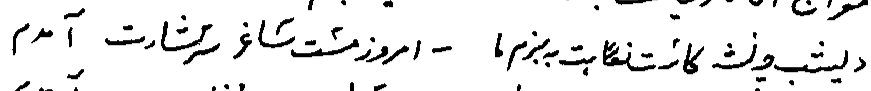

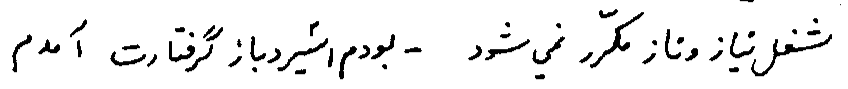

r

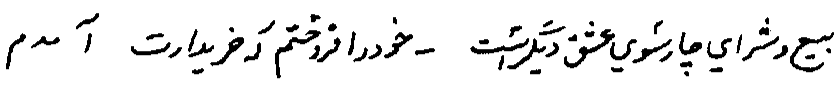

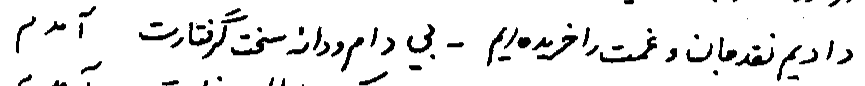

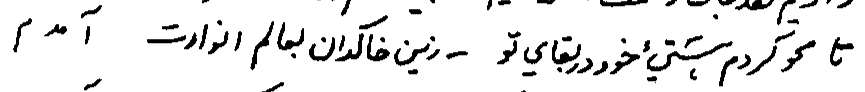

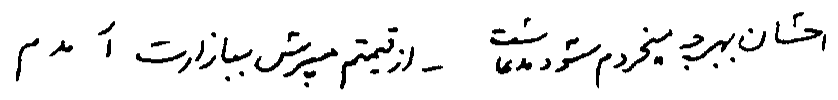

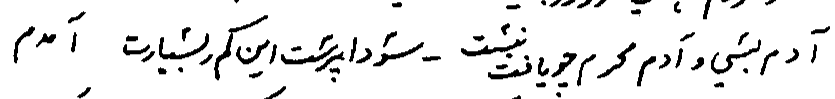

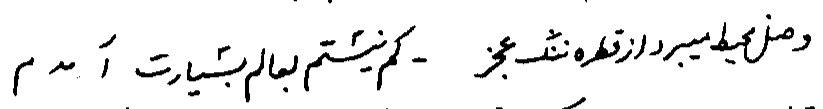

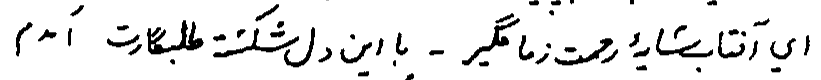

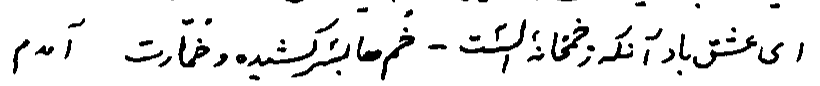

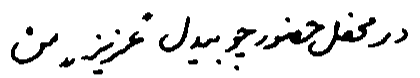

:

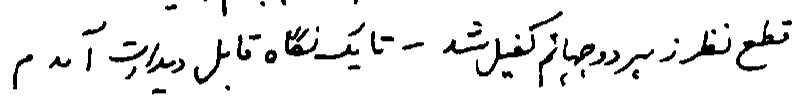

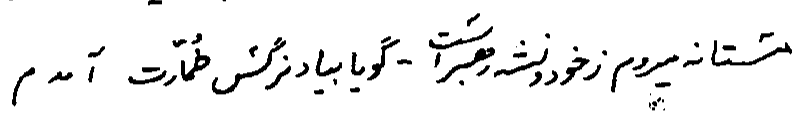

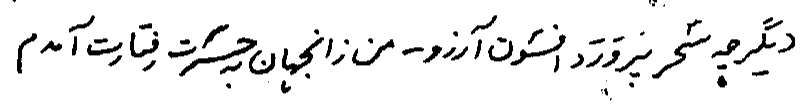

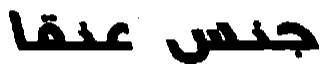

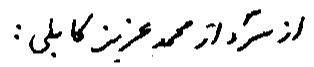

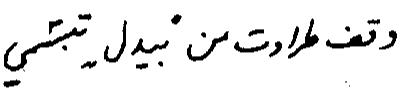

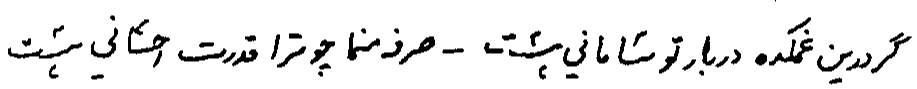

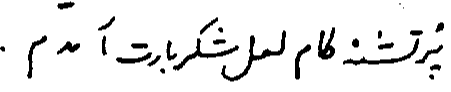

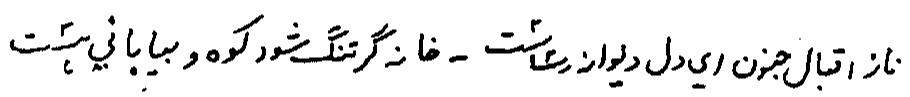

دل

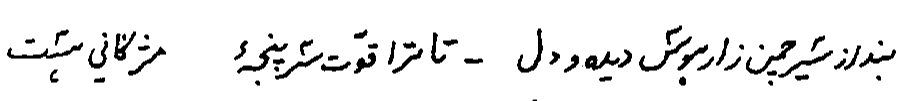

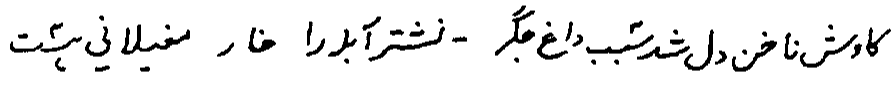

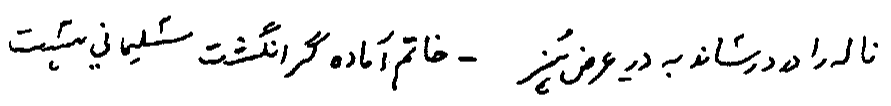

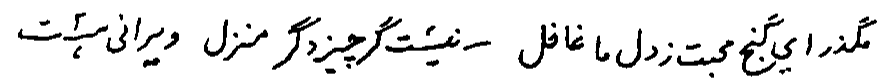

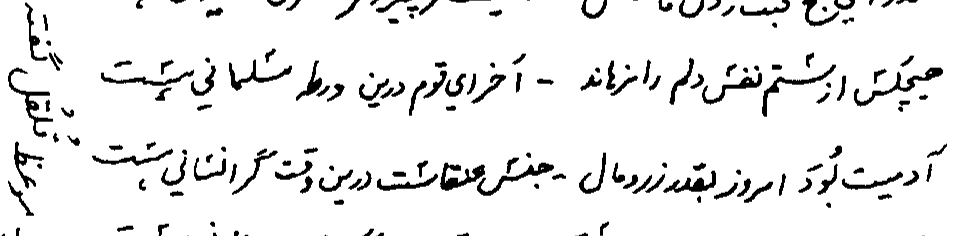

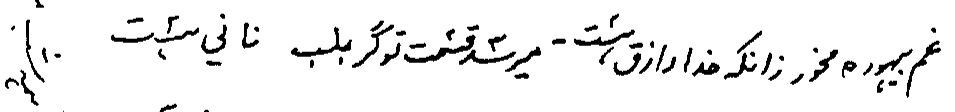

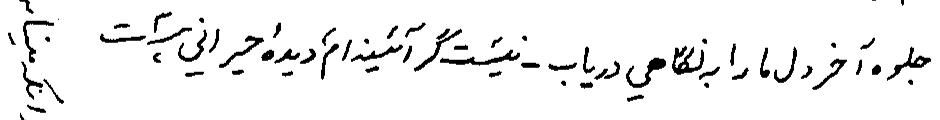

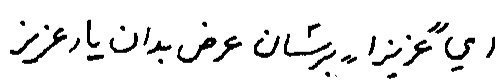

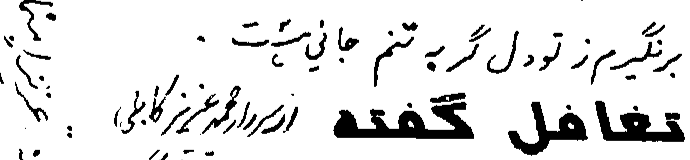

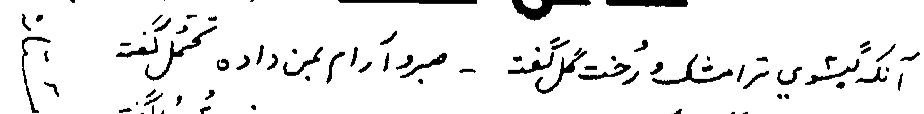

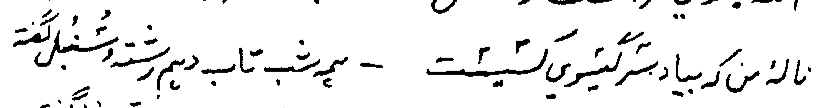

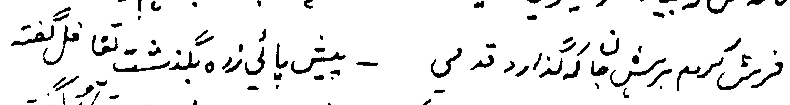

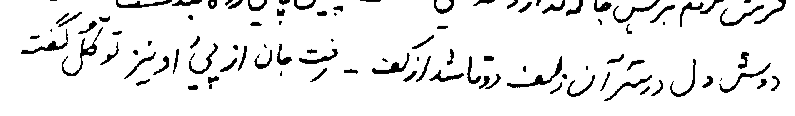

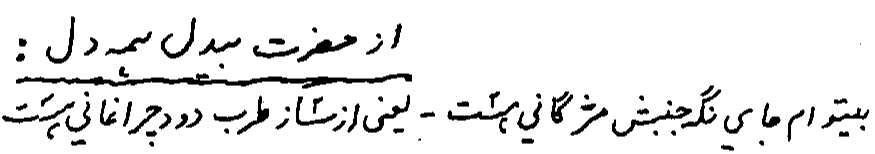

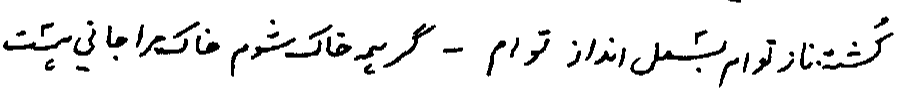

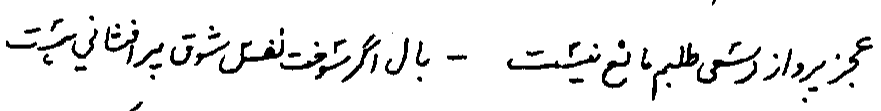

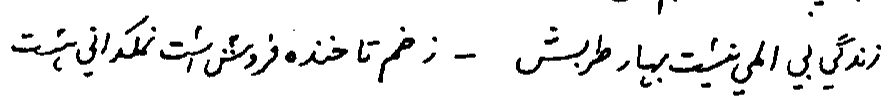

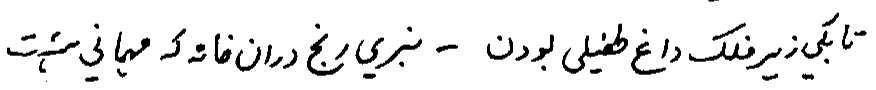

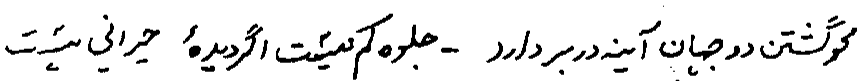

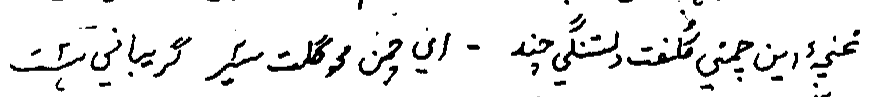

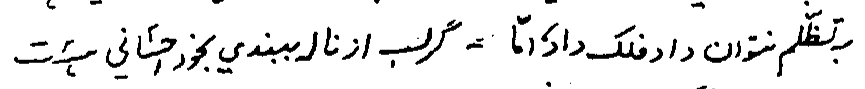

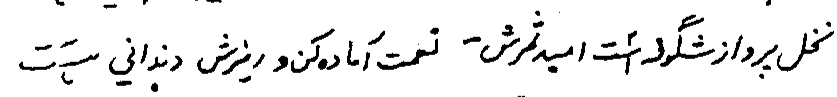

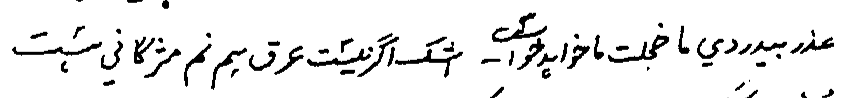

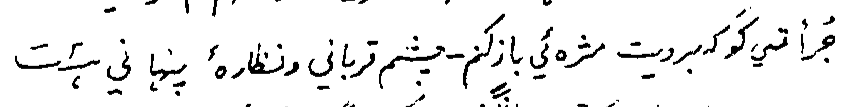

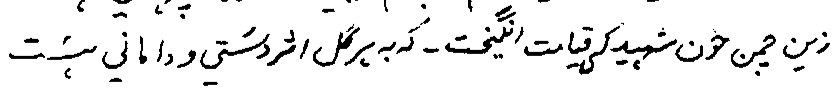

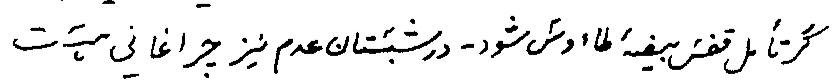

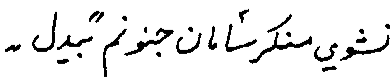

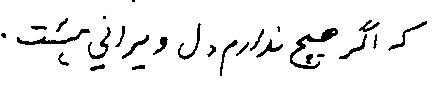




\section{0

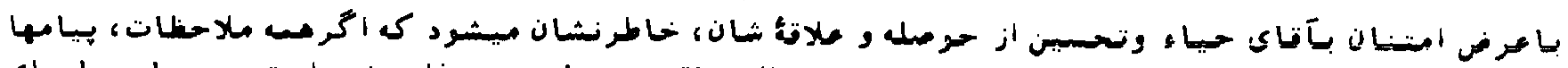

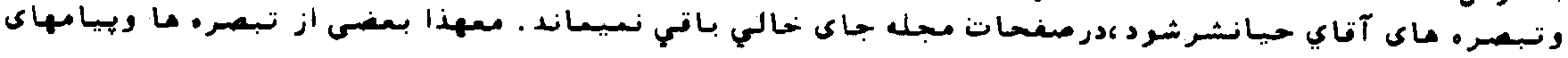

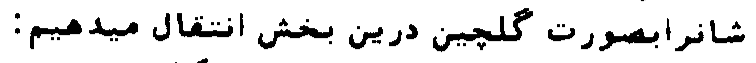

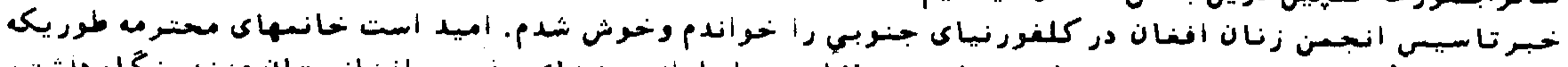

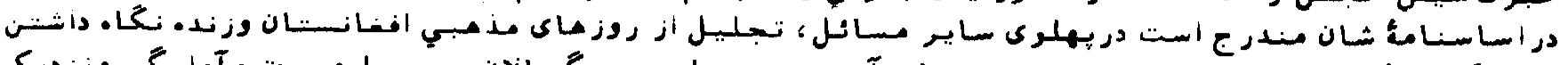

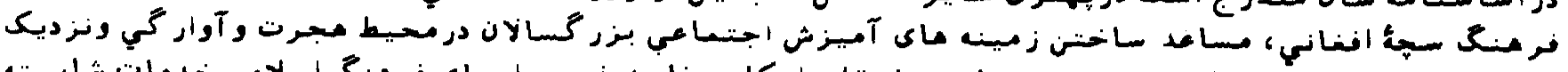

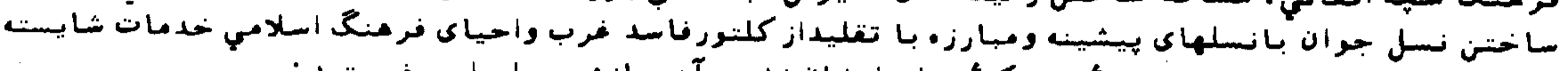

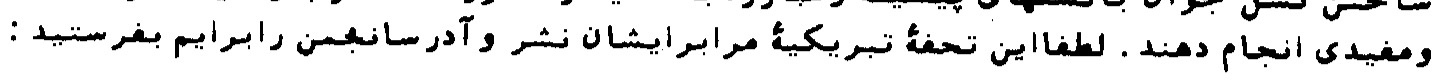

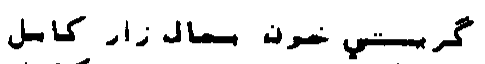

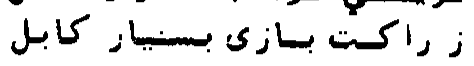

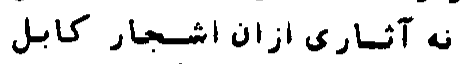

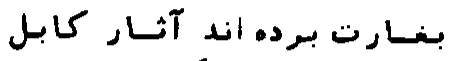

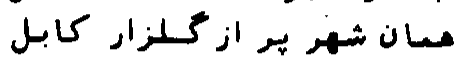

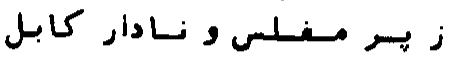

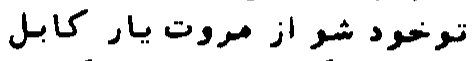

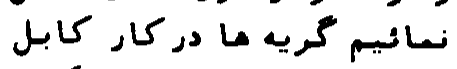

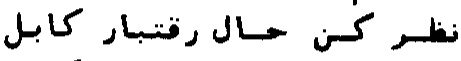

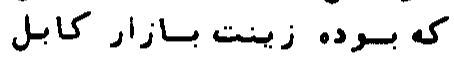

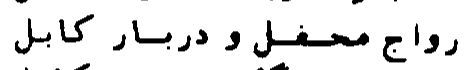

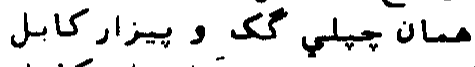

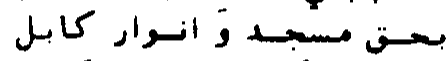

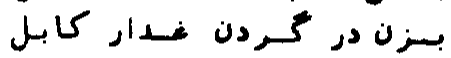

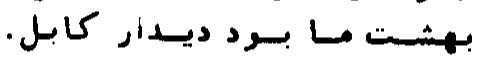

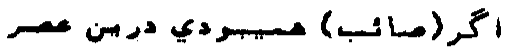

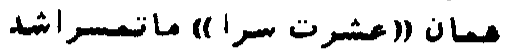

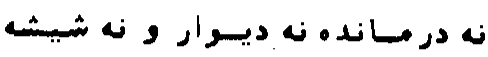

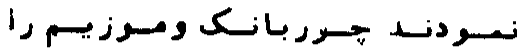

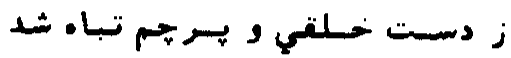

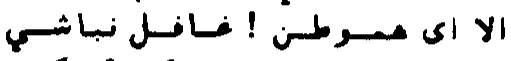

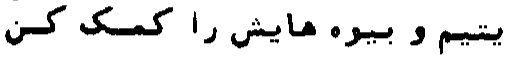

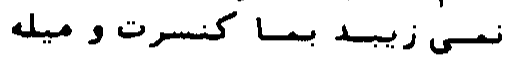

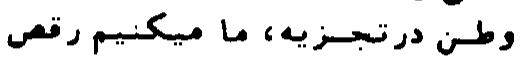

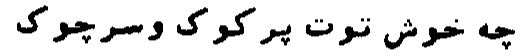

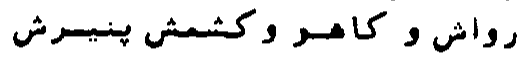

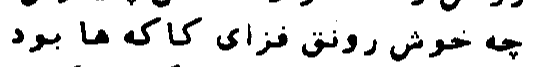

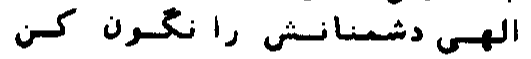

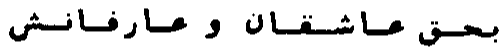

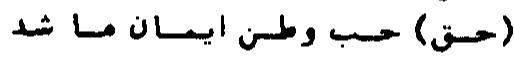

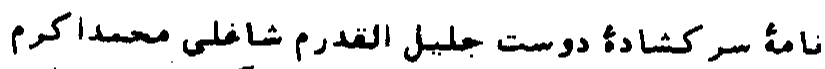

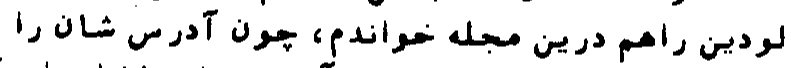

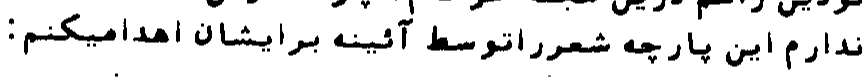

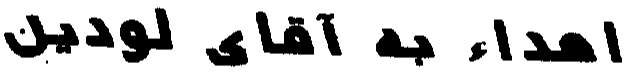

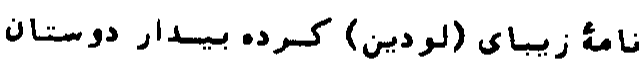

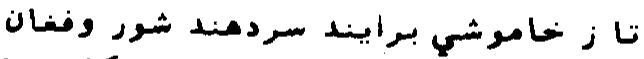

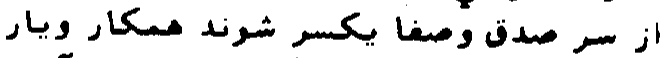

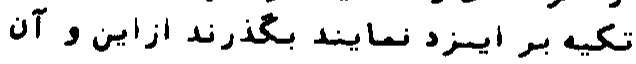

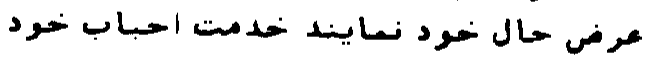

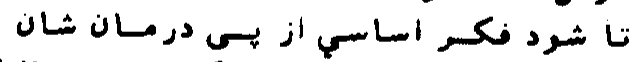

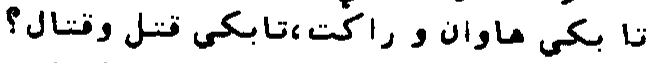

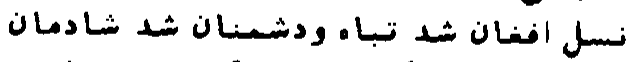

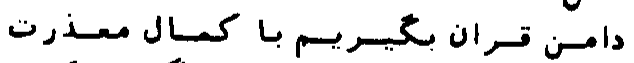

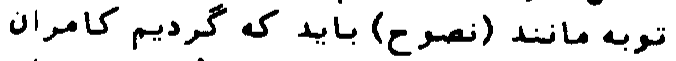

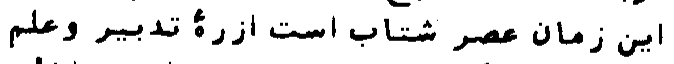

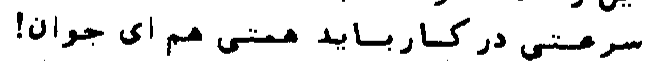

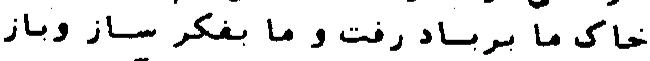

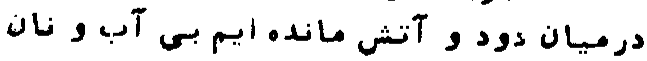

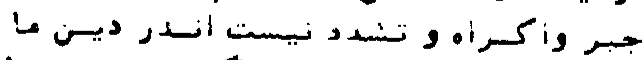

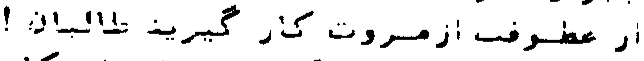

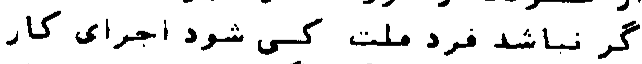

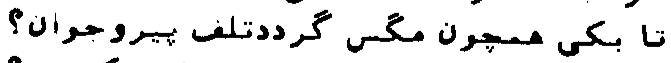

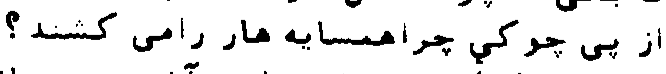

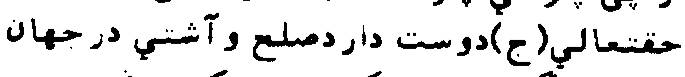

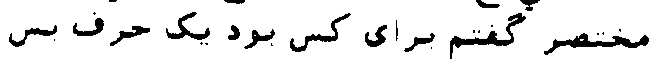

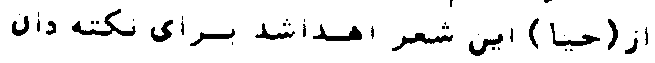

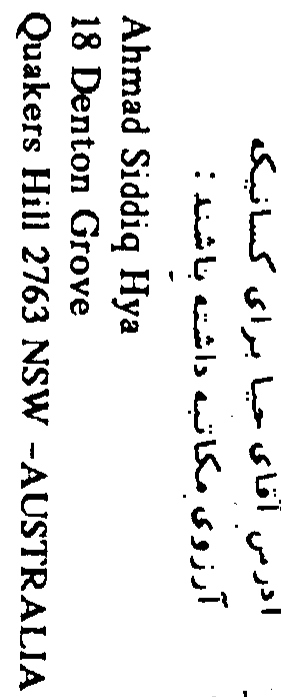

alon isango

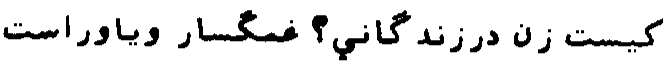

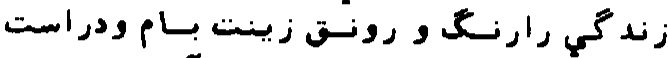

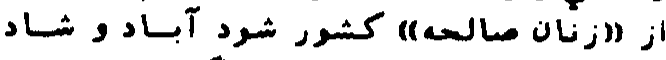

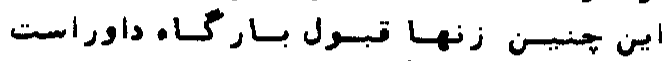

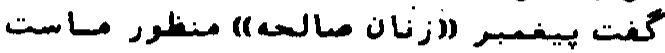

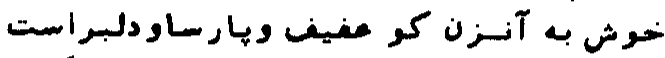

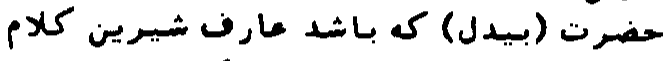

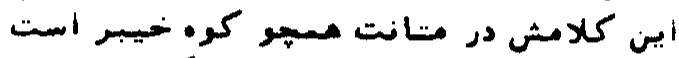

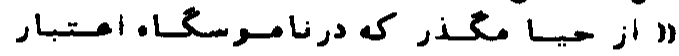

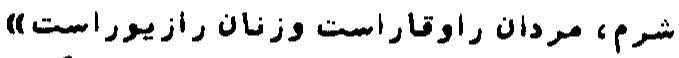

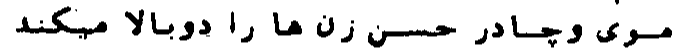

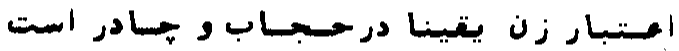

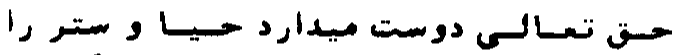

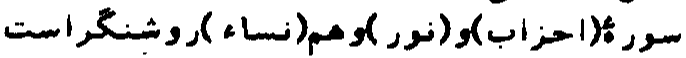

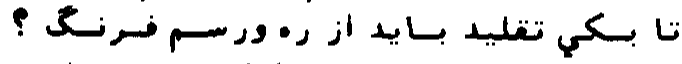

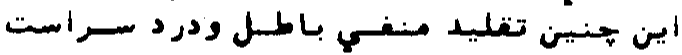

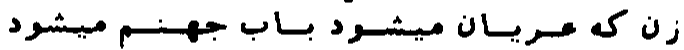

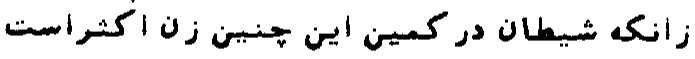

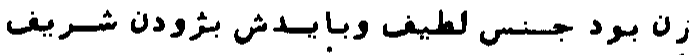

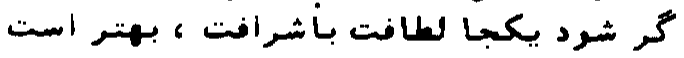

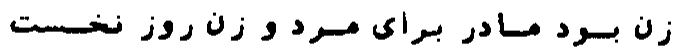

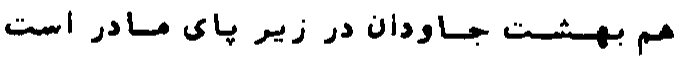

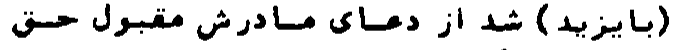

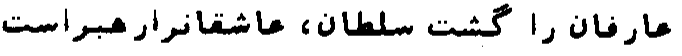

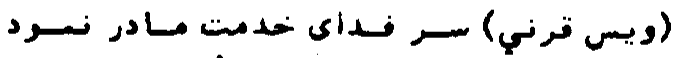

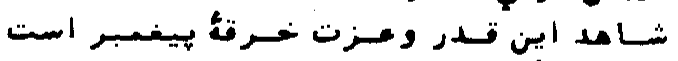

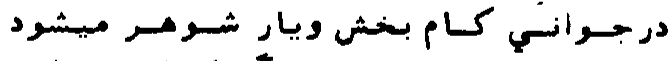

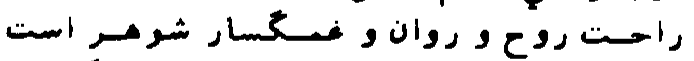

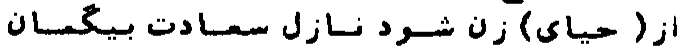

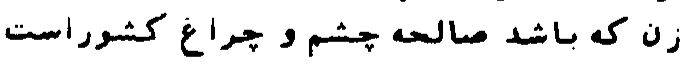

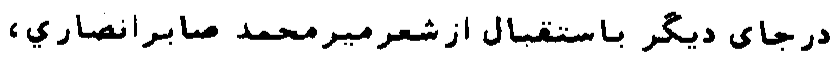

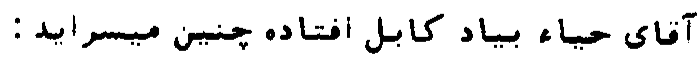
كالزال كابل

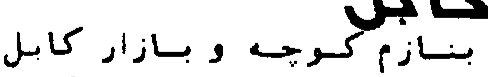

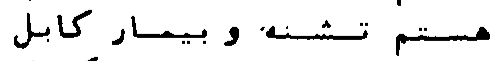

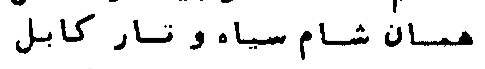

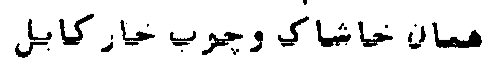

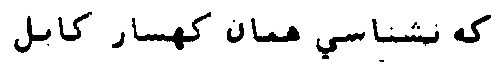

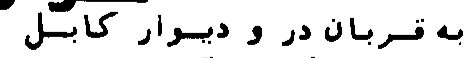

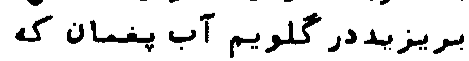

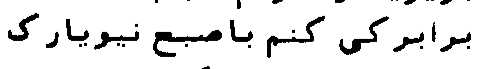

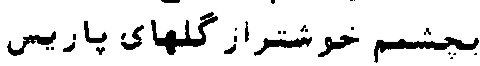

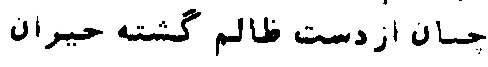




\section{1}

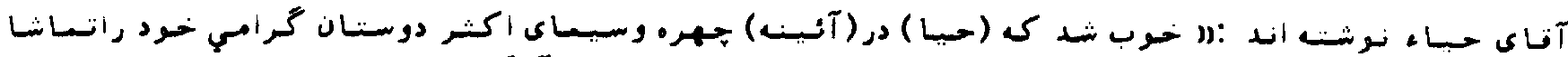

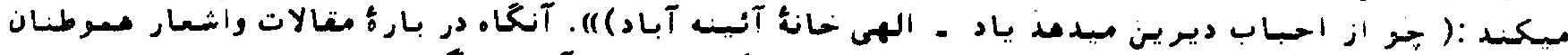

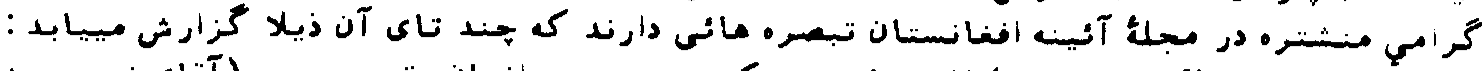

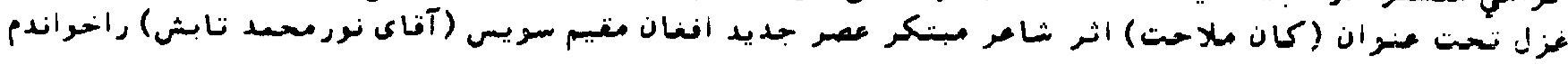

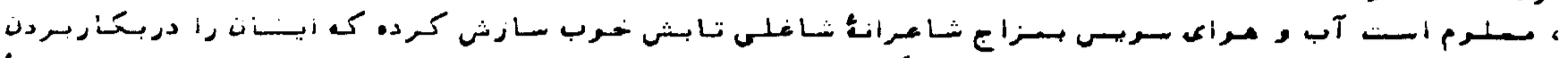

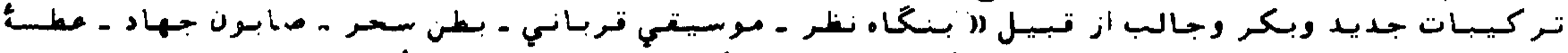

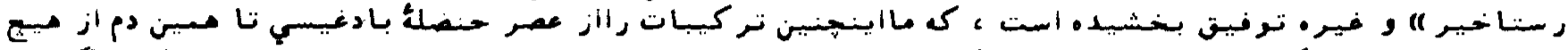

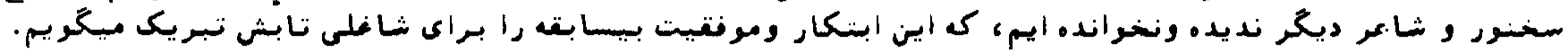

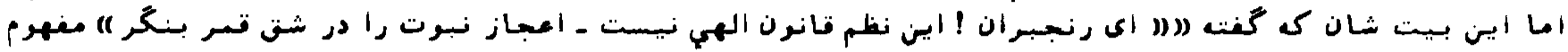

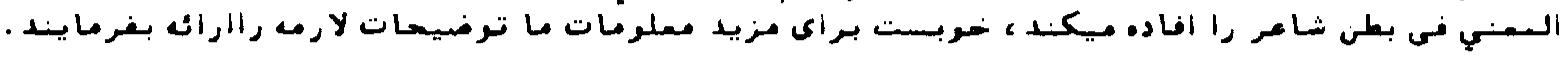

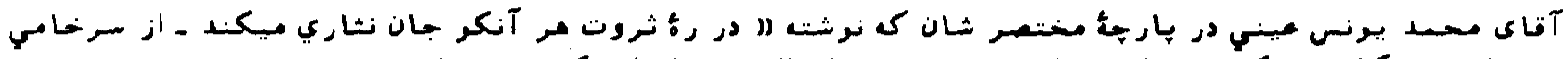

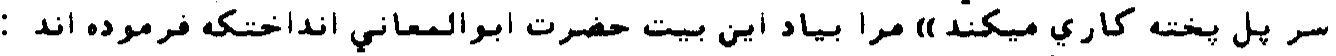

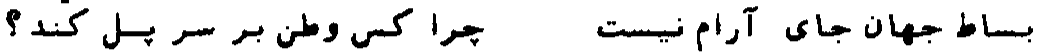

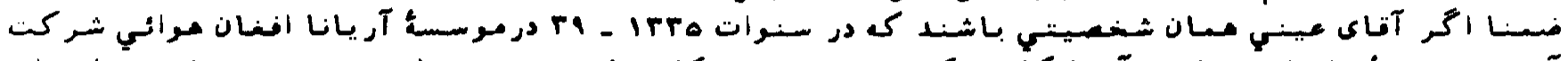

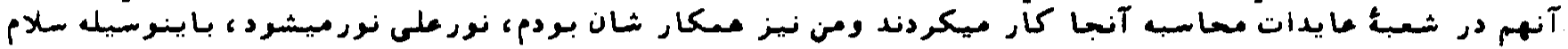

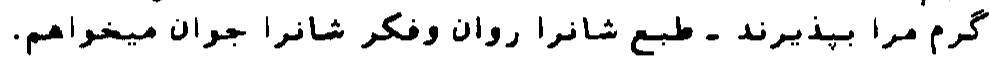

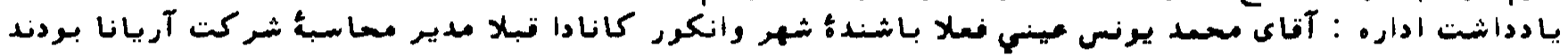

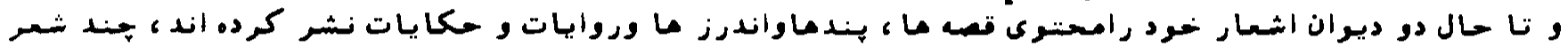

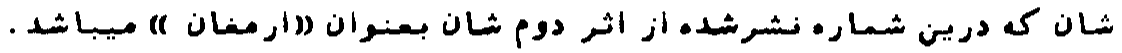

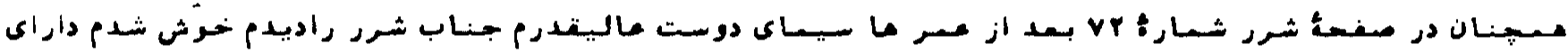

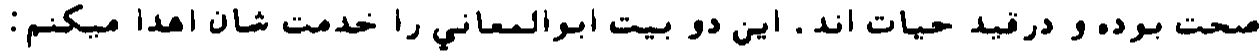

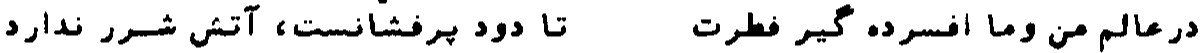

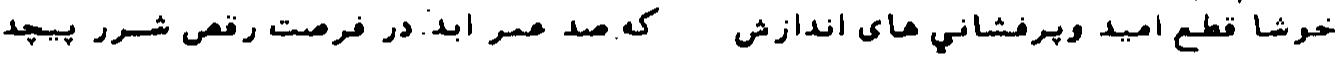

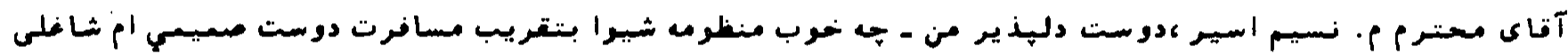

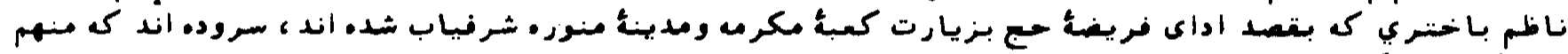

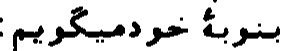

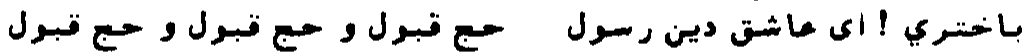

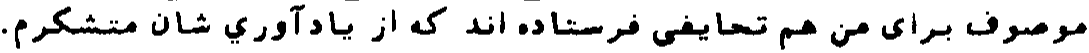

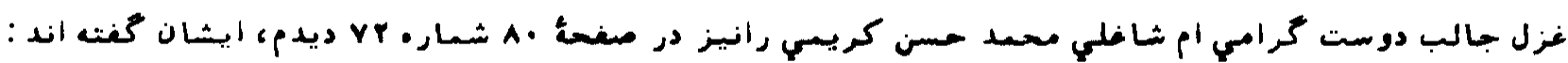

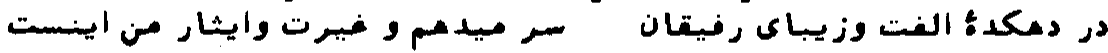

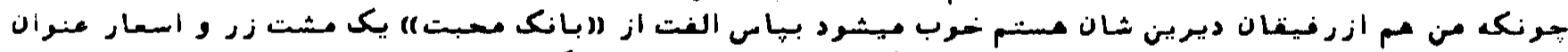

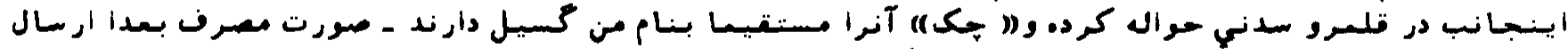

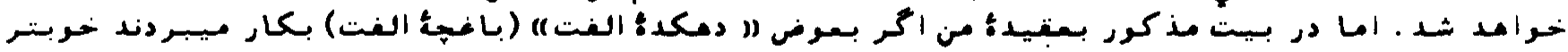

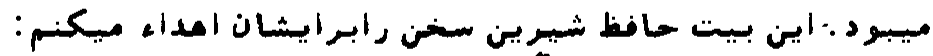

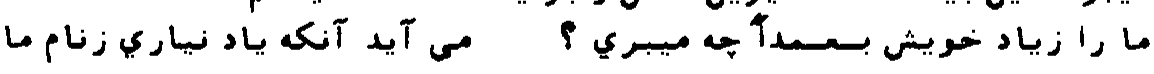

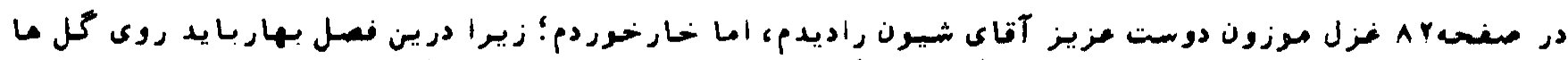

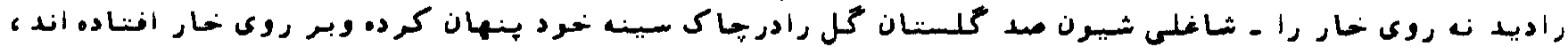

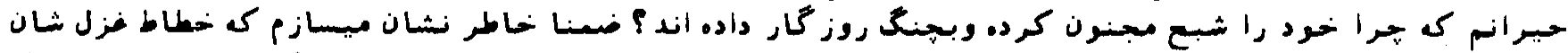

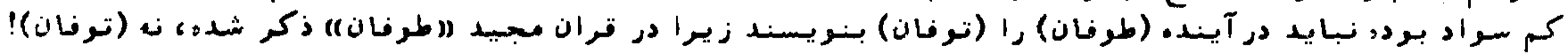

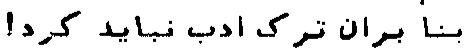

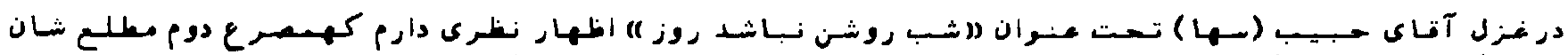

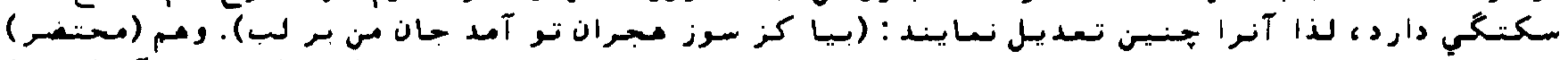

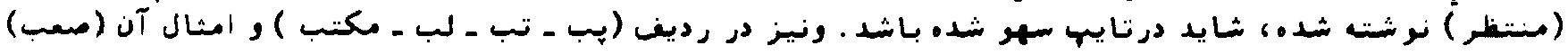

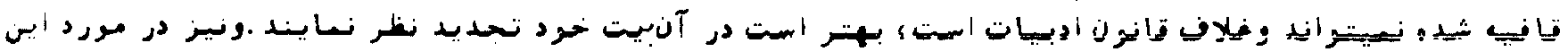




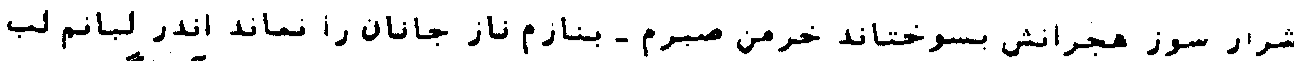

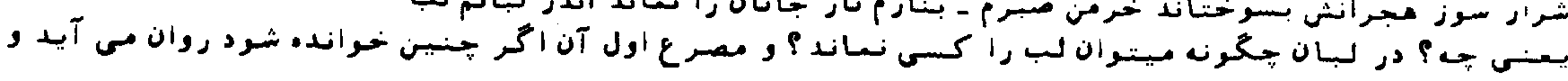

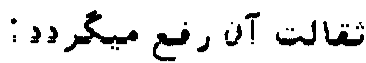

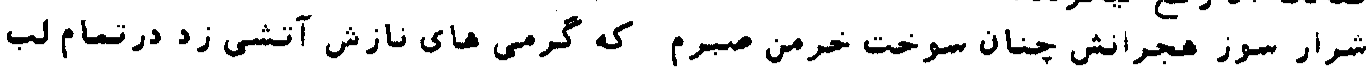

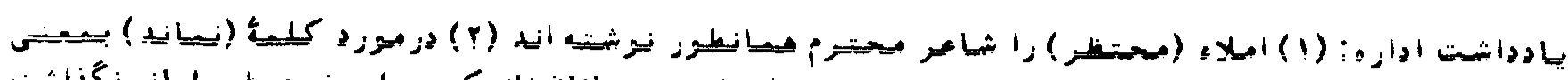

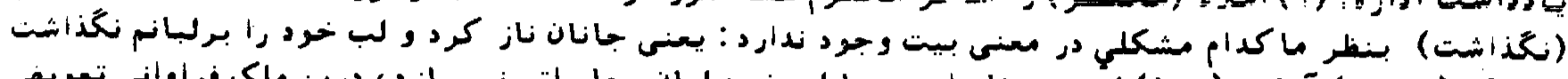

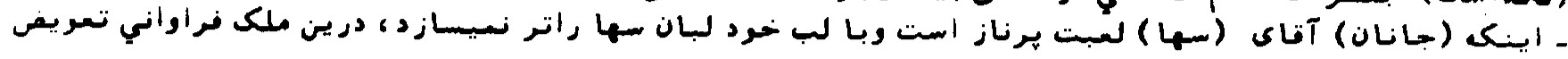

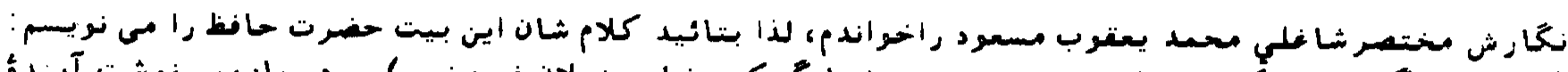

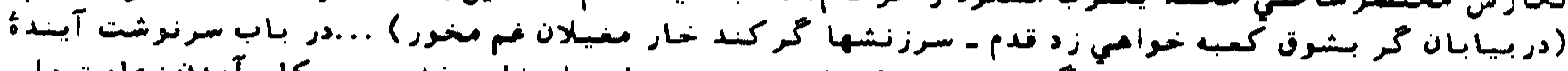

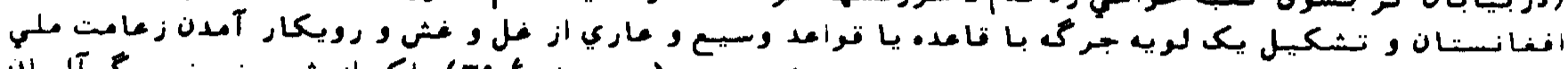

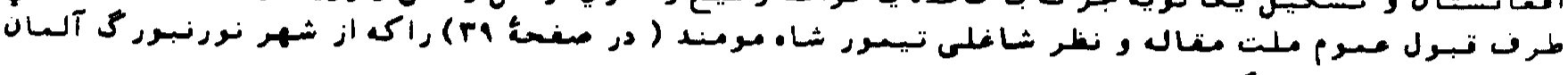

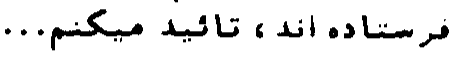

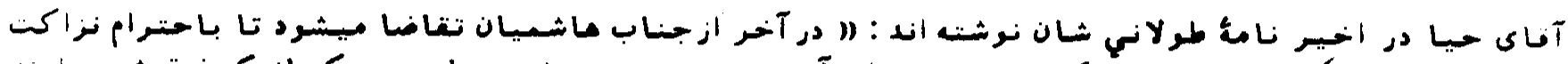

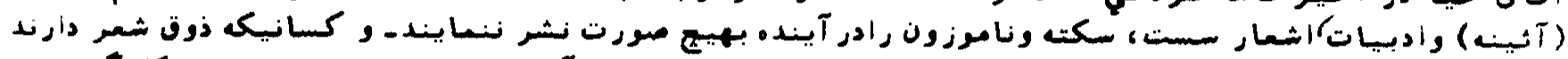

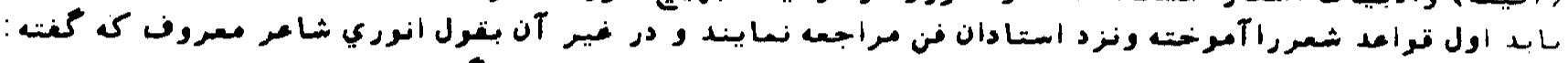

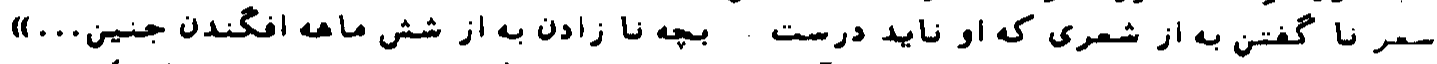

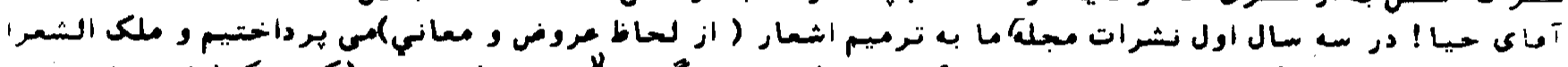

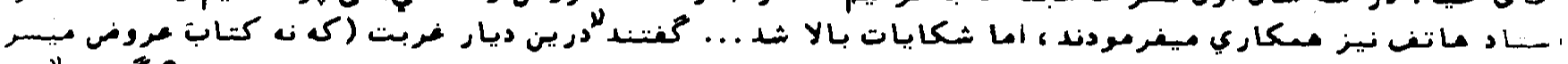

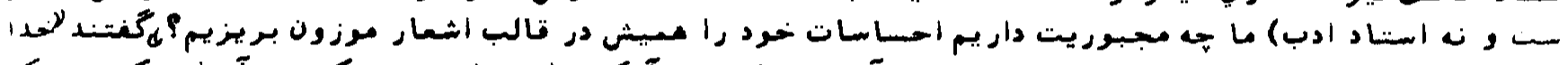

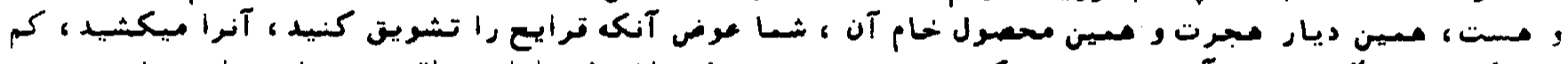

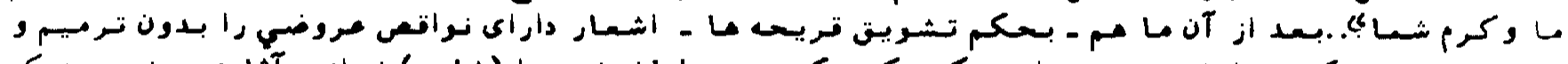

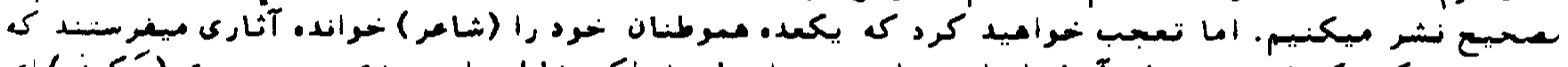

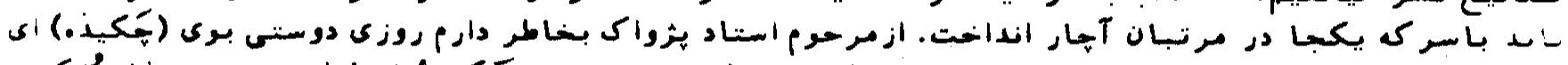

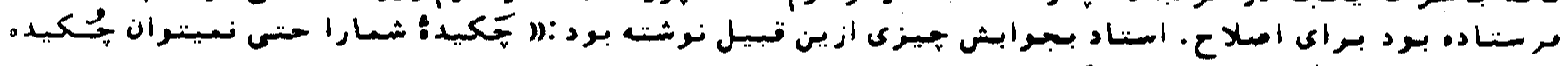

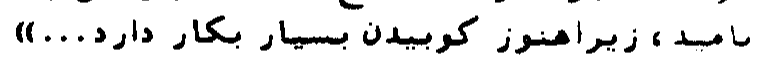

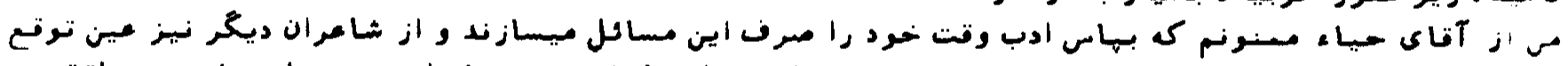

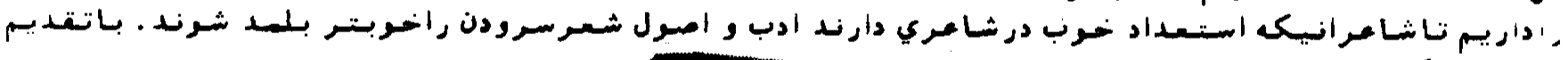

\section{Iا Iا}

ازئر

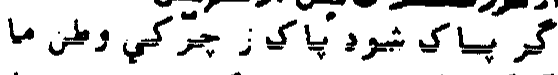

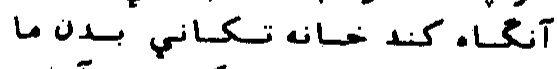

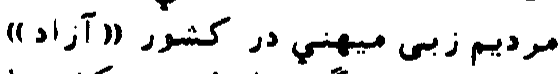

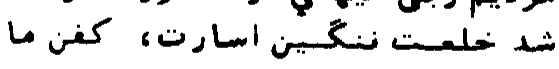

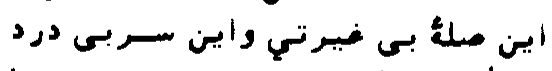

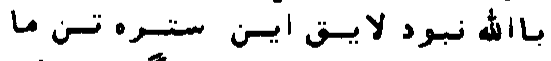

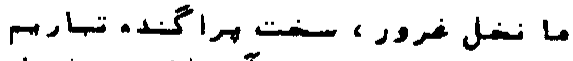

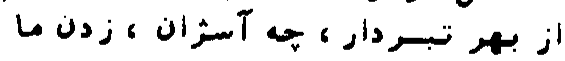

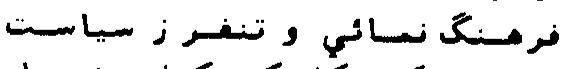

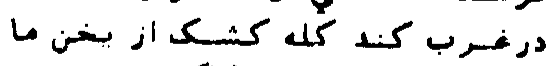

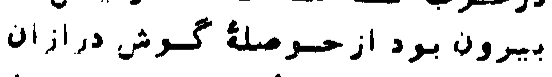

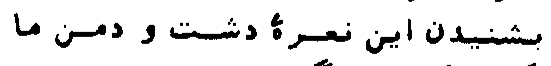

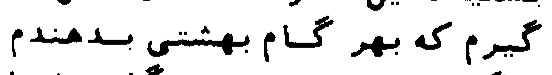

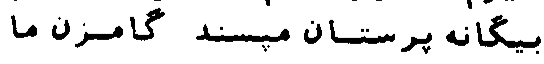

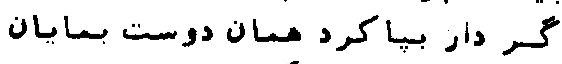

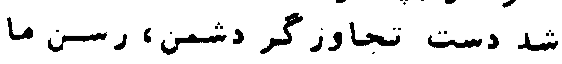
'ب

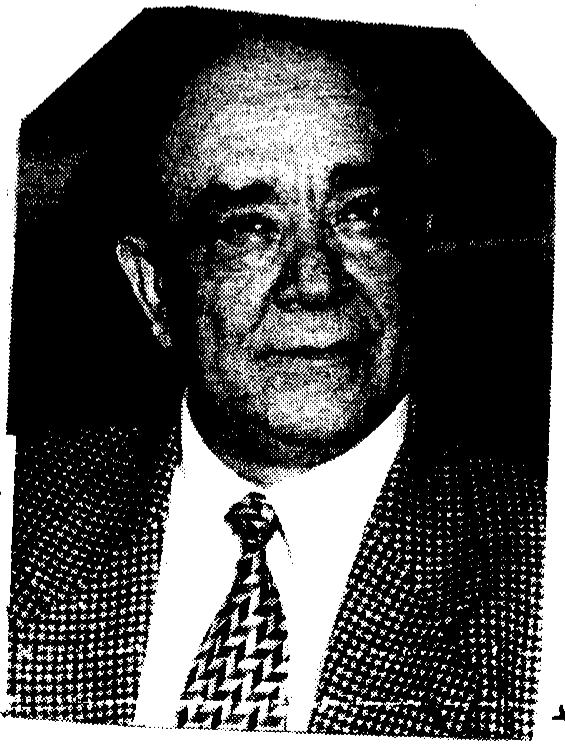

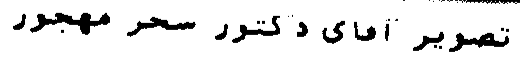
r i-

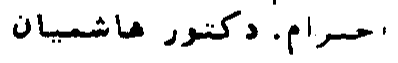

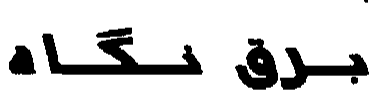

از دكتور سـدر ممسبــور

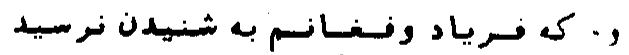

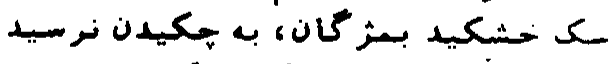

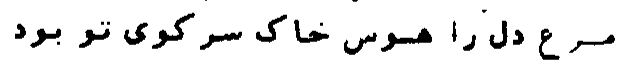

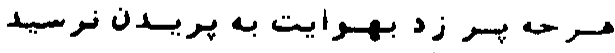

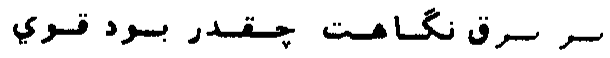

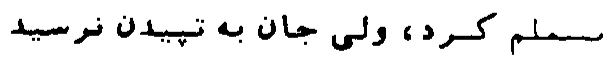

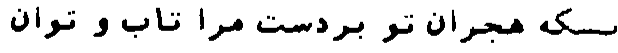

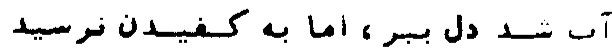

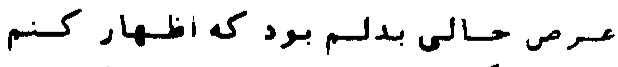

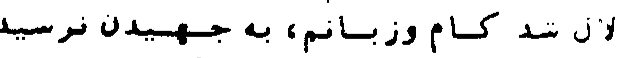

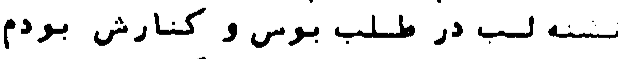

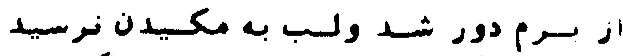

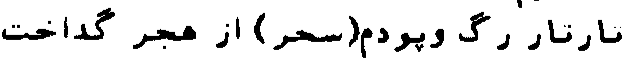

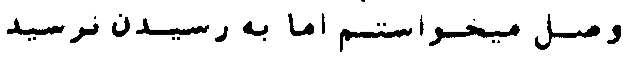


120 (2)

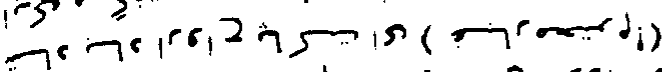

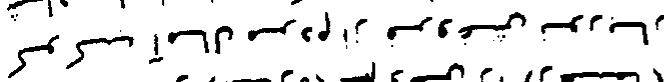

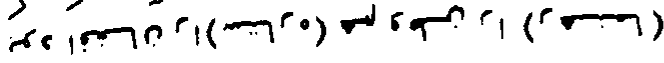

(r)

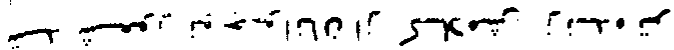

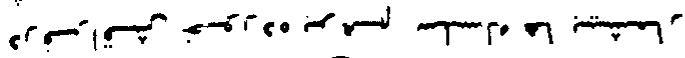

,

נו

(" s s

is

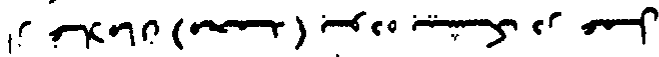

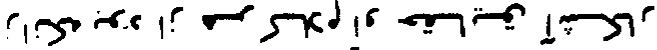

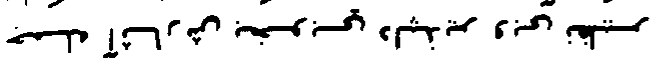

cm

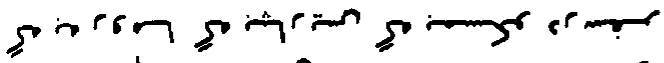

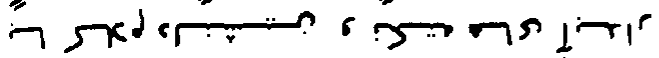

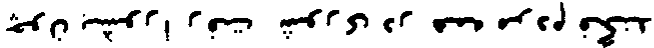

r

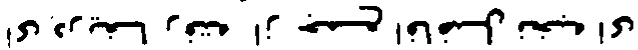

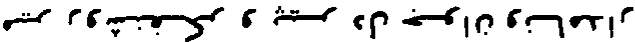

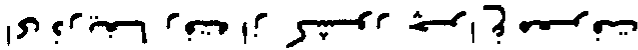

ח

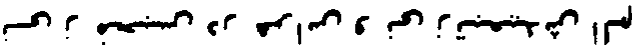

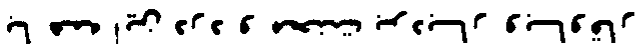

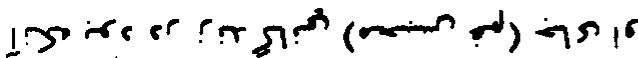

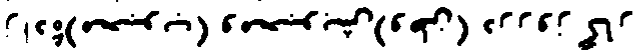

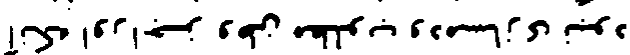

רים

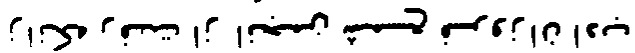

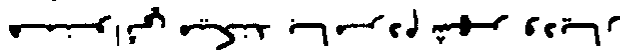

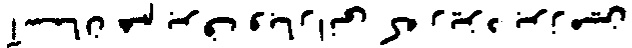

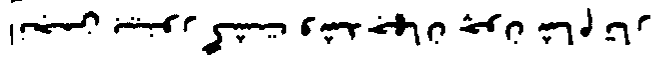

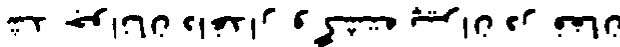

|

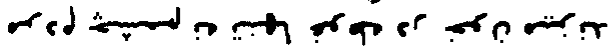

רים

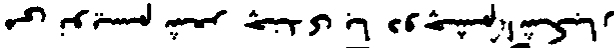

\section{etrin ${ }^{\circ}$}

תו

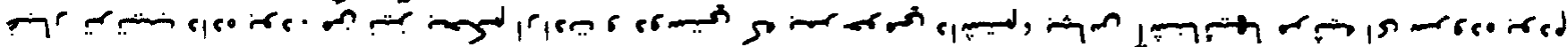

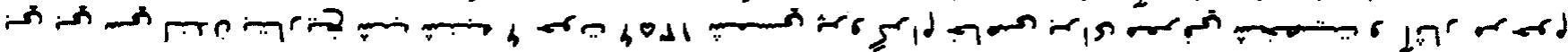

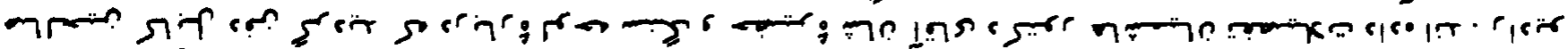

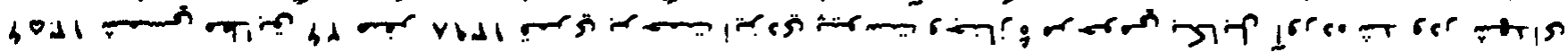

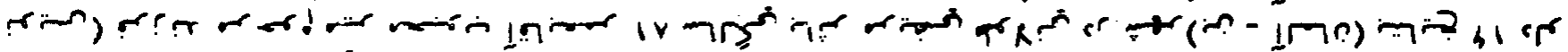

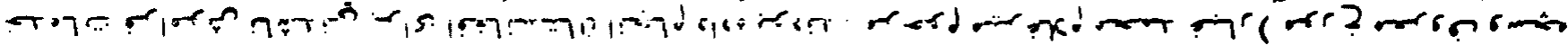

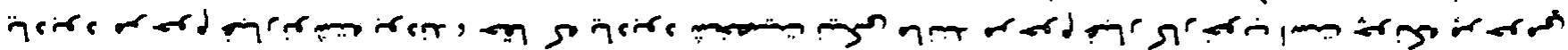

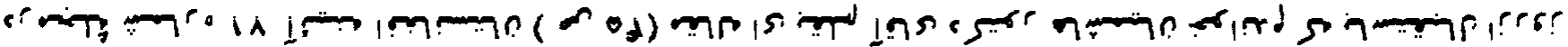

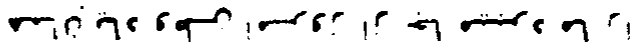
is 2 s a צ'

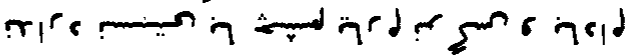

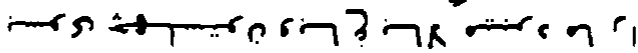

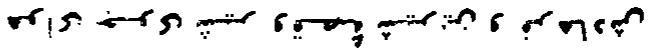

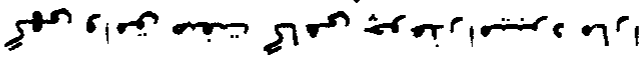
ra

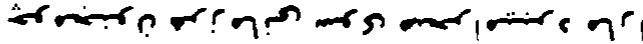
ח

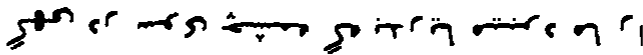

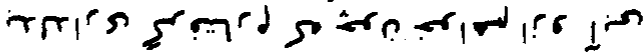
क्ष

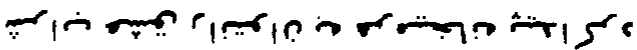

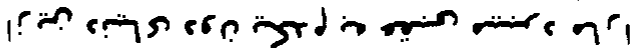
sing

\section{ne6re}

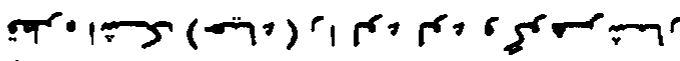
arn?

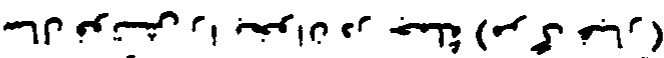

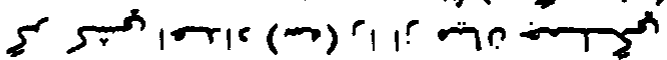

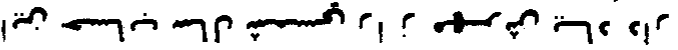
רים "

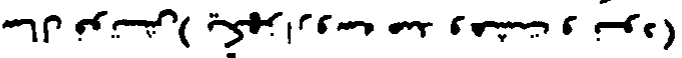

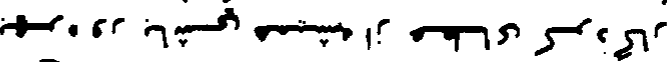

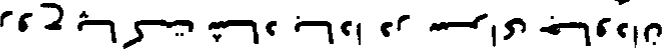

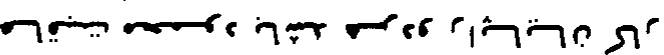

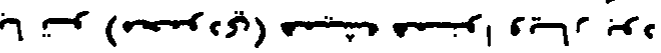

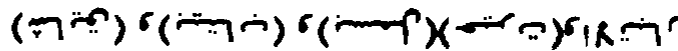

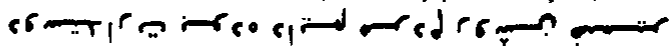

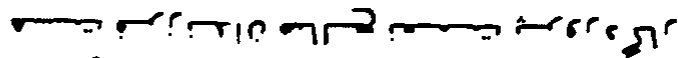

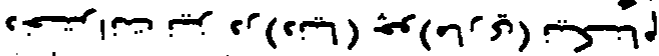

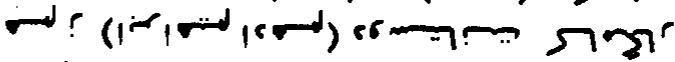
(1) is (x)

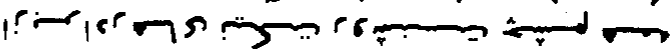
"2rem

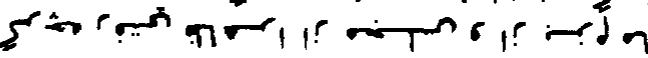

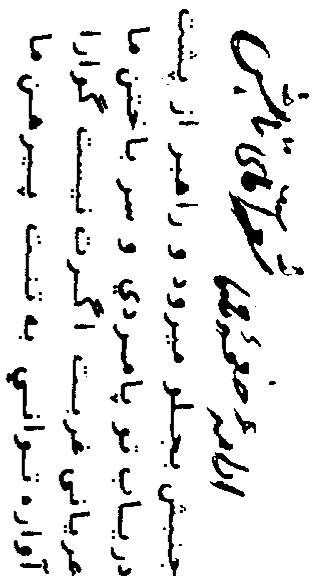




\section{صفحذ هاعد تواناهصهد نسيم السيد 65 \\ غنيست است

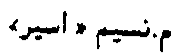 \\ باور عشئ}

باتتديم احترام و سلام. بعرض ميرسانم كه. دفاعية آتاى عيدالسمدومابزاده فرياررا

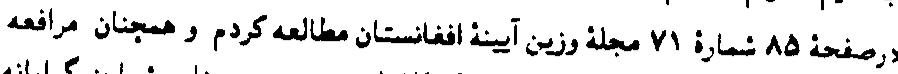

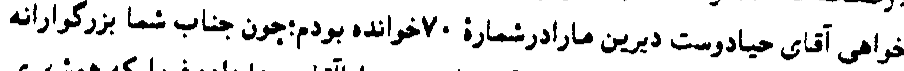

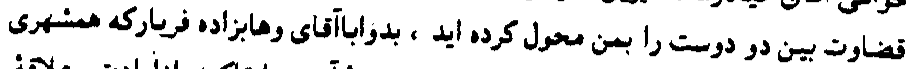

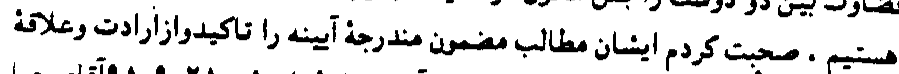

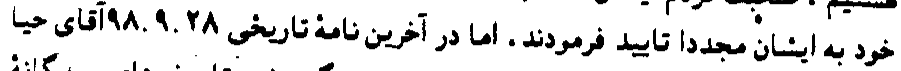

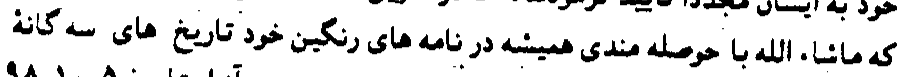

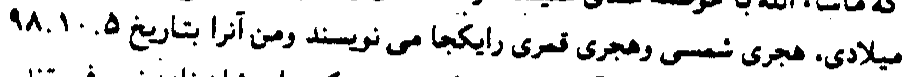

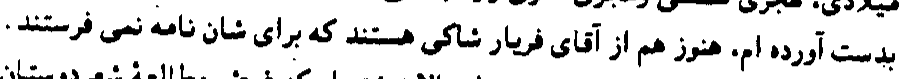

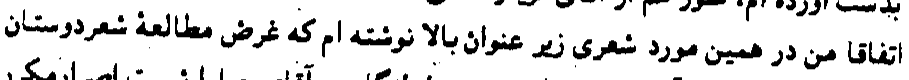

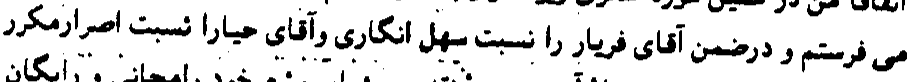

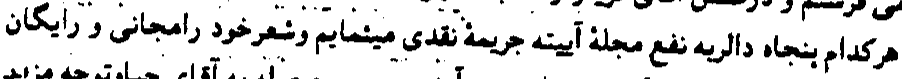

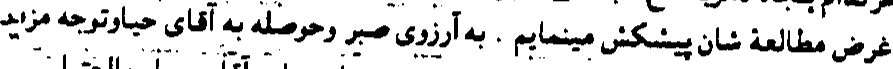

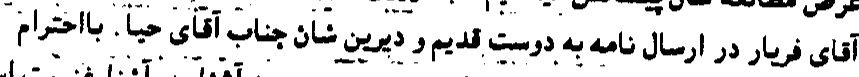

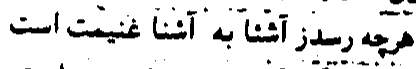

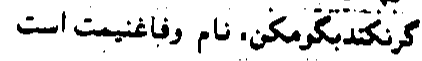

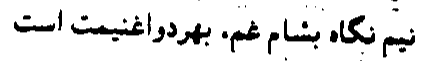

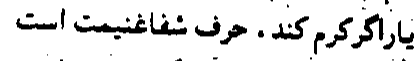

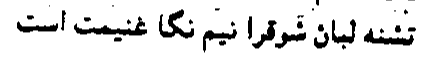

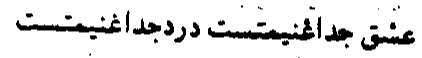

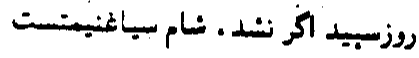

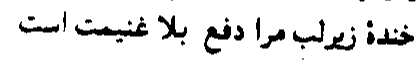

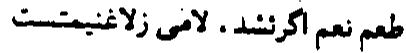

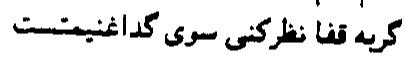

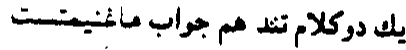

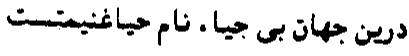

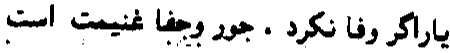

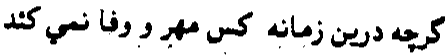

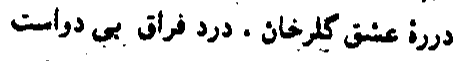

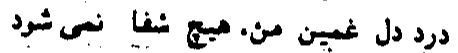

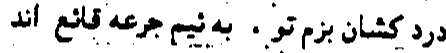

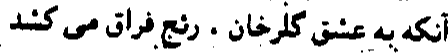

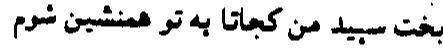
كرجه زقهرت هرزمان، سيل بلا مبي رسي

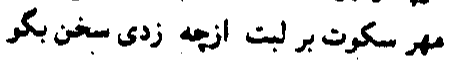

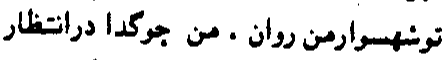

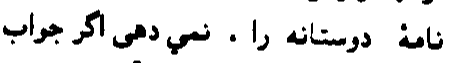

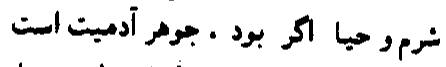

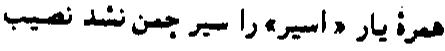

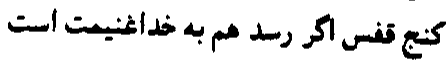
بمتاسبت يرتاب راكت امريكا.

\section{درافغانستان وسودان}

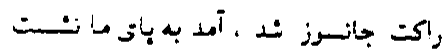

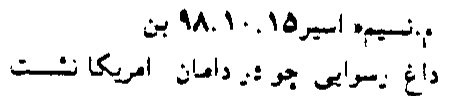

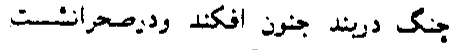

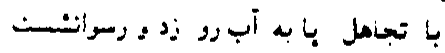

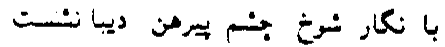

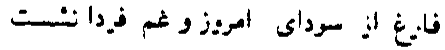

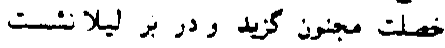

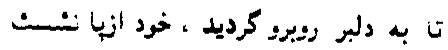

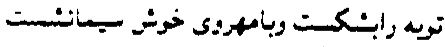

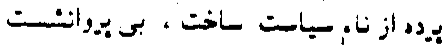

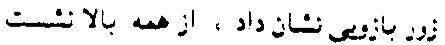

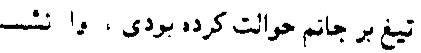

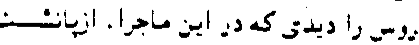

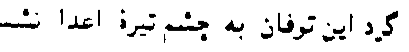

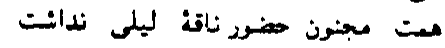

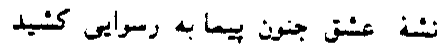

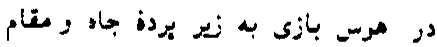

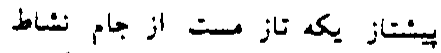

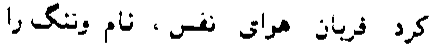

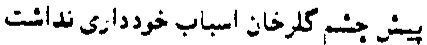

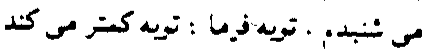

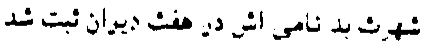

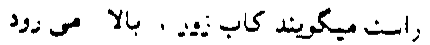

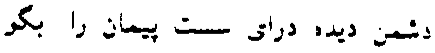

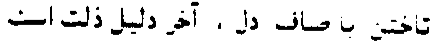

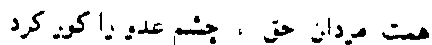

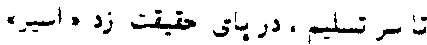

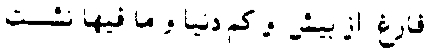

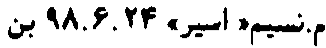
كنيـده سرجوخورثبد جهان أزخاورعنتم

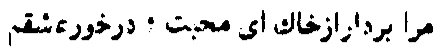

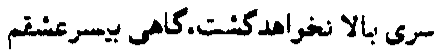

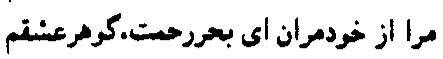

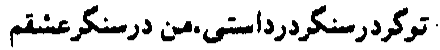
كيرافزوده ساقى جرعه أى درساغرعنيتم جه بيجا ناز فرمايى كه من در آخر عنداني

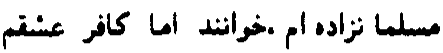

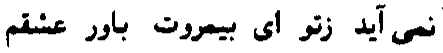

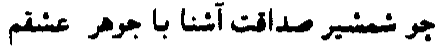

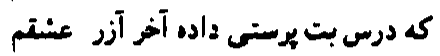

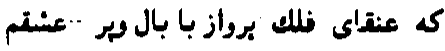

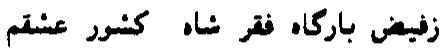

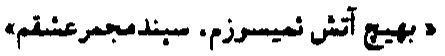

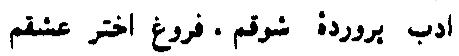

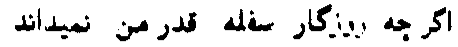
غمث مبران توكيه زمينكير و خرابم كرد

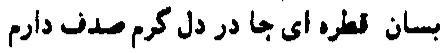

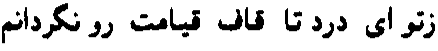

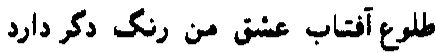
جه بيجا جلوه بغرونس كه تو دراول نازى ئي بنسوربيخودى مشسرون كفرودين نسيكنجد

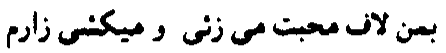

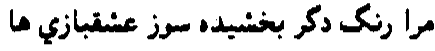

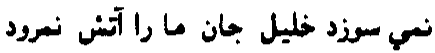

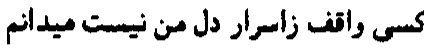

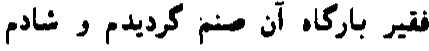
دل از آتش زبانيهاى بيدل ندكه ميكميد

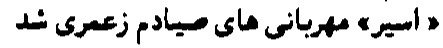
كه المكنده ابت تار الفتى در نهمبرعنتم

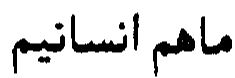

رأى امتسداد با تأييد سبحانى كند

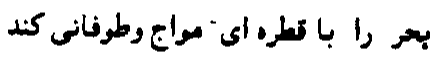

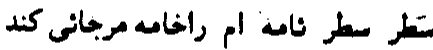

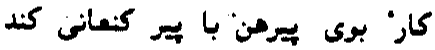

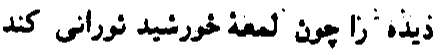

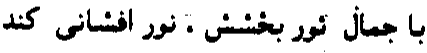

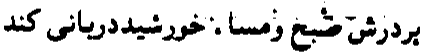

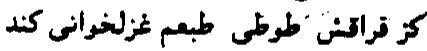

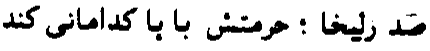

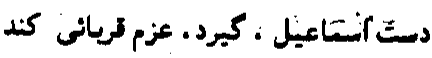

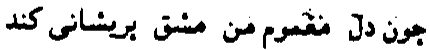

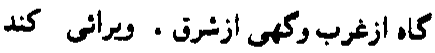

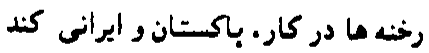

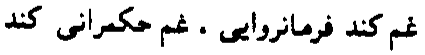

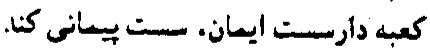
مهد اسلام است ودعواى مسلسانى كند

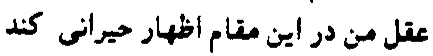

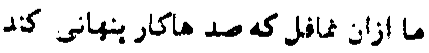

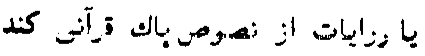
منـكل انزونى كذارد ـ منسكل آسانى كند آند

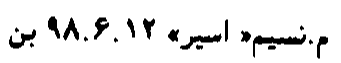
طاير ظبعم اكر عزم نير افنسانى كند

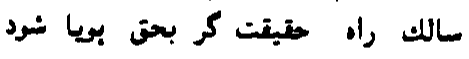

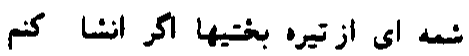
يوسف كمكنته اي دارم كه يادن با دلم كر مبا عطرى ازان ككنتنه آرد بر مسنام

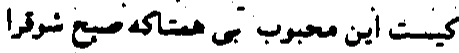

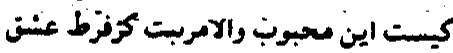

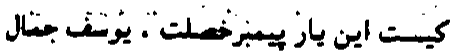

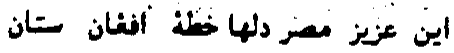

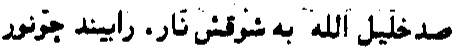

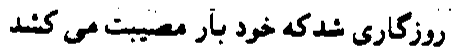

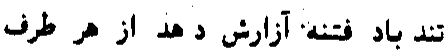
باك و نا باكم نده سر بار كنتى مراد دراد

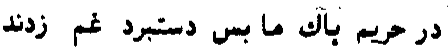
كعبه فم درسوكى ماكياسيه بونبيده است

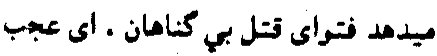
زمد وتسبيح وردا ـ اين بعله تذوير وريا

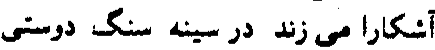

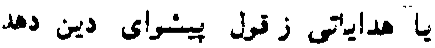

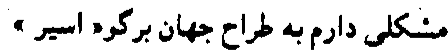

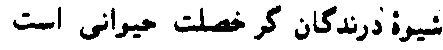
ماخود انسانيم •با ما كار انسانى كند كند 


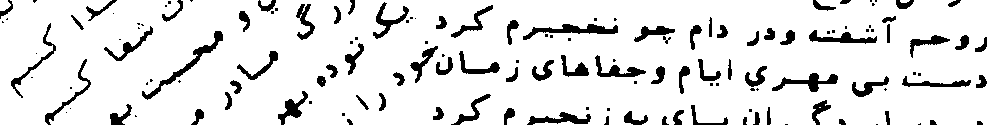

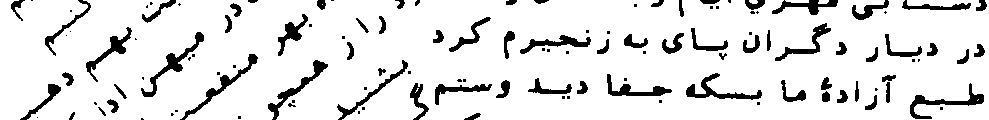

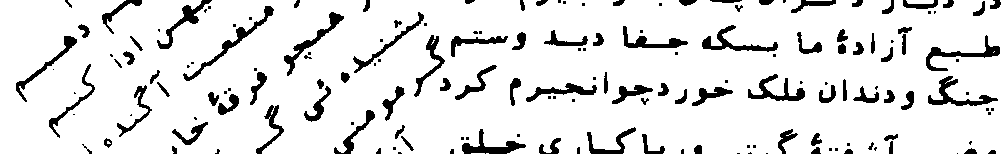

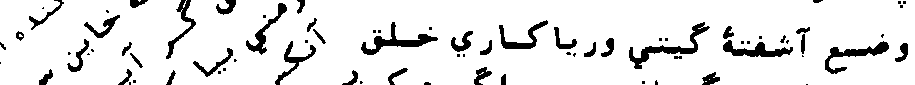

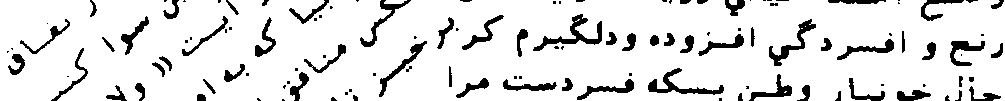

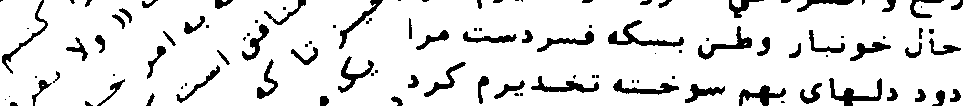

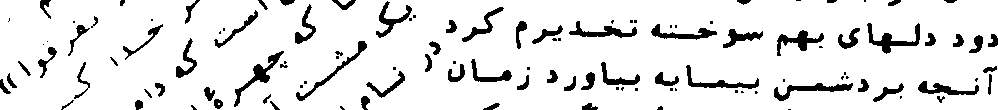

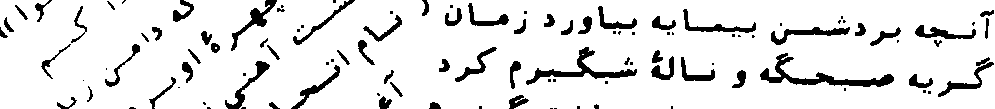

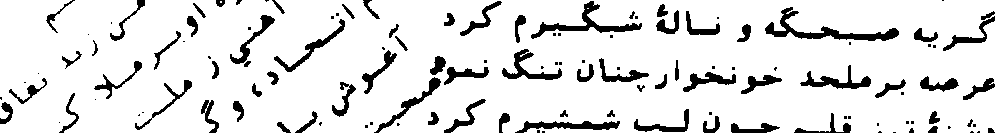

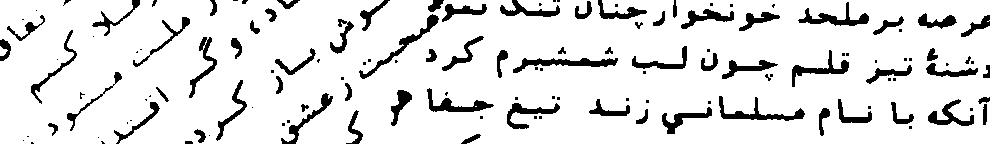

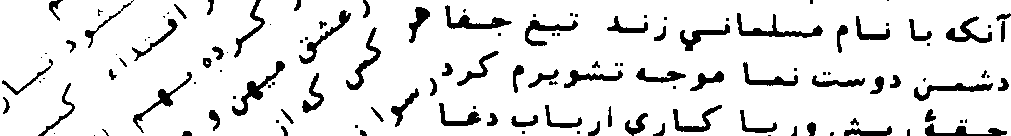

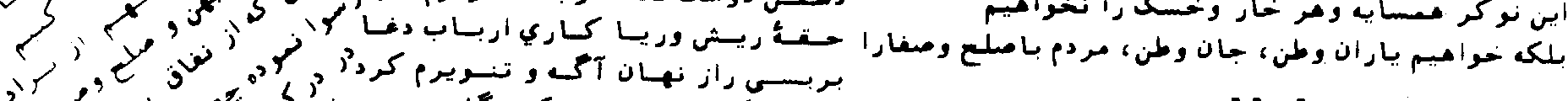

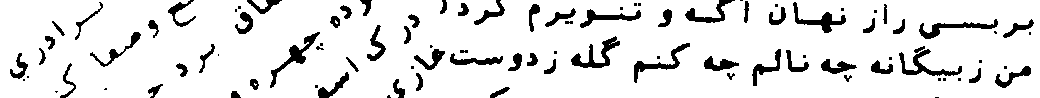

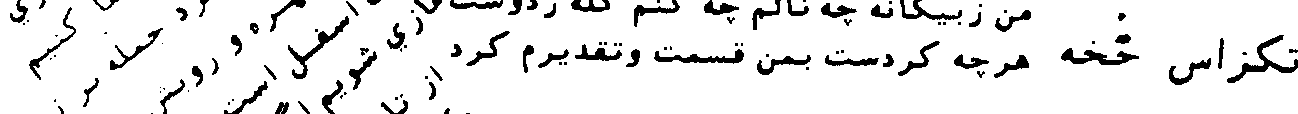

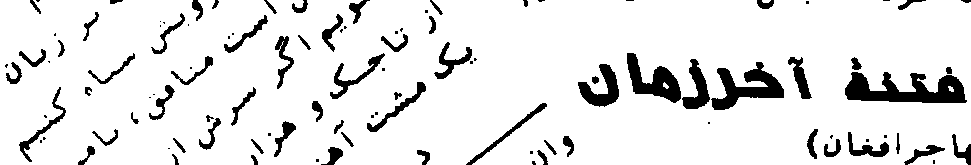

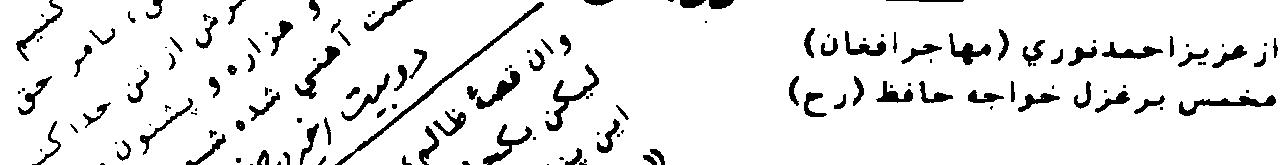

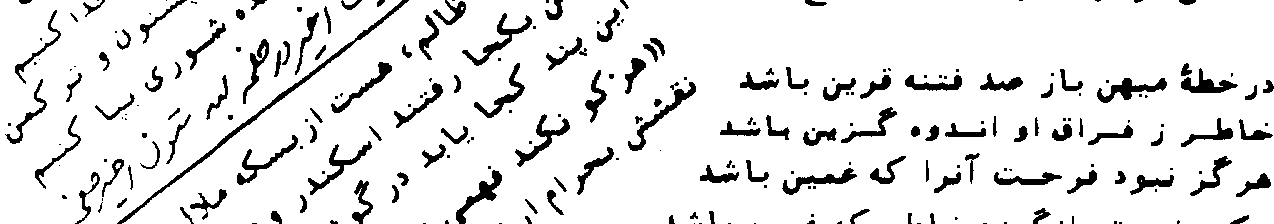

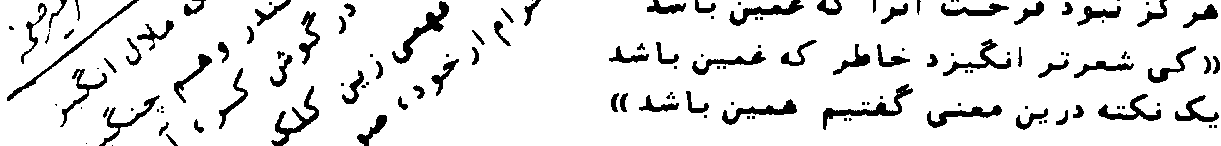

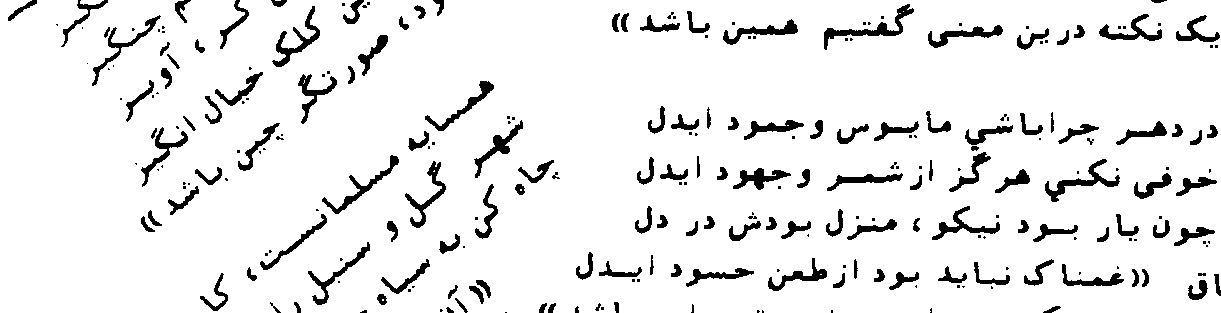

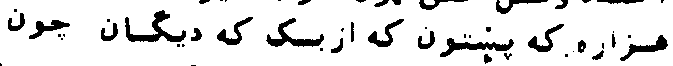

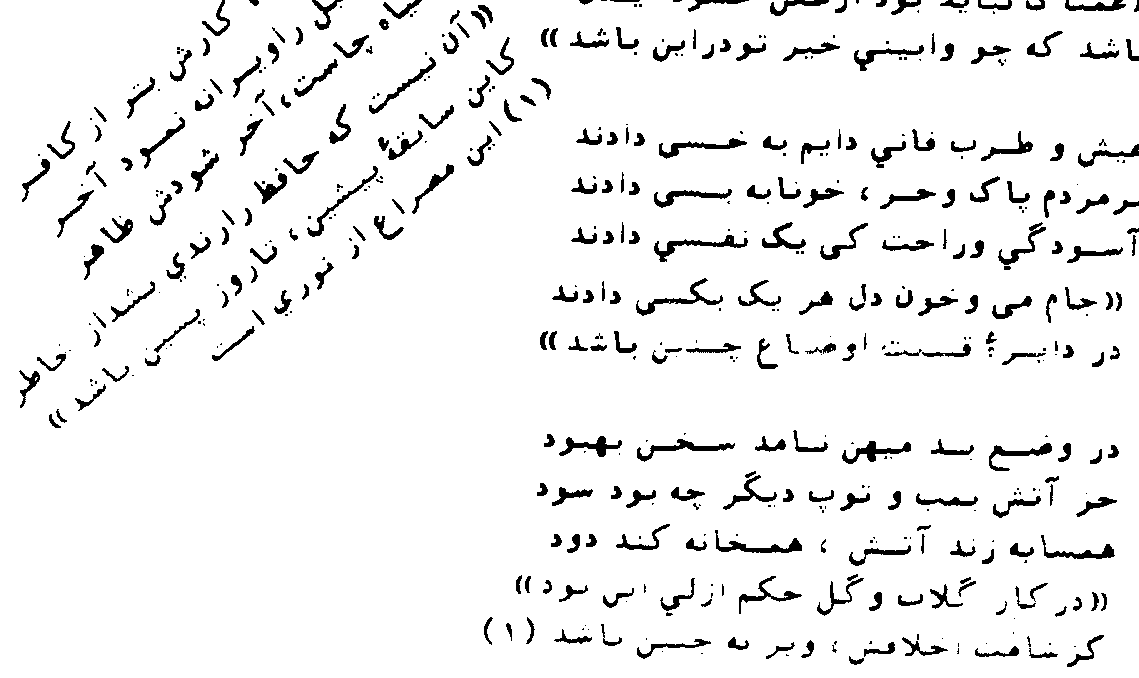

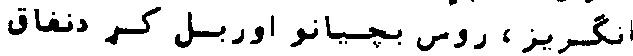

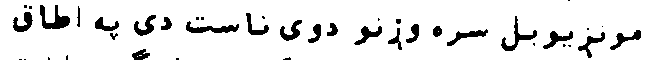

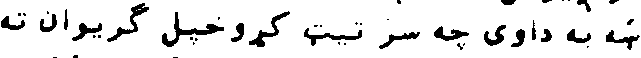

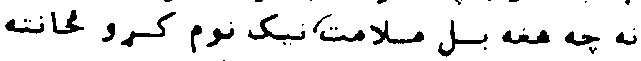

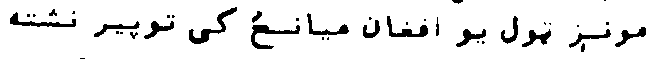

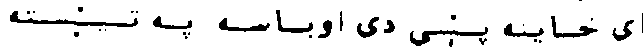

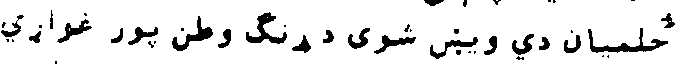

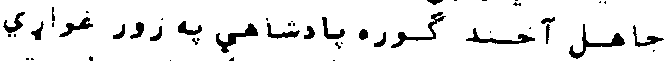

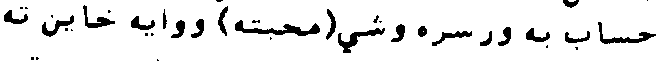

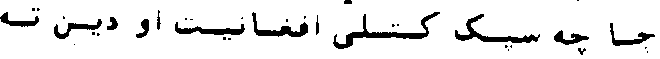




\section{صفحف شاعرتواناالحاج خليل الله شاظم باختلي

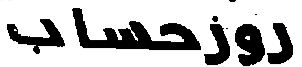

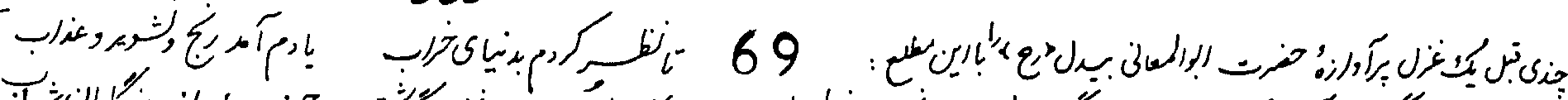

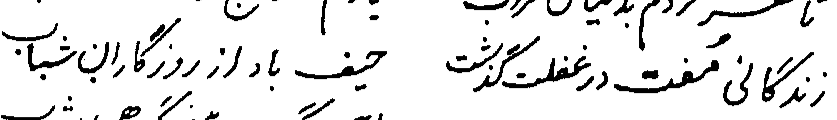

و و

برن

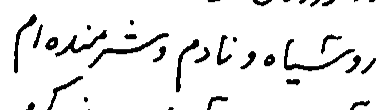

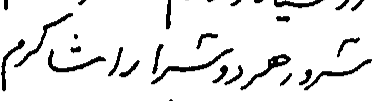

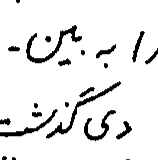

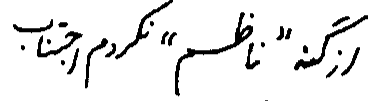

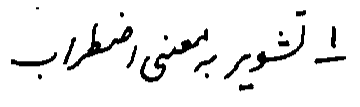

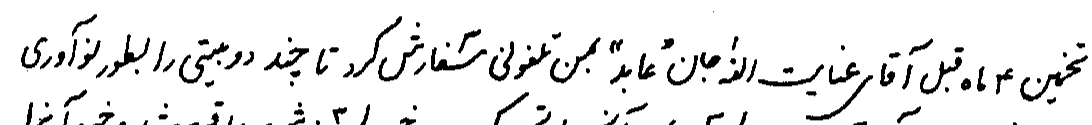

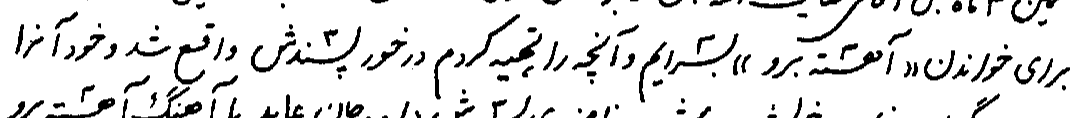

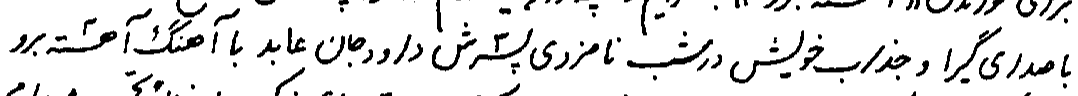

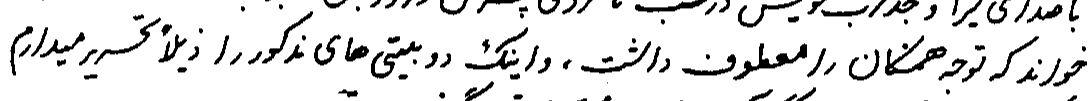

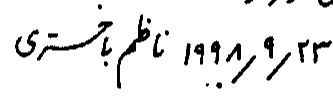

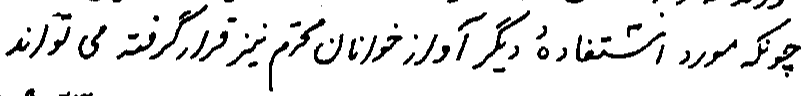

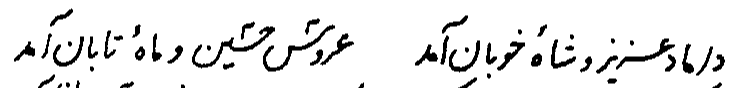

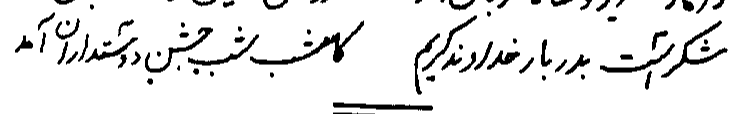

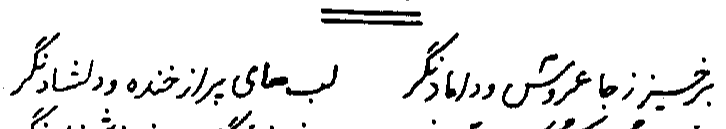

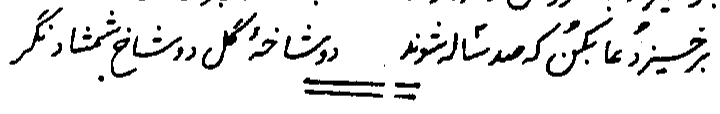

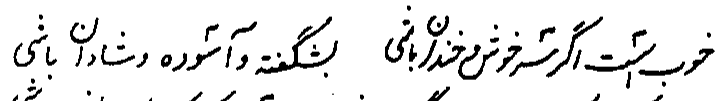

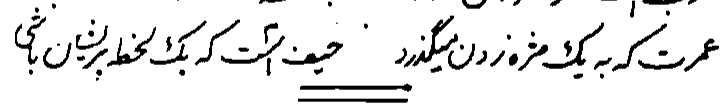

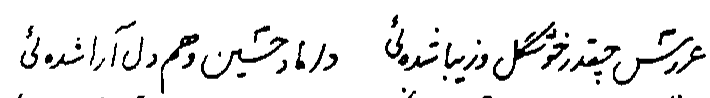

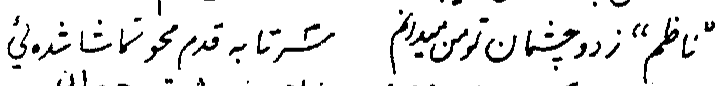

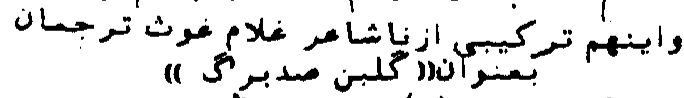

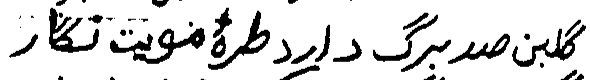

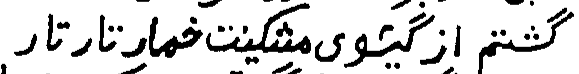

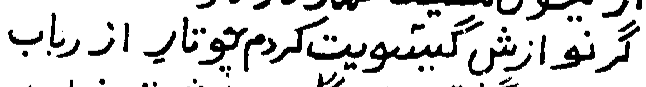

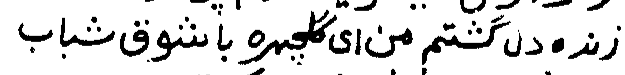

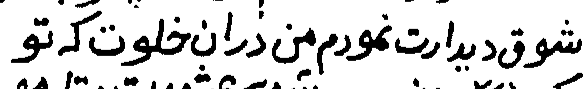

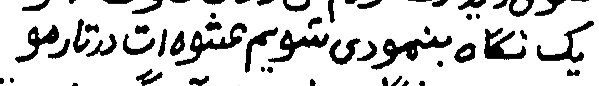

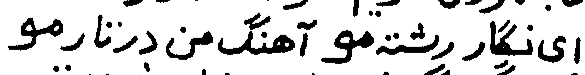

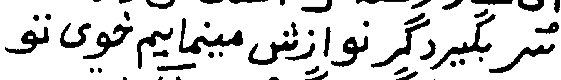

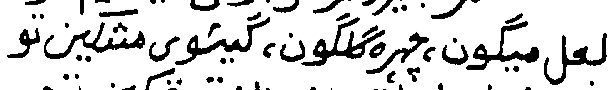

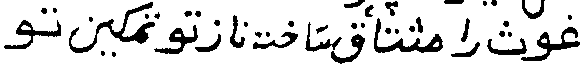

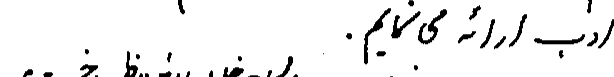

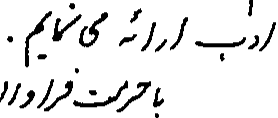

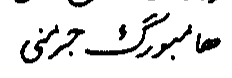

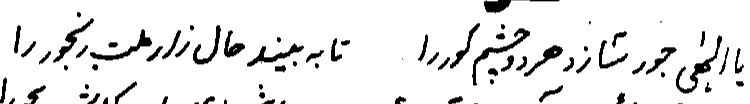

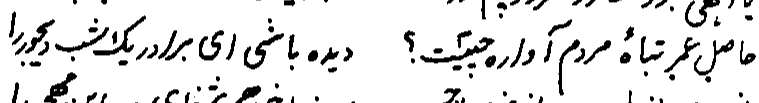

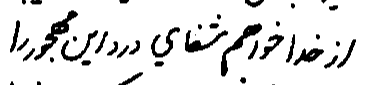

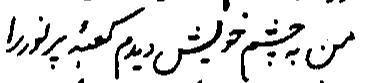

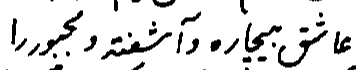

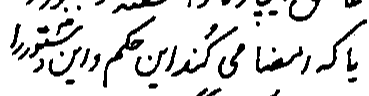
ק ب ه

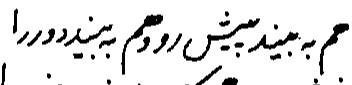

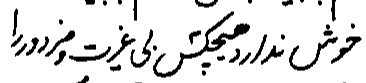

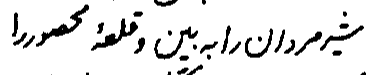

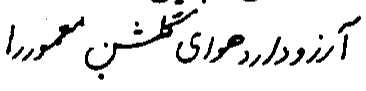

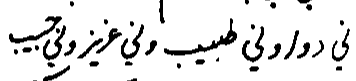
ת 行的割; بكن:

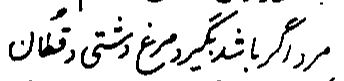

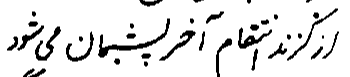
(B)

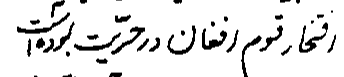
䒠

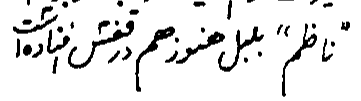

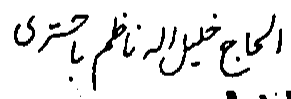

\section{عفو هجازات}

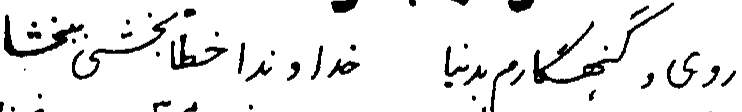
.

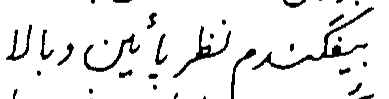

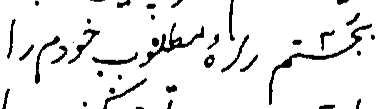

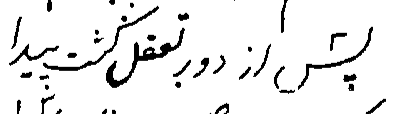

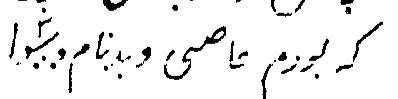

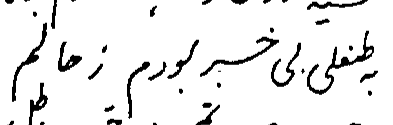

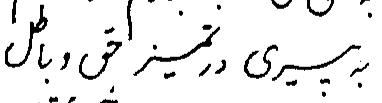
بـ 0 .

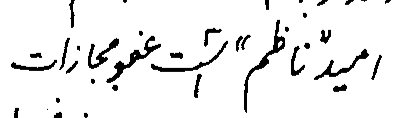

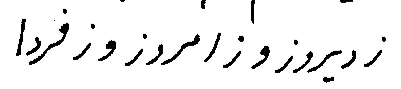

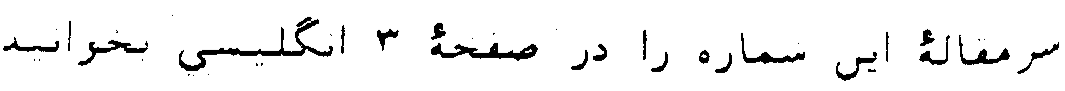




\section{كَدَ'}

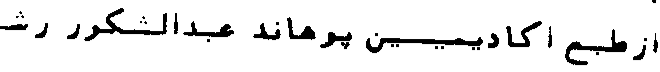

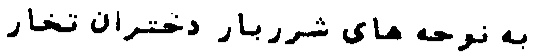

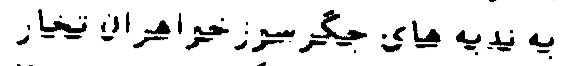

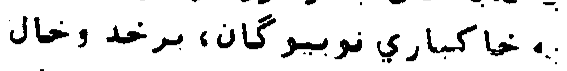

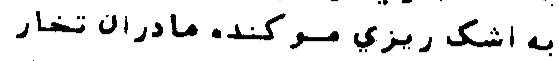

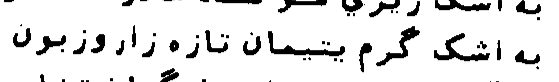

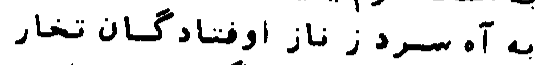

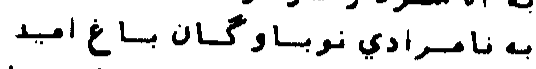

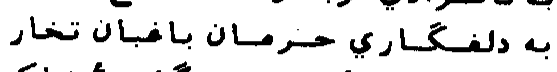

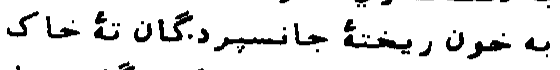

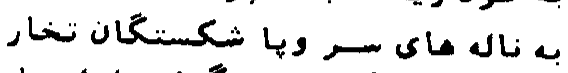

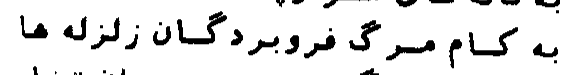

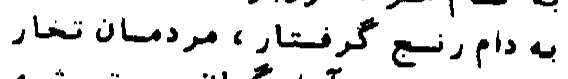
به

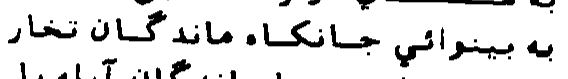

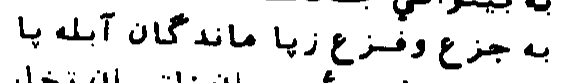

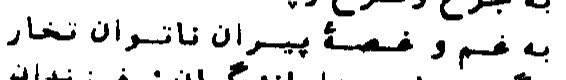

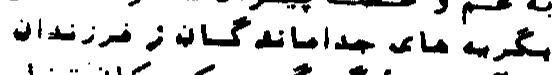

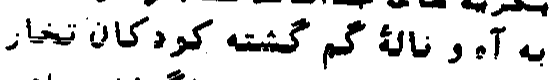

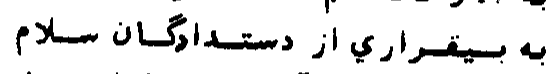

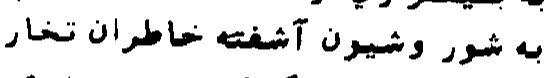

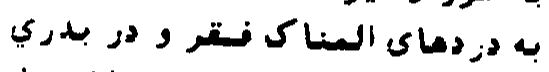

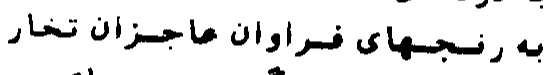

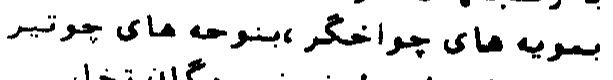

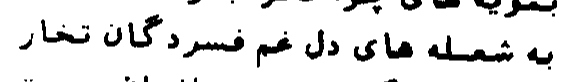

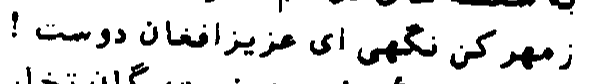

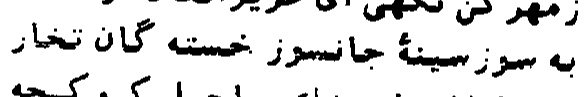

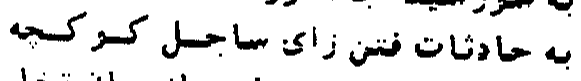

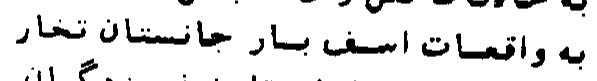

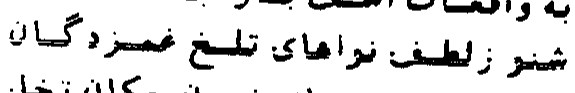

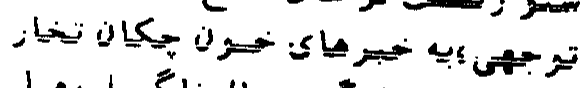

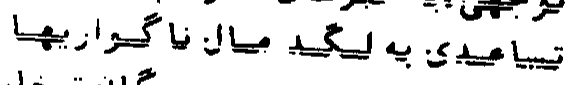

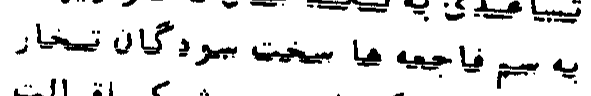

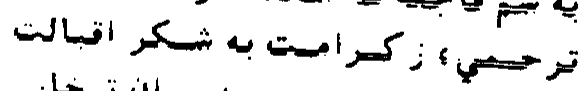

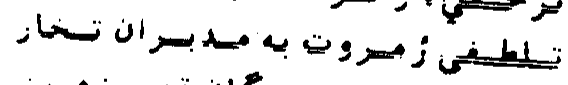

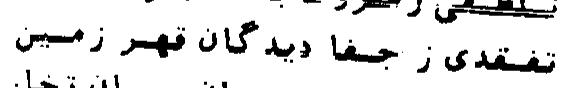

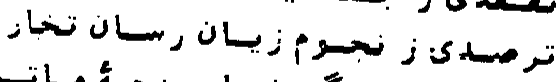

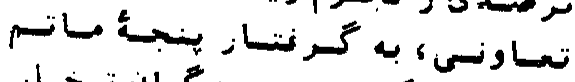

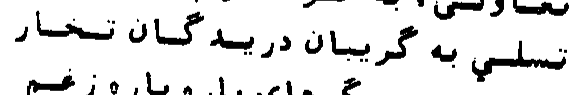

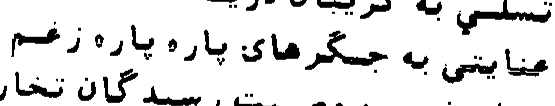

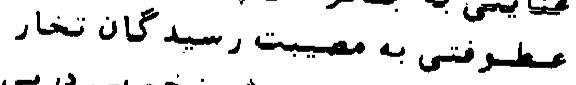

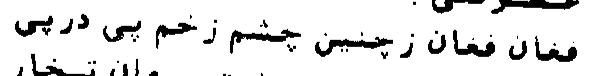

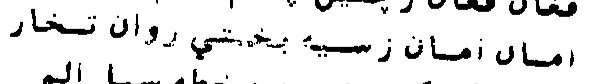

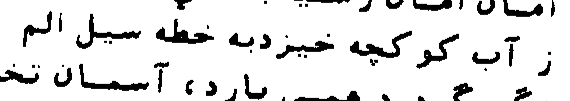

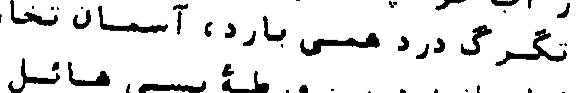

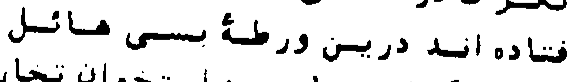

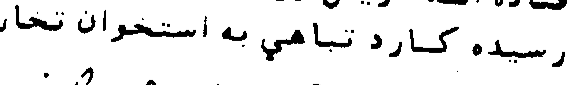

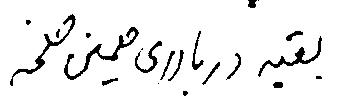

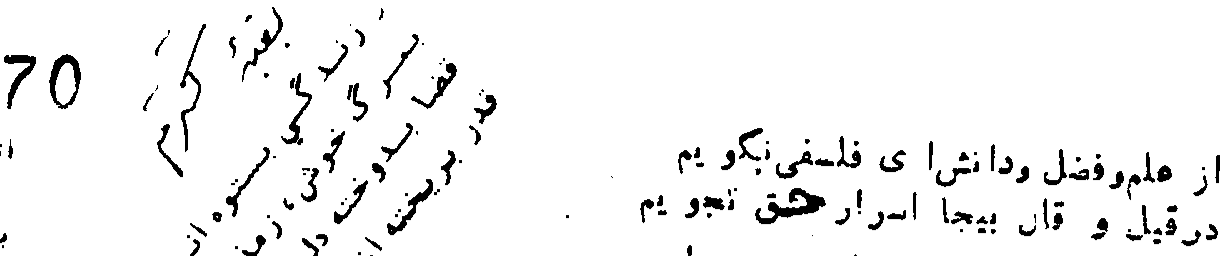

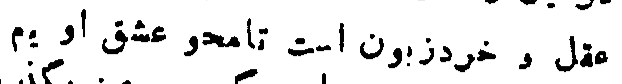

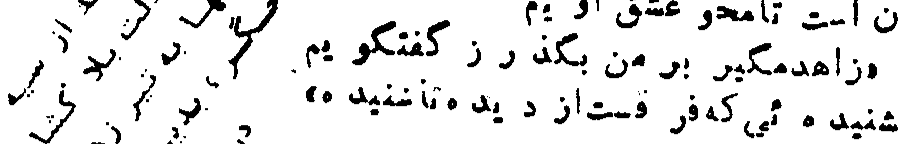

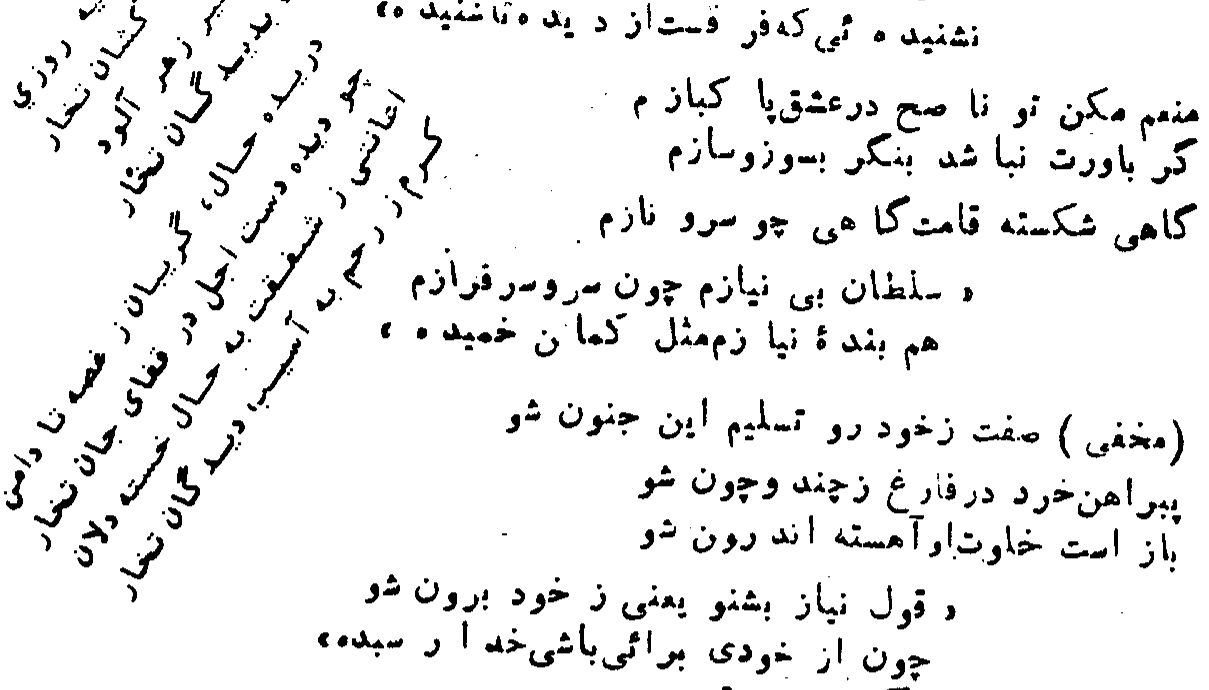

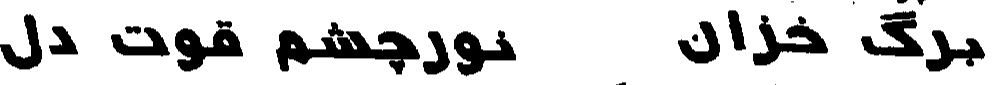

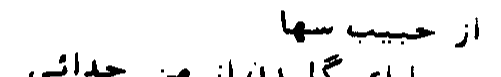

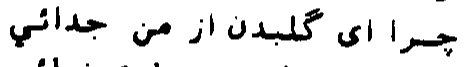

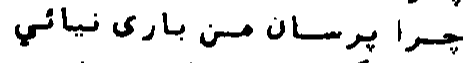

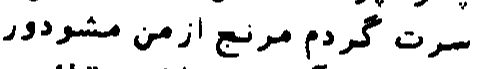

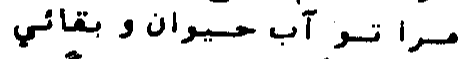

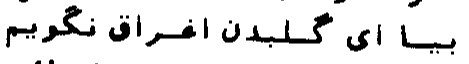

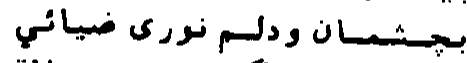

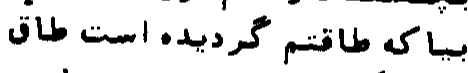

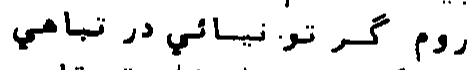

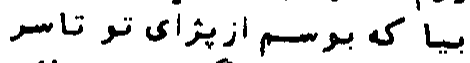

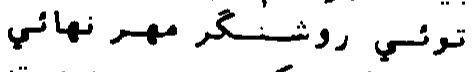

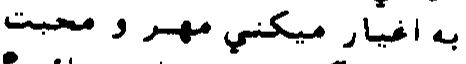

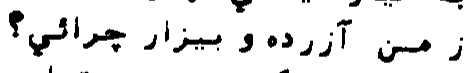
بهر وضعى كع مسى بينهم ترامن

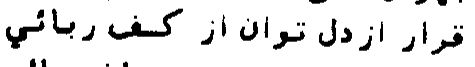

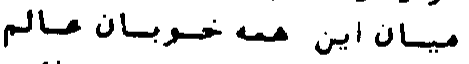

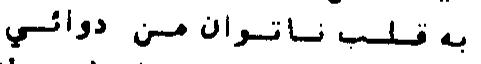

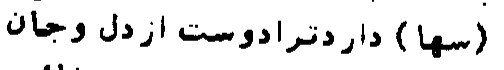

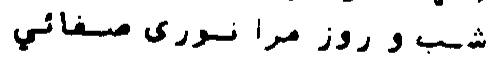

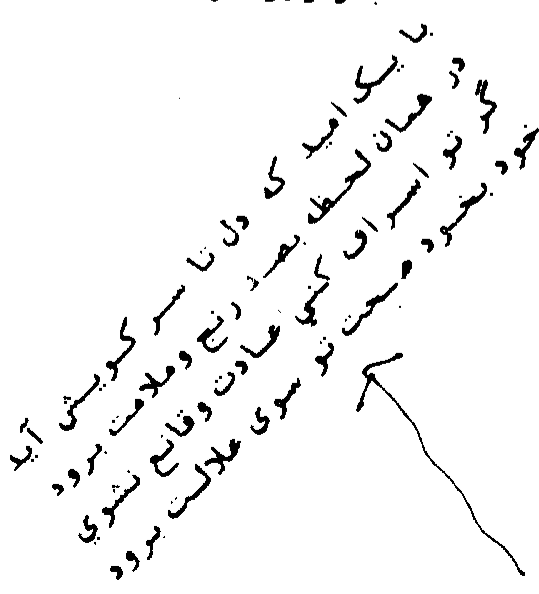

ازميرمن مخغي حسينهي كابلمي

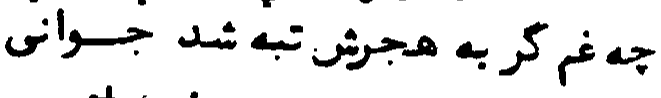

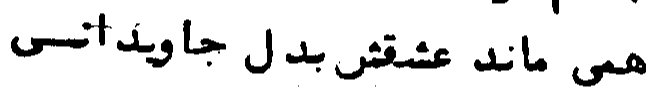

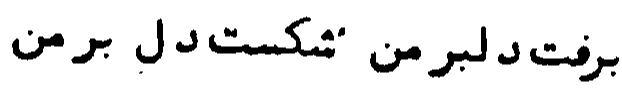

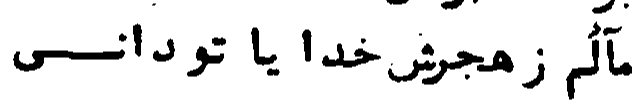

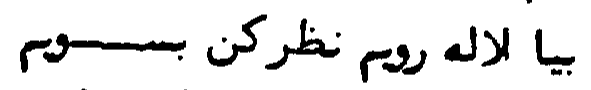

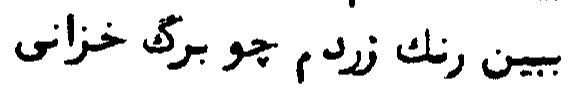

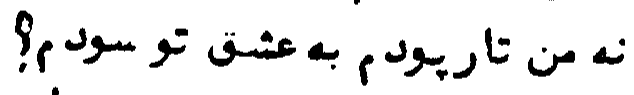

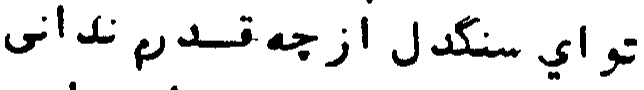

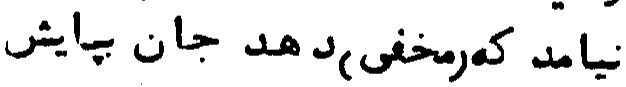

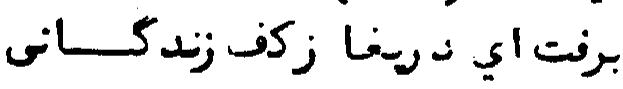

\section{عامبس السرافي}




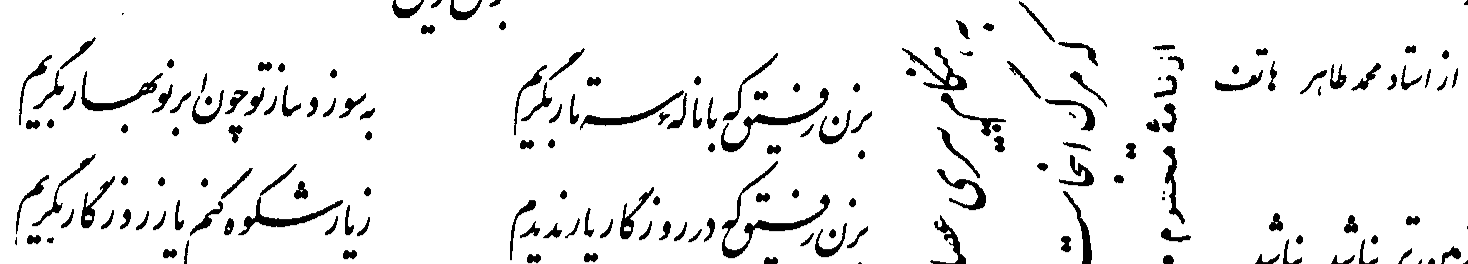

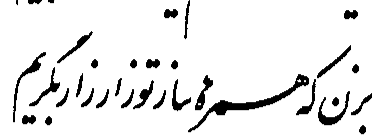

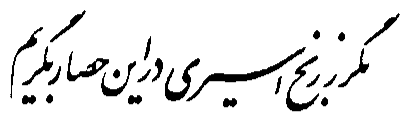

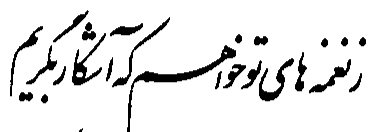

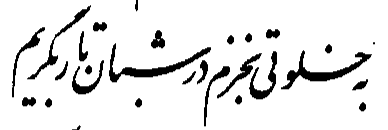

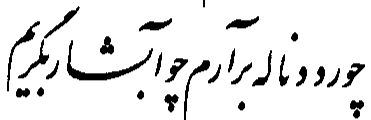

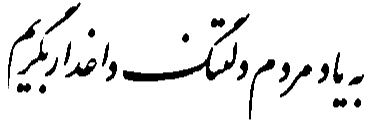

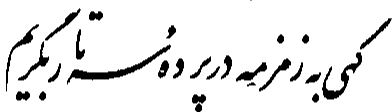

كأن

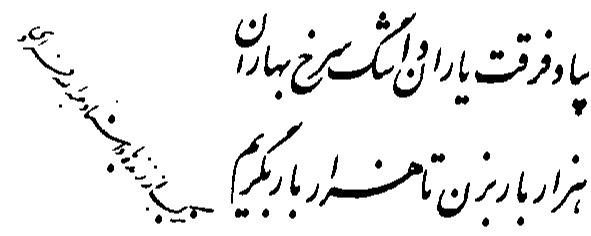

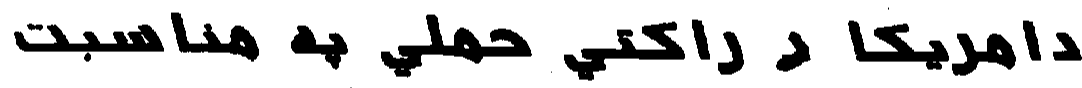

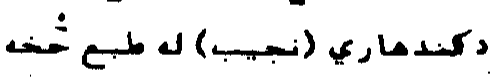

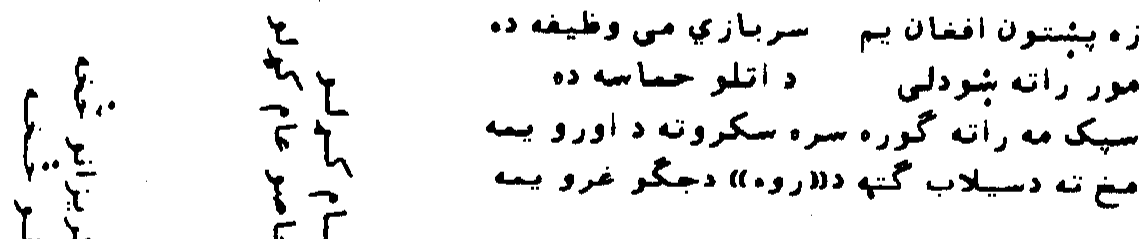

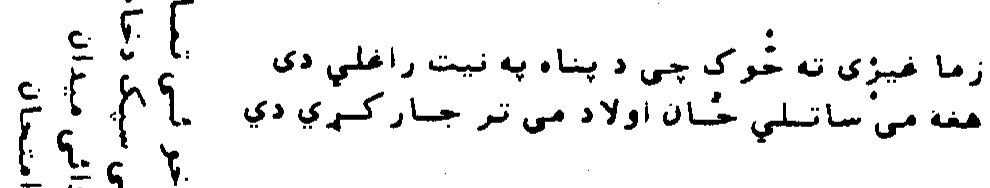
$\underline{2}=q$

En the $=q_{1}$ ع

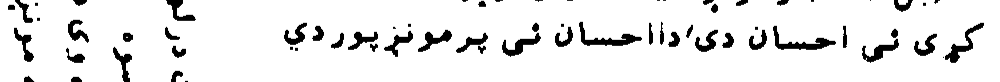
c I. $=8$, iq $q$ q $\therefore \operatorname{sic}$

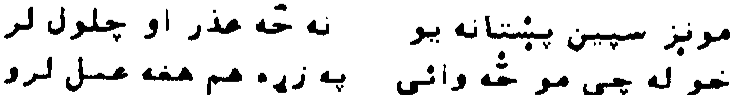

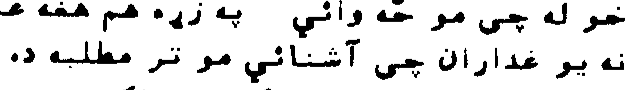

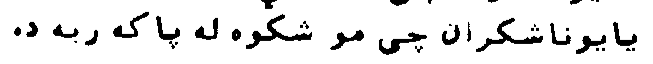

is

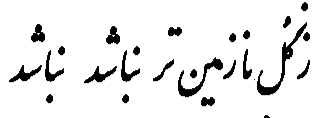

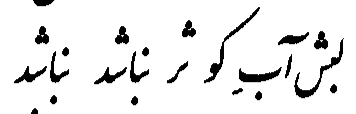

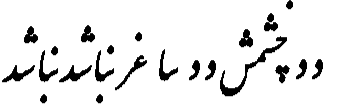

is

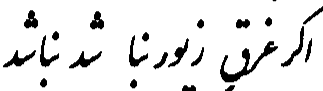

\section{م,}

\&

人ر

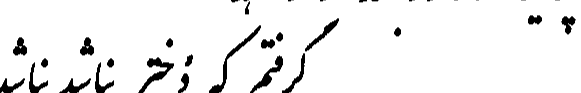
ر

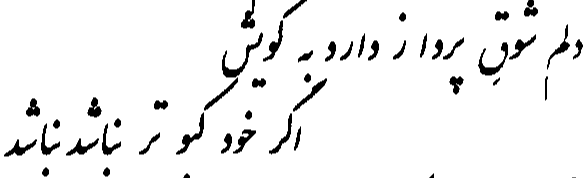



$$
\text { • }
$$

ק

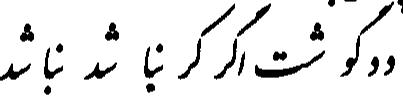

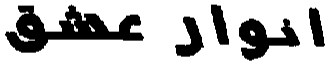

ازعبدالصسا ومابزاده( فريار )

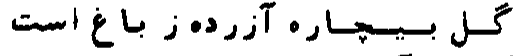

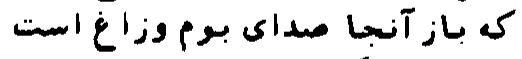

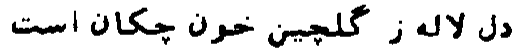

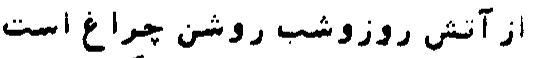

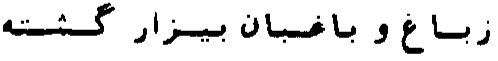

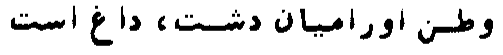

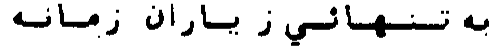

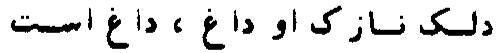

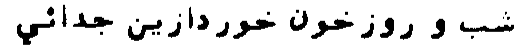

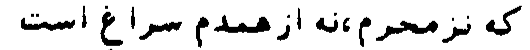

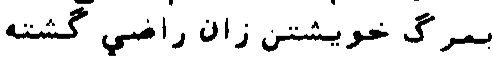

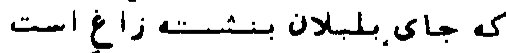

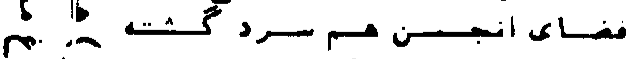

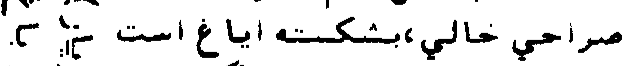

خ

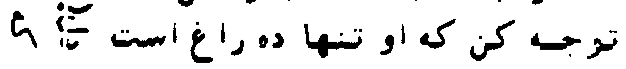




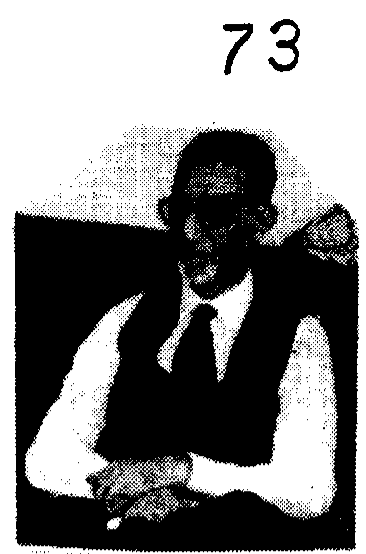

\title{
تماريه بر آثار واJده
}

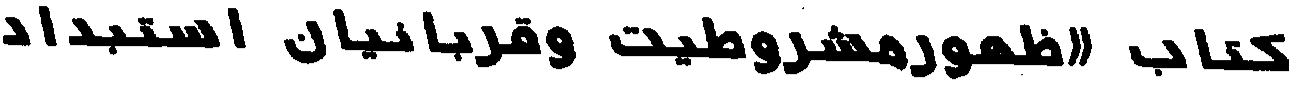

\author{
IJLentodidg \\ مرلف : سيـد مسعود يو منسيار
}

:0بn

ADDRESS: MR. POHANYAR: BROMBERGER STR. \#4, 5880 LUDENSCHEID - GERMANY

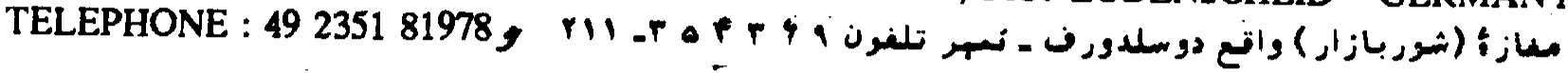

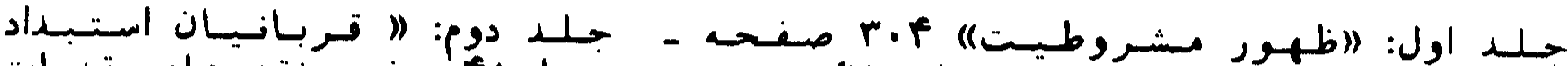

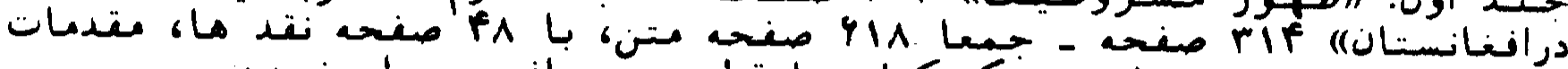

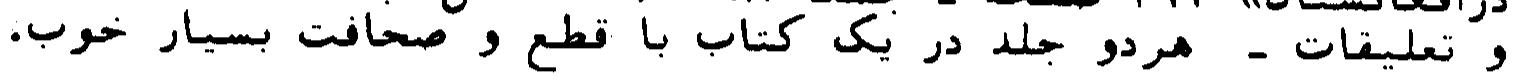

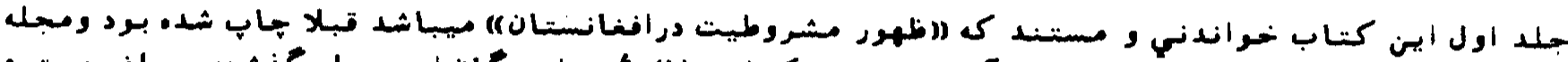

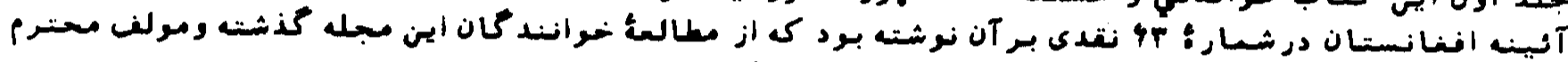

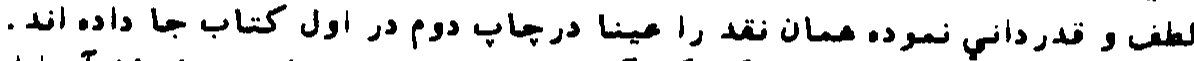

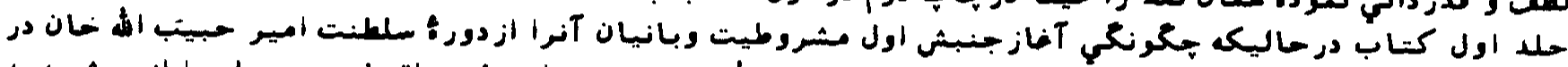

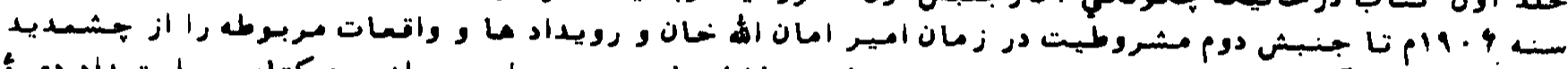

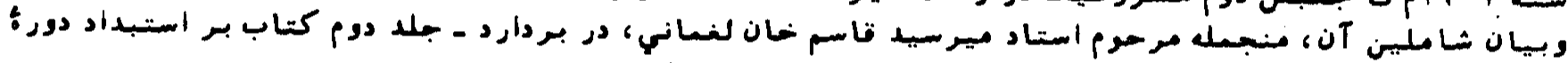

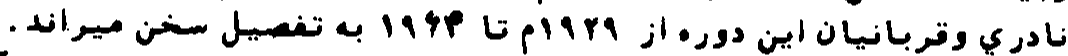

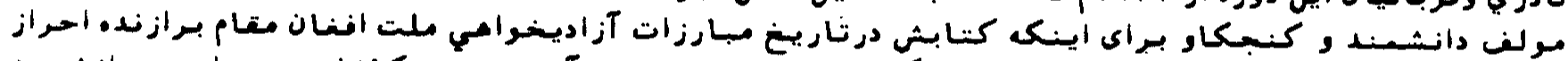

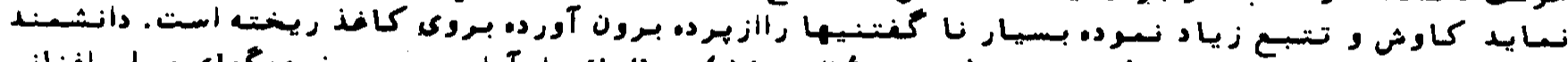

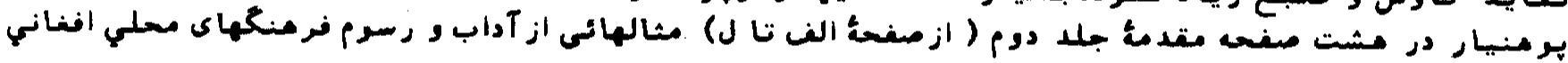

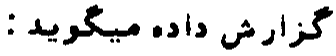

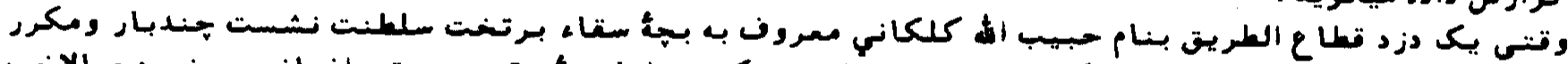

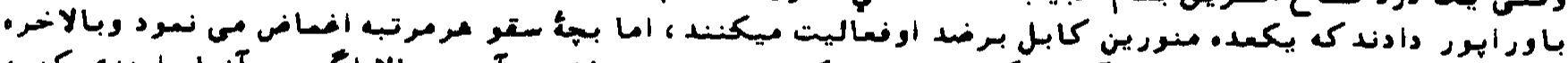

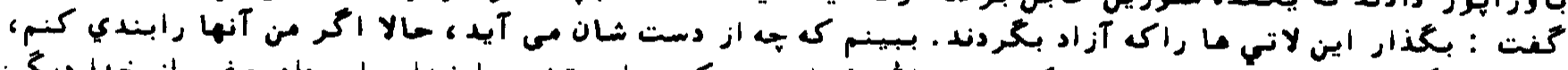

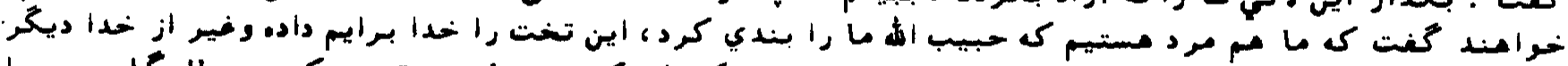

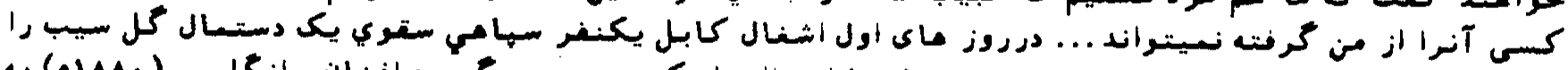

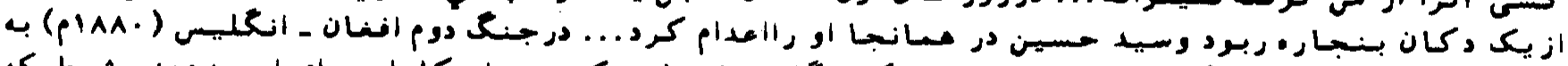

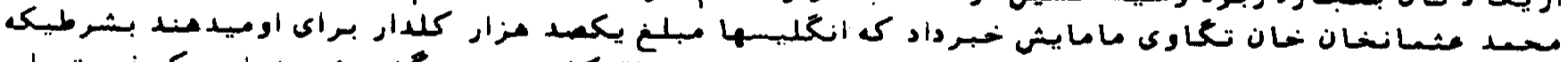

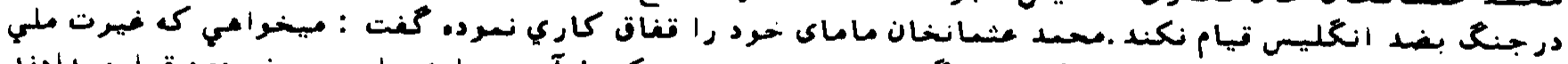

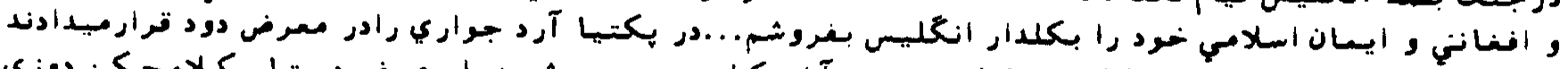

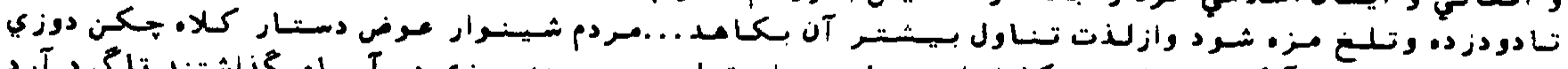

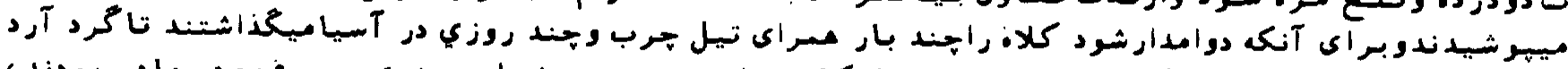

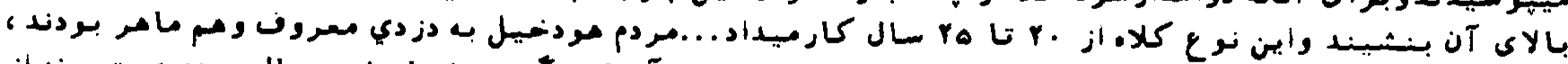

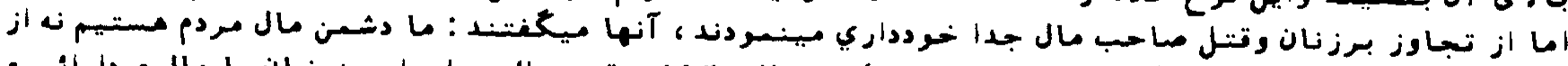

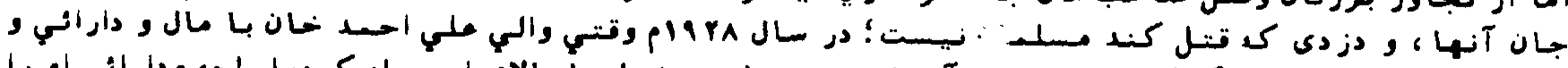

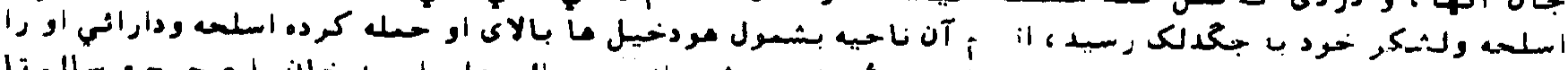

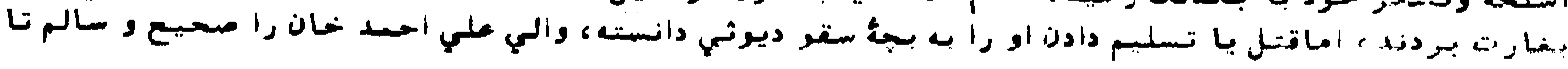

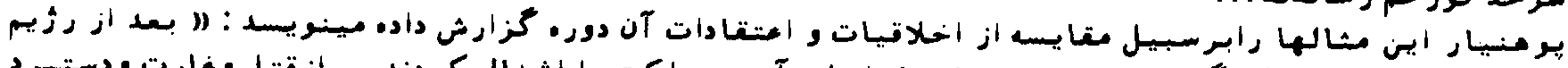

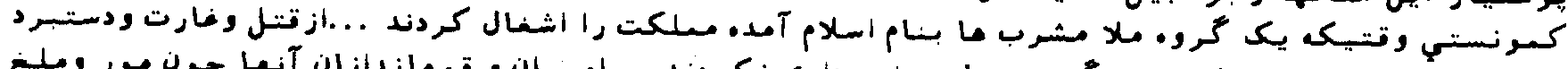

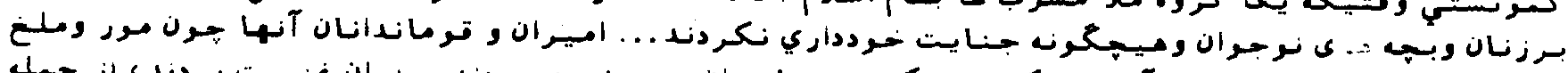

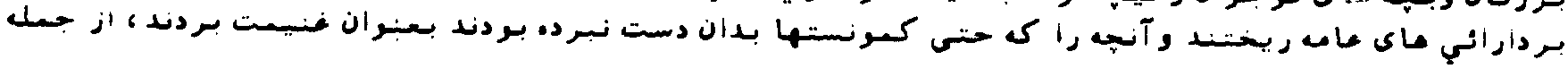




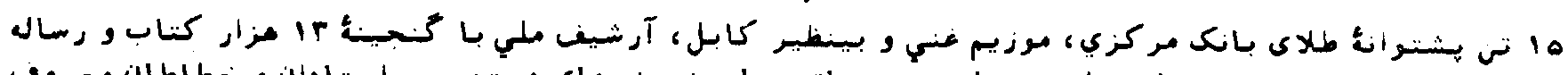

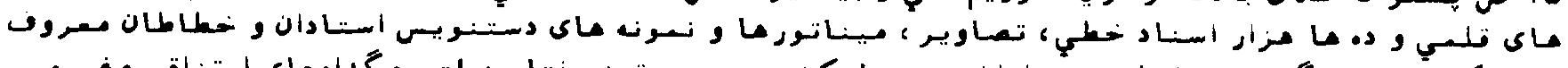

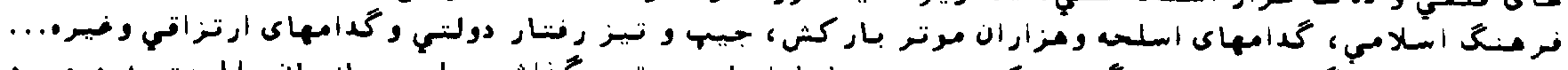

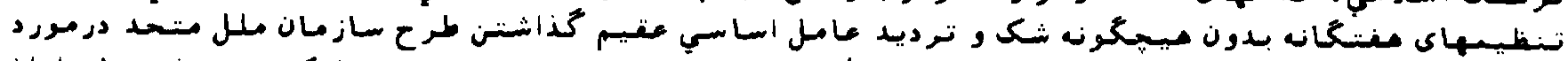

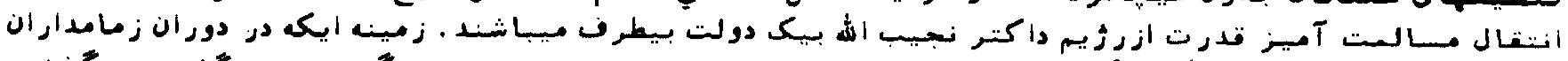

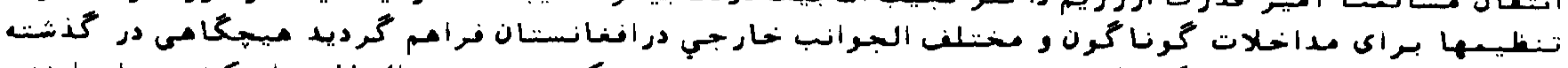

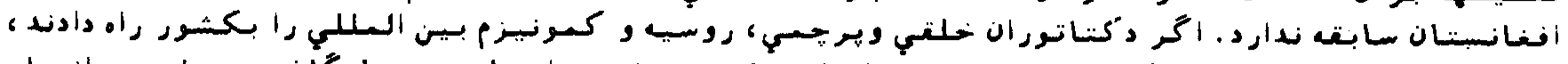

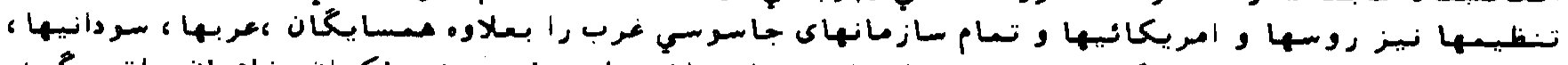

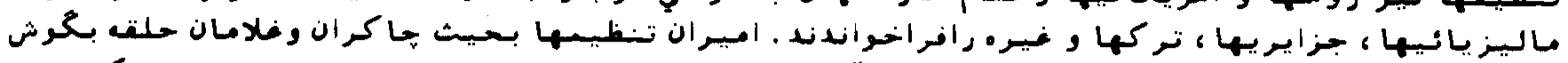

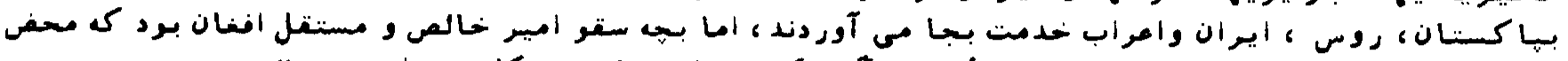

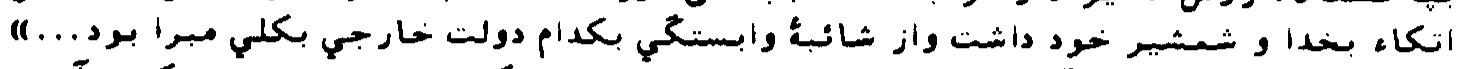

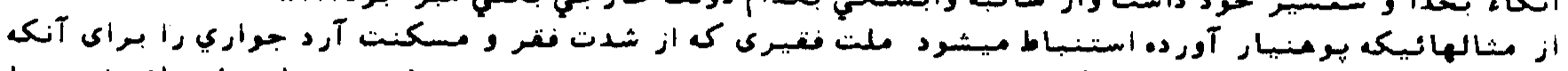

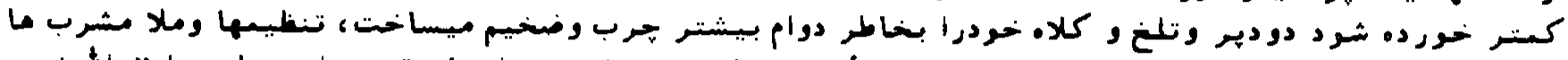

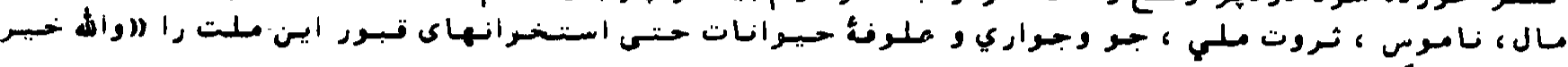

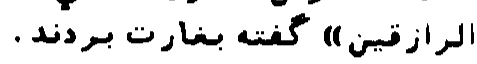

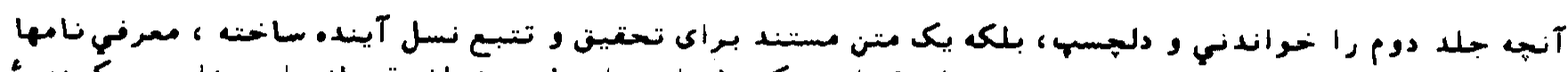

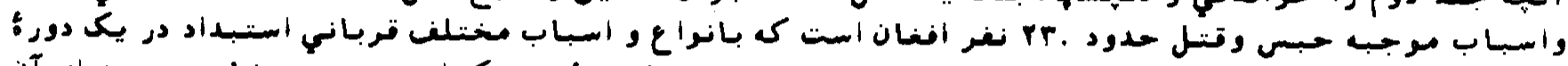

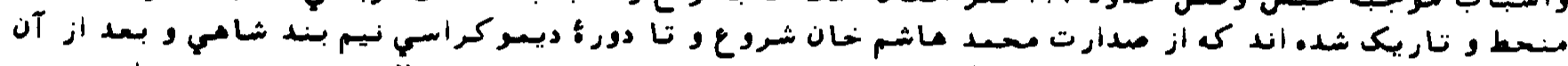

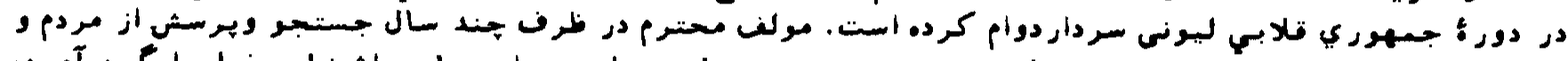

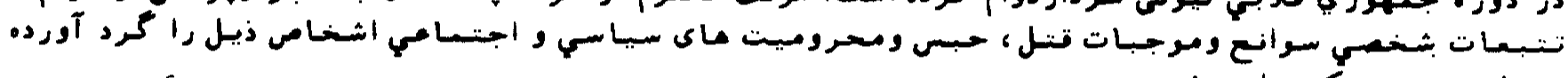

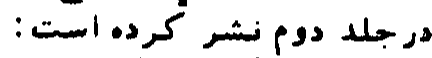

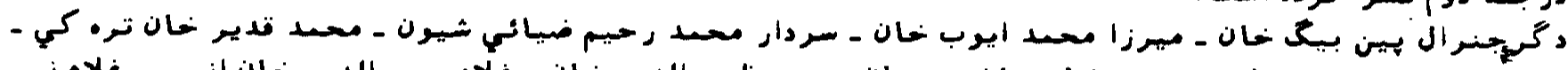

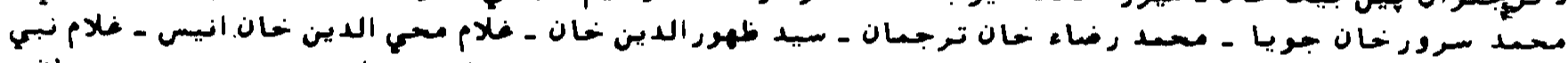

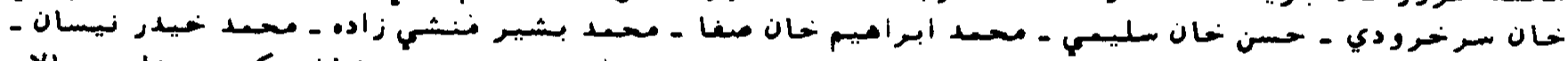

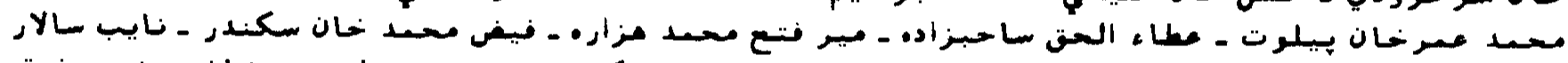

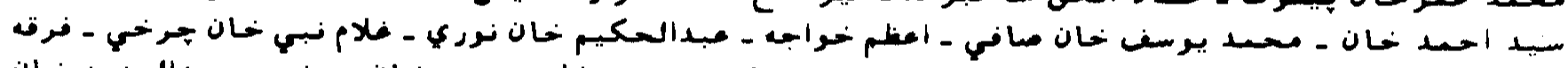

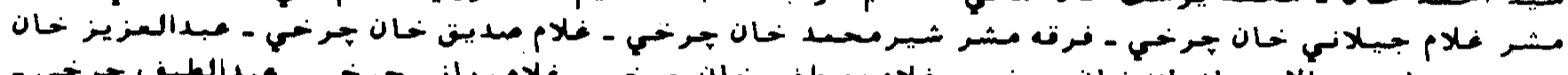

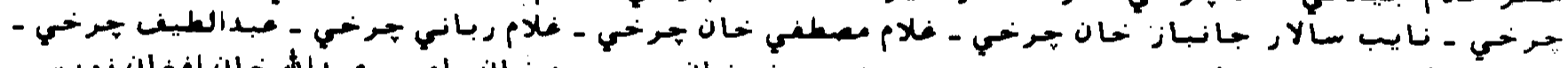

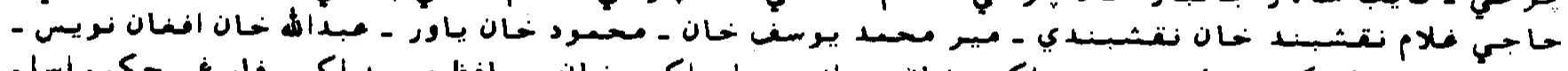

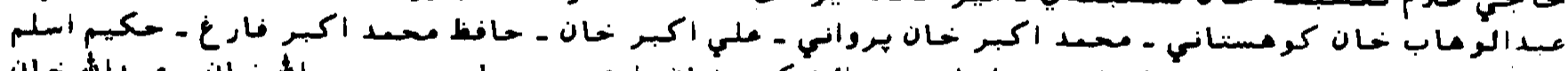

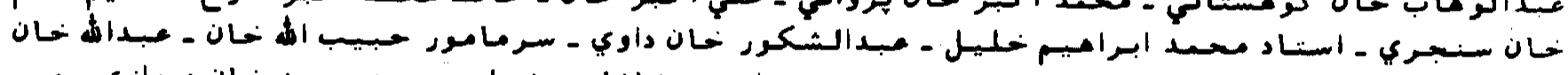

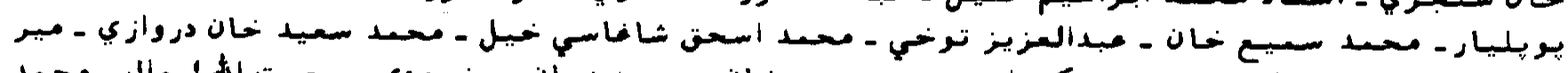

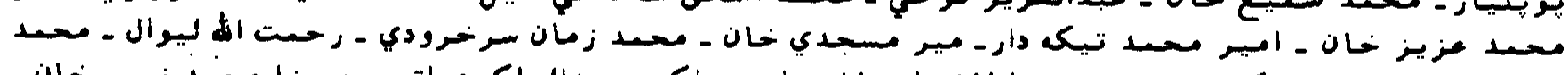

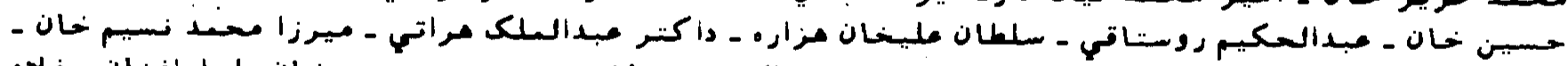

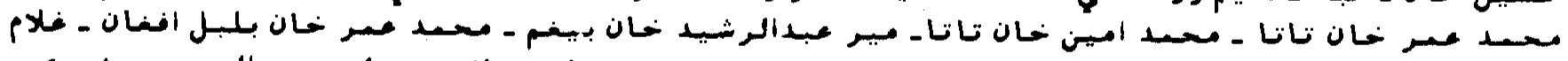

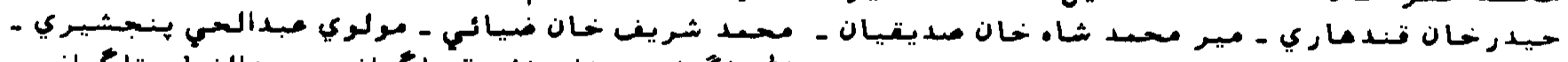

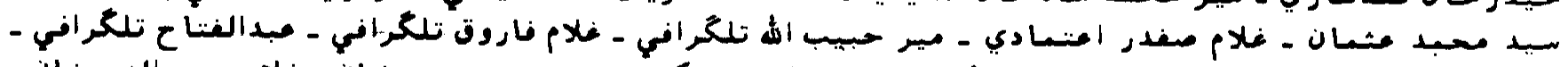

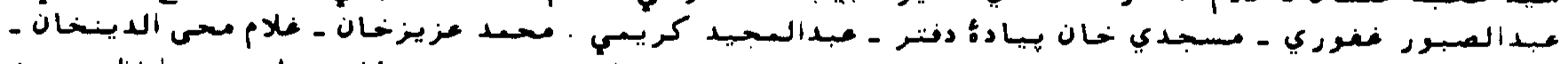

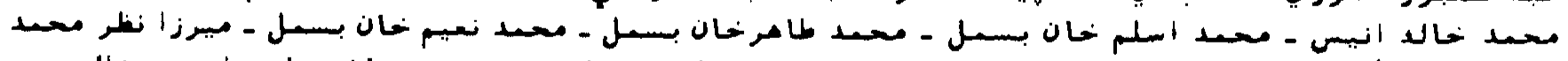

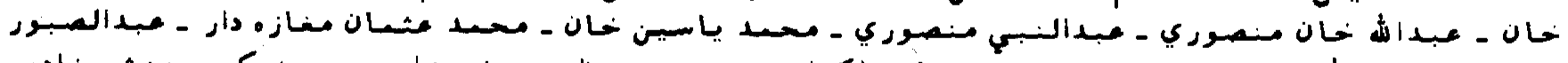

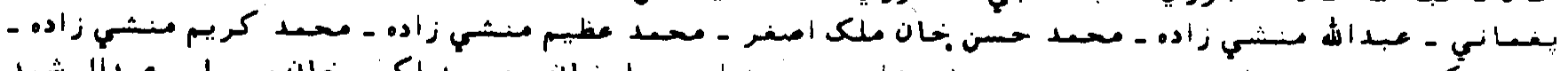

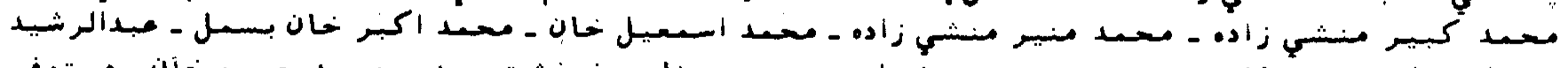

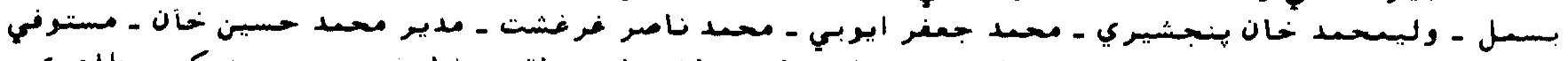

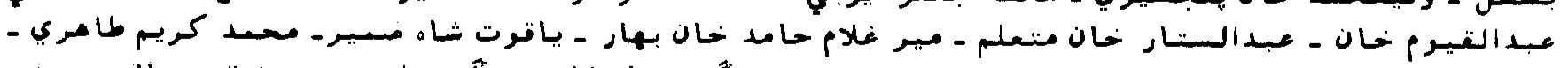

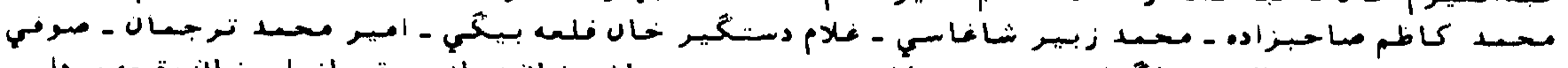

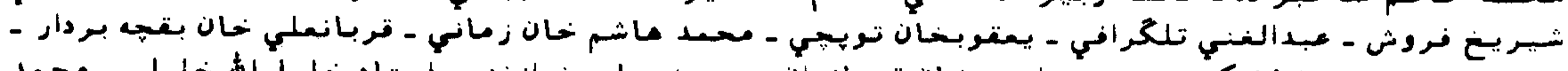

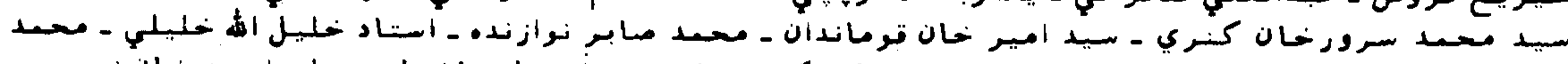

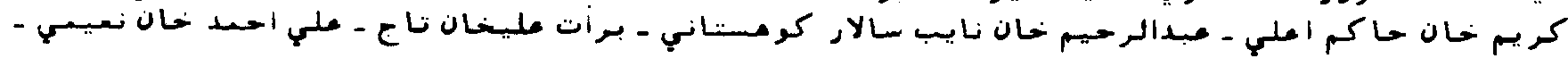

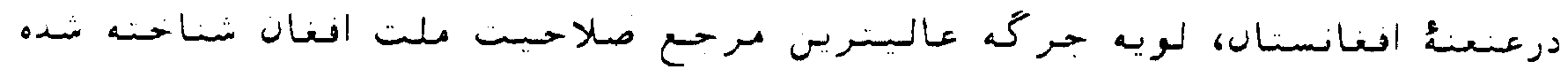




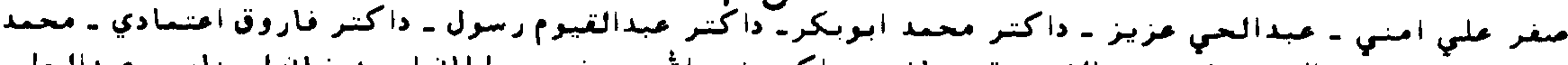

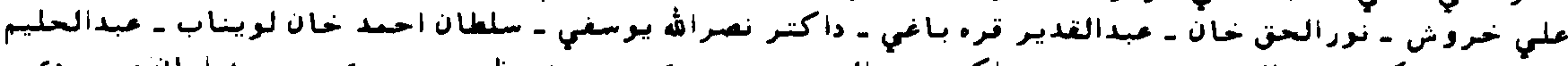

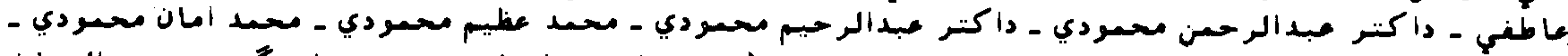

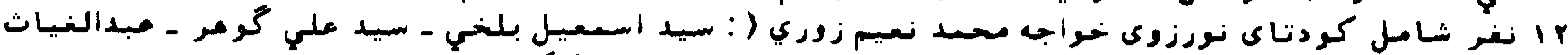

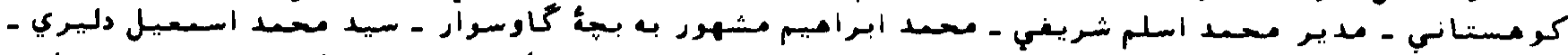

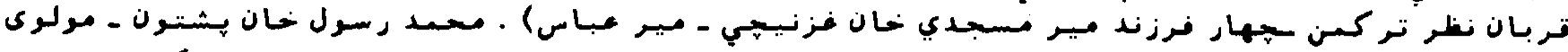

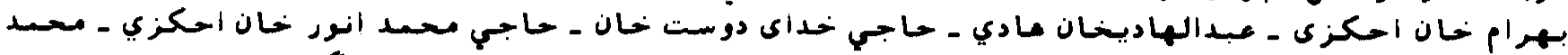

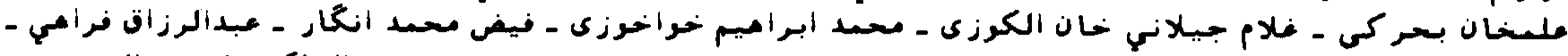

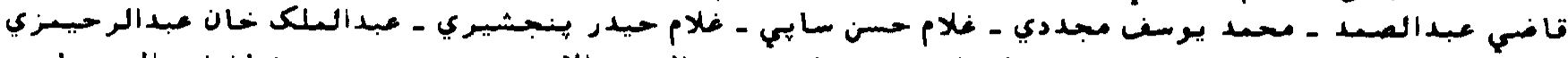

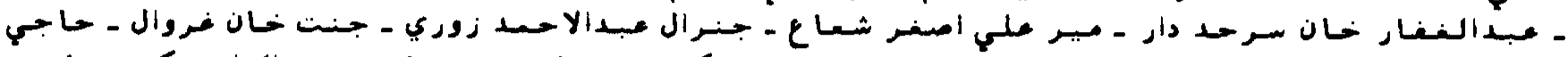

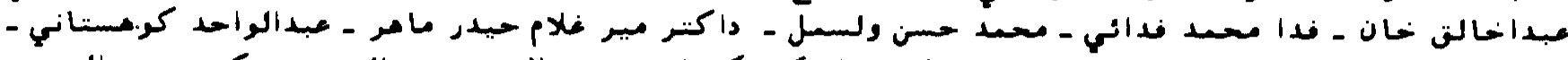

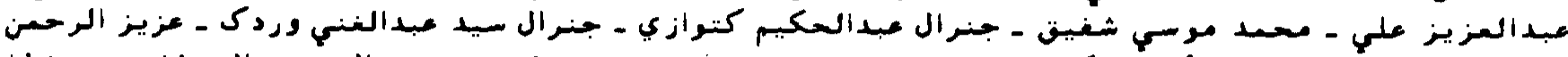

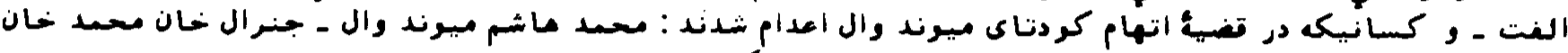

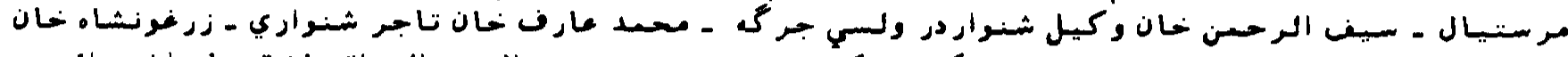

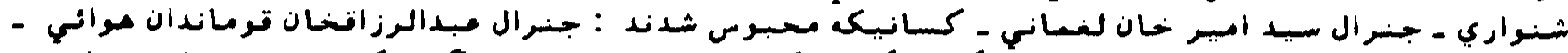

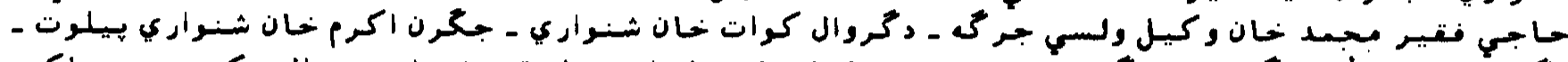

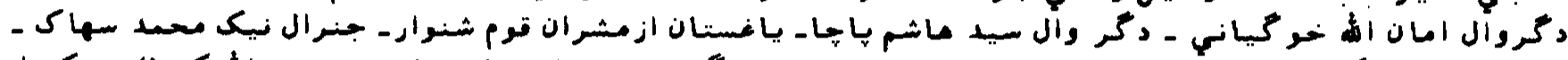

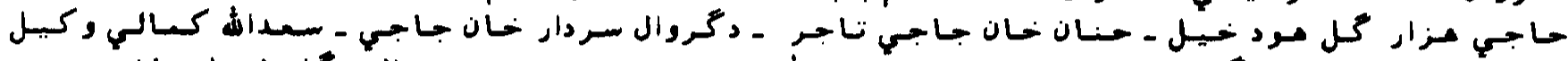

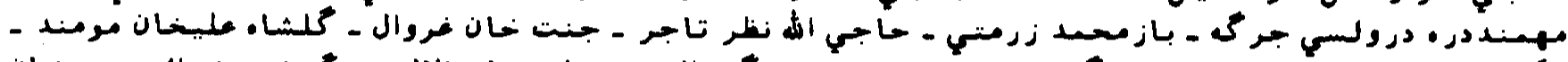

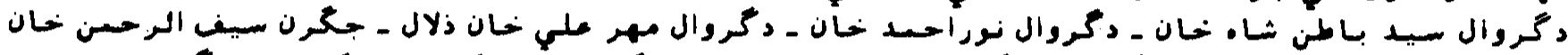

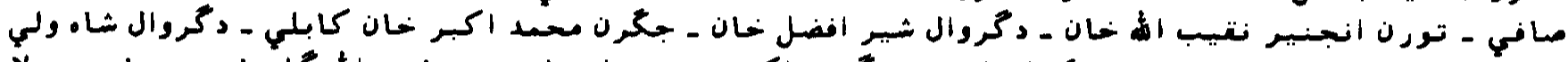

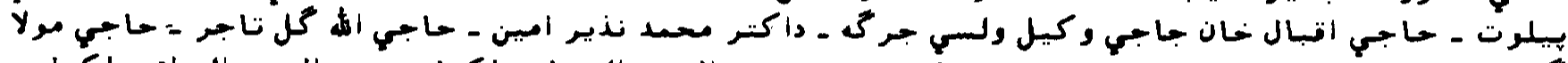

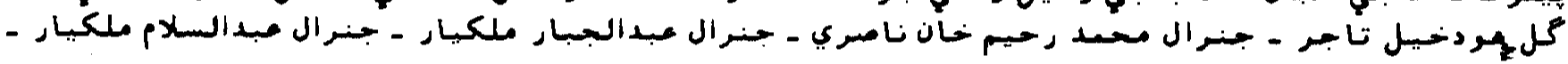

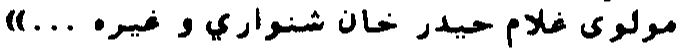

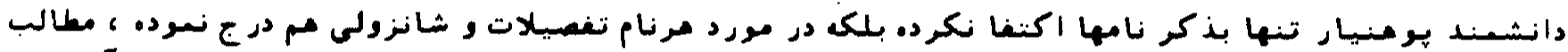

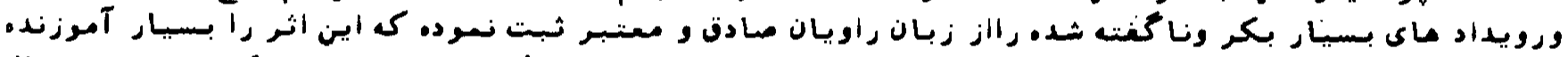

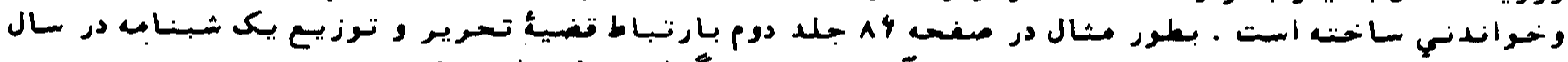

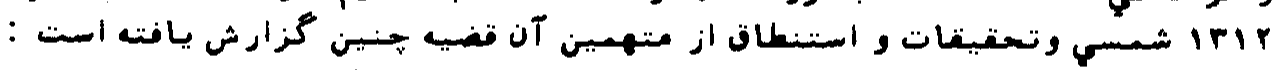

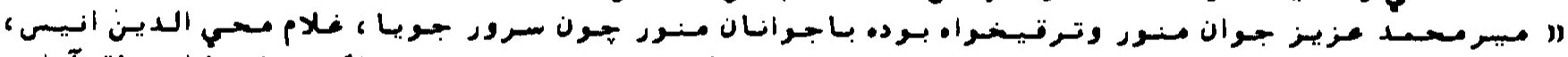

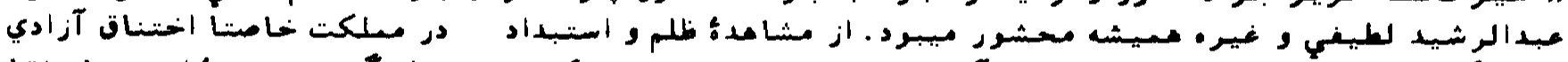

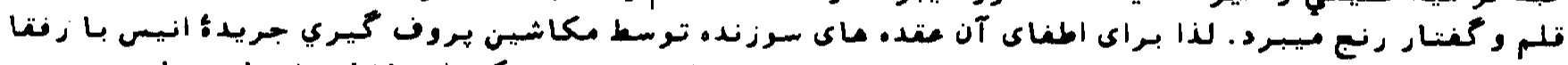

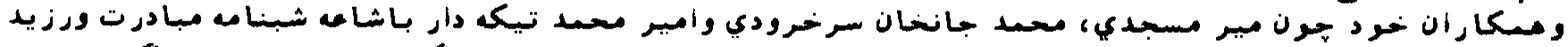

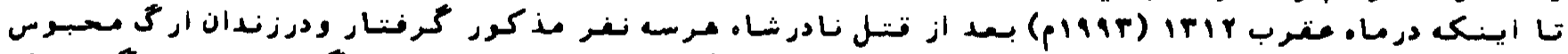

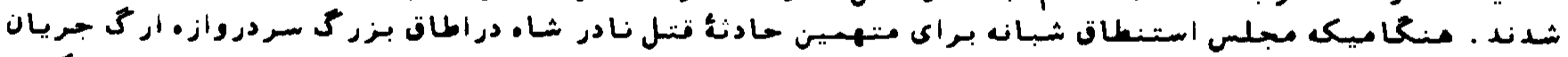

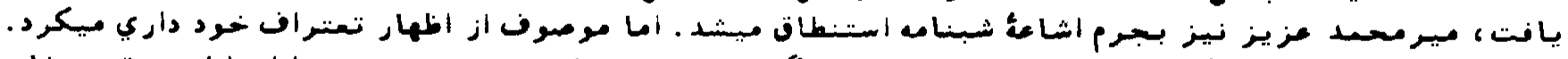

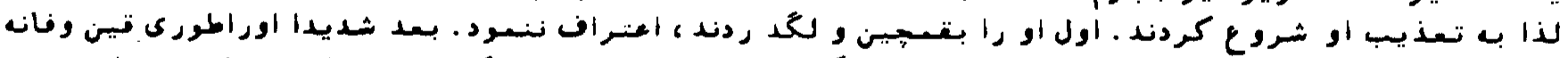

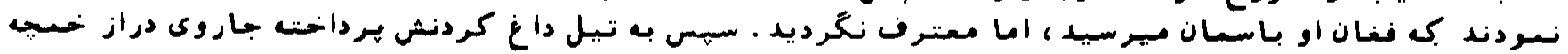

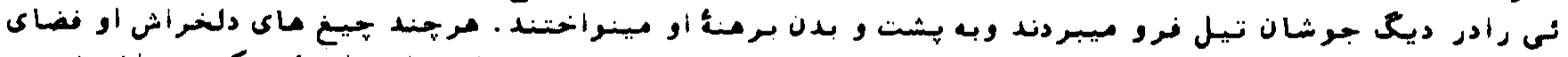

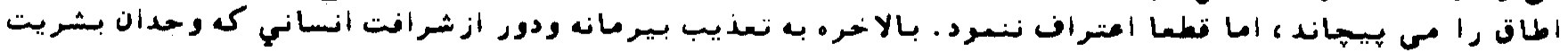

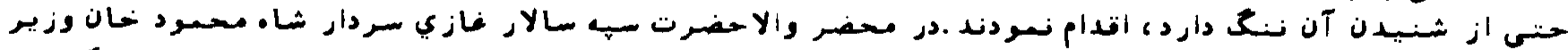

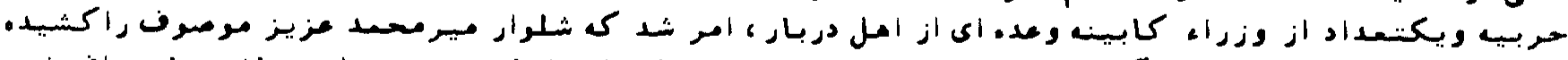

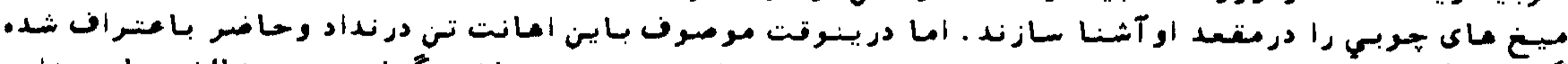

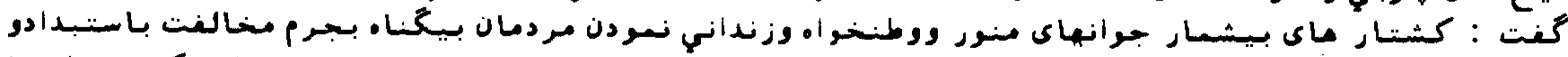

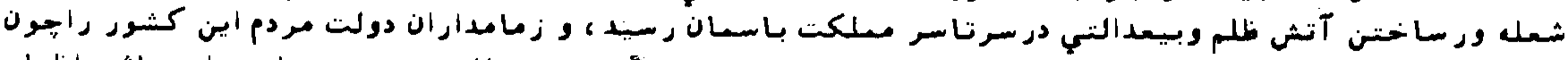

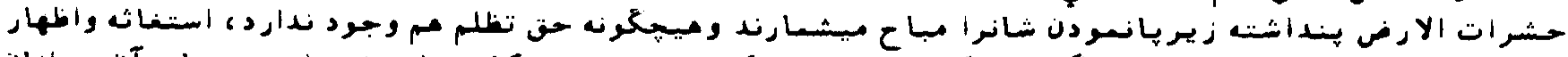

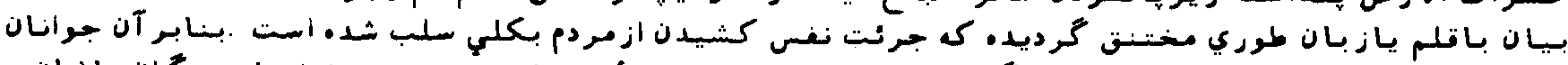

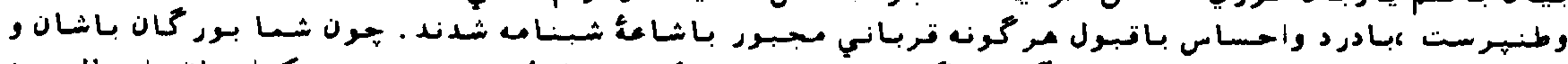

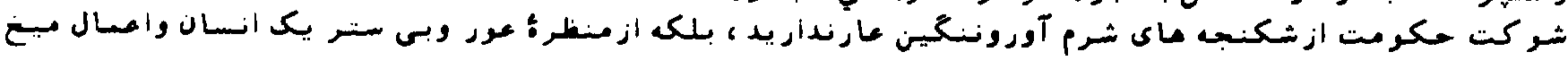




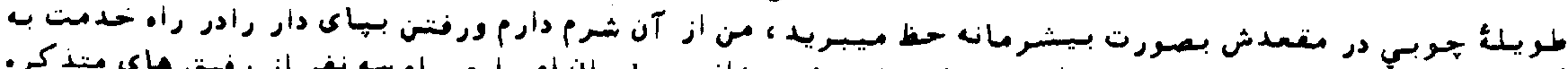

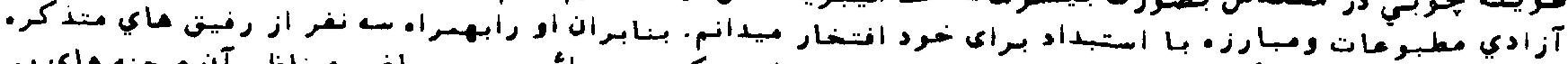

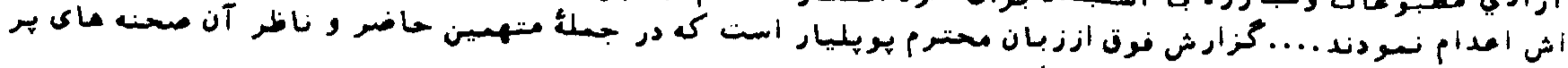

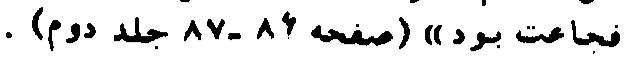

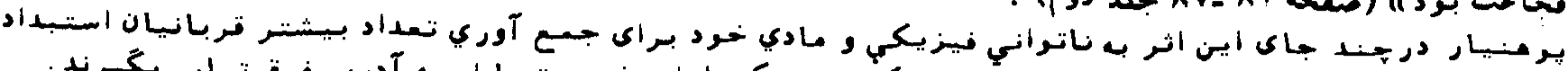

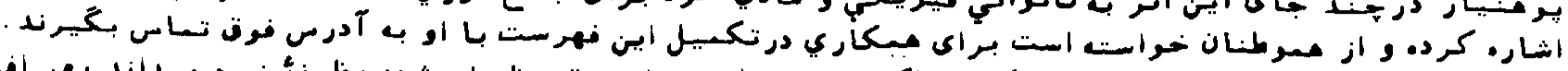

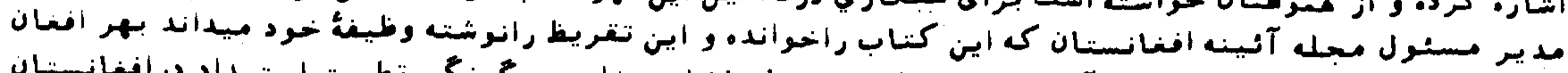

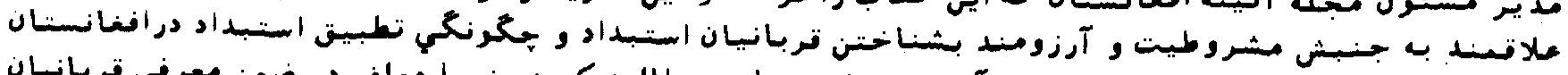

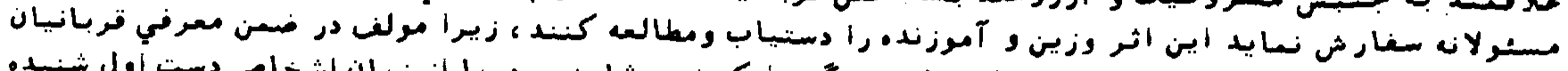

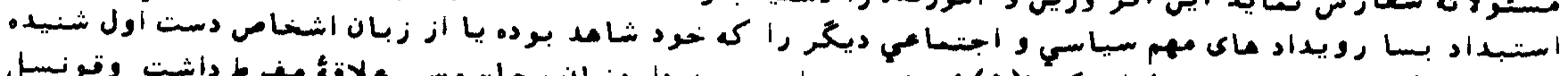

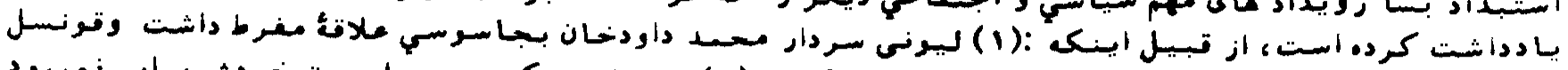

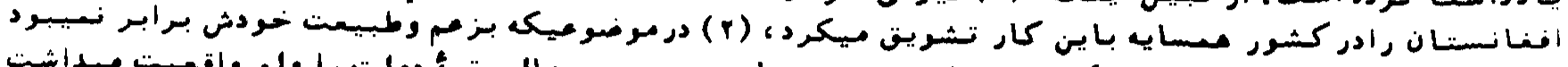

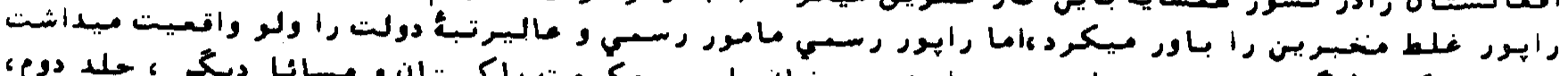

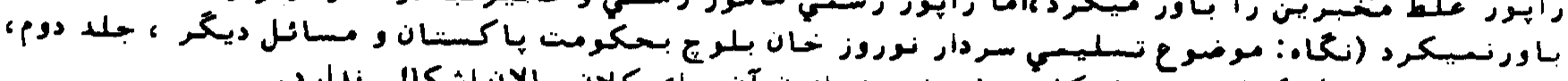

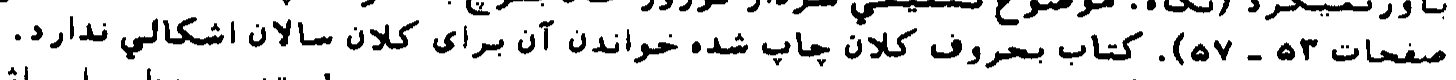

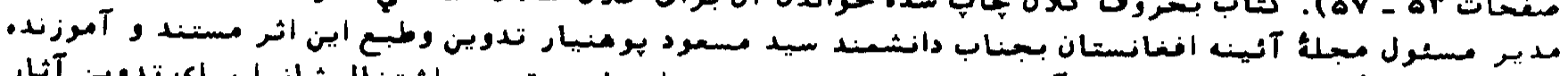

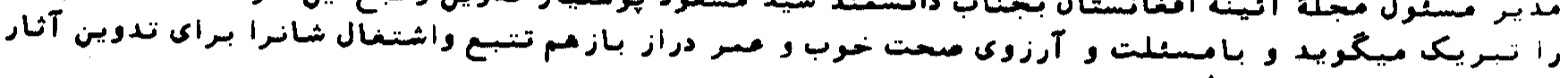

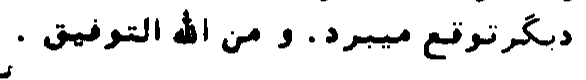

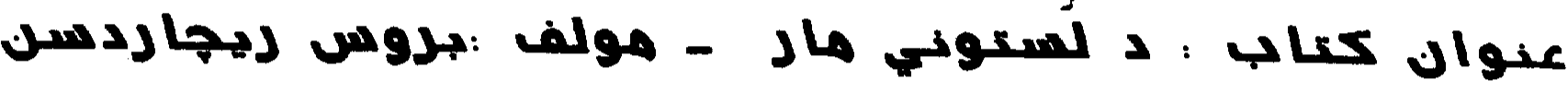

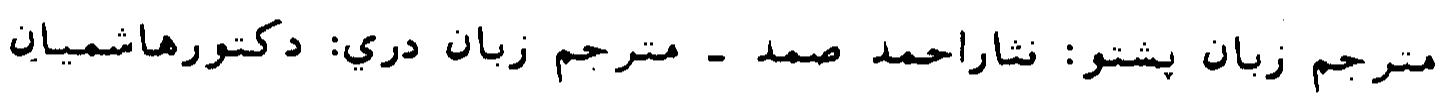

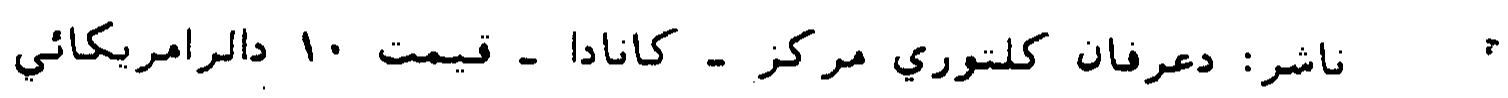

Address: Irfan Cultural Centre- C/O 2075 Warden Ave,Unit 27 - Scarborough,ONT.M1T-3R1 CANADA

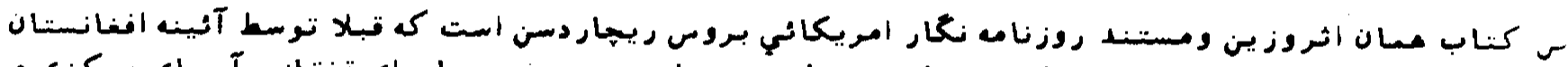

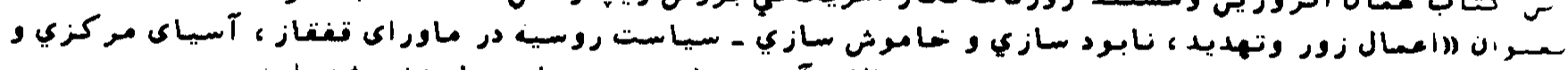

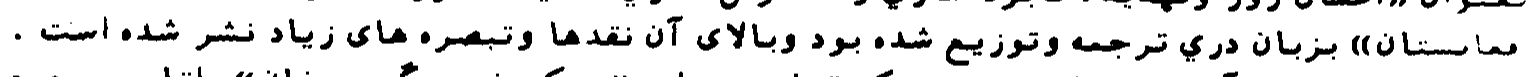

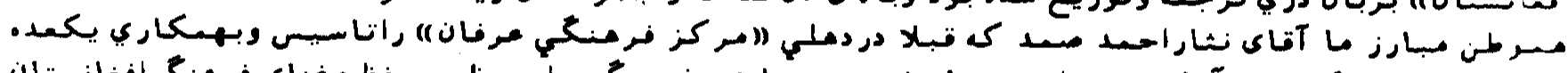

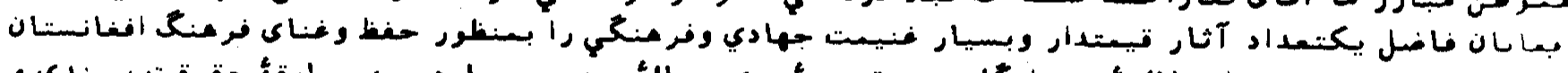

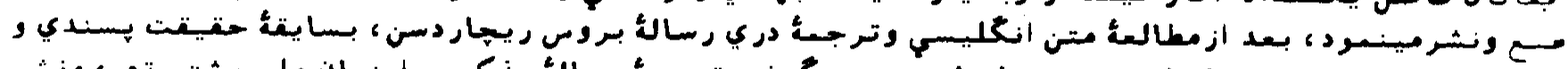

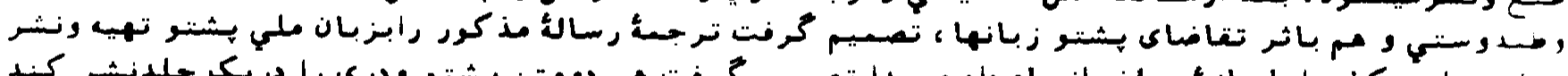

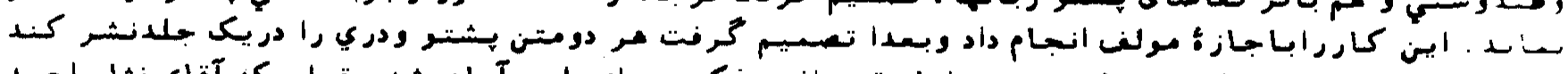

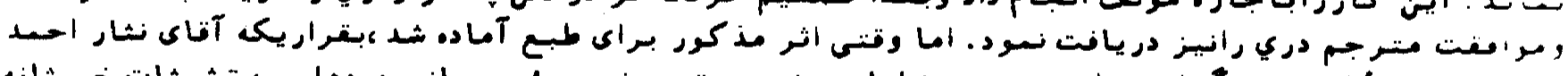

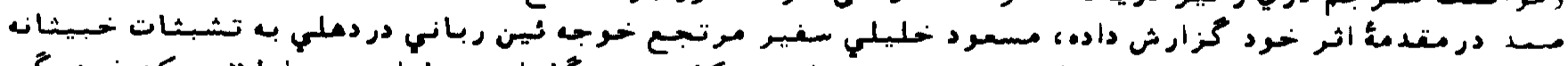

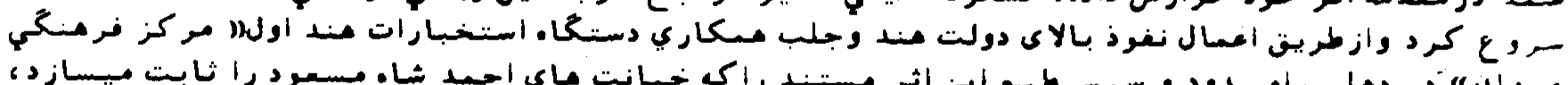

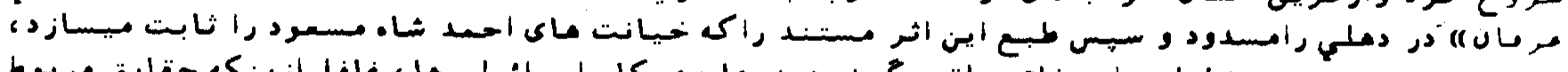

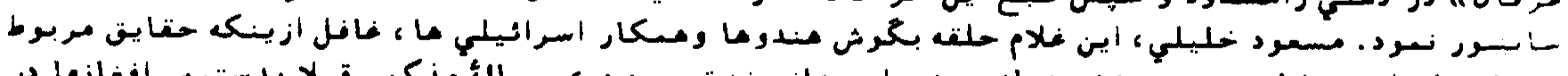

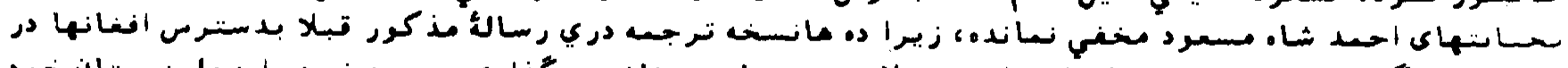

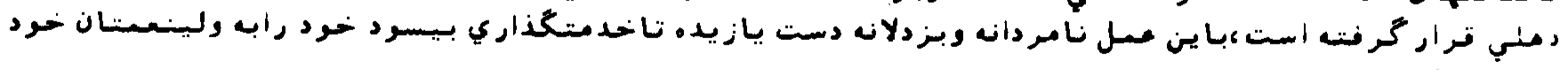

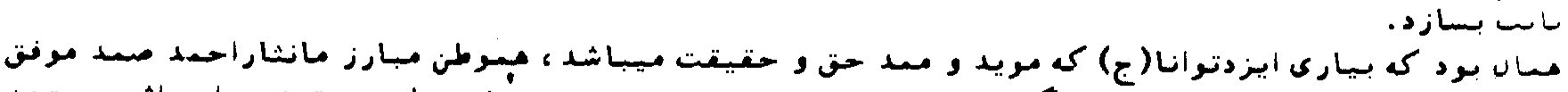

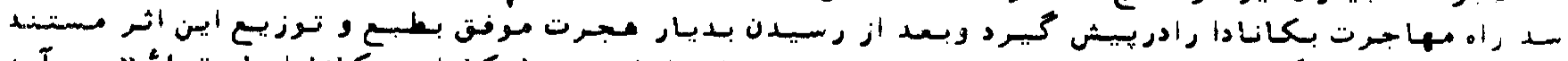

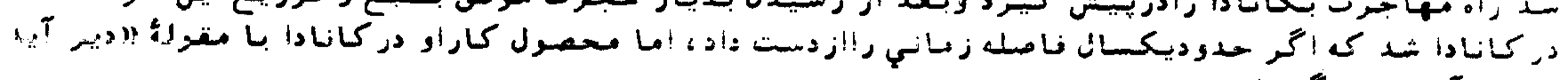

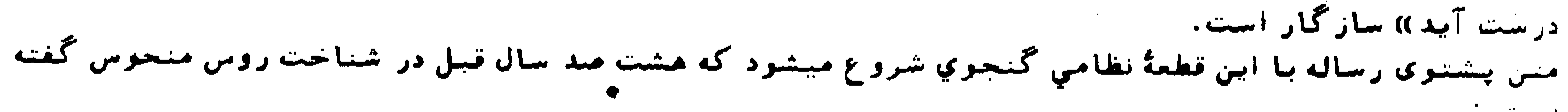

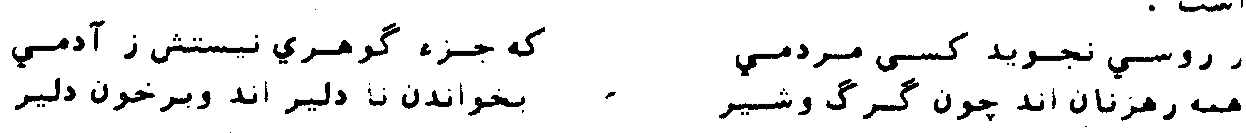




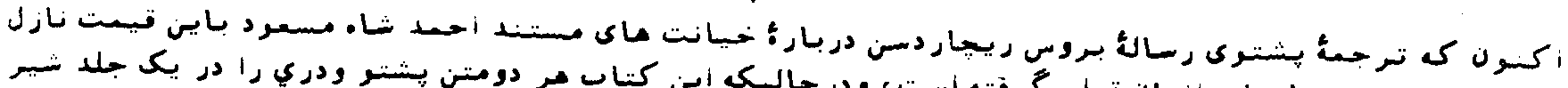

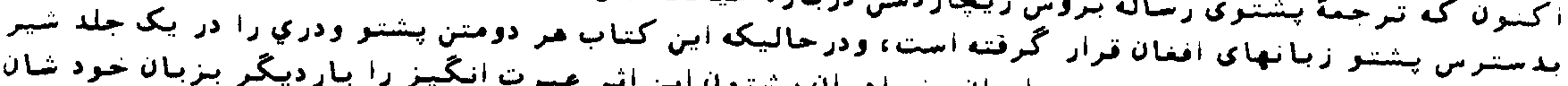

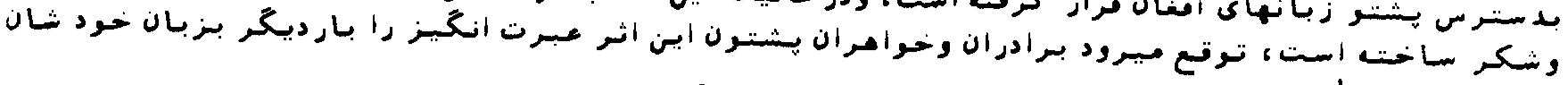

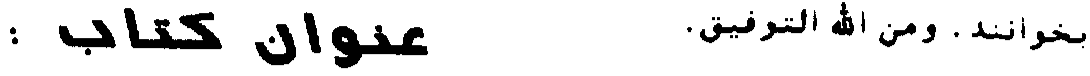

\section{THE WARLORD ABDUL RASHEED DOSTUM \\ A BLOOD-STAINED CHAPTER IN THE HISTORY OF AFGHANISTAN}

جنگ مالار عبدالرشيد دوستم - صفحُ خون آلود درتاريخ افنانستان

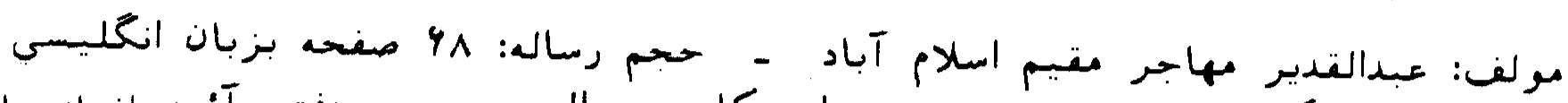

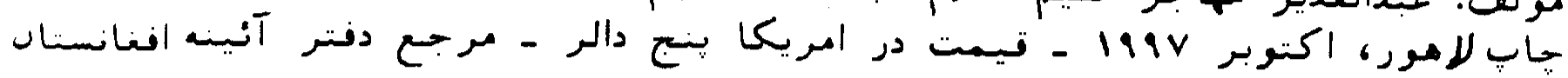

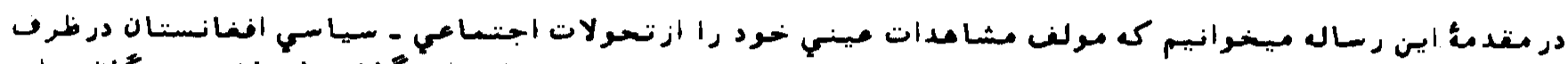

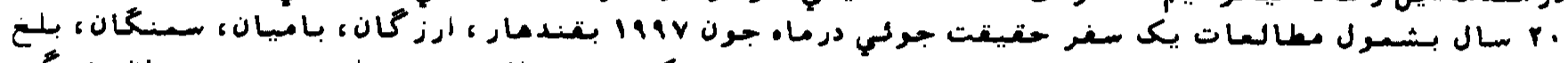

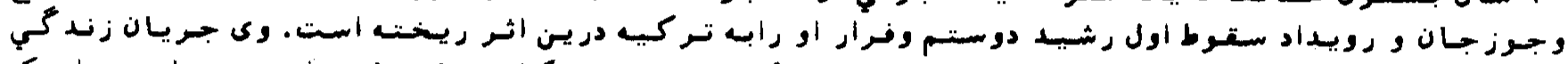

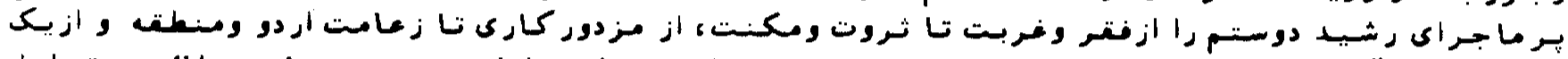

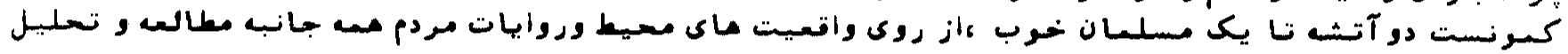

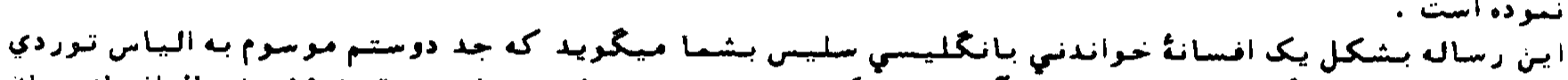

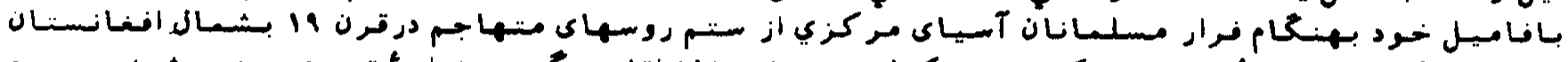

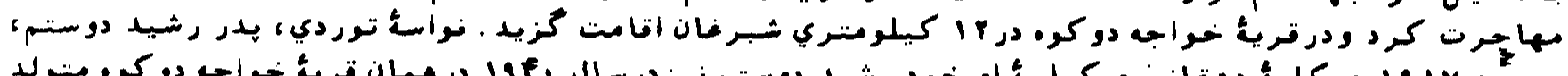

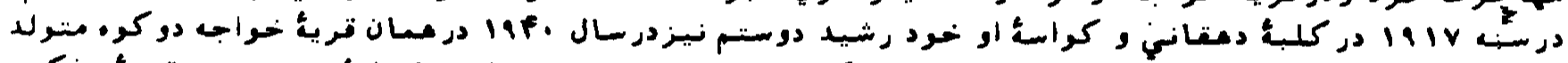

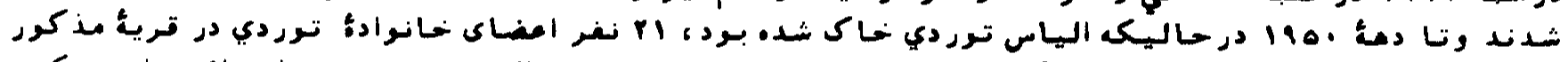

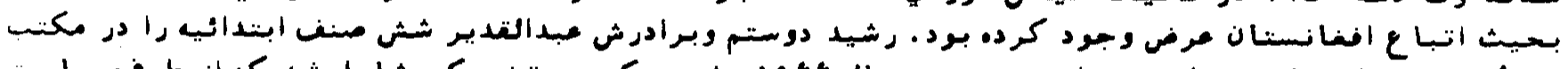

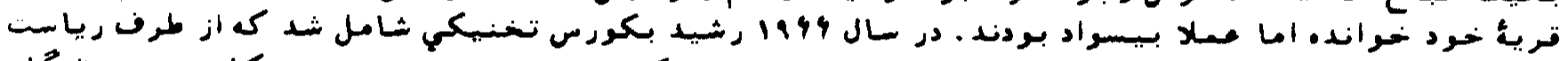

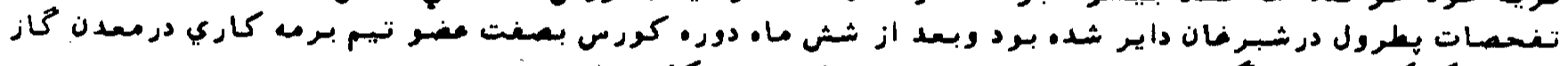

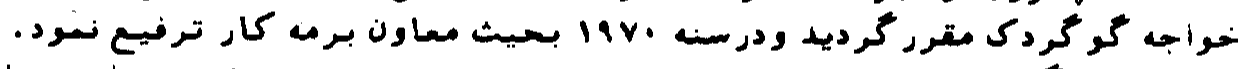

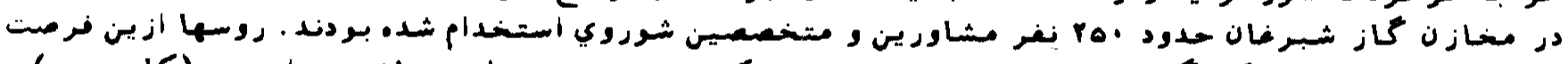

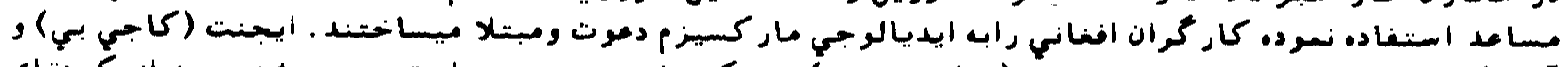

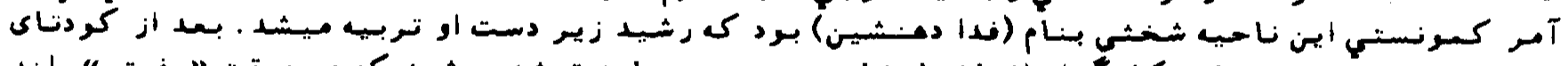

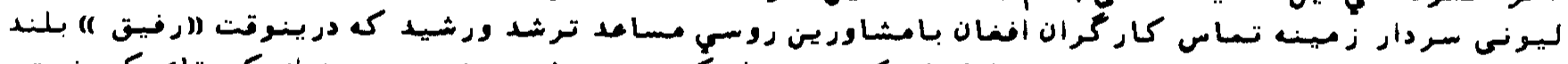

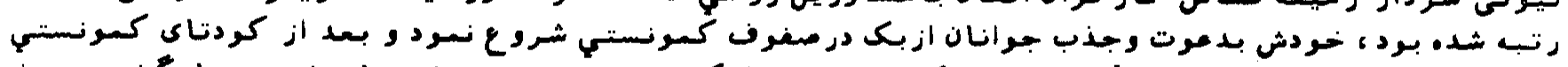

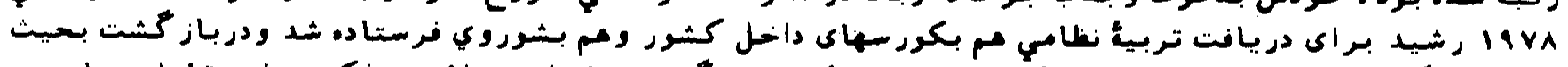

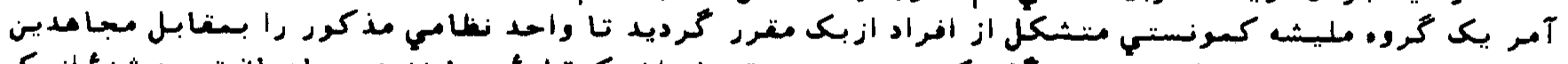

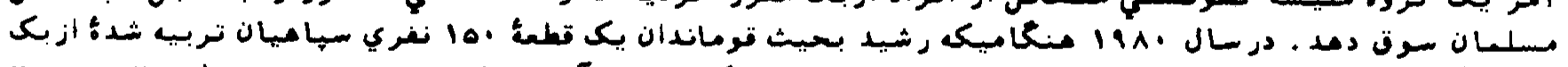

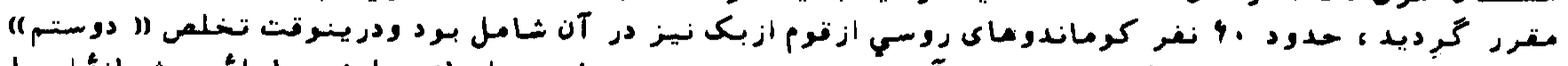

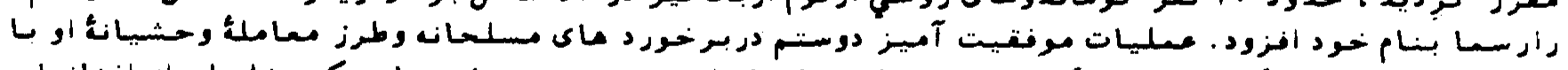

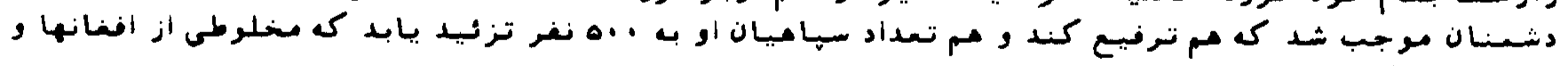

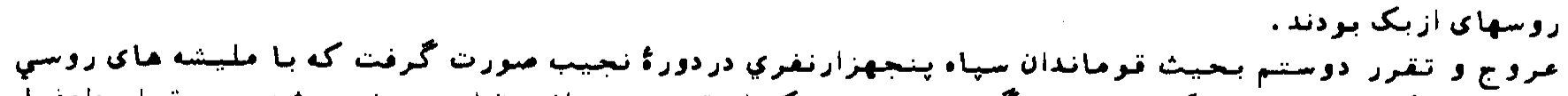

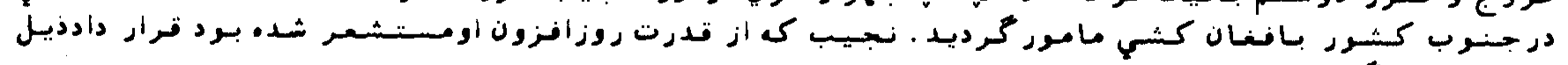

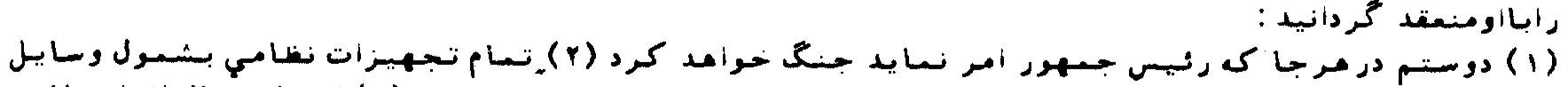

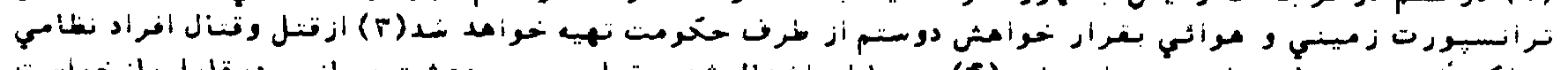

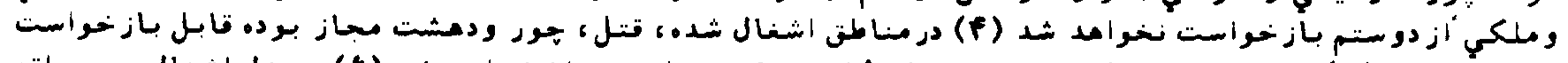

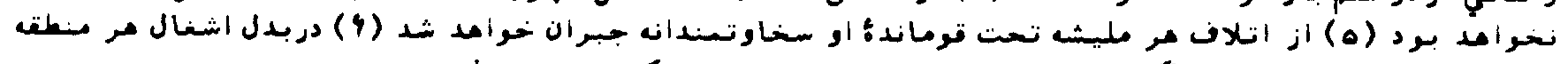

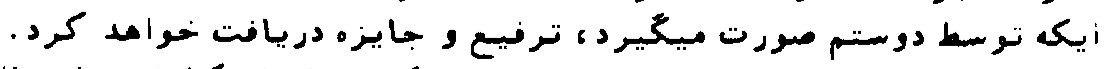

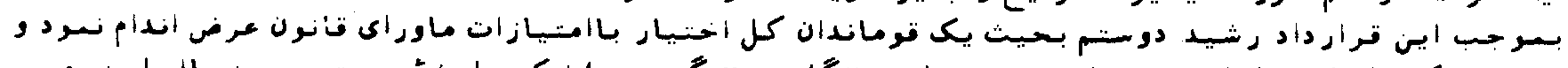

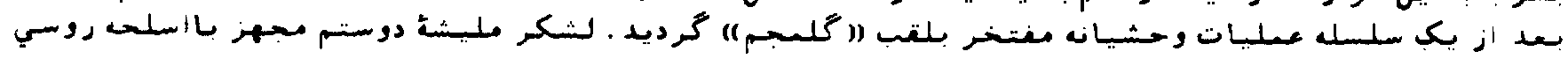




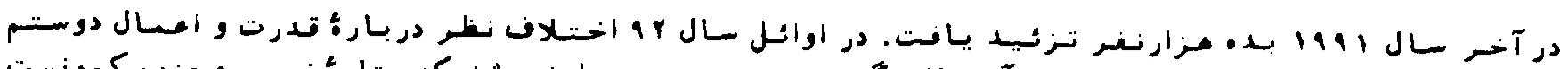

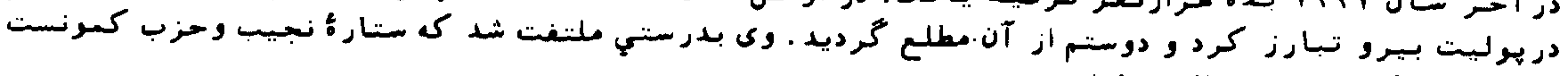

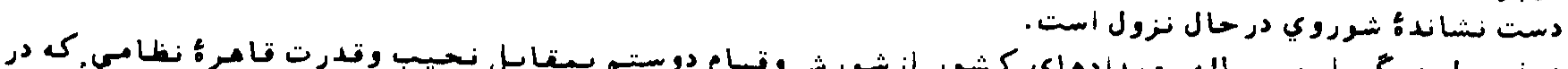

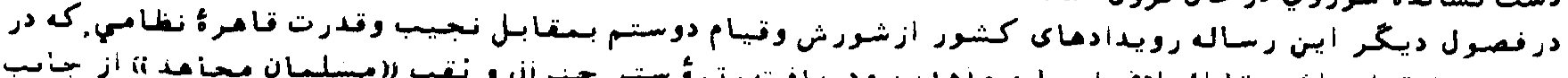

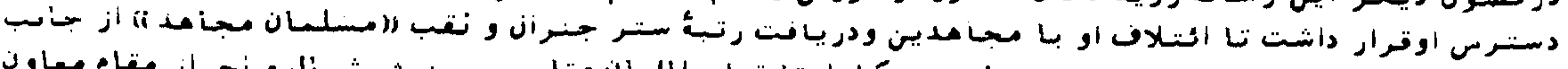

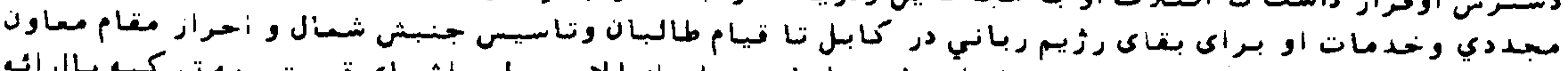

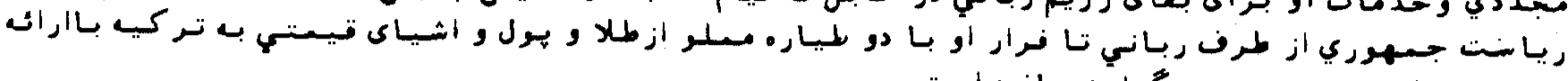

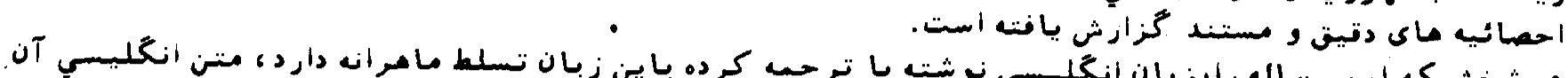

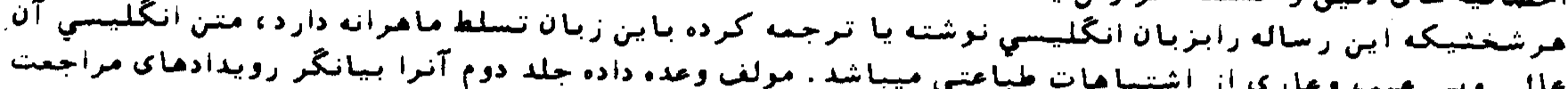

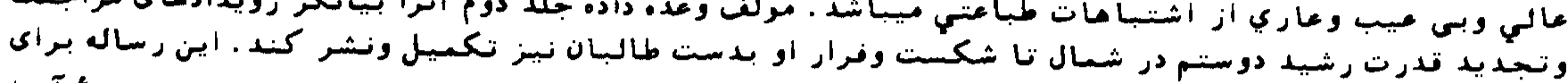

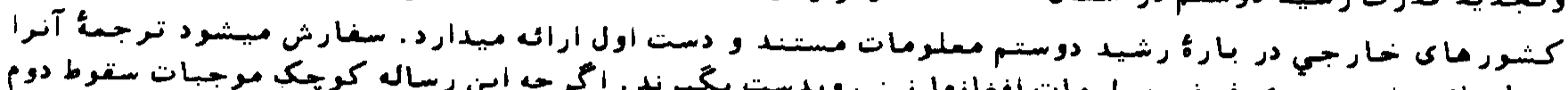

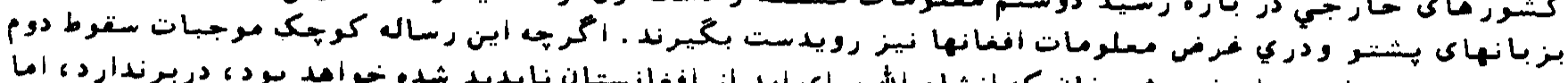

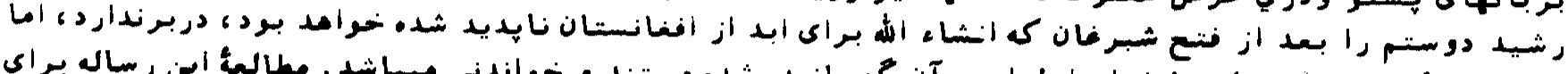

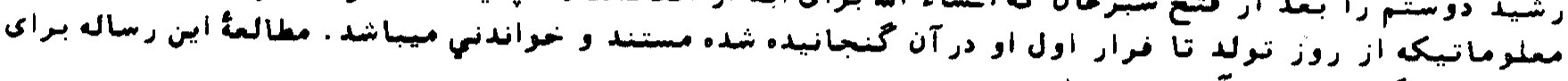

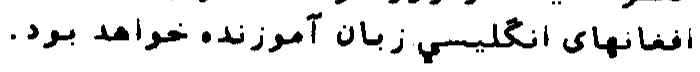

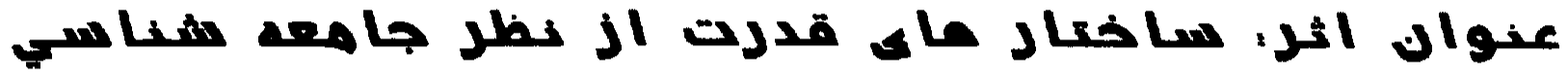

\author{
مولف : يوهنوال دكتور معسم عثمان روستار تره كي

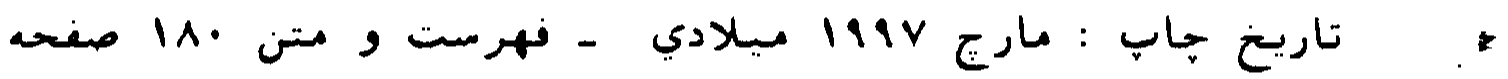

Dr. Rustar - 14 Avenue de L'Europe - 69140 Rillieux-La-Pape - France

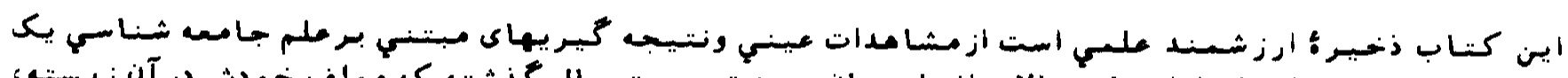

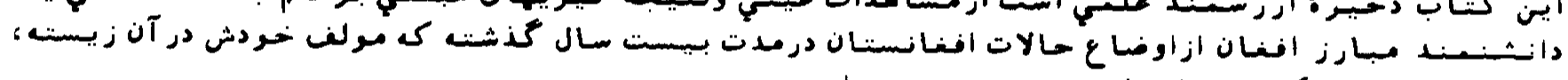

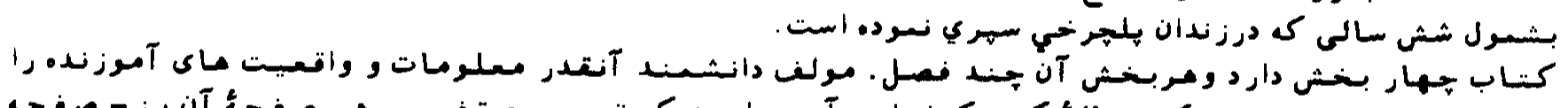

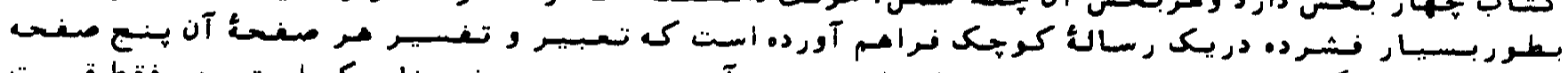

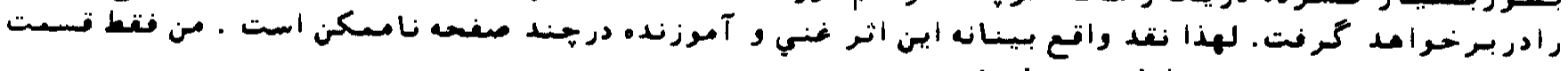

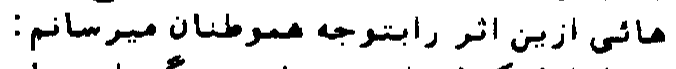

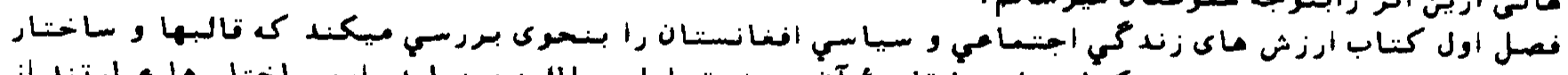

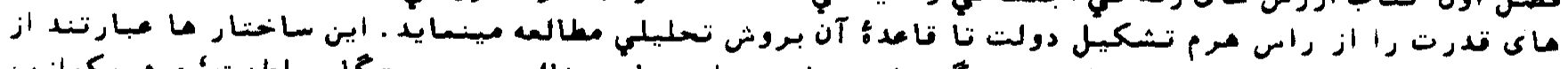

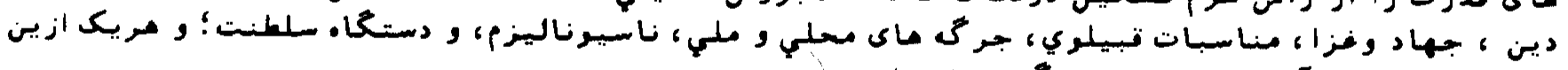

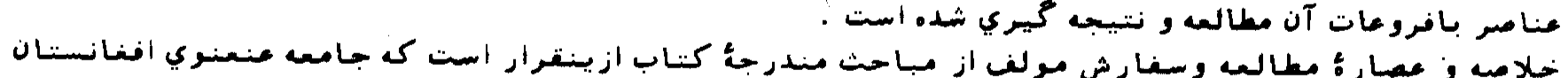

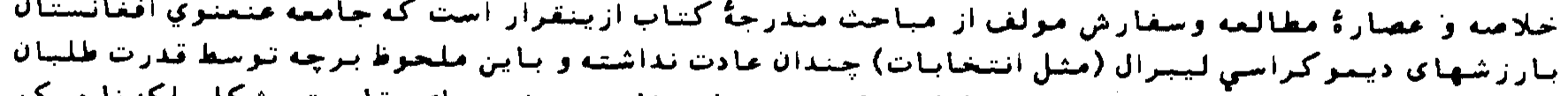

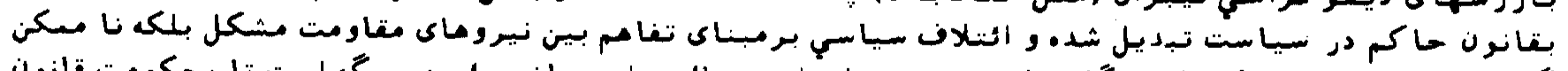

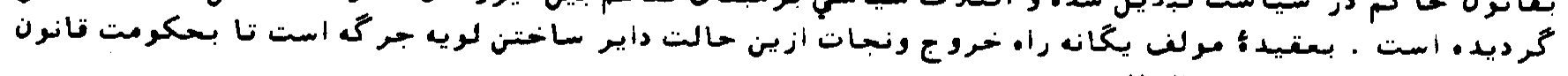

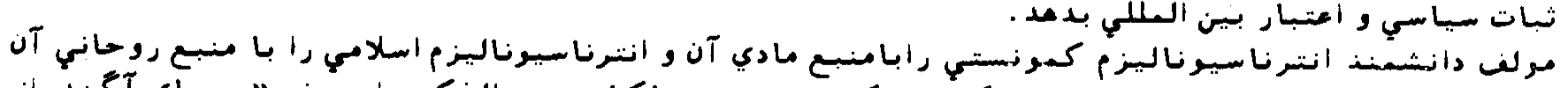

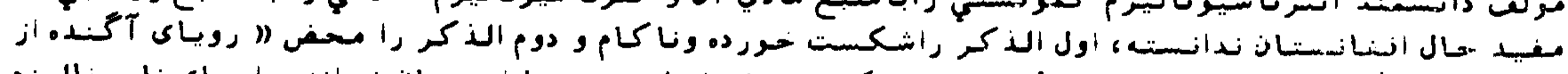

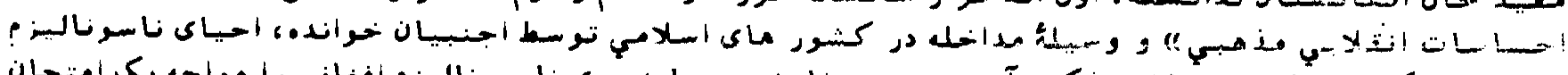

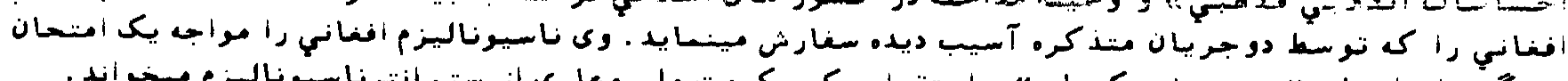

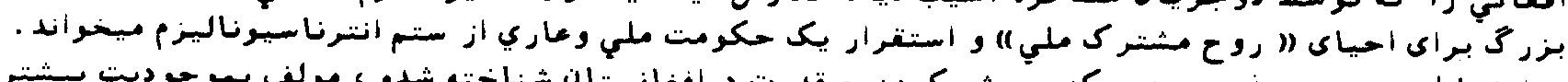

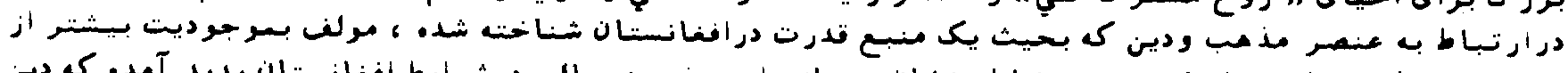

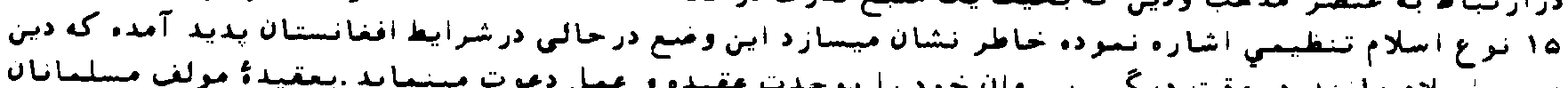

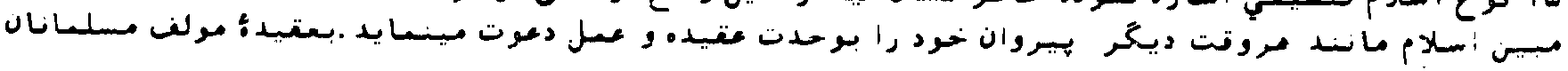




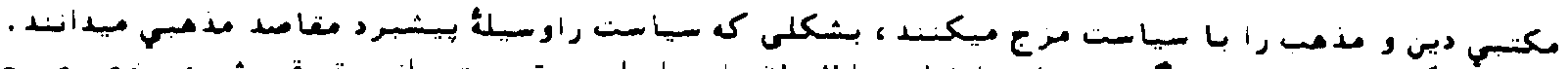

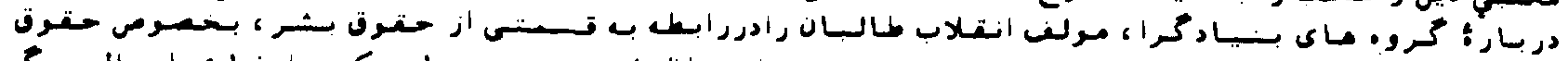

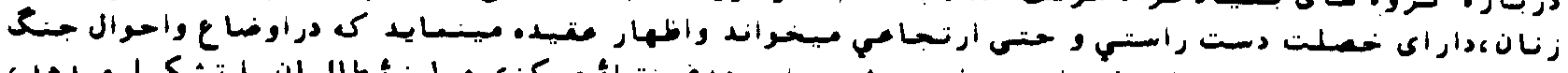

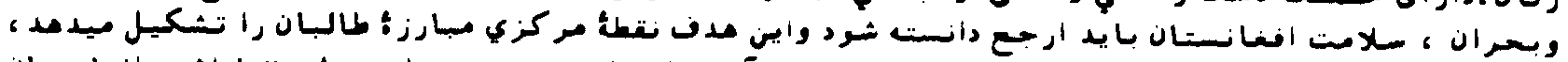

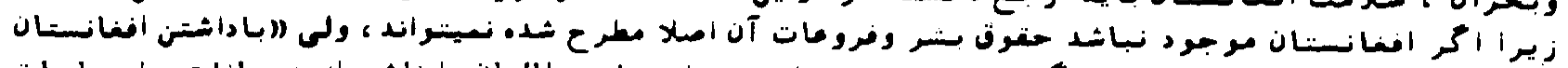

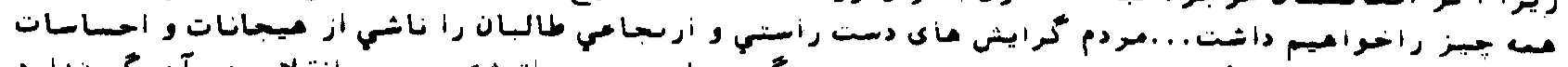

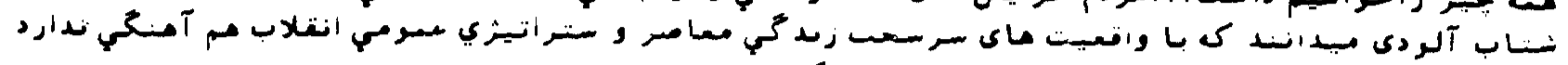

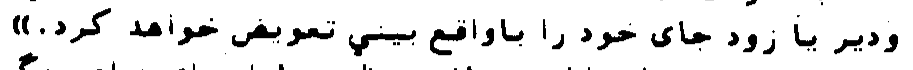

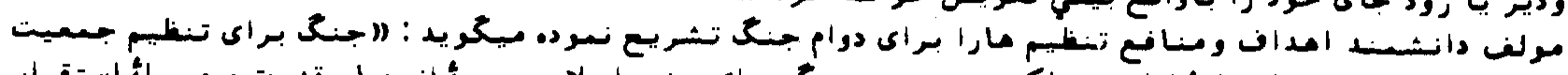

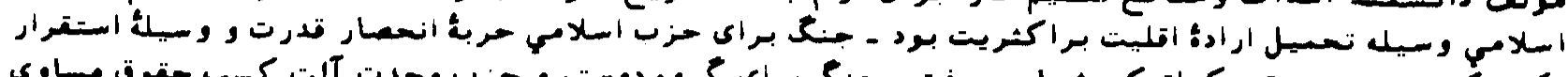

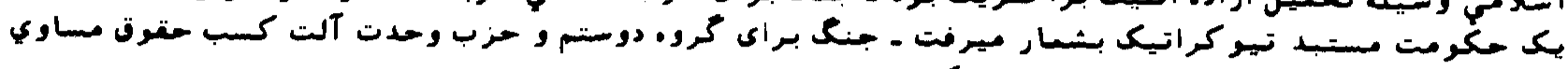

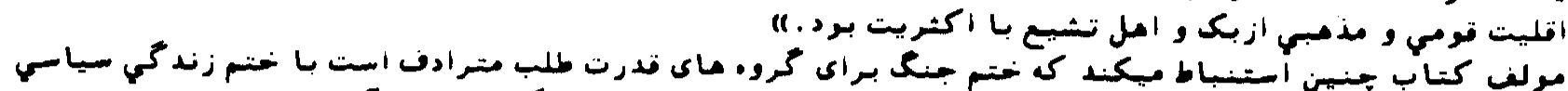

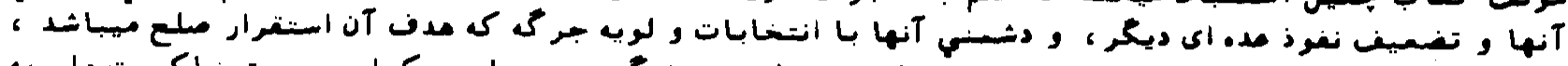

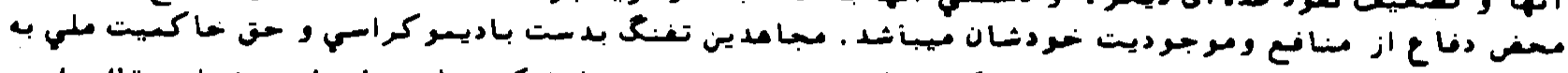

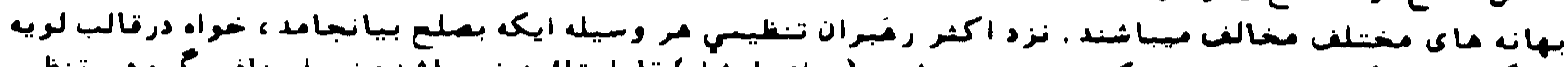

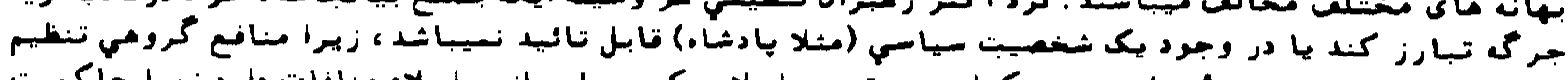

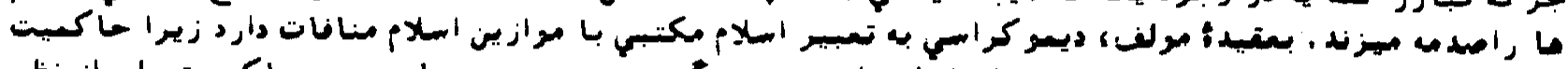

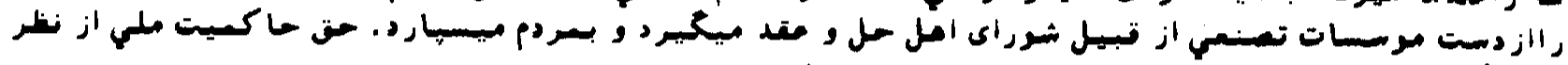

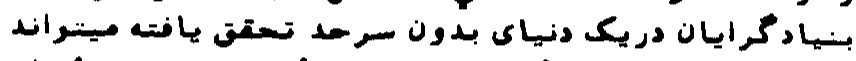

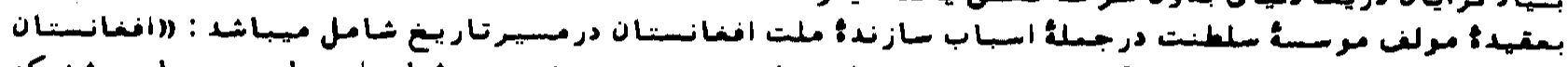

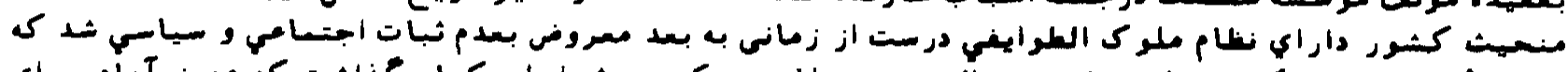

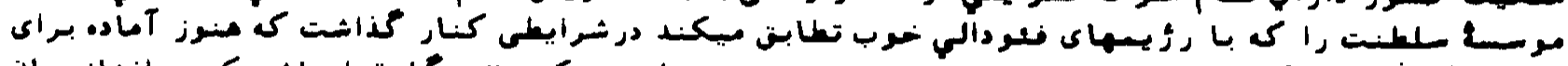

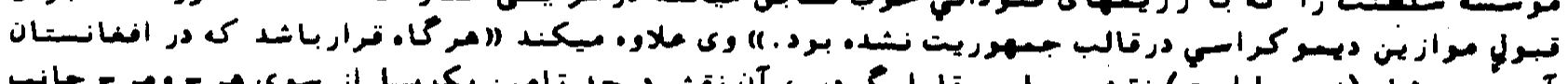

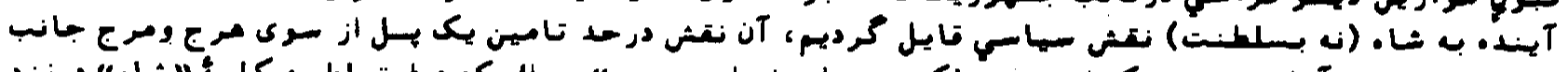

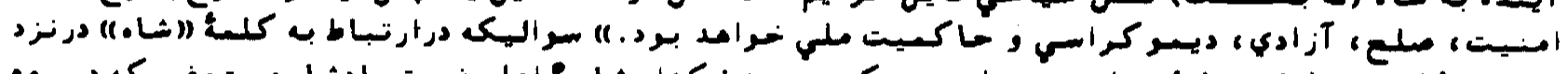

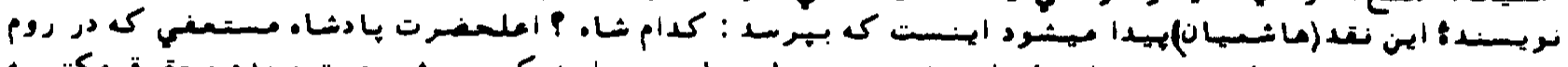

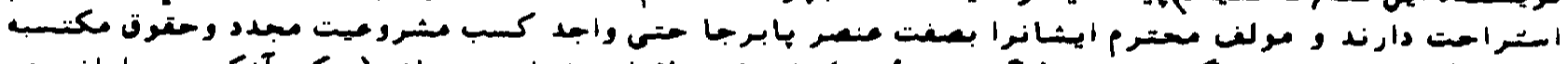

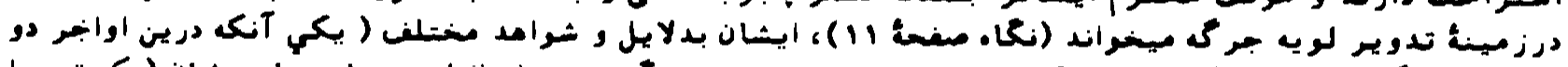

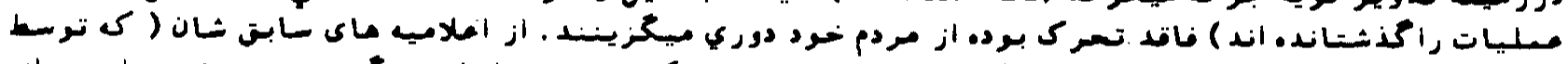

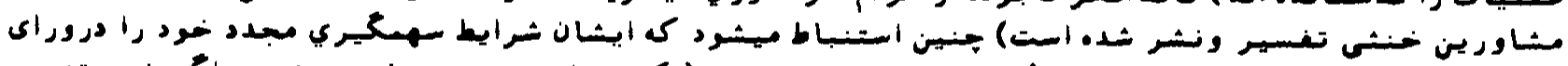

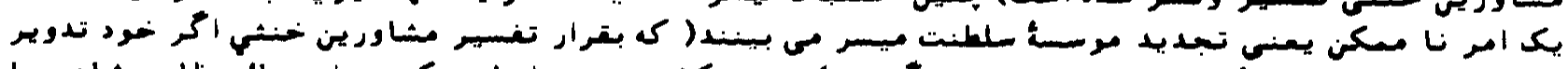

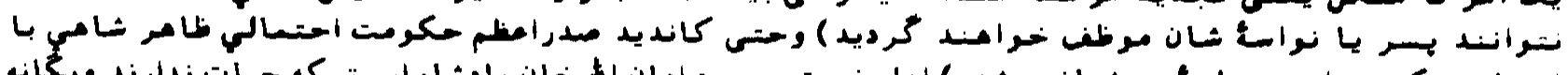

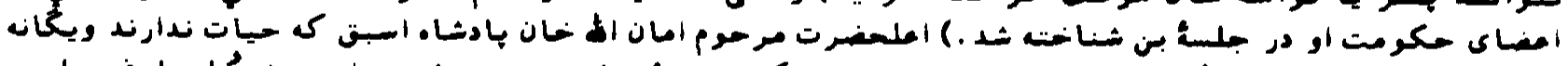

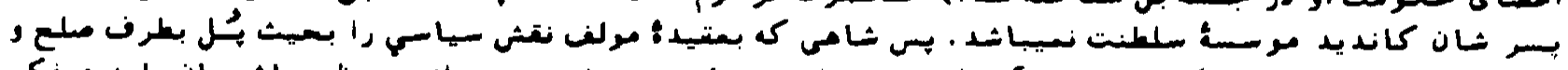

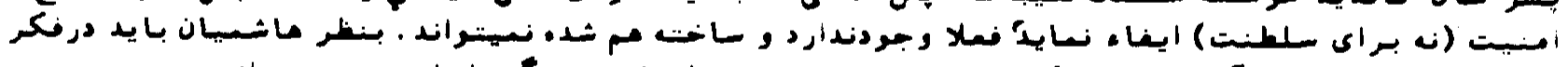

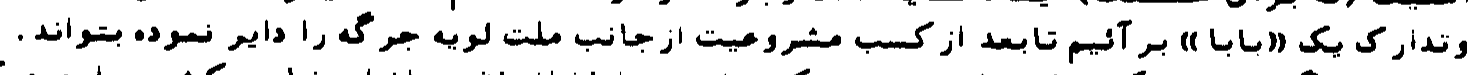

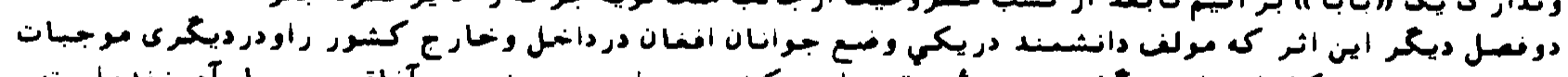

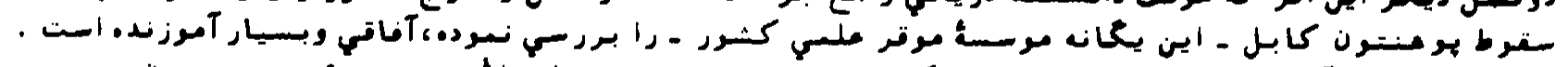

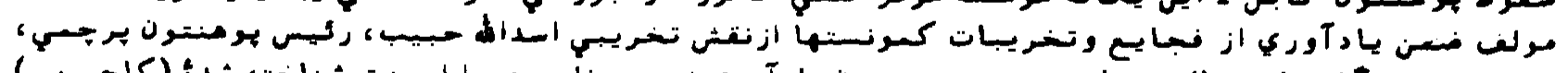

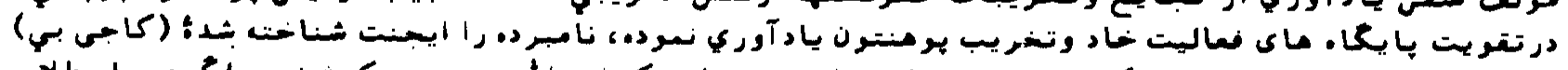

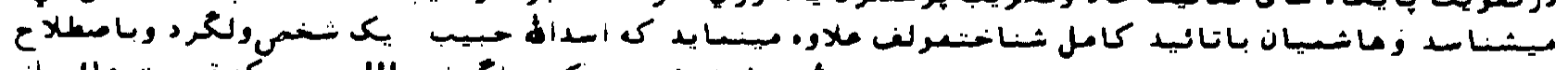

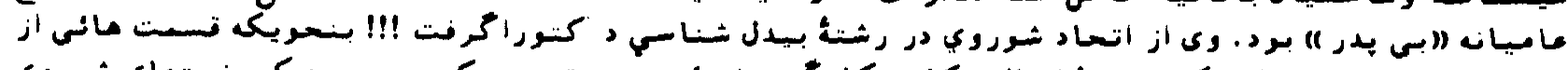

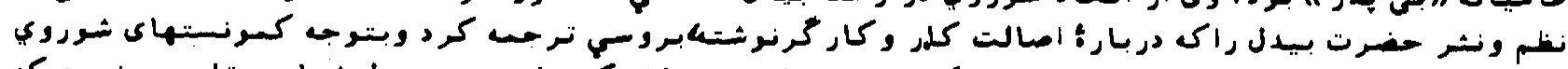

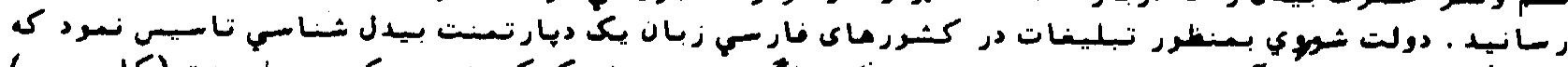

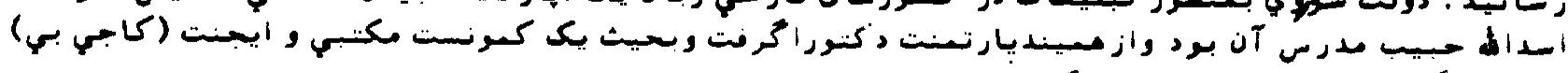

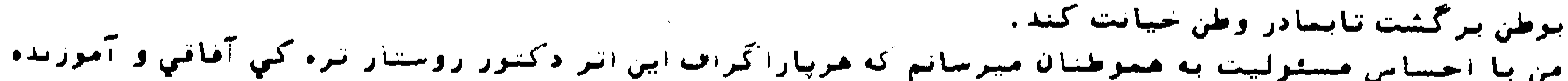

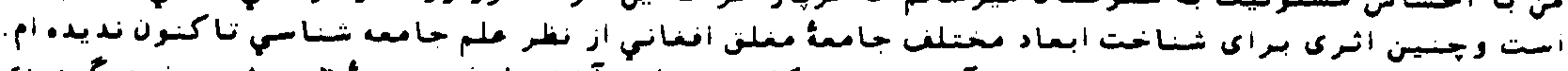

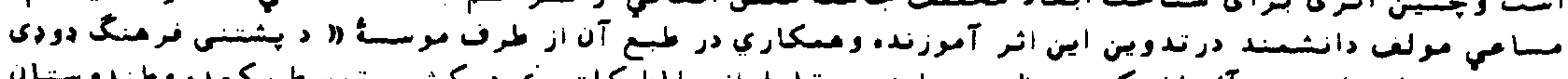

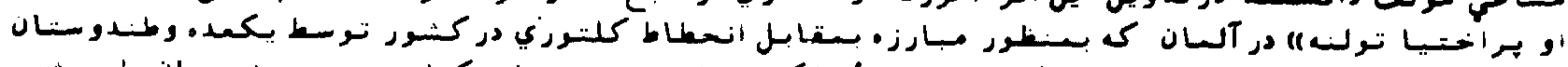

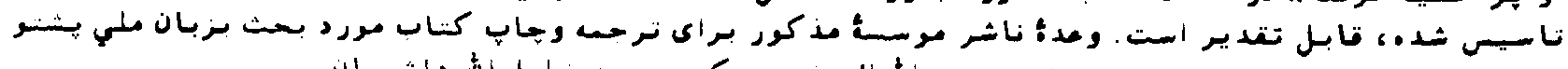

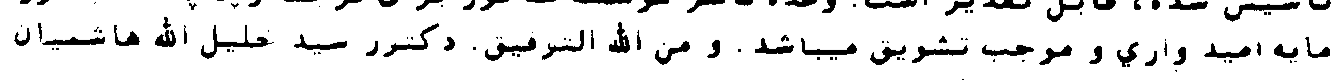




\section{0

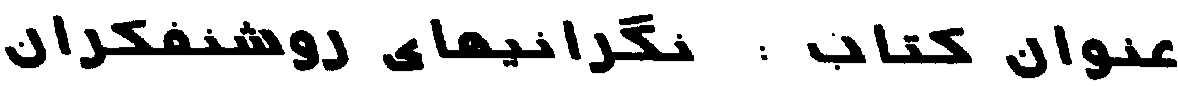

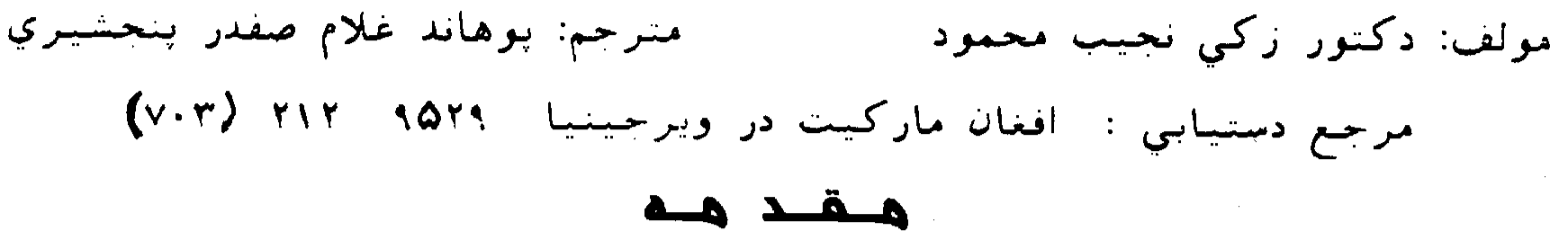

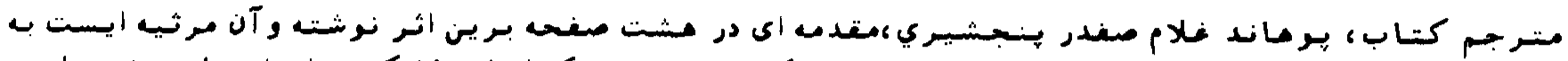

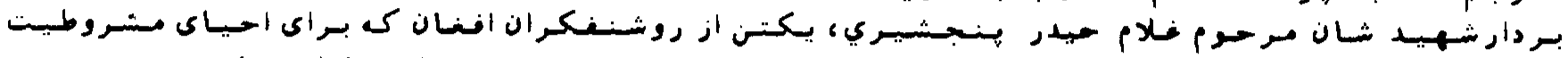

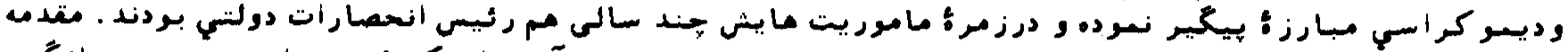

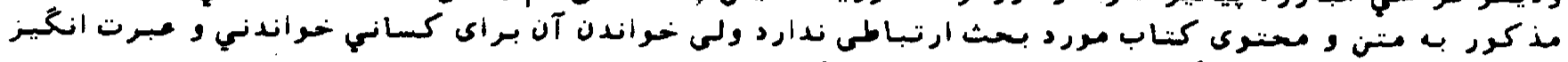

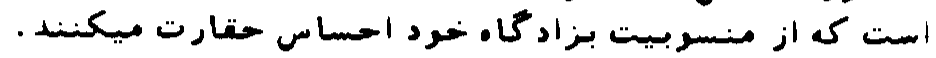

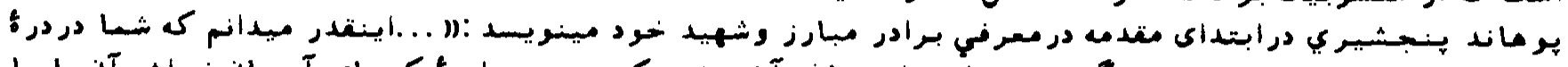

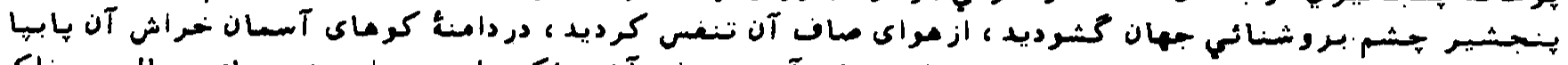

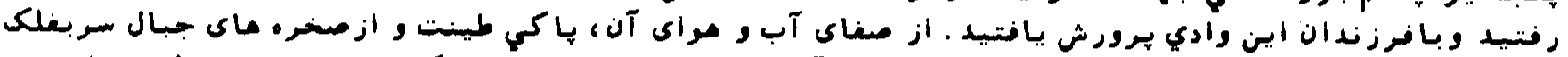

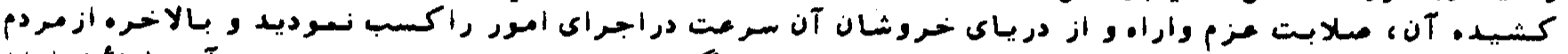

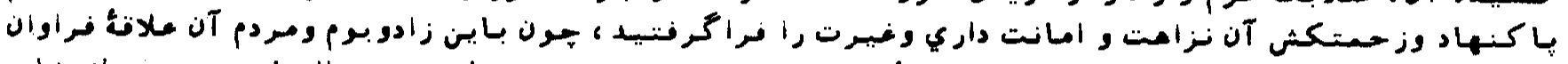

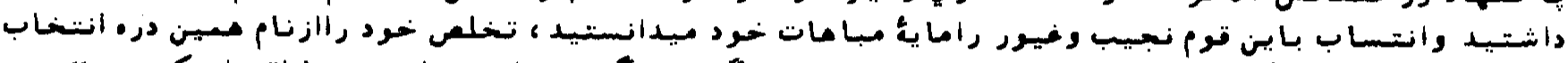

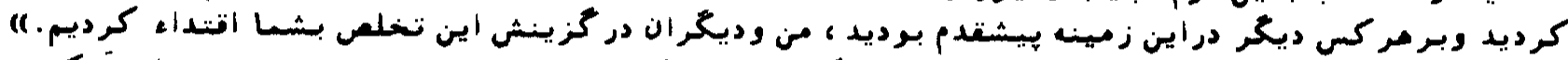

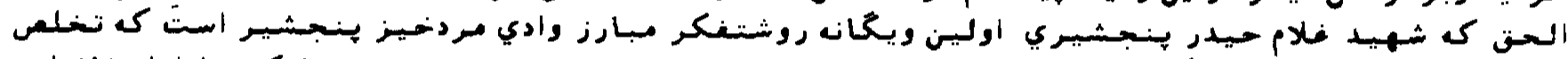

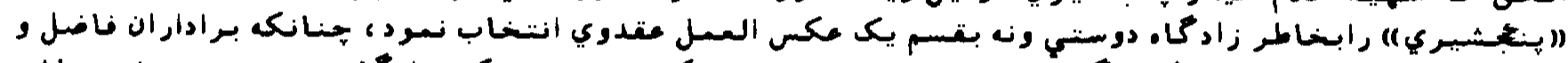

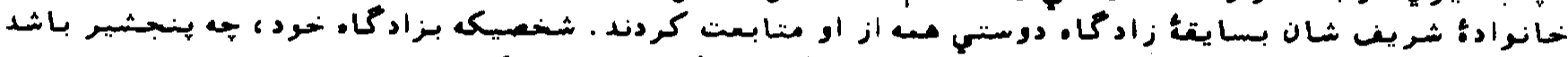

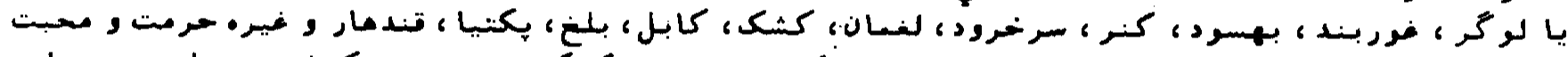

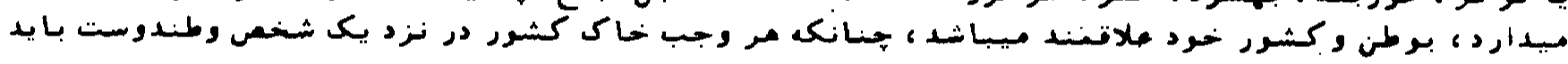

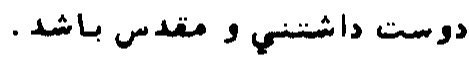

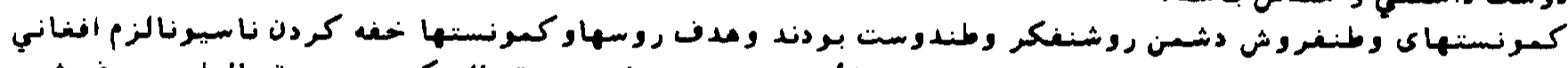

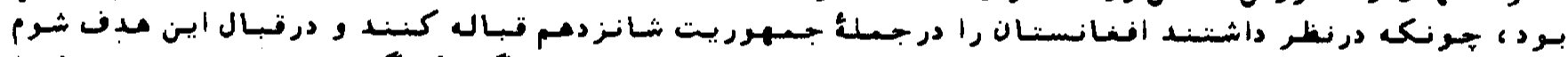

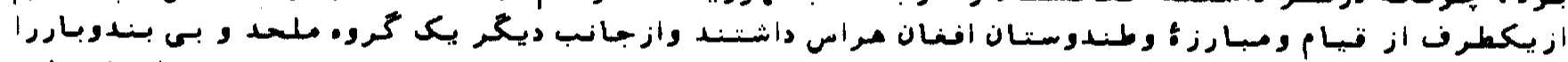

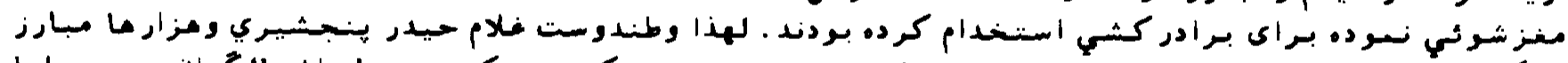

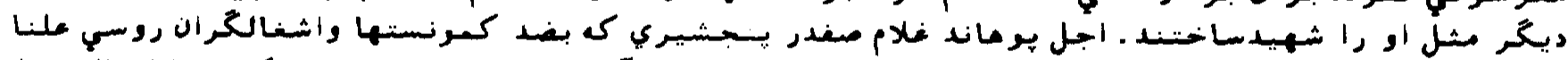

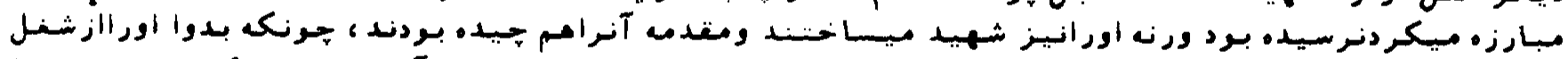

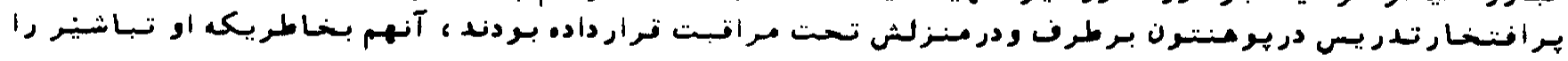

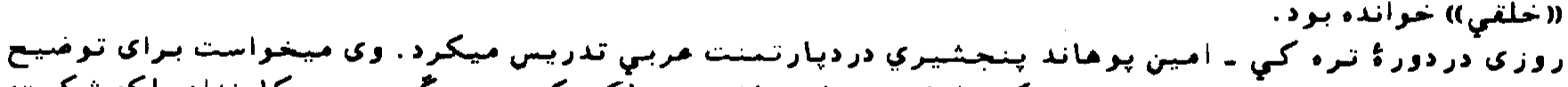

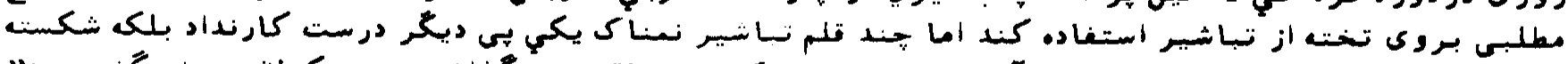

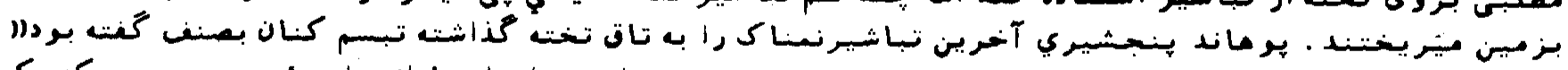

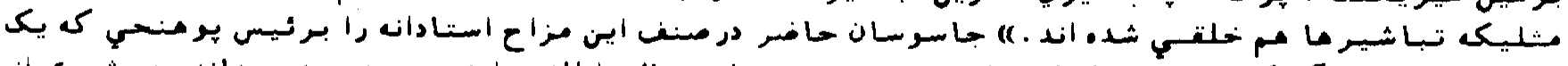

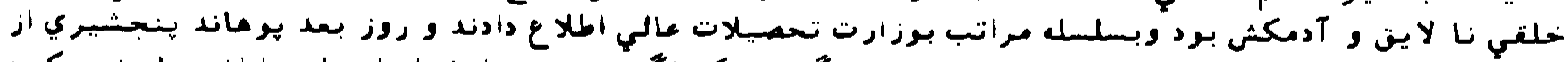

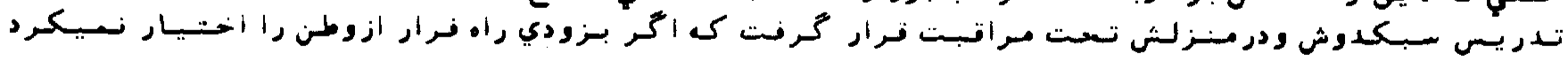

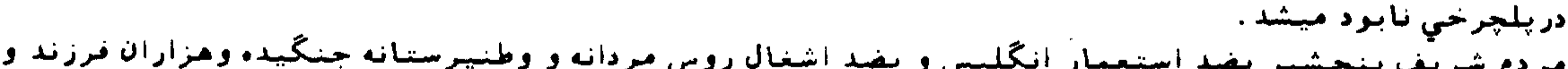

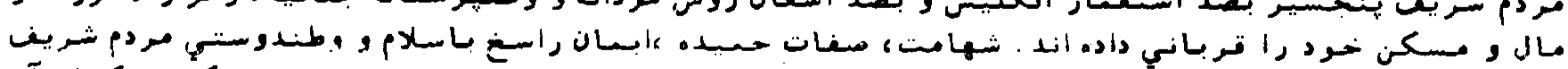

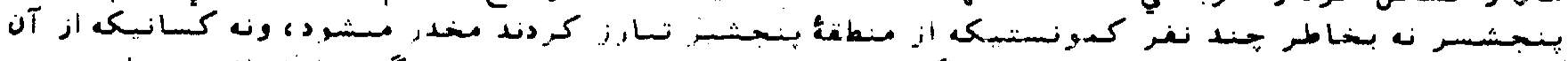

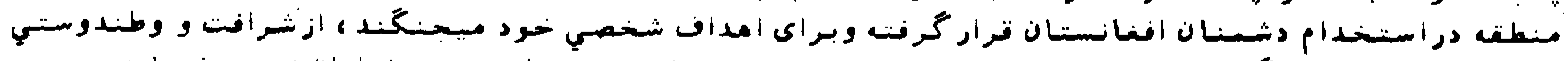

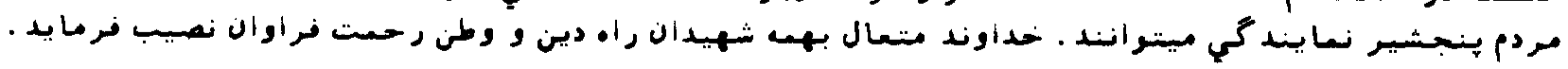

اكر طالبان قدرت سياسي رابمهردم انتقال ندهند، بمسشكلات بيستنر مراجه خراهند شد 


\section{كسمــ}

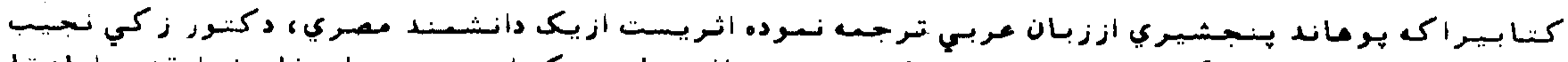

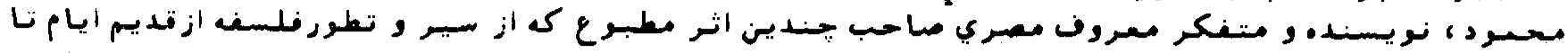

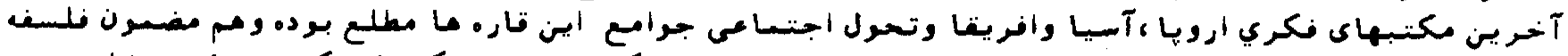

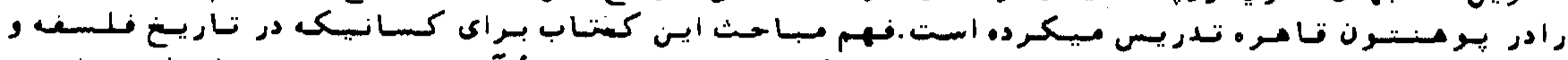

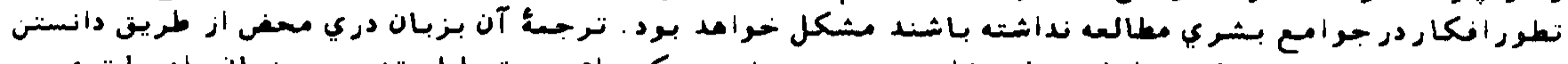

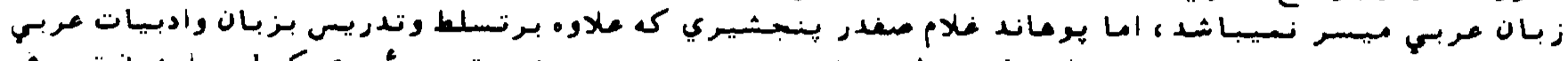

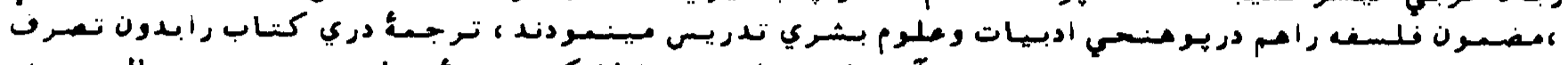

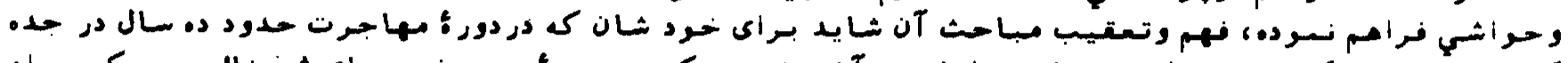

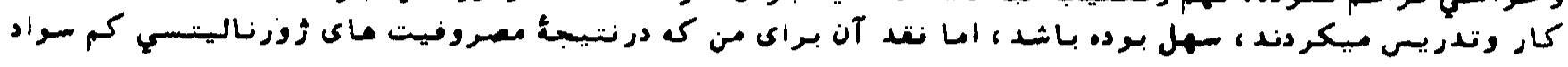

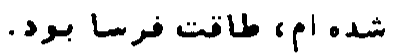

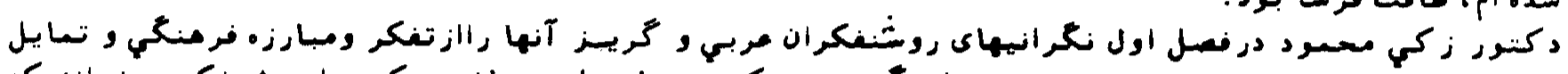

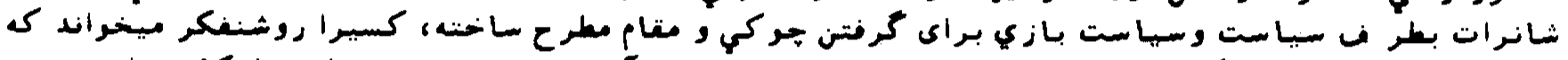

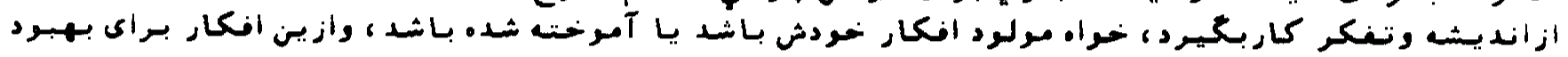

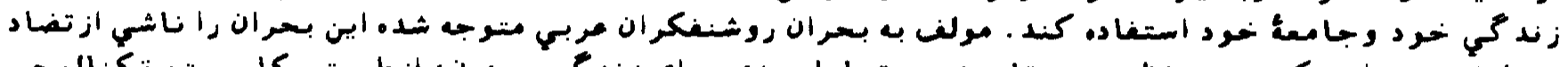

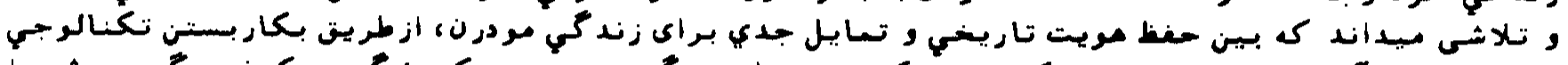

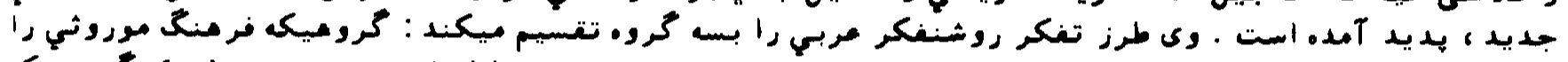

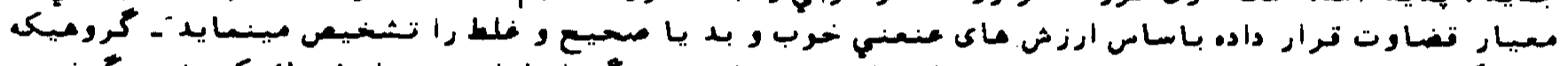

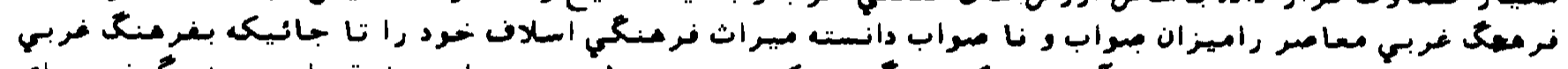

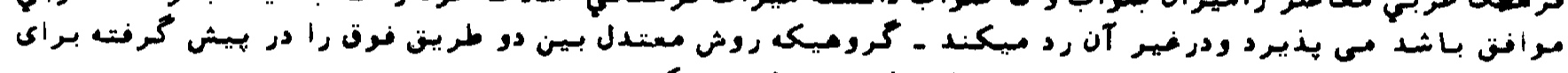

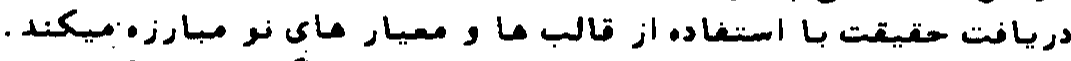

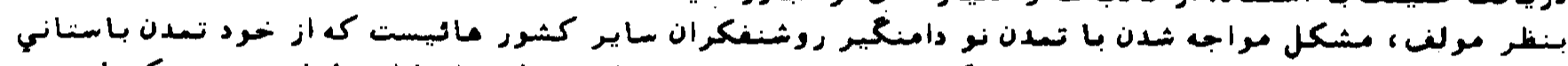

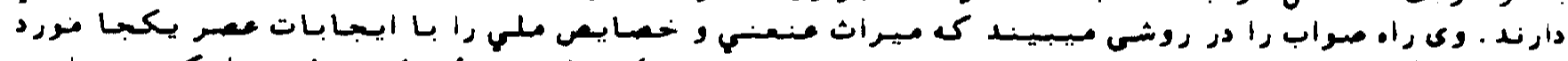

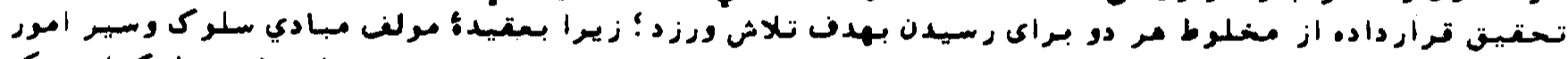

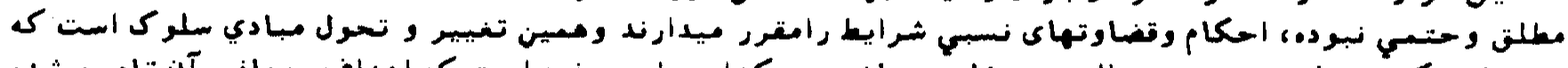

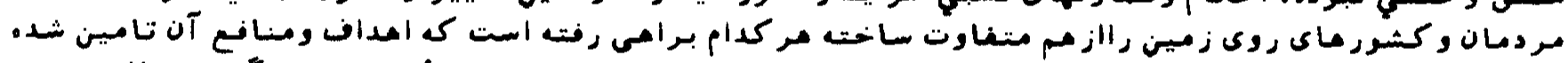

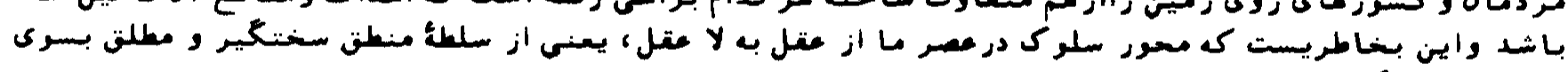

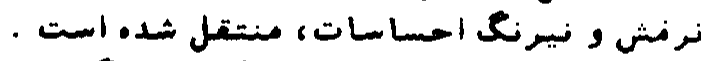

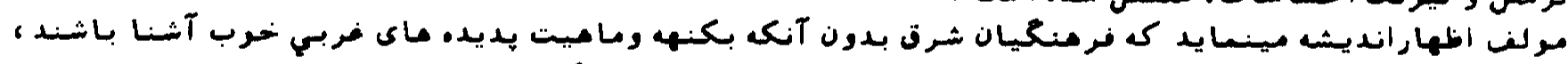

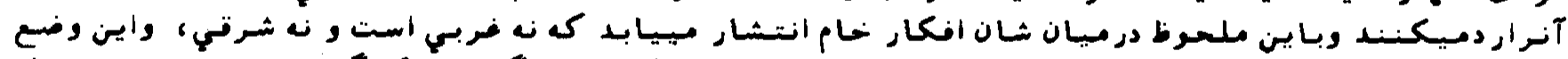

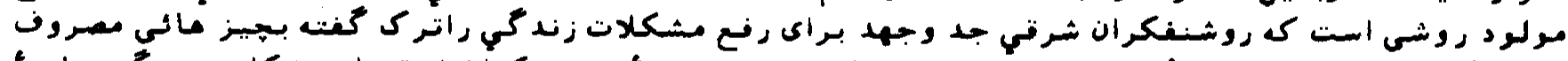

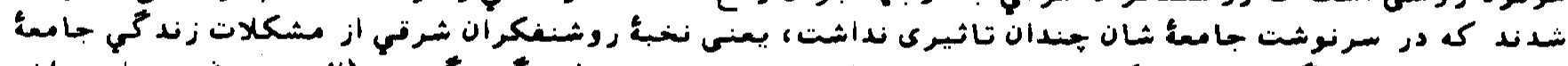

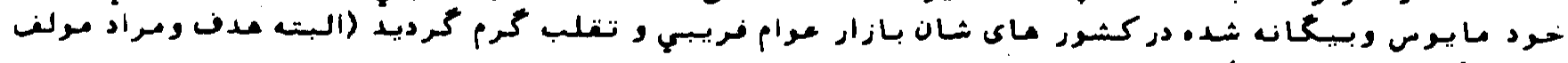

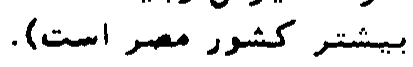

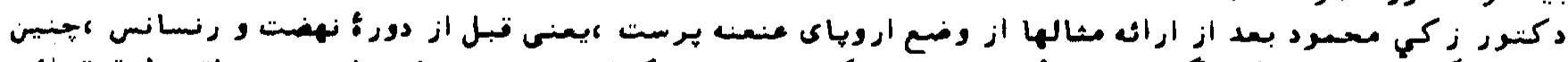

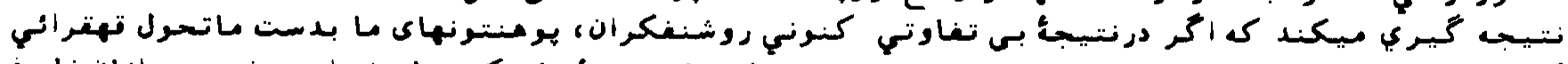

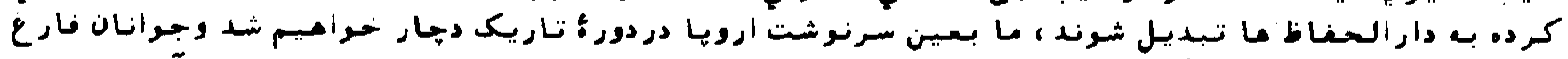

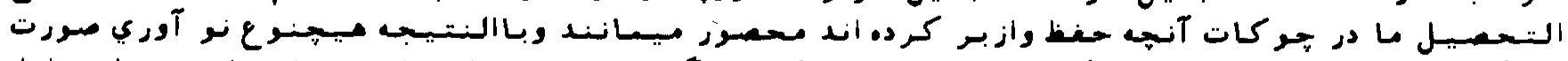

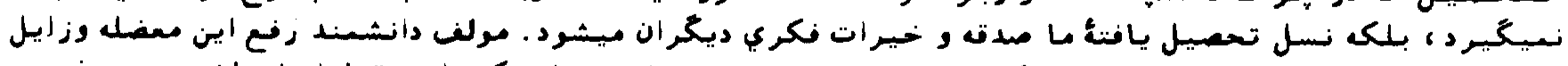

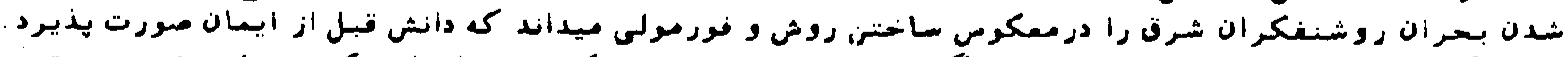

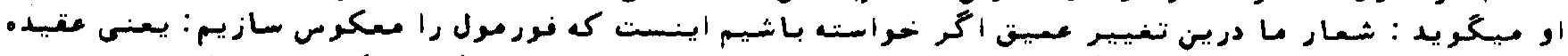

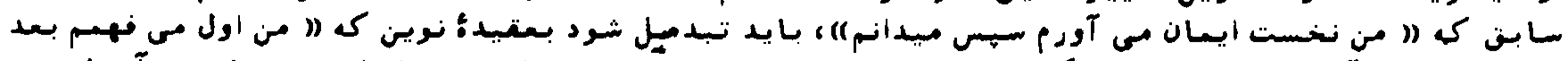

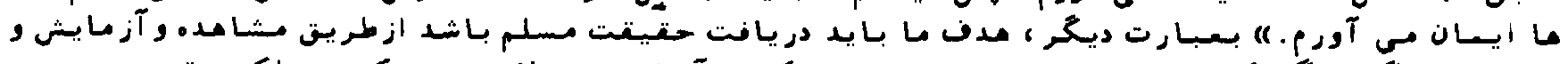

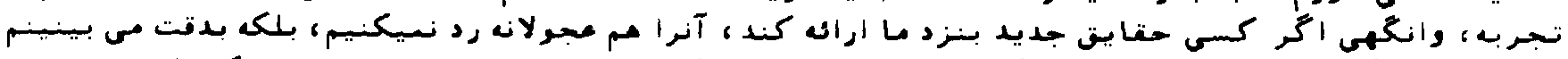

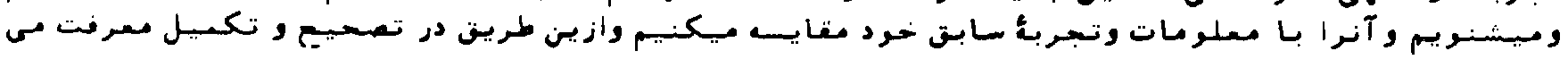




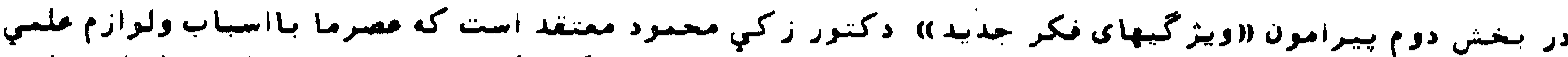

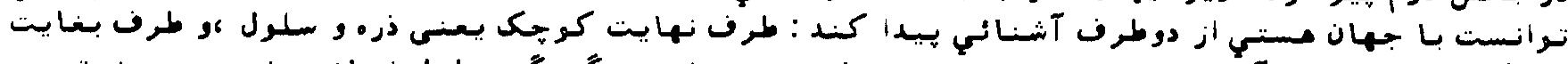

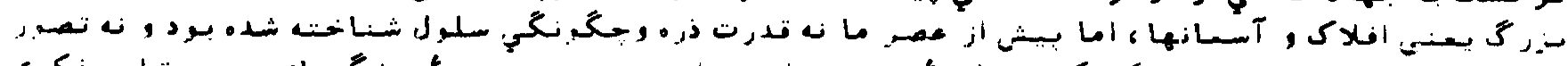

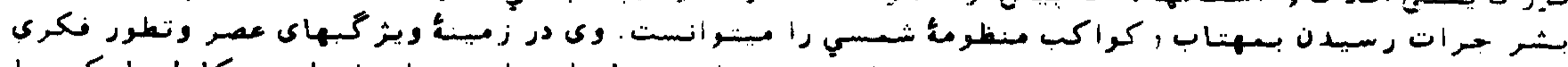

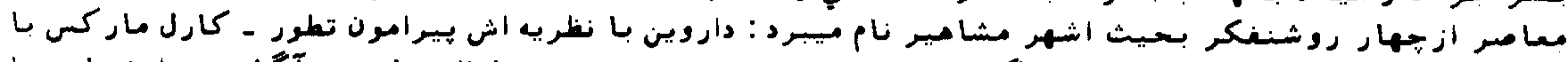

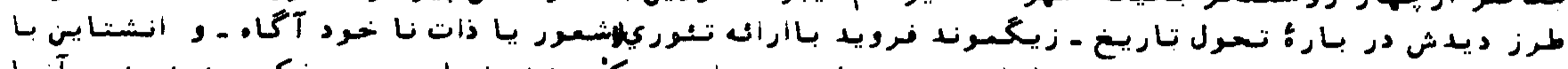

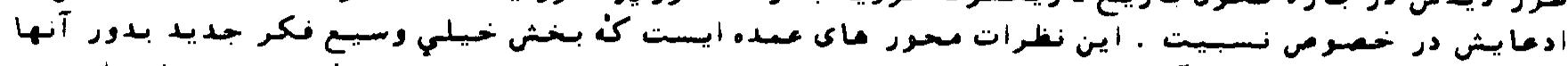

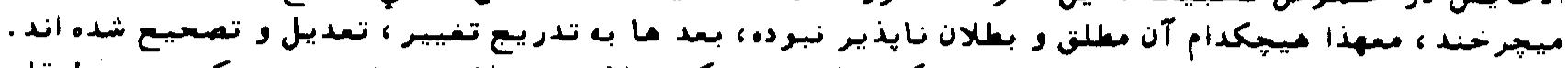

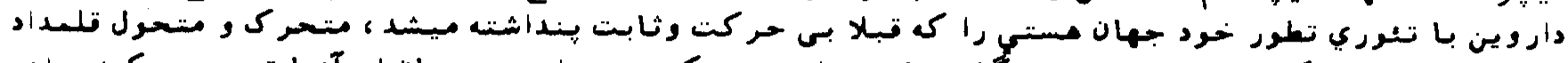

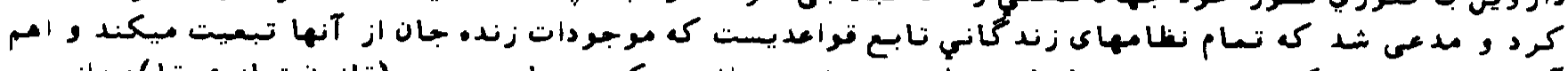

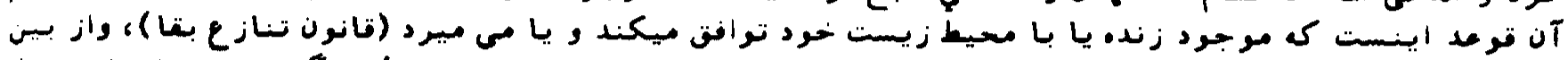

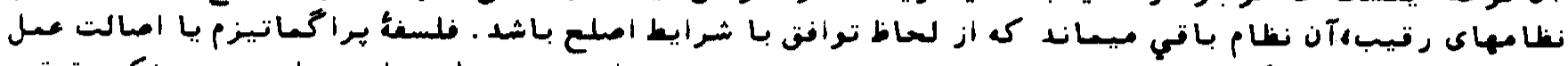

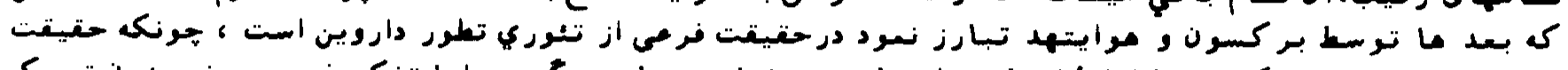

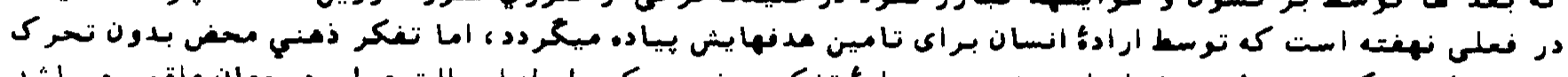

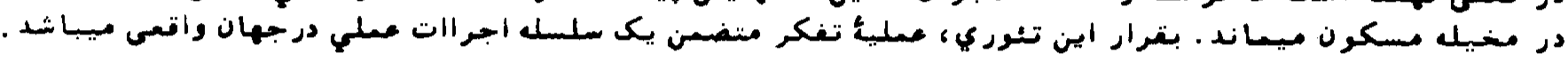

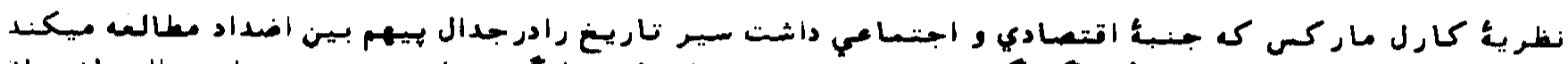

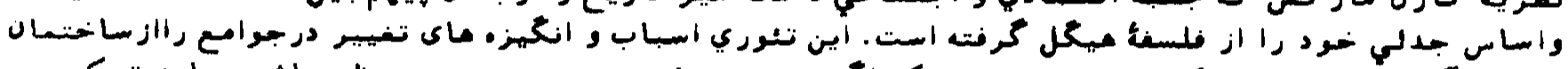

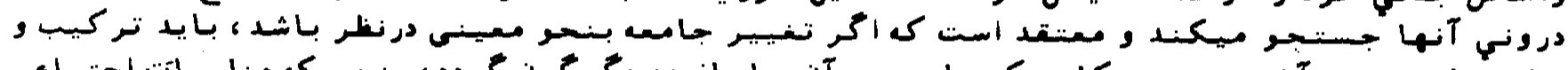

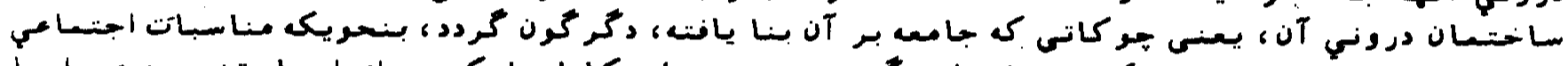

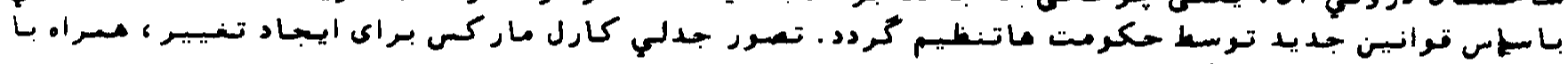

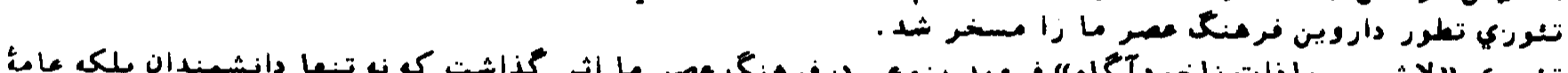

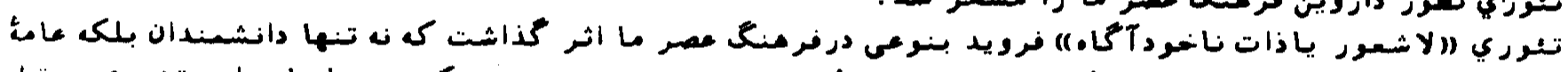

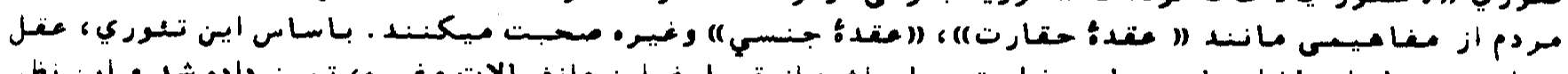

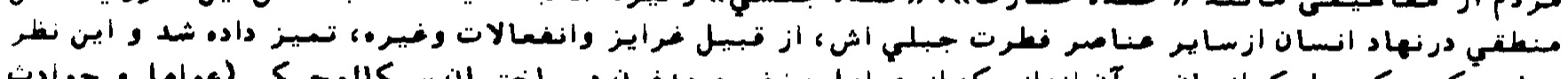

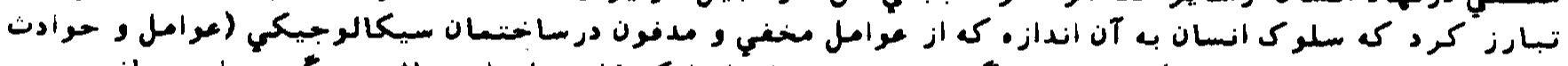

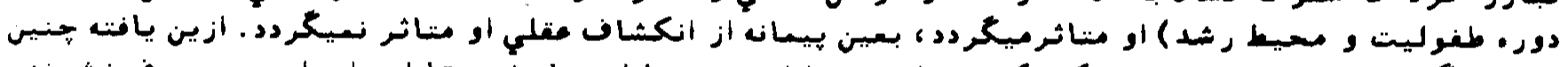

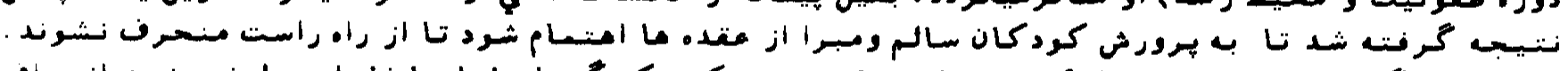

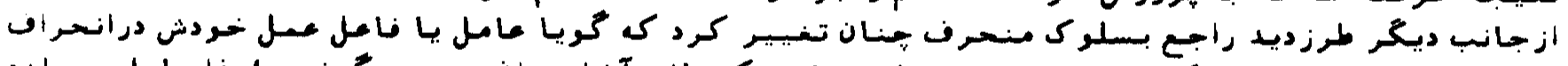

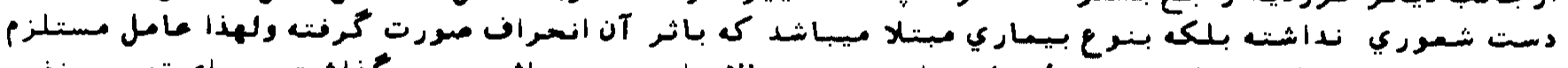

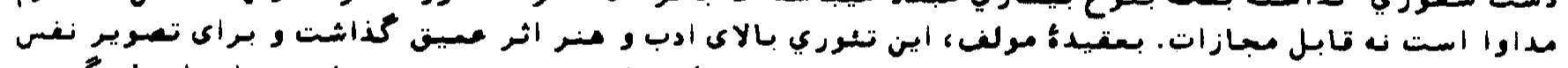

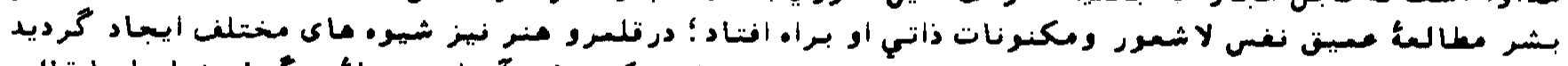

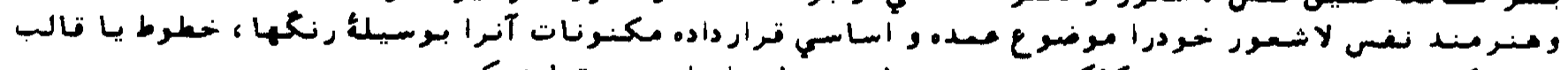

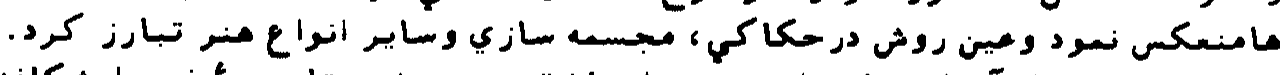

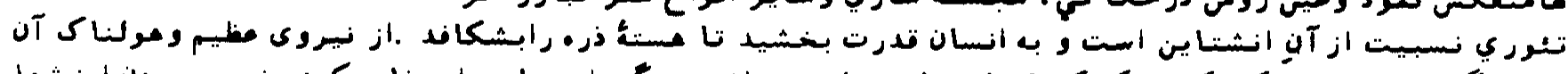

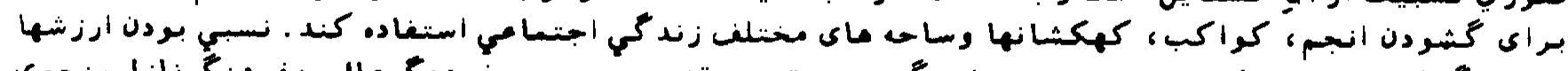

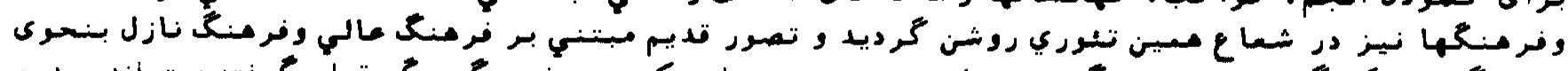

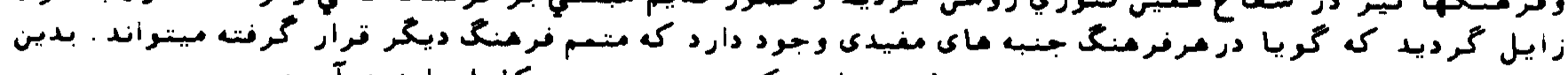

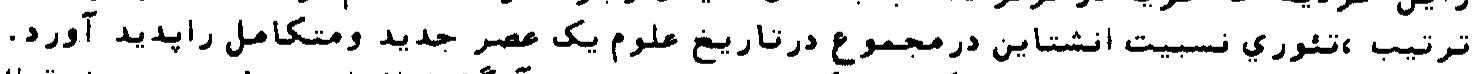

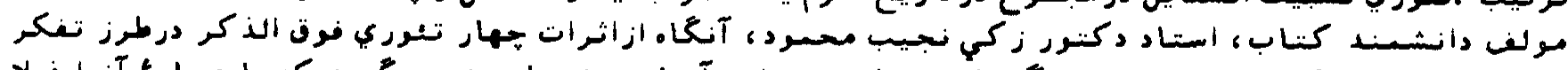

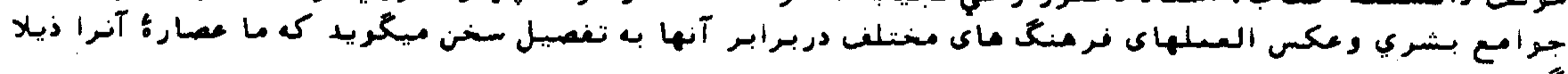

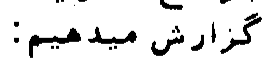

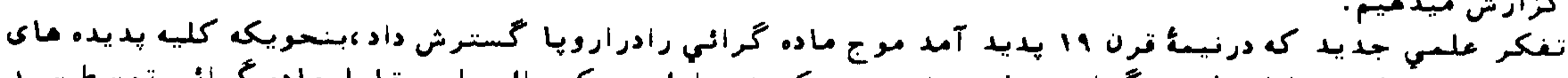

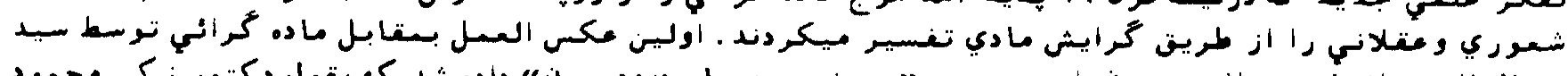

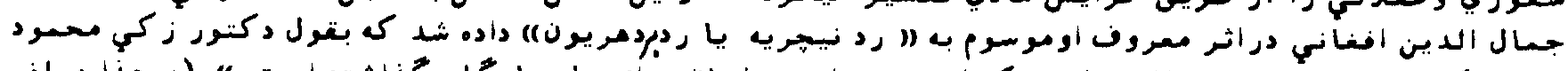

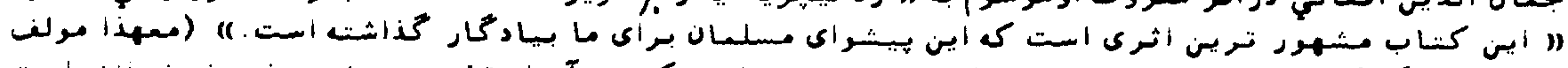

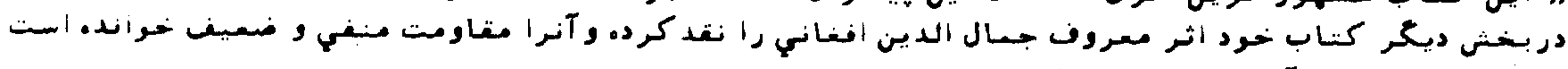

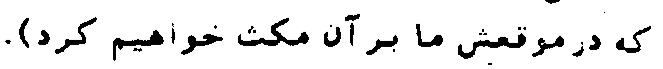




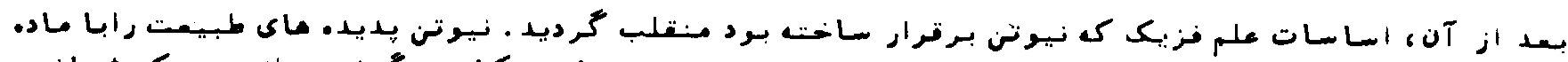

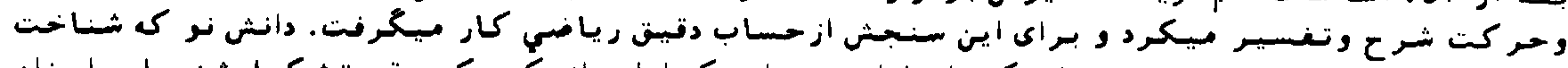

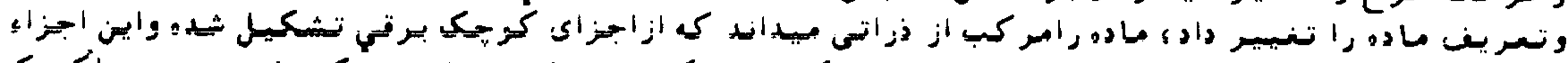

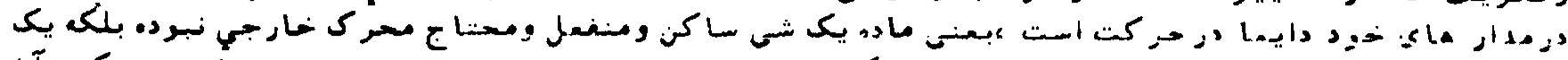

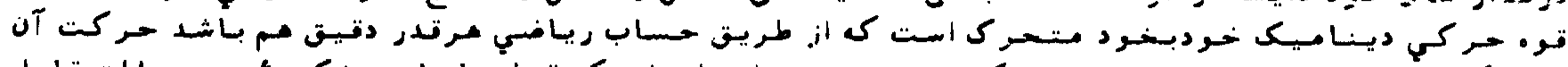

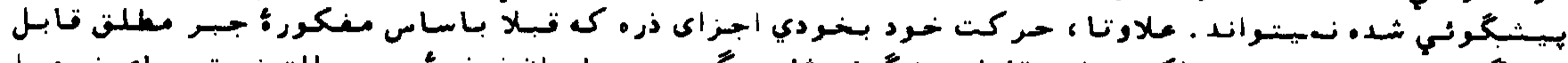

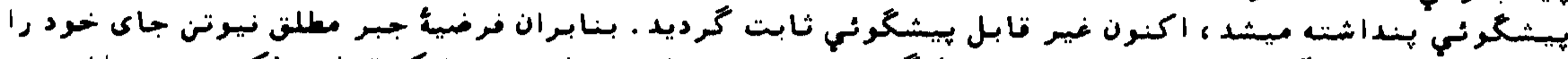

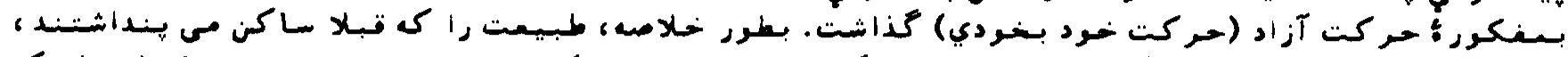

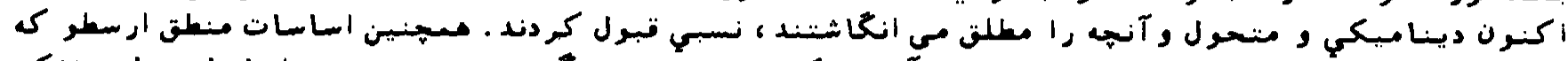

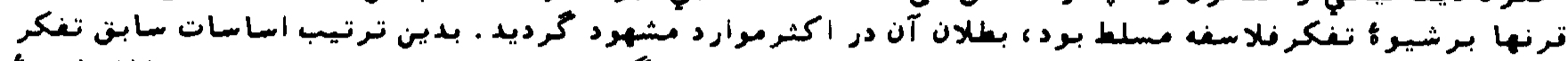

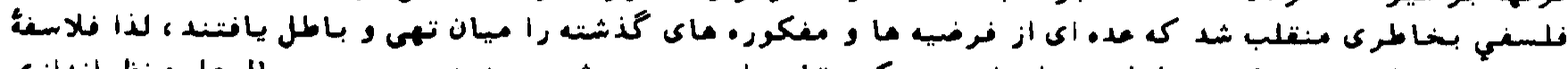

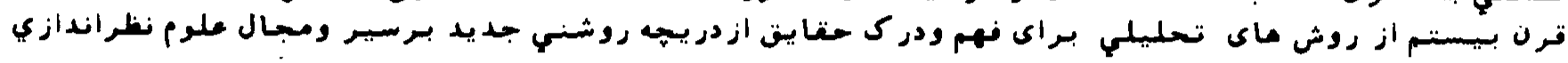

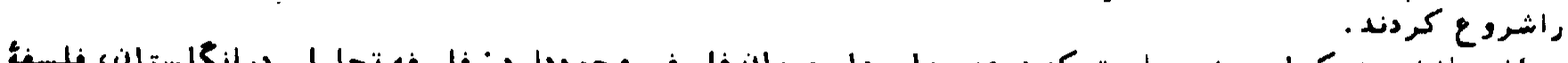

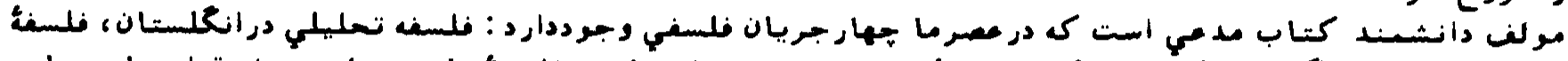

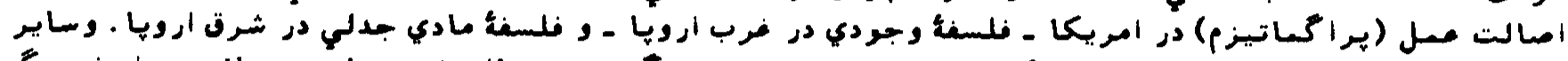

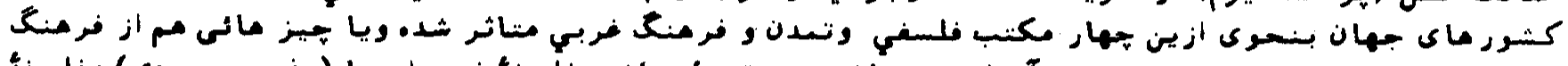

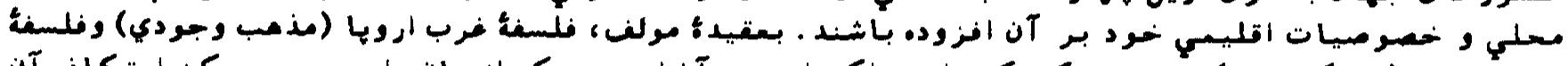

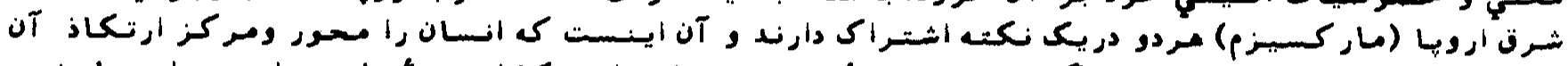

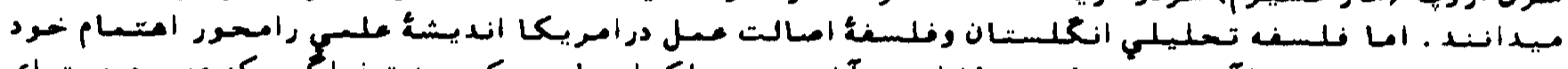

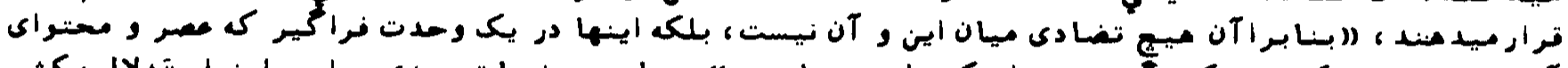

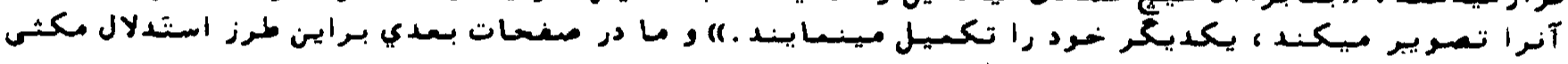

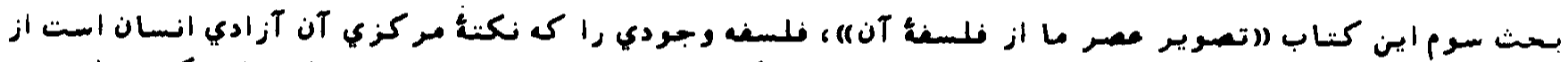

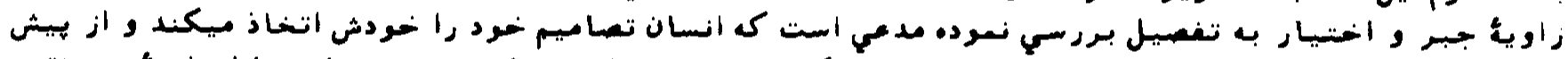

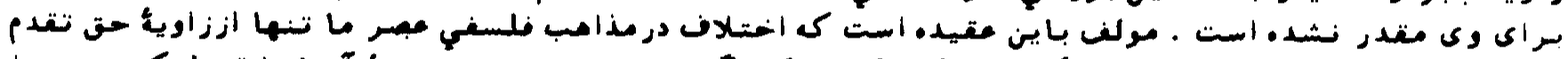

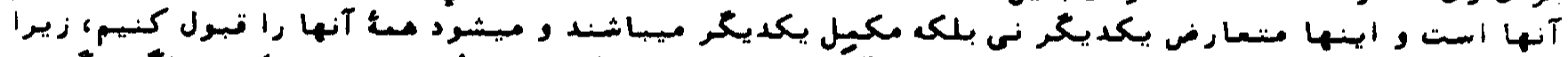

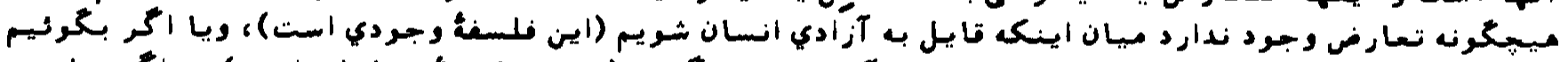

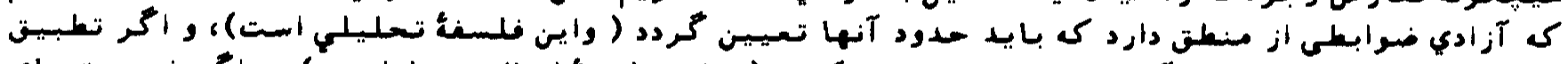

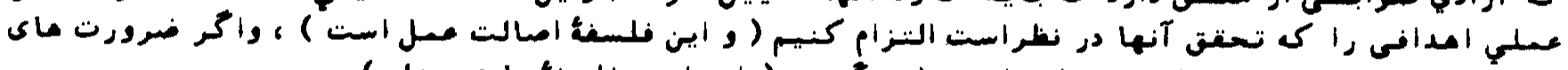

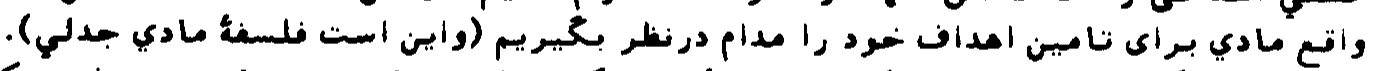

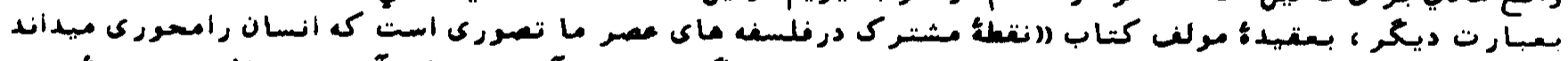

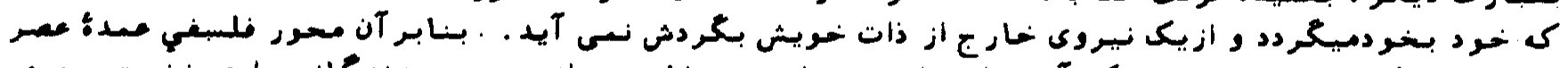

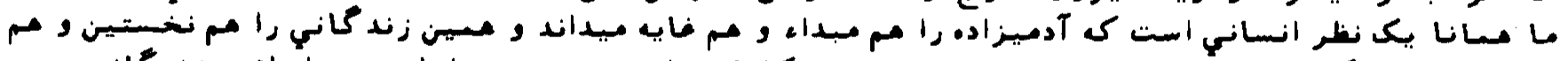

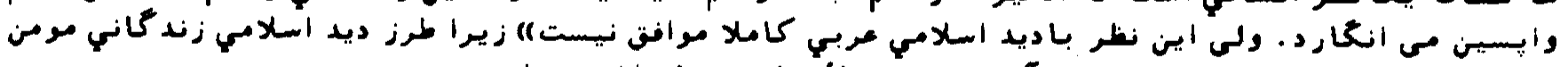

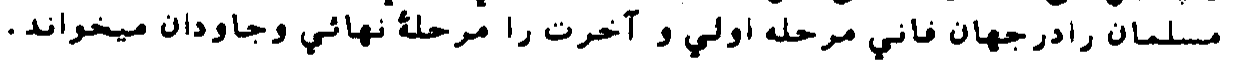

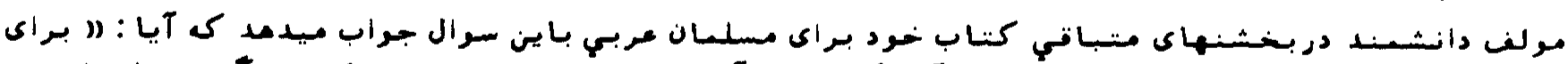

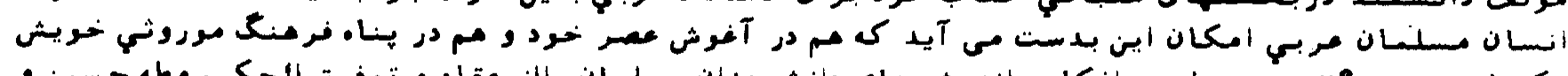

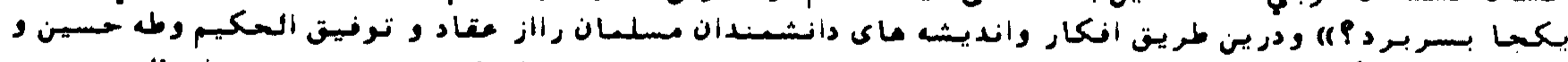

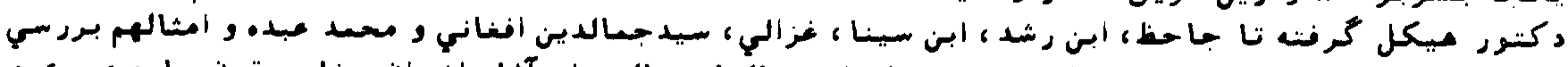

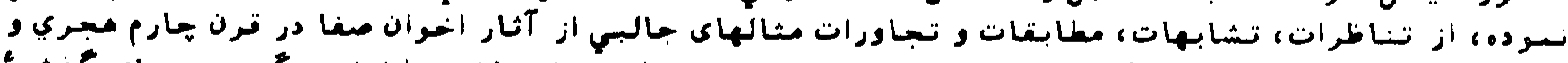

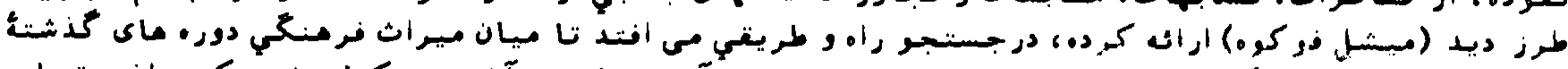

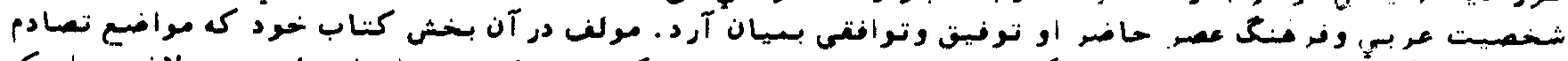

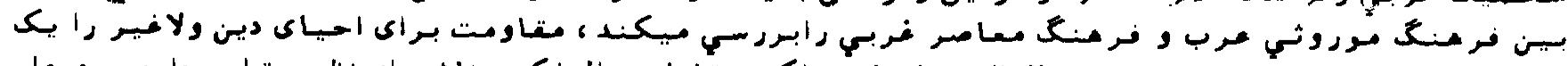

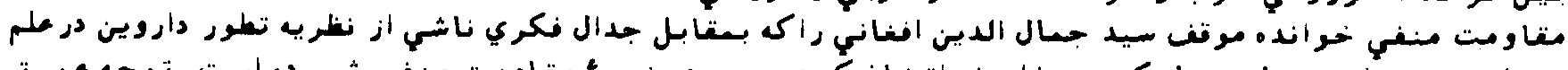

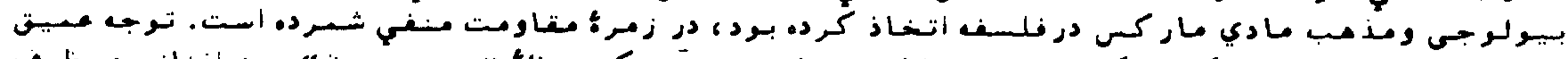

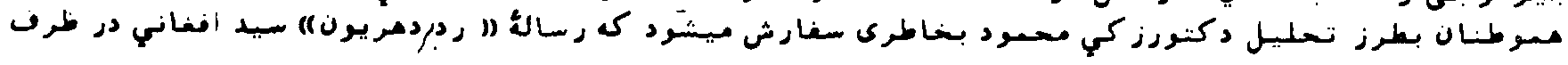




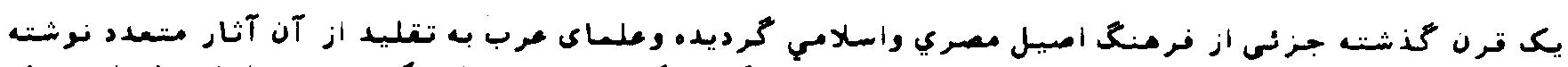

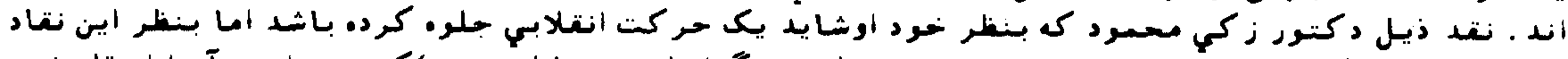

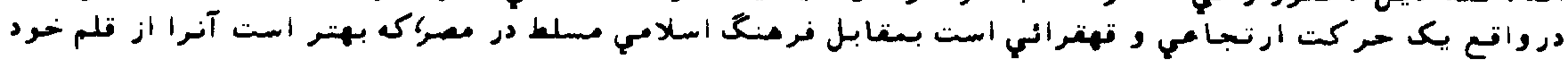

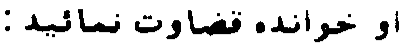

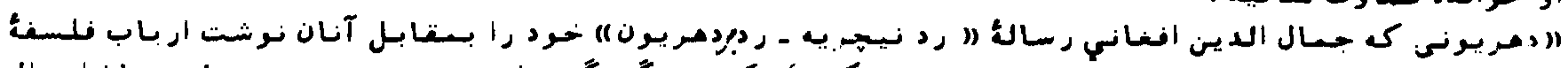

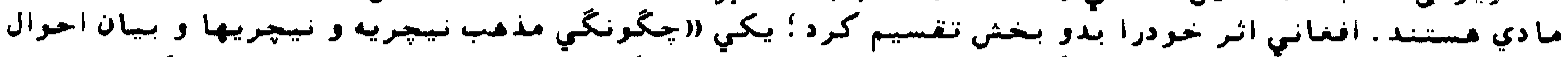

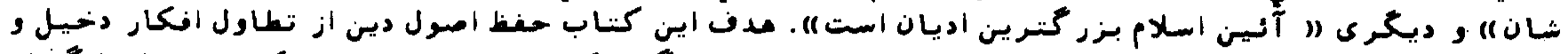

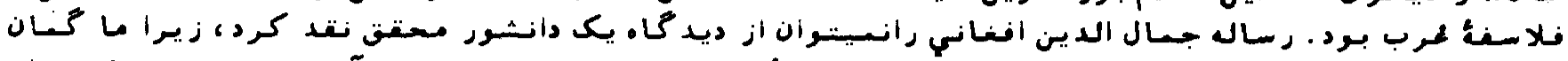

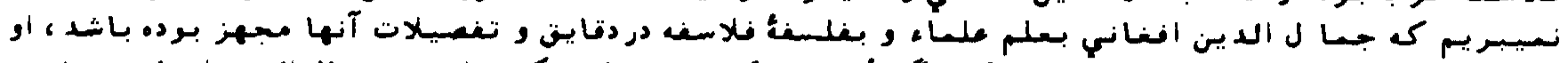

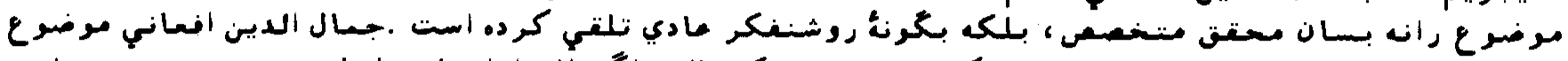

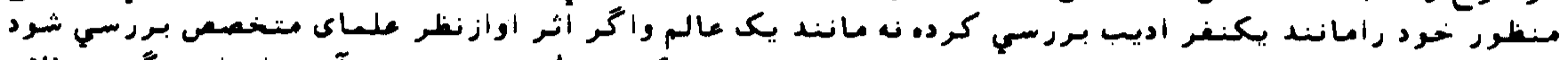

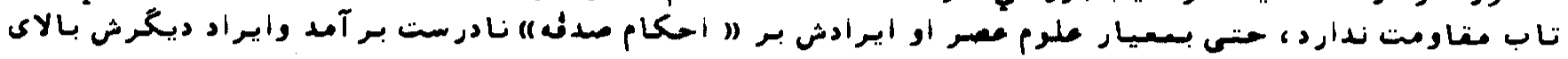

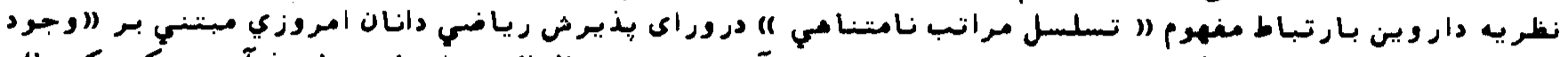

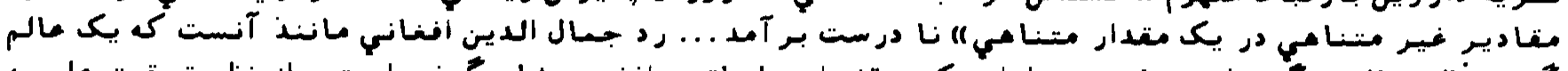

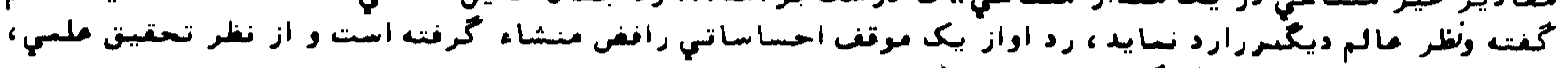

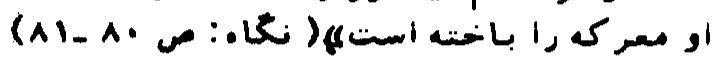

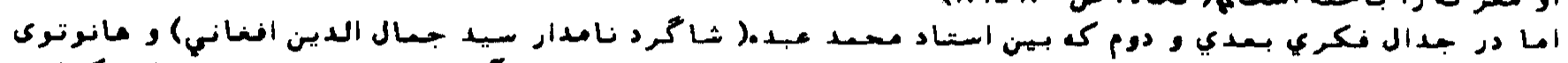

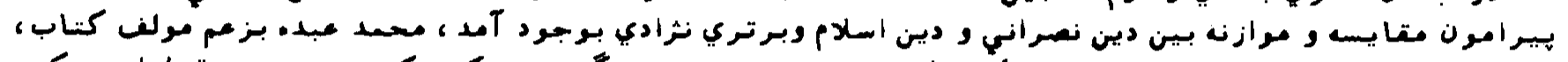

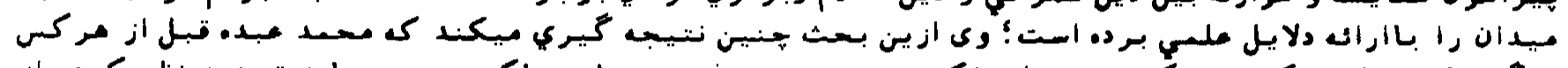

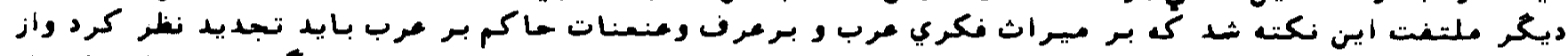

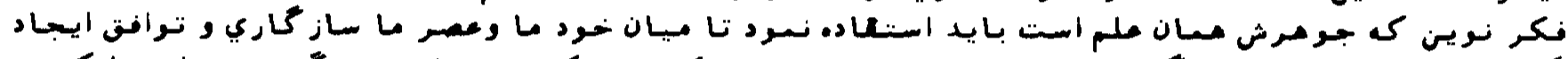

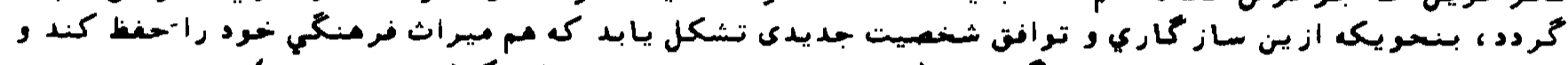

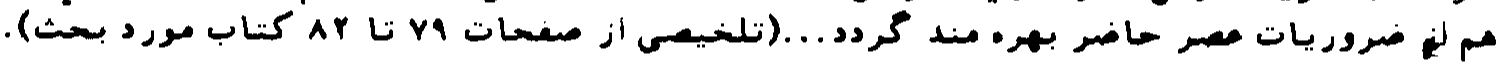

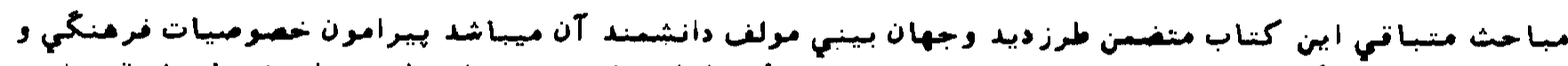

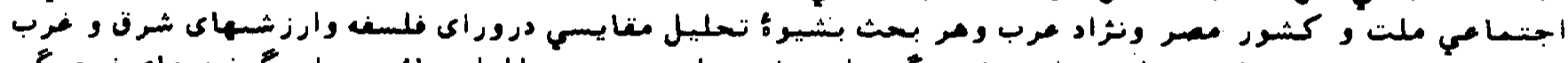

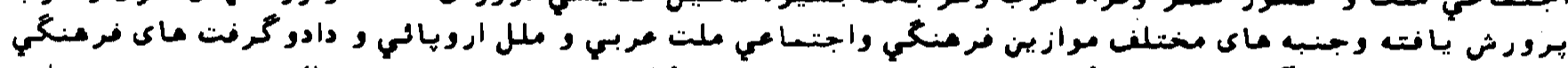

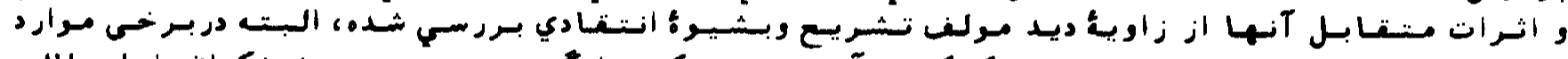

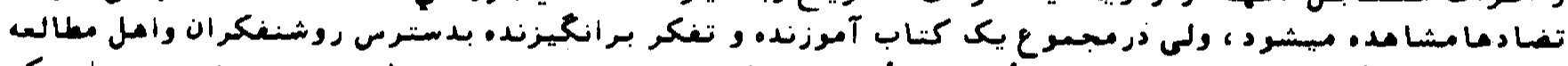

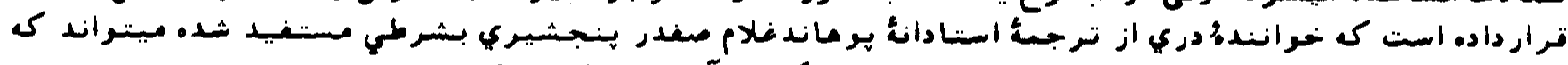

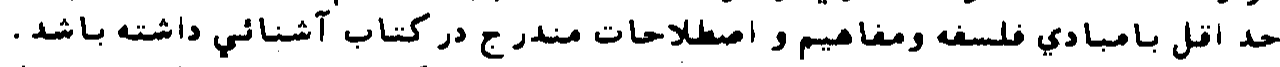

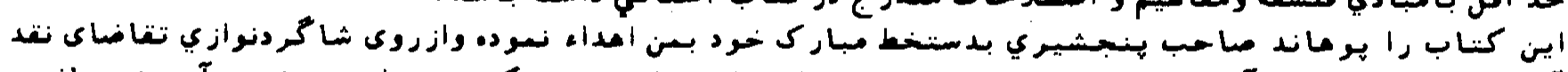

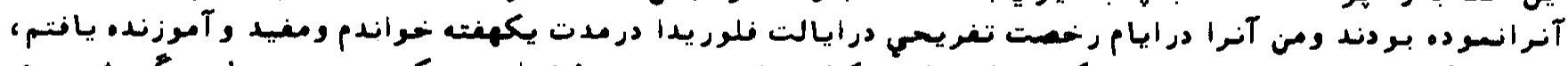

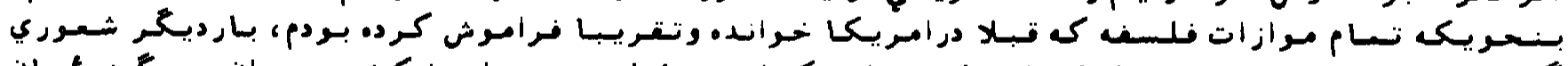

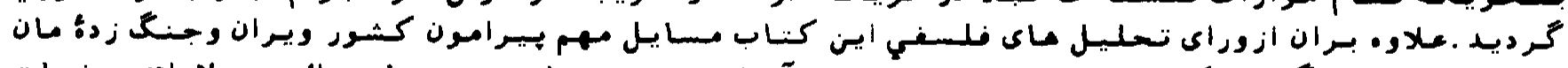

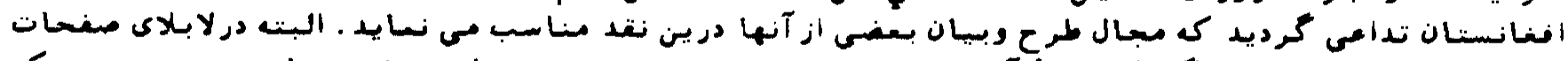

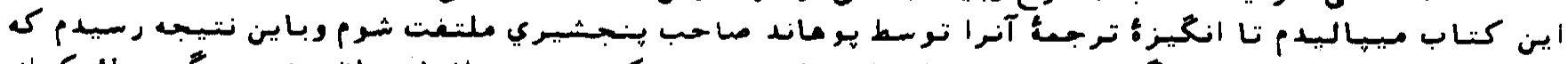

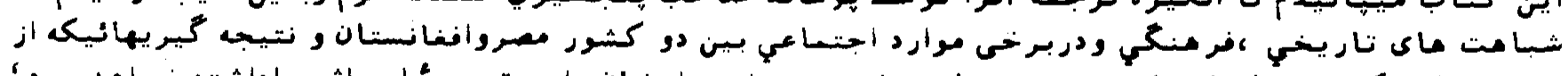

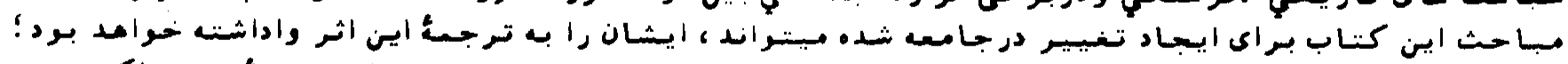

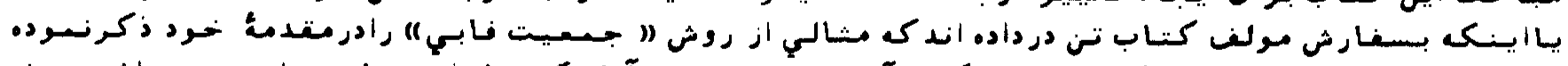

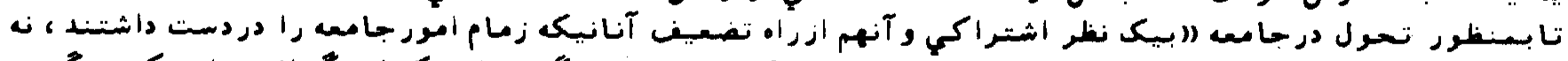

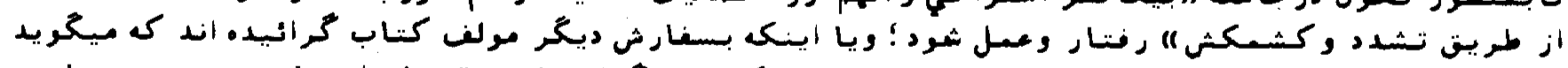

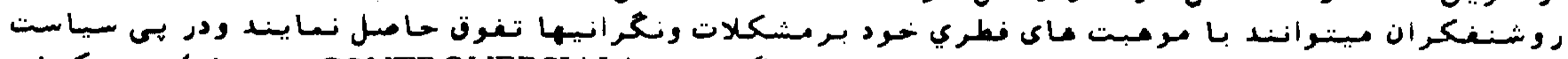

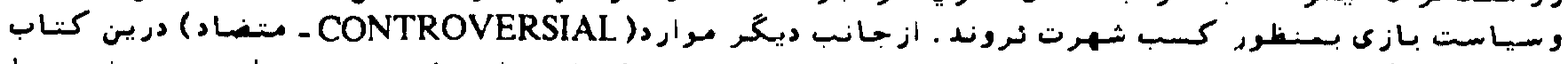

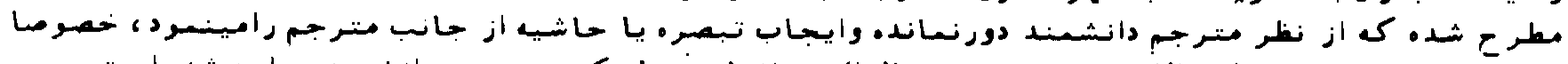

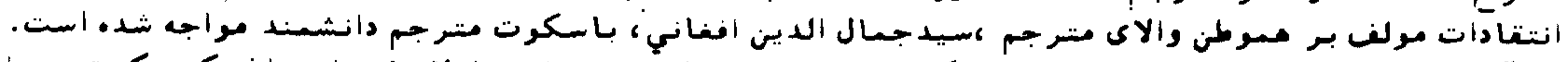

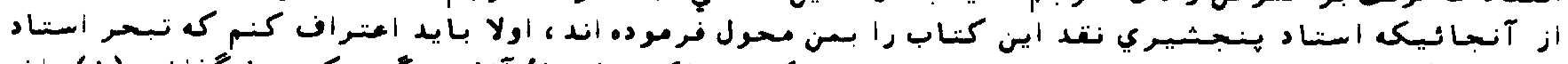

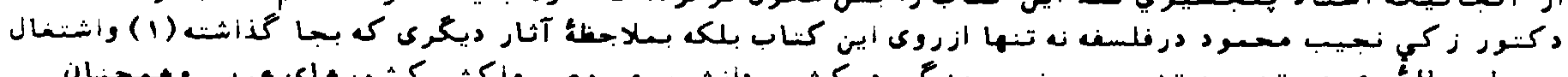

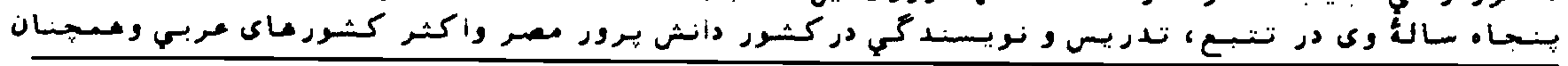




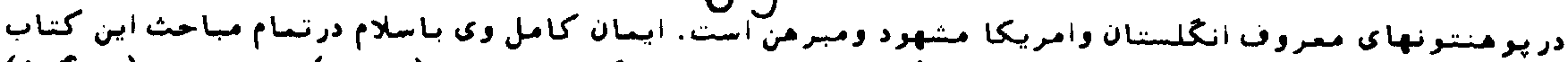

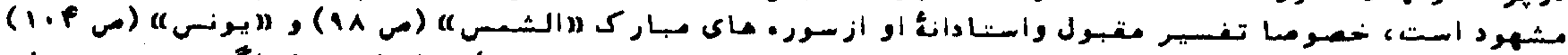

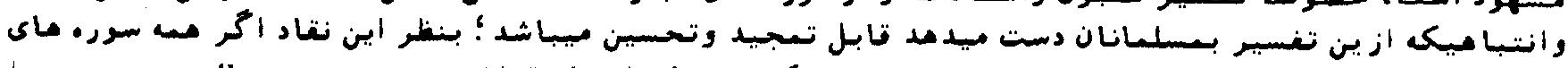

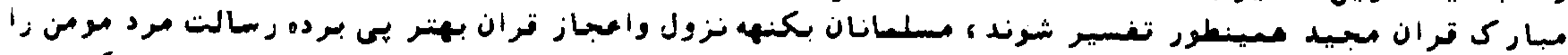

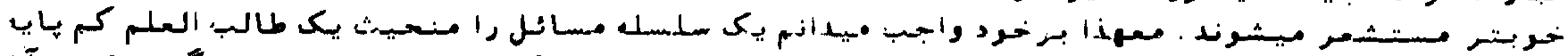

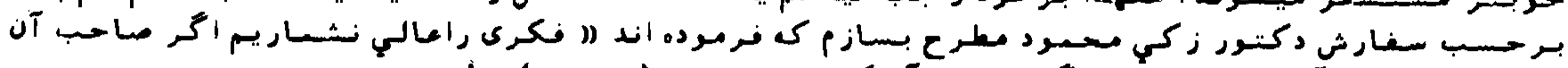

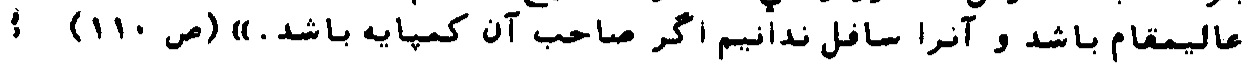

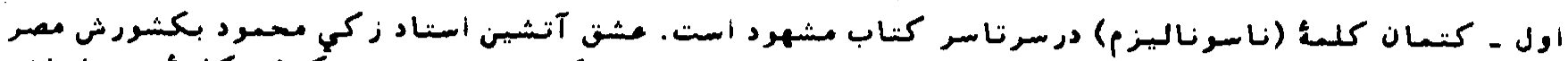

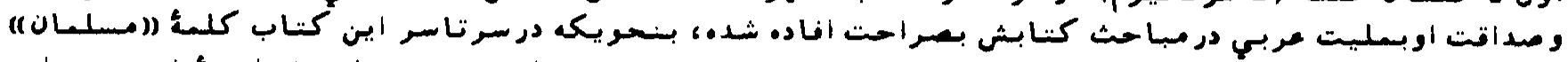

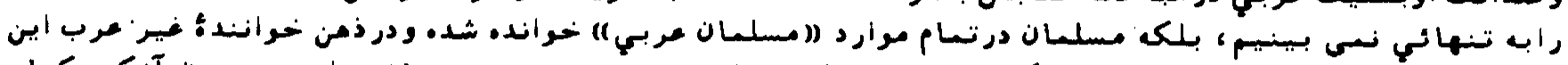

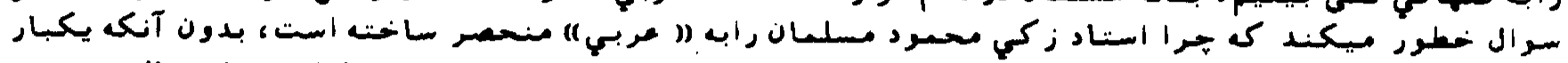

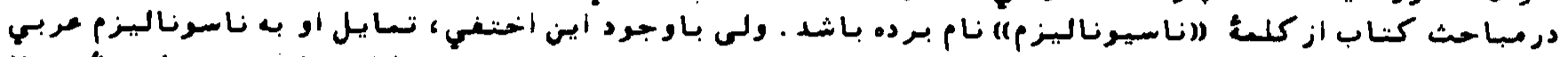

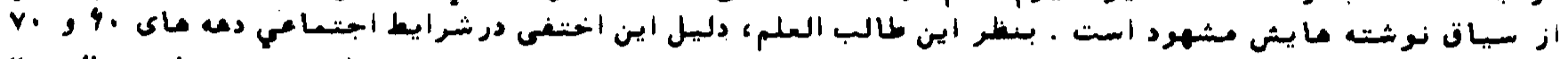

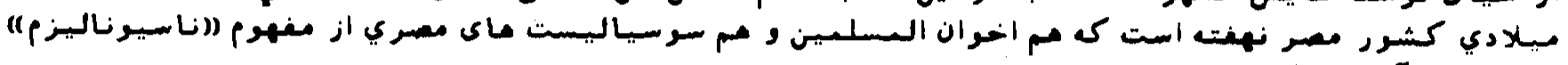

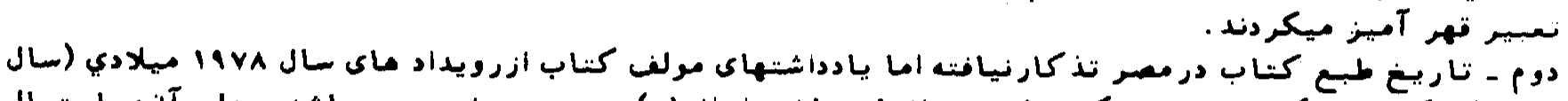

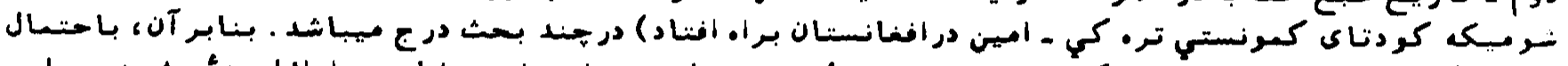

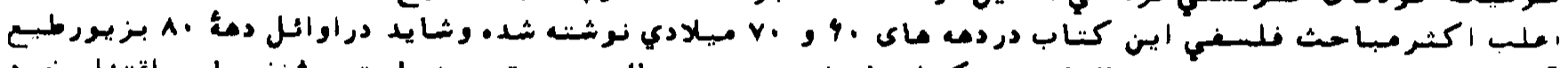

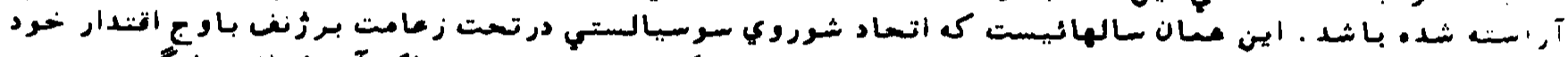

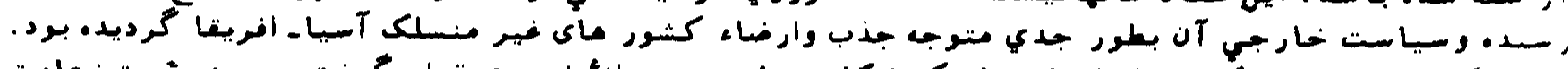

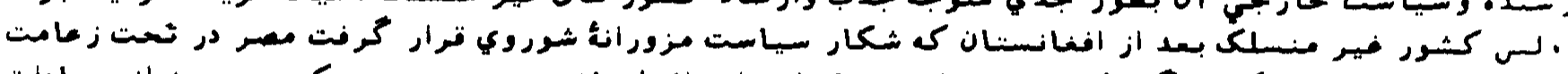

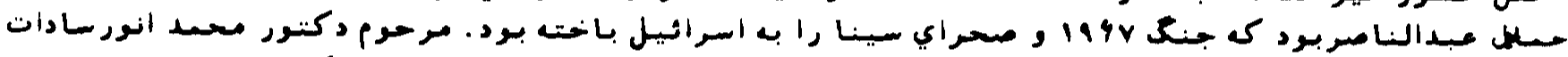

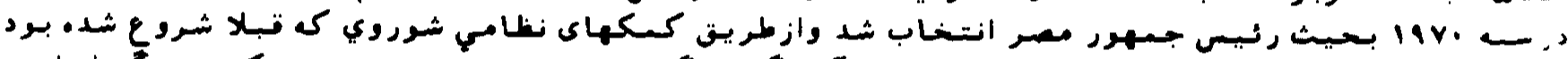

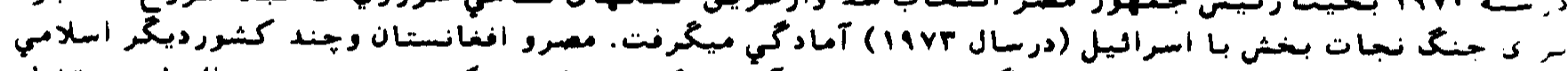

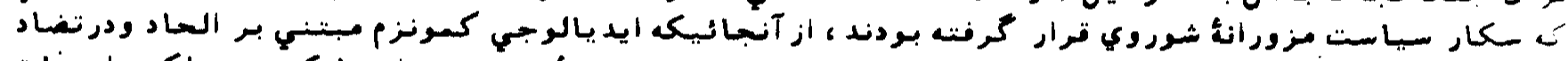

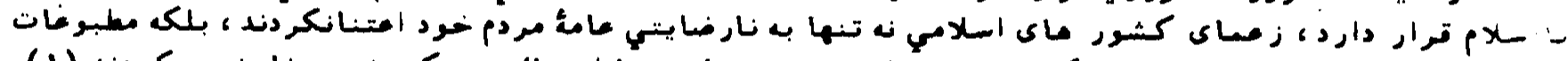

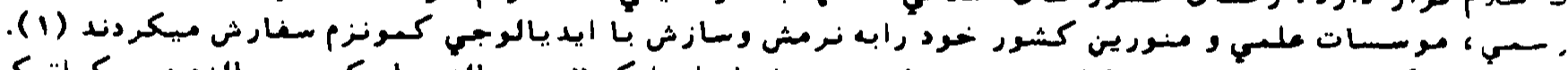

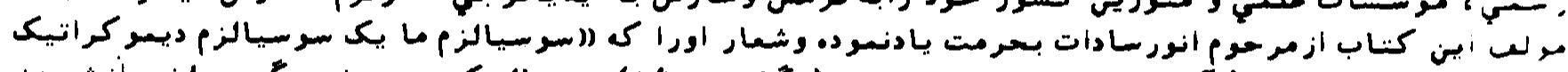

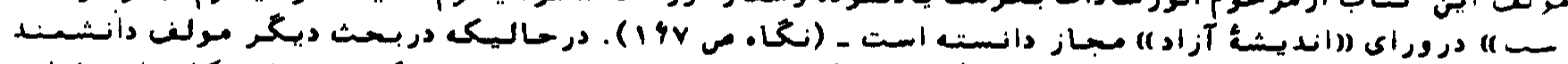

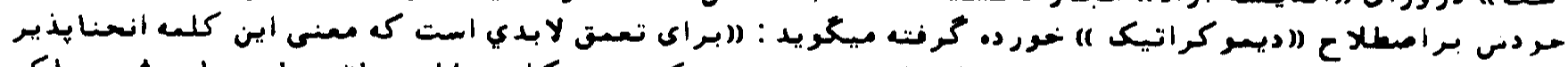

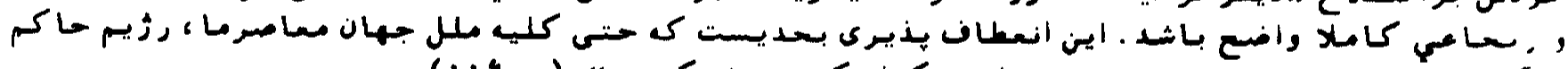

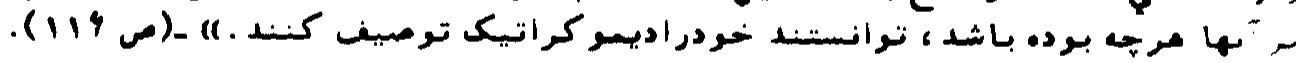

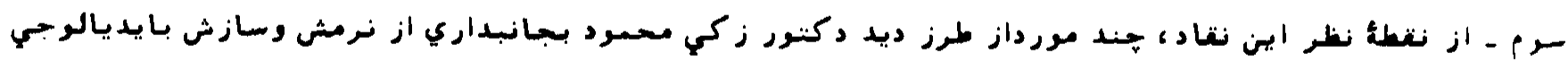

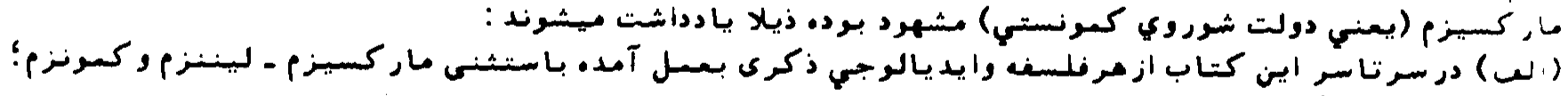

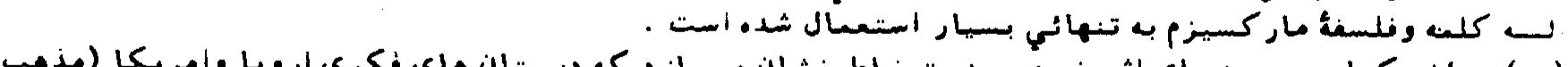

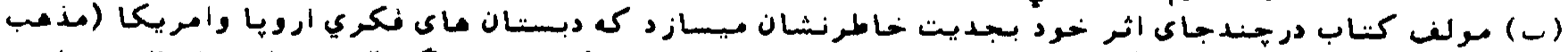

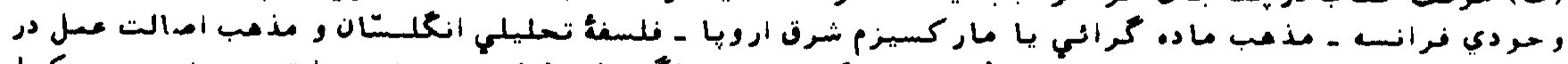

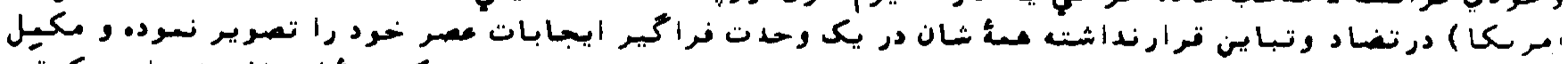

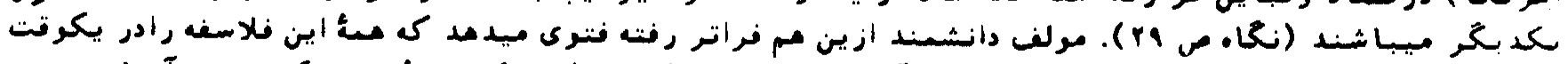

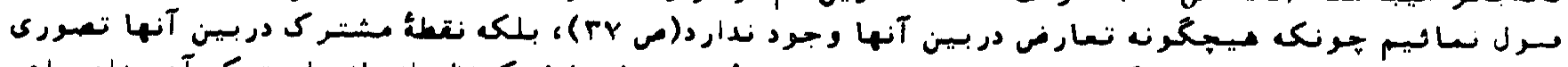

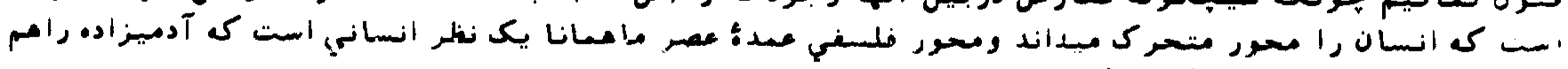

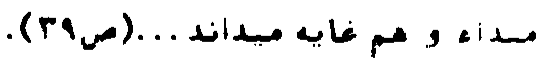

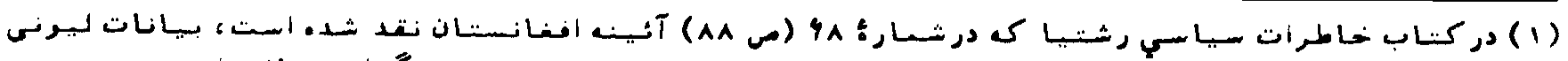

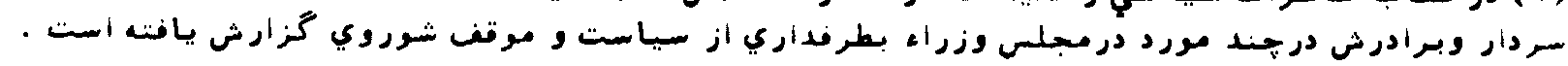




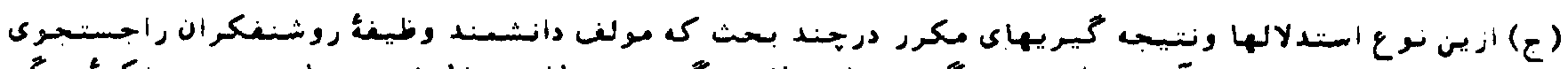

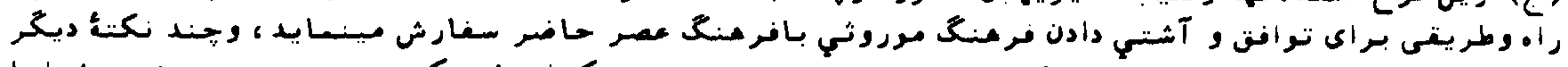

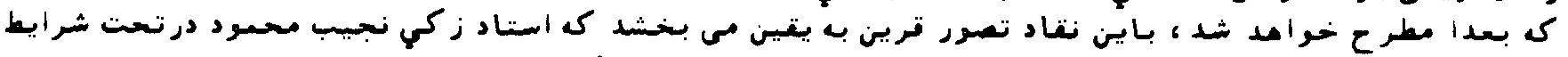

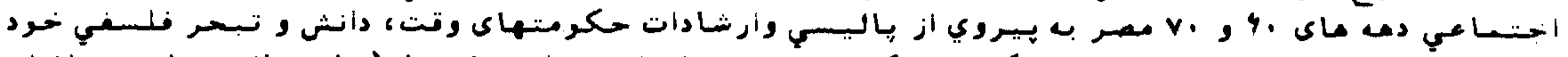

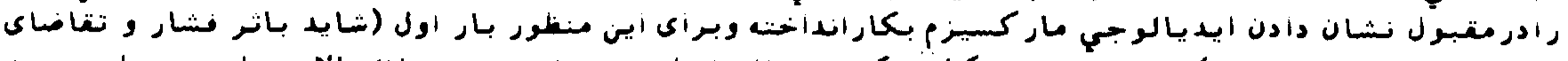

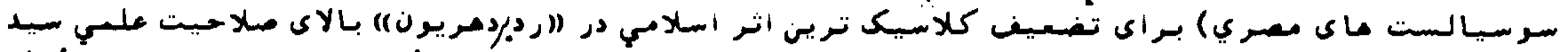

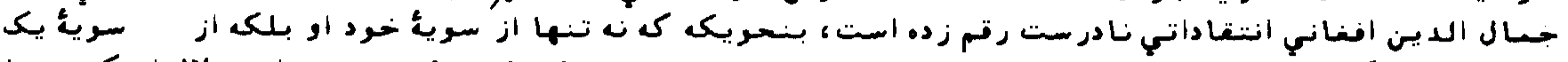

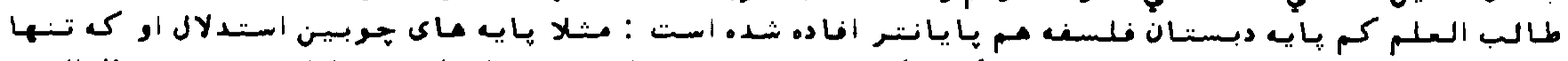

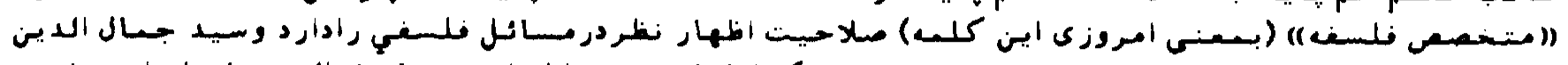

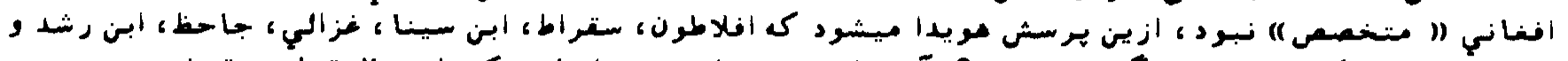

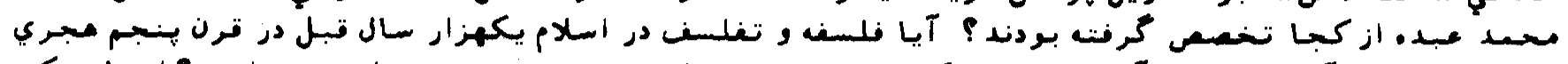

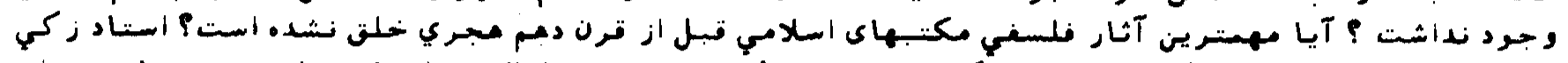

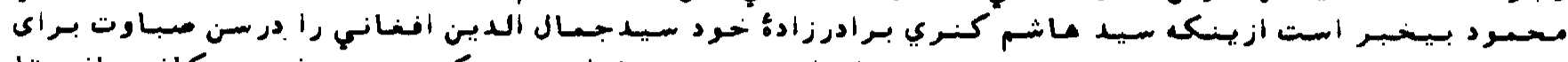

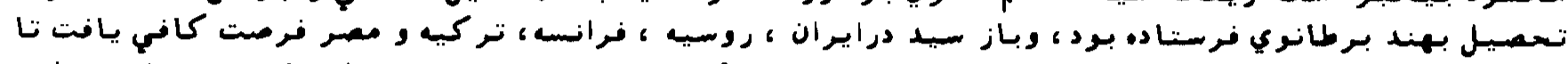

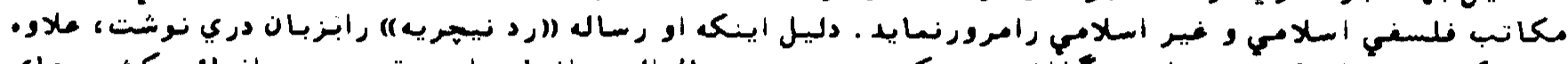

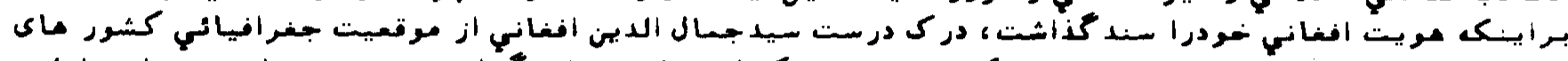

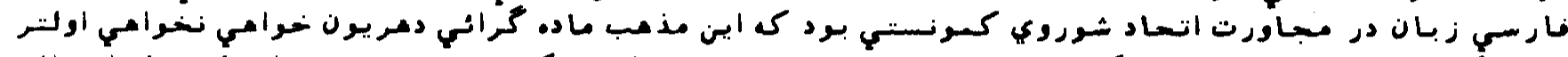

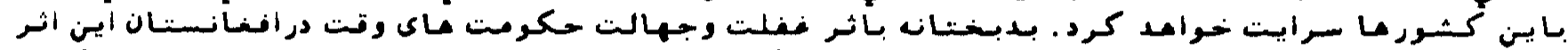

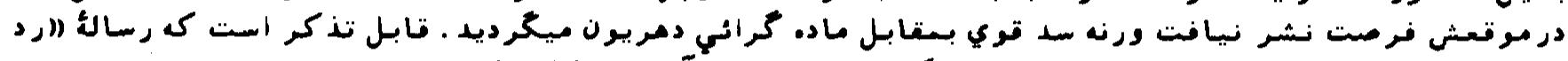

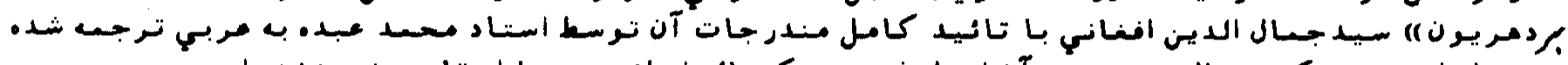

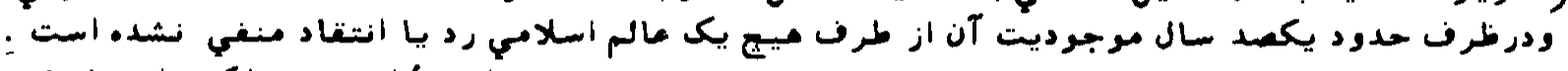

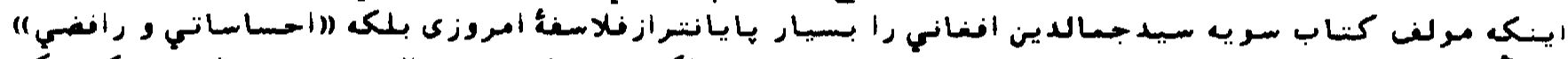

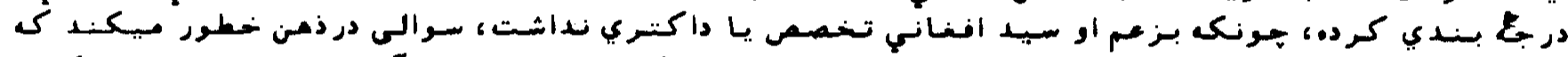

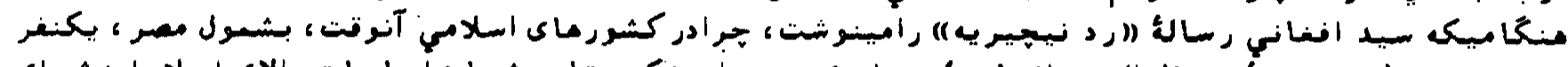

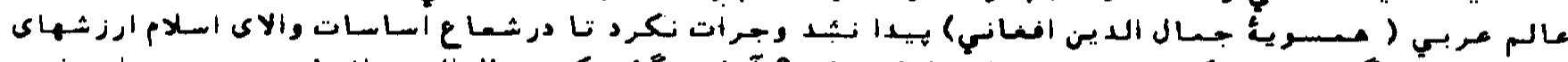

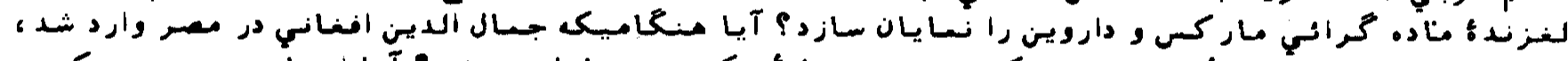

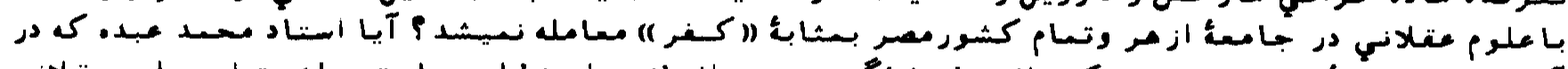

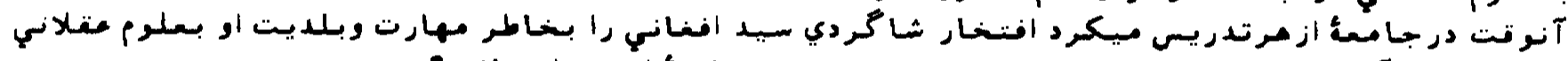

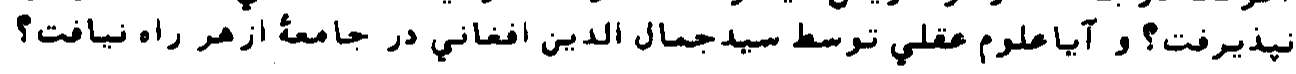

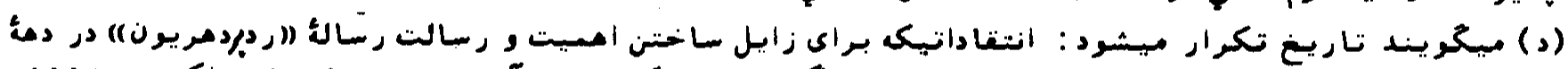

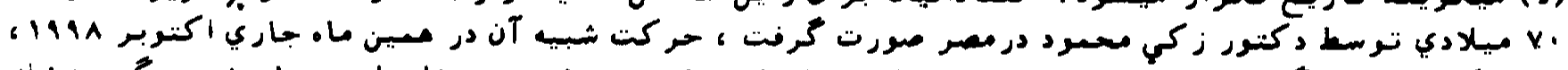

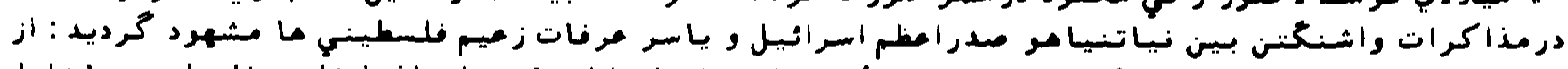

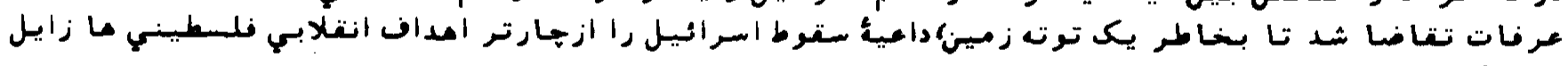

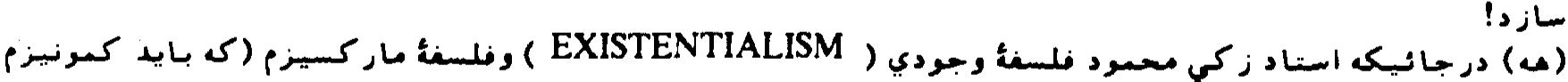

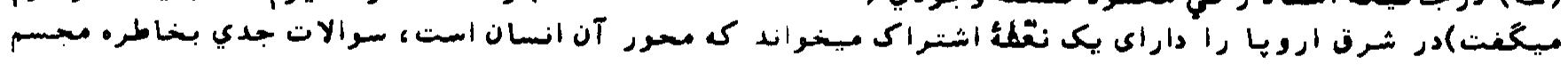

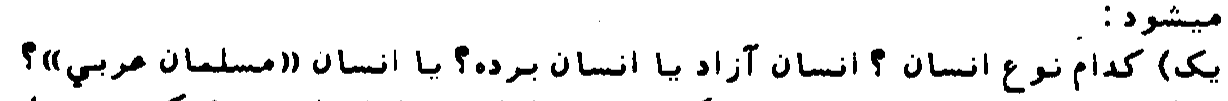

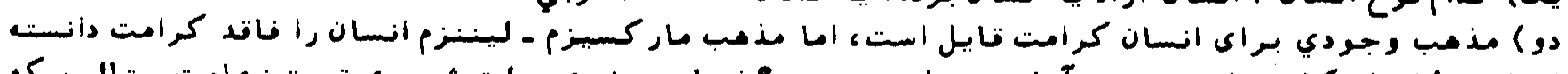

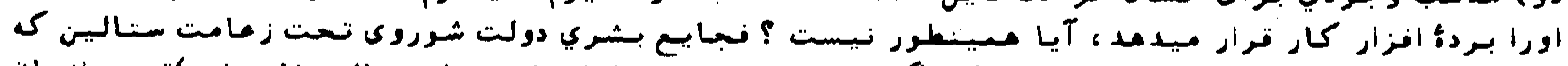

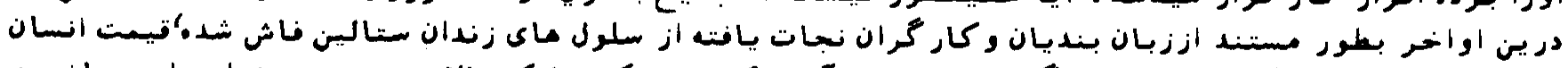

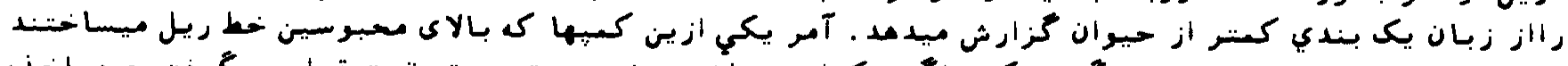

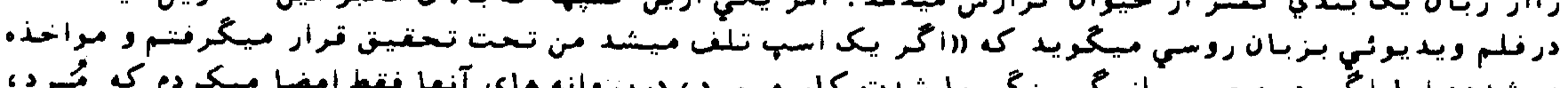

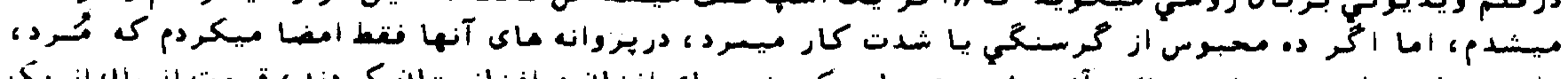

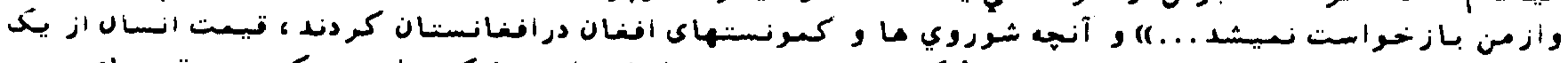

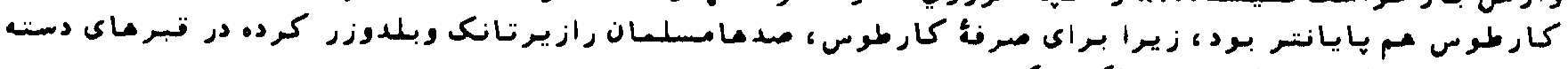

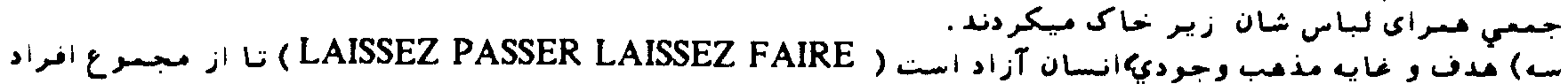

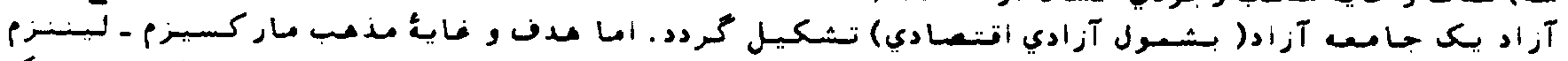

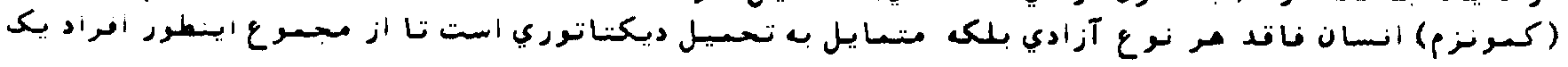




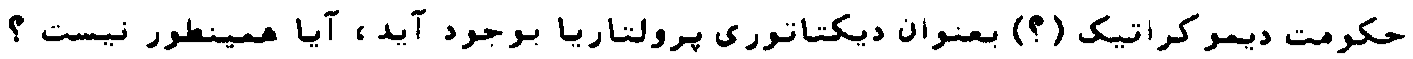

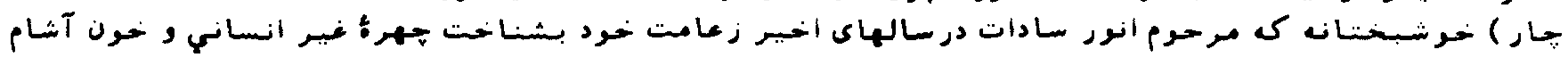

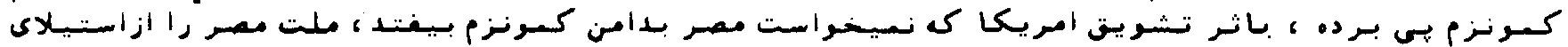

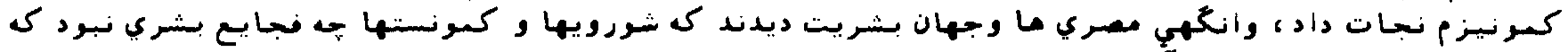

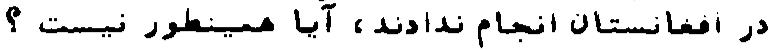

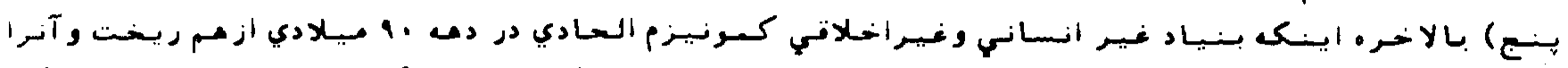

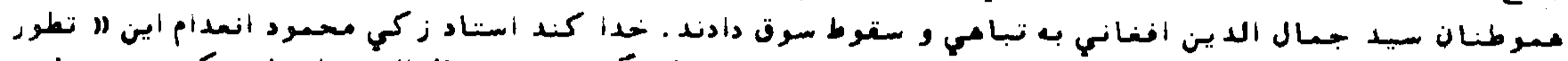

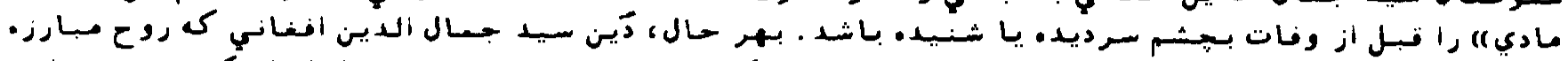

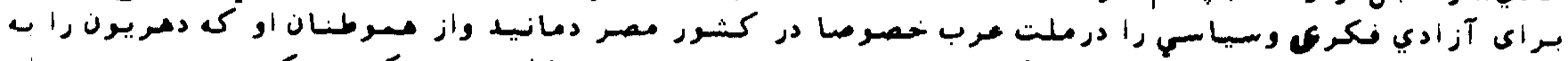

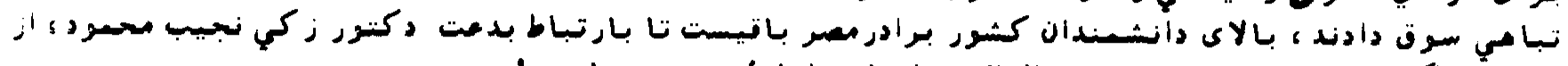

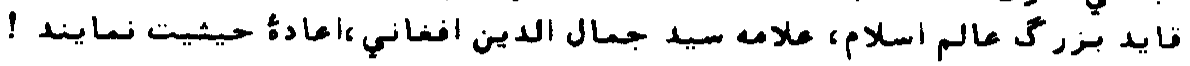

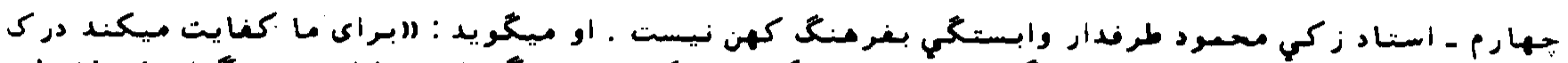

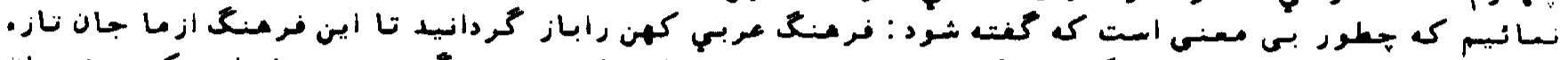

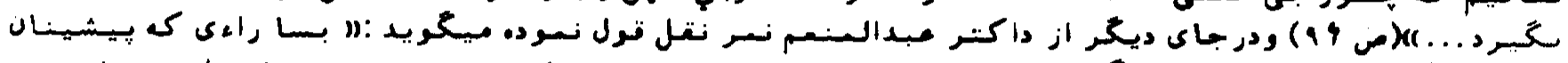

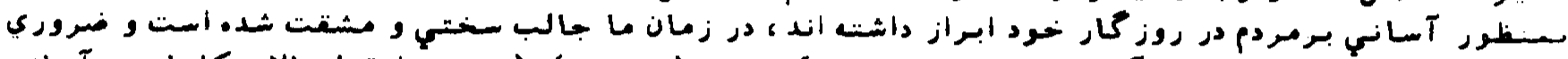

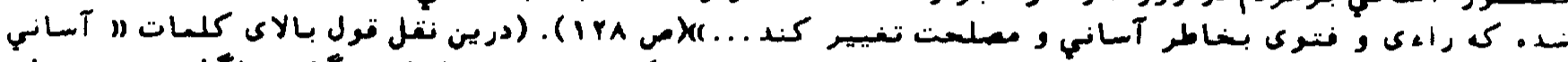

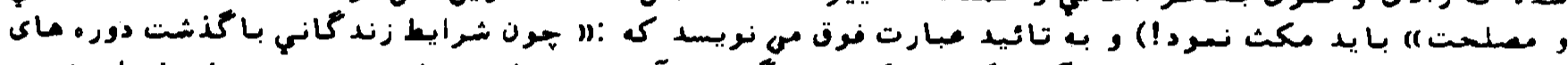

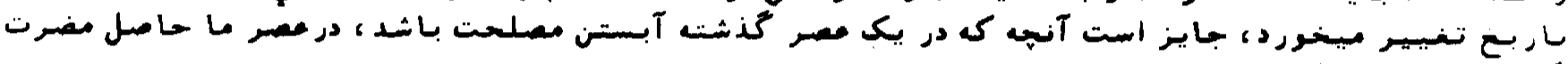

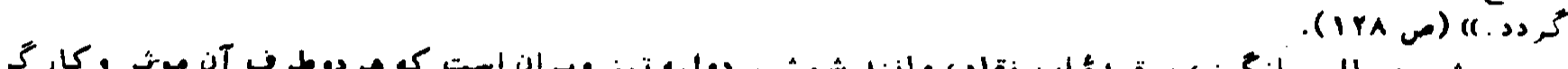

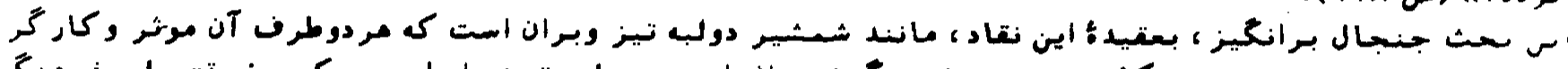

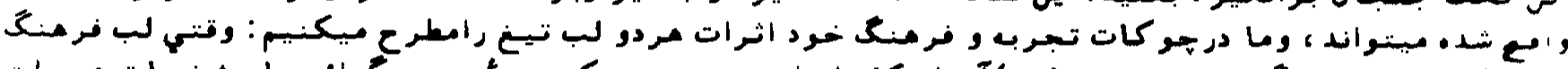

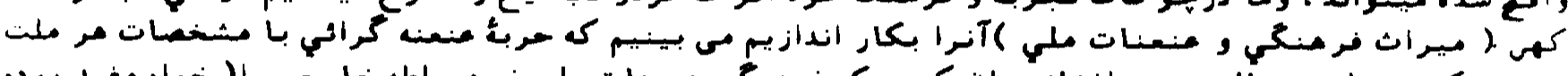

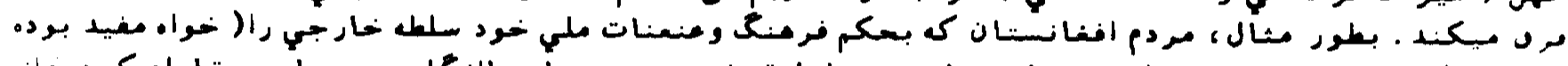

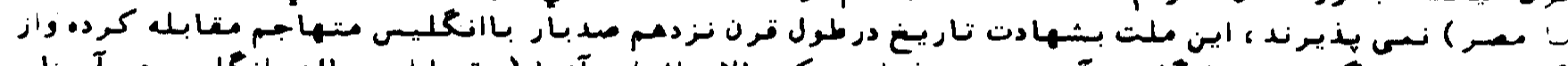

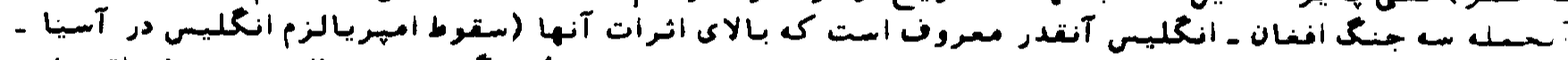

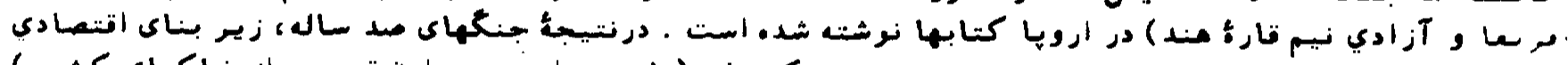

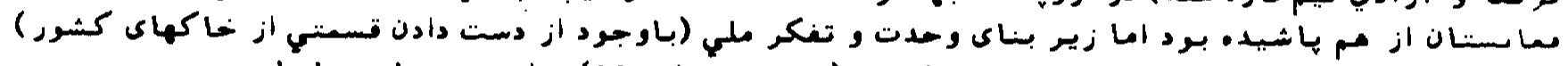

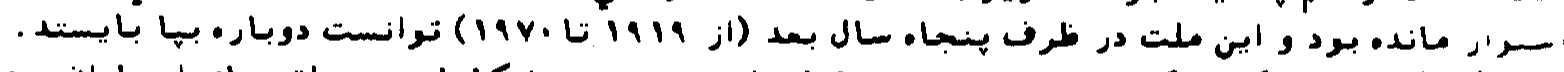

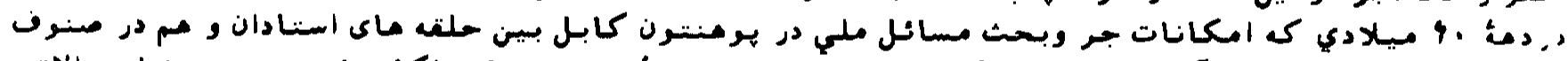

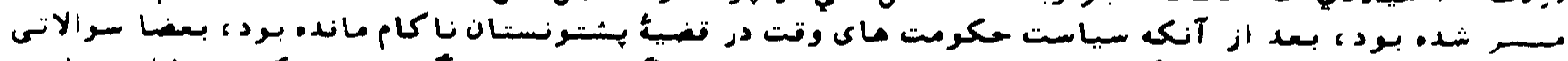

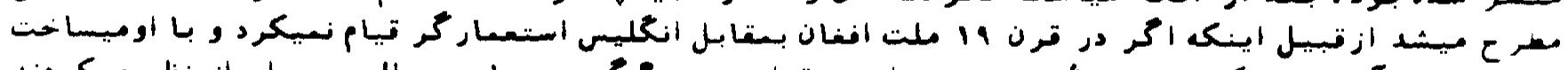

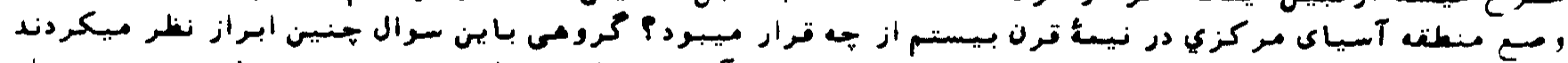

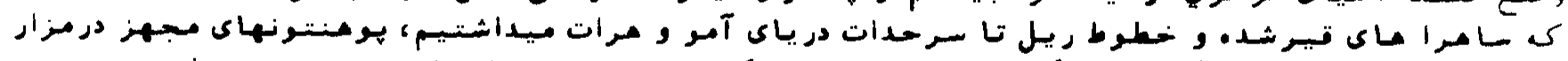

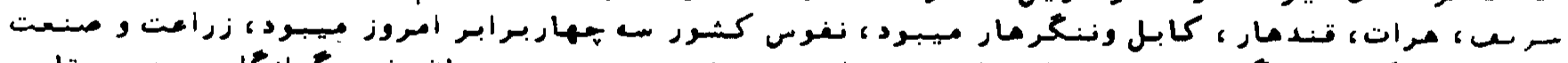

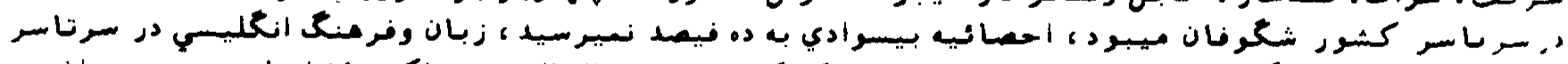

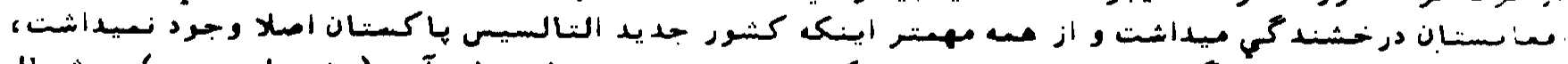

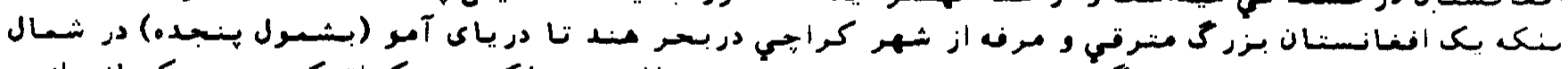

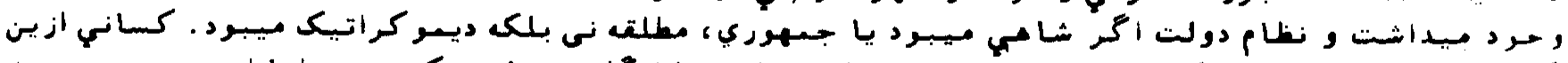

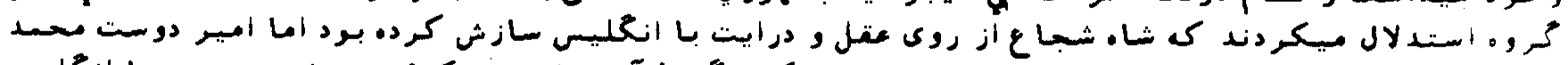

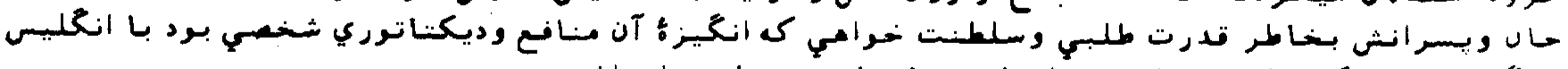

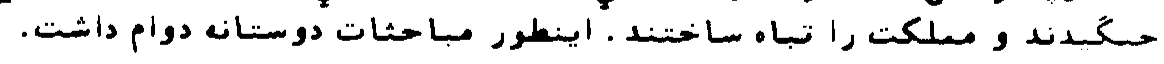

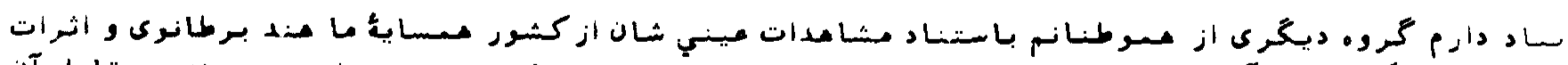

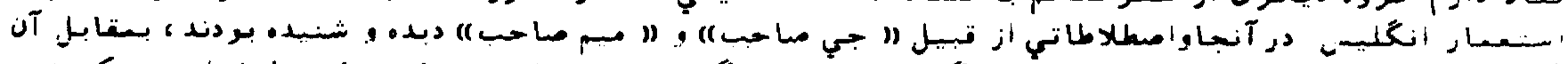

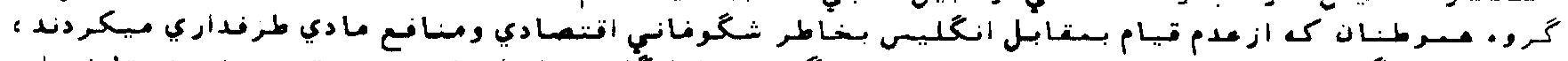

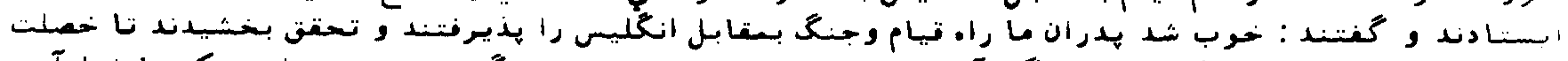

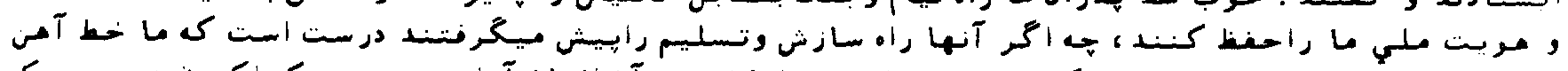

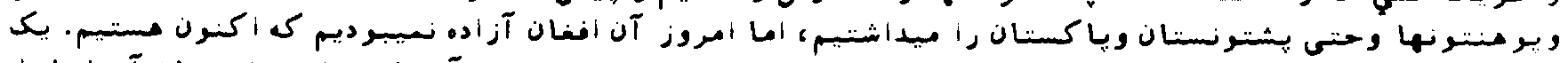

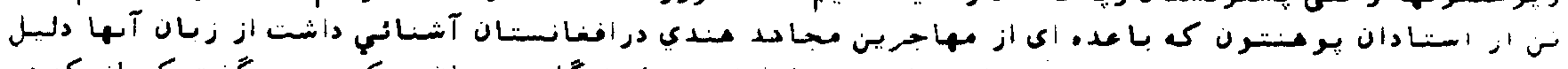

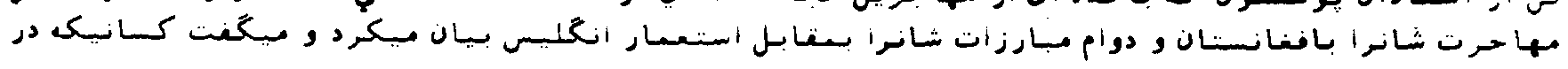




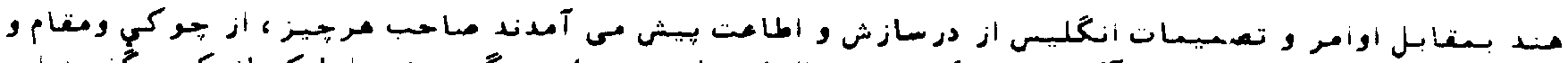

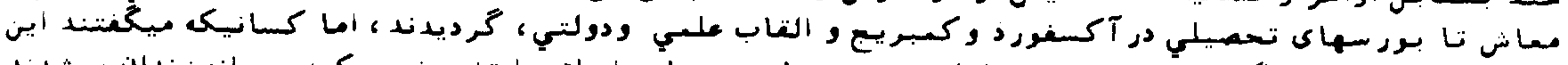

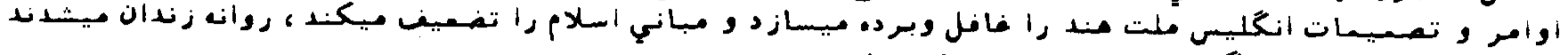

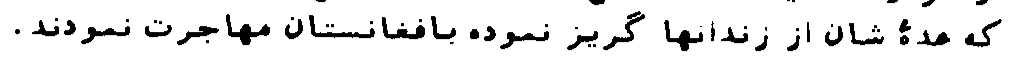

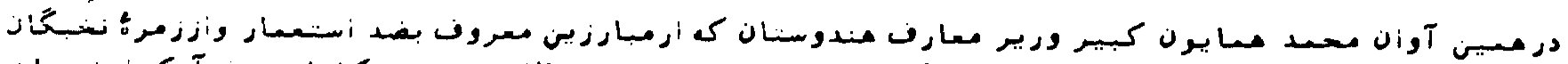

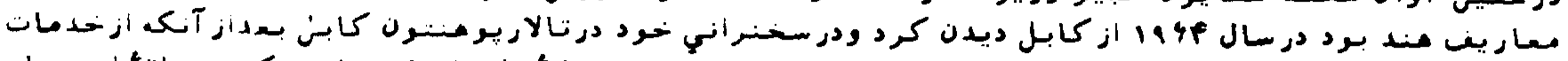

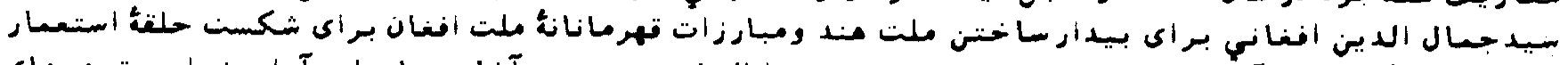

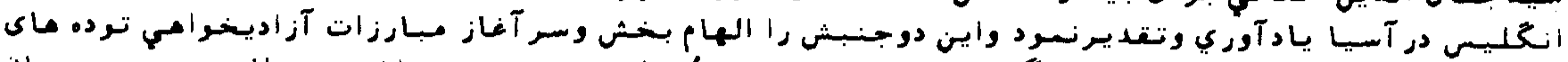

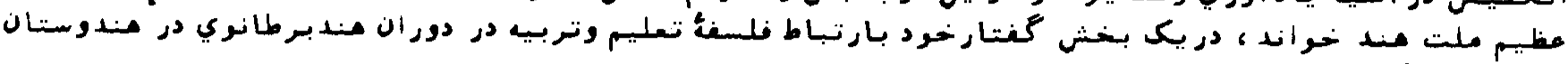

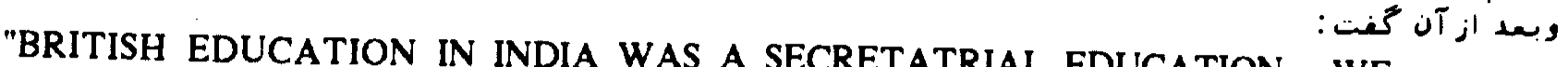
ARE TRYING TO EDUCANEN INDIA WAS A SECRETATRIAL EDUCATION. WE

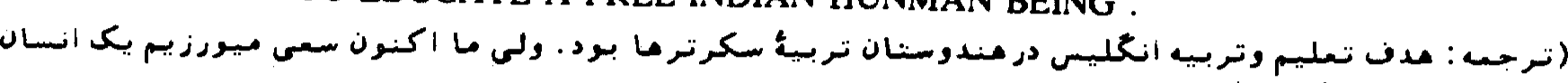

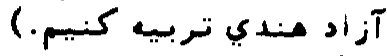

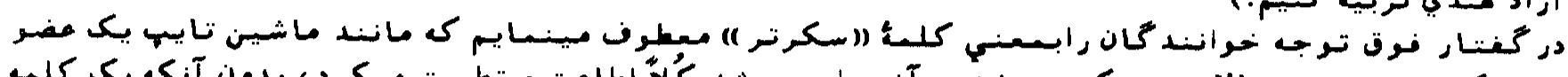

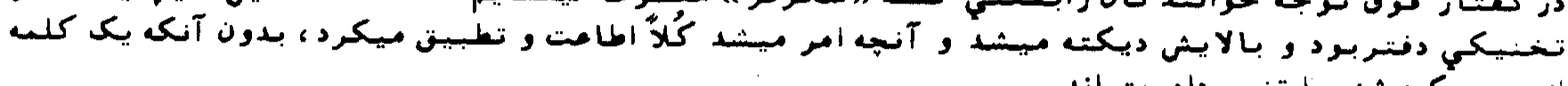

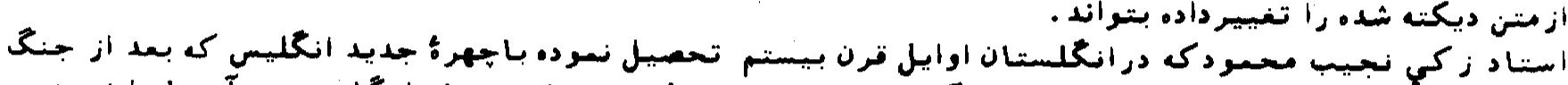

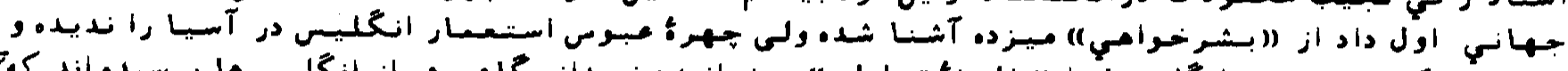

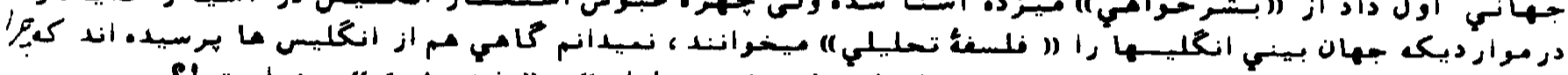

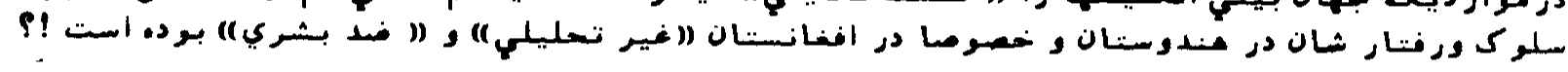

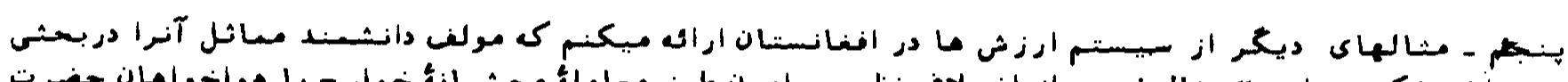

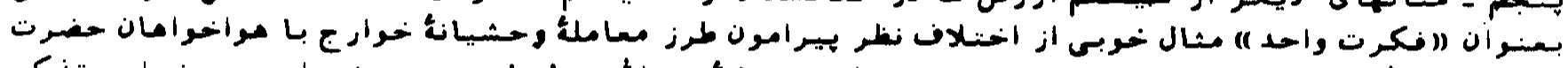

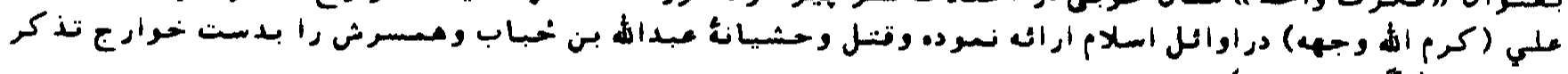

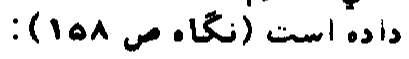

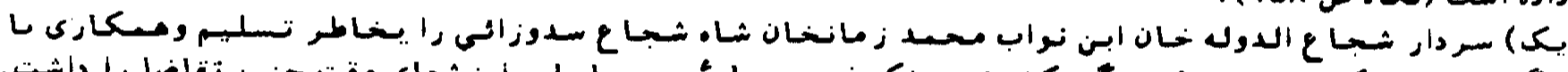

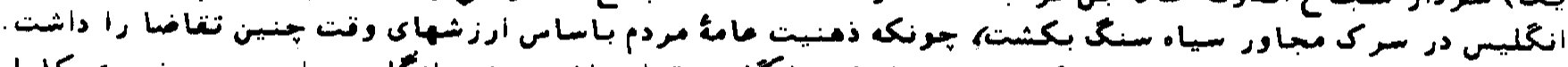

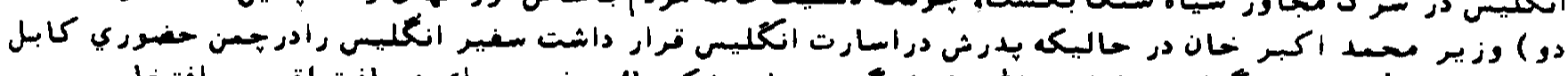

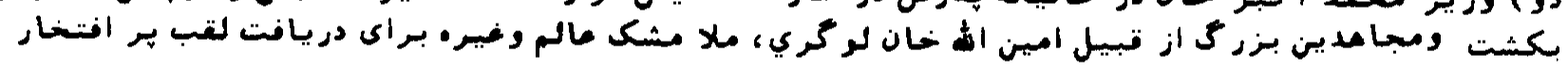

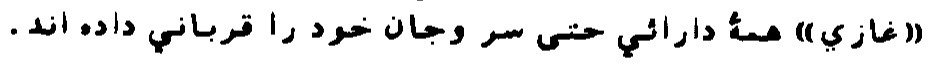

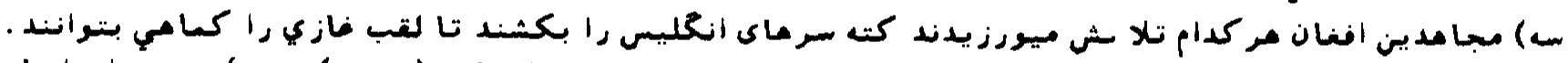

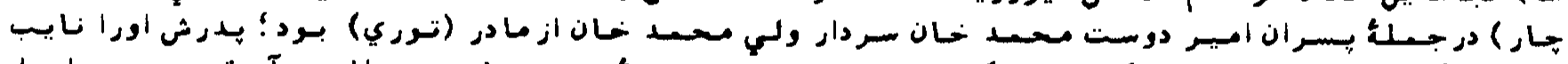

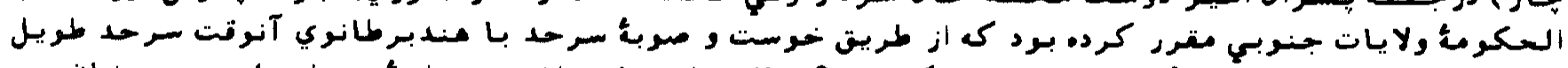

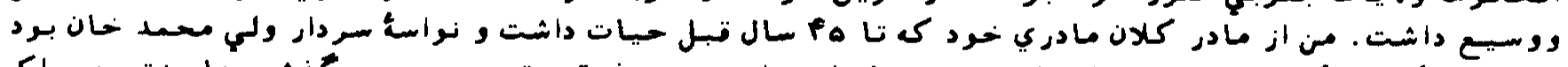

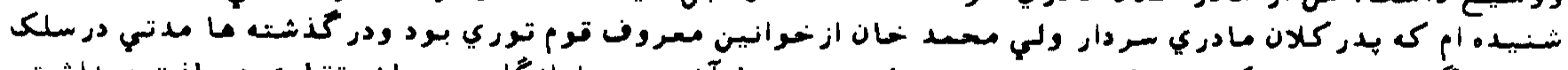

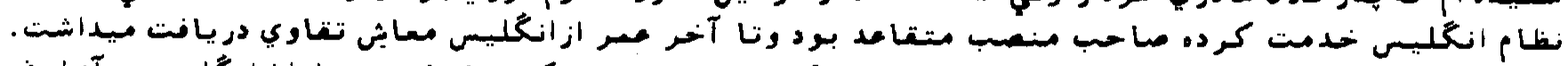

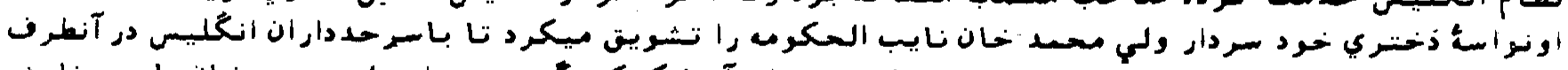

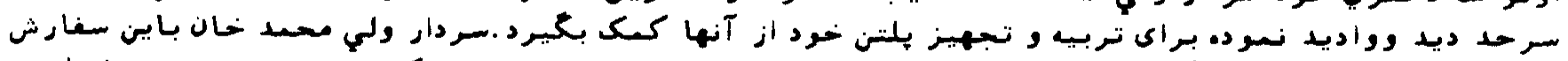

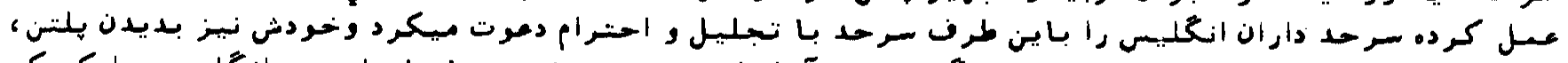

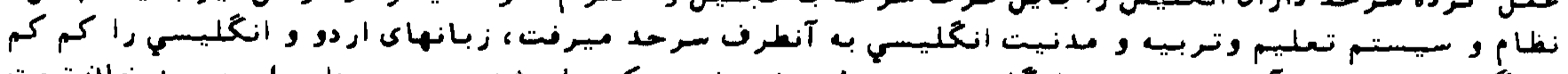

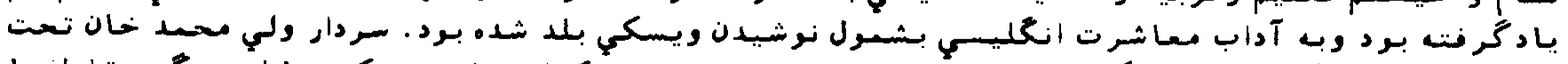

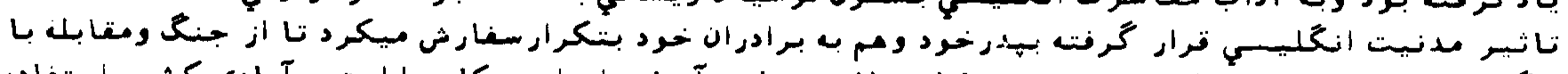

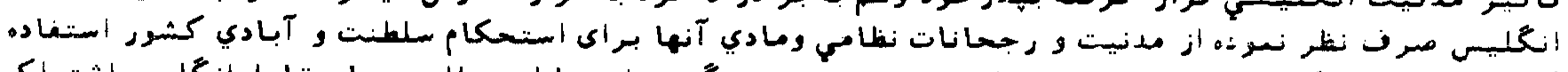

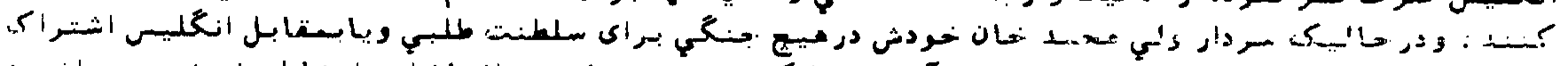

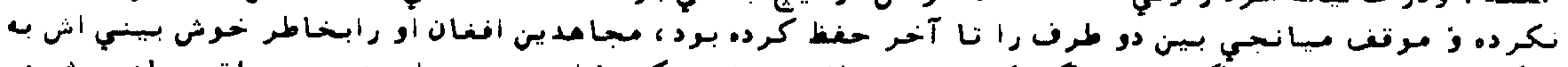

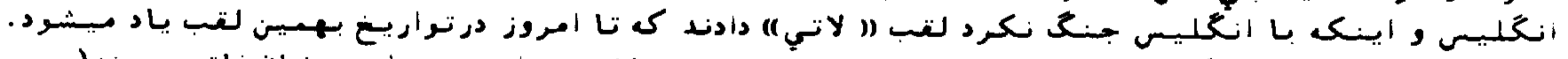

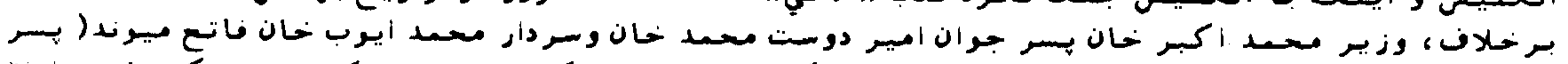

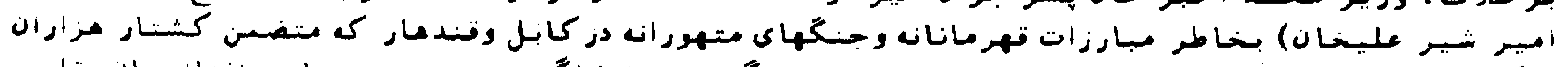

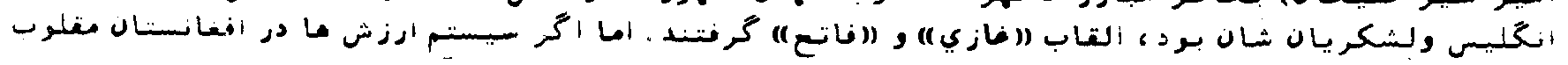




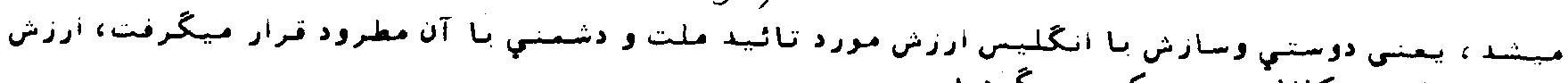

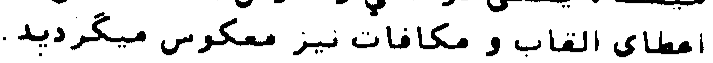

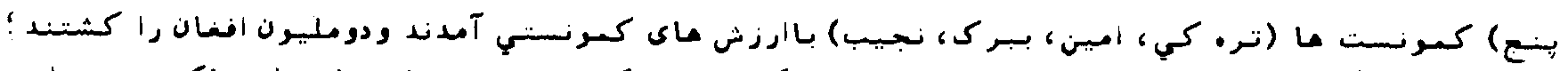

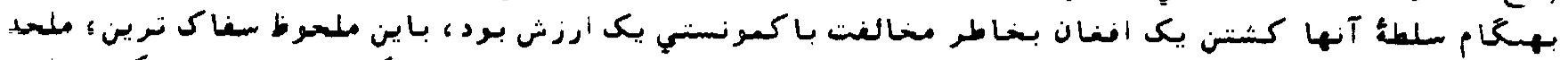

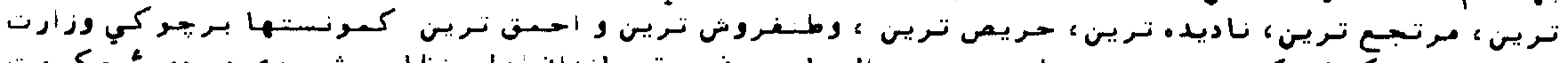

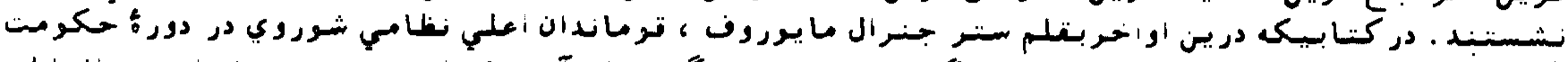

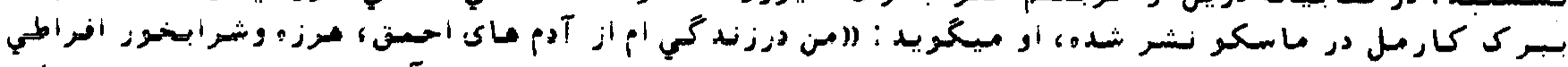

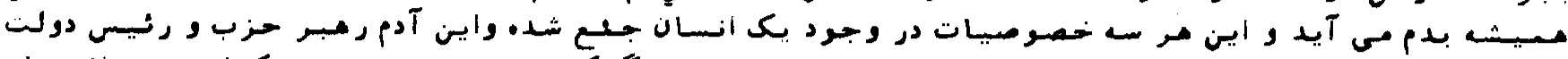

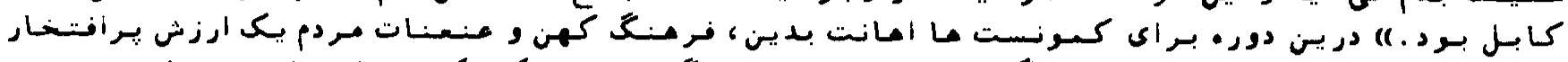

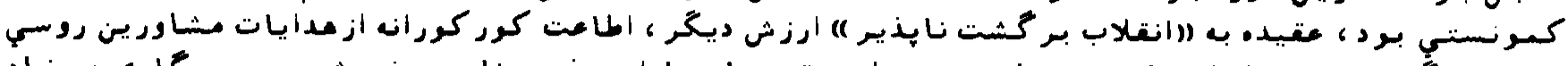

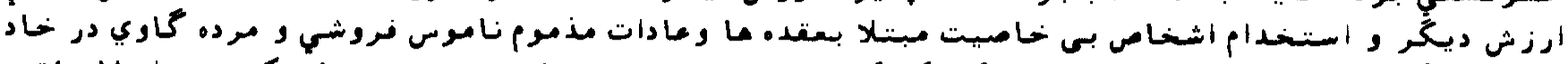

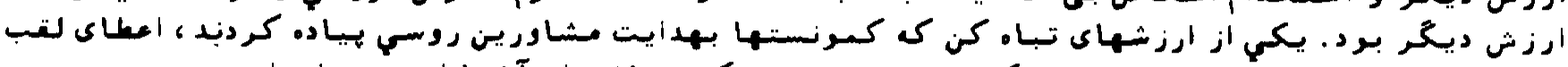

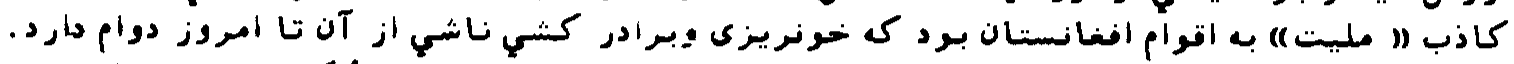

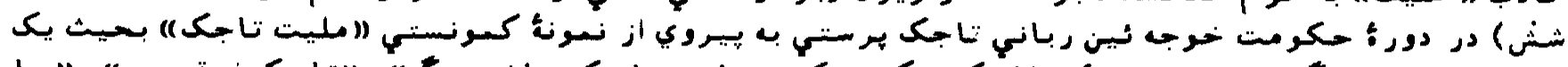

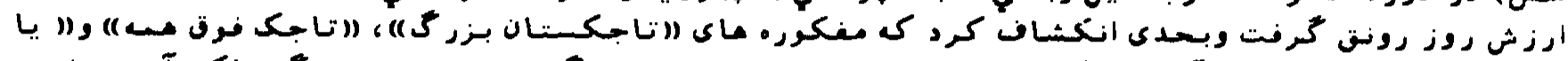

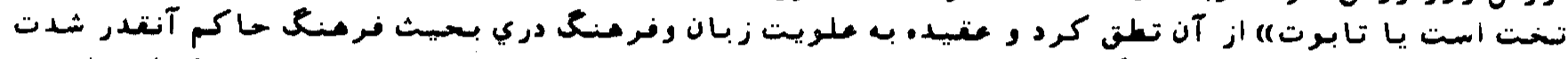

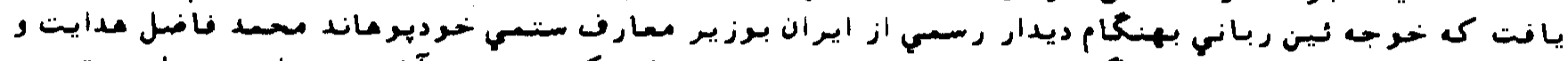

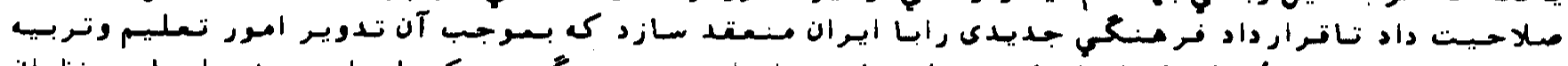

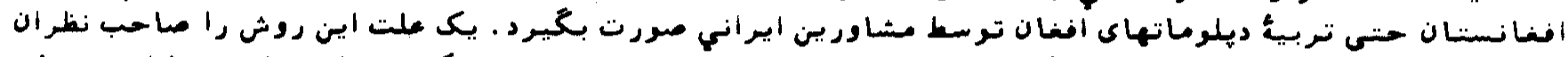

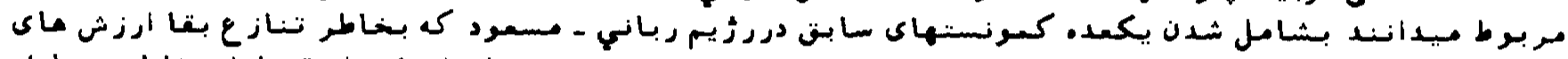

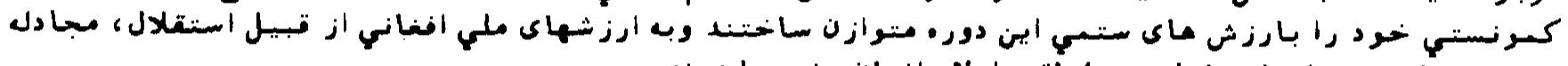

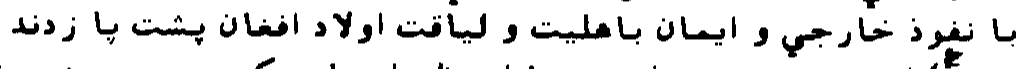

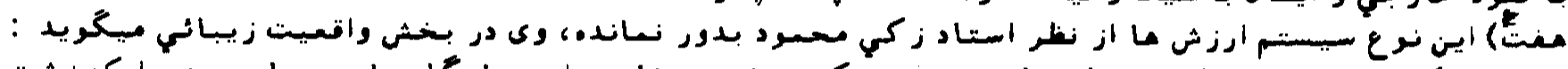

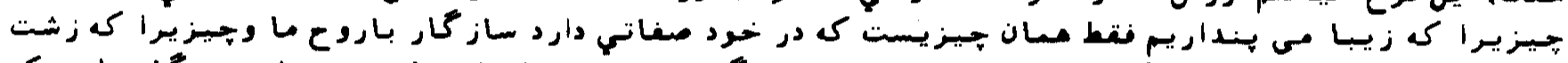

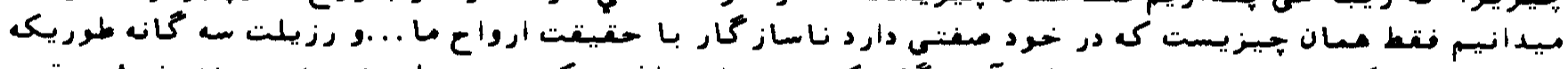

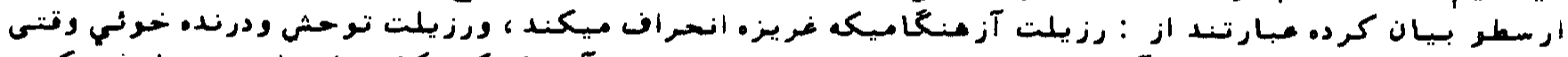

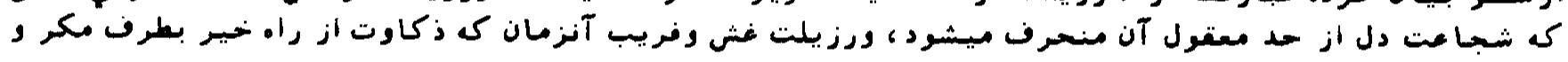

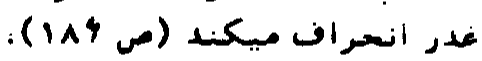

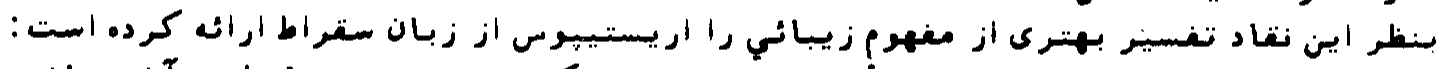

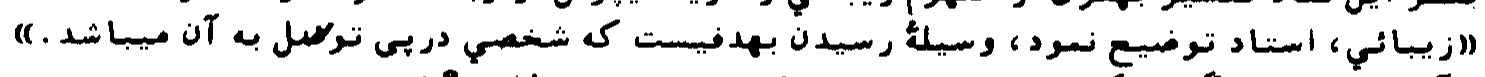

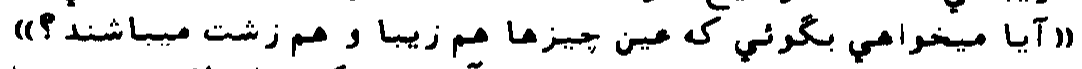

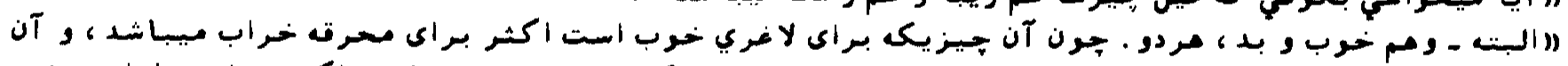

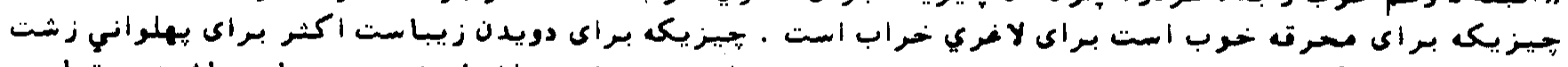

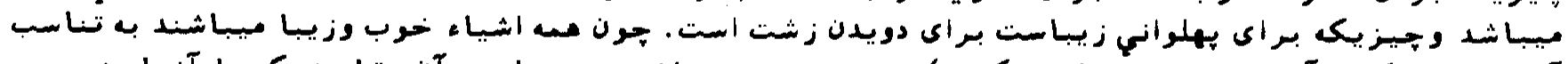

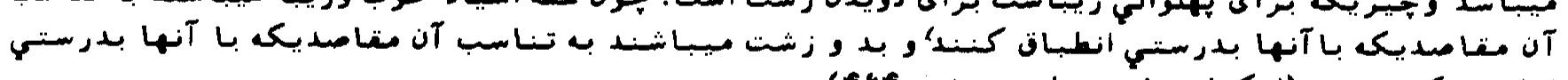

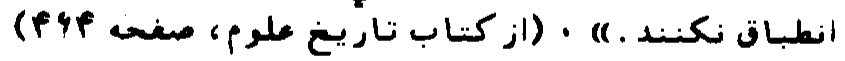

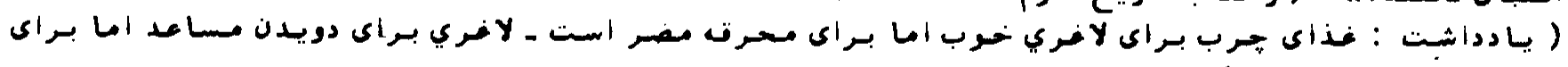

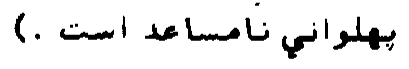

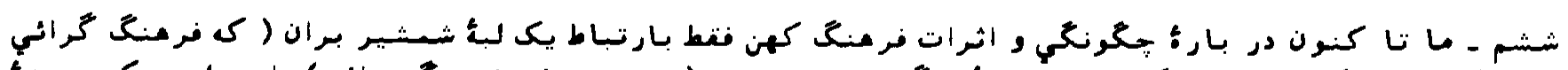

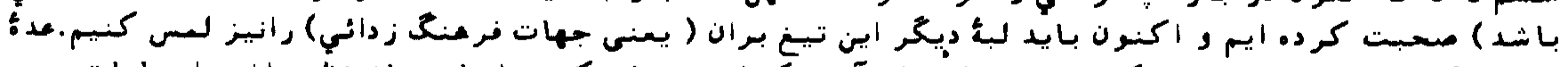

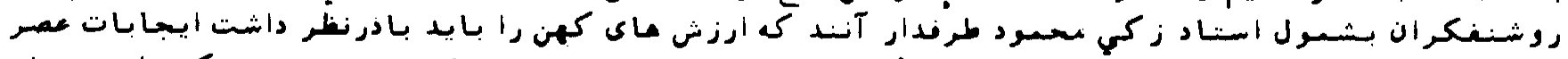

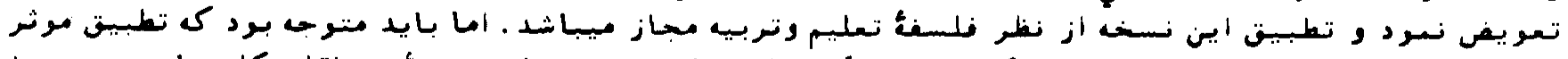

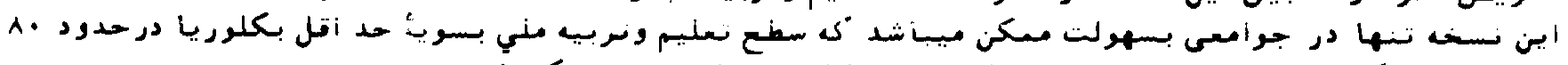

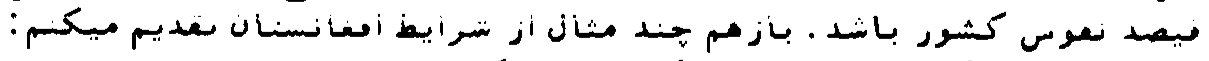

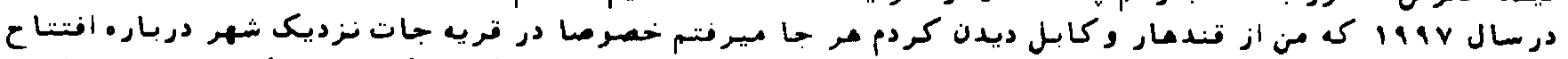

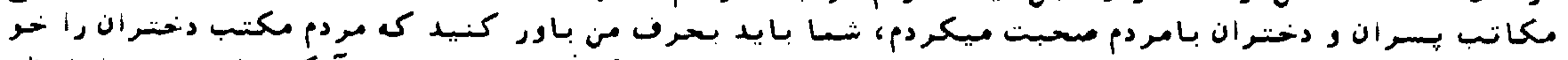

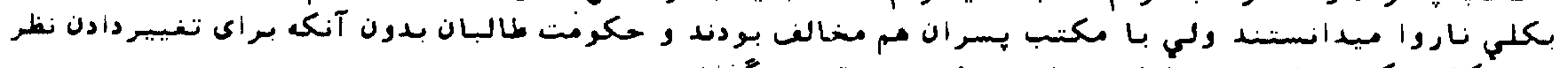

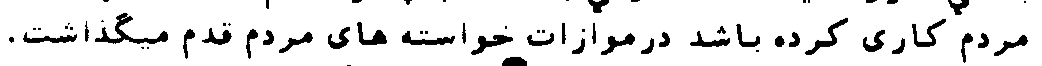

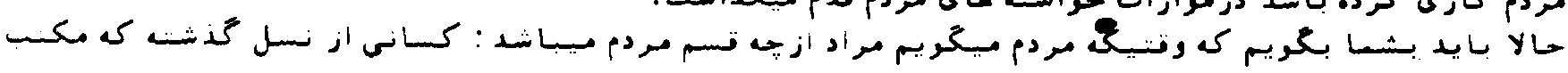




\section{0}

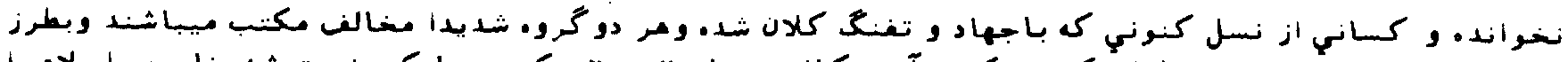

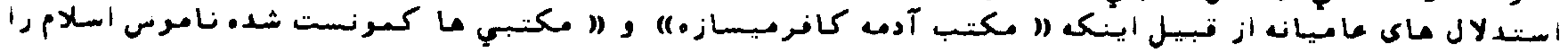

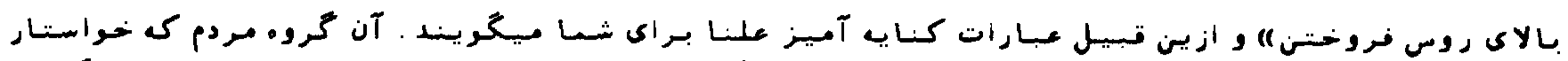

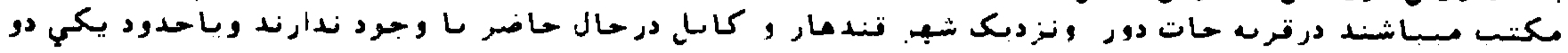

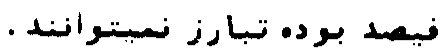

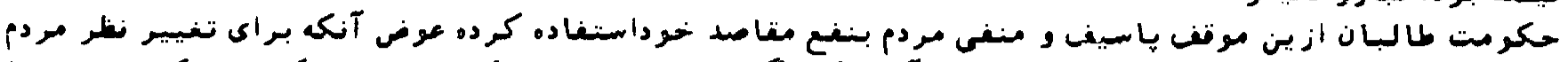

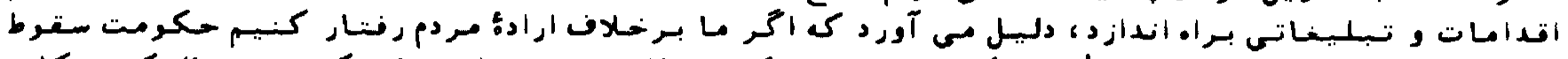

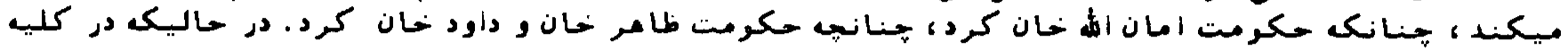

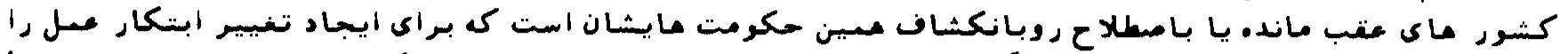

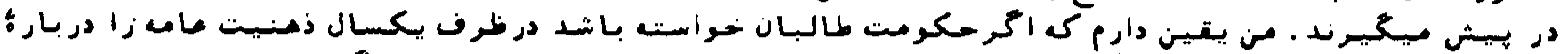

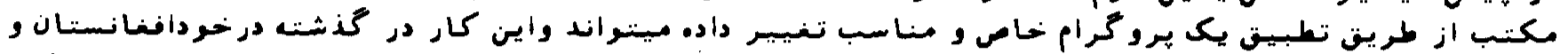

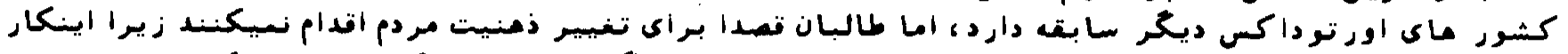

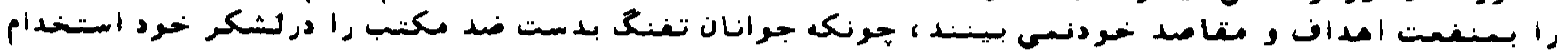

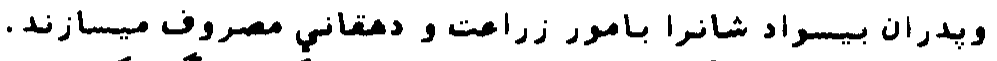

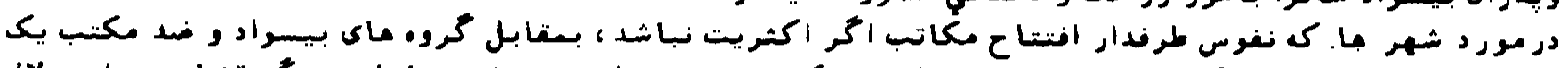

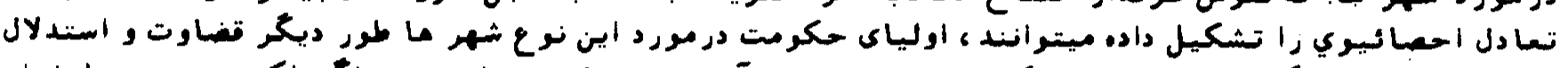

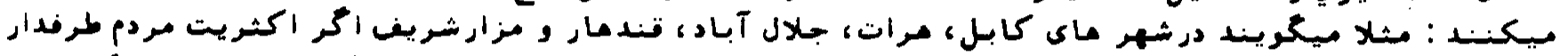

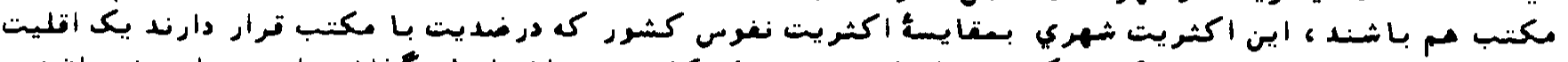

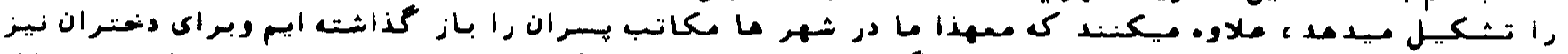

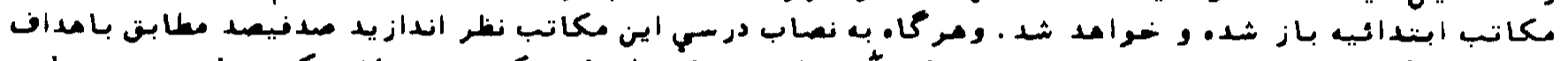

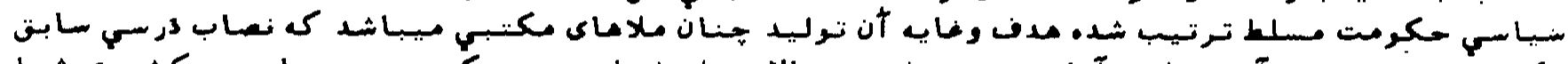

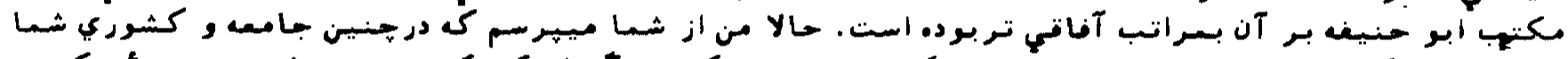

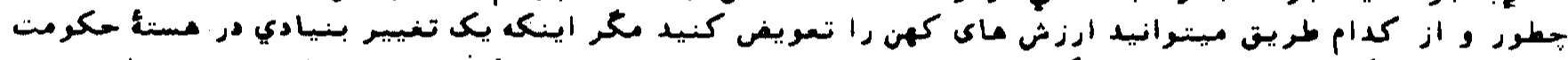

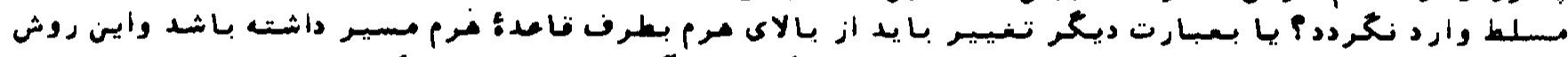

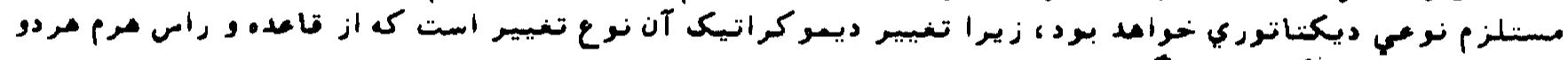

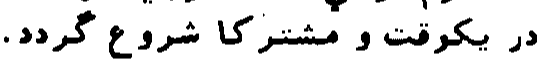

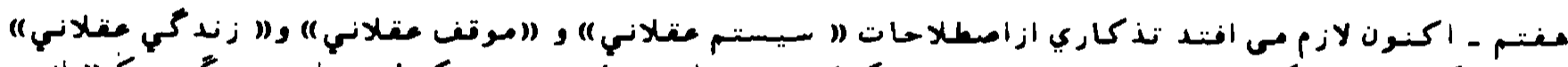

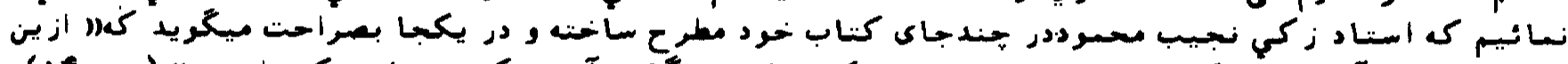

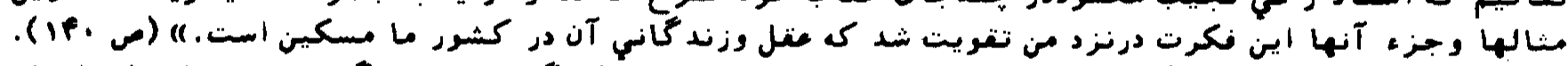

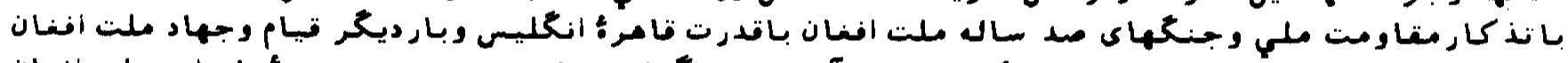

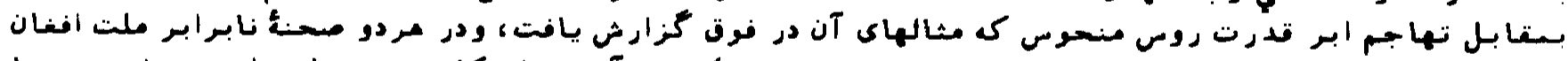

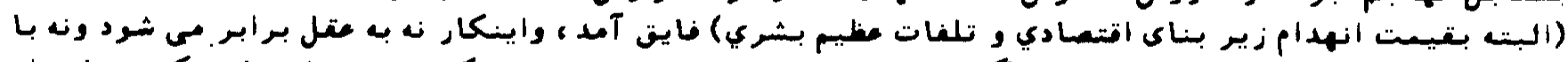

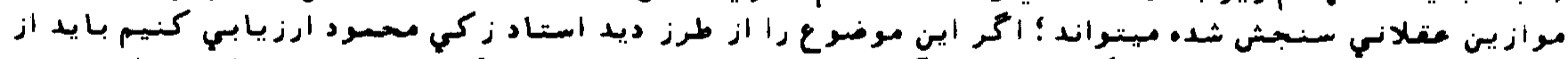

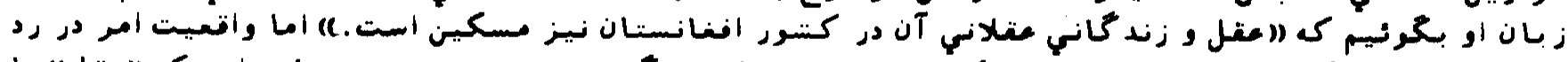

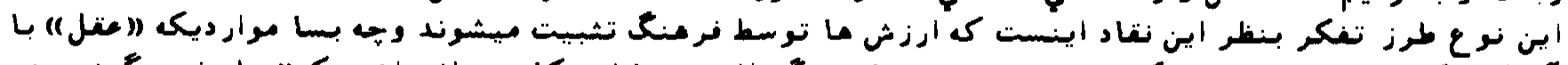

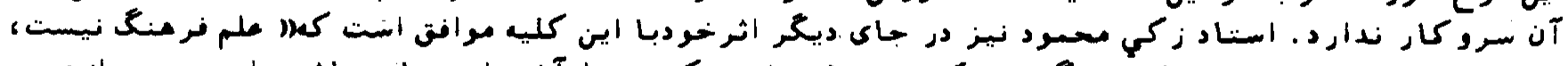

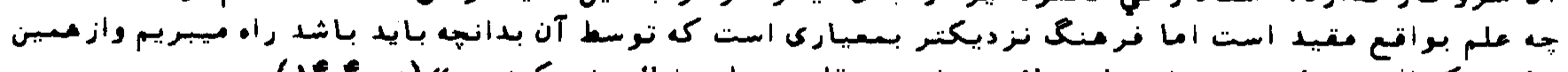

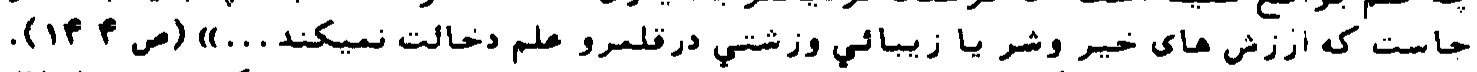

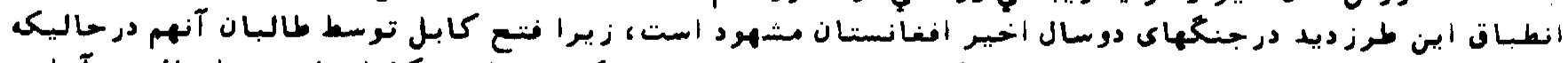

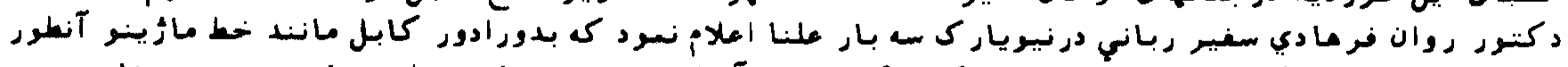

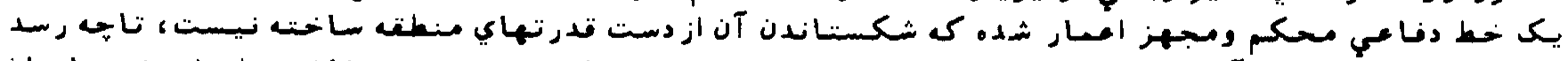

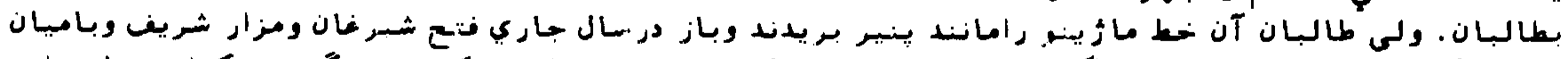

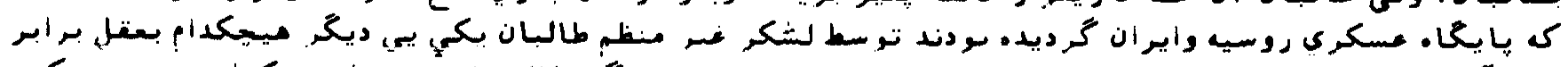

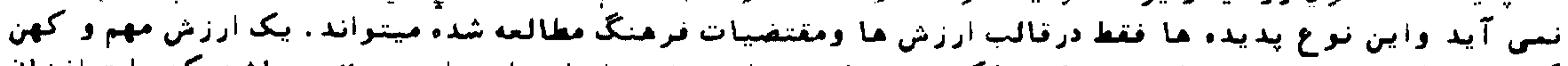

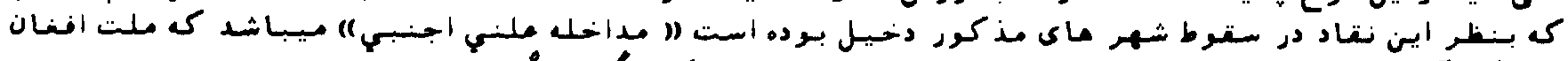

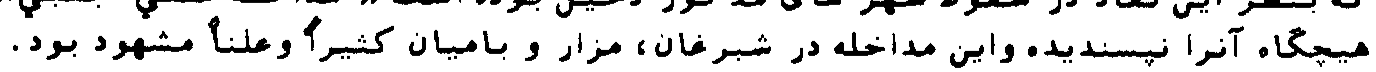

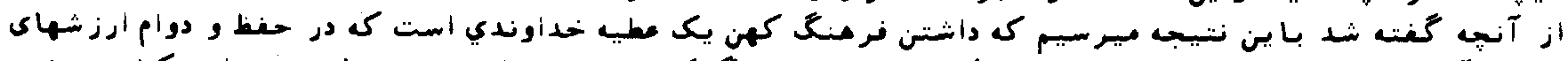

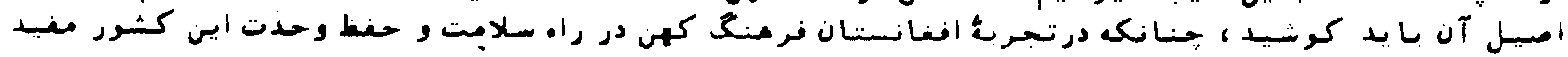




\section{1}

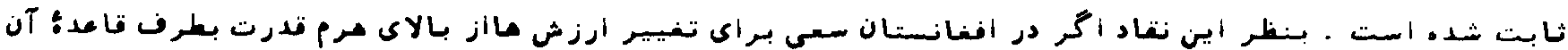

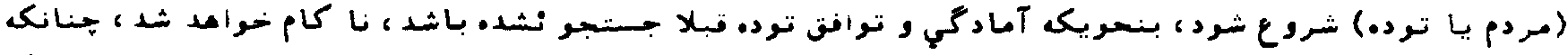

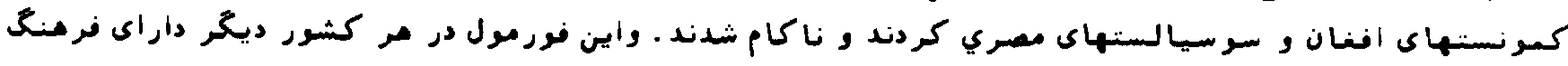

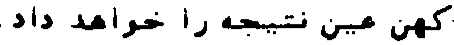

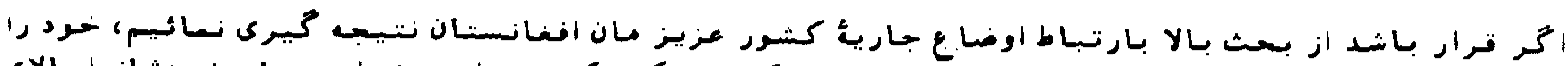

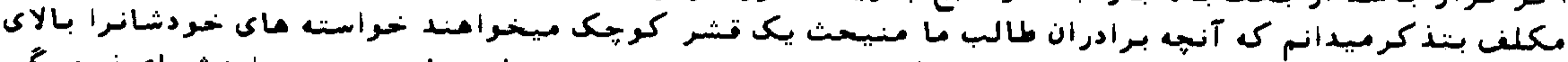

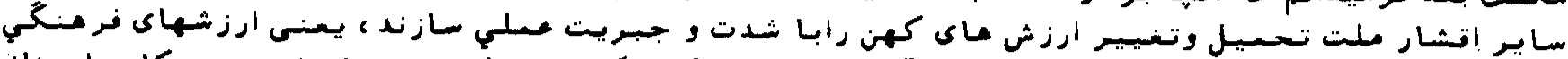

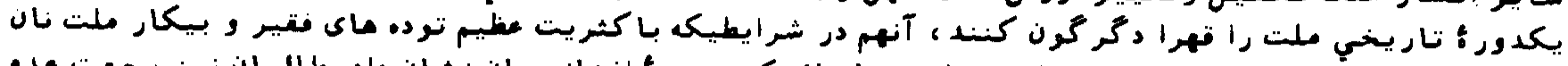

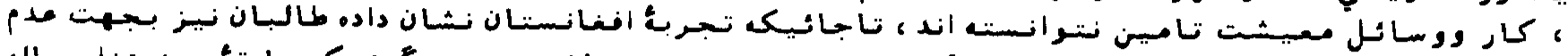

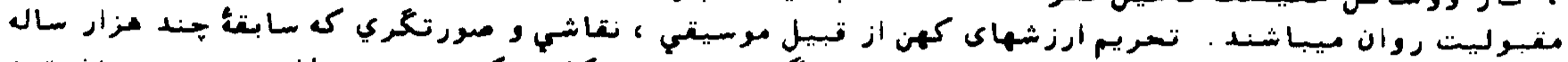

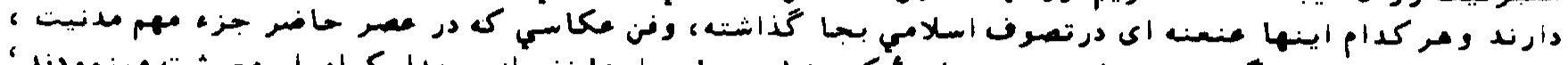

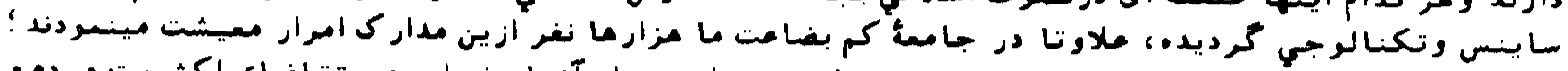

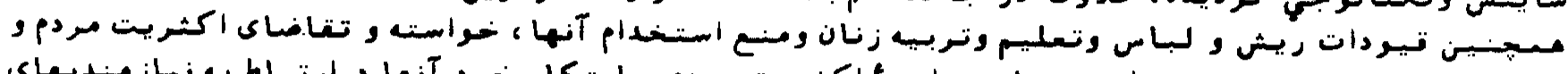

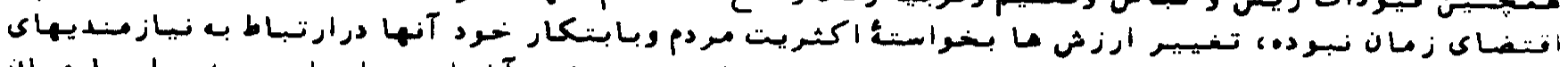

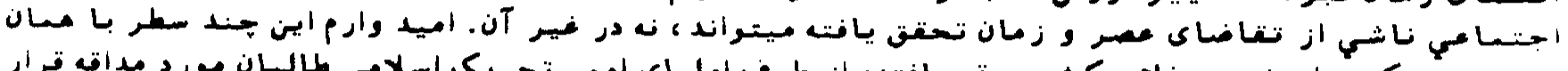

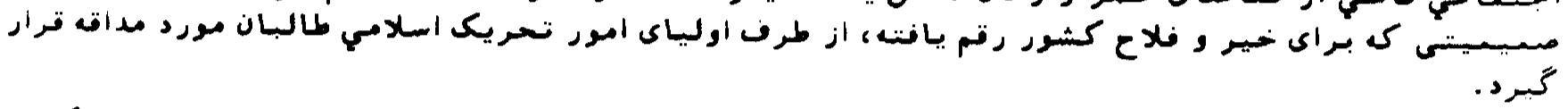

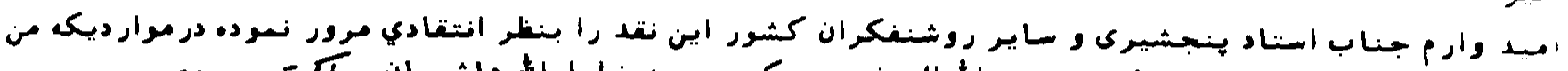

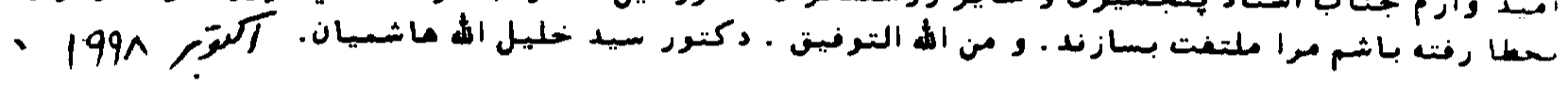

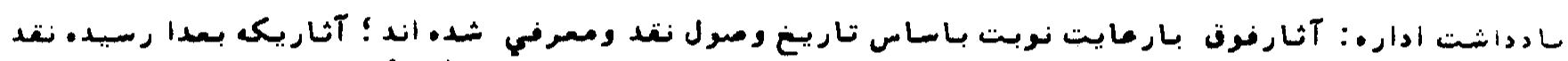

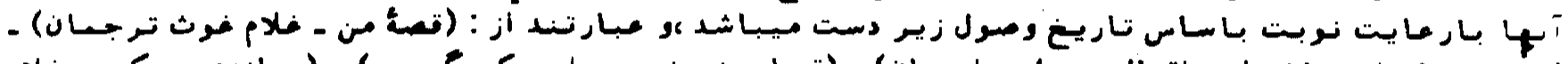

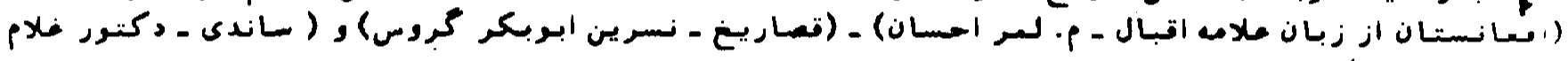
معطفي رسولي).

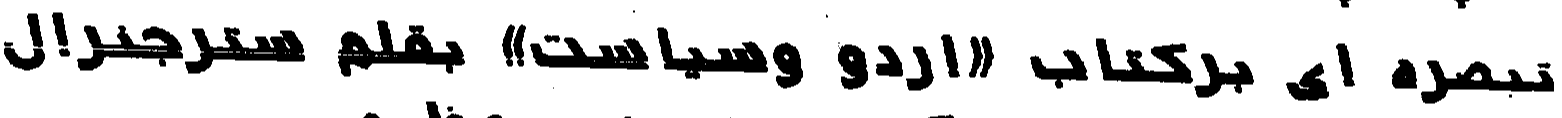 كangas}

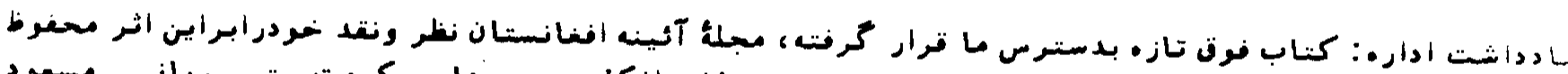

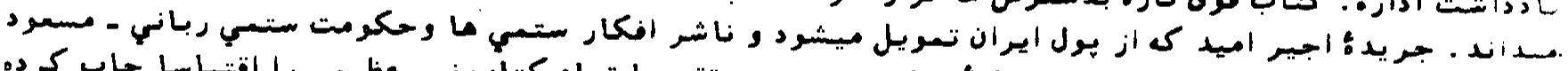

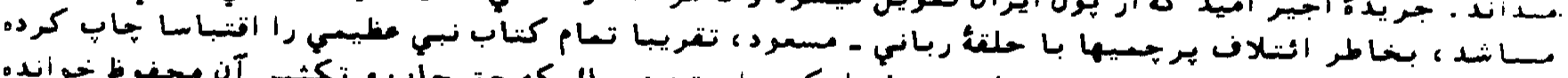

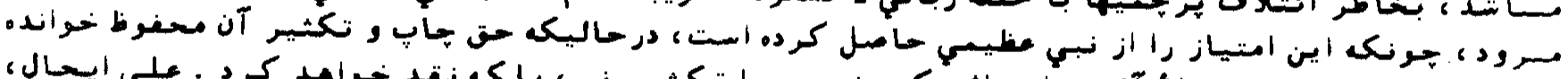

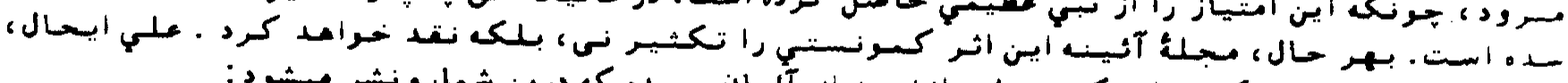

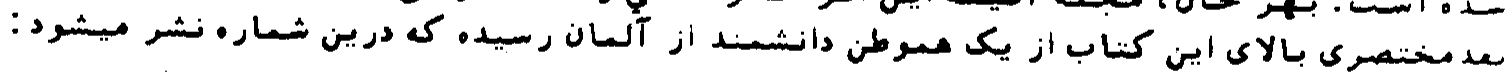

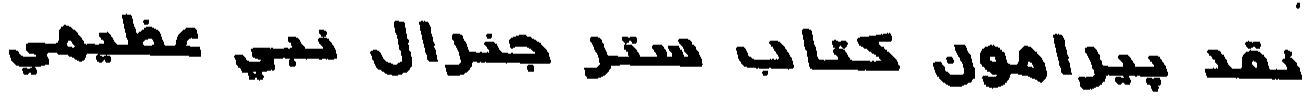

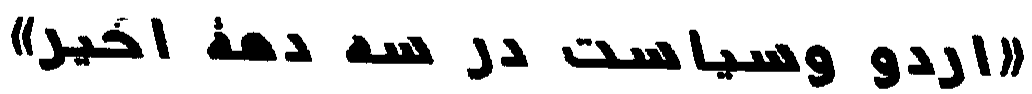

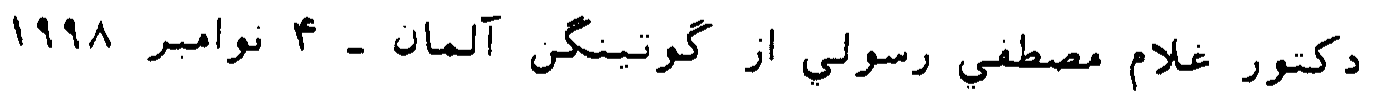
بتشش اول

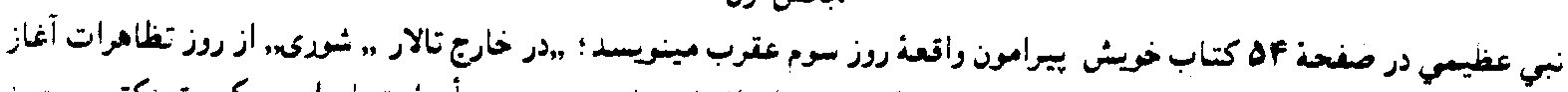

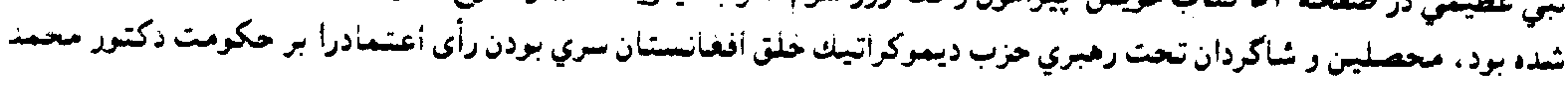

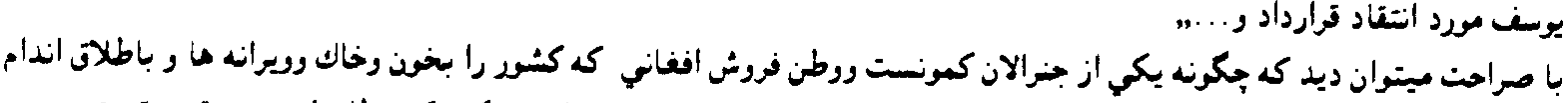

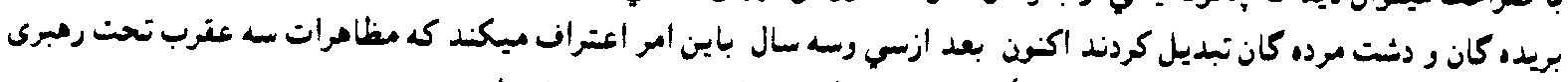

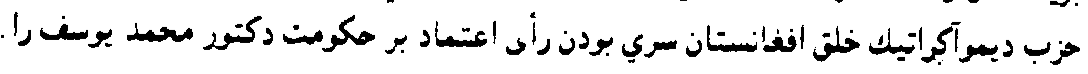




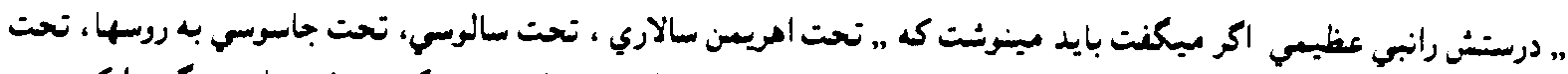

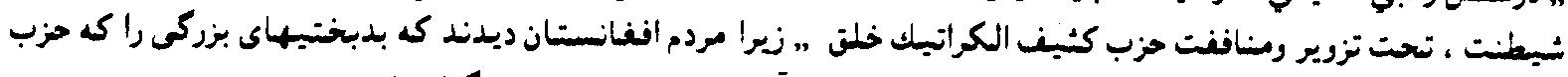

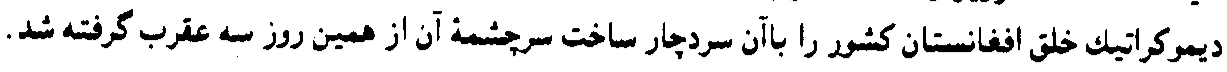

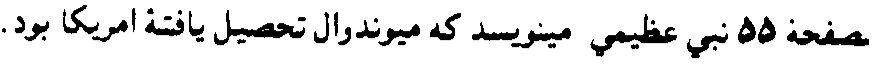
جه دروغ شاخداري!

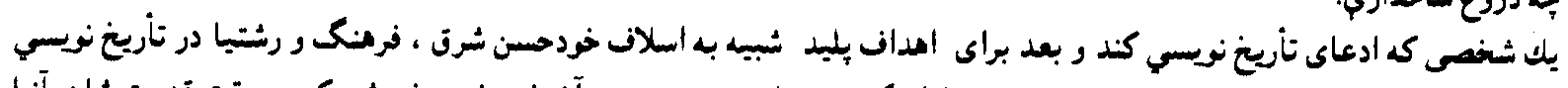

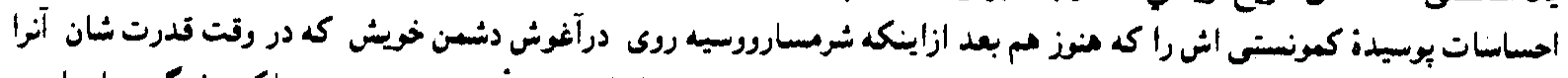

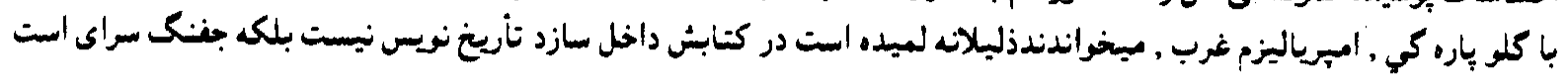

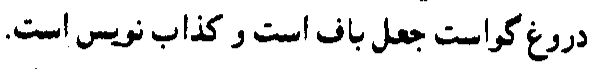

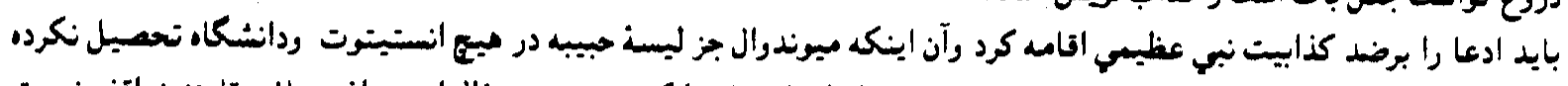

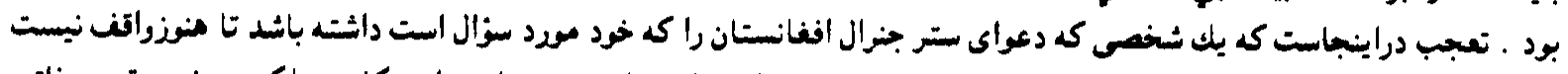

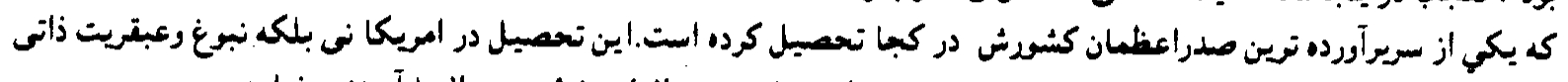

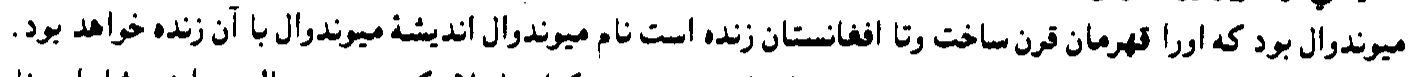

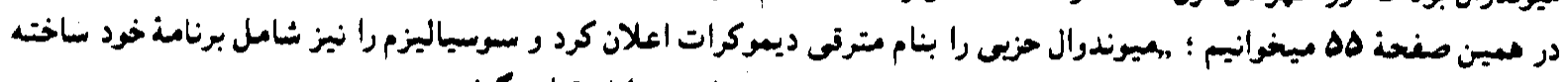

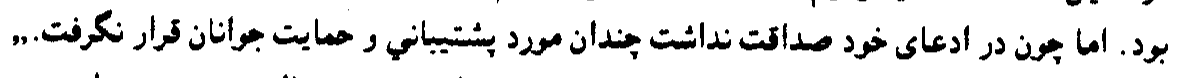

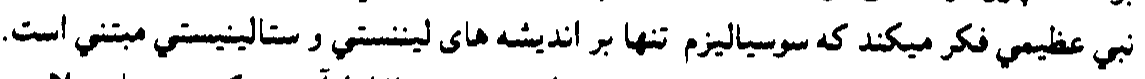

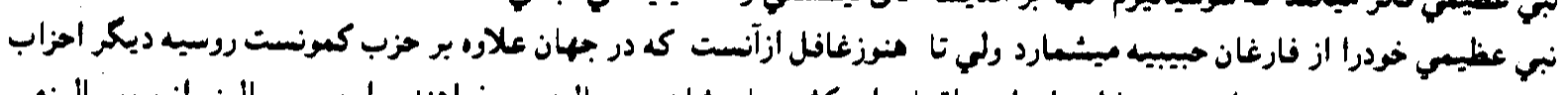

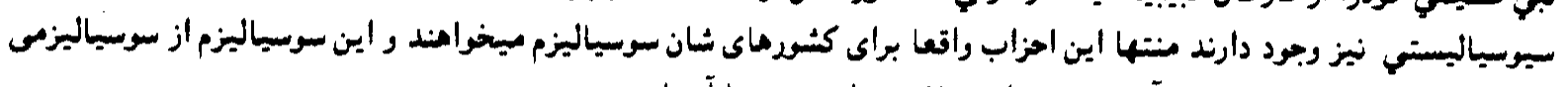

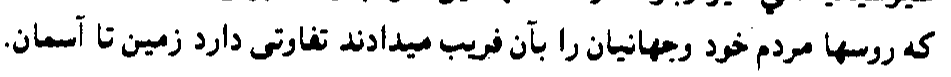

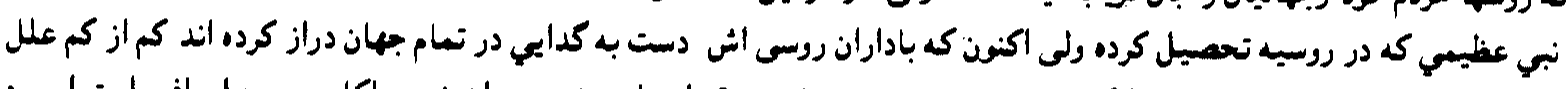

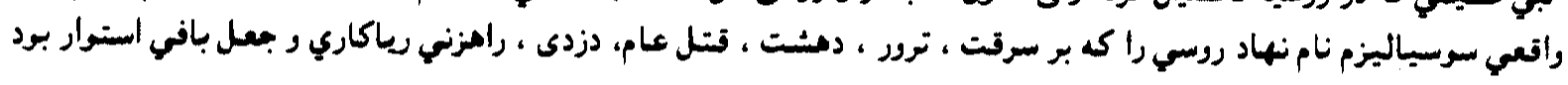

مطانعه ميكرد.

نبي عظيمي بايد بداند كه سوسياليزمى را كه لينن با كودتاى خونين V نومبر سال 1917 در روسيه اعلان كرد ترور ودمشت كرايي

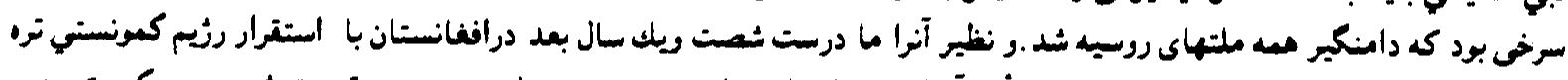

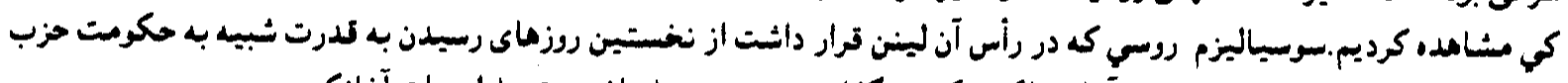

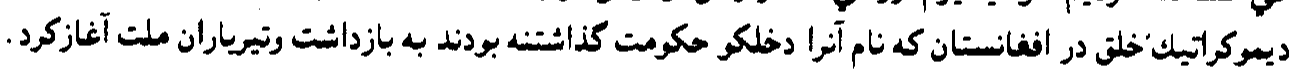

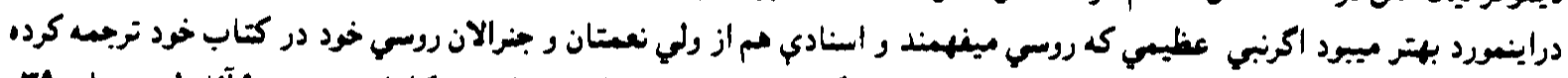

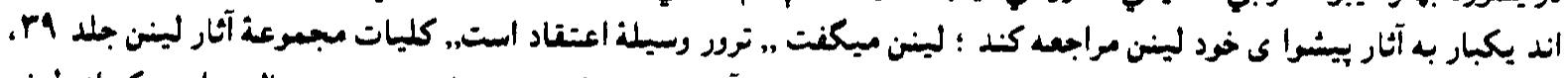

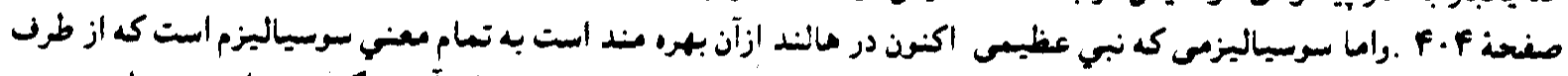

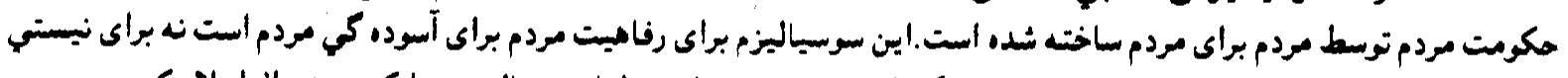

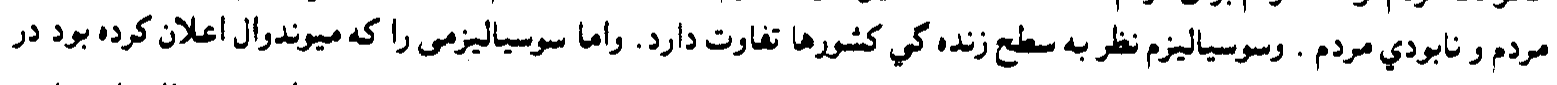

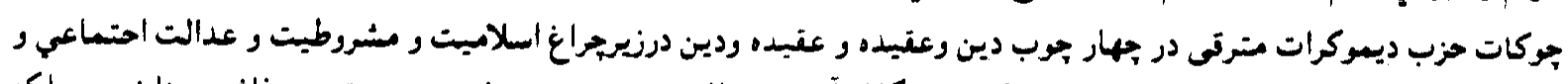

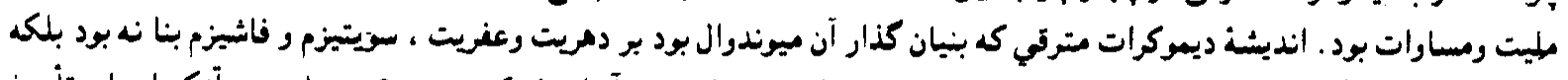

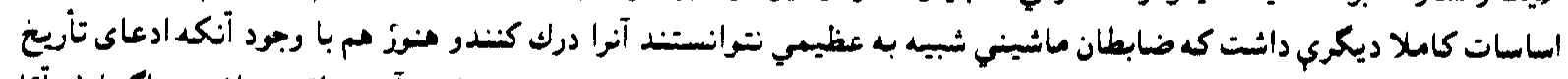

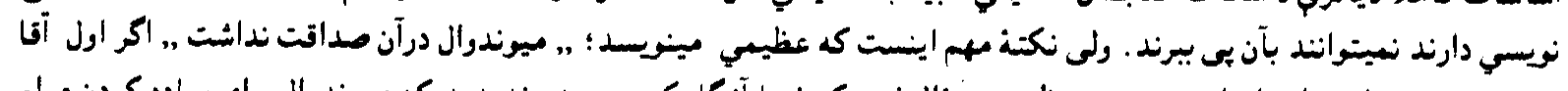

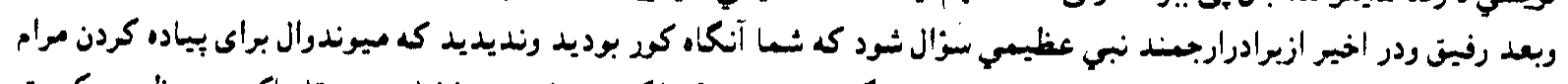

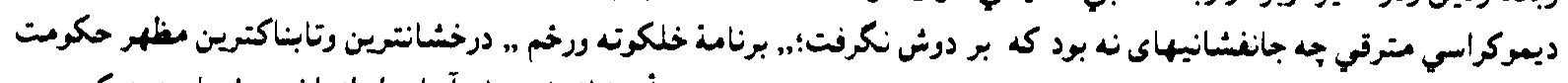

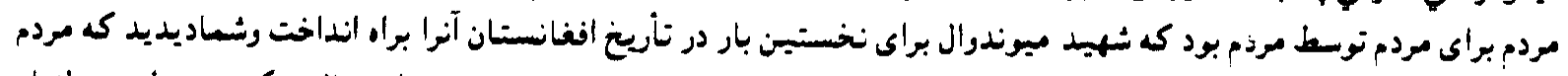

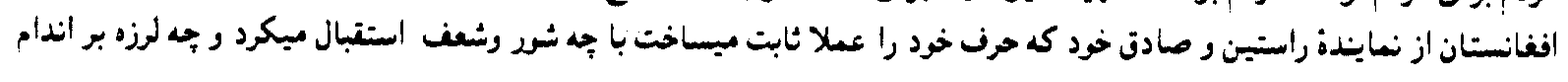

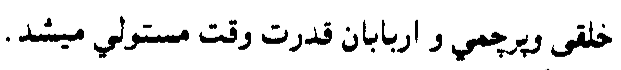

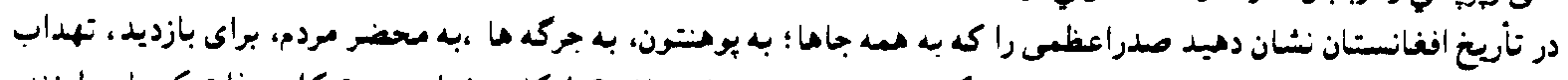

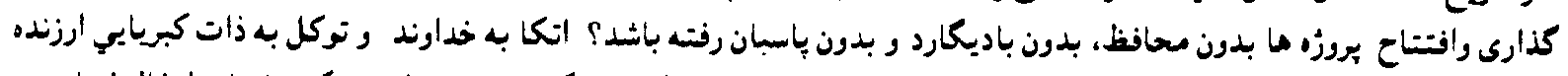

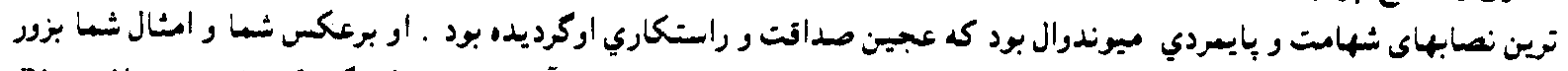

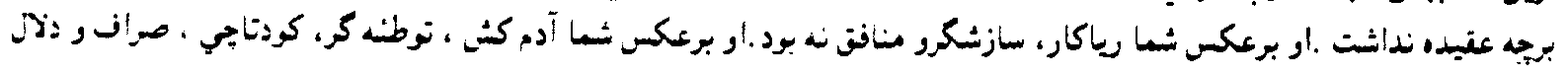




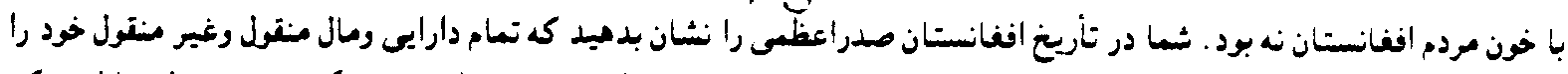

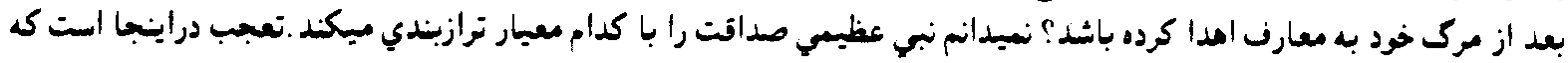

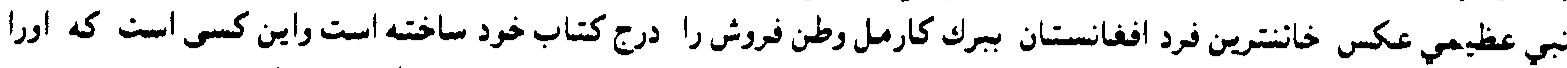

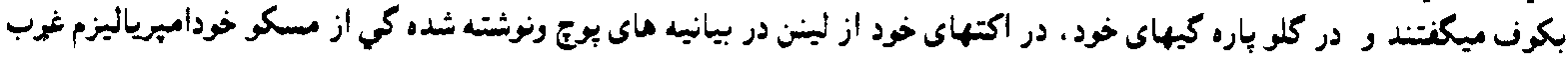

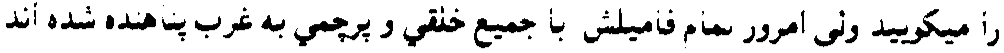

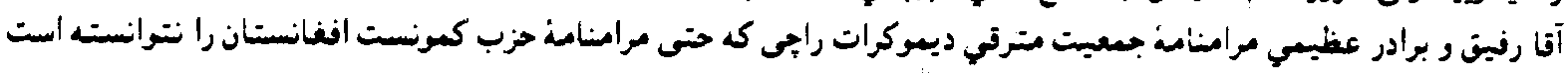

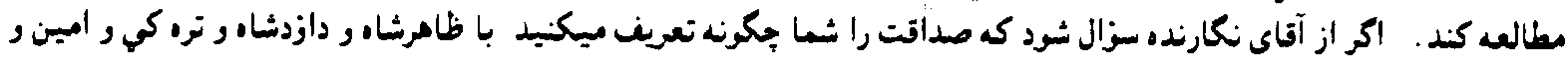

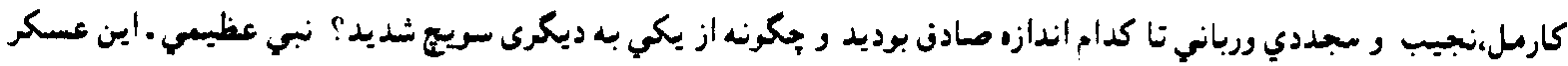

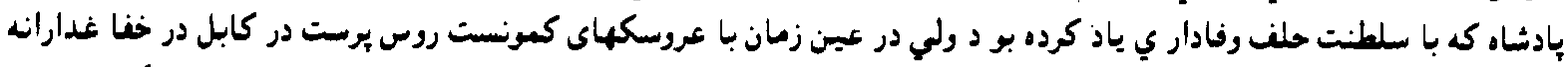

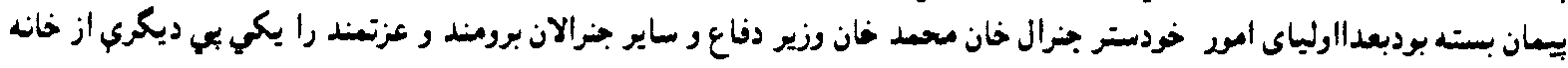

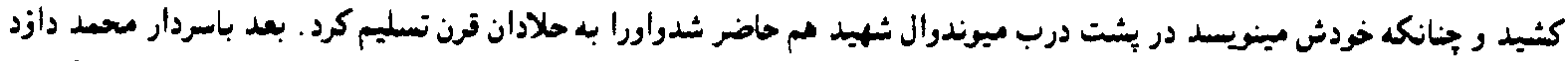

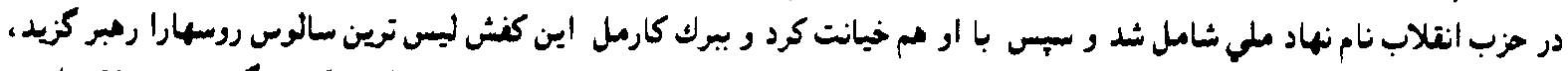

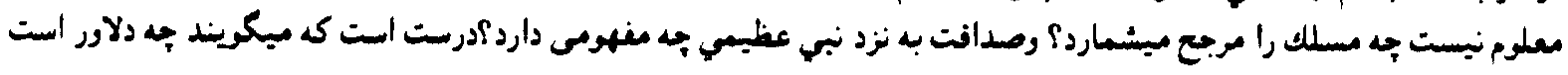

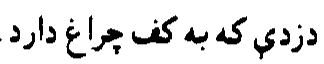

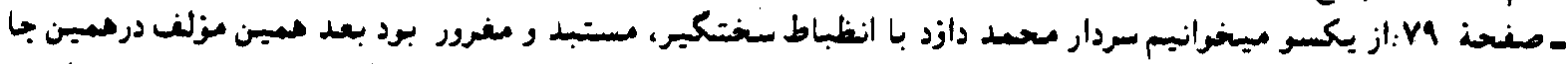

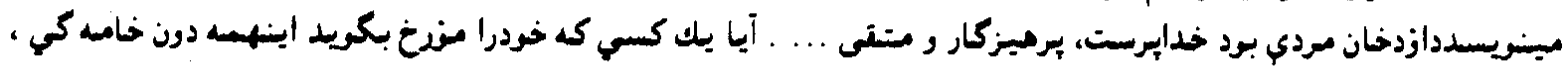

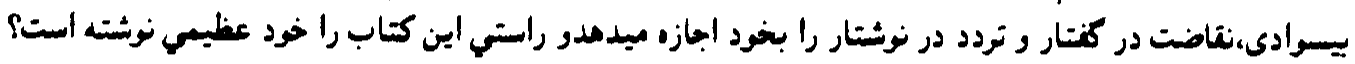

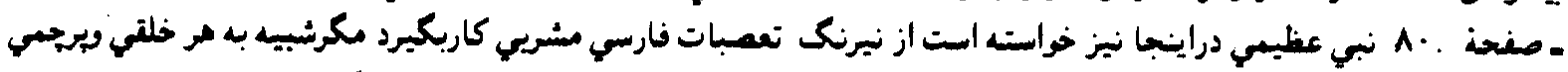

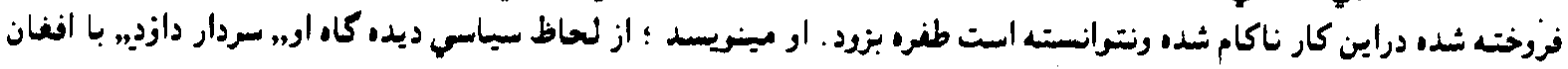

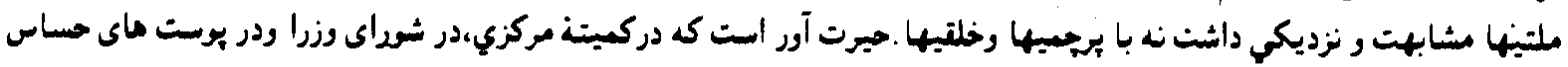

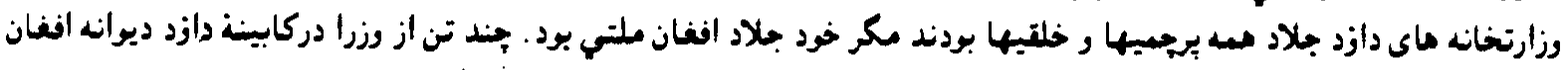

$$
\text { ملتي بودند؟ }
$$

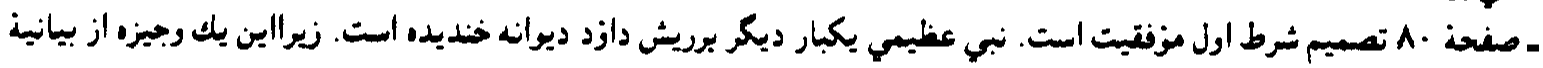

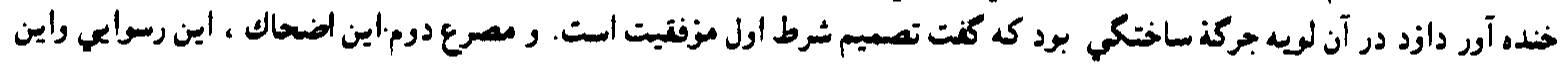

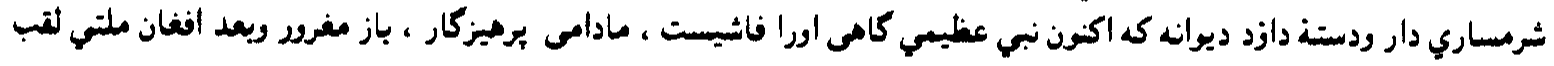

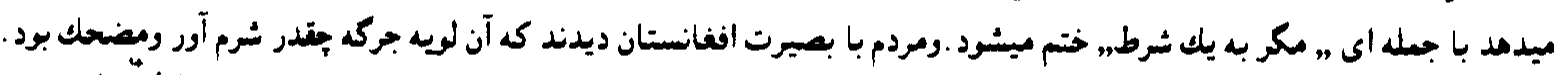
()

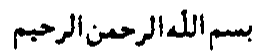

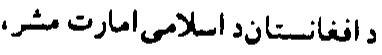

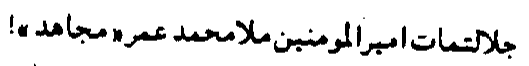
السلام علبكم ورحة اللهو بركانه.

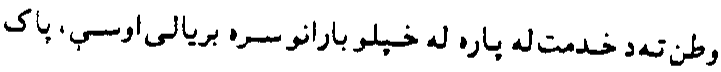

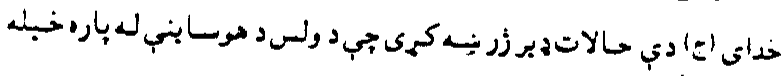
اسلامى دندداو وظابف ترسردكي.

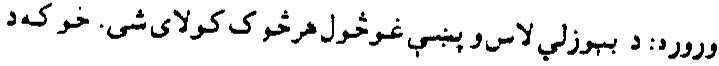

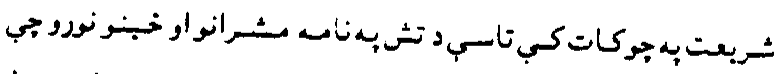

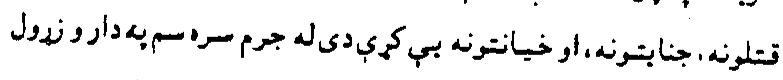

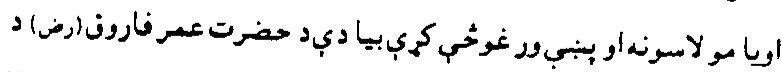

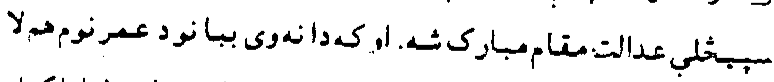
زيانىدى تهنكدي بوىشريعتاو د نارون اعظم نهاو ، ودان، خبلداكىاز بو موتي دي وى أفغانستـان.

20

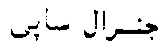

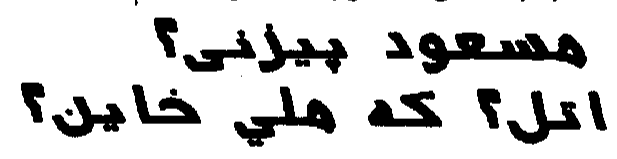

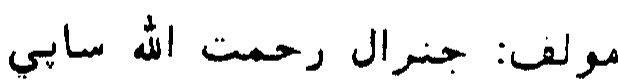

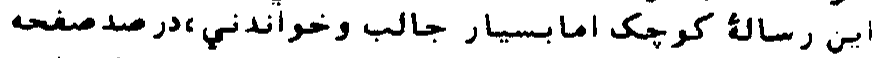

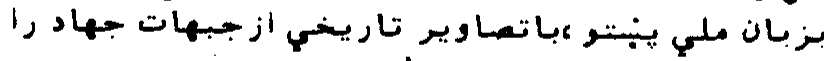

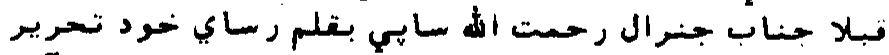

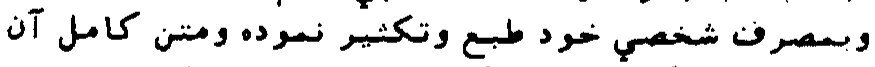

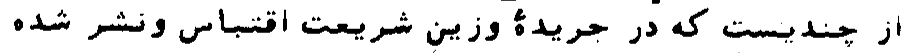

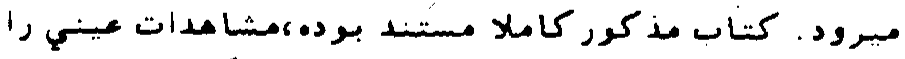

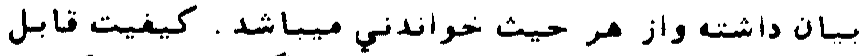

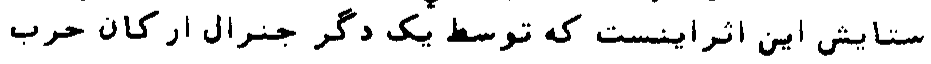

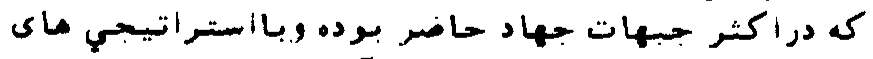

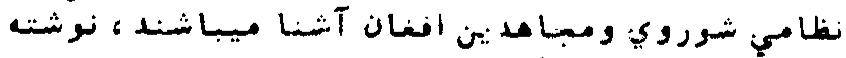

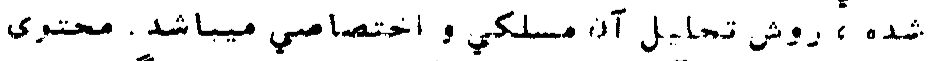

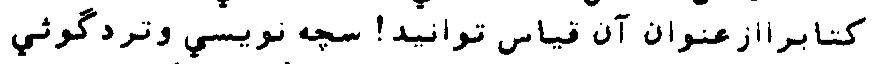

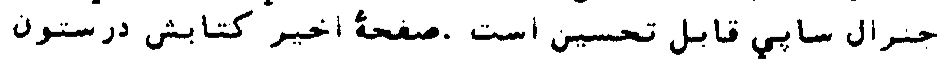

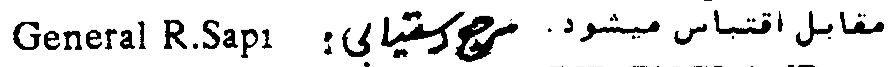
26 First Ave - London, W3 - 7JR,ENGLAND phone:441817437581 


\section{زنما بهقاجل طالبان تورخودده...!!}

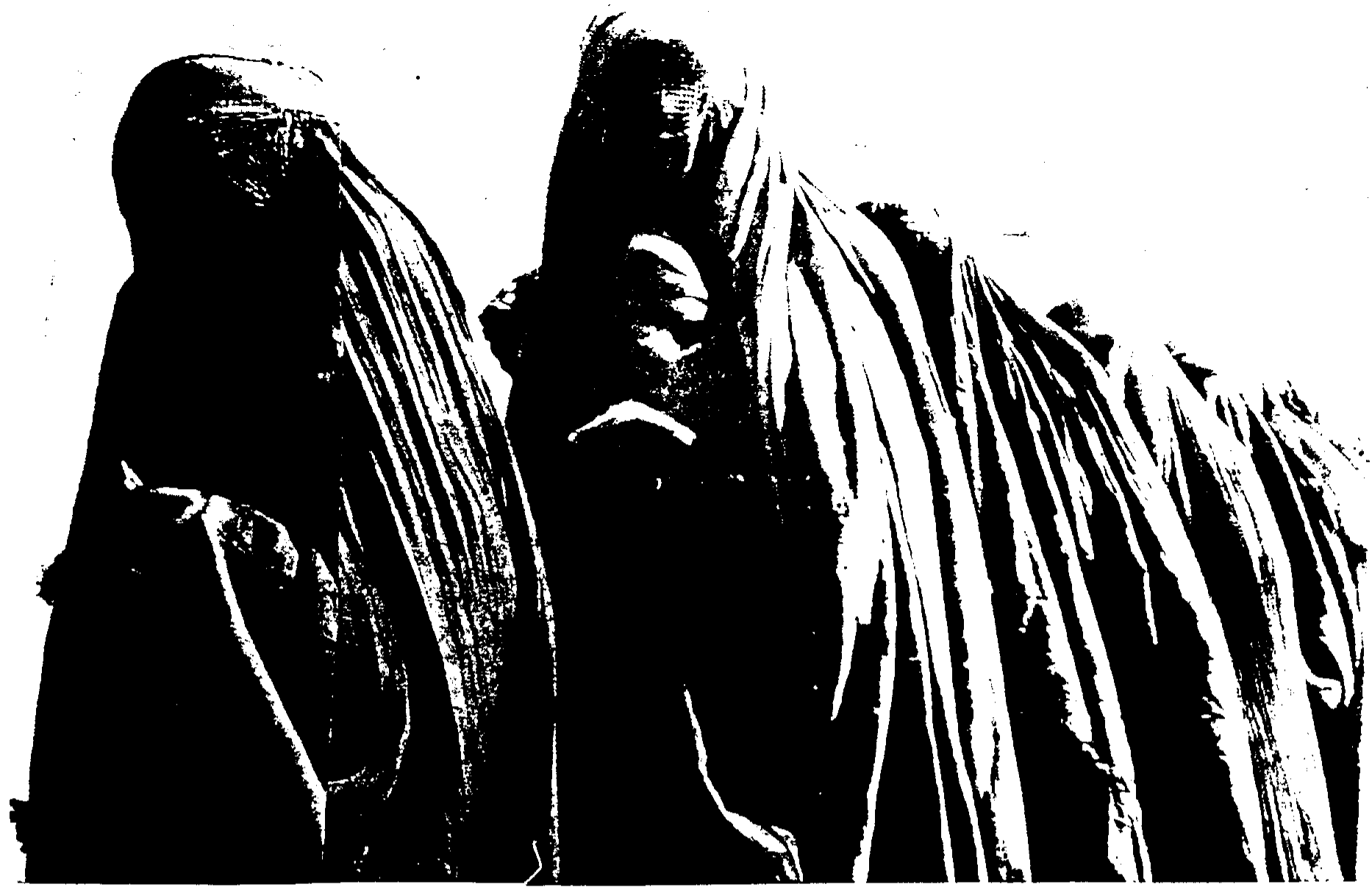

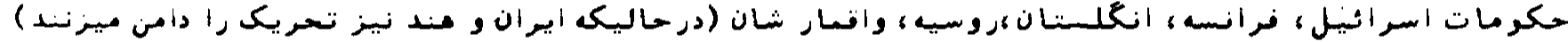

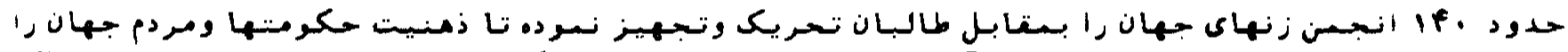

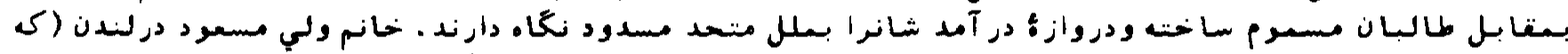

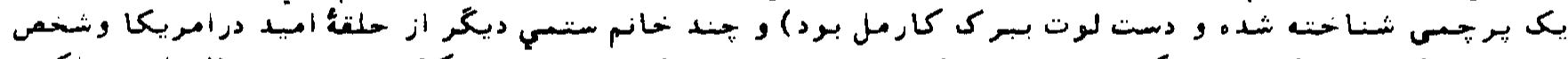

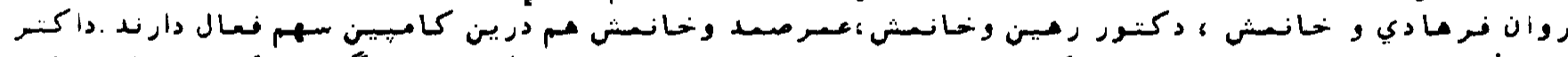

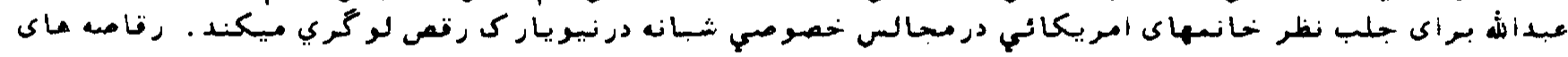

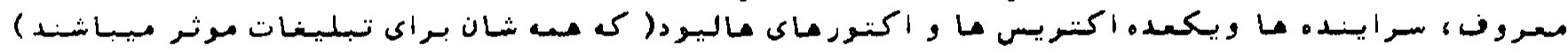

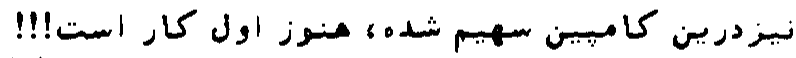

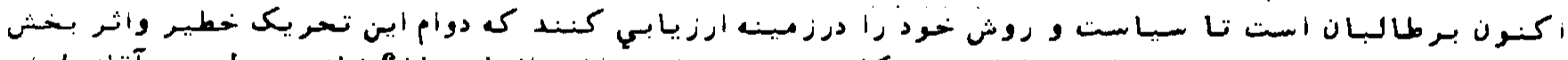

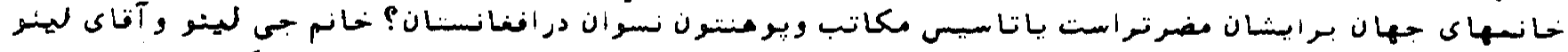

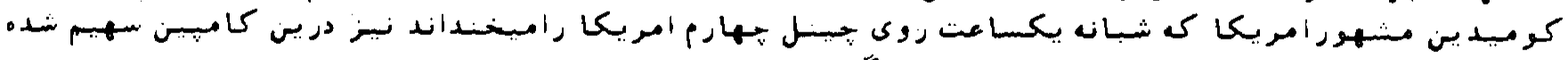

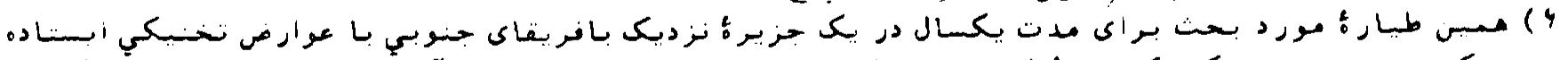

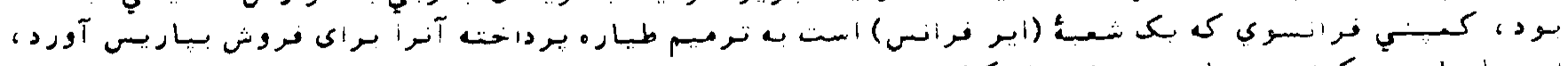

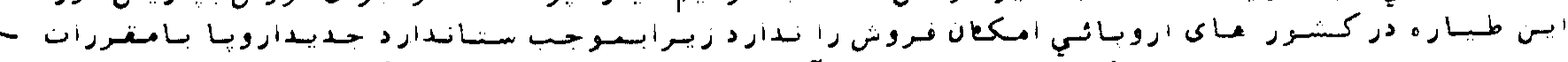

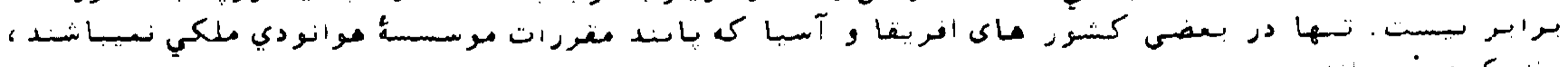

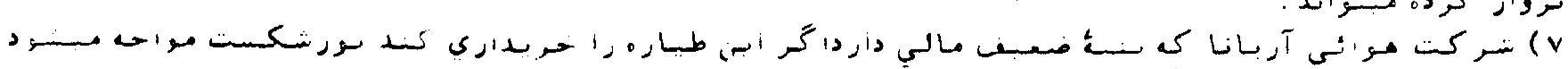

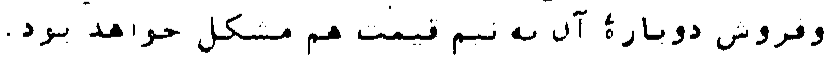

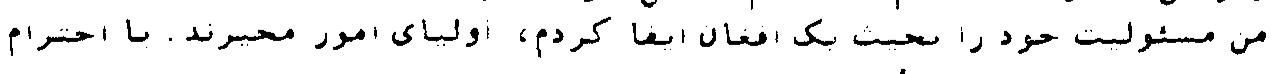

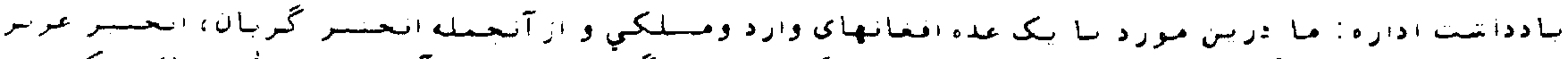

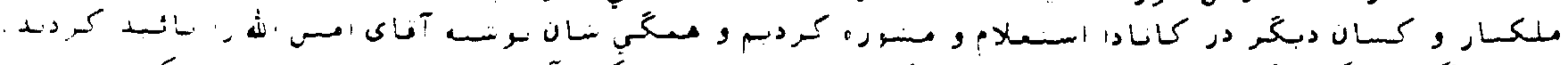

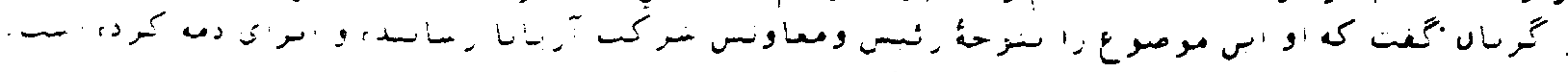


Wali, minister for the Promotion of her office demanding that they be Virtue and the Prevention of Vice.

Wali, like other Taliban mem. bers, relies on an extreme interpretation of Islam to mete out punishments for such sins as drinking. gambling. drugs, watching television and listening to music. Women are prohibited from wearing white . litely and without publicity, so as to ankles. Homosexuals are buried not to provoke their leaders.

under toppled walls of brick. Adulterers are killed with stones.

"On the surface, it looks like I have a difficult job." Wali says. "But I am willing and happy in my work."

\section{Former News Anchor} Must Now Wear Veil

Shafiqa Mahmood Habibi was once Afghanistan's most popular news anchor. During the 1970s and 1980s, she and her husband, a former government minister, enjoyed a favored spot among the country's elite. With her striking good looks and professional success. Habibi stood as a symbol for everything that Afghanistan, an impoverished and illiterate country. might one day become.

On Sept. 27. 1996, the Taliban army took control of Kabul after a fight that devastated the capital.

"At 1 o'clock, the Taliban came into the city." Habibi recalls. "At $60^{\circ}$ clock. they announced on the radio that women had to stay in the house and could not come out. I first thought this was a temporary measure because of the fighting. Then I came to realize that it was permanent."

Today. Habibi spends most of her time doing chores around the house. But there isn't so much of that, she says, since two of her three children have fled the country. On the rare occasion when Habibi ventures outside, her face-once among the most well known in the country -is covered by a veil.

"All the time I cook," she says with a cracked smile.

Inside her apartment. Habibi carries on a quiet rebellion. In a room off the foyer. the walls are adorned with family members portraits-illegal under the Taliban. which considers images of living things sacrilege. And Habibi still keeps an album of photos that show her smiling with movie stars and heads of state.

"When the Taliban some to this house, we take them to another room." she says.

Habibi is careful not to criticize the country's leaders, but she and a handful of other professional women are beginning to push the says.

Taliban to change.

Shortly after she was fired from her job. she and a group of female colleagues staged a protest outside paid. Four months later, after consulting Mullah Omar, the Taliban agreed.

Habibi and 11 other professional women recently sent a petition to the Taliban asking that they be allowed to return to work. The women presented their request po"The Taliban think everything is $\mathrm{OK}$ and that we, are havov sittine in our rooms," Habibi says. "But we want them to know that we are not happy. It is not the money. There is a need to rebuild the country, and our experience is being wasted."

As a Muslim. Habibi says she has hope that the clerics will ease up She is less certain of the support she enjoys among other Afghan women

"For the lower-class women, life is as it has always been." she says. "They are happy. Prices are stable. And there is peace."

\section{Militias of Past Looted and Extorted}

Jilani Humayum remembers when-Kabul was divided among five warring militias. In the early 1990 s. the fighting raged almost every night, and soldiers of each army looted and extorted in the fiefdoms they controlled.

"If you were walking down the street carrying a kilogram of meat, someone holding a Kalashnikov would charge you a tax." Huma. yum says. "If you drove down the street, they charged you a tax. Even common people were joining in. taking their Kalashnikovs and demanding bribes."

Humayum, a businessman, fled Kabul in 1992 and went to Pakistan. Two months ago, he returned to the Afghan city of Kandahar. where he has set up a dairy farm and a store selling milk and butter

"Now you can walk the streets without fear," he says.

Humayum's enthusiasm for the Taliban ends with the peace. He doesn't care for their harsh brand of Islam or the way they are imposing it. Not long ago. Taliban entorcers decided that his uncle's hair was too long. and they pulled him aside and shaved his head without water.

Huinajum wouid iike io see nis young daughter go to school.

need to do more. We need to put (the government to rellow will we were starting. She's testified allow girls to attend classes only before Congress, demonstrated. led a when the country is able to build delegation to protest Unocal's pro. separate schools and give the girls posed pipeine /through Afghan
Leno, she says, "got active just as

What they consider appropriate

Sadiquedions.

Sadiqullah Siddiqui, a taxi driver. dhink that will ever come. He's taking his two sons and wo daughters to Pakistan, where to read and writc.

He thinks he knows why the Taliban has made education a low priority.

"The Taliban does not want our children going to school." says Kandahar's driving his cab down cause if you get an education. you won't fight."

\section{Luxury Hotel Faded and Dusty}

Sher Ahmed, the food and beverage manager, holds a color brochure that the Hotel Intercontinental printed 30 years ago. It pool. couples playing tennis and Ahmed as a young man, sporting a red dinner jacket and a wide smile.

Ahmed. now wearing a gray the deserted dance floor. As a young man. he used to sneak up to the fifth loor to watch the couples dance.

Today, the bar is closed, the dance floor is dusty. and Ahmed rarely
ventures up anymore. "I used to love this place." he says.

\section{Continied Krmp.4}

"These women have had their ives stolen from them." Leno says you see it all over the news. This is whole country. $11^{1 / 2}$ million omen. gone."

She is spearheading the Feminist Majority Foundation's Campaign to Stop Gender Apartheid. which is demanding that the U.S. treat Af ghanistan as it did South Africa
under apartheid-refusing to recognize the Taliban as a legitimate vernment and trying to convince business interests to stay out until we began a campaign of public Katherine Spillar, the foundation's national coordinator. "And the U.S. has taken a position-both President Clinton and Secretary of State [Madeleine) Albright have been outspoken in therr condemnation. But we tore these women's rights." Policy-wise, it's working "In 1996

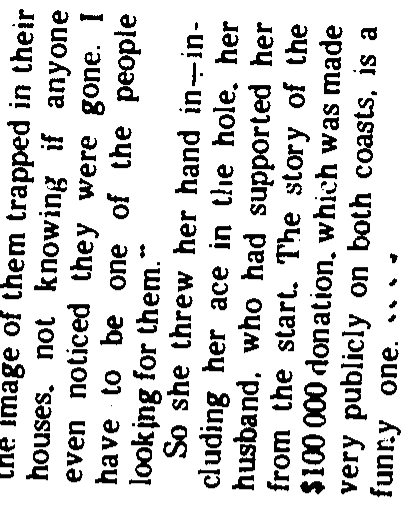

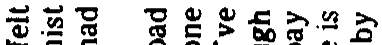

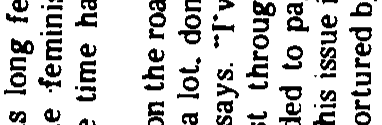
롤

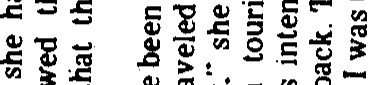

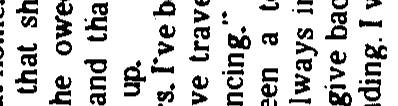

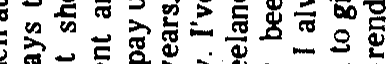

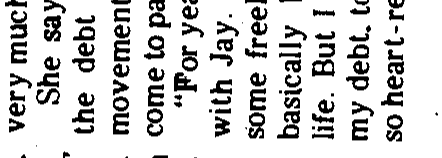

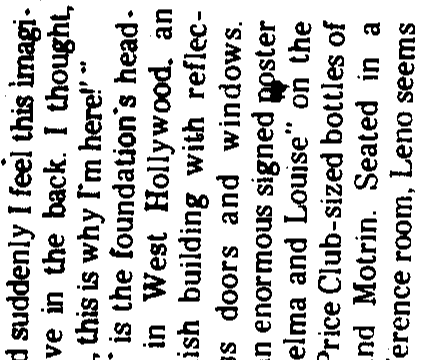

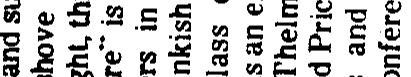

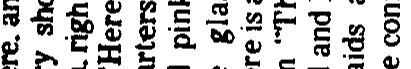
岁壱
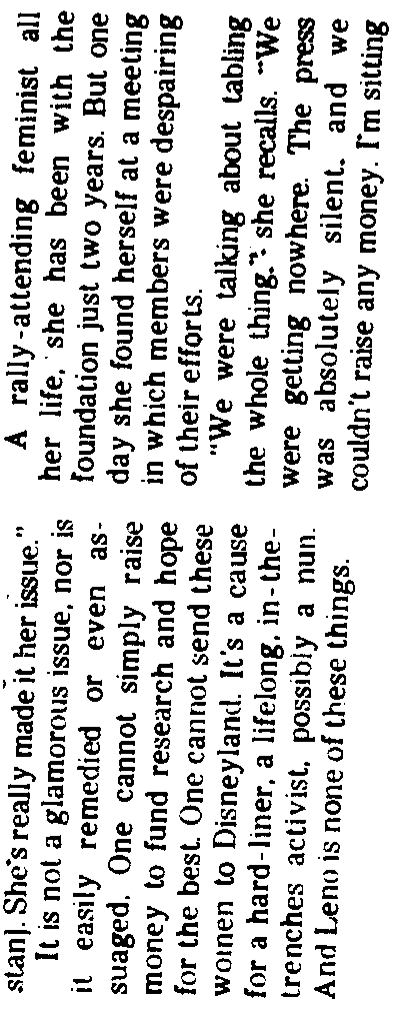


\section{'In Revenge, There Is Life,' Taliban Says}

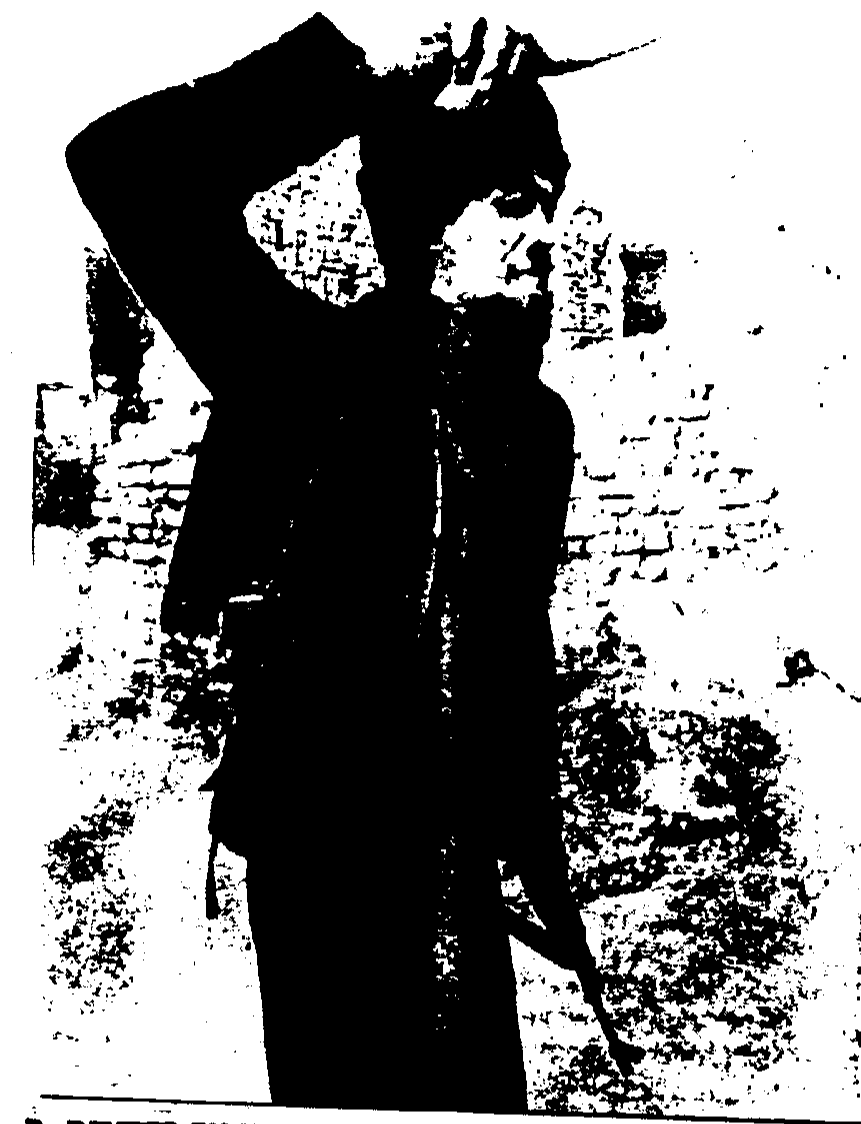

D) DEXTER PILKINS

TIMES STAFP WRTTER

KABUL, Aghanistan-The Ho? - tel Intercontinental, gem of a ru. ined city, exiudes a medieval charm.

The lobby walls surrendered their photos and portrates to the Taliban religious police two years 8go. The drtnking and danctrit that drew sultans and kings have been replaced by ice water and prayer. At night, the breeze wants into the. Pamir Supper Club through a hole made by a missile, the gap framing a city of twnkling lights and curfew calm.

"The Taliban took everything." ays Sher Ahmed, the sad-eyed manager of food and beverages. "But they brought the fighting to an end.

Peace has come to Afghanistan with a puritanical touch. After nine years of civil war. the soldiers of the Taliban religious army have tamed the cities and conquered the cointijisiute, Uringing this nation a measure of order and quietude it he not known in more than a guarter century.

meir triumph, sealed this sum: mer during a remarkable string of victories over their rivals, efinds to more than $95 \%$ of Afghan
But the price of peace horptiven tyranny and fear, impoeed itrian Irlamic movement so extreme that those who violate its ways are often killed or maimed in public ceremonite that echo with verses trom the Koran Members of the Taliban militia patrol the cities with switches and whips, dispersing crowds and smooping for diswent.

: The onset of calm has given rise io the first sigms of discontent often expressed at the risk of arrest or death. Couples complain that their daughters are prohibited from attending school. Many women their identities cloaked behind guaids carrofing owtches thrivh

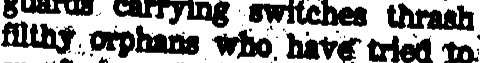

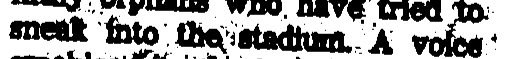

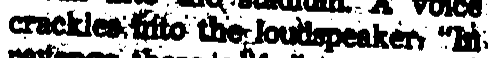
revienge, there to bfe."

The condemned man, Atqullah. is actod cordo-lesed at the far end of the football field, blindforted One of the anreons hands 2 Rathobnitrot aseaut the to the murder victim's brother."

The crowd soed silent. The brother crouches and takes alm.

"In revenge, there is tfe." the voice sayr.

The brother fires into Atiqullab's head Atiqullah linger motionless for a second, then collapoes in

\section{'We Emerged}

\section{to Restore Order}

"I dor': know whai we hive done to earn the enmity of so mavy countries," says Mullah Mohammed Hassan, a senior Taliban leader. "What have we done?"

Hassan offers a bit of history to juetify the Taliban's harah regime: Afghanistan's glorious victory in 1969, ending 10 years of Soviet

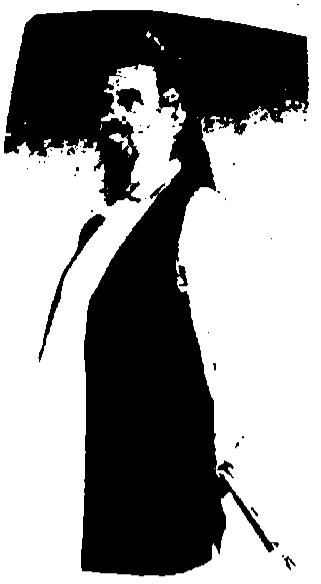

Agence Prance.Preas on one abother. Anarchy was Sher Ahmed. the a d e: manager of lood and beverages.

occupation, soured when the many factions of freediom nighiers turned looved; aind Afghanistan wa deThe brother otand over Atigul- came an opportunity for one of the
The lies aim and fires agitn. The body where reald up a chectpoint. around Then the brotber walls o pay bribes and the orees forced a flal ahot Spect crocichoes and fires laughtera and wives.

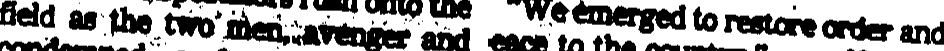

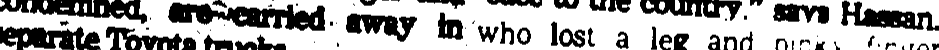

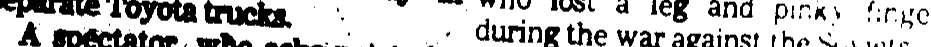

identefied, explains that not to be Inspired by a young ritric-a

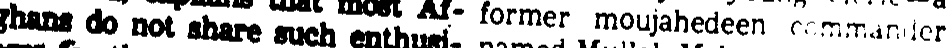
and the public executions but named Mullah Mohammes 1)marStadthey fill the Kabul Sport the Taliban swept across Aighanistimum on Fridays because there fan earlier this decade a in a $\infty 0$ little else to do. in America, you have televidion fighters rolled over the pro: isusily unbeatable militias and cupiured unbeatable m.in
Kabul in 1996.

To answer the anarchy. !he T.1. ban imposed a series of rules so sweeping that they touch evcry aspect of daily life. Men must wear their mustaches just above their lips and their bangs neatiy trimmed along their forcheads. Women must don burkas that cover the entire body and provide only a screen-covered opening in front of the eyes.

If a woman refuses?

"Maybe we beat her with a stick." says Mullah Mohammed 


\section{Trying to Save Women Buried Alive}

Tf you were a woman or a girl in Afghanistan. here Taliban

There is no school for you, not even elemeniary most of the university professars half the teachers and most of the university professors. now you can neither

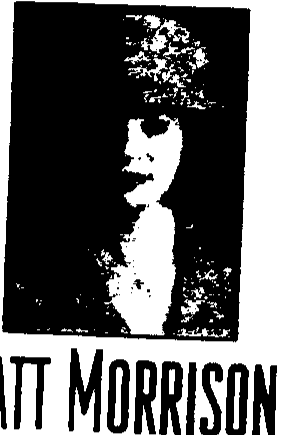

learn nor teach. If your kids get sick, if you go into labor. male doctors cannot so much as take your temperature. and almost no female doctors still practice. So you may stay home. and you might just die.

At home. your windows must be painted over lest a passing male glimpse a female form. If you go out. you wear a burka. an unwieldy swaddling of cloth with only the eyes exposed through a small mesh screen. like the one in the door of a solitary confinement cell. But woe betide you if the Afghan winds - so brisk that kite-flying used to be a national pas:ime until it was banned as irreligious - whip the taric and expose eyen an inch of skin. Youll be chis sed and beaten. maybe to death.

The same will happen if you set foot outside your dur with anyone but your brother. husband. father or $\mathrm{s} n$. Yet many men died in the civil war: perhaps jurs are among them. and you cannot leave home a! all. But why should you? You can't have a job.

Today in New York and tomorrow in L.A.. writer

Mavis Leno (ves. as in that Leno) is taking the lead in a Feminist Majority Foundation campaign to make the Clinton administration and Congress act with the same force against Afghanistan's "gender apartheid" as was used in South Africa, flexing policy and that powerful crowbar called the dollar.

"If we do not call this human rights abuse." Leno says. "then we have a problem calling women human. or we don't call 'abuse' anything that doesn't involve killing somebody."

In their desolation and isolation, Afghan women remind her of her father. trapped years ago in a mine cave-in. He came out of it with broken ribs, but he told his daughter that "the most terrible thing was that he had no way of knowing if anyone was looking for him." So it is now: "These women have been buried alive. and they don' $\mathrm{t}$ know if anybody even knows they re there and is coming for them. We need to let them know we re coming.

This is no "women's thing." no "liberal thing. " Jay Leno will try to enlist Bob and Elizabeth Dole. One of Orange County's GOP congressmen, Dana Rohrabacher. is ferocious on the topic. In this he has no bigger supporter than Hassan Nouri, an Afghan American engineer who set up war-zone medical clinics and opposed Communists and the Taliban alitre.

The Taliban. Nouri says. distorts Islam to "violate human rights in the name of religion." After a woman was raped by dozens of rival soldiers and died. the Taliban pledged to stop such treatment of women. In a way. says Nouri acidly, they did. "They put them all in prison.
$\mathrm{O}$ nce the beneficiary of $U$.S. support. the rebel Afghanistan lik more Islamic than the poitcilitration camp; it is no Shepard's than the people who cheered Matthew Madeleine murder are Christian. Secretary of State Madeleine Albright calls its dealings with women "harsh and back ward." Sen. Dianne Feinstein has Taliban at arm' into its abuses. Even Iran keeps the Taliban at arm's length, like a bad fish. If it were a virus-and some fear it is politically contagious would have been quarantined long ago.

So where. people ask. is government pressure on its rich ally, Saudi Arabia? On its neighbor Pakistan? I have a question. just as good - where are the power brokers who are laboring to whip up moral outrage on a sex scandal, when here truly is an outrageous offense against a whole gender, and all they have to
do to see it is to lift a veil?

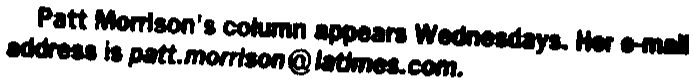

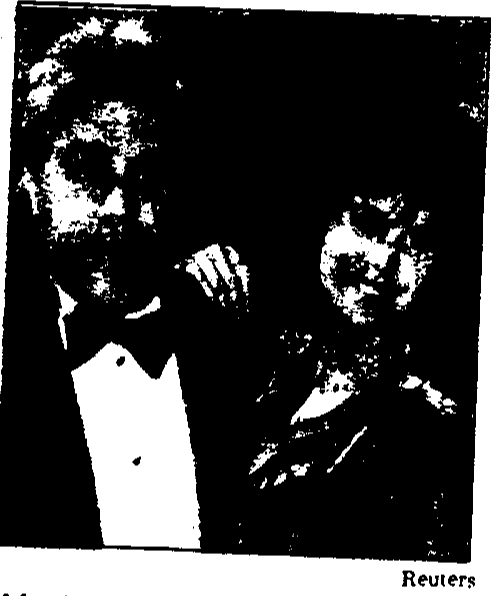

Mavis Leno, with Jay. could not turn her back on Afghani women.

\section{Stealing Back the} Lives of Women

- Rights: Mavis Leno is leading the charge against Afghanistan` Taliban, who are subjugating women and girls.
By MARY MCNAMARA TIMES STAFF WRITER

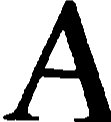
$s$ a cause, women are never chic. Rain forests ailing children. vanishing are the issues that generate philanthropic and activist heat. Women in need . . . well. the fuzzy-warm factor is pretty low. What assails women is usually some pervasive system. The social order. The malc psyche. The government. Who wants to confront such behemoths? Mavis Leno, it would seem. Ma vis Leno, wife of Jay. Her life has been, she says. "a very fun, boho kind of existence." but now she is making her high-profile activist debut-on behalf of women. And Afghani women at that.

Last week. she and her husband douated $\$ 100.000$ to the Feminist Majority Fund, of which she is a board member. in an effort to increase awareness of women living under the Taliban.

A rebel army of religious zeatots. the Taliban wrested control of Afghanistan two years ago. At the time. the Muslim majority. including women. had been leading modern if war-torn lives. Women went to college. held jobs outside the home; they made up $70 \%$ of the country's teachers. $40 \%$ of its doc. tors. They moved freely. Unoer tine Taliban. all that changed.

An annotated list of the new restrictions and reprisals reads like something from "The Handmaid's Tale." Women no longer are allowed to hold jobs or attend school or college. They may not be treated by male doctors. They may not leave their homes unless accompanied by a male relative. and then only if 
distortion is not always easy to discern, neither for those who transmit the untruth nor for us who try to detect them Like everyone else, reporters and editors either sincerely share in the political ideology that makes it so easy for them to believe the news they produce. or they go along with because they know on which side their bread is buttered. footnotes.

For the continuity of civilization, history and the lessons derived therefrom must be more than propaganda with -Bruce Richardson-

\section{Notes}

1 Edward S. Herman.The Political Economy of the Mass Media. New York. Pantheon. 1988. pp 106-117. 2 Ibid. 3 Ibid. 4 Ibid.

5 Ali-in A. Snyder. Warriors of Disinformation. New York. Arcade. 1995, pp 15-18. 6 Ibid. 7 Ibid.

8 Alvin A. Snyder. Warriors of Disinformation. New York. Arcade. 1995. pp 112-119. 9 Ibid.

10 Kar Bird and Max Holland. "The Philippines: Official News," Washington. Nation. June 1994, pp 33-37. 11 Ibid. 12 Ibid.

13 David Wise. The Politics of Lying. New York, Vintage. 1973. pp 319-322. 14 Ibid. 15 Ibid. 16 Ibid

17 Lee Solomon. Unreliable Sources. New York, St. Martin. 1995, pp 122-133. 18 Ibid. 19 Ibid. 20 Ibid. 21 Ibid. 22 Ibid.

23 Ibid. 24 Ibid.

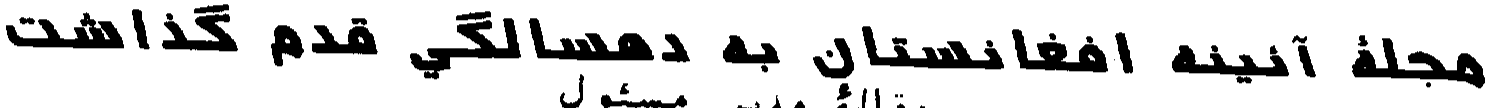

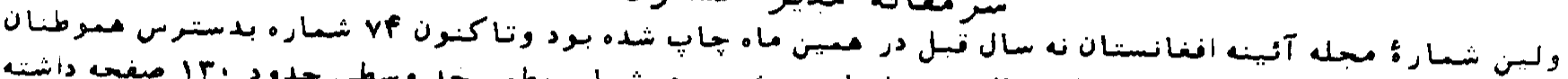

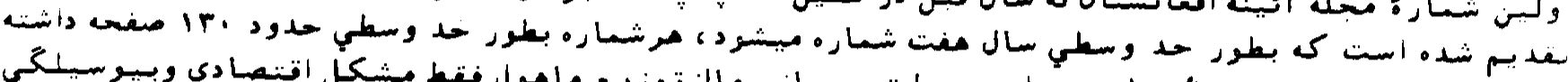

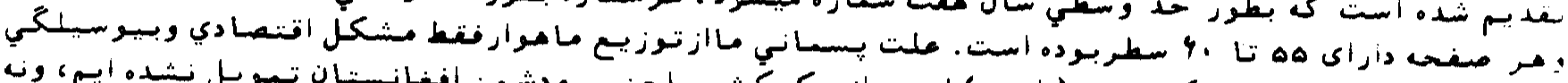

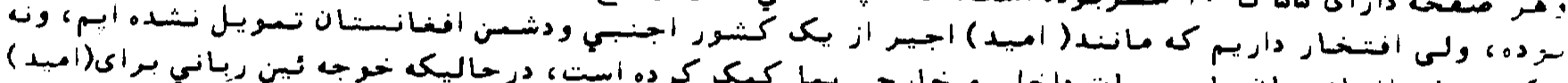

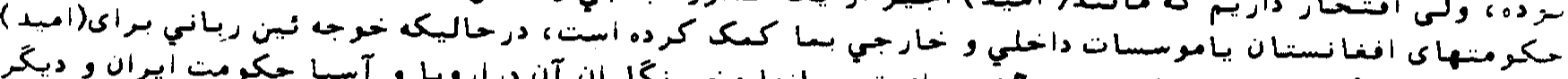

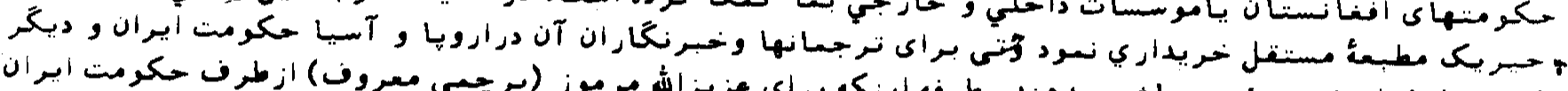

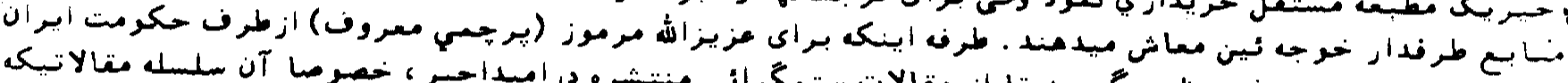

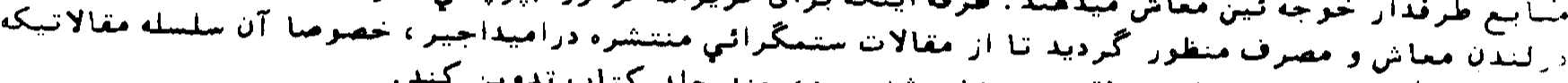

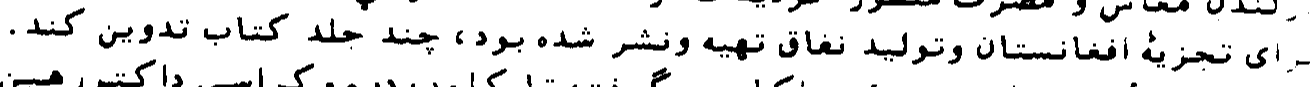

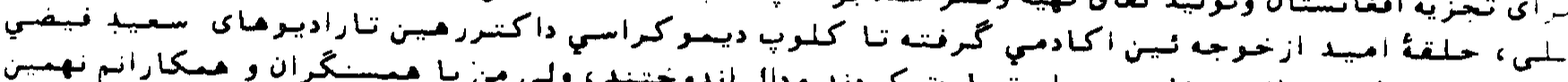

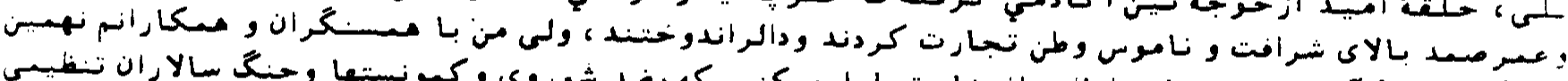

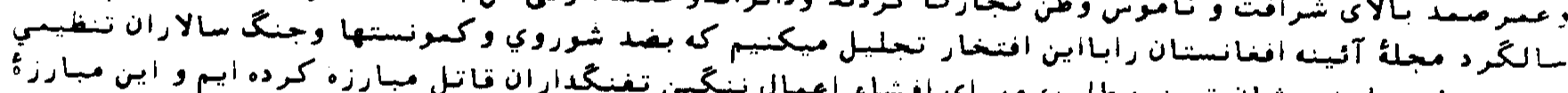

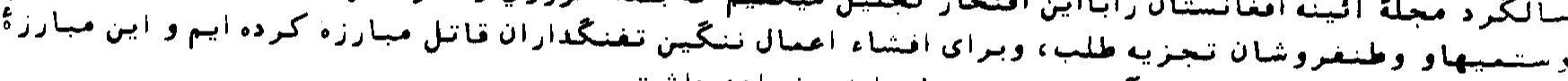

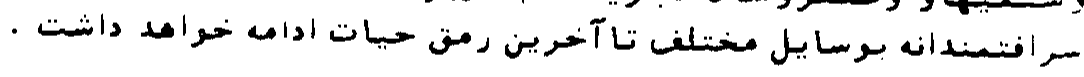

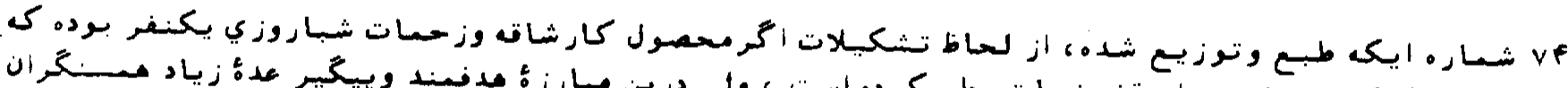

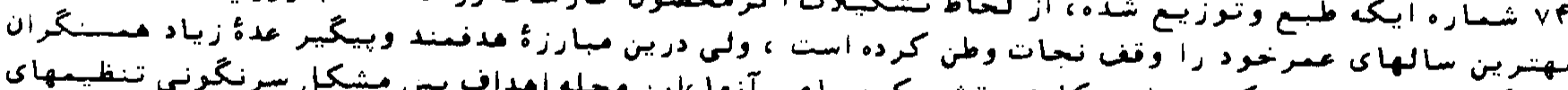

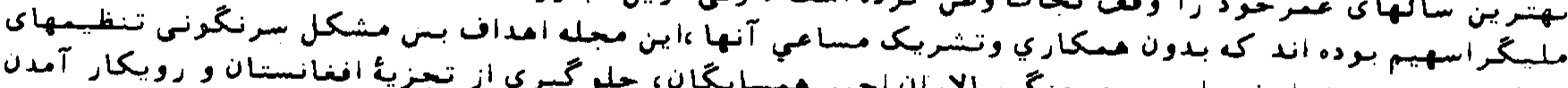

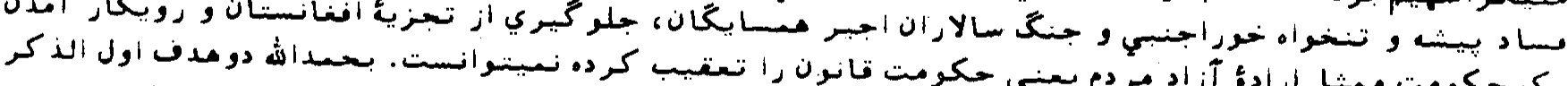

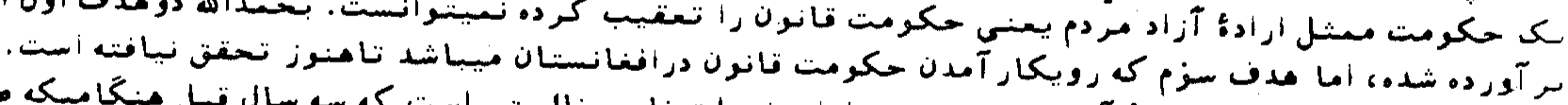

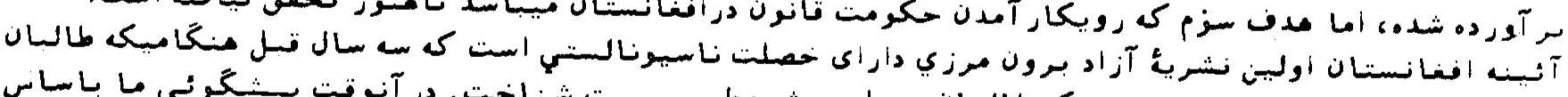

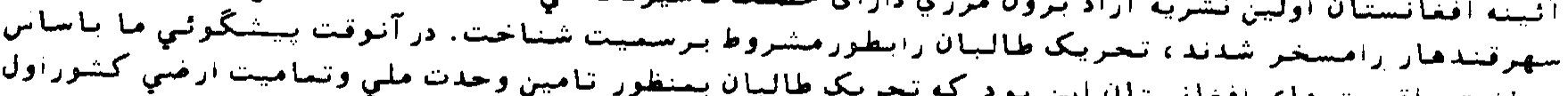

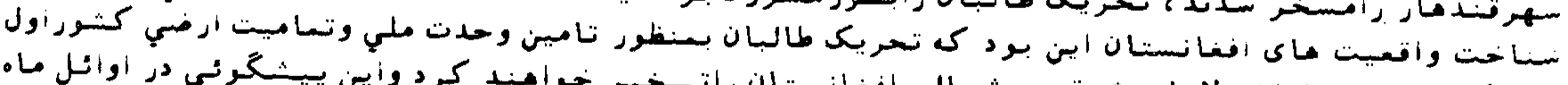

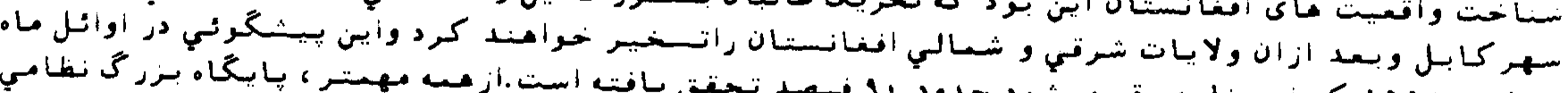

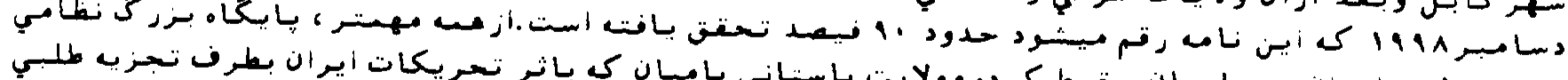

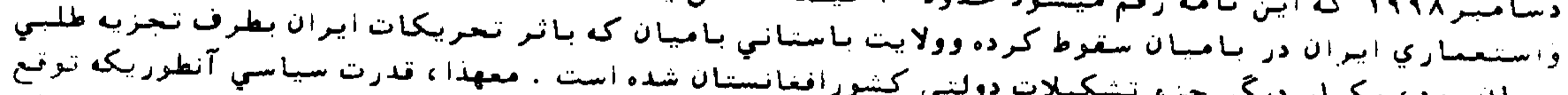

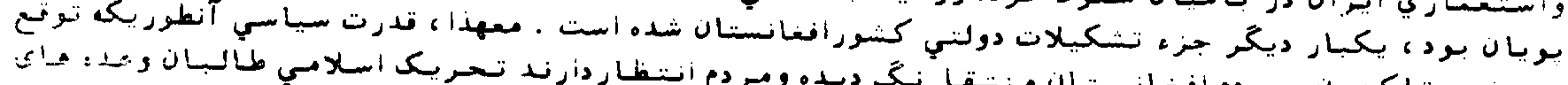

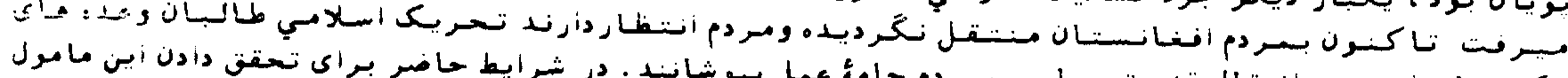

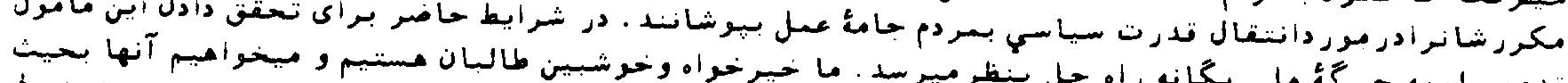

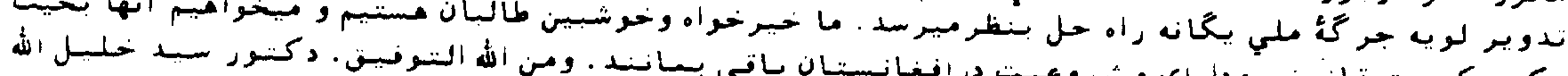
معاشعبان. 


\section{2}

Thus, government and corporate interests not only structure the way the media report reality, they structure much of reality itself. [15] Along with owners and advertisers, government leaders exercise a substantial influence over what become news. Aside from this coincidence of ideological perspectives, news people generally are attracted to power, finding it more gratifying and rewarding to stand with than against it. [16] Those who wield words often hope that they might exercise a determinant influence over those who wield power. Aware that news people are ready to be seduced, rulers are not above enticing publishers, editors, and journalists with invitations into the circles of power.

In addition to cozy off-the-record receptions with top officials, there are gala events at the highest levels of government featuring not only leaders of industry, diplomats, congressional leaders, and entertainment celebrities but also a selection of journalists, editors, and publishers. The government exercises control over news organizations through its control over the product to market: information (and misinformation). Daity assemblages of proposals, tips. press releases, documents, and interviews roll out of the White House and various federal agencies and departments. In a metter of hours, sometimes minutes, the major media are telling the public what the government wants them to hear. The State Department alone spends millions of dollars annually disseminating information that fits its view of the world. It employs a public relations staff of over three thousand people, many of whom can supply news saippets to cooperative journalists, or nothing at all to those proscribed as uncooperative. [17]

Every morning in the nation's capital, the White House senior staff meet to decide as one former participant put it, "What do we want the press to cover today and how?" Within minutes after the decisions are made, the "line of the day" is sent out via computer to all senior administration officials and to thousands of government public relations people and press secretaries, covering agencies that deal with both domestic and foreign affairs. [18] As a follow-up, officials call each network fifteen minutes prior to the evening news telecast to ascertain what will appear. As a former chief of staff once remarked about the self-serving stories he repeatedly planted in the press: "We fed it to them and they ate it." [19]

Government leaders have ways of retaliating against unfavorable treatment by the news media. Officials routinely deny interviews: withold access to information, give exclusive material to favored journalists, and misleading information to disfavored ones, and award prestigious positions to especially cooperative newspeople. When cooption of the media into this exclusive realm fails, however, government is not above utilizing the police powers of the state. for example: the FBI has and does harass news people who persist in writing troublesome stories, and on repeated occasions the goverument has subpoenaed documents, tapes, and other materials used by news organizations. [20] Obviously, such Orwellien measures impose a "chilling effect" on the press, and encourages self-censorship.

\section{Propaganda Disguised as News}

The Pentagon sends out hundreds of stories and slanted editorials cach week that are routinely picked up by newspapers and broadcast stations across the country and presented to the public as trustworthy products of independent journalism. [21] According to officials at the United States Information Service (USIS), the government has teams of propagandists in Washington who create stories that are wired daity to USIS's 206 offices in 127 countries. [22] Many of these news plants appear in the foreigo press then retum as "blowback" that is, they are picked up by US correspondents abroad and transmitted to an unsuspecting American public. [23]

What this "manufactured" news is not likely to do is inform us of the economic class interests that underlie the US global military machine. [24] It will not tell us, for example, about US involvement in the repressive political and military policies employed to subordinate Afghanistan to the dictates of Washington. In sum. those who see the news as being the outcome of objective reportage by professionally trained, independent journalists are missing much of the picture. What passes for "news" is a product of many forces, involving the dominant political culture and powerful economic and government institutions, all dedicated to maintaining an ideological monopoly: controlling the flow of information and opinion in ways that best advance their interests. That so many journalists fail to see the evidence of how thoroughty they themselves are part of the news-manufacturing process.

\section{Effective Propaganda}

The most effective propaganda is that which relies on framing rather than falsehood. By bending the truth rather than breaking it. using emphasis. nuance. innuendo. and peripheral embellishments as communicators can create a desired impression without resorting to explicit advocacy and without departing too far from the appearance of objectivity: One common method is to select labels and other vocabulary designed to convey politically loaded images. These labels and phrases. like masks in a Greek drama. convey negative cues regarding events and persona. often without benefit of. and usually as a substitute for. supportive information. The persistent. fallacious characterization of the Taliban as oppressive. religious zealots devoid of bumanity represents an excellent case in point. Thus. during a CBS Sixn Minutes re-broadcast. Christiane Amanpour referred retrospectively to the Taliban as "the most repressive government in the historn of Afghanistan." how different an impression would have been created had she been factual and labeled them as a grass-roots movement for national unity or as a social phenomenon that rose from the ashes of twenty years of war and in response to the horror of previous administrations.

When political struggles are never explained in their substance. we are left with the presumption that the conflict is caused by an innately hostile adversary. By slighting content and framing detrils as in the Sixn Minutes telecast. the media are able to neutralize the trutb while giving an appearance of baving thoroughty and objectrivel. treated the subject. Misunformation is sometimes so widespread that the line betweén intentional and unintentional 
The foregoing reflects a belief, based on many years of study of the workings of the media, and for the pupose of this inquiry, the attendant news coverage of Afghanistan, that they serve to mobilize support for the special interests that dominate the state and private activity, and that their choices, emphases, and omissions can often be understood best, and sometimes with striking clarity and insight, by analyzing them in such terms. Leaders of the media claim that their news choices rest on unbiased professional and objective criteria, and they have support for this contention in the intellectual community. [1] If, however, the powerful are able to fix the premises of discourse, to decide what the general populace is allowed to see, hear, and think about, and to manage public opinion by regular propaganda campaigns, the standard view of how the system works is at serious odds with reality. [2] Walter Lippmann writing in the 1920s: claimed that propaganda had already become "a regular organ of popular government:" and was steadily increasing in sophistication and importance. [3] There are those among us who contend that this is not all the massmedia do, but many believe the propaganda function to be a very important aspect of their overall service. Contrary to the usual image of the press as cantankerous, obstinate, and ubiquitous in its search for the truth, we will endeavor to depict how an undertying elite consensus largely structures all facets of the news.

For many people an issue does not exist until it appears in the news media. Indeed, what we even define as an issue or event, what we see and hear, and what we do not see and hear are greatly determined by those who control the communications world, be it separatist movement in Kosovo. alleged terrorist attacks against US interests, or UN assertions of Taliban transgressions, few of us know things except as they are defined in the news. Even when we don't believe what the media say, we are still hearing or reading their viewpoint rather than some other. They, therefore, are still setting the agenda, defining what it is we must believe or disbelieve, accept or reject. The media exert a persistent influence in defining the scope of respectable political discourse. [4] The press's misrepresentations are not usually accidental, nor merely the result of the complexity of actual events or the maladroitness of poorly informed journalists. While those kinds of problems exist, another kind of distortion predominates, one not due to chance or to the idiosyncratic qualities of news production or news people. [5] The major distortion are repeatable and systemic, the product not only of deliberate manipulations but of the ideological and economic intercourse under which the media operate. [6]

Be this as it may, growing numbers of people are becoming increasingly aware that the media are neither objective nor consistently accurate in their portrayal of issues and events. There has emerged a growing understanding that we need to defend ourselves by challenging the misinformation we are fed. [7] The question then becomes: how: much of what the news media tell us is true, and how does it control our view of the world, and how does the media influence and manipulate the public's perception of reality?

The mass-media serve as a system for communicating messages and symbols to the general populace. It is

4 their function to amuse, entertain, and inform, and to inculcate individuals with the values, beliefs, and codes of behavior that will integrate them into the institutional structures of the larger society. [8] In a world of concentrated wealth and major conflicts of class interest, to fulfill this role requires systemic propaganda. [9] A propaganda model focuses on this inequality of wealth and power and its multilevel effects on mass-media interests and choices. It traces the route by which money and power are able to filter out the news they deem fit to print marginalize dissent, and allow the government and dominant private interests to get their message across to the public: the essential ingredients of our propaganda model, or set of news "filters" fall under the following headings: (1) the size; concentrated ownership: owner-wealth; and profit orientation of the dominant mass-media firms; (2) advertising as the primary income source of the mass-media; (3) the reliance of the media on information provided by government, business, and "experts" funded and approved by these primary sources and agents of power; (4) coercive measures taken by the power brokers as a means of disciplining the media; and (5) "antiterrorism" replacing communism as a national religion and control mechanism. [10] These elements interact with and reinforce one another. The raw material of news must pass through successive filters. leaving only the cleansed residue fit to print. Thus, they mold the premise of discourse and interpretation. and the definition of what is newsworthy in the first place, and they explain the basis and operation of what amount to propaganda campaigns. [11]

The elite domination of the media and marginalization of dissidents that results from the operation of these filters occurs so naturally (such is the case with reportage covering events in Afghanistan) that news people. frequently operating with complete integrity and goodwill. are able to convince themselves that they choose and interpret the news "objectively" and on the basis of professional news value. [12] In assessing the newsworthiness of the U.S government's urgent claims that Afghanistan has become the center for international terrorism. the media do not stop to ponder the bias inherent in the priority assigned to government supplied raw material or the possibility that the government might be manpulating the news imposing its oun agenda. and deliberatel diverting attention from other materiai $[13 j$

$$
\text { The Myth of Objectivin. }
$$

As articulated by the journalism profession. "objectivity" means reporters should not render their oun opinions or biases into their reporting. But the wortby ideal has been debased in practice to mean that reporters must discard their critical thinking and questioning. They must not press too deeply into areas that might canse discomfort to those of power. wealth and conservative ideolog. In so tring to neutralize themselves. they often succeed in neutralizing their subject manter Corporate and government entities not onl own the media but control much of the world's

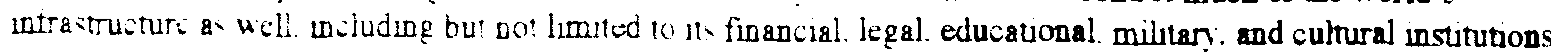




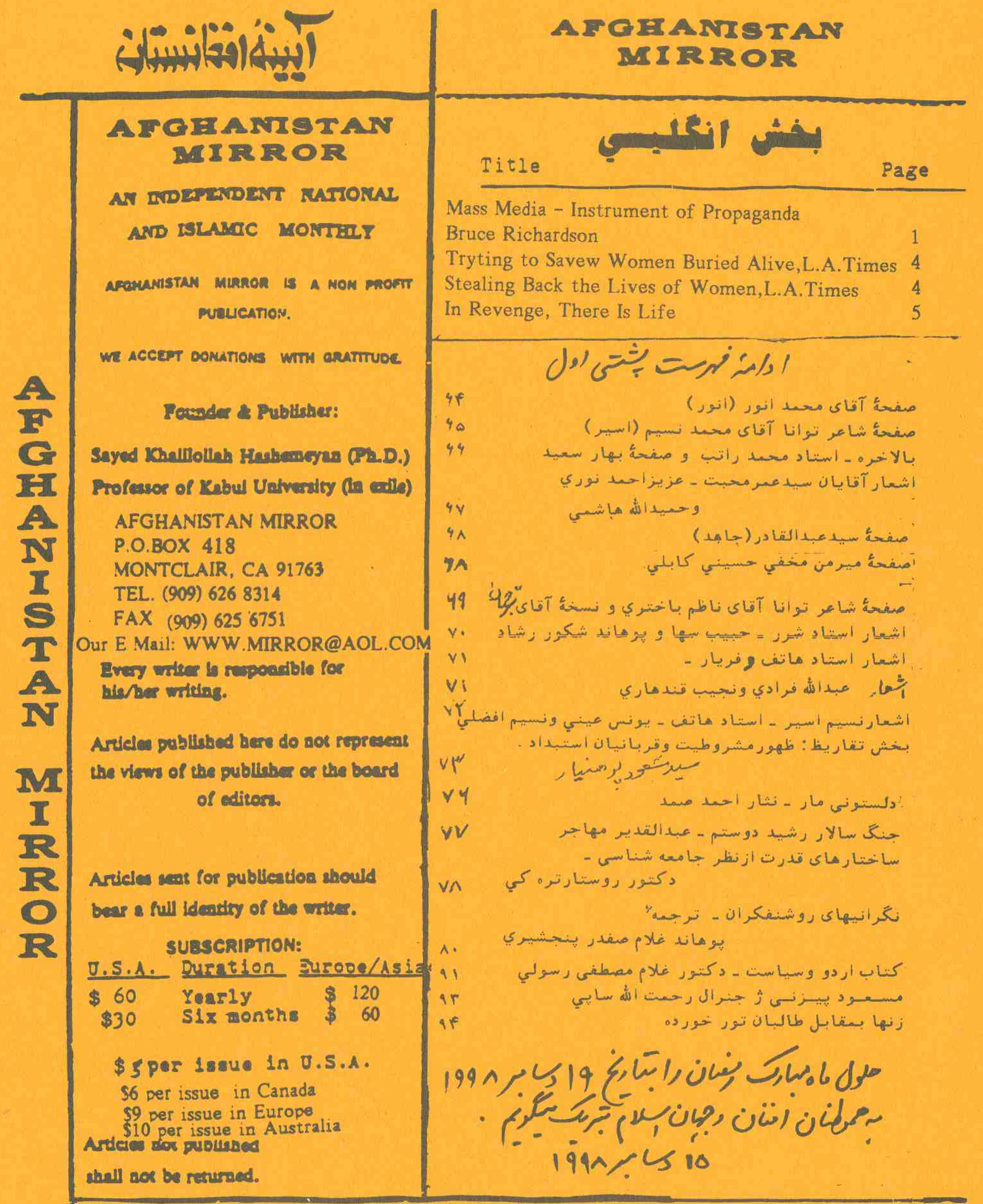


ACKU

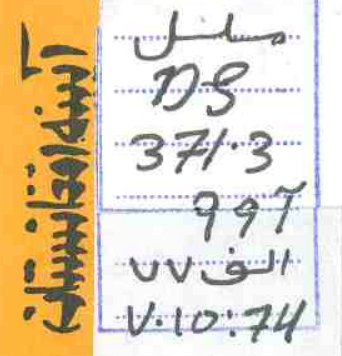

A.
F.
G.
HI 1

A

N?

$I \frac{1}{3}$

T.

A

ME

$\mathbf{I}^{\mathbf{5}}$

0

$\mathbf{R}$

\%

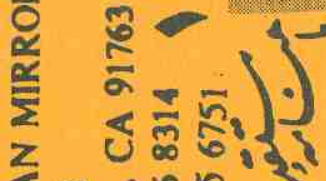

₹

들 ₹

爻额

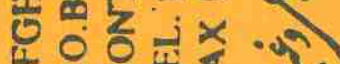

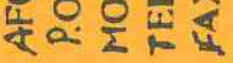

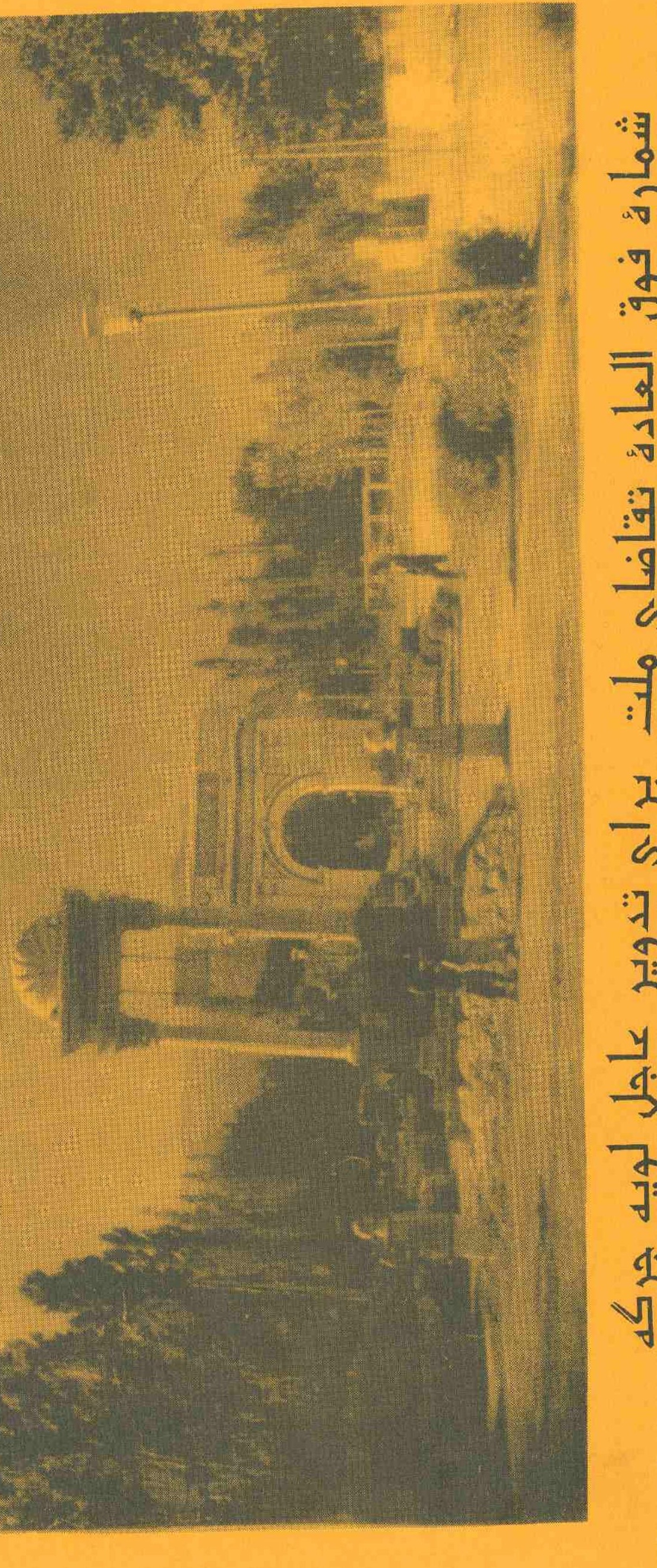

